\title{
Refinement of the List of Constituents for Groundwater Monitoring at M-Area
}

by

D. G. Wells

Westinghouse Savannah River Company

Savannah River Site

Aiken, South Carolina 29808

This paper was prepared in connection with work done under the above contract number with the U. S.

Department of Energy. By acceptance of this paper, the publisher and/or recipient acknowledges the U. S. Government's right to retain a nonexclusive, royalty-free license in and to any copyright covering this paper, along with the right to reproduce and to authorize others to reproduce all or part of the copyrighted paper. 


\section{DISCLAIMER}

This report was prepared as an account of work sponsored by an agency of the United States Government. Neither the United States Government nor any agency thereof, nor any of their employees, makes any warranty, express or implied, or assumes any legal liability or responsibility for the accuracy, completeness, or usefulness of any information, apparatus, product, or process disclosed, or represents that its use would not infringe privately owned rights. Reference herein to any specific commercial product, process, or service by trade name, trademark, manufacturer, or otherwise does not necessarily constitute or imply its endorsement, recommendation, or favoring by the United States Government or any agency thereof. The views and opinions of authors expressed herein do not necessarily state or reflect those of the United States Government or any agency thereof.

This report has been reproduced directly from the best available copy.

Available to DOE and DOE contractors from the Office of Scientific and Technical Information, P. O. Box 62, Oak Ridge, TN 37831; prices available from (423) 576-8401.

Available to the public from the National Technical Information Service, U. S. Department of Commerce, 5285 Port Royal Road, Springfield, VA 22161. 


\section{DISCLAIMER}

Portions of this document may be illegible electronic image products. Images are produced from the best available original document. 


\section{TABLE OF CONTENTS}

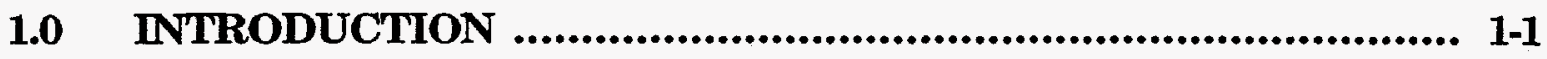

1.1 METHOD OF STUDY ...................................................... 1-1

1.2 DISCUSSION OF RESULTS.................................................... 1-3

1.3 RECOMMENDATIONS........................................................... 1-9

\section{LIST OF APPENDICES}

APPENDIX I PLOTS .............................................................. I-I

APPENDIX II DATA........................................................... II-I 


\section{INTRODUCTION}

For several years Westinghouse Savannah River Company (WSRC) has been examining ways of reducing monitoring costs. Most of these efforts have been aimed at reducing the number of wells sampled or reducing sample frequency. With regards to monitoring around the M-Area Settling Basin, we are now examining a possible reduction in the number of constituents analyzed. It is our opinion that many constituents can be dropped entirely. Several others should be dropped from analyses in the plume definition wells, while retained for analyses at the point of compliance (POC) wells.

Constituents that can be dropped entirely are nonhazardous inorganics generally referred to as water quality indicators. Monitoring for these parameters is sensible when a facility is in detection monitoring, but it is much less useful at a facility like the M-Area Basin. The water quality indicators are helpful in detecting whether or not a facility has impacted the environment. But their concentrations are not important in themselves. At M-Area, it is well documented that the facility has impacted groundwater quite seriously with a known group of hazardous constituents. So the concentrations of the nonhazardous constituents are of little interest.

Obviously, monitoring for the hazardous constituents should continue, but it should only continue at wells that are likely to yield useful data. At M-Area there are 41 Point of Compliance (POC) wells monitoring an area of about .25 square miles and about 236 plume definition wells monitoring the surrounding 4 square miles. The locations of well clusters and the point of compliance are shown in figure 1. The POC wells and plume definition wells are intended to serve entirely different purposes and should not, necessarily, be monitored for the same things.

The POC wells form a picket line around the facility and are intended to detect any constituents leaching from it. They are also intended to determine whether such constituents exceed action levels. Plume definition wells are added to define the plume created by a particular set or subset of contaminants. They can also be used to monitor the effectiveness of corrective action upon a particular set of constituents. But one cannot assume that plume definition wells installed to monitor a plume of contaminants A and B are located appropriately to monitor all other possible contaminants.

The M-Area plume definition wells were installed in several phases over a ten year time span as SRS struggled to define the extent of a large plume of TCE and PCE. These wells were not located for the purpose of monitoring the numerous inorganics and radionuclides on the unit's monitoring list. Many of the inorganics and radionuclides are relatively immobile in groundwater and cannot be expected to appear in the widely scattered TCE/PCE plume definition wells.

\section{METHOD OF STUDY}

In order to determine how useful the various constituents are and where they are useful, a study has been made of their spatial distributions. The concentrations of 22 nonhazardous, hazardous and radiological constituents were plotted on maps of A-M Area. Plots were made for both the M-Area Aquifer and the Upper Lost Lake Aquifer using well data from third quarter 1996. In most cases plots were also made for first quarter 1995.

Since it was expected that contouring of the data would be meaningless in many cases, 
WSRC-TR-00347

November, 1997

Section 1-2

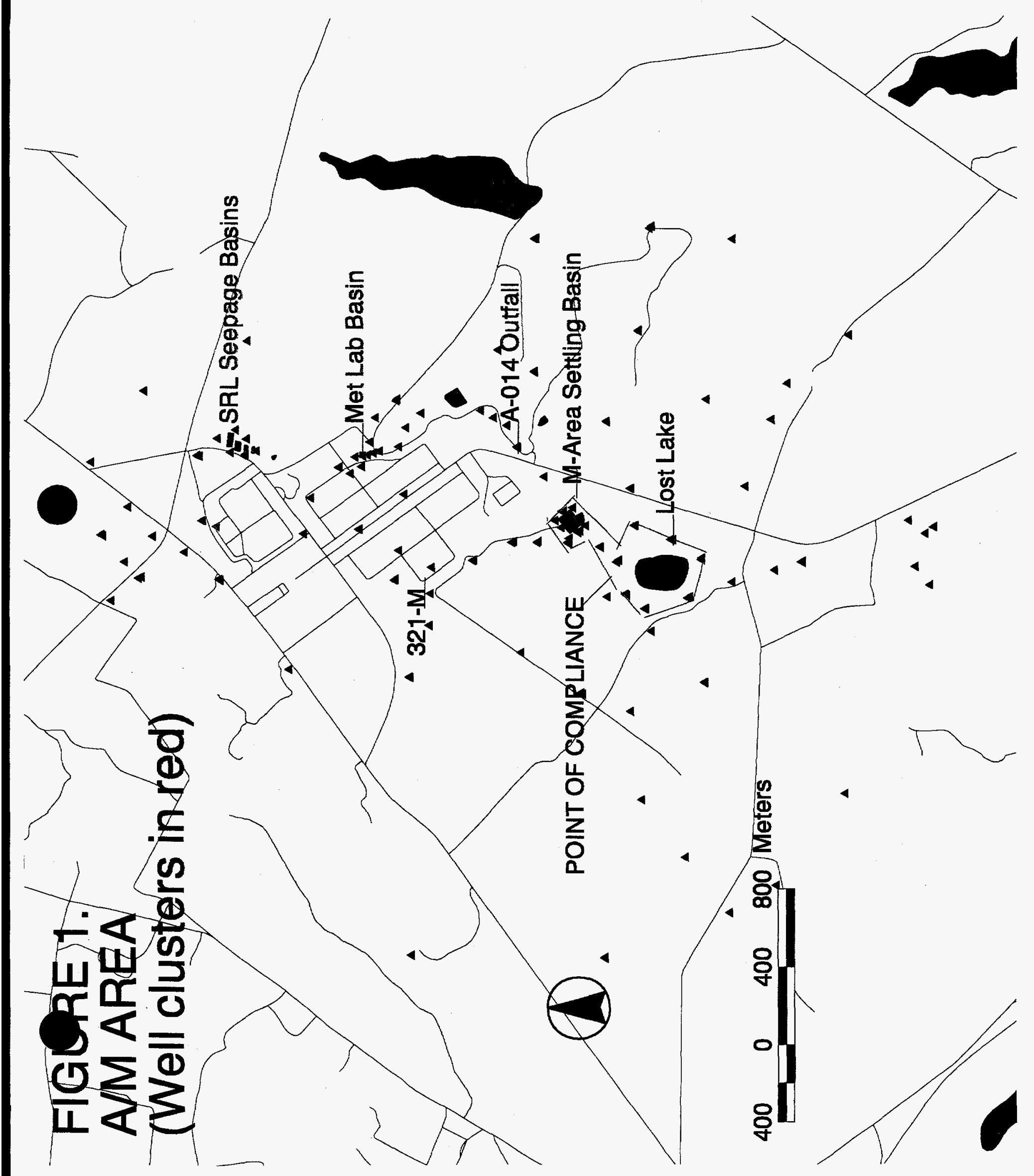


contouring routines were not used. Instead the values were grouped, color coded, and plotted as colored dots. The spectrum was used for the color graduation with low values at the red and yellow end and high values at the blue end. The plot of TCE (figure 2) shows what an organized plume of contamination should look like. Moving away from the basin, the dots go from blue to green to yellow to red. If any of the other 22 constituents have been released in significant amounts, their plots should show similar patterns.

The legends for most 1995 plots contain detection limit (DL) information while the 1996 plots refer to estimated quantitation limits (EQL's). Values below these limits were condidered below detection. It should be noted that the limits change from year to year. The legends also refer to background values. In most cases the background values used are the background limits from table E-5 of the approved application, but in many cases the background value had to be set equal to the DL or EQL.

The plots are compiled in Appendix I. They are arranged in the same order in which they are discussed below.

\section{DISCUSSION OF RESULTS}

\section{Hazardous Constituents}

The list of groundwater monitoring constituents contained in Appendix IIIB-A of the M Area HWMF Part B permit is quite lengthy and somewhat confusing (table 1). The list is divided into two sections and five subsections, and the origin and purpose of some of the sections is difficult to understand. The most easily understood section of the list is titled "261 Appendix VIII/264 Appendix IX Hazardous Constituents". It is divided into inorganic and organic sections. These constituents are those that were at some point found to be statistically elevated above background at the point of compliance. The inorganic section is composed of barium, cyanide, nickel, lead and selenium.

\section{Barium}

Plots of barium concentrations show that barium occurs naturally throughout the area. While it tends to occur above the calculated background near the basin, it should be noted that above background concentrations are scattered across the area. Still, the frequency of elevated results around the basin indicates that barium may have been released and should be monitored at the point of compliance. But since even the elevated levels are far below the maximum contaminant level (MCL) there is no need for more widespread monitoring.

\section{Cyanide}

Plots of cyanide concentrations show no detections in either quarter in either aquifer. Detections of cyanide at A/M-Area have been rare over the years. But statistical analysis done in 1988 showed it to be elevated above background in well MSB-3A. MSB-3A went dry shortly thereafter and cyanide has not been detected in the replacement well. So it is questionable whether or not cyanide should be on the GWPS list. But even assuming that cyanide should be on the list, there is no evidence that it has spread beyond the point of compliance. Therefore, cyanide monitoring should be discontinued at the plume definition wells.

\section{Nickel}

Only two wells appear to yield consistent detections. There are a handful of detections scattered 


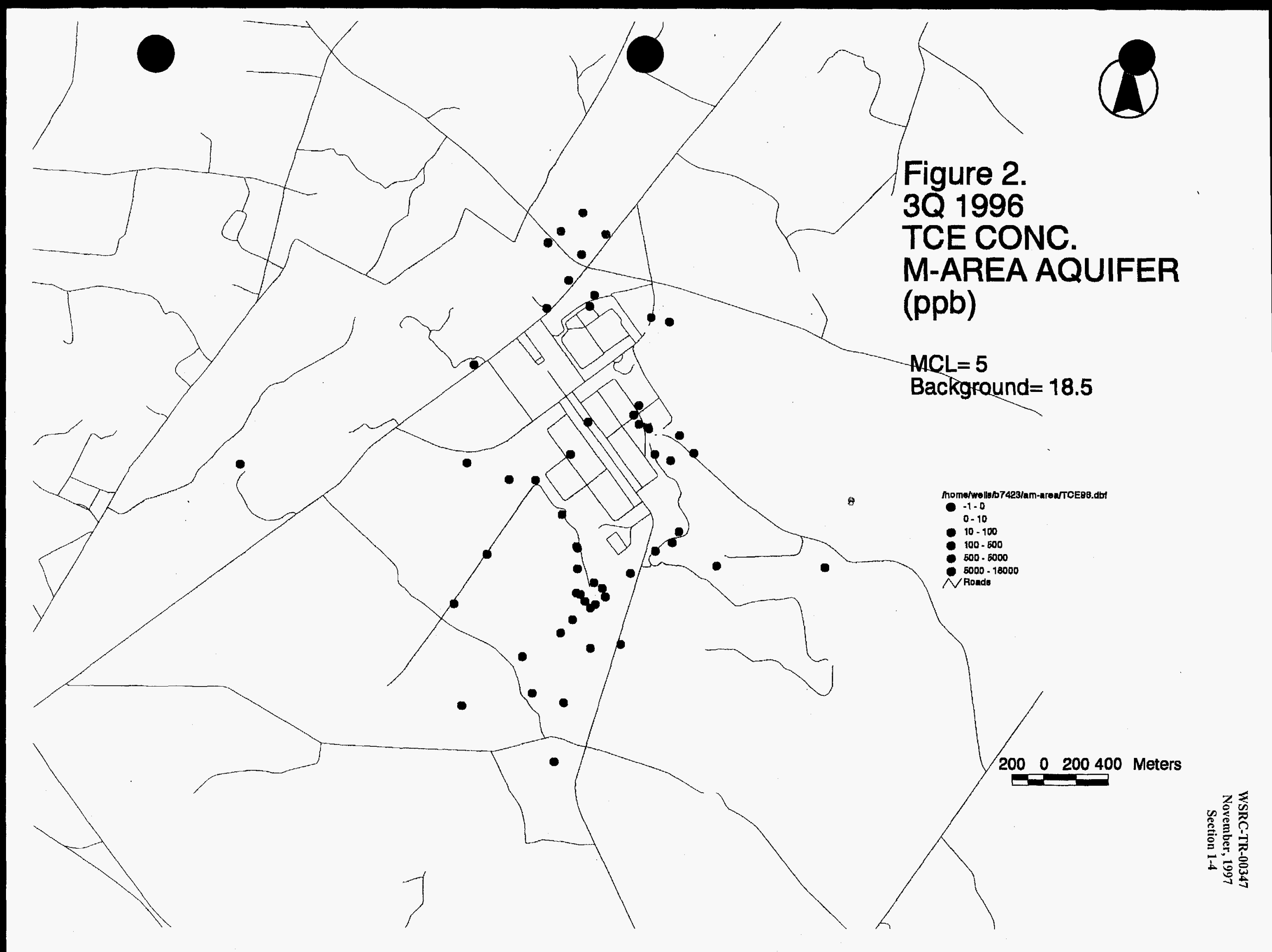




\section{Table 1.}

\section{APPENDIX IIB-A}

\section{SAVANNAH RIVER SITE M-AREA HWMF GROUNDWIATER PROTECTION STANDARD/MONITORING CONSTITUENTS}

\section{Groundwater Protection Standard}

\section{Appendix VIII / 264 Appendix IX Hazardous Constituents}

\section{Constituent}

Inorganics

Barium

Cyanide

Nickel

Lead

Selenium

\section{Concentration Limit}

2.0

$\mathrm{PQL}^{2}$

$0.1 \mathrm{mg} / 1^{1}$

$0.015 \quad \mathrm{mg} / \mathrm{1}^{3}$

$0.05 \mathrm{mg} / \mathrm{l}^{1}$

Organics

Chlorobenzene

1,1-Dichloroethane

1,1,1-Trichloroethane

1,1,2,2-Tetrachloroethane

1,1-Dichloroethene

Trans-1,2-Dichloroethene

Trichloroethene

Tetrachloroethene

Polychlorinated Biphenols
PQL

PQL

0.2

PQL

$0.007 \mathrm{mg} / \mathrm{l}^{1}$

$0.1 \mathrm{mg} / \mathrm{l}^{1}$

$0.005 \mathrm{mg} / 1^{1}$

$0.005 \quad \mathrm{mg} / \mathrm{l}^{1}$

$0.0005 \mathrm{mg} / 1^{1}$

1 MCL - Maximum Contaminant Level as established in the U.S. EPA Drinking Water Regulations and Health Advisories (updated 5/94)

2 PQL - Practical Quantitation Limit (PQL) as defined in R.61-79.264 Appendix IX

3 Action Level as established in the U.S. EPA Drinking Water Regulations and Health Advisories (updated 5/94)

II. Monitoring Constituents

Inorganics

$\underline{\text { Radionuclides }}$

Field Parameters
Aluminum, Chloride, Chromium, Cobalt, Copper, Fluoride, Manganese, Mercury, Nitrate/Nitrite-as nitrogen, Total Phosphate, Sodium, Sulfate, Uranium, Zinc

Gross-Alpha, Gross-Beta, Total Radium

$\mathrm{pH}$, Specific Conductance, Temperature 
among the plume definition wells, but there is clearly nothing that could be described as a plume. It should also be noted that the highest detection plotted is less than one half of the (MCL). So, while nickel is being detected close to the basin, there is no evidence that it is spreading beyond the immediate area, and it is present only in extremely low concentrations. Monitoring for nickel at the plume definition wells is serving no purpose and should be discontinued.

\section{Lead}

Lead concentration plots show that one POC well is consistantly above background and that concentrations in a few other wells may range from above the MCL to below detection depending on the quarter. Concentrations in the vast majority of wells are below detection. The plots do not present a very convincing argument that there is lead leaching from the basin. Monitoring for lead should continue only at the point of compliance.

\section{Selenium}

As in the case of cyanide, detections of selenium are rarely reported. Selenium was placed on the list because it was above background in well MSB-4A. Selenium was detected four times in that well between the first quarter of 1987 and the third quarter of 1988 . These detections were very close to the detection limit. The well went dry shortly thereafter and selenium has never been detected in the replacement well, MSB-4D. A detection was reported for third quarter of 1996 , but the result was below the EQL. If selenium cannot be detected in a well only a few feet from the selenium "hot spot", then monitoring for it at the plume definition wells serves no purpose and should be discontinued.

\section{Monitoring Constituents}

The other major section of the monitoring list is titled "Monitoring Constituents". Apparently this section was intended to list constituents that are not on the RCRA Appendix VIII list of hazardous substances (although some do appear on Appendix IX). The largest subset of the "Monitoring Constituents" is titled "Inorganics".

The majority of the "Inorganics" appear to have come from a list that first appeared as Table I in the July 9, 1987 Federal Register that established the Appendix IX monitoring list. Table I was a list of nonhazardous constituents that could be used along with Appendix IX on a case by case basis. They were considered to be useful indicators of water quality that might be used to detect an impact from a unit even before any hazardous constituents were found. It is unclear why they were added to the original M-Area list, but it is reasonable to say that they are no longer needed as early warning constituents since we already know that significant contamination has been released from the basin. The Table I constituents are aluminum, chloride, fluoride, manganese, nitrate, sodium, and sulfate.

\section{Aluminum}

In the case of aluminum, plotted data indicate that detection in low levels is more common adjacent to the M-Basin than elsewhere. However, detections are sprinkled throughout the area and often are found immediately adjacent to nondetects. Higher concentrations appear to be distributed more or less randomly. So the most that can be said is that the basin is somehow causing low levels of nonhazardous aluminum to be found in the wells nearest the fenceline. This information may be interesting, but, from an environmental protection standpoint, it is of no real value. 


\section{Chloride}

Most chloride concentrations above background are far from the basin. But the $1996 \mathrm{M}$-Area aquifer plot does seem to show a tendency for wells along the sewer line and around the basin to yield higher results than those farther out. Since all of these values are below background it is difficult to draw any conclusions. Chloride could be showing up as a breakdown product of the solvents, but that is uncertain. Monitoring of solvent breakdown can be done more efficiently by looking at the organic breakdown products found in the GWPS list. There does not appear to be any value in monitoring chloride concentrations.

\section{Fluoride}

Plotted fluoride data show that fluoride is rarely detected and the detections appear to be randomly distributed. The fluoride data are clearly of no value.

\section{Manganese}

Manganese is detectable in most wells. The wells adjacent to the basin appear to have higher concentrations, but there is no mappable plume. The manganese data are of no value.

\section{Nitrate}

The plots indicate that nitrate-nitrite concentrations are significantly elevated around the basin. M-Area Aquifer wells show levels slightly above background, but Upper Lost Lake concentrations are much higher. It appears that a plume of nitrate-nitrite has penetrated through the upper aquifer and now resides in the Upper Lost Lake. It also appears that little, if any, new nitrate-nitrite is being added to the upper aquifer. While the nitrate-nitrite results are interesting, it is not clear that they are of any real value. Nitrate monitoring may be warranted at the POC wells, but it should be discontinued at the plume definition wells.

\section{Phosphate}

The third quarter 1996 Upper Lost Lake plot shows a cluster of phosphate detections around Lost Lake. The other plots show nothing of interest. While the third quarter 1996 data gives some indication of a very slight phosphate impact, there is no real value in continuing to monitor phosphate.

\section{Sodium}

Sodium appears to be found in higher concentrations adjacent to the basin and around the edge of Lost Lake. It is unclear what this means. It probably shows that sodium is a good indicator of contamination, but since it is already known that contamination exists in the area, the sodium data are not useful. While interesting, the sodium monitoring is not of any real benefit and should be discontinued.

\section{Sulfate}

Sulfate concentrations near the M-Area Basin show no pattern. .However, they do appear to be slightly higher than background around the SRL Seepage Basins (which are being addressed through the CERCLA process) and around the Met Lab Basin (which is addressed by a separate Part B Permit module). It is not clear what, if anything, should be done to respond to high sulfate (there is no MCL), but it might be prudent to continue sulfate monitoring in the Met Lab 
wells while discontinuing it elsewhere. The Met Lab permit module calls for sulfate monitoring, so dropping it from the M-Area module would have no impact there. Monitoring at the SRL Seepage Basins will be worked out through the Federal Facility Agreement (FFA). Of course, SRS and SCDHEC representatives working on the FFA should be made aware of any impending changes to M-Area monitoring.

\section{Copper and Zinc}

The inorganics list also contains copper and zinc. Apparently, these metals were put on this part of the list because they are not Appendix VIII hazardous substances, even though they do appear in Appendix IX.

Copper was included on the list because it was statistically determined to be elevated in POC wells in 1989. In 1996, copper concentrations around the basin appeared to be similar to those in the background wells while higher values appear far from the basin. The area with the highest copper concentrations is far to the west of the basin where copper is elevated in the Upper Lost Lake. It would be difficult to make a case linking these copper concentrations to activities at the M-Basin.

Zinc was also determined to be elevated in 1988. There are zinc concentrations above background around the basin, but such concentrations occur throughout the area. Some of the highest concentrations are found far from the basin. So it is difficult to tell whether or not the basin has contributed zinc to the groundwater.

Since previous statistical analyses indicate that these metals are more prevalent in the POC wells than in background wells, monitoring at the POC wells may be warranted. If they ever exceed an action level at the point of compliance, then perhaps further sampling may be needed. But for now, the data yielded by sampling the entire well network is not useful.

\section{Mercury and Chromium}

Mercury and Chromium were originally on the GWPS but were dropped when the statistics were redone. The metals have been retained on the monitoring list because they were found in basin sludge. The plots demonstrate that detections of these metals are rare and don't form a pattern that would indicate contamination from the basin. It makes sense to monitor for these constituents at the point of compliance in case they do begin to leach from the sludge. But until they are found consistently in the wells surrounding the basin, there is no point in looking for them elsewhere.

\section{Uranium}

Uranium is present in the basin, but has almost never been detected in the groundwater. This is not surprising considering the immobility of uranium. There is no reason to monitor for uranium beyond the point of compliance.

\section{Cobalt}

Cobalt is on the list because it was detected in wells MSB-5A and MSB-22 in 1988 . Well 22 then yielded three straight nondetects before going dry in 1990. MSB-5A yielded one more cobalt detection in the third quarter of 1989. It is still sampled regularly, but has produced no more cobalt detections. Even if the cobalt is really present, there is no reason to believe it has migrated any distance from the basin. Monitoring beyond the point of compliance is not needed. 


\section{Radionuclides}

Another subset of analytes on the list is titled "Radionuclides". These constituents, gross alpha, gross beta and total radium were placed on the list by SRS as a "matter of comity". Total radium data is always difficult to interpret because background levels in the soil are often quite high. In M-Area it is difficult to discern a radium impact from the basin.

Plots of gross alpha and gross beta concentrations do indicate a basin impact. In the Upper Lost Lake Aquifer both parameters are elevated in wells around the basin and Lost Lake. While total radium monitoring can probably be dropped altogether, gross alpha and gross beta monitoring should continue at the point of compliance wells and a few plume definition wells.

\section{RECOMMENDATIONS}

To summarize, it is SRS's opinion that the groundwater monitoring program should be modified in the following ways. Semi-annual sampling for the inorganics in Section I of the Groundwater Protection Standards list (barium, cyanide, nickel, lead, and selenium) should only be performed at the POC and background wells. The "Inorganics" list in Section II should be shortened to include only nitrate, copper, zinc, chromium, mercury, uranium and cobalt. Annual monitoring for these constituents (with the exception of nitrate) should be continued only at the POC and background wells. Nitrate should be monitored at the POC wells, the background wells, and at well clusters MSB-17, MSB-18, and MSB-39 (figure 3).

Monitoring for radium should be discontinued. Monitoring for gross alpha and gross beta should continue only at the POC wells, the background wells and well clusters MSB-17, MSB-18 and MSB-39. 
WSRC-TR-00347

November, 1997

Section 1-10
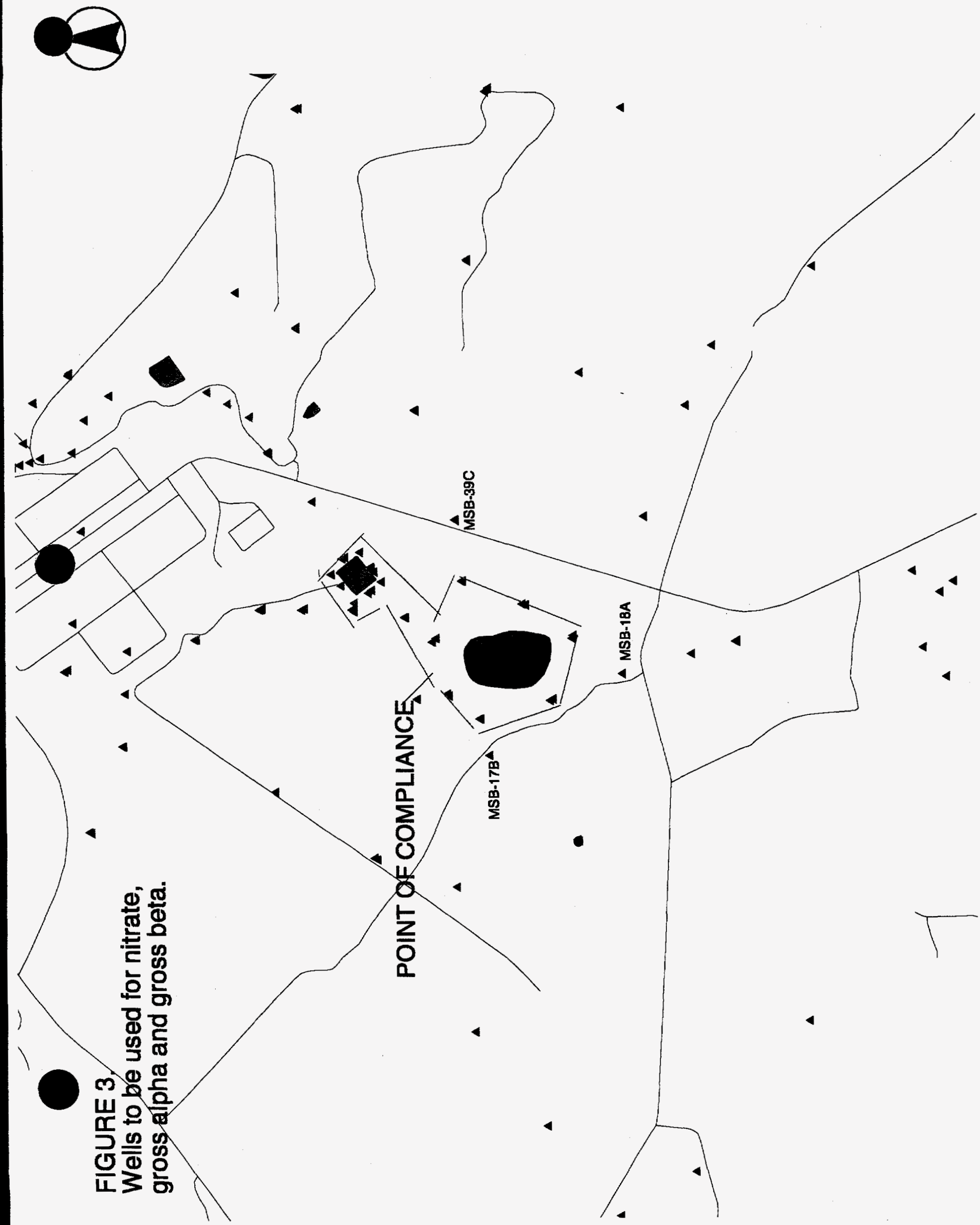
APPENDIX I

PLOTS 


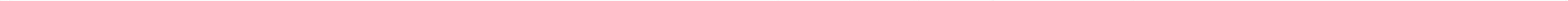




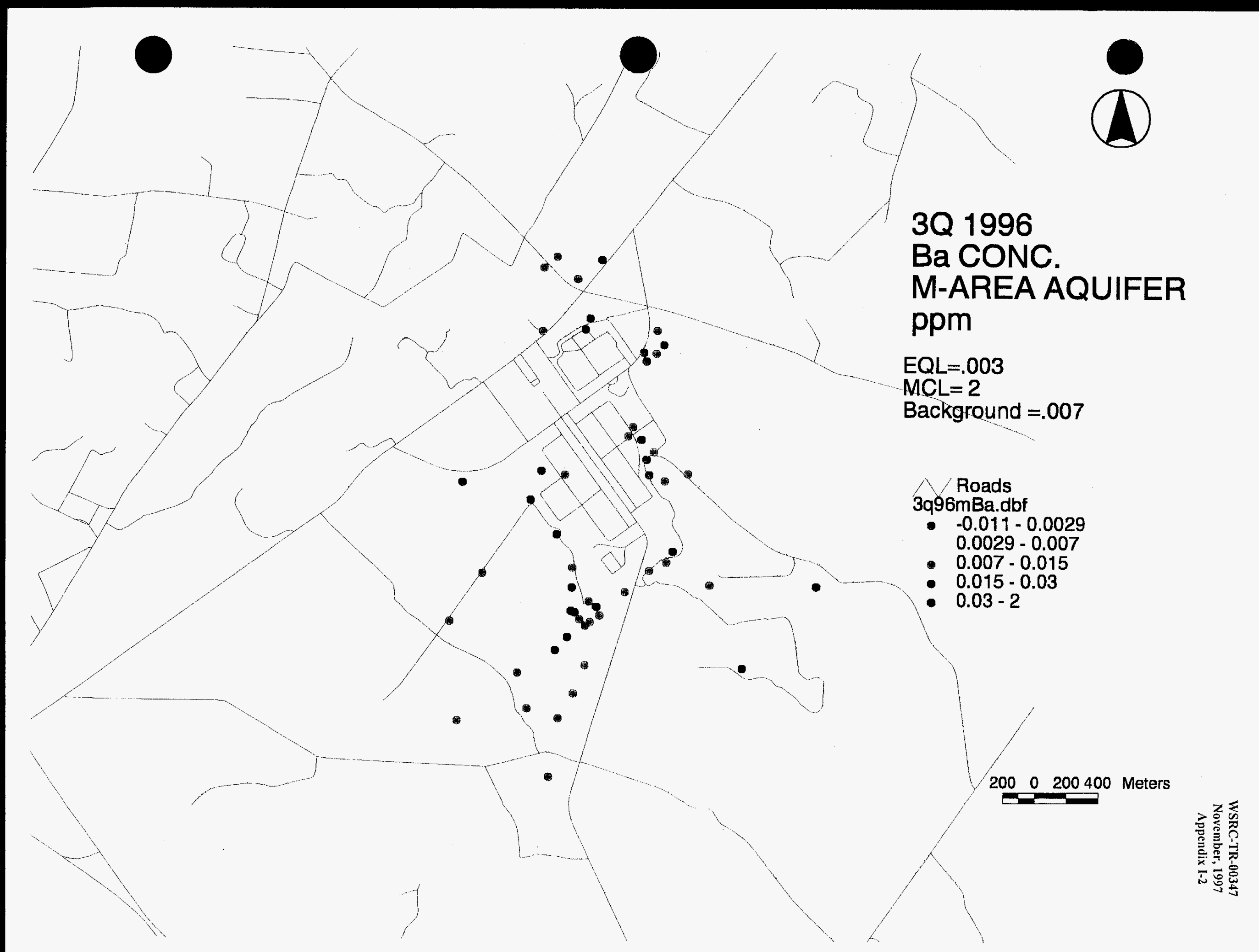




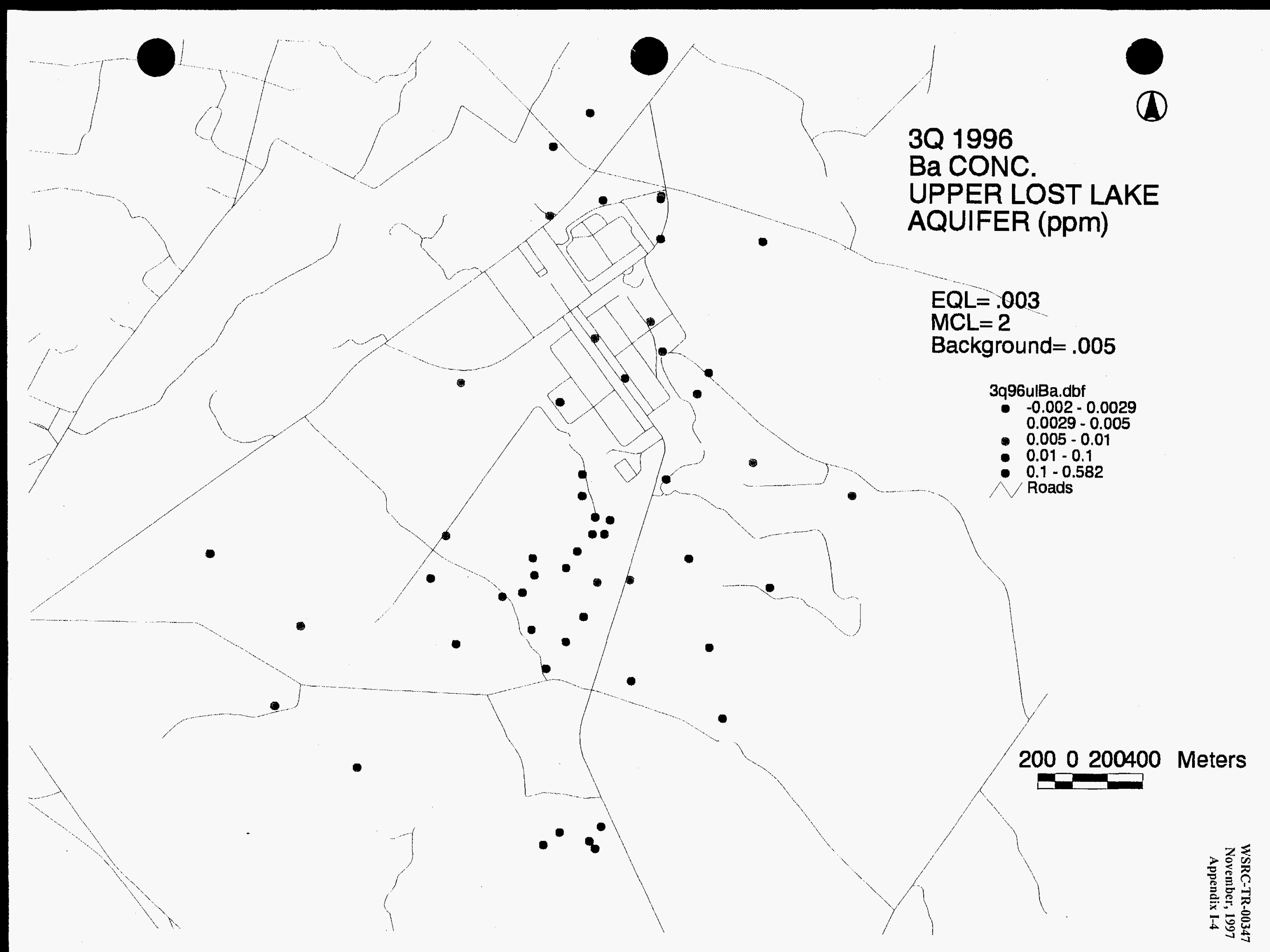




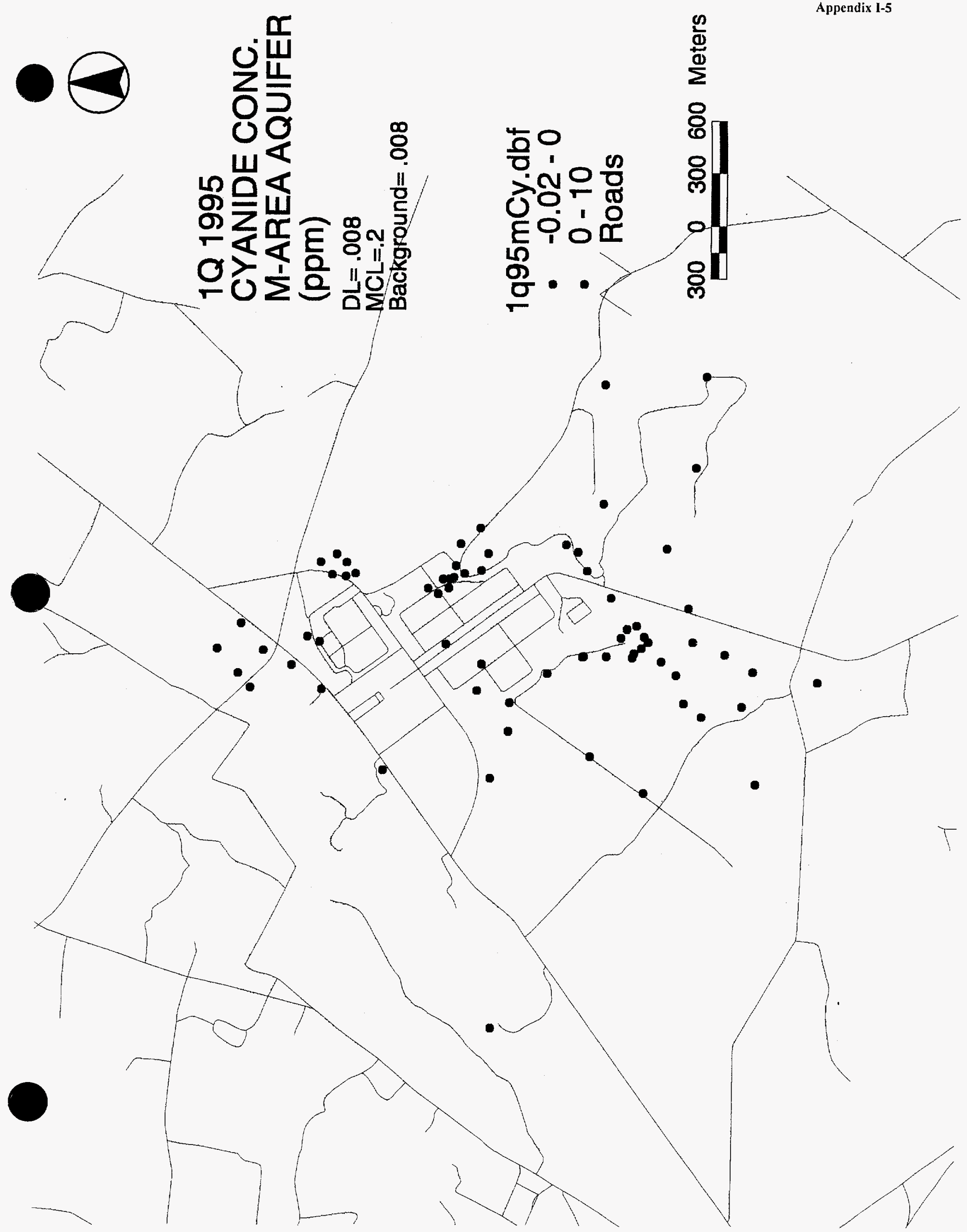




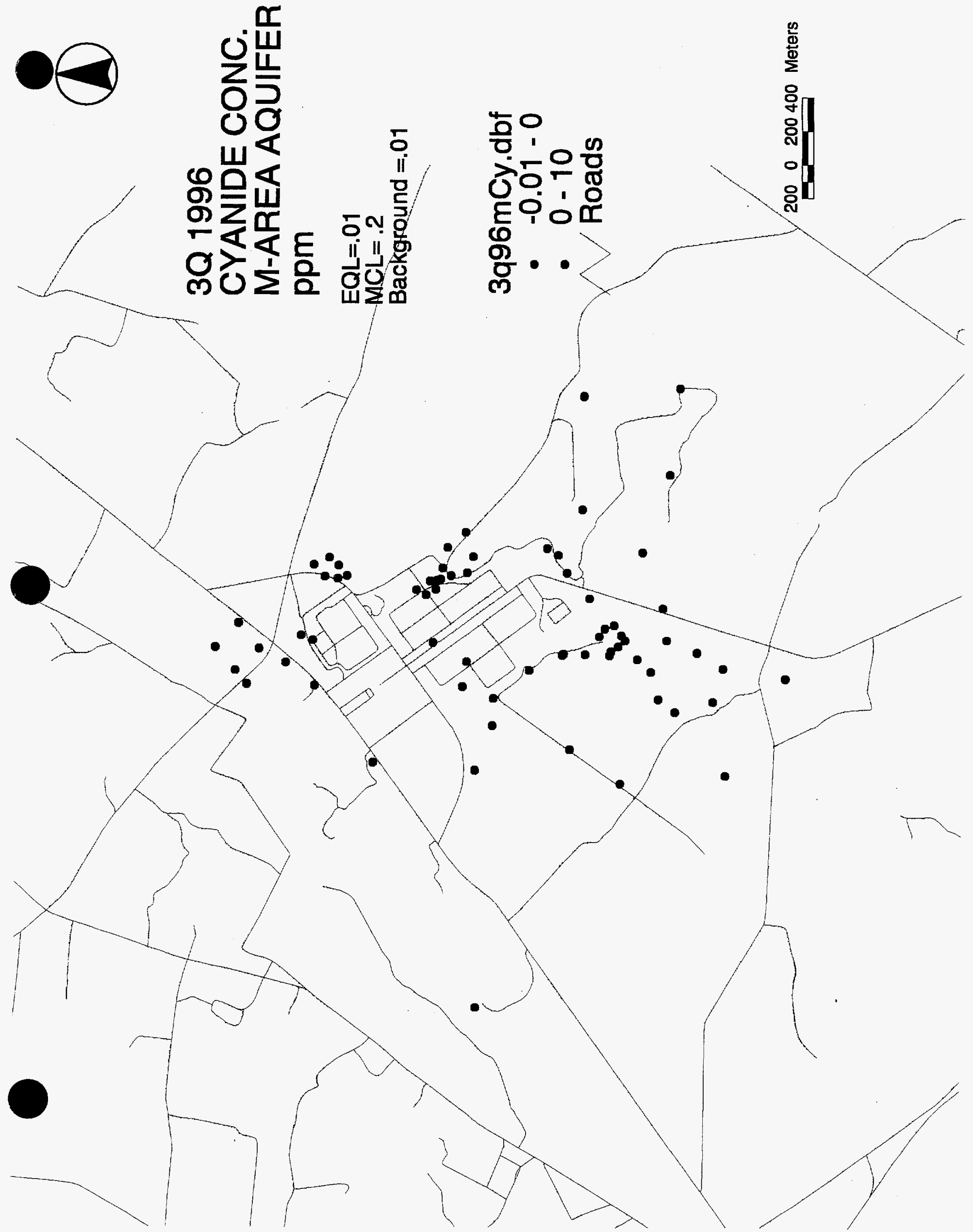




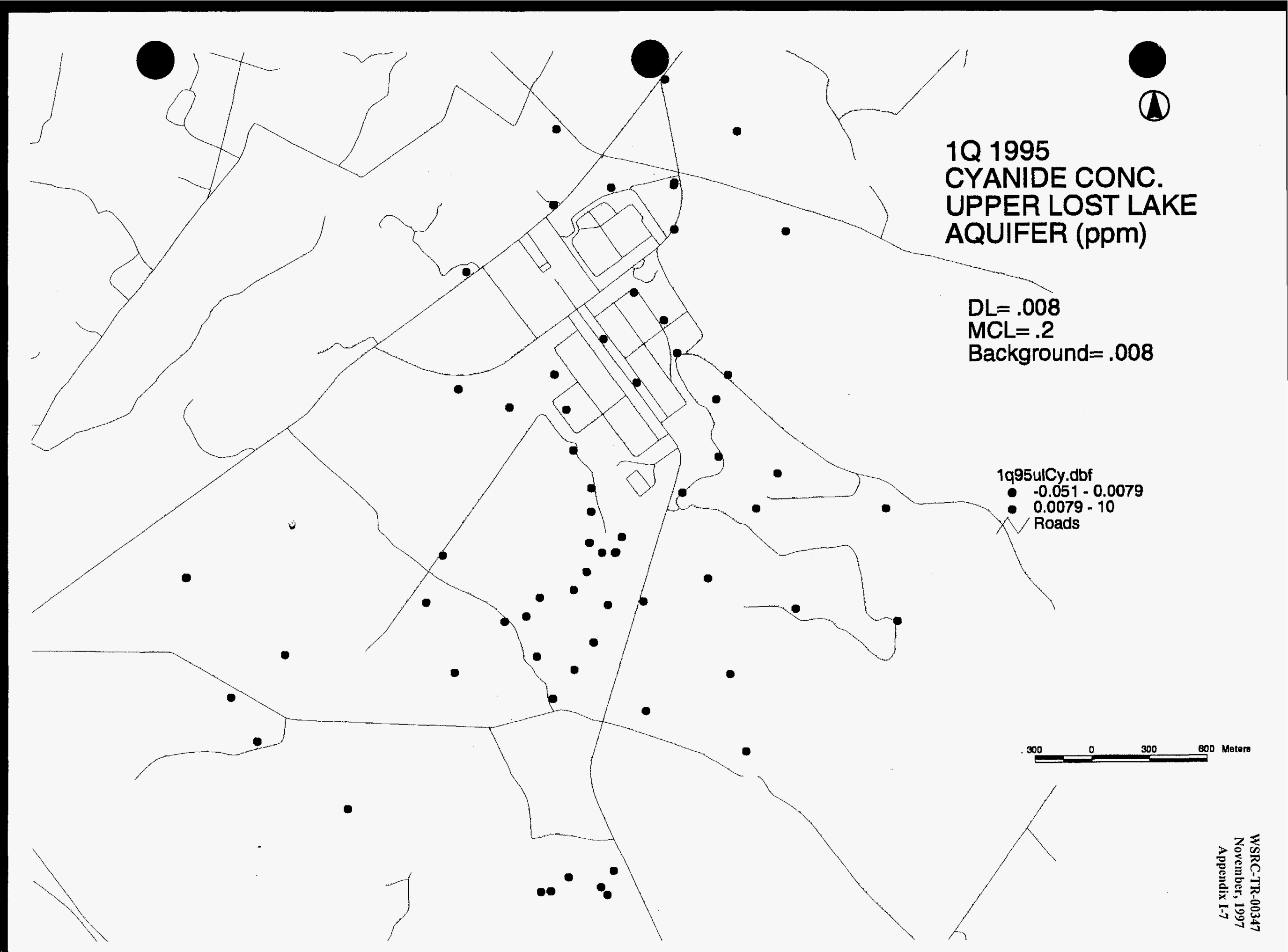




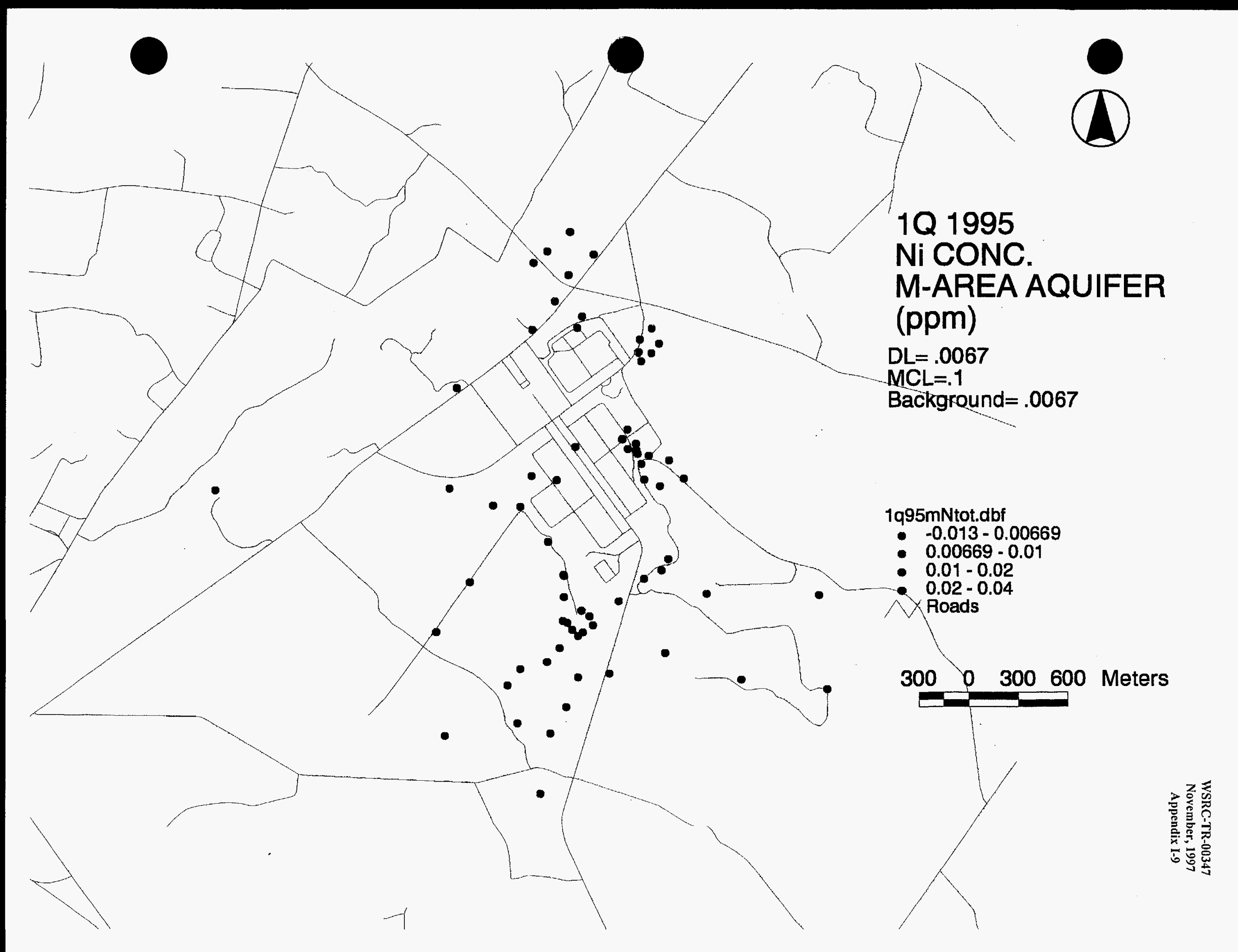




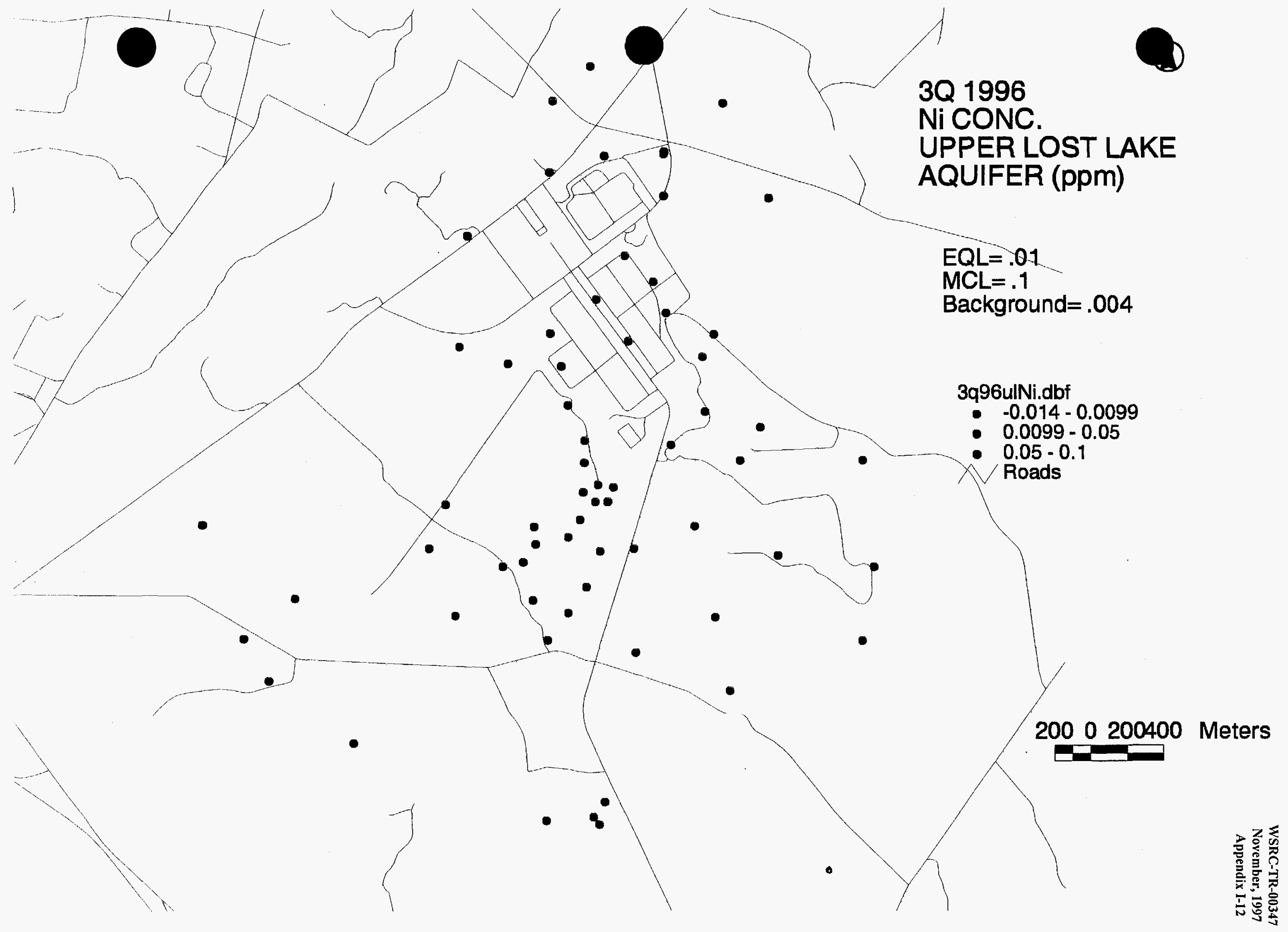




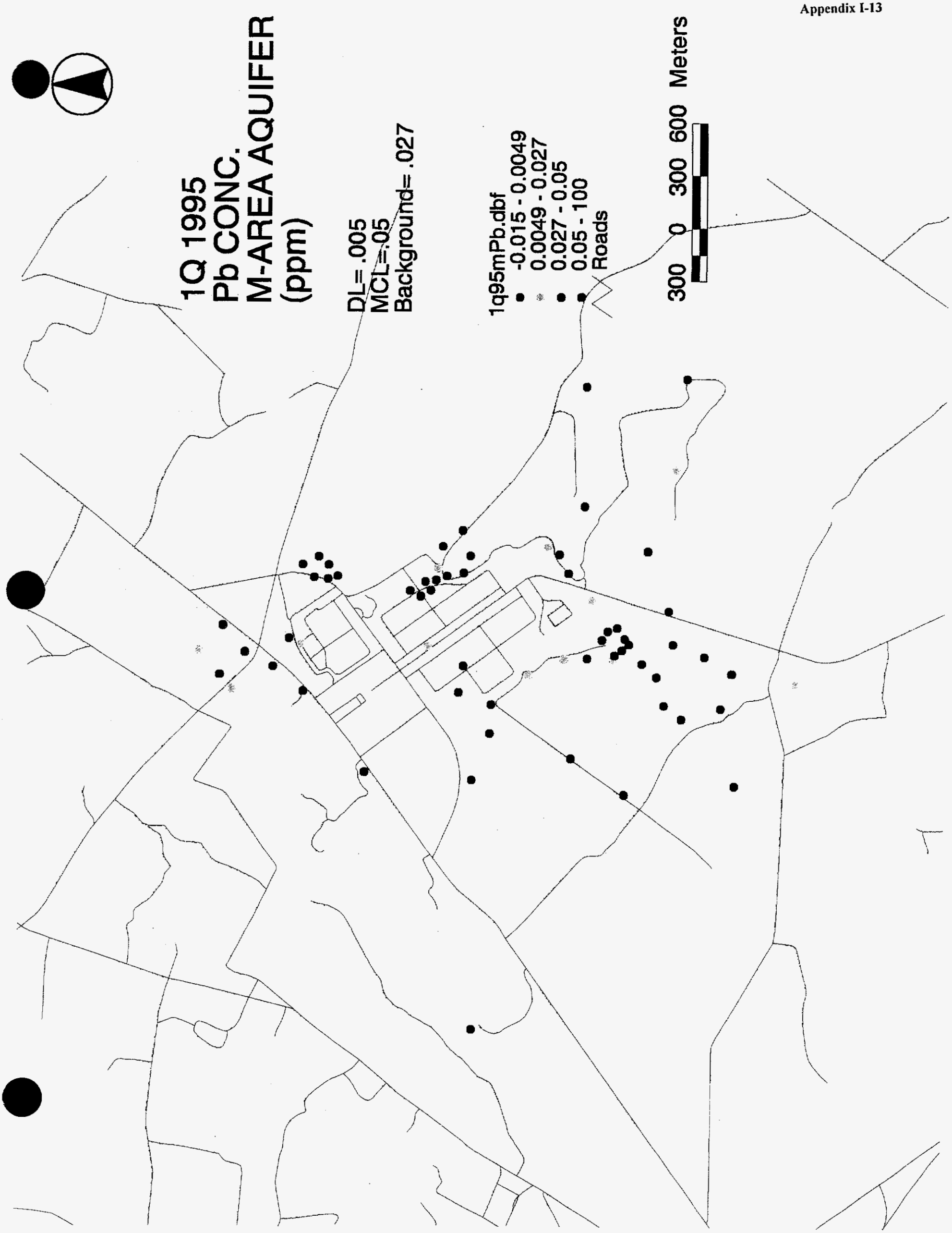




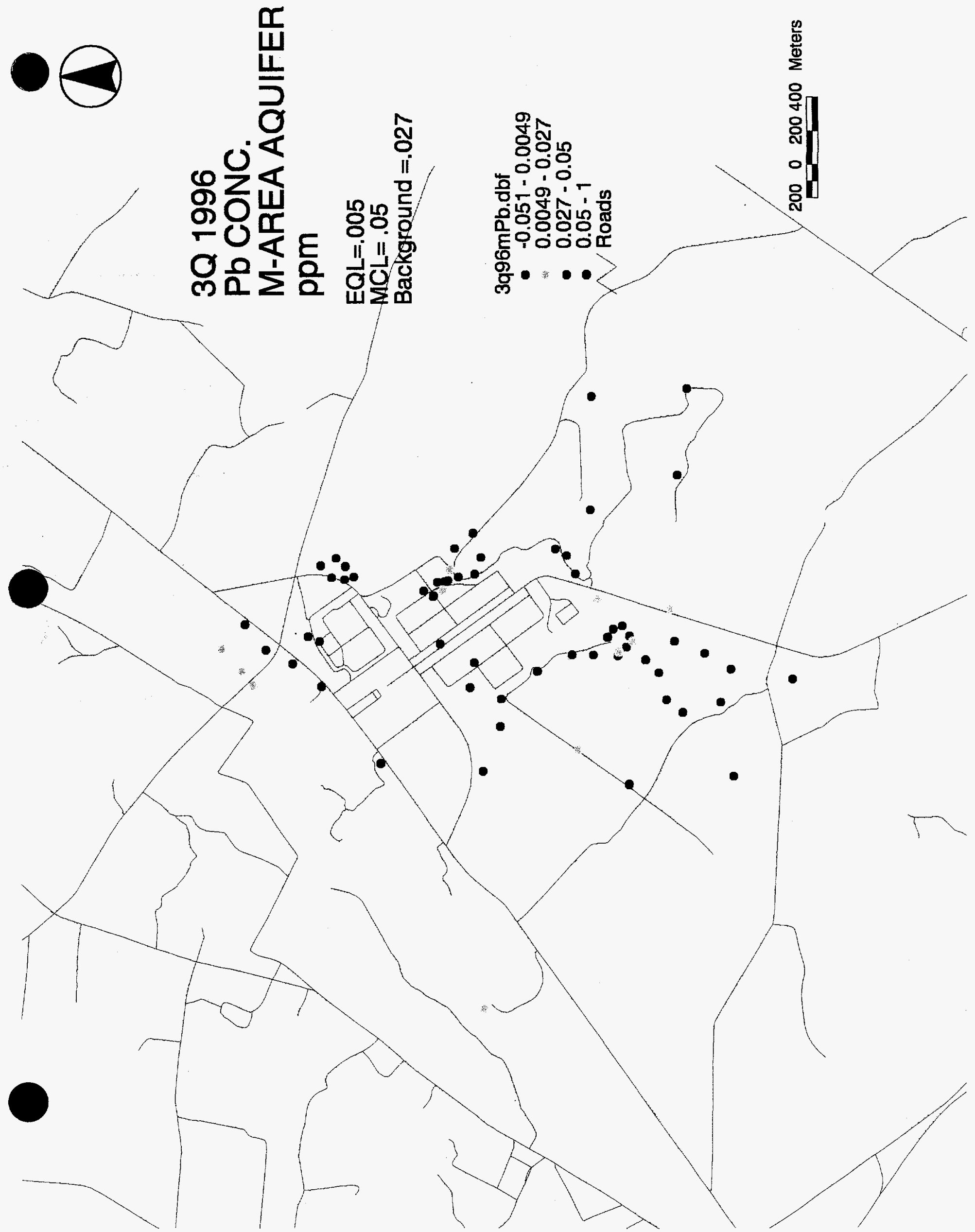




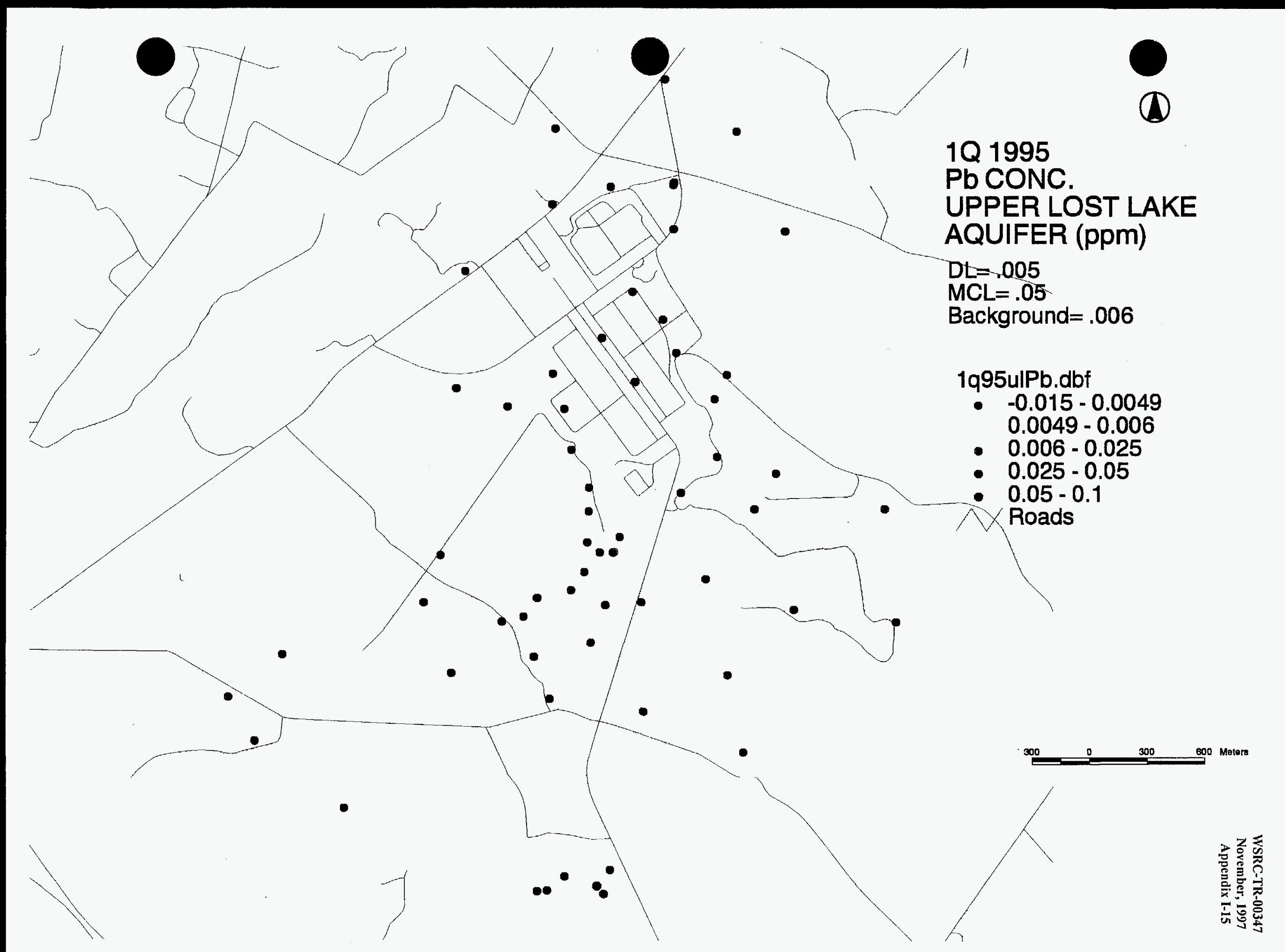




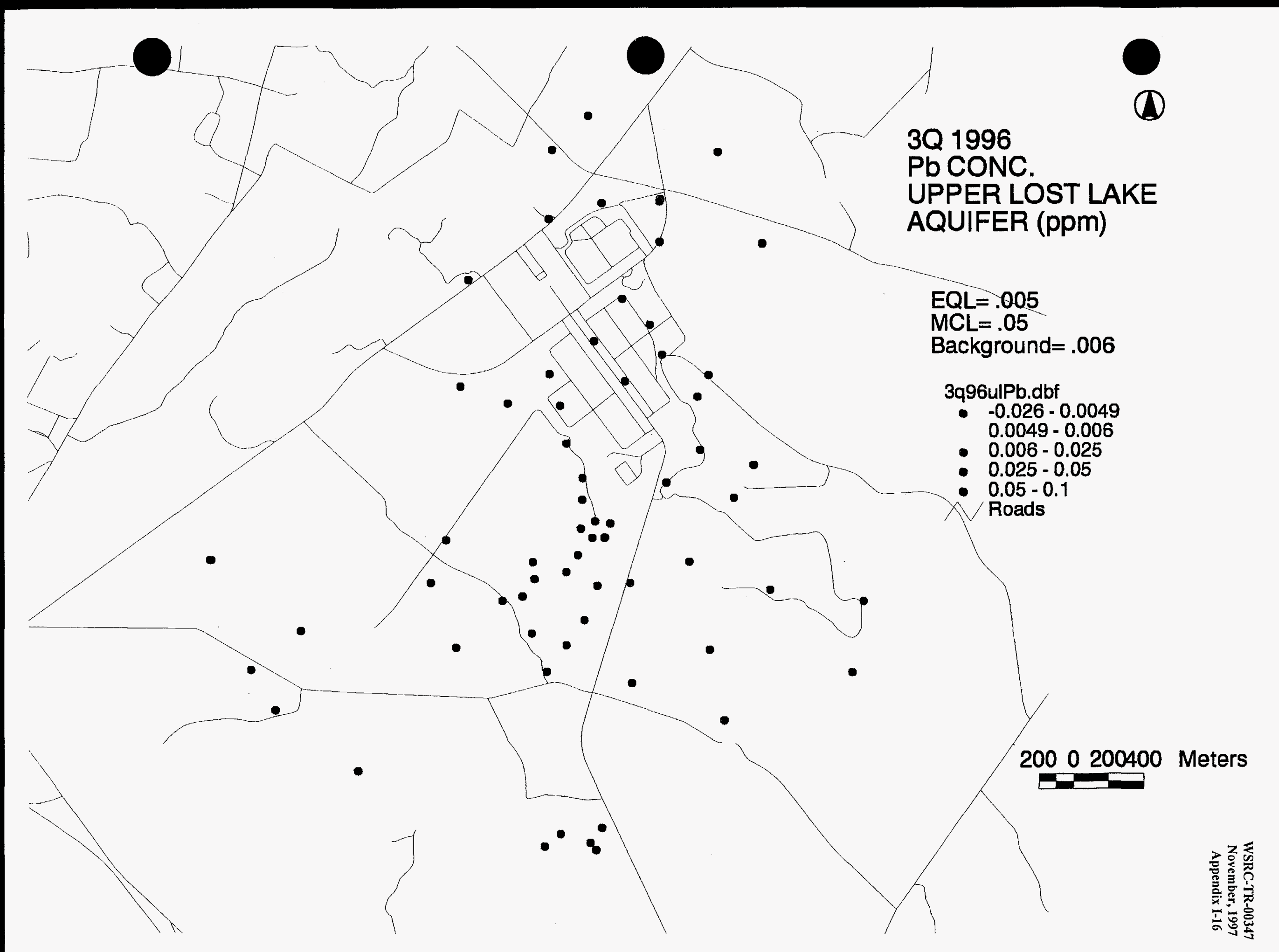




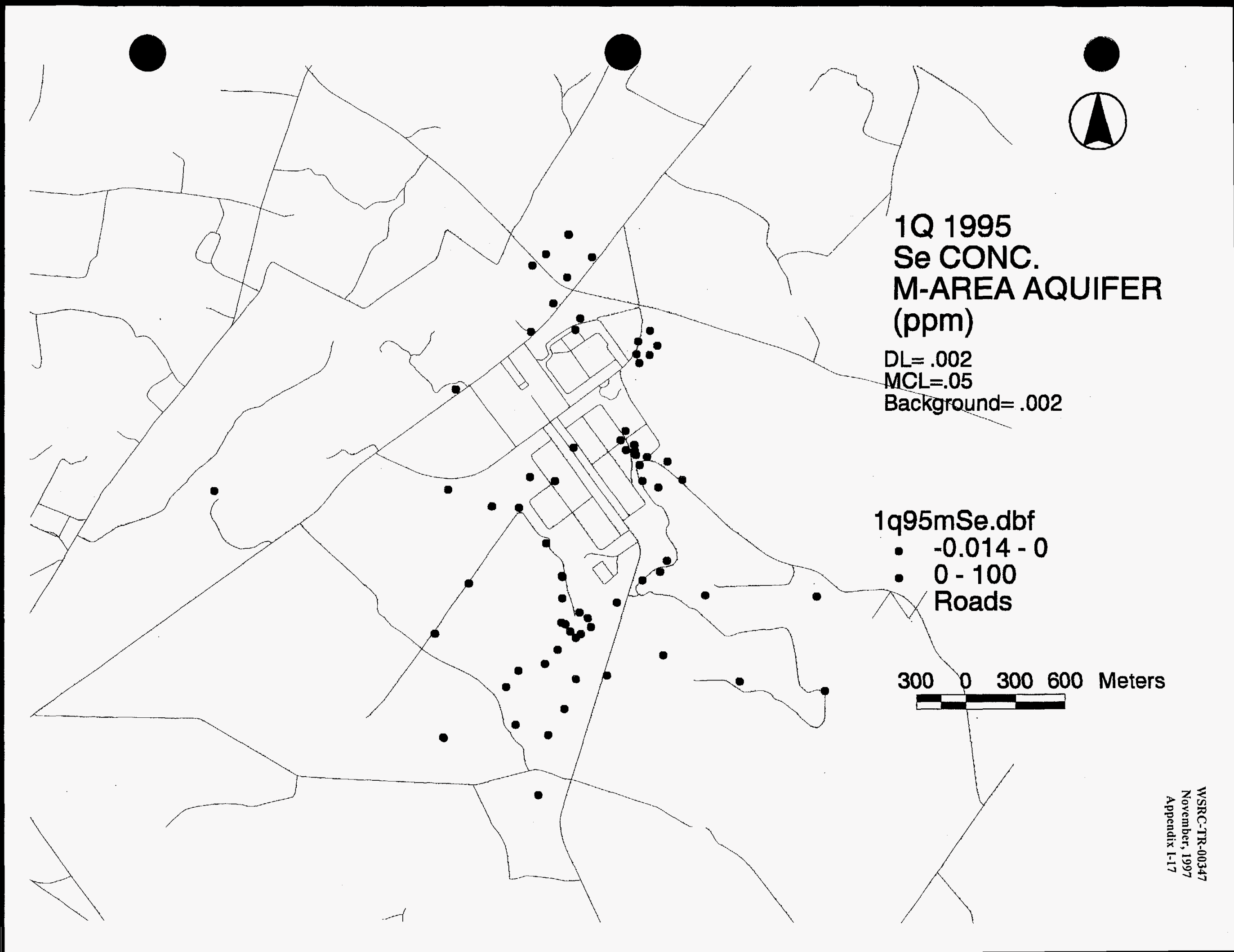




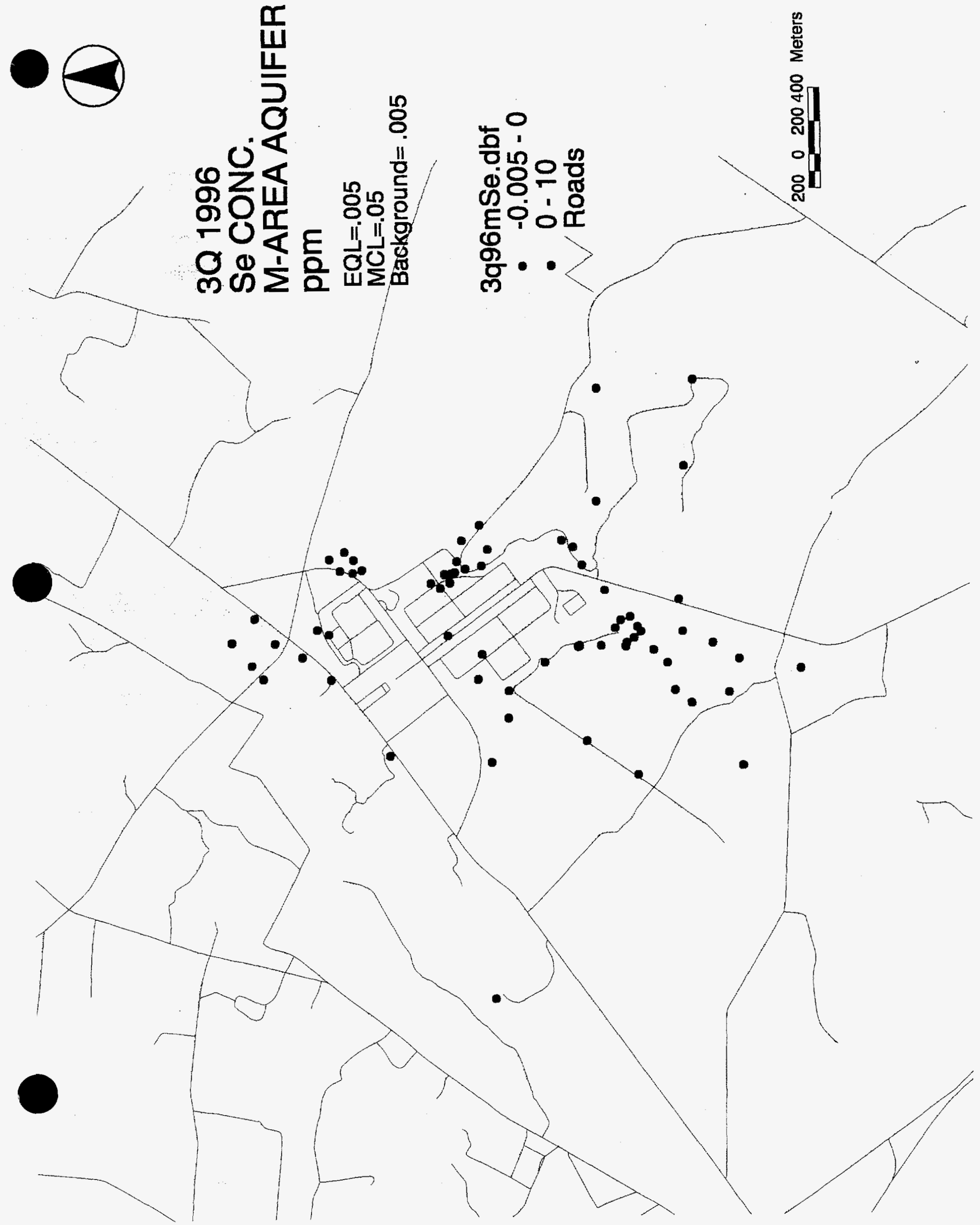




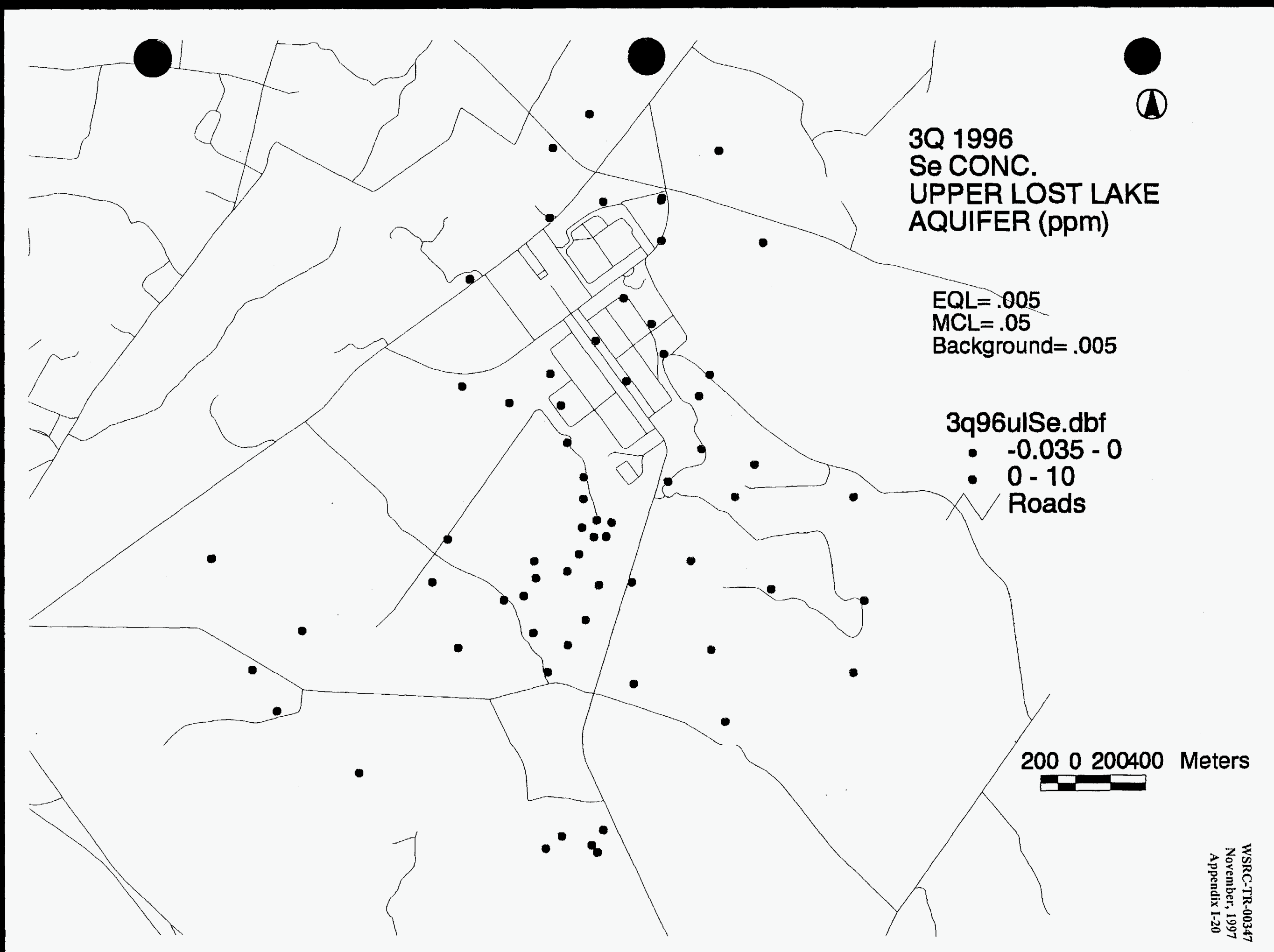




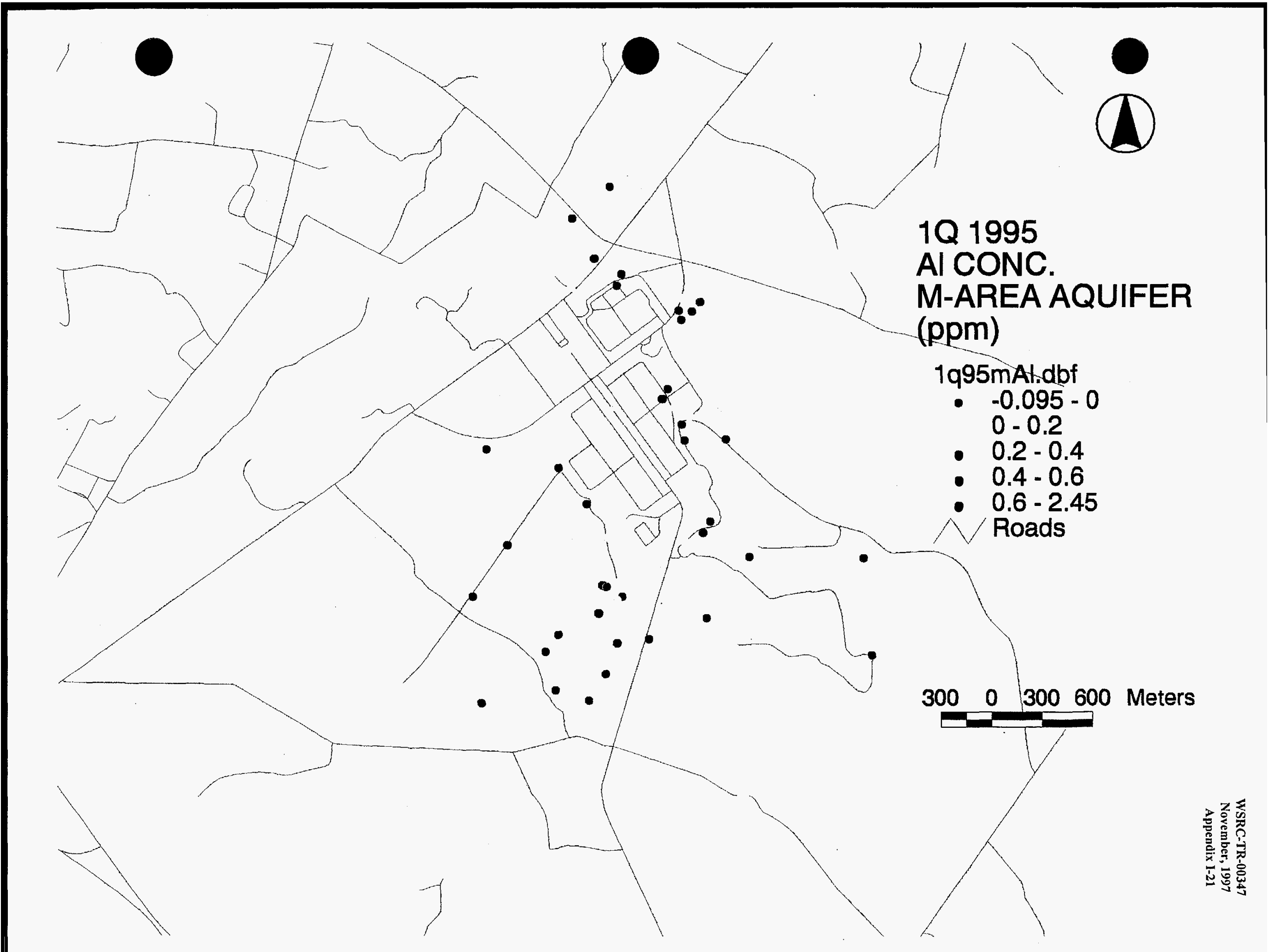




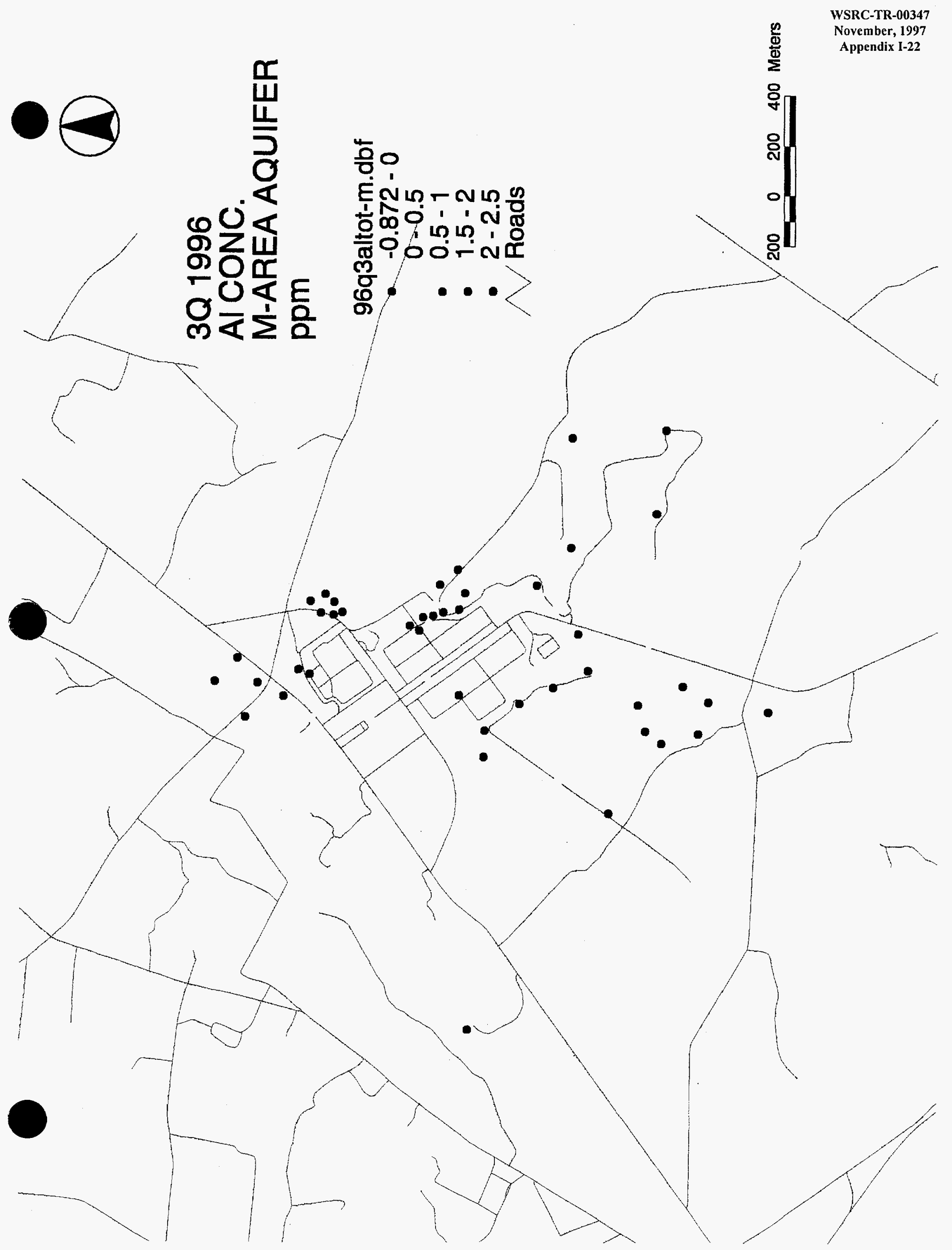



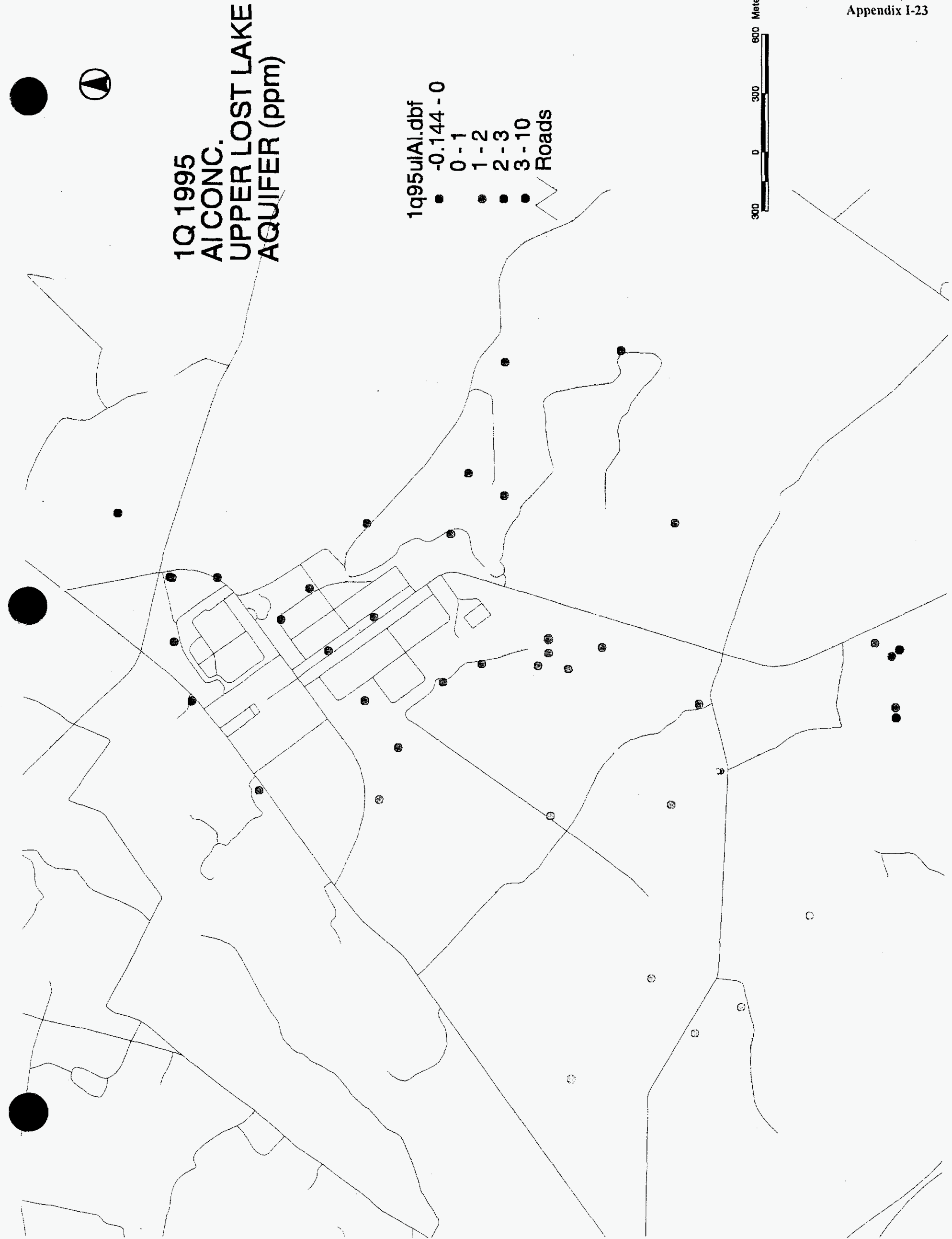


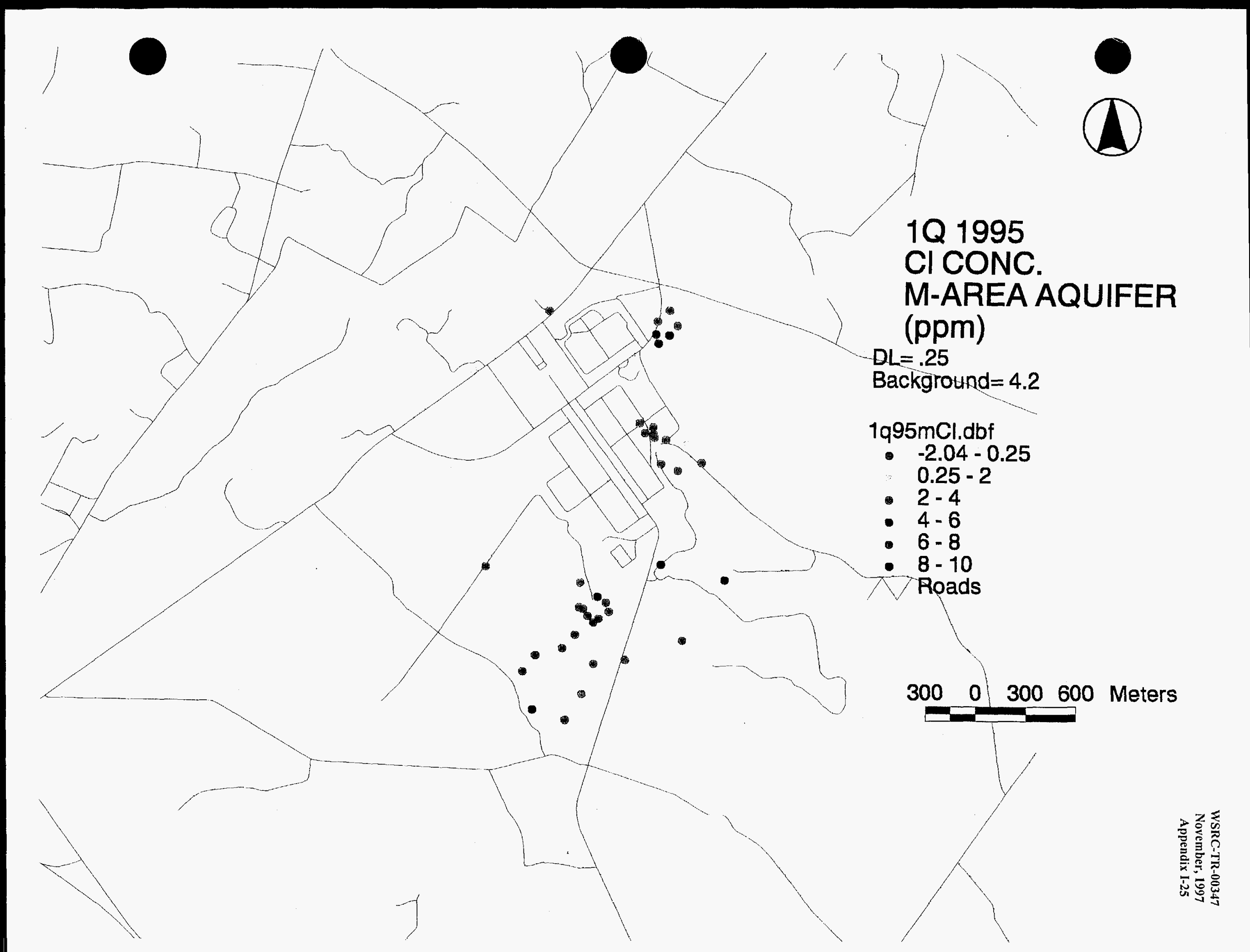



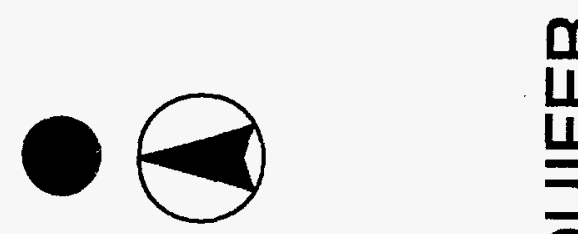

Appendix 1-26

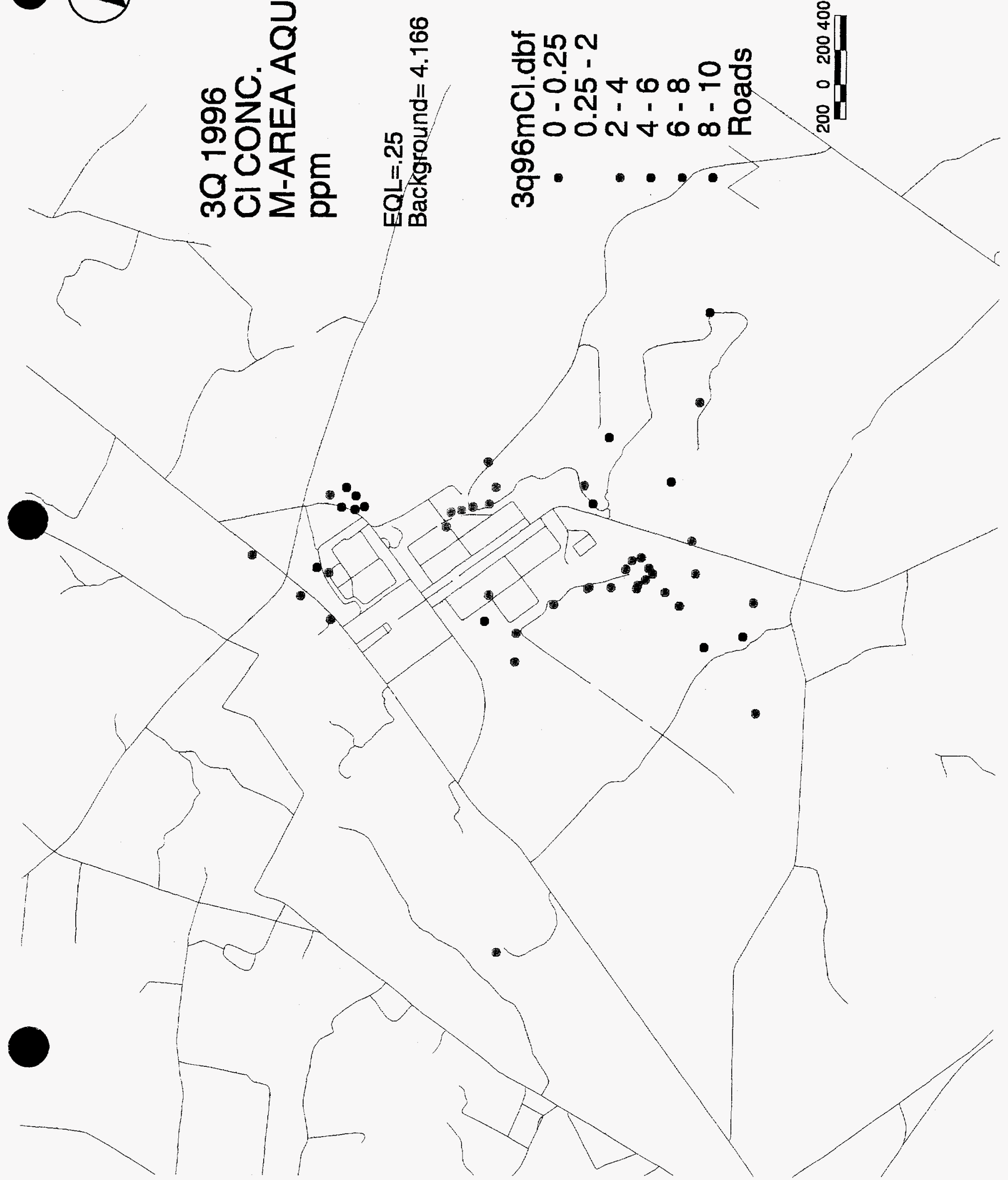




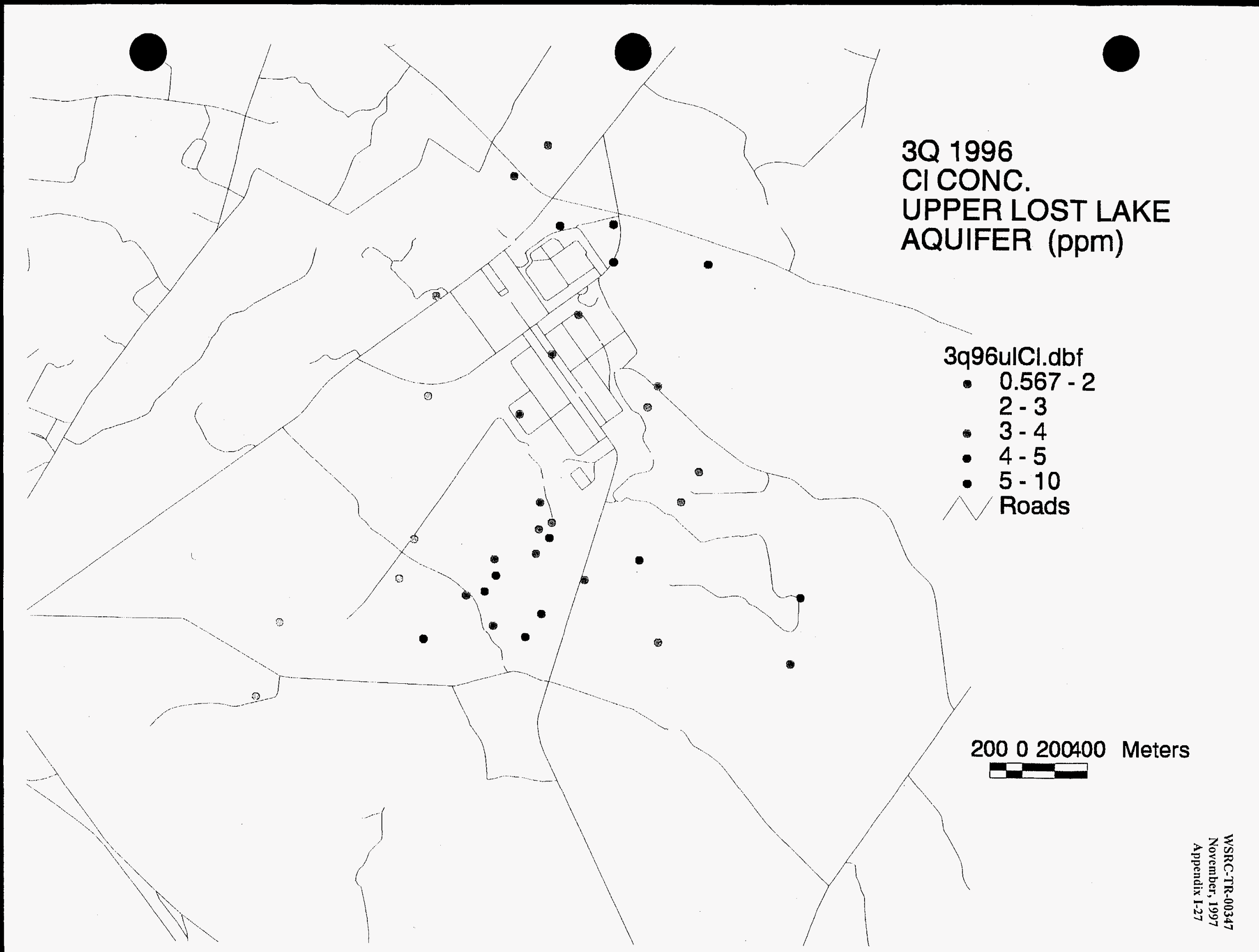



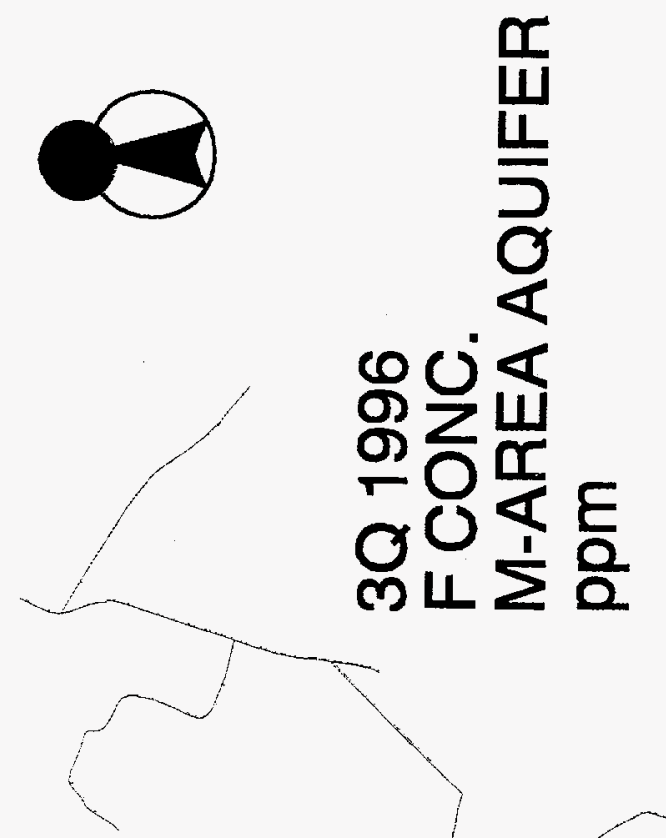


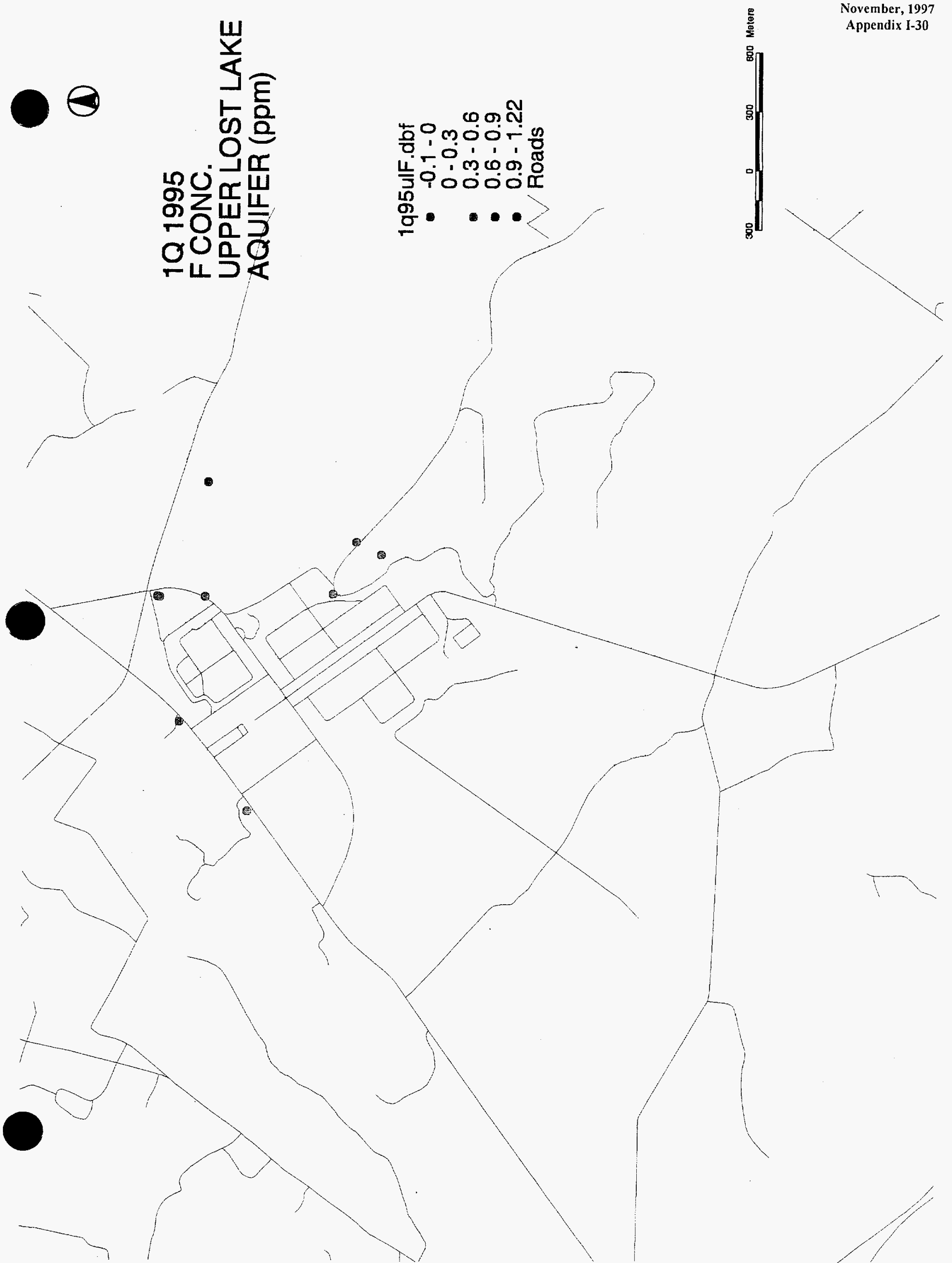




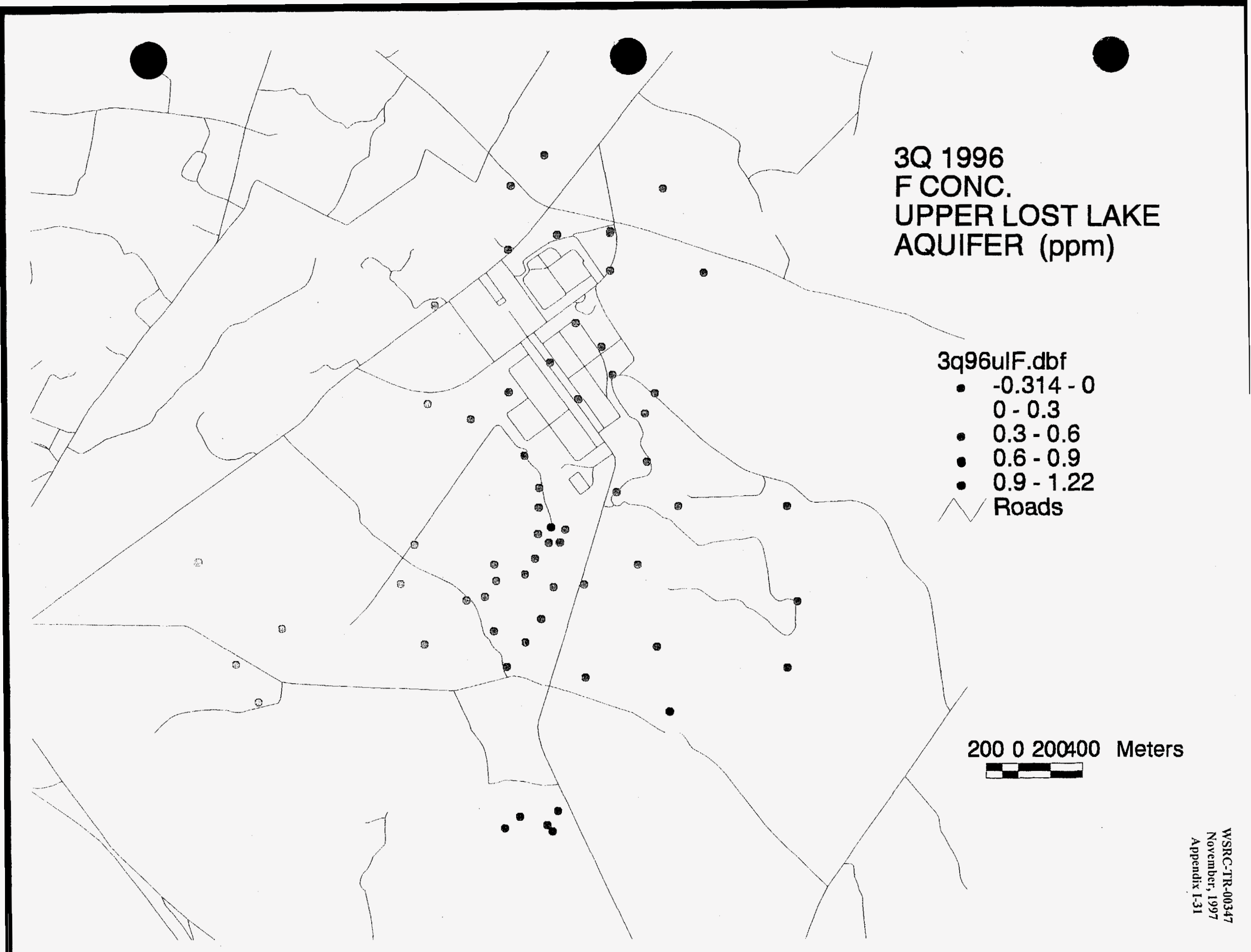




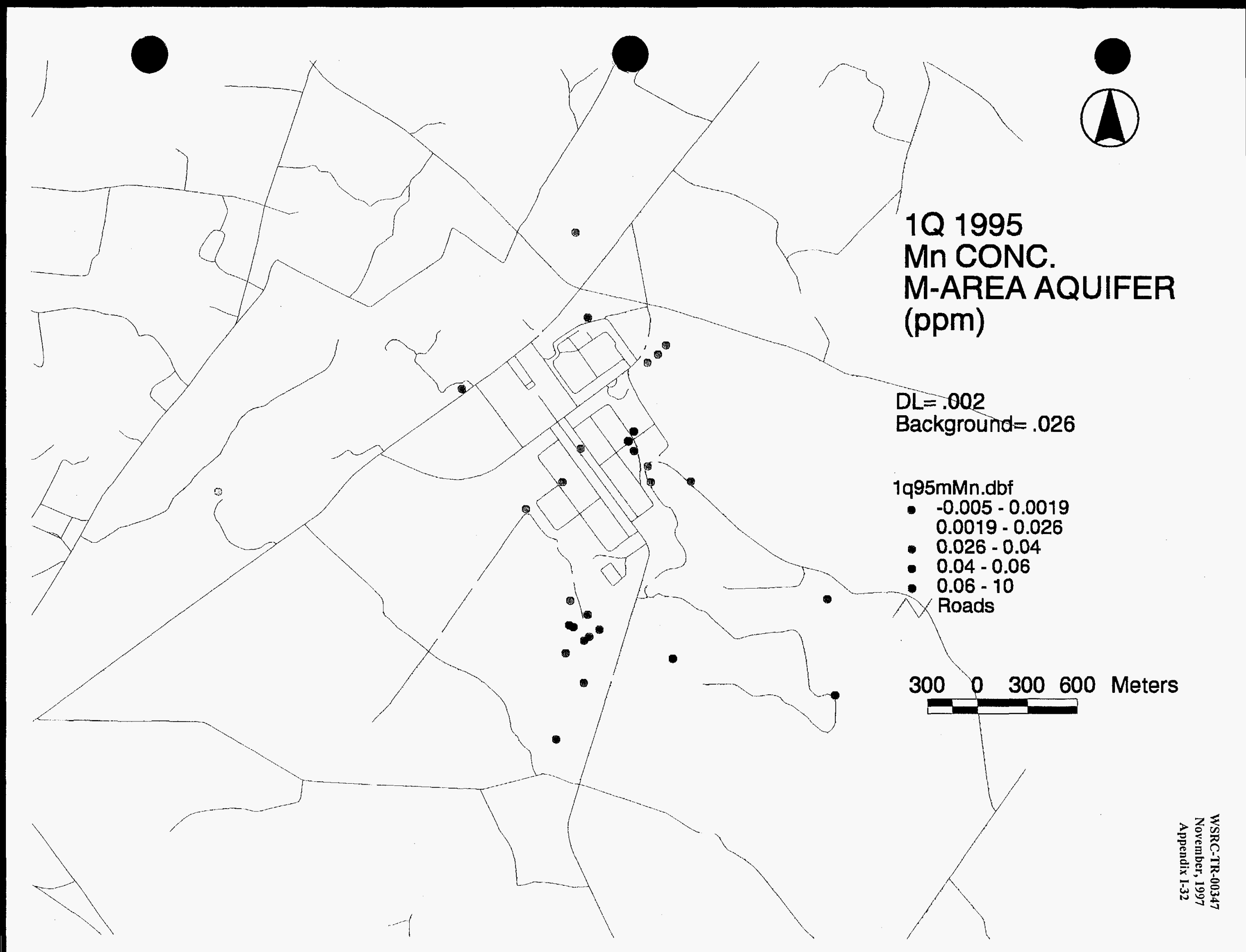




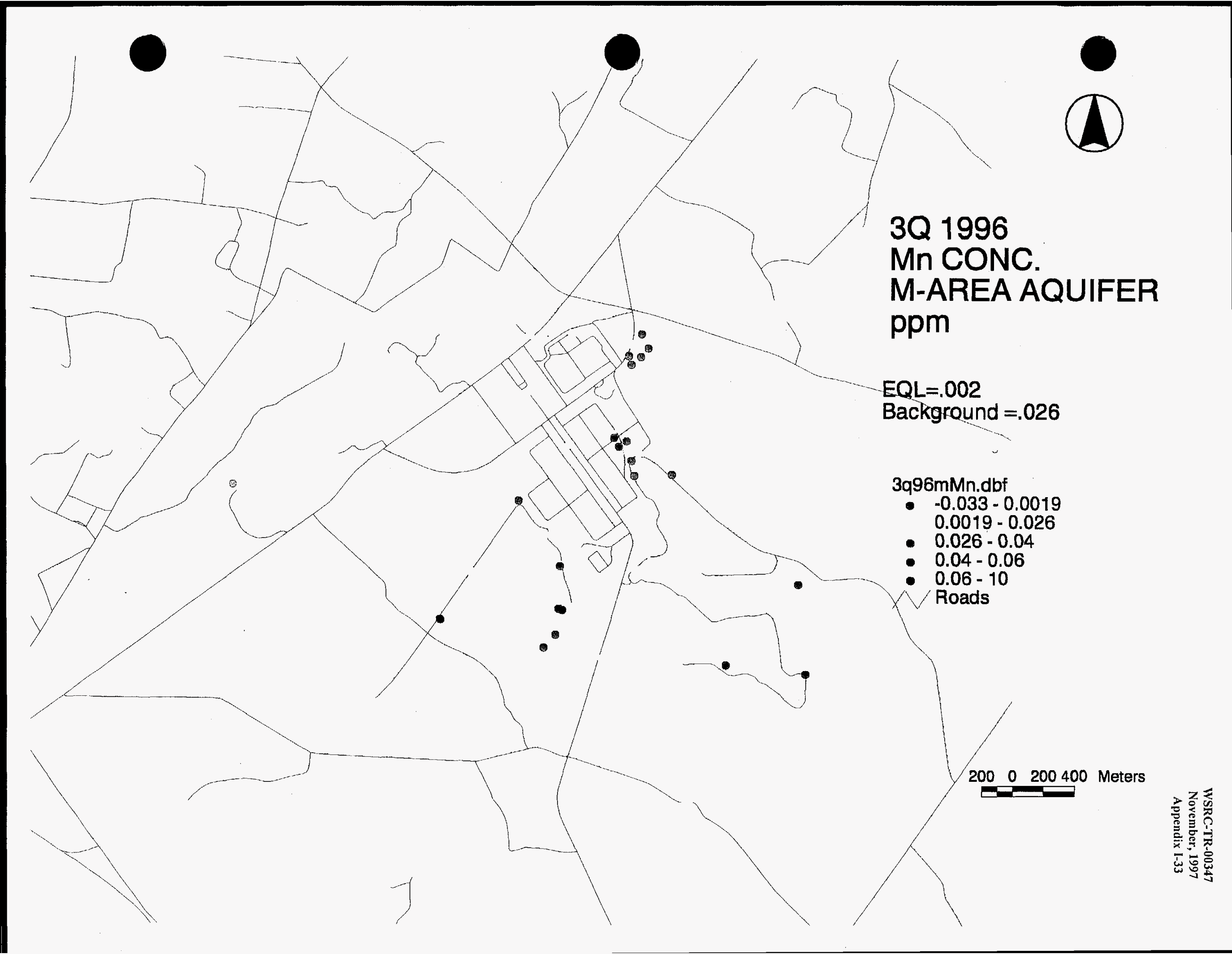




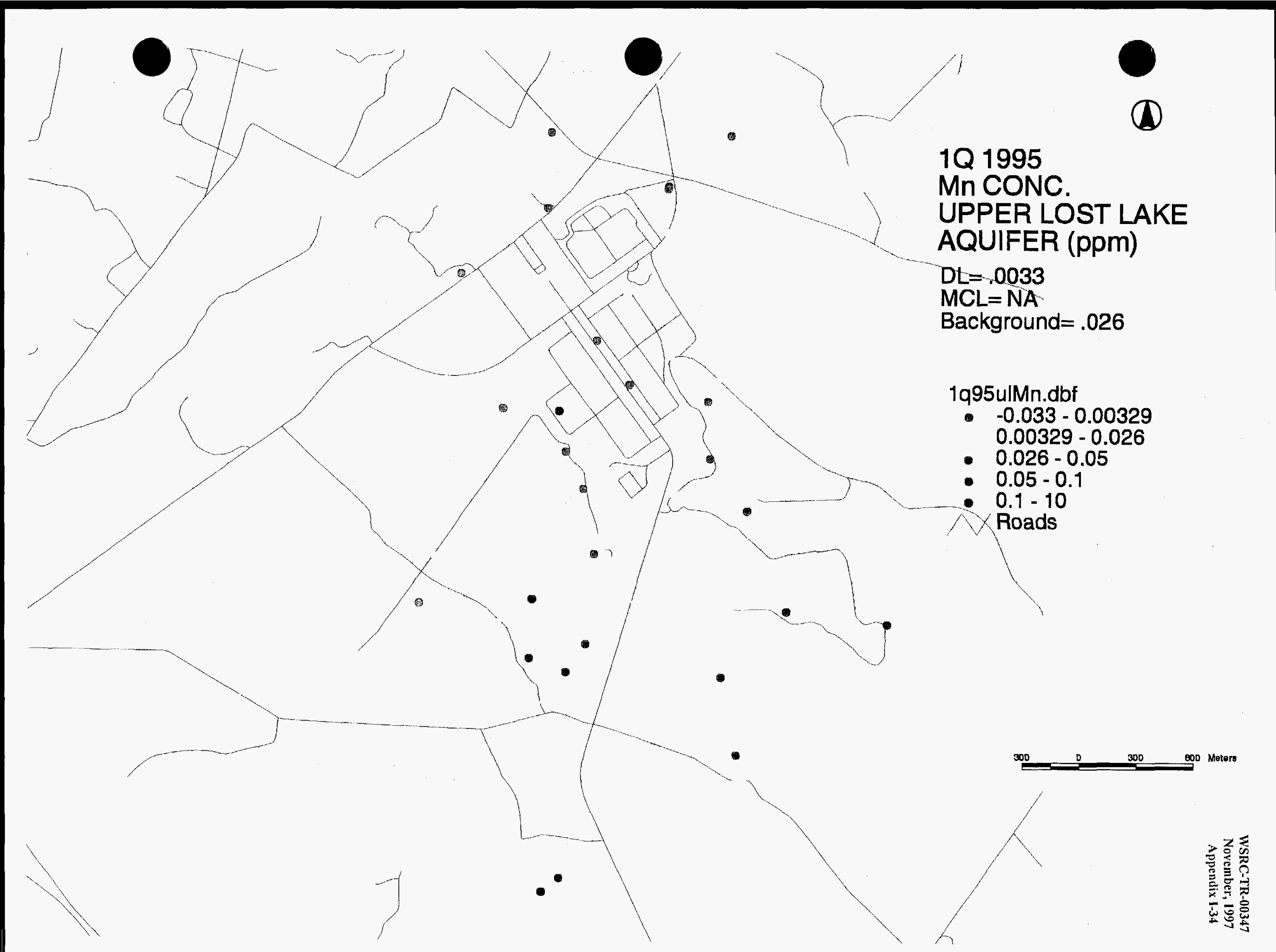




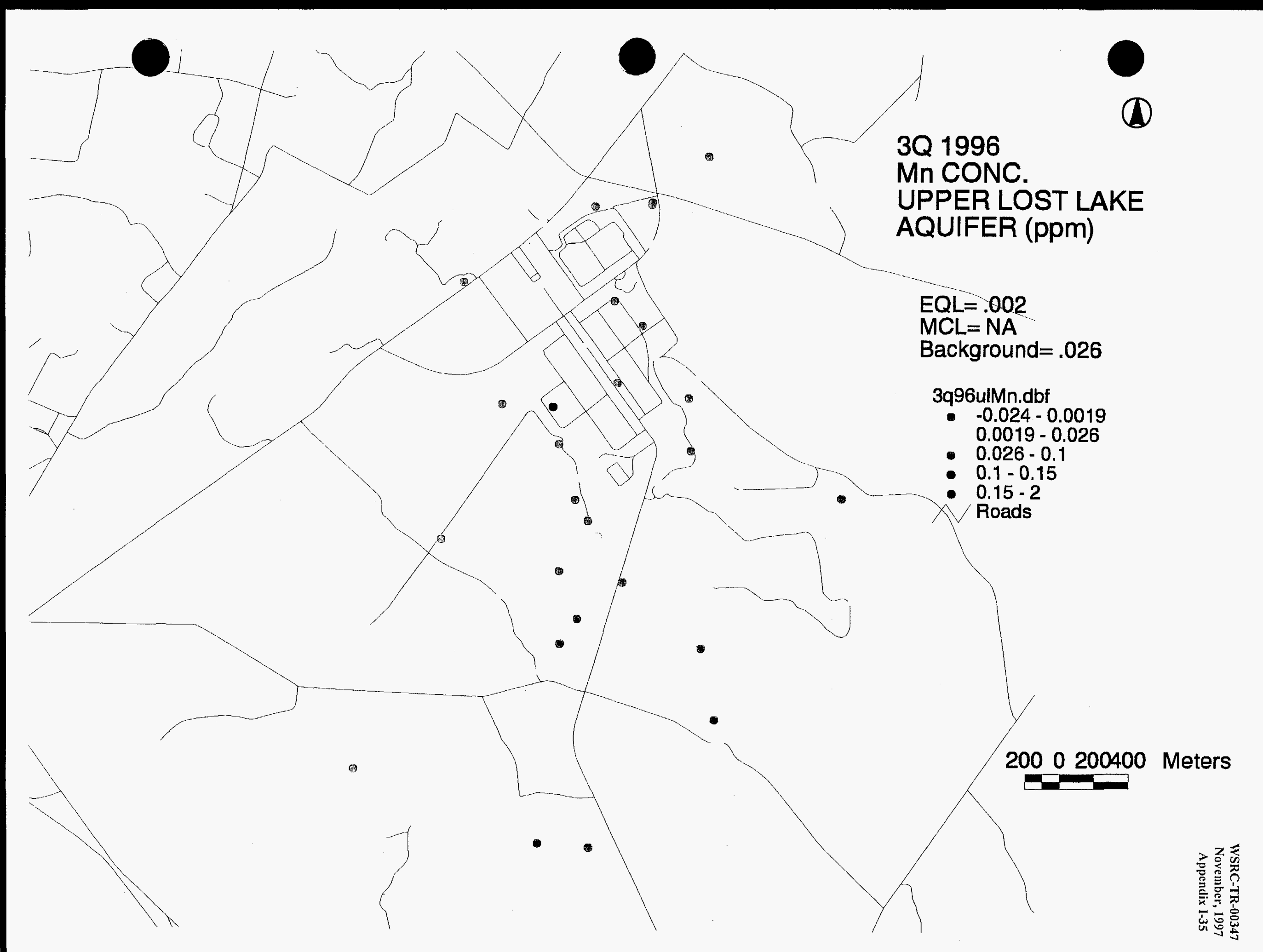




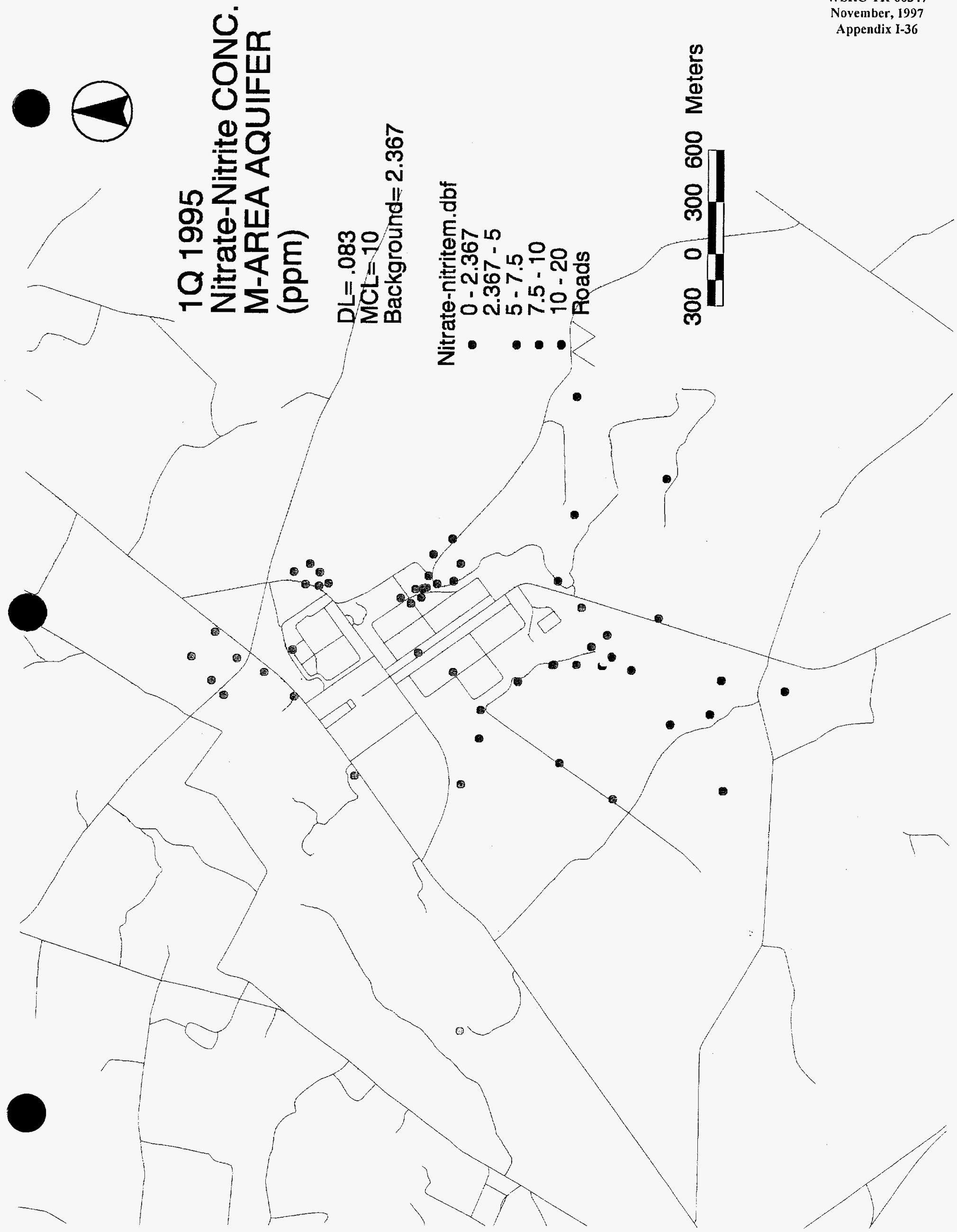




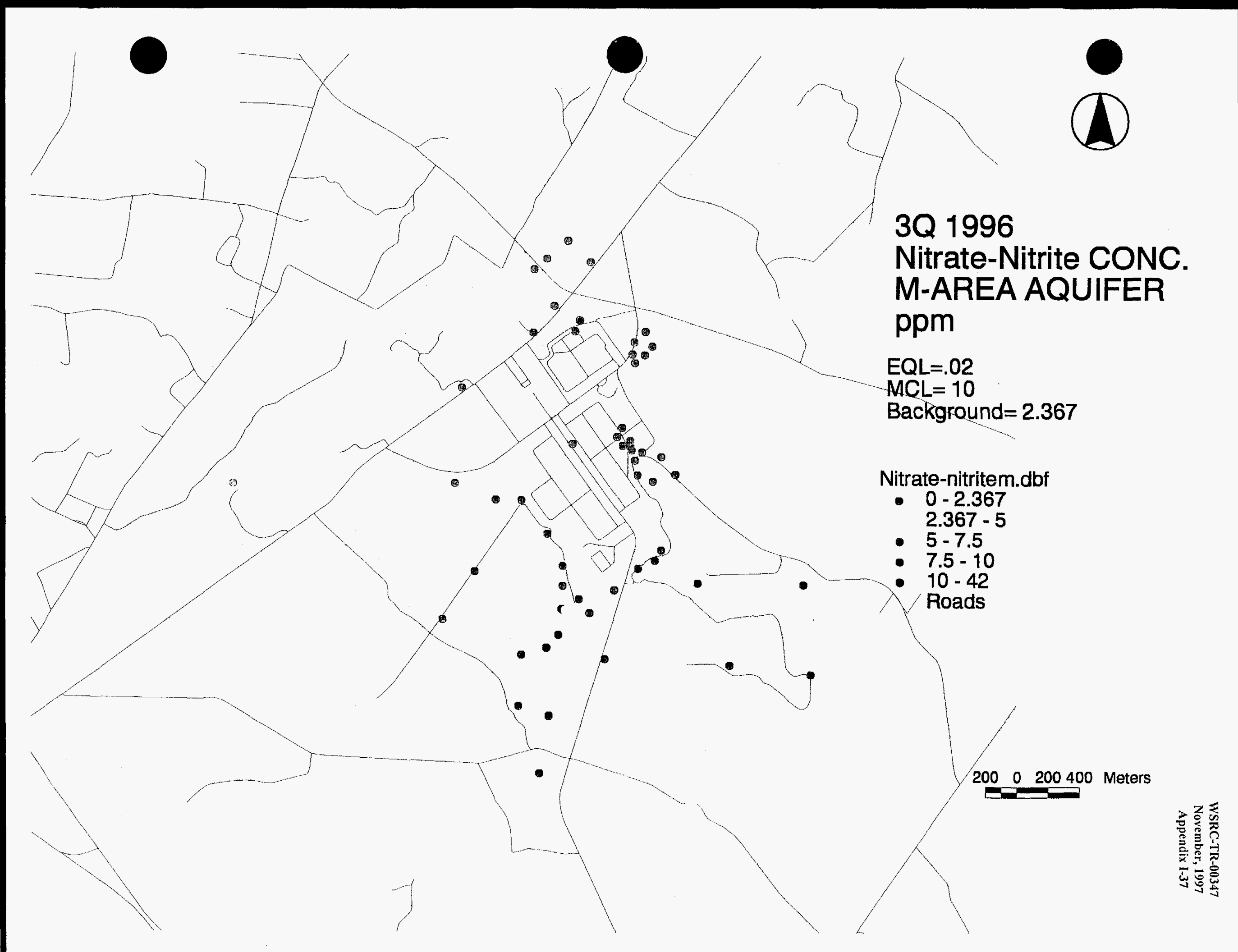




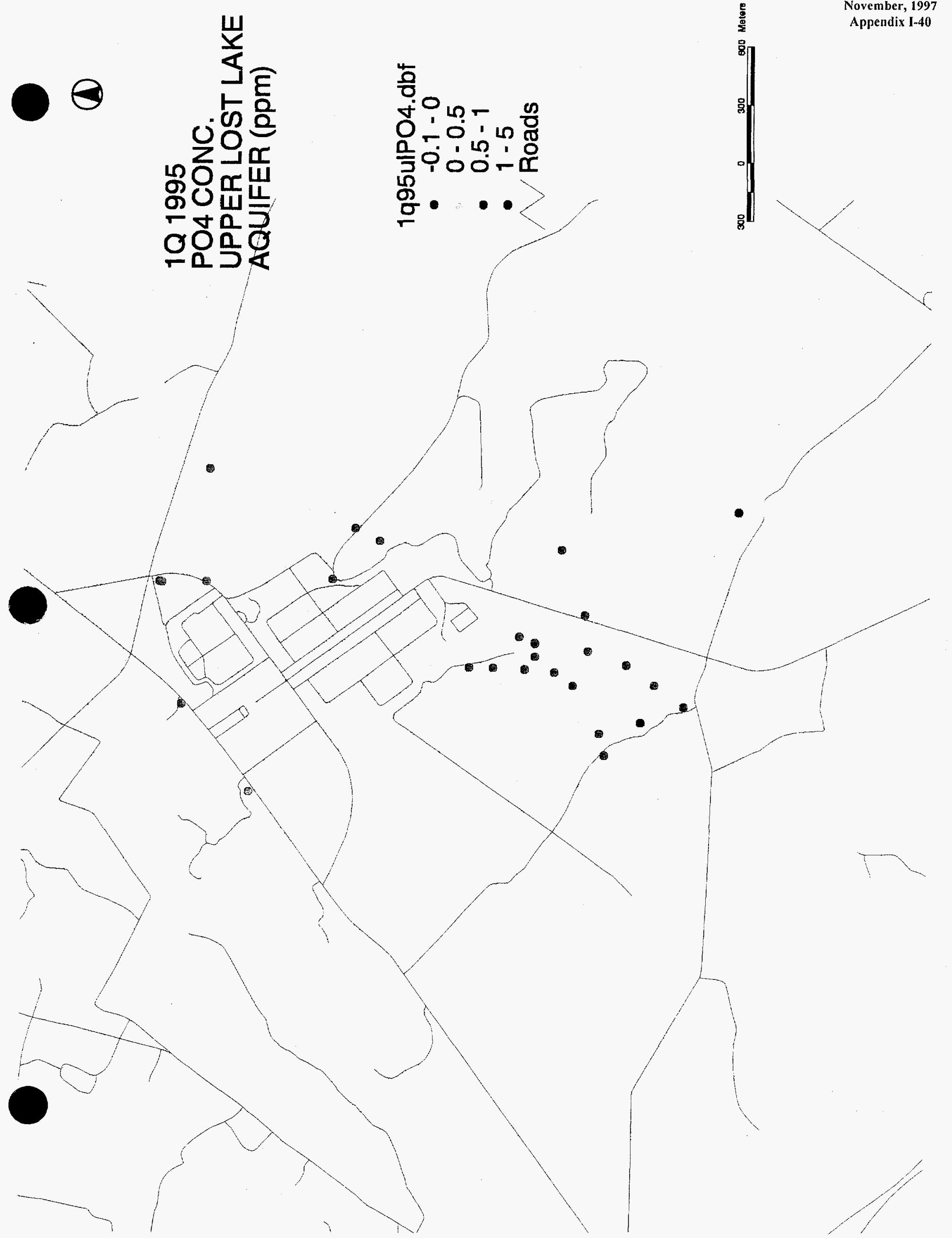




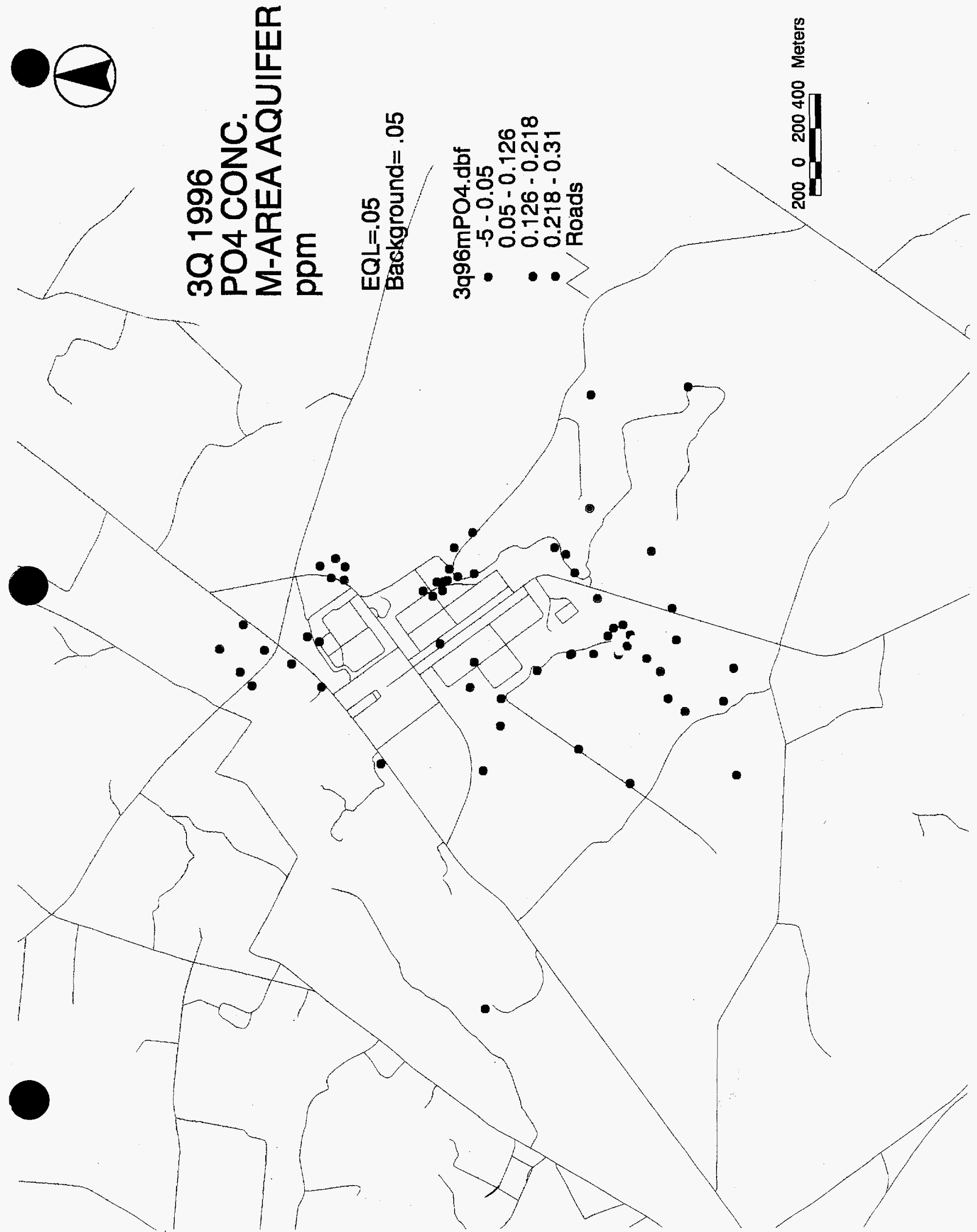




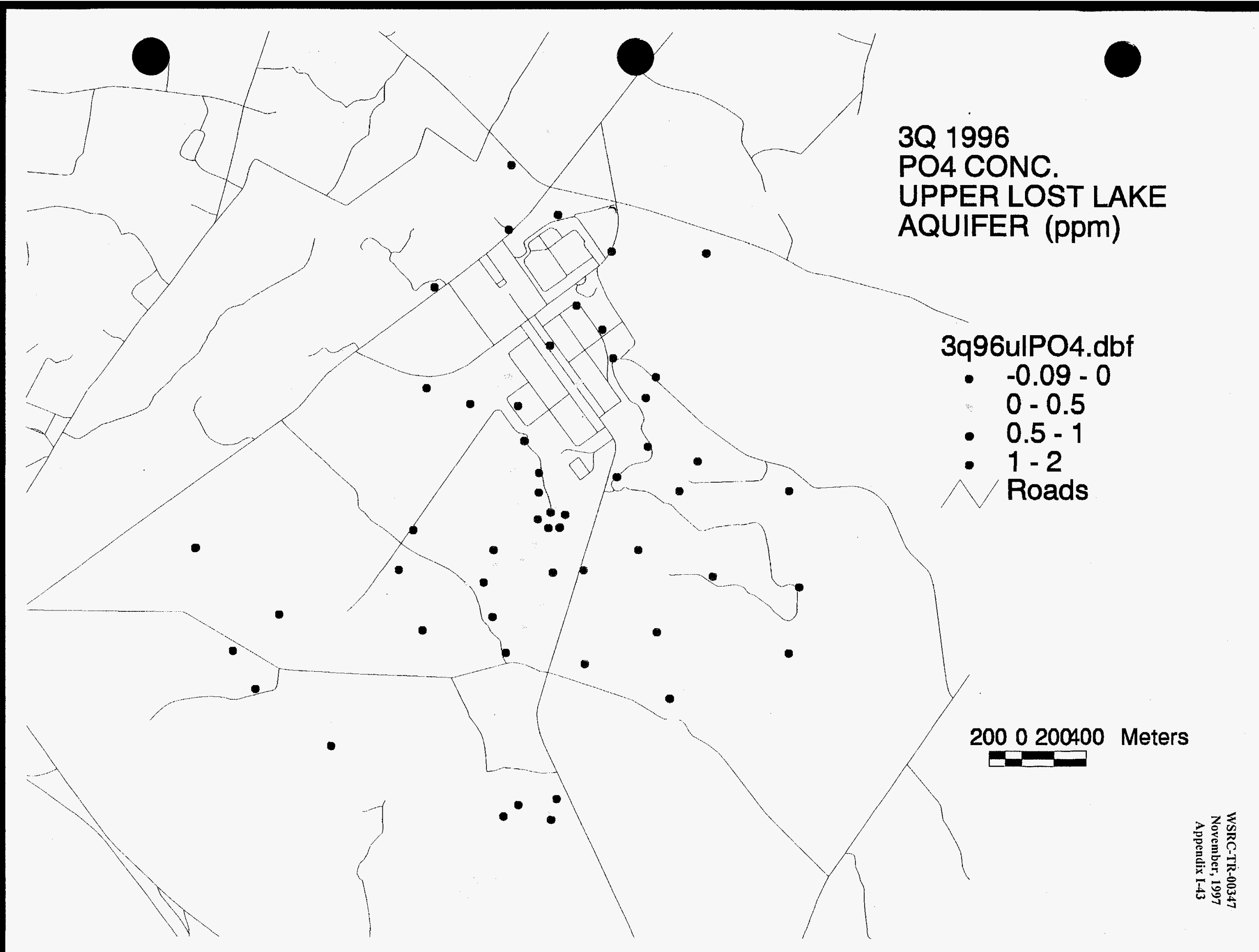




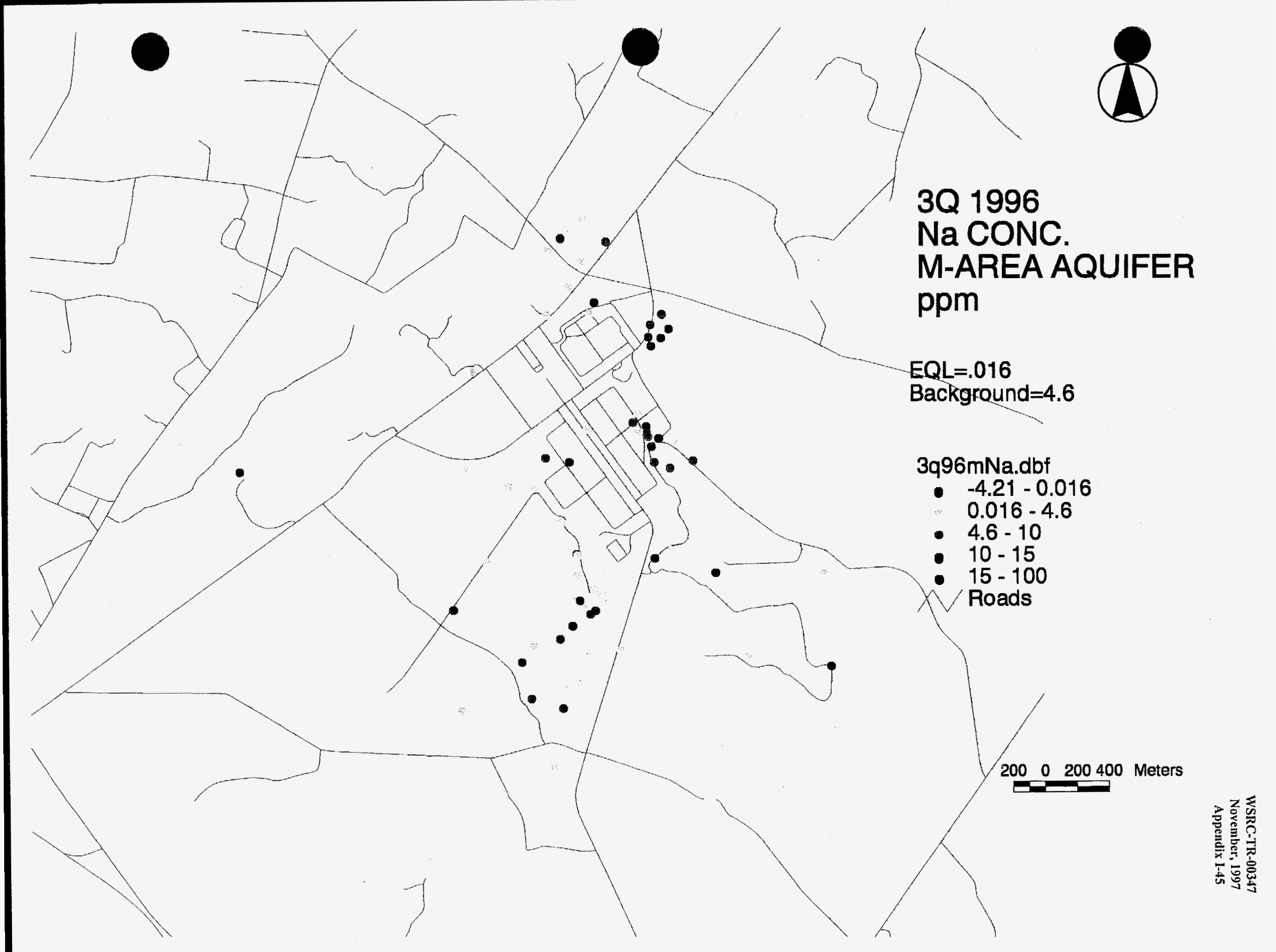




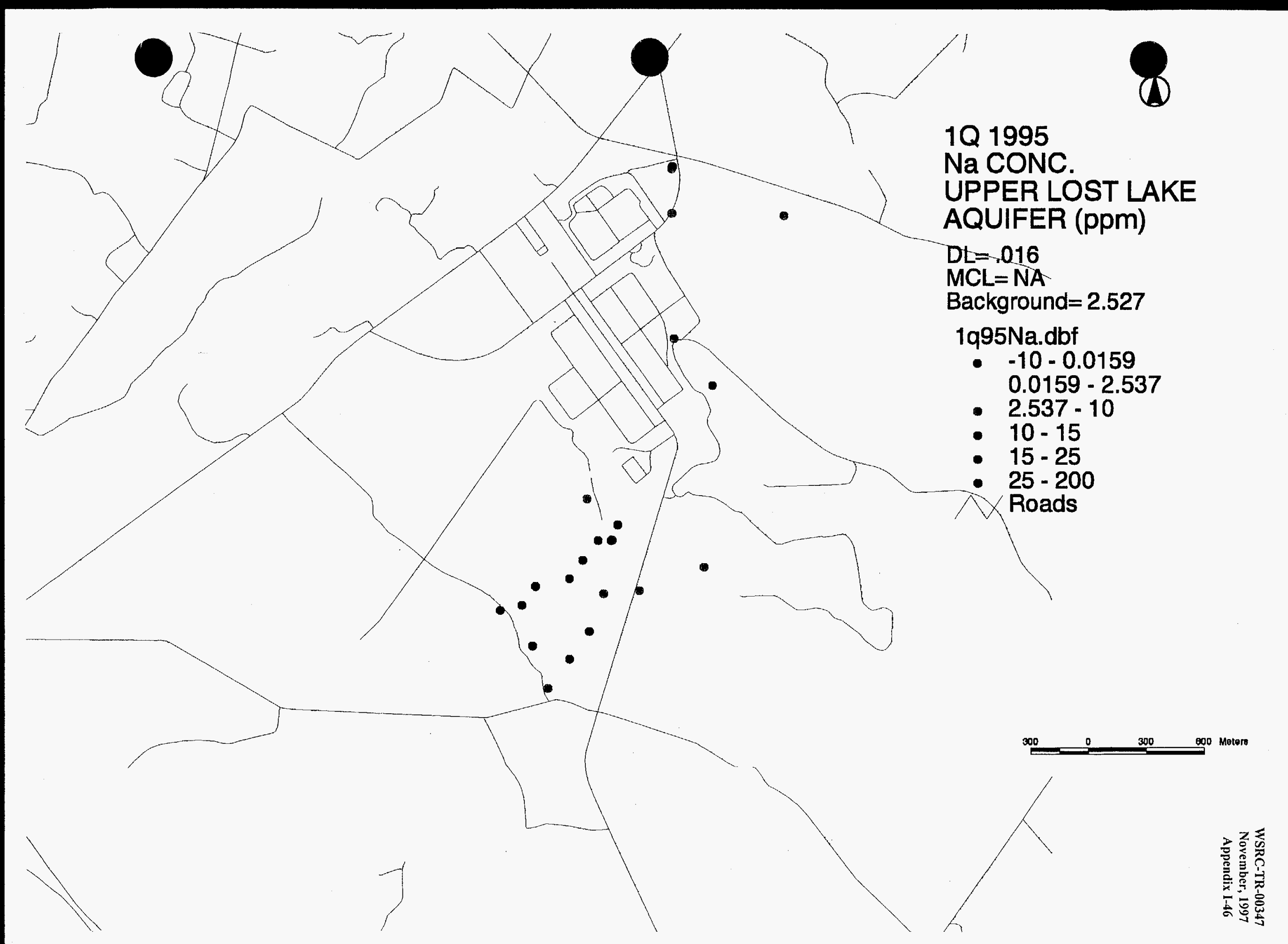




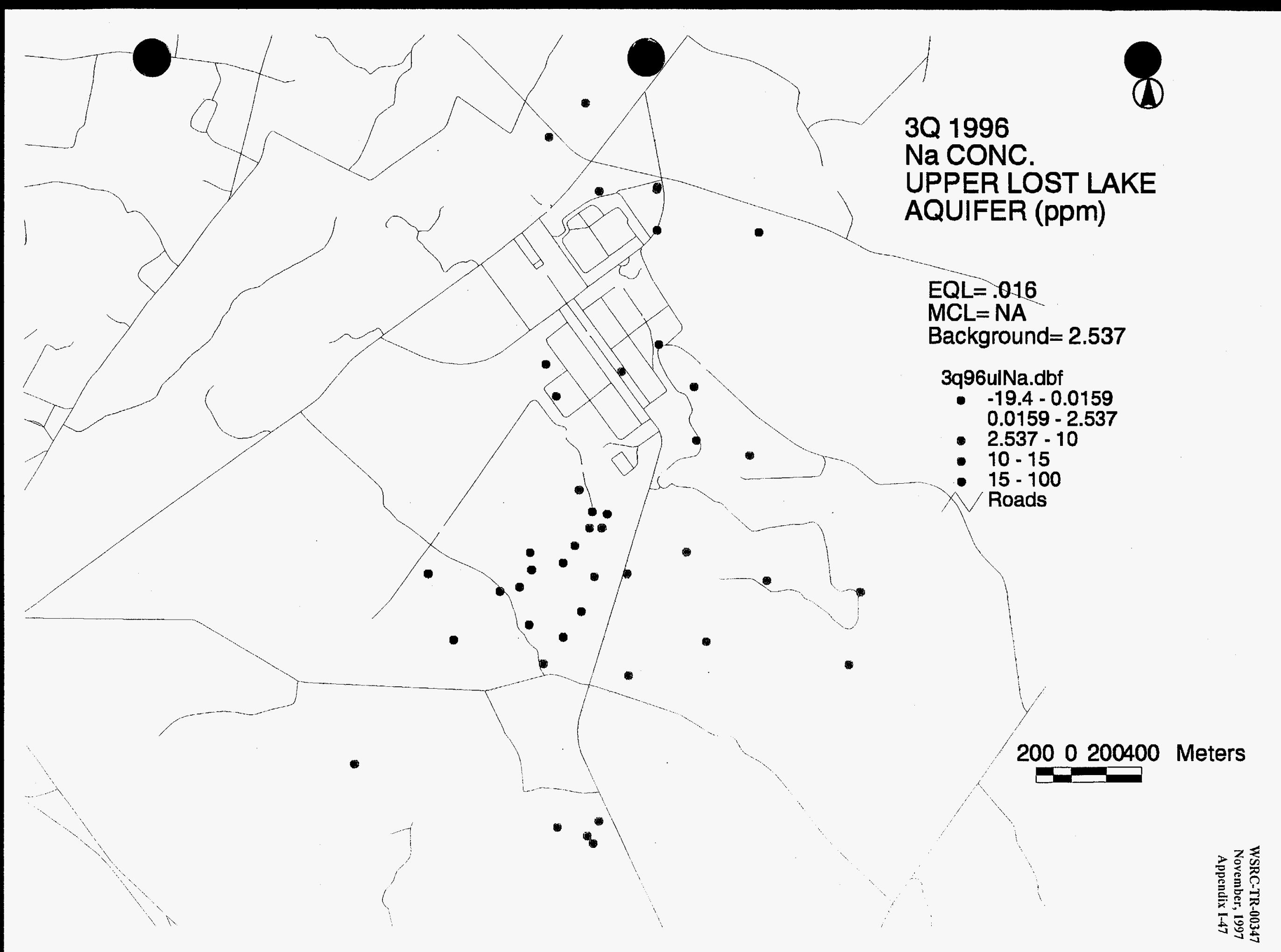




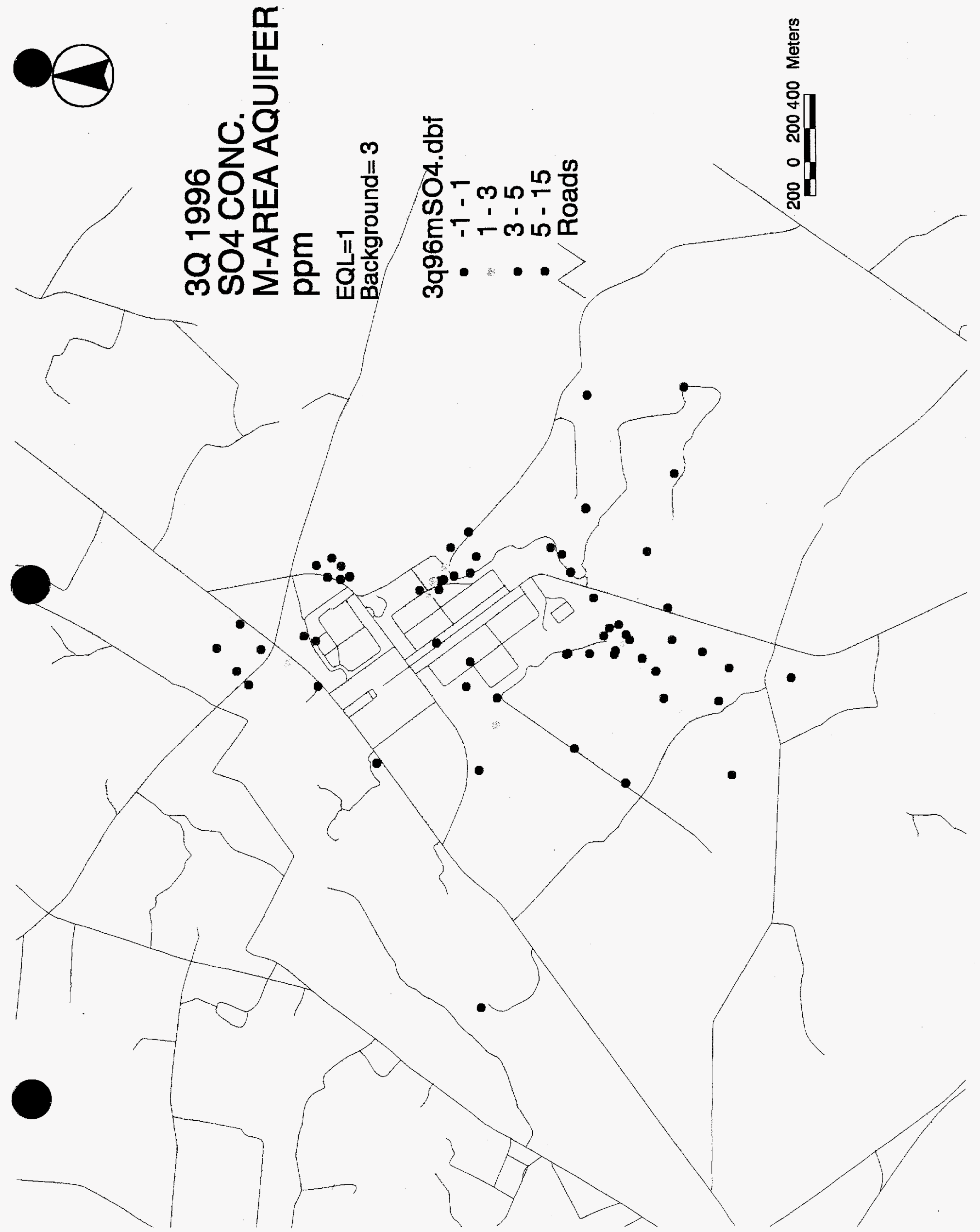




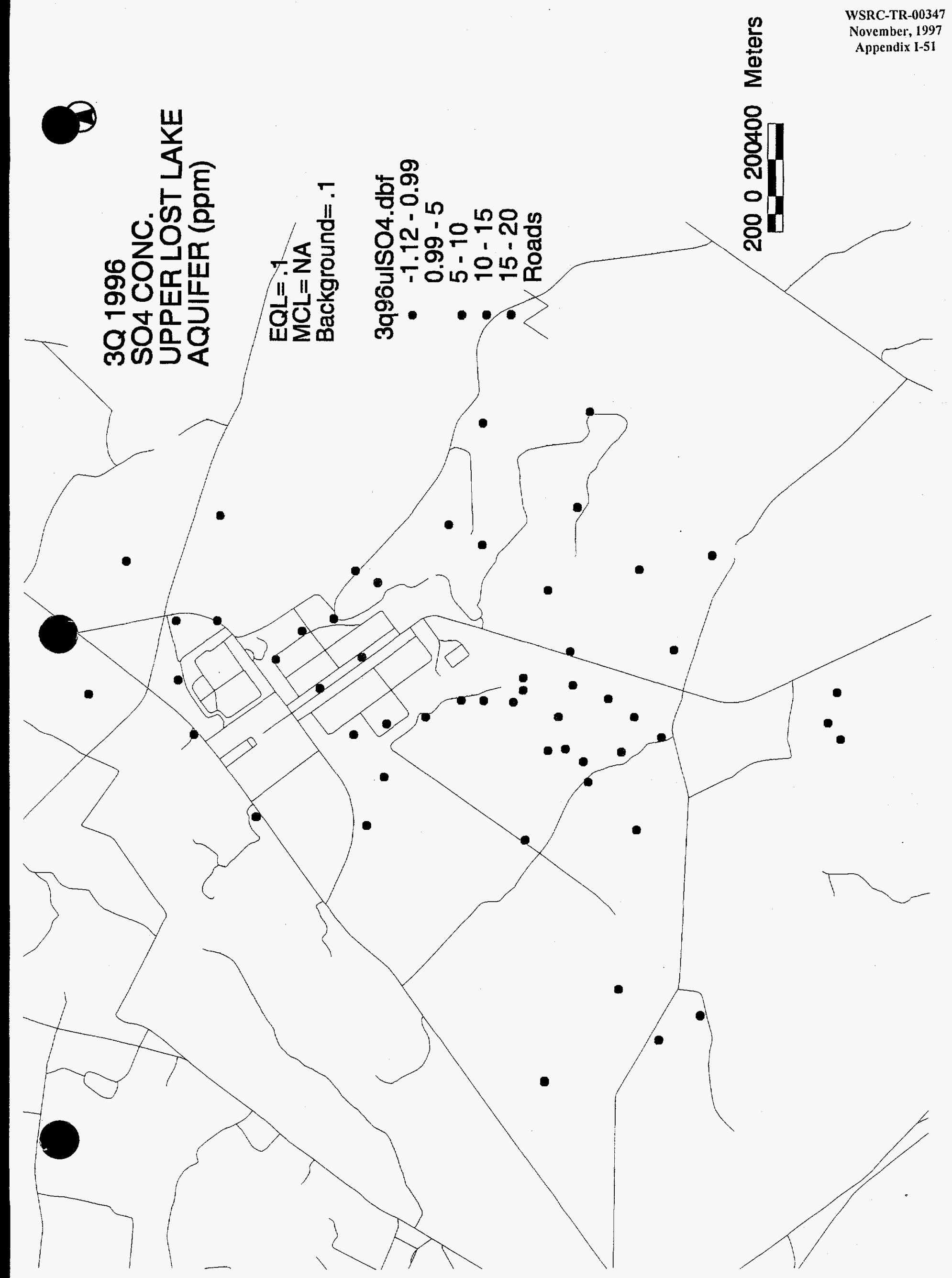




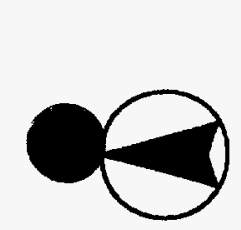

r

$\frac{\mathbf{L}}{\mathrm{L}} \quad \frac{0}{0}$

Appendix I-52

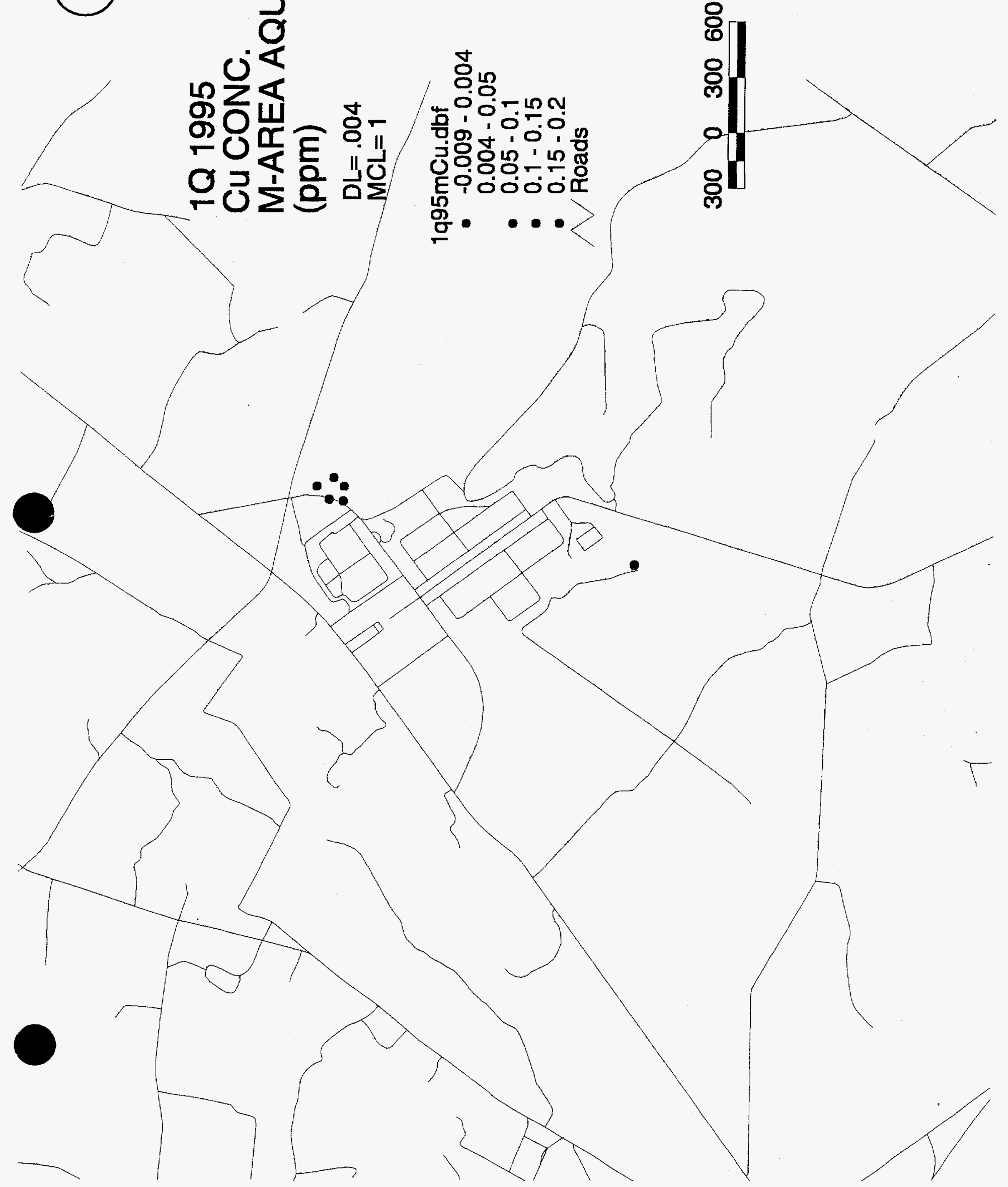




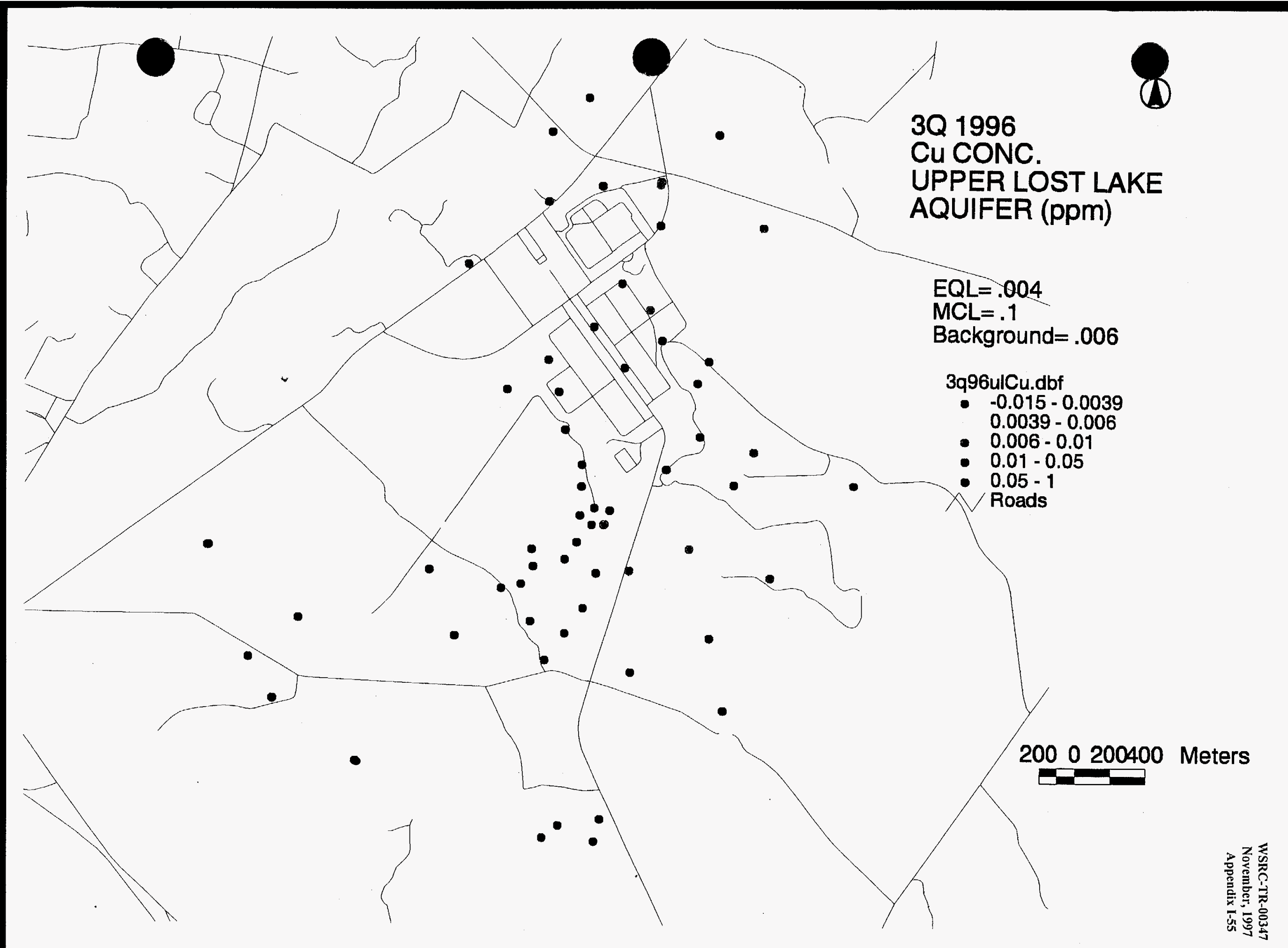




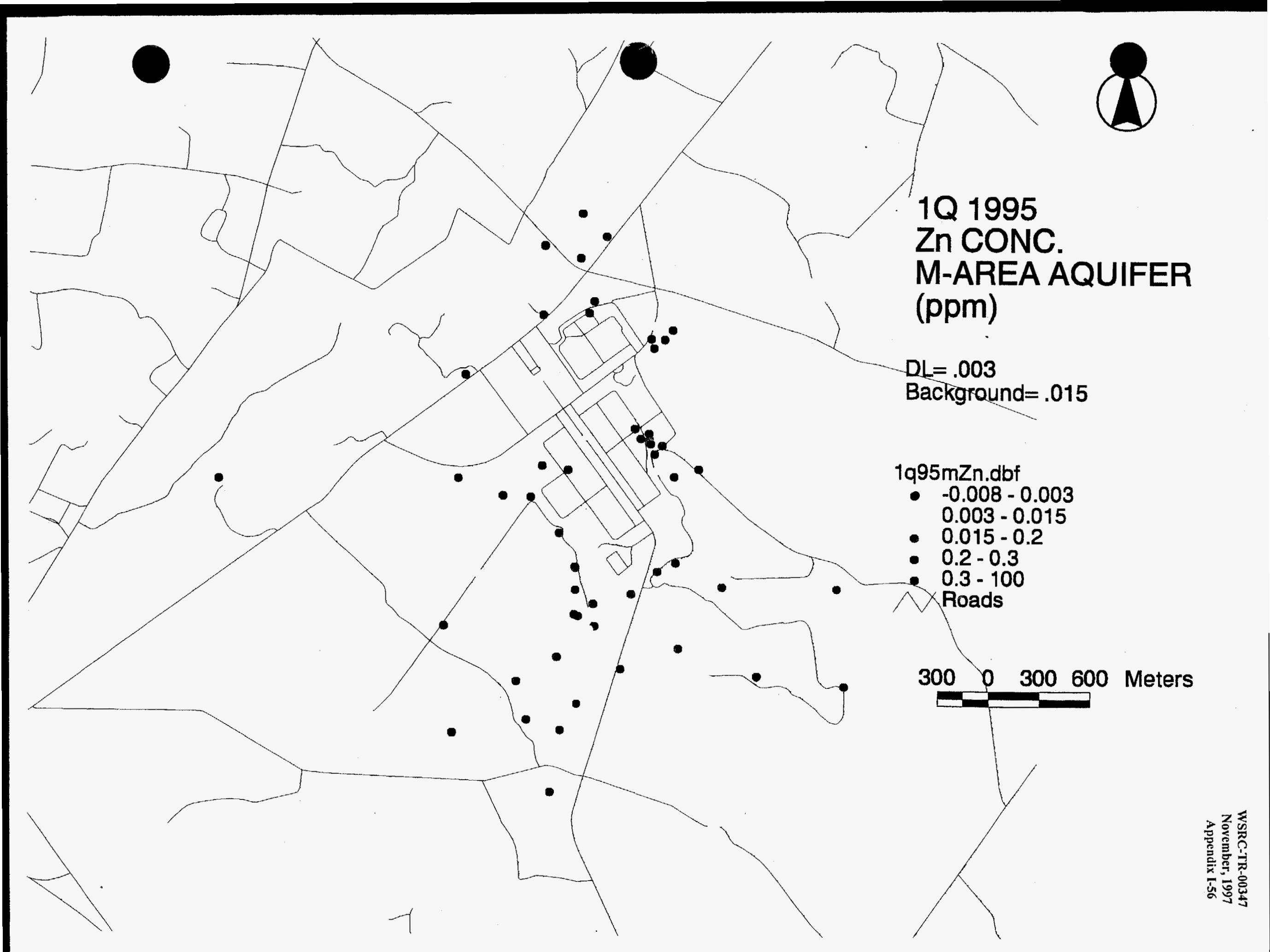




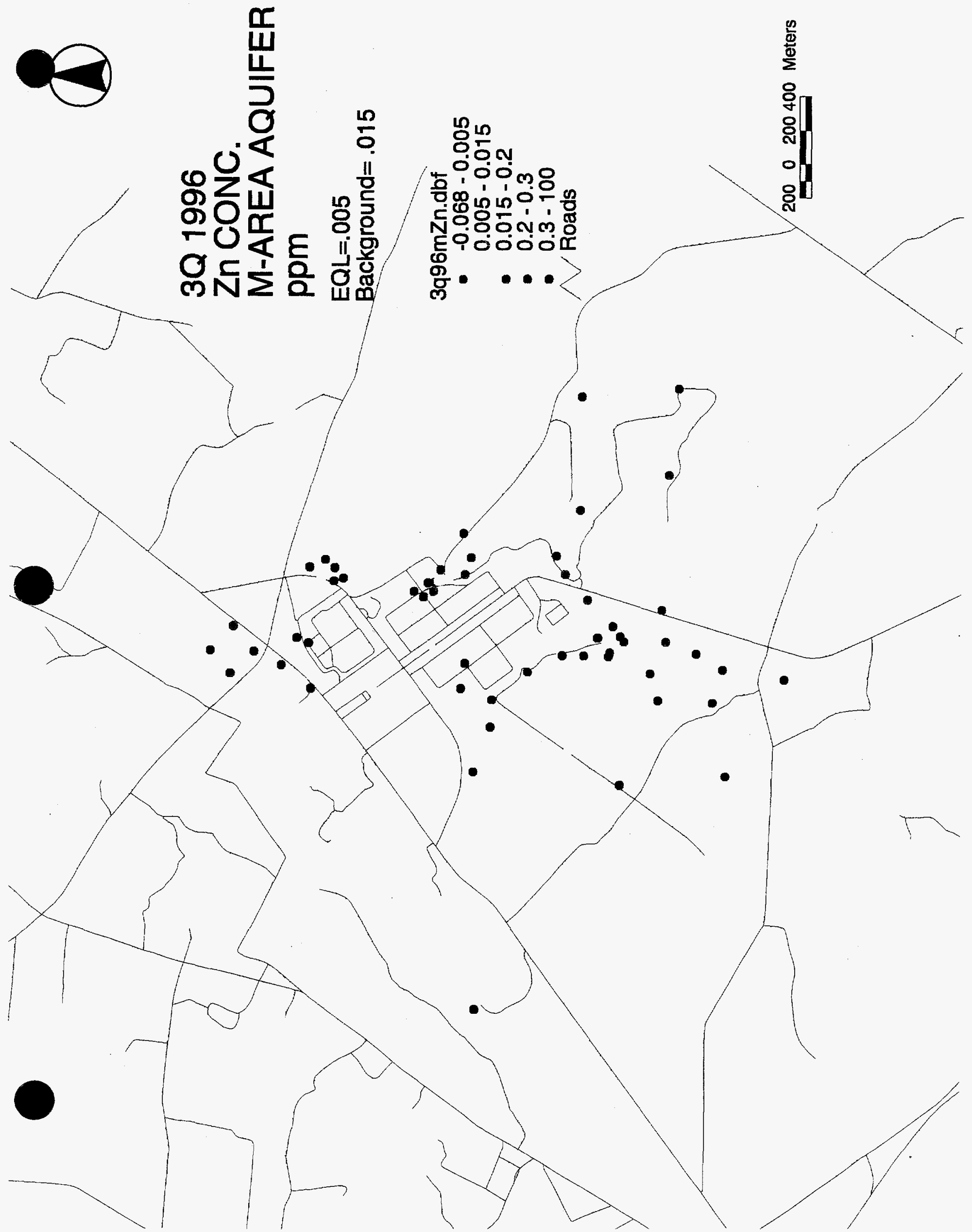




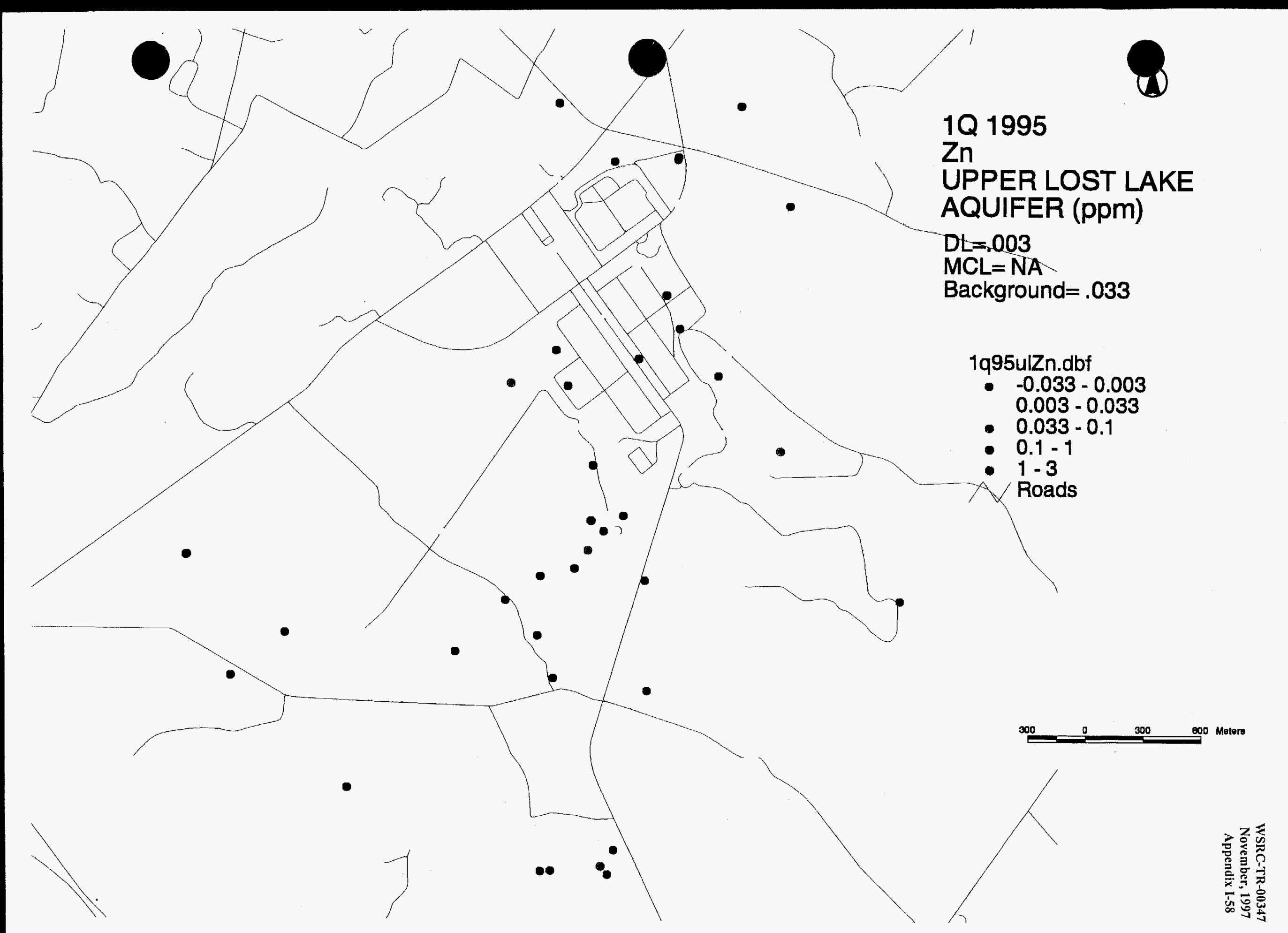




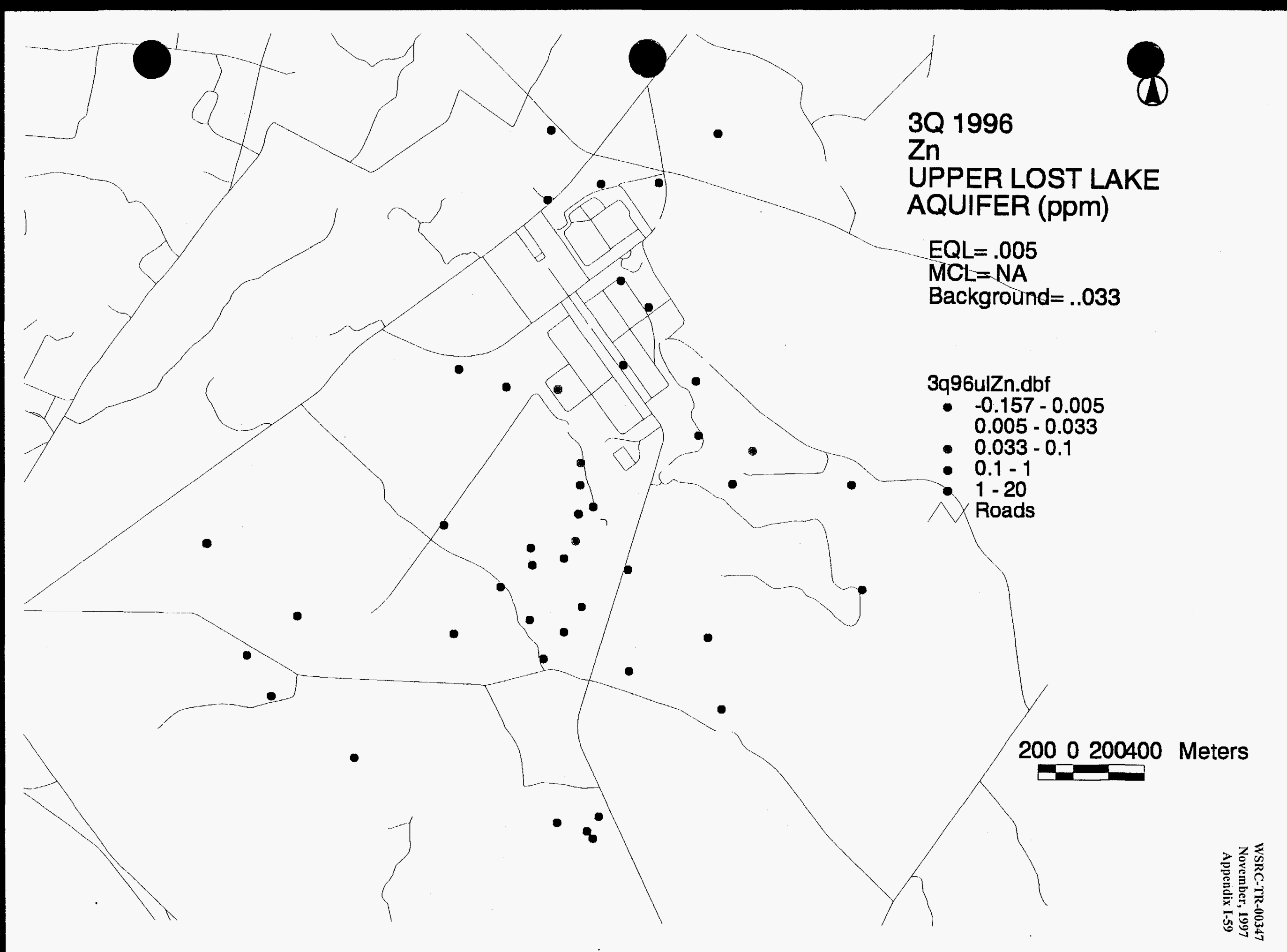




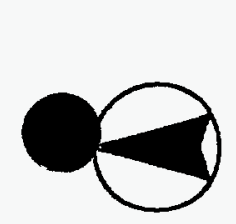

담

怘

Appendix I-60

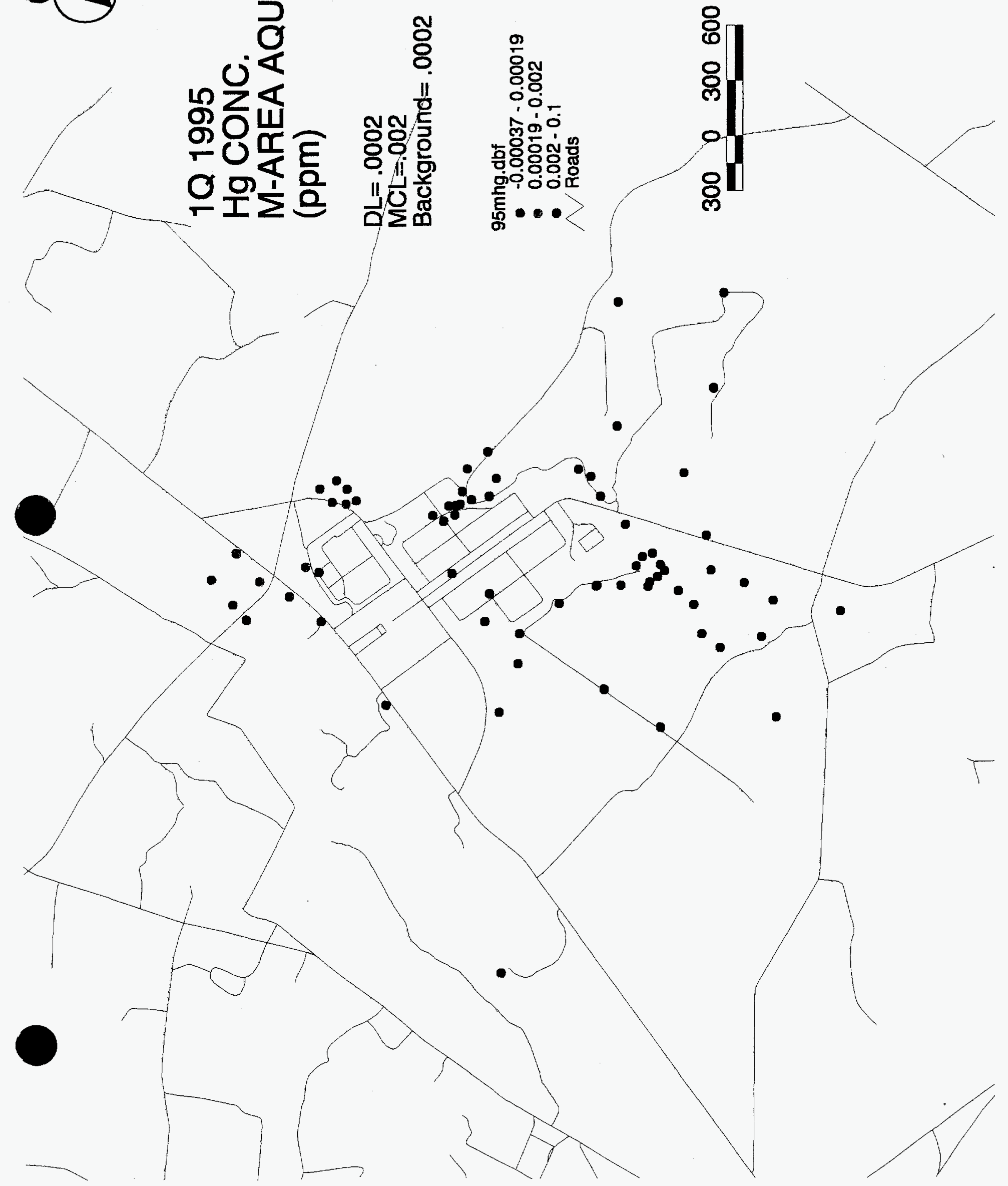




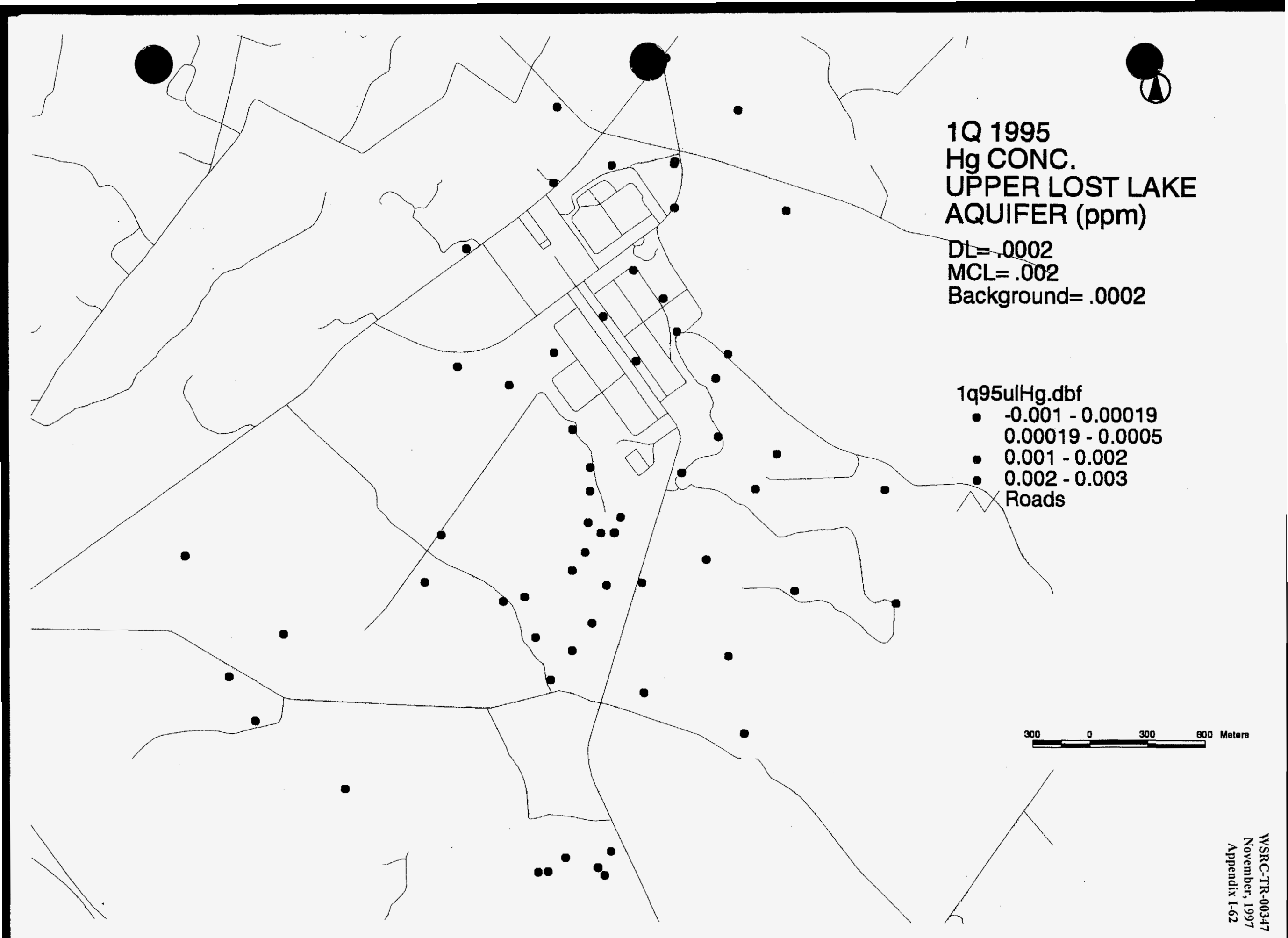




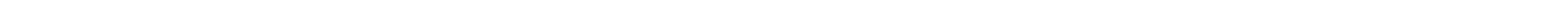




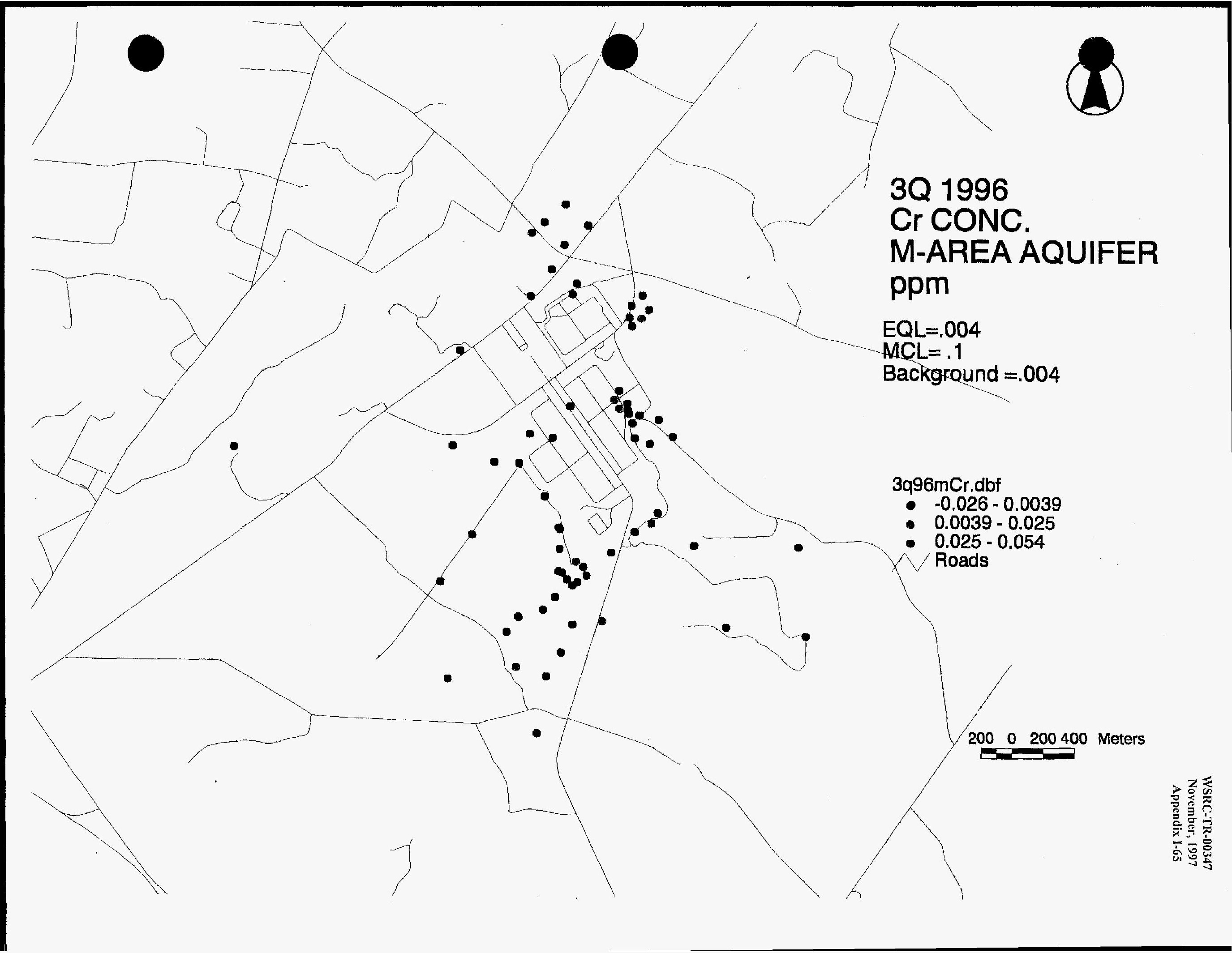




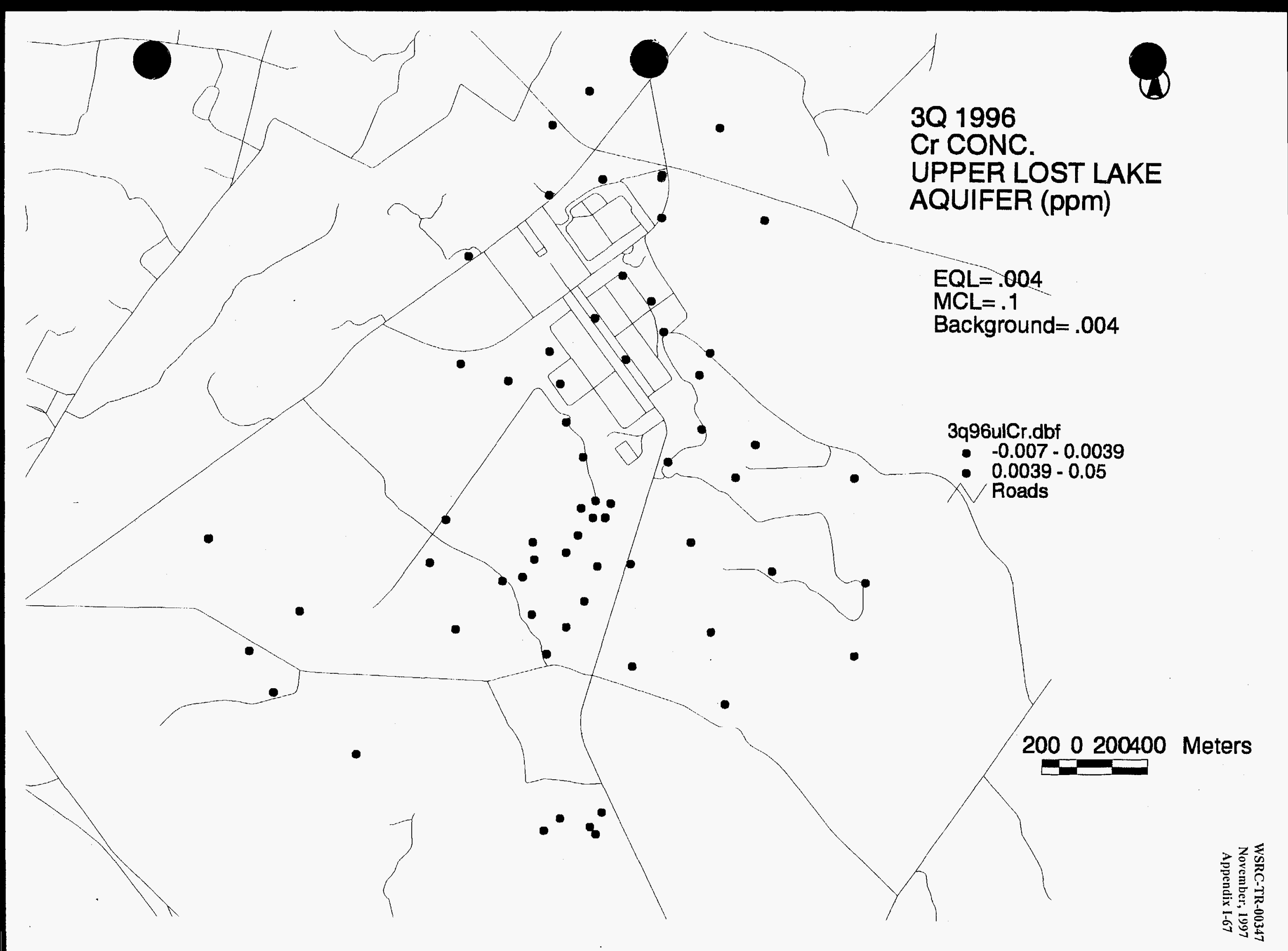




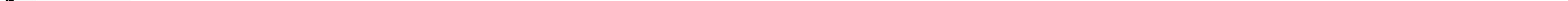




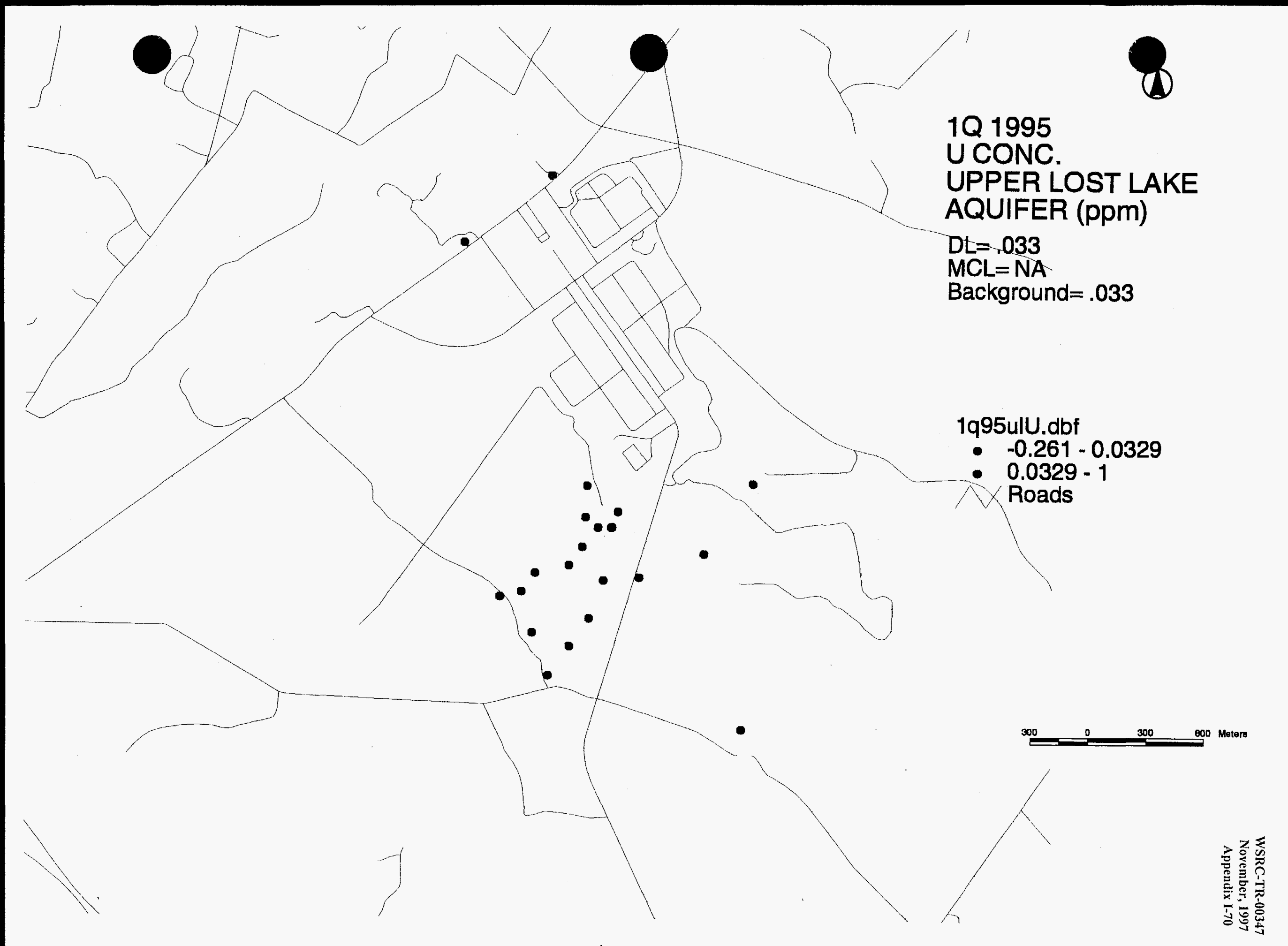




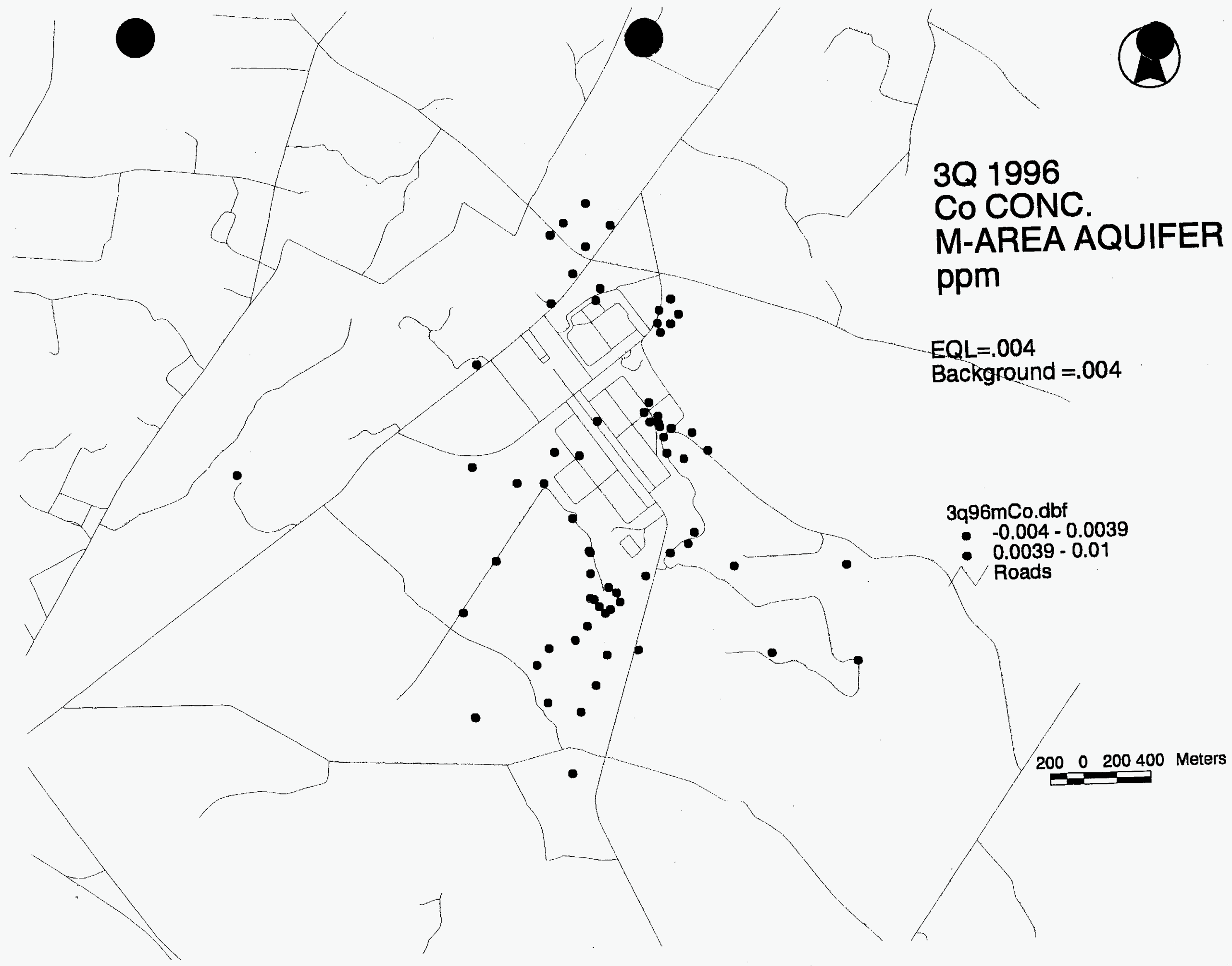



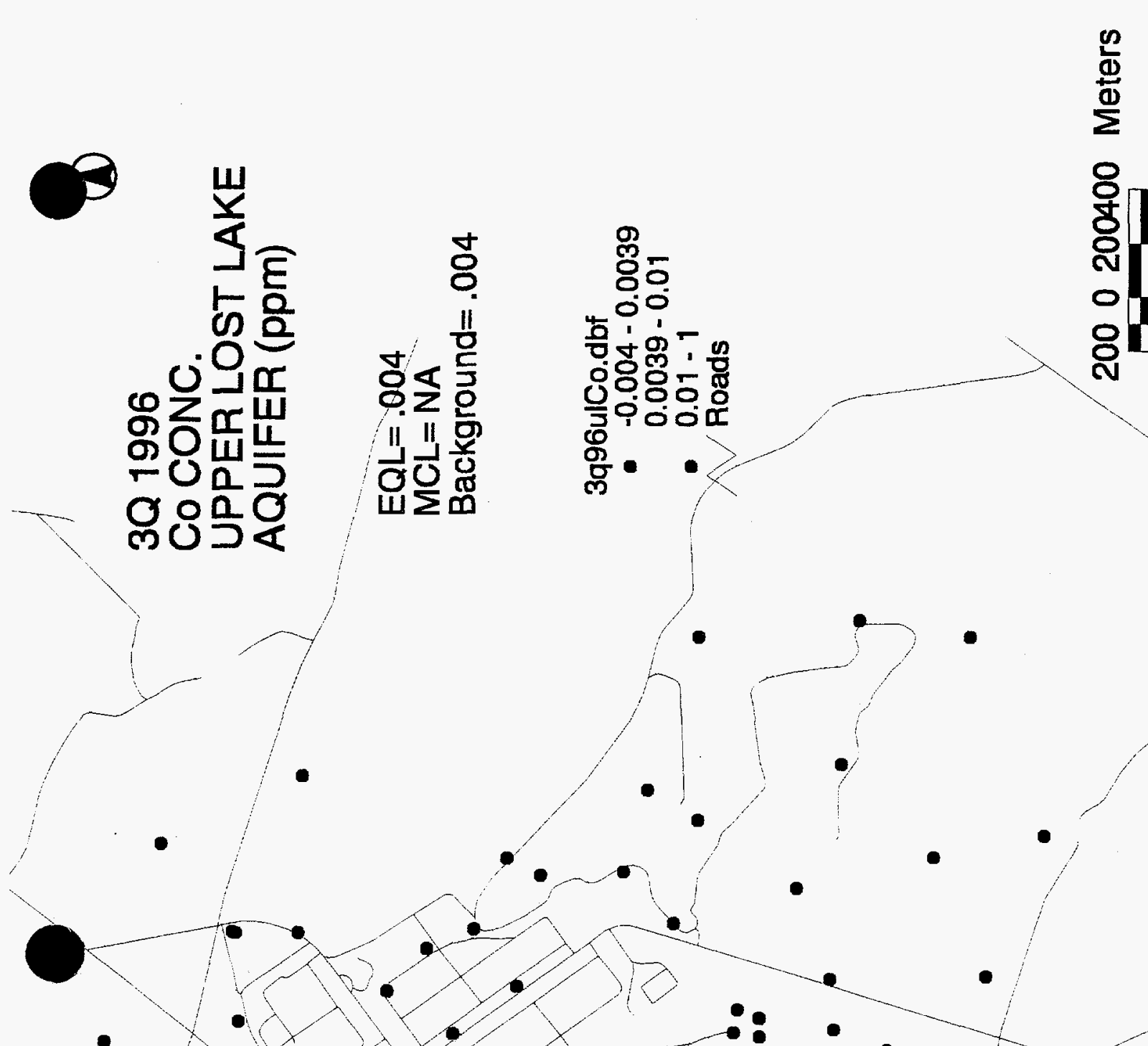


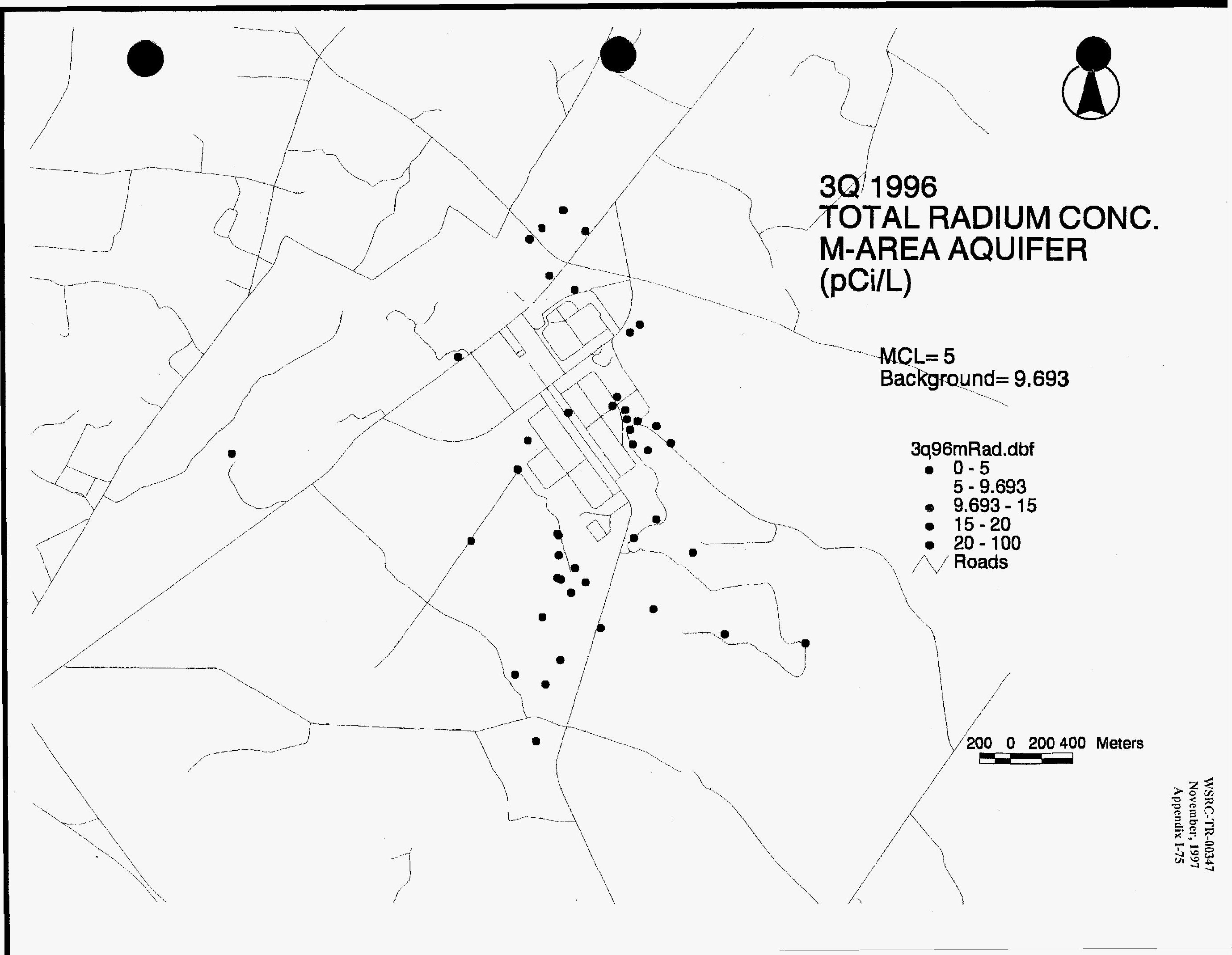




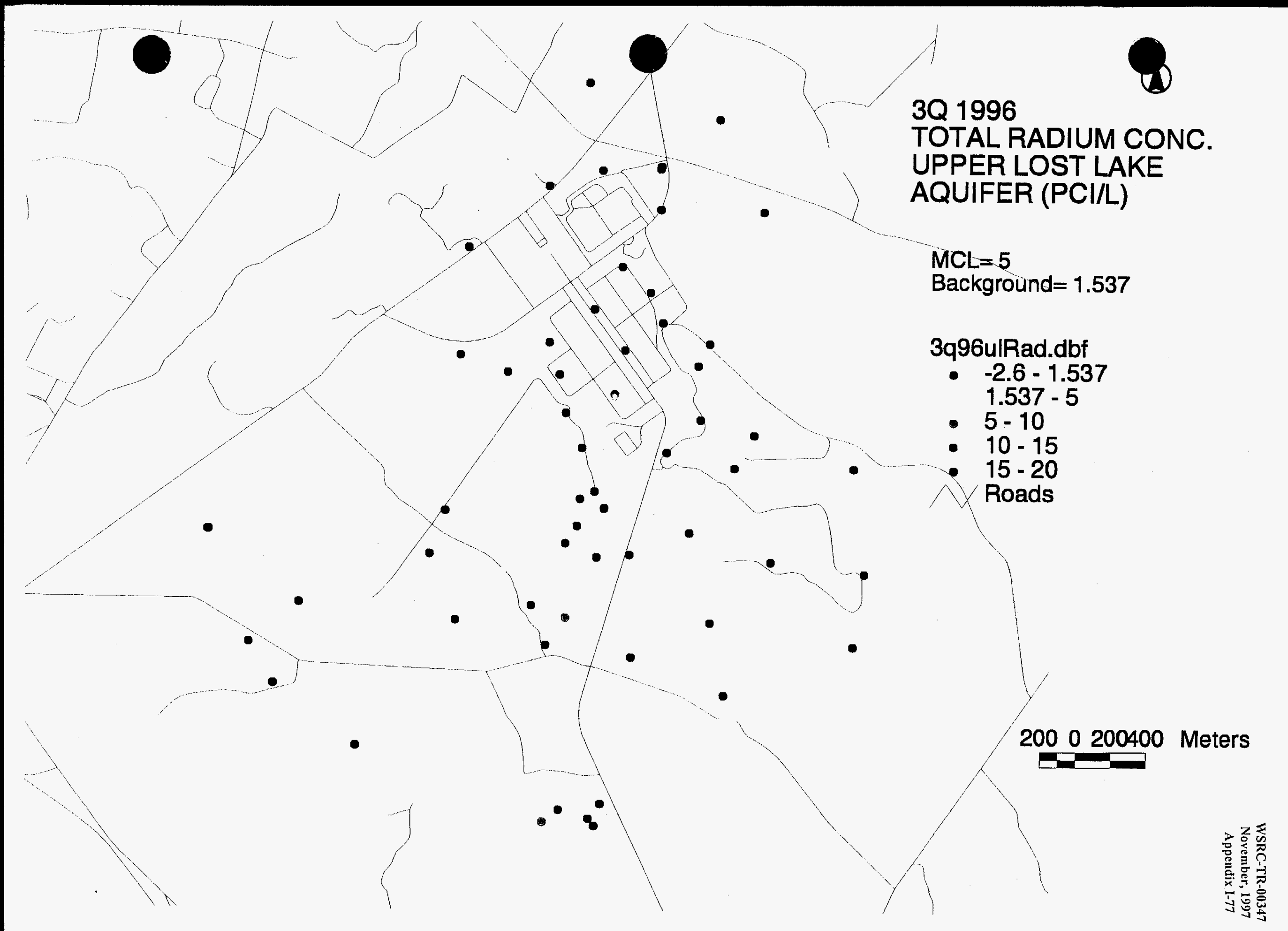




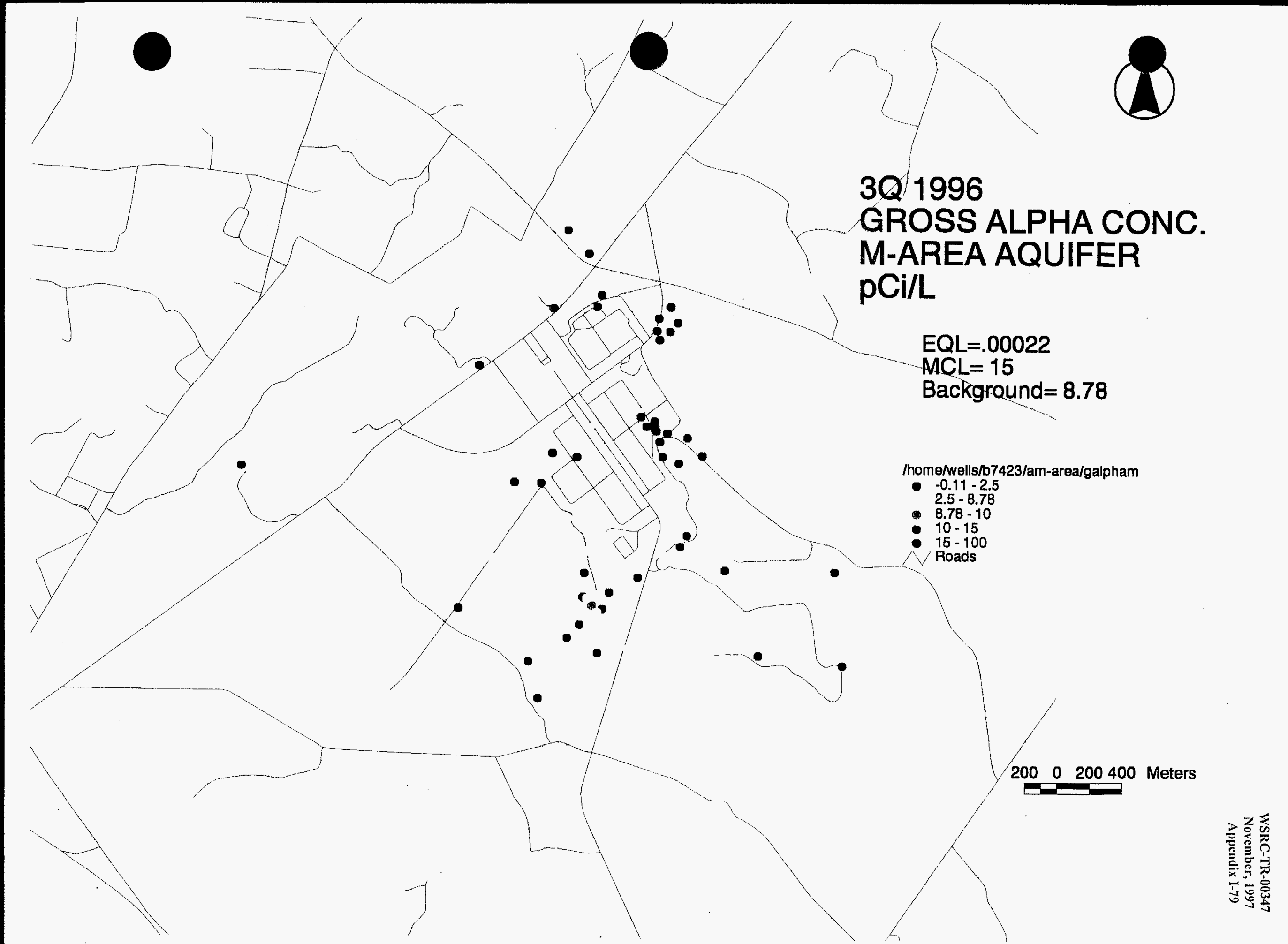




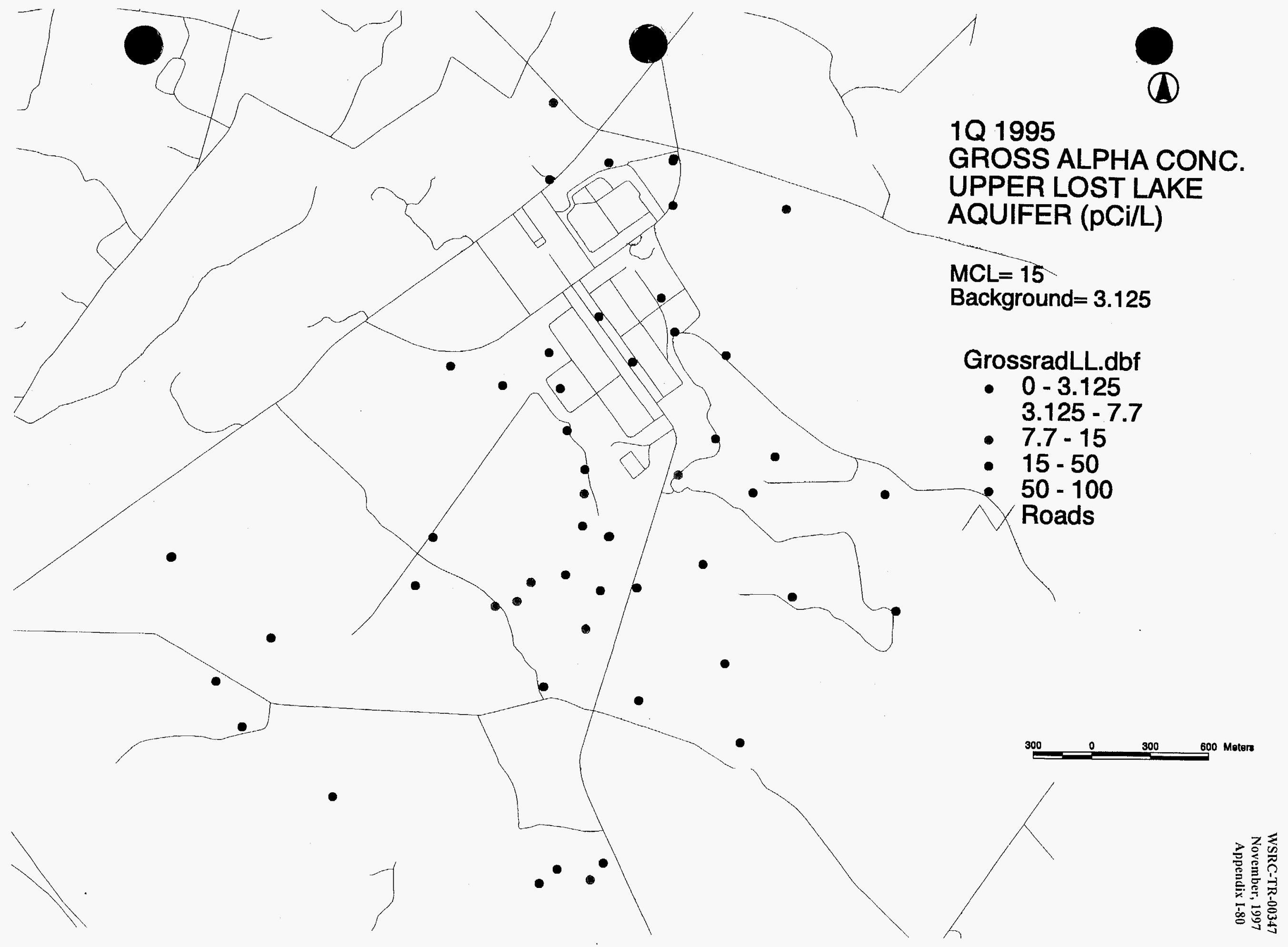




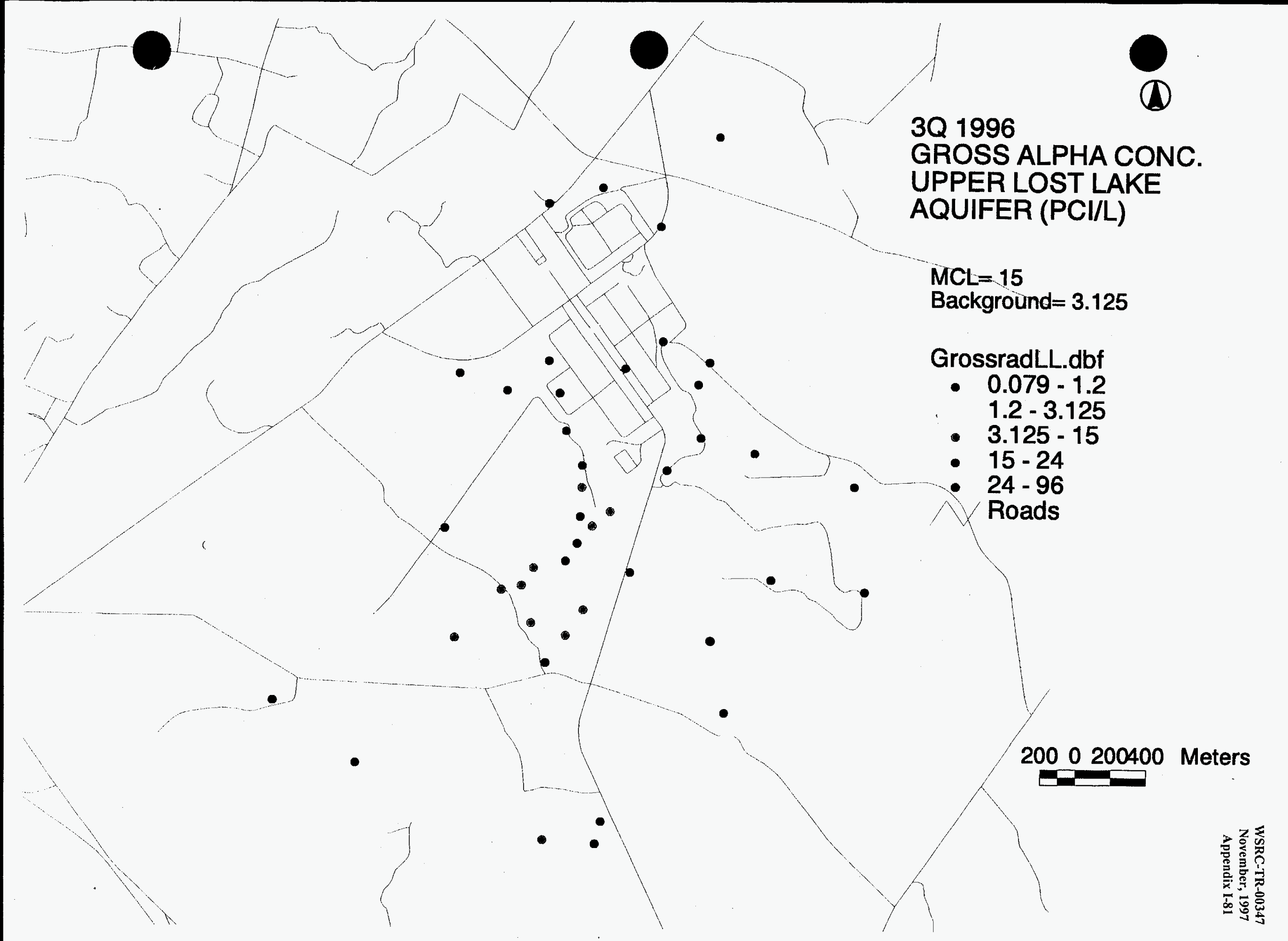




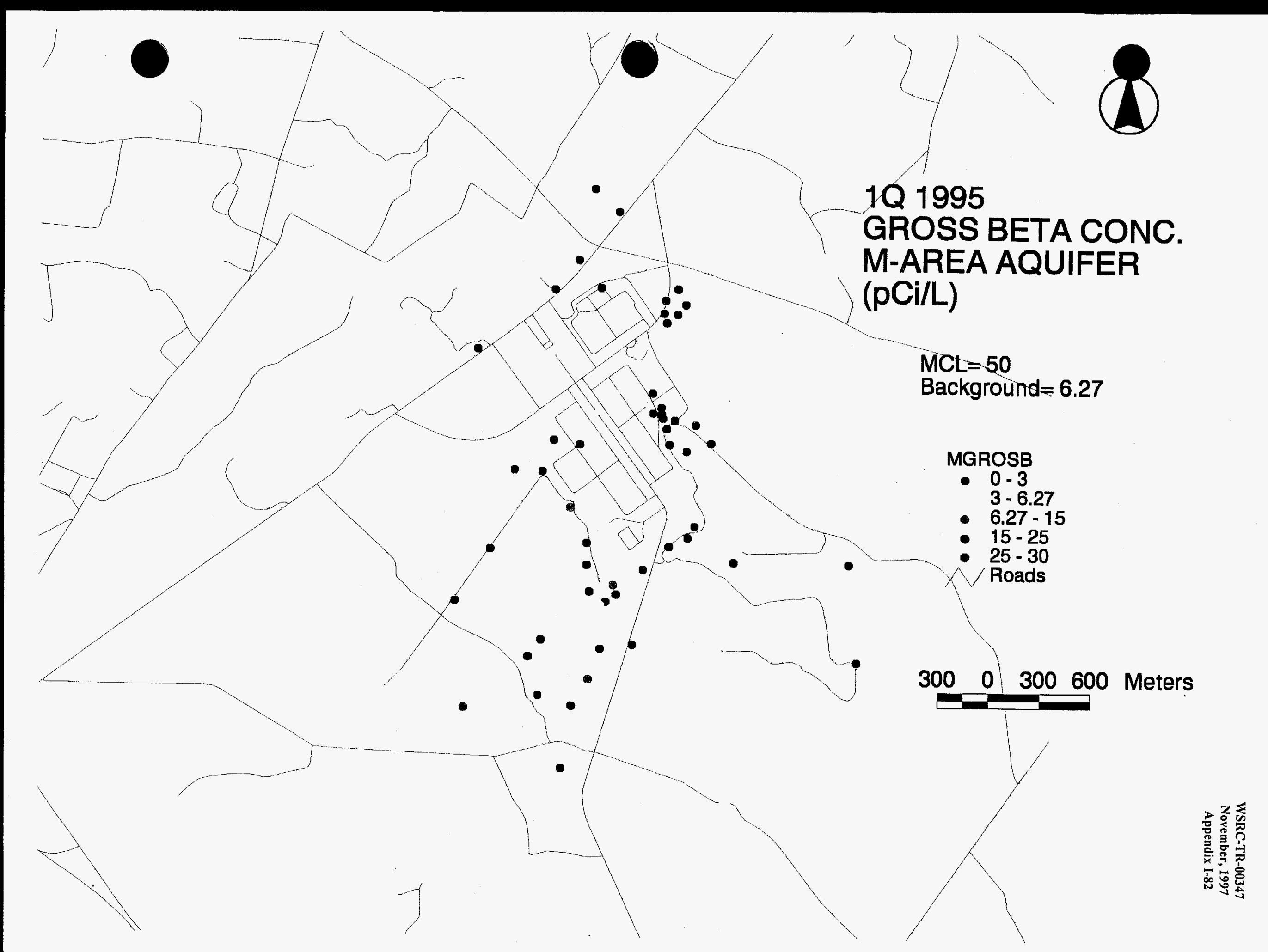




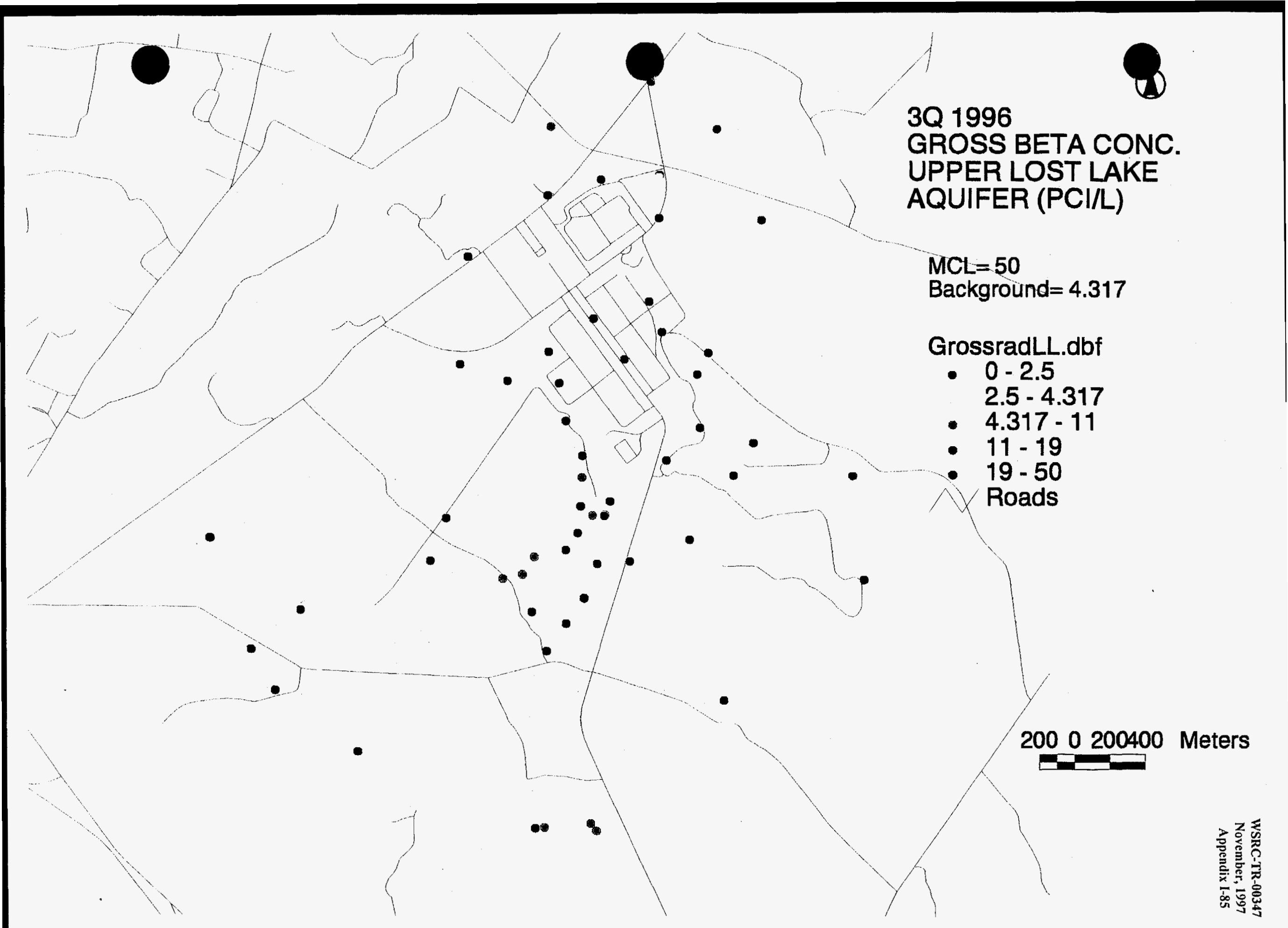


APPENDIX II

DATA 


\begin{tabular}{|c|c|c|c|c|c|c|}
\hline 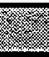 & 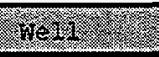 & 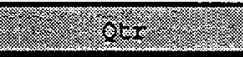 & 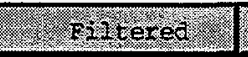 & 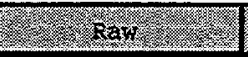 & 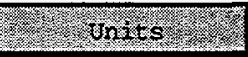 & 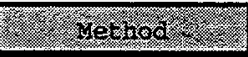 \\
\hline$A M B$ & $4 D$ & 9501 & 0.129000 & 0.129000 & MGL & EPA6010 \\
\hline Aw1 & 40 & 9501 & 0.00950 & 0.009950 & MGL & EPA6roipl: \\
\hline$A M B$ & 4D & 9501 & 3.450000 & 3.450000 & MGL & EPA 300.0 \\
\hline AMB & $4 D$ & 9501 & -0.006670 & -0.006670 & MGL & EPA6010 \\
\hline AMB & 40 & $95 Q 1$ & -0.008330 & -0.008330 & MGL & EPA335.3 \\
\hline AMB & 40 & $95 Q 1$ & -0.050000 & -0.050000 & MGL & EPA 300.0 \\
\hline AMB & $4 \mathrm{D}$ & $95 \mathrm{Q} 1$ & -0.000330 & -0.000333 & MGL & EPA7 470 \\
\hline AMB & 4D & $95 Q 1$ & 0.018200 & 0.018200 & MGL & EPA 6010 \\
\hline$A M B$ & $4 \mathrm{D}$ & $95 Q 1$ & -0.006670 & -0.006670 & MGL & EPA6010 \\
\hline AMB & $4 D$ & 9501 & 0.007810 & 0.007810 & MGL & EPA7421 \\
\hline AMB & $4 \mathrm{D}$ & $95 Q 1$ & -0.003330 & -0.003330 & MGI. & EPA7740 \\
\hline$A M B$ & $4 D$ & $95 Q 1$ & 8.600000 & 8.600000 & PCI & EPIA-010 \\
\hline AMB & $4 \mathrm{D}$ & 9501 & -0.083300 & -0.083300 & MGL & EPA.365.4 \\
\hline AMB & $4 D$ & 9501 & 0.022600 & 0.022600 & MGL & EPA 6010 \\
\hline AMB & 5 & 9501 & 0.111000 & 0.111000 & MGL & EPA6010 \\
\hline AMB & 5 & 9501 & -0.003460 & 0.003460 & MGL & EPA6010 \\
\hline AMB & 5 & 9501 & 3.250000 & 3.250000 & MGL & EPA 300.0 \\
\hline$A M B$ & 5 & 9501 & -0.006670 & -0.006570 & MGL & EPA6010 \\
\hline AMB & 5 & 9501 & -0.008330 & -0.008330 & MGL & EPA335.3 \\
\hline AMB & 5 & 9501 & -0.050000 & -0.050000 & MGL & EPA3 00.0 \\
\hline AMB & 5 & $95 \mathrm{Q} 1$ & -0.000330 & -0.000333 & MGL & EPA7470 \\
\hline AMB & 5 & $95 Q 1$ & 0.005070 & 0.005070 & MGL & EPA6010 \\
\hline AMB & 5 & $95 Q 1$ & -0.006670 & -0.006670 & MGL & EPA 6010 \\
\hline AMB & 5 & 9501 & -0.005000 & -0.005000 & MGL & EPA7421 \\
\hline$A M B$ & 5 & $95 Q 1$ & -0.003330 & -0.003330 & MGL & EPA7740 \\
\hline AMB & 5 & $95 Q 1$ & 2.900000 & 2.900000 & PCL & EPIA-010 \\
\hline AMB & 5 & 9501 & -0.083300 & -0.083300 & MGL & EPA.365.4 \\
\hline$A M B$ & 5 & 9501 & -0.003330 & -0.003330 & MGL & EPA6010 \\
\hline AMB & 7 & 9501 & 0.156000 & 0.156000 & MGL & EPA6010 \\
\hline AMB & 7 & 9501 & 0.008380 & 0.008380 & MGL & EPA 6010 \\
\hline AMB & 7 & 9501 & 2.070000 & 2.070000 & MGL & EPA300.0 \\
\hline AMB & 7 & 9501 & -0.006670 & -0.006670 & MGL & EPA6010 \\
\hline AMB & 7 & 9501 & -0.008330 & -0.008330 & MGL & EPA335.3 \\
\hline AMB & 7 & 9501 & -0.050000 & -0.050000 & MGL & EPA300.0 \\
\hline$A M B$ & 7 & 9501 & -0.000330 & -0.000333 & MGL & EPA7470 \\
\hline$A M B$ & 7 & 9501 & 0.003570 & 0.003570 & MGL & EPA 6010 \\
\hline AMB & 7 & $95 Q 1$ & -0.006670 & -0.006670 & MGL & EPA6010 \\
\hline$A M B$ & 7 & 9501 & 0.010800 & 0.010800 & MGL & EPA7421 \\
\hline$A M B$ & 7 & 9501 & -0.003330 & -0.003330 & MGL & ERA7740 \\
\hline AMB & 7 & 9501 & -0.100000 & -0.100000 & PCL & EPIA-010 \\
\hline$A M B$ & 7 & 9501 & -0.083300 & -0.083300 & MGL & EPA365.4 \\
\hline AMB & 7 & 9501 & 0.114000 & 0.114000 & MGL & EPA 6010 \\
\hline AMB & $8 \mathrm{D}$ & $95 Q 1$ & -0.033300 & -0.033300 & MGL & EPA6010 \\
\hline AMB & $8 \mathrm{D}$ & 9501 & -0.005000 & -0.005000 & MGL & EPA6010 \\
\hline AMB & $8 \mathrm{D}$ & 9501 & 2.940000 & 2.940000 & MGL & EPA300.0 \\
\hline $\mathrm{AMB}$ & $8 D$ & 9501 & -0.006670 & -0.006670 & MGL & EPA6010 \\
\hline AMB & $8 D$ & 9501 & -0.008330 & -0.008330 & MGL & EPA335.3 \\
\hline AMB & 80 & $95 \mathrm{Q} 1$ & -0.050000 & -0.050000 & MGL & EPA300.0 \\
\hline AMB & $8 D$ & 9501 & -0.000330 & -0.000333 & MGL & EPA7470 \\
\hline AMB & $8 \mathrm{D}$ & $95 Q 1$ & -0.003330 & -0.003330 & MGL & EPA 6010 \\
\hline AMB & $8 D$ & 9501 & -0.006670 & -0.006670 & MGL & EPA6010 \\
\hline AMB & $8 D$ & 9501 & -0.005000 & -0.005000 & MGL & EPA7421 \\
\hline AMB & $8 D$ & 9501 & -0.003330 & -0.003330 & MGL & EPA7740 \\
\hline AMB & 8D & 9501 & -0.300000 & -0.300000 & PCL & EPIA-010 \\
\hline
\end{tabular}


WSRC-TR-00347

November, 1997

Appendix II-2

\begin{tabular}{|c|c|c|c|c|}
\hline 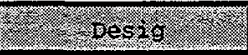 & rater & U. U⿺辶一 & (2) & F \\
\hline ALTOT & -0.033300 & 3689042.030000 & 431733.610000 & \\
\hline Barot & $17-0.005000$ & 0.0669042 .030000 & 437738.610000 & 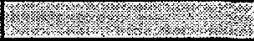 \\
\hline CL & -0.417000 & 3689042.030000 & 431733.610000 & \\
\hline CRTOT & -0.006670 & 3689042.030000 & 431733.610000 & \\
\hline CYN & -0.008330 & 3689042.030000 & 431733.610000 & \\
\hline$F$ & -0.050000 & 3689042.030000 & 431733.610000 & \\
\hline HGTOT & -0.000333 & 3689042.030000 & 431733.610000 & \\
\hline MNTOT & -0.003330 & 3689042.030000 & 431733.610000 & \\
\hline NITOT & -0.006670 & 3689042.030000 & 431733.610000 & \\
\hline PBTOT & -0.005000 & 3689042.030000 & 431733.610000 & \\
\hline SETOT & -0.003330 & 3689042.030000 & 431733.610000 & \\
\hline TOTRAD & -0.100000 & 3689042.030000 & 431733.610000 & \\
\hline TPOS & 0.043000 & 3689042.030000 & 431733.610000 & \\
\hline ZNTOT & -0.003330 & 3689042.030000 & 431733.610000 & \\
\hline ALTOT & & 3689020.560000 & 431741.020000 & \\
\hline BATOT & & 3689020.560000 & 431741.020000 & \\
\hline $\mathrm{CL}$ & -0.417000 & 3689020.560000 & 431741.020000 & \\
\hline CRTOT & & 3689020.560000 & 431741.020000 & \\
\hline $\mathrm{CYN}$ & -0.008330 & 3689020.560000 & 431741.020000 & \\
\hline$F$ & -0.050000 & 3689020.560000 & 431741.020000 & \\
\hline HGTOT & 0.000124 & 3689020.560000 & 431741.020000 & \\
\hline MNTOT & & 3689020.560000 & 431741.020000 & \\
\hline NITOT & & 3689020.560000 & 431741.020000 & \\
\hline PBTOT & -0.005000 & 3689020.560000 & 431741.020000 & \\
\hline SETOT & -0.003330 & 3689020.560000 & 431741.020000 & \\
\hline TOTRAD & & 3689020.560000 & 431741.020000 & \\
\hline TPO4 & -0.083300 & 3689020.560000 & 431741.020000 & \\
\hline ZNTOT & & 3689020.560000 & 431741.020000 & \\
\hline ALTOT & -0.033300 & 3689008.580000 & 431809.180000 & \\
\hline BATOT & -0.005000 & 3689008.580000 & 431809.180000 & \\
\hline$C L$ & -0.417000 & 3689008.580000 & 431809.180000 & \\
\hline CRTOT & -0.006670 & 3689008.580000 & 431809.180000 & \\
\hline CYN & -0.008330 & 3689008.580000 & 431809.180000 & \\
\hline $\mathrm{F}$ & -0.050000 & 3689008.580000 & 431809.180000 & \\
\hline HGTOT & -0.000333 & 3689008.580000 & 431809.180000 & \\
\hline MNTOT & -0.003330 & 3689008.580000 & 431809.180000 & \\
\hline NITOT & -0.006670 & 3689008.580000 & 431809.180000 & \\
\hline PBTOT & -0.005000 & 3689008.580000 & 431809.180000 & \\
\hline SETOT & -0.003330 & 3689008.580000 & 431809.180000 & \\
\hline TOTRAD & -0.100000 & 3689008.580000 & 431809.180000 & \\
\hline TPO4 & 0.043000 & 3689008.580000 & 431809.180000 & \\
\hline ZNTOT & -0.003330 & 3689008.580000 & 431809.180000 & \\
\hline ALTOT & & 3688957.180000 & 431762.020000 & \\
\hline BATOT & & 3688957.180000 & 431762.020000 & \\
\hline CL & -0.417000 & 3688957.180000 & 431762.020000 & \\
\hline CRTOT & & 3688957.180000 & 431762.020000 & \\
\hline CYN & -0.008330 & 3688957.180000 & 431762.020000 & \\
\hline$F$ & -0.050000 & 3688957.180000 & 431762.020000 & \\
\hline HGTOT & 0.000124 & 3688957.180000 & 431762.020000 & \\
\hline MNTOT & & 3688957.180000 & 431762.020000 & \\
\hline NITOT & & 3688957.180000 & 431762.020000 & \\
\hline PBTOT & -0.005000 & 3688957.180000 & 431762.020000 & \\
\hline SETOT & -0.003330 & 3688957.180000 & 431762.020000 & \\
\hline TOTRAD & & 3688957.180000 & 431762.020000 & \\
\hline
\end{tabular}


WSRC-TR-00347

November, 1997

Appendix II-3

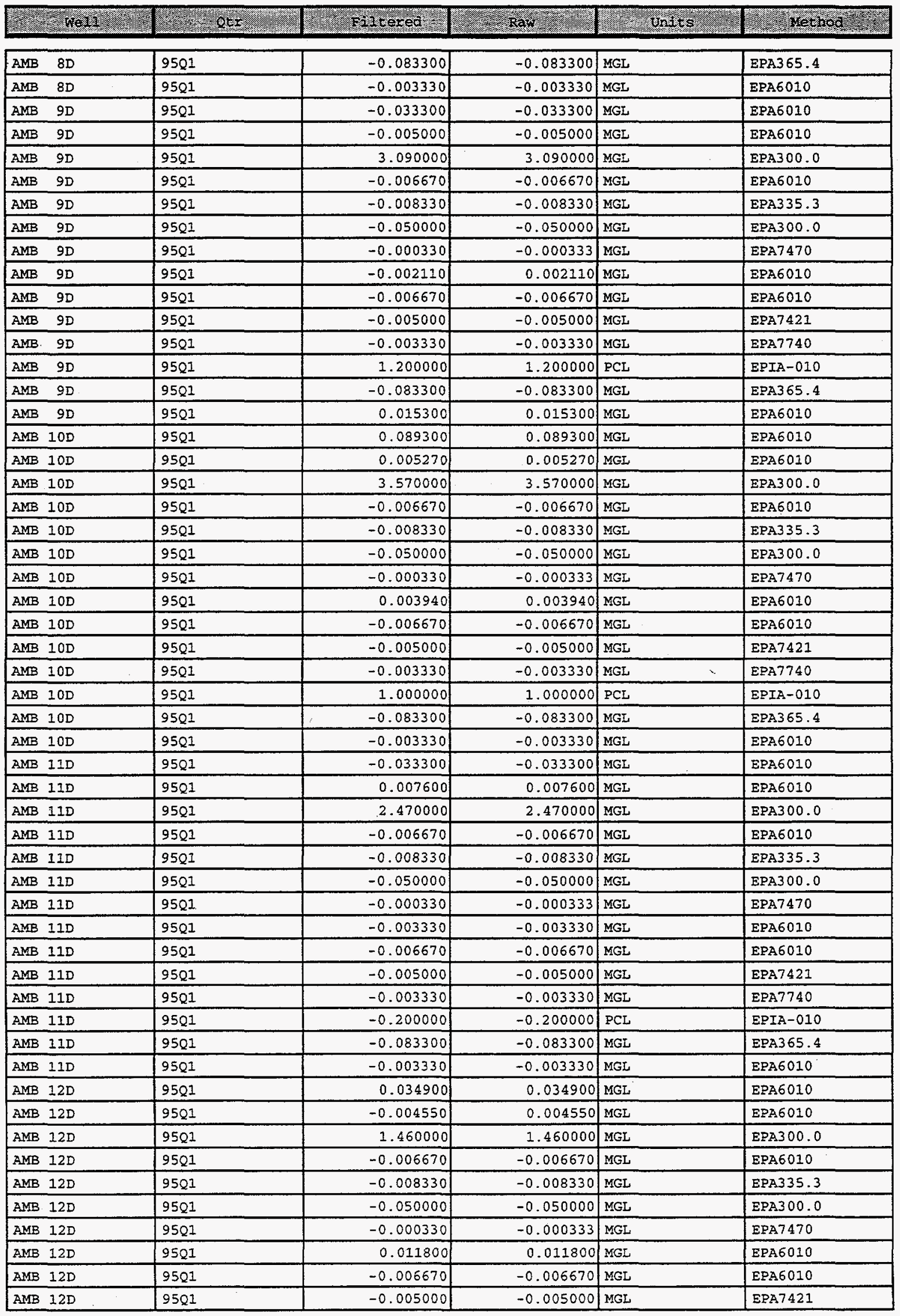




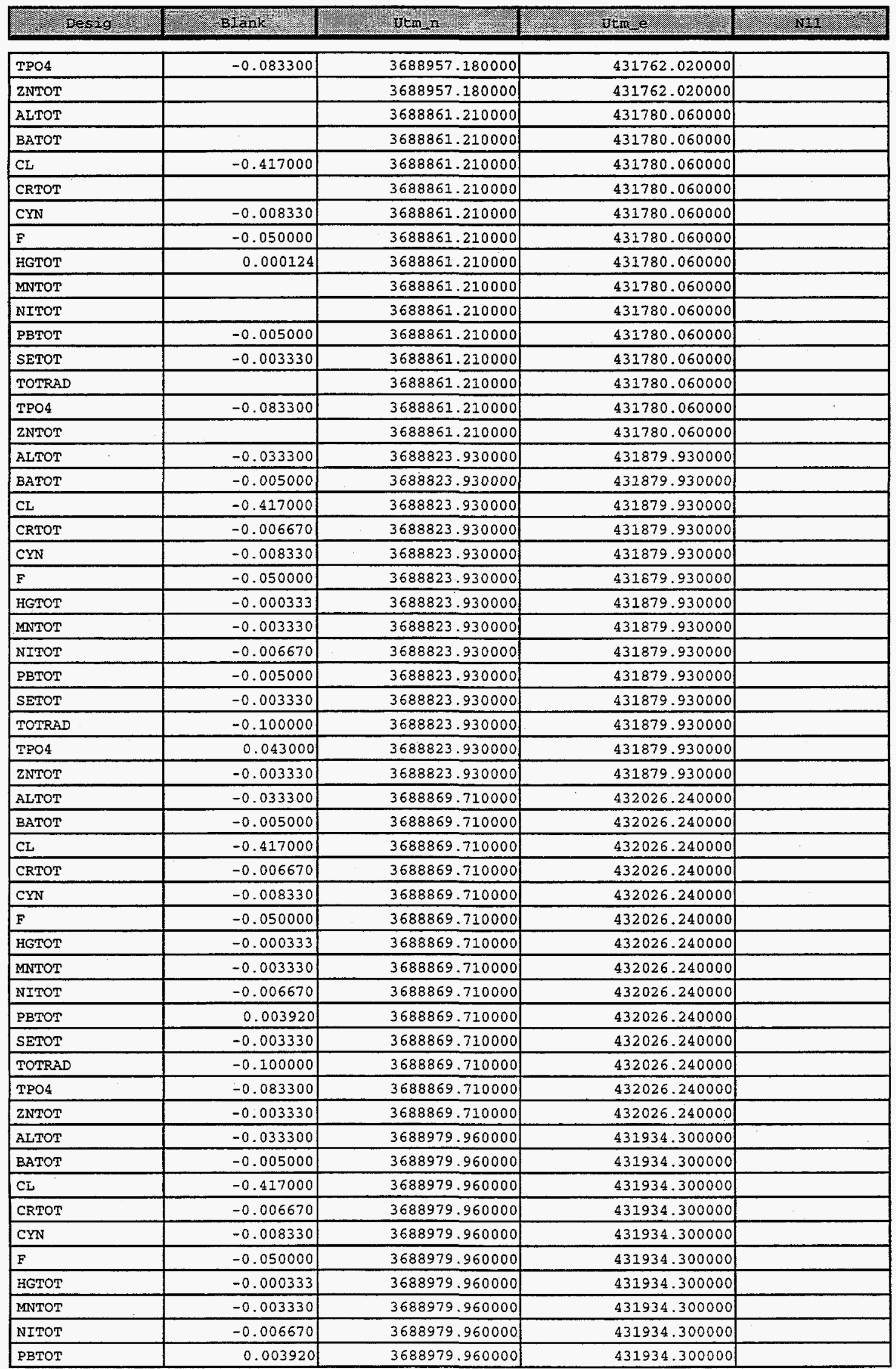


WSRC-TR-00347

November, 1997

Appendix II-5

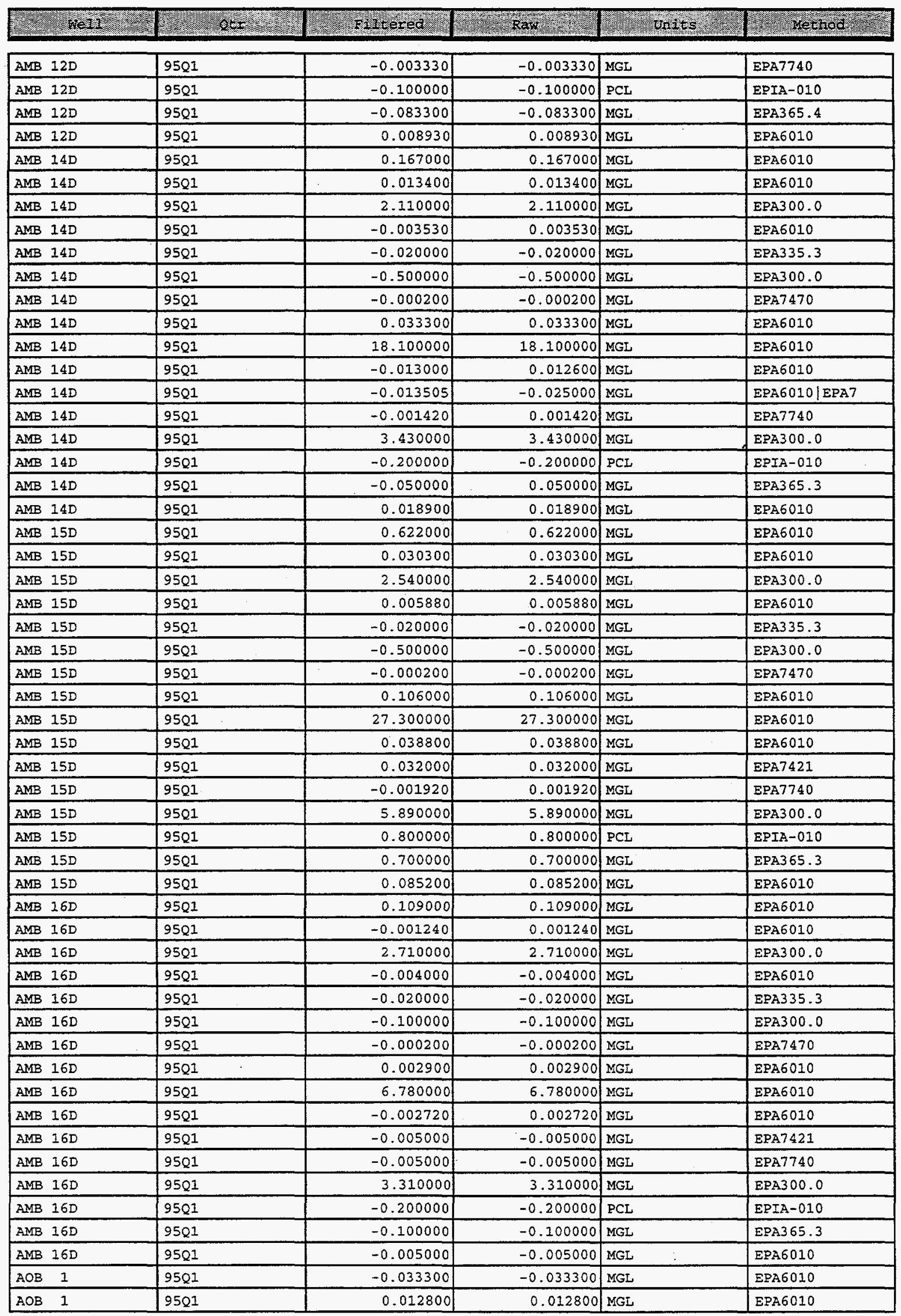


WSRC-TR-00347

November, 1997

Appendix II-6

\begin{tabular}{|c|c|c|c|c|}
\hline $2=0$ & Btani - I & (2) $6 \ln _{\mathrm{n}} \mathrm{n}$ & - & 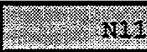 \\
\hline SETOT & -0.003330 & 3688979.960000 & 431934.300000 & \\
\hline TOTRAD & -0.100000 & 3688979.960000 & 431934.300000 & \\
\hline TPO4 & -0.083300 & 3688979.960000 & 431934.300000 & \\
\hline ZNTOT & -0.003330 & 3688979.960000 & 431934.300000 & \\
\hline ALTOT & 0.018700 & 3689049.590000 & 431679.790000 & \\
\hline BATOT & 0.000220 & 3689049.590000 & 431679.790000 & \\
\hline $\mathrm{CL}$ & -0.250000 & 3689049.590000 & 431679.790000 & \\
\hline CRTOT & -0.004000 & 3689049,590000 & 431679.790000 & \\
\hline CYN & -0.020000 & 3689049.590000 & 431679.790000 & \\
\hline$F$ & -0.100000 & 3689049.590000 & 431679.790000 & \\
\hline HGTOT & -0.000200 & 3689049.590000 & 431679.790000 & \\
\hline MNTOT & -0.002000 & 3689049.590000 & 431679.790000 & \\
\hline NATOT & -0.100000 & 3689049.590000 & 431679.790000 & \\
\hline NITOT & 0.002440 & 3689049.590000 & 431679.790000 & 2.000000 \\
\hline PETOT & -0.005000 & 3689049.590000 & 431679.790000 & 2.000000 \\
\hline SETOT & 0.001340 & 3689049.590000 & 431679.790000 & \\
\hline 504 & -1.000000 & 3689049.590000 & 431679.790000 & \\
\hline TOTRAD & & 3689049.590000 & 431679.790000 & \\
\hline TPO4 & -0.100000 & 3689049.590000 & 431679.790000 & \\
\hline ZNTOT & -0.005000 & 3689049.590000 & 431679.790000 & \\
\hline ALTOT & 0.004650 & 3689108.380000 & 431645.660000 & \\
\hline BATOT & 0.000258 & 3689108.380000 & 431645.660000 & \\
\hline$C L$ & -0.250000 & 3689108.380000 & 431645.660000 & \\
\hline CRTOT & -0.004000 & 3689108,380000 & 431645.660000 & \\
\hline CYN & -0.020000 & 3689108.380000 & 431645.660000 & \\
\hline$F$ & -0.100000 & 3689108.380000 & 431645.660000 & \\
\hline HGTOT & -0.000200 & 3689108.380000 & 431645.660000 & \\
\hline MNTOT & -0.002000 & 3689108.380000 & 431645.660000 & \\
\hline NATOT & -0.100000 & 3689108.380000 & 431645.660000 & \\
\hline NITOT & -0.010000 & 3689108.380000 & 431645.660000 & \\
\hline PBTOT & -0.005000 & 3689108.380000 & 431645.660000 & \\
\hline SETOT & 0.001340 & 3689108.380000 & 431645.660000 & \\
\hline 504 & -1.000000 & 3689108.380000 & 431645.660000 & \\
\hline TOTRAD & & 3689108.380000 & 431645.660000 & \\
\hline TPO4 & -0.100000 & 3689108.380000 & 431645.660000 & \\
\hline ZNTOT & -0.005000 & 3689108.380000 & 431645.660000 & \\
\hline ALTOT & 0.004650 & 3689082.430000 & 431730.020000 & \\
\hline BATOT & 0.000258 & 3689082.430000 & 431730.020000 & \\
\hline$C L$ & -0.250000 & 3689082.430000 & 431730.020000 & \\
\hline CRTOT & -0.004000 & 3689082.430000 & 431730.020000 & \\
\hline CYN & -0.020000 & 3689082.430000 & 431730.020000 & \\
\hline$F$ & -0.100000 & 3689082.430000 & 431730.020000 & \\
\hline HGTOT & -0.000200 & 3689082.430000 & 431730.020000 & \\
\hline MNTOT & 0.000240 & 3689082.430000 & 431730.020000 & \\
\hline NATOT & -0.100000 & 3689082.430000 & 431730.020000 & \\
\hline NITOT & -0.010000 & 3689082.430000 & 431730.020000 & \\
\hline PBTOT & -0.005000 & 3689082.430000 & 431730.020000 & \\
\hline SETOT & 0.001340 & 3689082.430000 & 431730.020000 & \\
\hline 504 & -1.000000 & 3689082.430000 & 431730.020000 & \\
\hline TOTRA.D & & 3689082.430000 & 431730.020000 & \\
\hline TPO4 & -0.100000 & 3689082.430000 & 431730.020000 & \\
\hline ZNTOT & -0.005000 & 3689082.430000 & 431730.020000 & \\
\hline ALTOT & 0.020000 & 3688309.330000 & 431888.900000 & \\
\hline ВАTOT & -0.005000 & 3688309.330000 & 431888.900000 & \\
\hline
\end{tabular}


WSRC-TR-00347

November, 1997

Appendix II-7

\begin{tabular}{|c|c|c|c|c|c|c|}
\hline 20. & Writ & 10 & tistrered & 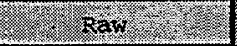 & (1080 & Uevisad \\
\hline $\mathrm{AOB}$ & 1 & 9501 & -0.006670 & -0.006670 & MGL & EPA 6010 \\
\hline$A O B$ & 1 & 9501 & -0.008330 & -0.008330 & MGL & EPA 335.3 \\
\hline$A O B$ & 1 & 9501 & -0.000330 & -0.000333 & MGL & EPA7470 \\
\hline $\mathrm{AOB}$ & 1 & 9501 & -0.006670 & -0.006670 & MGL & EPA6010 \\
\hline $\mathrm{AOB}$ & 1 & 9501 & -0.005000 & -0.005000 & MGL & EPA7421 \\
\hline$A O B$ & 1 & 9501 & -0.003330 & -0.003330 & MGL & EPA7740 \\
\hline$A O B$ & 1 & 9501 & 0.900000 & 0.900000 & PCL & EPIA-010 \\
\hline$A O B$ & 1 & 9501 & -0.003330 & -0.003330 & MGL & EPA6010 \\
\hline $\mathrm{AOB}$ & 2 & 9501 & -0.033300 & -0.033300 & MGL & EPA6010 \\
\hline$A O B$ & 2 & 9521 & -0.003520 & 0.003520 & MGL & EPA 6010 \\
\hline $\mathrm{AOB}$ & 2 & $95 Q 1$ & -0.006670 & -0.006670 & MGL & EPA6010 \\
\hline $\mathrm{AOB}$ & 2 & $95 Q 1$ & -0.008330 & -0.008330 & MGL & EPA335.3 \\
\hline $\mathrm{AOB}$ & 2 & 9501 & -0.000330 & -0.000333 & MGL & EPA7470 \\
\hline $\mathrm{AOB}$ & 2 & 9501 & -0.004670 & 0.004670 & MGL & EPA6010 \\
\hline $\mathrm{AOB}$ & 2 & 9501 & 0.005050 & 0.005050 & MGL & EPA 7421 \\
\hline$A O B$ & 2 & 9501 & -0.003330 & -0.003330 & MGL & EPA7740 \\
\hline $\mathrm{AOB}$ & 2 & 9501 & 0.800000 & 0.800000 & PCL & EPIA-010 \\
\hline$A O B$ & 2 & 9501 & 0.014900 & 0.014900 & MGL & EPA6010 \\
\hline ASB & $1 \mathrm{~A}$ & 9501 & -0.033300 & -0.033300 & MGL & EPA6010 \\
\hline ASB & $1 \mathrm{~A}$ & 9501 & 0.013600 & 0.013600 & MGL & EPA6010 \\
\hline ASB & $1 \mathrm{~A}$ & $95 Q 1$ & 4.510000 & 4.510000 & MGL & EPA300.0 \\
\hline $\mathrm{ASB}$ & $1 \mathrm{~A}$ & $95 Q 1$ & -0.006670 & -0.006670 & MGL & EPA6010 \\
\hline ASB & $1 \mathrm{~A}$ & 9501 & 0.017600 & 0.017600 & MGL & EPA 6010 \\
\hline$A S B$ & $1 \mathrm{~A}$ & 9501 & -0.008330 & -0.008330 & MGL & EPA335.3 \\
\hline ASB & $1 \mathrm{~A}$ & 9501 & -0.050000 & -0.050000 & MGL & EPA300.0 \\
\hline ASB & $1 \mathrm{~A}$ & 9501 & -0.000330 & -0.000333 & MGL & EPA7470 \\
\hline$A S B$ & 18 & 9501 & -0.002190 & 0.002190 & MGI & EPA6010 \\
\hline $\mathrm{ASB}$ & $1 \mathrm{~A}$ & 9501 & 10.300000 & 10.300000 & MGL & EPA6010 \\
\hline$A S B$ & $1 \mathrm{~A}$ & 9501 & -0.006670 & -0.006670 & MGL & EPA6010 \\
\hline ASB & $1 \mathrm{~A}$ & 9501 & -0.015000 & -0.015000 & MGL & EPA6010|EPA7 \\
\hline ASB & $1 \mathrm{~A}$ & 9501 & -0.003330 & -0.003330 & MGL & EPA7740 \\
\hline ASB & $1 \mathrm{~A}$ & 9501 & 6.690000 & 6.690000 & MGL & EPA 300.0 \\
\hline $\mathrm{ASB}$ & $1 \mathrm{~A}$ & 9501 & 1.200000 & 1.200000 & PCL & EPIA-010 \\
\hline$A S B$ & $1 \mathrm{~A}$ & 9501 & -0.083300 & -0.083300 & MGL & EPA 365.4 \\
\hline $\mathrm{ASB}$ & $1 \mathrm{~A}$ & 9501 & -0.003330 & -0.003330 & MGL & EPA6010 \\
\hline ASB & $2 \mathrm{AR}$ & 9501 & -0.037867 & 0.032317 & MGL & EPA200.7|EPA \\
\hline ASB & $2 \mathrm{AR}$ & 95Q1 & 0.011100 & 0.011300 & MGI & EPA200.7|EPA \\
\hline $\mathrm{ASB}$ & $2 A R$ & 9501 & 4.360000 & 4.380000 & MGL & EPA 300.0 \\
\hline $\mathrm{ASB}$ & $2 A R$ & $95 Q 1$ & -0.006020 & 0.006020 & MGL & EPA200.7|EPA \\
\hline ASB & $2 \mathrm{AR}$ & 9501 & -0.008923 & -0.018000 & MGL & EPA2 $00.7 \mid \mathrm{EPA}$ \\
\hline ASB & $2 \mathrm{AR}$ & 9501 & -0.013365 & -0.013365 & MGL & EPA335.2/EPA \\
\hline $\mathrm{ASB}$ & $2 \mathrm{AR}$ & 9501 & -0.050000 & 0.041650 & MGL & EPA300.0|EPA \\
\hline ASB & $2 \mathrm{AR}$ & 9501 & -0.000237 & 0.000182 & MGL & EPA245.1|EPA \\
\hline ASB & $2 \mathrm{AR}$ & 95Q1 & -0.004643 & 0.004088 & MGL & EPA200.7|EPA \\
\hline ASB & $2 \mathrm{AR}$ & 9501 & 10.790000 & 10.306667 & MGL & EPA200.7|EPA \\
\hline ASB & $2 \mathrm{AR}$ & 9501 & -0.002753 & 0.001642 & MGL & EPA200.7|EPA \\
\hline ASB & $2 A R$ & 9501 & 0.301000 & 0.295000 & MGL & EPA353.2 \\
\hline ASB & $2 \mathrm{AR}$ & 9501 & -0.007733 & -0.013000 & MGL & EPA200.7 EPA \\
\hline ASB & $2 A R$ & 9501 & -0.013777 & -0.013777 & MGL & ERA200.7/EPA \\
\hline ASB & $2 \mathrm{AR}$ & 9501 & 7.355000 & 7.372500 & MGL & EPA300.0 \\
\hline ASB & $2 A R$ & 9501 & 0.716667 & 0.833333 & PCL & EPA903. OMOD \\
\hline ASB & $2 A R$ & 9501 & -0.036016 & 0.019356 & MGI & EPA365.2 $\mid \mathrm{EPA}$ \\
\hline ASB & $2 \mathrm{AR}$ & $95 Q 1$ & -0.003143 & 0.002588 & MGL & EPA200.7/EPA \\
\hline ASB & $3 A R$ & $95 \mathrm{Q1}$ & -0.033300 & -0.033300 & MGL & EPA6010 \\
\hline
\end{tabular}


WSRC-TR-00347

November, 1997

Appendix II-8

\begin{tabular}{|c|c|c|c|c|}
\hline 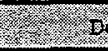 & Blonk & Uan & $2.346 \mathrm{n}=$ & 11 \\
\hline CRTOT & -0.006670 & 3688309.330000 & 431888.900000 & \\
\hline $\mathrm{CnN}$ & -0.008330 & 3688309.330000 & 431888.900000 & \\
\hline HGTOT & -0.000333 & 3688309.330000 & 431888.900000 & \\
\hline NITOT & -0.006670 & 3688309.330000 & 431888.900000 & \\
\hline PBTOT & -0.005000 & 3688309.330000 & 431888.900000 & \\
\hline SETOT & -0.003330 & 3688309.330000 & 431888.900000 & \\
\hline TOTRAD & & 3688309.330000 & 431888.900000 & \\
\hline ZNTOT & 0.002000 & 3688309.330000 & 431888.900000 & \\
\hline ALTOT & -0.033300 & 3688376.570000 & 431929.960000 & \\
\hline BATOT & -0.005000 & 3688376.570000 & 431929.960000 & \\
\hline CRTOT & -0.005670 & 3688376.570000 & 431929.960000 & \\
\hline $\mathrm{CYN}$ & -0.008330 & 3688376.570000 & 431929.960000 & \\
\hline HGTOT & -0.000333 & 3688376.570000 & 431929.960000 & \\
\hline NITOT & -0.006670 & 3688376.570000 & 431929.960000 & \\
\hline PBTOT & -0.005000 & 3688376.570000 & 431929.960000 & \\
\hline SETOT & -0.003330 & 3688376.570000 & 431929.960000 & \\
\hline TOTRAD & -0.300000 & 3688376.570000 & 431929.960000 & \\
\hline ZNTOT & -0.003330 & 3688376.570000 & 431929.960000 & \\
\hline ALTOT & -0.033300 & 3689583.820000 & 431763.230000 & \\
\hline BATOT & -0.005000 & 3689583.820000 & 431763.230000 & \\
\hline $\mathrm{CL}$ & -0.417000 & 3689583.820000 & 431763.230000 & \\
\hline CRTOT & -0.006670 & 3689583.820000 & 431763.230000 & \\
\hline CUTOT & -0.006670 & 3689583.820000 & 431763.230000 & \\
\hline $\mathrm{CYN}$ & -0.008330 & 3689583.820000 & 431763.230000 & \\
\hline$F$ & 0.040000 & 3689583.820000 & 431763.230000 & \\
\hline HGTOT & -0.000333 & 3689583.820000 & 431763.230000 & 2.000000 \\
\hline MNTOT & -0.003330 & 3689583.820000 & 431763.230000 & \\
\hline NATOT & -0.015700 & 3689583.820000 & 431763.230000 & \\
\hline NITOT & -0.006670 & 3689583.820000 & 431763.230000 & \\
\hline PBTOT & -0.015000 & 3689583.820000 & 431763.230000 & 2.000000 \\
\hline SETOT & -0.003330 & 3689583.820000 & 431763.230000 & \\
\hline SO4 & -1.670000 & 3689583.820000 & 431763.230000 & \\
\hline TOTRAD & -0.200000 & 3689583.820000 & 431763.230000 & \\
\hline TPO4 & -0.083300 & 3689583.820000 & 431763.230000 & \\
\hline ZNTOT & -0.003330 & 3689583.820000 & 431763.230000 & \\
\hline ALTOT & 0.024000 & 3689635.650000 & 431826.400000 & 3.000000 \\
\hline BATOT & -0.006600 & 3689635.650000 & 431826.400000 & 3.000000 \\
\hline $\mathrm{CL}$ & -0.308500 & 3689535.650000 & 431826.400000 & 4.000000 \\
\hline CRTOT & -0.010000 & 3689635.650000 & 431826.400000 & 3.000000 \\
\hline CUTOT & -0.004000 & 3689635.650000 & 431826.400000 & 3.000000 \\
\hline CYN & -0.013365 & 3689635.650000 & 431826.400000 & 4.000000 \\
\hline$F$ & -0.038600 & 3689635.650000 & 431826.400000 & 4.000000 \\
\hline HGTOT & 0.003329 & 3689535.650000 & 431826.400000 & 3.000000 \\
\hline MNTOT & -0.004910 & 3689635.650000 & 431826.400000 & 3.000000 \\
\hline NATOT & 0.012933 & 3689635.650000 & 431826.400000 & 3.000000 \\
\hline NITOT & 0.001565 & 3689635.650000 & 431826.400000 & 3.000000 \\
\hline $\mathrm{NO} 3$ & -0.060000 & 3689635.650000 & 431826.400000 & 2.000000 \\
\hline PBTOT & 0.004197 & 3689635.650000 & 431826.400000 & 3.000000 \\
\hline SETOT & 0.002155 & 3689635.650000 & 431826.400000 & 3.000000 \\
\hline $\mathrm{SO4}$ & -1.185000 & 3689635.650000 & 431826.400000 & 4.000000 \\
\hline TOTRAD & 0.300000 & 3689635.650000 & 431826.400000 & 3.000000 \\
\hline TPO4 & -0.051320 & 3689635.650000 & 431826.400000 & 5.000000 \\
\hline zNTOT & 0.002267 & 3689635.650000 & 431826.400000 & 3.000000 \\
\hline ALTOT & 0.020000 & 3689690.930000 & 431874.090000 & \\
\hline
\end{tabular}


WSRC-TR-00347

November, 1997

Appendix II-9

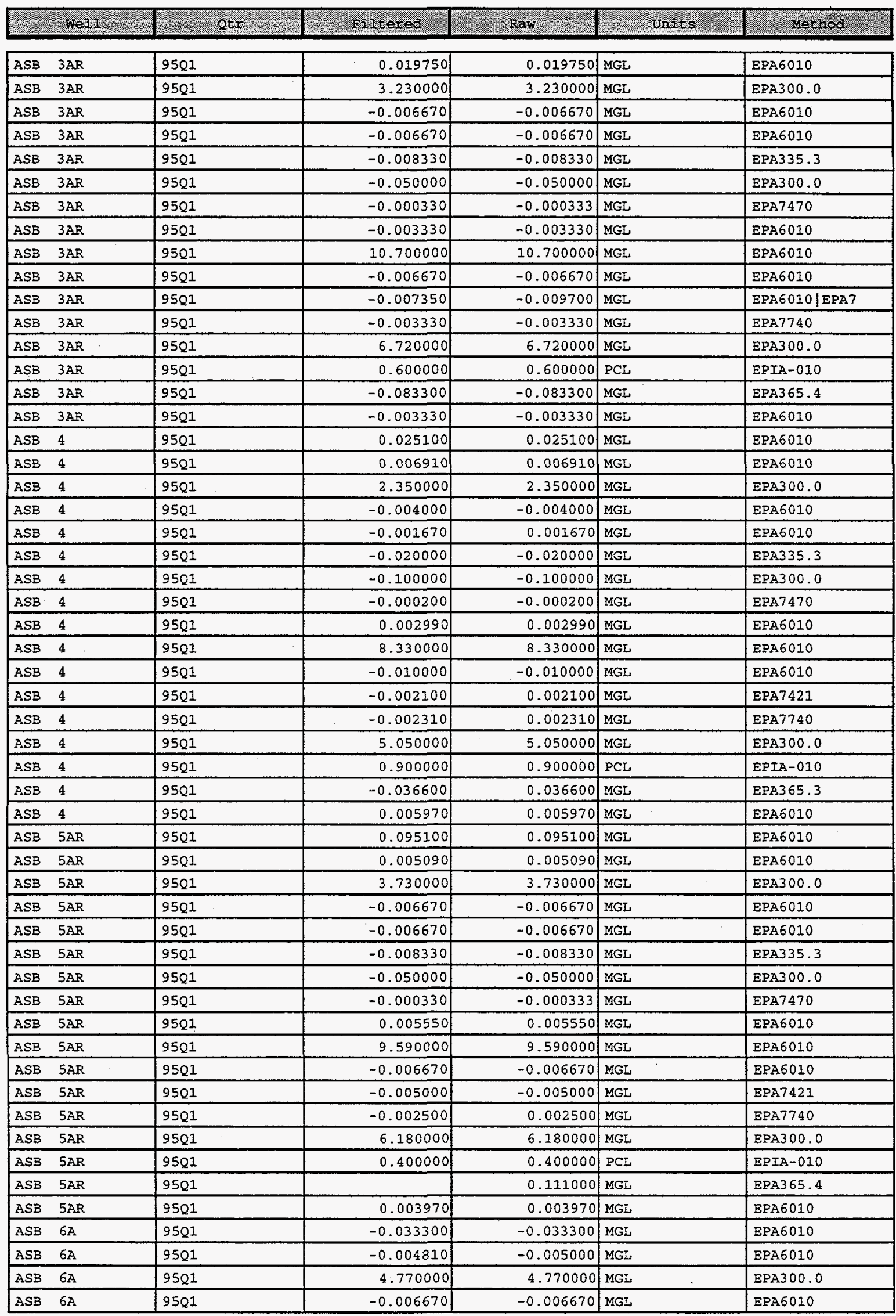




\begin{tabular}{|c|c|c|c|c|}
\hline CL & -0.417000 & 3689690.930000 & 431874.090000 & \\
\hline CRTOT & 0.004000 & 3689690.930000 & 431874.090000 & \\
\hline CUTOT & -0.006670 & 3689690.930000 & 431874.090000 & \\
\hline CYN & -0.008330 & 3689690.930000 & 431874.090000 & \\
\hline$E$ & -0.050000 & 3689690.930000 & 431874.090000 & \\
\hline HGTOT & -0.000333 & 3689690.930000 & 431874.090000 & \\
\hline MNTOT & -0.003330 & 3689690.930000 & 431874.090000 & 2.000000 \\
\hline NATOT & 0.010000 & 3689690.930000 & 431874.090000 & \\
\hline NITOT & -0.006670 & 3689690.930000 & 431874.090000 & 2.000000 \\
\hline PBTOT & -0.011300 & 3689690.930000 & 431874.090000 & 2.000000 \\
\hline SETOT & -0.003330 & 3689690.930000 & 431874.090000 & \\
\hline SO4 & -1.670000 & 3689690.930000 & 431874.090000 & \\
\hline TOTRAD & 0.000000 & 3689690.930000 & 431874.090000 & \\
\hline TPO4 & -0.083300 & 3689690.930000 & 431874.090000 & \\
\hline ZNTOT & 0.002000 & 3689690.930000 & 431874.090000 & \\
\hline ALTOT & -0.020000 & 3689783.530000 & 431830.120000 & \\
\hline BATOT & 0.000380 & 3689783.530000 & 431830.120000 & \\
\hline$C L$ & -0.250000 & 3689783.530000 & 431830.120000 & \\
\hline CRTOT & -0.004000 & 3689783.530000 & 431830.120000 & \\
\hline CUTOT & -0.004000 & 3689783.530000 & 431830.120000 & \\
\hline CYN & -0.020000 & 3689783.530000 & 431830.120000 & \\
\hline$F$ & -0.100000 & 3689783.530000 & 431830.120000 & \\
\hline HGTOT & 0.000053 & 3689783.530000 & 431830.120000 & \\
\hline MNTOT & -0.002000 & 3689783.530000 & 431830.120000 & \\
\hline NATOT & -0.100000 & 3689783.530000 & 431830.120000 & \\
\hline NITOT & -0.010000 & 3689783.530000 & 431830.120000 & \\
\hline PBTOT & -0.005000 & 3689783.530000 & 431830.120000 & \\
\hline SETOT & -0.005000 & 3689783.530000 & 431830.120000 & \\
\hline SO4 & -1.000000 & 3689783.530000 & 431830.120000 & \\
\hline TOTRAD & -0.200000 & 3689783.530000 & 431830.120000 & \\
\hline TPO4 & 0.042400 & 3689783.530000 & 431830.120000 & \\
\hline 2NTOT & -0.005000 & 3689783.530000 & 431830.120000 & \\
\hline ALTOT & -0.033300 & 3689716.970000 & 431756.910000 & \\
\hline BATOT & -0.005000 & 3689716.970000 & 431756.910000 & \\
\hline CL & -0.417000 & 3689716.970000 & 431756.910000 & \\
\hline CRTOT & -0.006670 & 3689716.970000 & 431756.910000 & \\
\hline CUTOT & -0.006670 & 3689716.970000 & 431756.910000 & \\
\hline CYN & -0.008330 & 3689716.970000 & 431756.910000 & \\
\hline$F$ & -0.050000 & 3689716.970000 & 431756.910000 & \\
\hline HGTOT & -0.000333 & 3689716.970000 & 431756.910000 & \\
\hline MNTOT & -0.003330 & 3689716.970000 & 431756.910000 & \\
\hline NATOT & -0.016700 & 3689716.970000 & 431756.910000 & \\
\hline NITOT & -0.006670 & 3689716.970000 & 431756.910000 & \\
\hline PBTOT & 0.003920 & 3689716.970000 & 431756.910000 & \\
\hline SETOT & -0.003330 & 3689716.970000 & 431756.910000 & \\
\hline 504 & -1.670000 & 3689716.970000 & 431756.910000 & \\
\hline TOTRAD & -0.100000 & 3689716.970000 & 431756.910000 & \\
\hline TPO4 & 0.043000 & 3689716.970000 & 431756.910000 & \\
\hline ZNTOT & -0.003330 & 3689716.970000 & 431756.910000 & \\
\hline ALTOT & 0.020000 & 3689639.510000 & 431746.030000 & \\
\hline BATOT & -0.005000 & 3689639.510000 & 431746.030000 & 2.000000 \\
\hline CL & -0.417000 & 3689639.510000 & 431746.030000 & \\
\hline CRTOT & 0.004000 & 3689639.510000 & 431746.030000 & \\
\hline
\end{tabular}




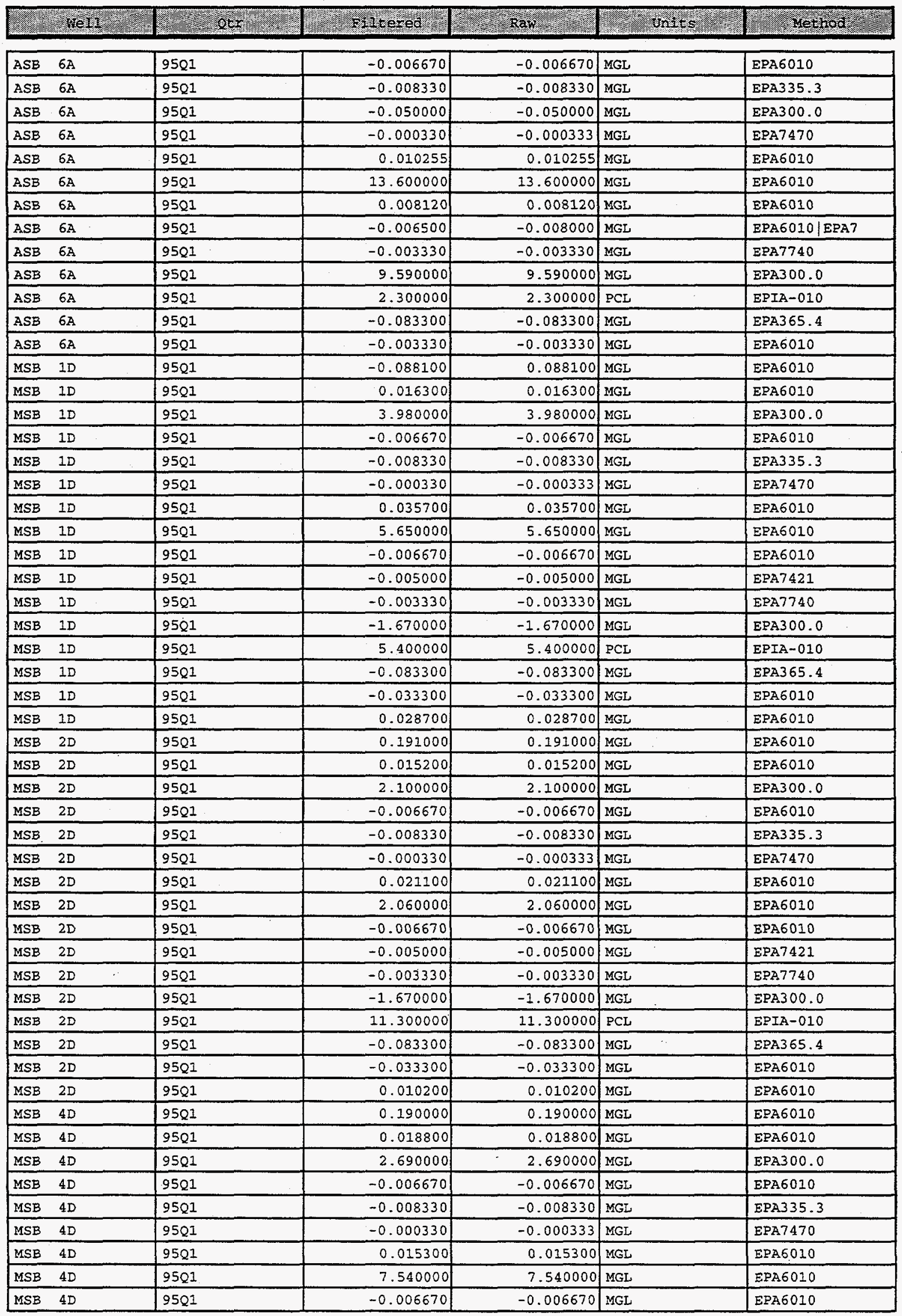


WSRC-TR-00347

Novem ber, 1997

Appendix II-12

\begin{tabular}{|c|c|c|c|c|}
\hline CUTOT & 0.004000 & 3689639.510000 & 431746.030000 & \\
\hline $\mathrm{CYN}$ & -0.008330 & 3689639.510000 & 431746.030000 & \\
\hline $\mathrm{F}$ & -0.050000 & 3689639.510000 & 431746.030000 & \\
\hline HGTOT & -0.000333 & 3689639.510000 & 431746.030000 & \\
\hline MNTOT & -0.003330 & 3689639.510000 & 431746.030000 & 2.000000 \\
\hline NATOT & 0.010000 & 3689639.510000 & 431746.030000 & \\
\hline NITOT & -0.006670 & 3689639.510000 & 431746.030000 & 2.000000 \\
\hline PBTOT & 0.007150 & 3689639.510000 & 431746.030000 & 2.000000 \\
\hline SETOT & 0.002000 & 3689639.510000 & 431746.030000 & \\
\hline SO4 & -1.670000 & 3689639.510000 & 431746.030000 & \\
\hline TOTRAD & 0.000000 & 3689639.510000 & 431746.030000 & \\
\hline TPO4 & -0.083300 & 3689639.510000 & 431746.030000 & \\
\hline ZNTOT & 0.002000 & 3689639.510000 & 431746.030000 & \\
\hline ALTOT & 0.020000 & 3687925.590000 & 431401.750000 & \\
\hline BATOT & -0.005000 & 3687925.590000 & 431401.750000 & \\
\hline $\mathrm{CL}$ & -0.417000 & 3687925.590000 & 431401.750000 & \\
\hline CRTOT & 0.004000 & 3687925.590000 & 431401.750000 & \\
\hline CYN & -0.008330 & 3687925.590000 & 431401.750000 & \\
\hline HGTOT & -0.000333 & 3687925.590000 & 431401.750000 & \\
\hline MNTOT & -0.003330 & 3687925.590000 & 431401.750000 & \\
\hline NATOT & 0.010000 & 3687925.590000 & 431401.750000 & \\
\hline NITOT & -0.006670 & 3687925.590000 & 431401.750000 & \\
\hline PBTOT & -0.005000 & 3687925.590000 & 431401.750000 & \\
\hline SETOT & -0.003330 & 3687925.590000 & 431401.750000 & \\
\hline SO4 & -1.570000 & 3687925.590000 & 431401.750000 & \\
\hline TOTRAD & 0.000000 & 3687925.590000 & 431401.750000 & \\
\hline TPO4 & -0.083300 & 3687925.590000 & 431401.750000 & \\
\hline UTOT & -0.033300 & 3687925.590000 & 431401.750000 & \\
\hline ZNTOT & 0.002000 & 3687925.590000 & 431401.750000 & \\
\hline AITOT & -0.033300 & 3688024.500000 & 431444.130000 & \\
\hline BATOT & -0.005000 & 3688024.500000 & 431444.130000 & \\
\hline $\mathrm{CL}$ & -0.417000 & 3688024.500000 & 431444.130000 & \\
\hline CRTOT & 0.004000 & 3688024.500000 & 431444.130000 & \\
\hline $\mathrm{CYN}$ & -0.008330 & 3688024.500000 & 431444.130000 & \\
\hline HGTOT & -0.000333 & 3688024.500000 & 431444.130000 & \\
\hline MNTOT & -0.003330 & 3688024.500000 & 431444.130000 & \\
\hline NATOT & -0.016700 & 3688024.500000 & 431444.130000 & \\
\hline NITOT & -0.006670 & 3688024.500000 & 431444.130000 & \\
\hline PBTOT & -0.005000 & 3688024.500000 & 431444.130000 & \\
\hline SETOT & -0.003330 & 3688024.500000 & 431444.130000 & \\
\hline SO4 & -1.670000 & 3688024.500000 & 431444.130000 & \\
\hline TOTRAD & -0.300000 & 3688024.500000 & 431444.130000 & \\
\hline TPO4 & -0.083300 & 3688024.500000 & 431444.130000 & \\
\hline UTOT & -0.033300 & 3688024.500000 & 431444.130000 & \\
\hline ZNTOT & -0.003330 & 3688024.500000 & 431444.130000 & \\
\hline ALTOT & -0.033300 & 3687943.280000 & 431335.920000 & \\
\hline BATOT & -0.005000 & 3687943.280000 & 431335.920000 & \\
\hline $\mathrm{CL}$ & -0.417000 & 3687943.280000 & 431335.920000 & \\
\hline CRTOT & -0.006670 & 3687943.280000 & 431335.920000 & \\
\hline CYN & -0.008330 & 3687943.280000 & 431335.920000 & \\
\hline HGTOT & -0.000333 & 3687943.280000 & 431335.920000 & \\
\hline MNTOT & -0.003330 & 3687943.280000 & 431335.920000 & \\
\hline NATOT & -0.016700 & 3687943.280000 & 431335.920000 & \\
\hline NITOT & -0.006670 & 3687943.280000 & 431335.920000 & \\
\hline
\end{tabular}


WSRC-TR-00347

November, 1997

Appendix II-13

\begin{tabular}{|c|c|c|c|c|c|c|}
\hline$\sqrt{17 \times 28}$ & n: & (2) a & 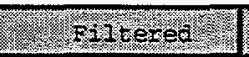 & 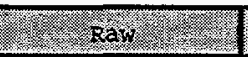 & M & 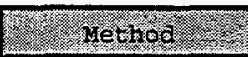 \\
\hline MSB & $4 D$ & 9501 & -0.005000 & -0.005000 & $\overline{M G L}$ & EPA7421 \\
\hline MSB & $4 D$ & $95 Q 1$ & -0.003330 & -0.003330 & MGL & EPA7740 \\
\hline MSB & 4D & 9501 & 4.020000 & 4.020000 & MGL & EPA 300.0 \\
\hline MSB & $4 \mathrm{D}$ & 9501 & 6.800000 & 6.800000 & PCL & EPIA-010 \\
\hline MSB & $4 D$ & 9501 & -0.083300 & -0.083300 & MGL & EPA365.4 \\
\hline MSB & $4 \mathrm{D}$ & 9501 & -0.033300 & -0.033300 & MGE & EPA6010 \\
\hline MSB & $4 \mathrm{D}$ & 9501 & 0.003790 & 0.003790 & MGL & EPA6010 \\
\hline MSB & $5 \mathrm{~A}$ & $95 Q 1$ & -0.033300 & -0.033300 & MGL & EPA6010 \\
\hline MSB & $5 \mathrm{~A}$ & 9501 & -0.005000 & -0.005000 & MGL & E2A 6010 \\
\hline MSB & $5 \mathrm{~A}$ & $95 Q 1$ & 2.260000 & 2.260000 & MGL & EPA300.0 \\
\hline MSB & $5 A$ & 9501 & -0.006670 & -0.006670 & MGL & EPA6010 \\
\hline MSB & $5 \mathrm{~A}$ & 9501 & -0.008330 & -0.008330 & MGL & EPA335.3 \\
\hline MSB & $5 \mathrm{~A}$ & 9501 & -0.000330 & -0.000333 & MGL & EPA7470 \\
\hline MSB & $5 \mathrm{~A}$ & 9501 & 0.003670 & 0.003670 & MGL & EPA6010 \\
\hline MSB & $5 \mathrm{~A}$ & 9501 & 6.240000 & 6.240000 & MGL & EPA6010 \\
\hline MSB & $5 A$ & 9501 & -0.006670 & -0.006670 & MGL & EPA6010 \\
\hline MSB & $5 \mathrm{~A}$ & 9501 & -0.005000 & -0.005000 & MGL & EPA7421 \\
\hline MSB & $5 \mathrm{~A}$ & $95 Q 1$ & -0.003330 & -0.003330 & MGL & EPA7740 \\
\hline MSB & $5 A$ & $95 Q 1$ & 1.810000 & 1.810000 & MGL & EPA300.0 \\
\hline MSB & $5 A$ & $95 Q 1$ & 0.500000 & 0.500000 & PCL & EPIA-010 \\
\hline MSB & $5 A$ & 9501 & -0.074200 & 0.074200 & MGL & EPA 365.4 \\
\hline MSB & $5 A$ & $95 Q 1$ & -0.033300 & -0.033300 & MGL & EPA6010 \\
\hline MSB & $5 A$ & 9581 & 0.006920 & 0.006920 & MGL & EPA6010 \\
\hline MSB & $6 \mathrm{~A}$ & 9501 & -0.031100 & 0.031100 & MGL & EPA6010 \\
\hline MSB & $6 \mathrm{~A}$ & 9501 & 0.006130 & 0.006130 & MGL & EPA6010 \\
\hline MSB & $6 \mathrm{~A}$ & 9501 & 4.820000 & 4.820000 & MGL & EPA300.0 \\
\hline MSB & $6 \mathrm{~A}$ & 9501 & -0.006670 & -0.006670 & MGL & EPA6010 \\
\hline MSB & $6 \mathrm{~A}$ & 9521 & -0.008330 & -0.008330 & MGL & EPA335.3 \\
\hline MSB & $6 \mathrm{~A}$. & $95 Q 1$ & -0.000330 & -0.000333 & MGL & EPA7470 \\
\hline MSB & $6 \mathrm{~A}$ & 9501 & 0.006950 & 0.006950 & MGL & EPA6010 \\
\hline MSB & $6 \mathrm{~A}$ & 9501 & 5.600000 & 5.600000 & MGL & EPA6010 \\
\hline MSB & $6 A$ & $95 Q 1$ & -0.006670 & -0.006670 & MGL & EPA6010 \\
\hline MSB & $6 \mathrm{~A}$ & $95 Q 1$ & -0.005000 & -0.005000 & MGL & EPA7421 \\
\hline MSB & $6 \mathrm{~A}$ & $95 Q 1$ & -0.003330 & -0.003330 & MGL & EPA7740 \\
\hline MSB & $6 \mathrm{~A}$ & 9501 & -1.640000 & 1.640000 & MGL & EPA 300.0 \\
\hline MSB & $6 \mathrm{~A}$ & 9501 & 1.700000 & 1.700000 & PCL & EPIA-010 \\
\hline MSB & $6 \mathrm{~A}$ & 9501 & -0.083300 & -0.083300 & MGL & EPA365.4 \\
\hline MSB & $6 \mathrm{~A}$ & 9501 & -0.033300 & -0.033300 & MGL & EPA6010 \\
\hline MSB & $6 \mathrm{~A}$ & 9501 & -0.003330 & -0.003330 & MGL & EPA6010 \\
\hline MSB & $7 \mathrm{~A}$ & 9501 & -0.033300 & -0.033300 & MGL & EPA6010 \\
\hline MSB & $7 A$ & 9501 & 0.014200 & 0.014200 & MGL & EPA6010 \\
\hline MSB & $7 A$ & 9501 & 3.680000 & 3.680000 & MGL & EPA300.0 \\
\hline MSB & $7 \mathrm{~A}$ & 9501 & -0.006670 & -0.006670 & MGL & EPA6010 \\
\hline MSB & $7 \mathrm{~A}$ & 9501 & -0.008330 & -0.008330 & MGL & EPA335.3 \\
\hline MSB & $7 \mathrm{~A}$ & 9501 & -0.000330 & -0.000333 & MGL & EPA7470 \\
\hline MSB & $7 \mathrm{~A}$ & $95 Q 1$ & 0.027000 & 0.027000 & MGL & EPA6010 \\
\hline MSB & $7 \mathrm{~A}$ & $95 Q 1$ & 3.960000 & 3.960000 & MGL & EPA6010 \\
\hline MSB & $7 \mathrm{~A}$ & 9501 & -0.006670 & -0.006670 & MGL & EPA6010 \\
\hline MSB & $7 \mathrm{~A}$ & $95 Q 1$ & -0.005000 & -0.005000 & MGL & EPA7421 \\
\hline MSB & $7 \mathrm{~A}$ & 9501 & -0.003330 & -0.003330 & MGL & EPA7740 \\
\hline MSB & $7 \mathrm{~A}$ & 9501 & -1.420000 & 1.420000 & MGL & EPA300.0 \\
\hline MSB & $7 \mathrm{~A}$ & $95 Q 1$ & 3.300000 & 3.300000 & PCL & EPIA-010 \\
\hline MSB & $7 \mathrm{~A}$ & 9501 & -0.083300 & -0.083300 & MGL & EPA365.4 \\
\hline MSB & $7 \mathrm{~A}$ & 9501 & -0.033300 & -0.033300 & MGL & EPA6010 \\
\hline
\end{tabular}


WSRC-TR-00347

November, 1997

Appendix II-14

\begin{tabular}{|c|c|c|c|c|}
\hline SETOT & -0.003330 & 3687943.280000 & 431335.920000 & \\
\hline SO4 & -1.670000 & 3687943.280000 & 431335.920000 & \\
\hline TOTRAD & & 3687943.280000 & 431335.920000 & \\
\hline TPO4 & -0.083300 & 3687943.280000 & 431335.920000 & \\
\hline UTOT & -0.033300 & 3687943.280000 & 431335.920000 & \\
\hline ZNTOT & -0.003330 & 3687943.280000 & 431335.920000 & \\
\hline ALTOT & -0.033300 & 3687698.960000 & 431018.910000 & \\
\hline BATOT & -0.005000 & 3687698.960000 & 431018.910000 & \\
\hline $\mathrm{CL}$ & -0.417000 & 3687698.960000 & 431018.910000 & \\
\hline CRTOT & -0.006670 & 3687698.960000 & 431018.910000 & \\
\hline CYN & -0.008330 & 3687698.960000 & 431018.910000 & \\
\hline HGTOT & -0.000333 & 3687698.960000 & 431018.910000 & \\
\hline MNTOT & -0.003330 & 3687698.960000 & 431018.910000 & \\
\hline NATOT & -0.016700 & 3687698.960000 & 431018.910000 & \\
\hline NITOT & -0.006670 & 3687698.960000 & 431018.910000 & \\
\hline PBTOT & 0.002780 & 3687698.960000 & 431018.910000 & \\
\hline SETOT & -0.003330 & 3687698.960000 & 431018.910000 & \\
\hline SO4 & -1.670000 & 3687698.960000 & 431018.910000 & \\
\hline TOTRAD & & 3687698.960000 & 431018.910000 & \\
\hline TPO4 & -0.083300 & 3687698.960000 & 431018.910000 & \\
\hline UTOT & -0.033300 & 3687698.960000 & 431018.910000 & \\
\hline ZNTOT & -0.003330 & 3687698.960000 & 431018.910000 & \\
\hline ALTOT & -0.033300 & 3687370.860000 & 431001.900000 & \\
\hline BATOT & -0.005000 & 3687370.860000 & 431001.900000 & \\
\hline CL & -0.417000 & 3687370.860000 & 431001.900000 & \\
\hline CRTOT & -0.006670 & 3687370.860000 & 431001.900000 & \\
\hline CXN & -0.008330 & 3687370.860000 & 431001.900000 & \\
\hline HGTOT & -0.000333 & 3687370.860000 & 431001.900000 & \\
\hline MNTOT & -0.003330 & 3687370.860000 & 431001.900000 & \\
\hline NATOT & -0.016700 & 3687370.860000 & 431001.900000 & \\
\hline NITOT & -0.006670 & 3687370.860000 & 431001.900000 & \\
\hline PBTOT & -0.005000 & 3687370.860000 & 431001.900000 & \\
\hline SETOT & -0.003330 & 3687370.860000 & 431001.900000 & \\
\hline SO4 & -1.670000 & 3687370.860000 & 431001.900000 & \\
\hline TOTRAD & & 3687370.860000 & 431001.900000 & \\
\hline TPO4 & -0.083300 & 3687370.860000 & 431001.900000 & \\
\hline UTOT & -0.033300 & 3687370.860000 & 431001.900000 & \\
\hline ZNTOT & -0.003330 & 3687370.860000 & 431001.900000 & \\
\hline ALTOT & -0.033300 & 3687308.670000 & 431200.260000 & \\
\hline BATOT & -0.005000 & 3687308.670000 & 431200.260000 & \\
\hline $\mathrm{CL}$ & -0.417000 & 3687308.670000 & 431200.260000 & \\
\hline CRTOT & -0.006670 & 3687308.670000 & 431200.260000 & \\
\hline CYN & -0.008330 & 3687308.670000 & 431200.260000 & \\
\hline HGTOT & -0.000333 & 3687308.670000 & 431200.260000 & \\
\hline MATTOT & -0.003330 & 3687308.670000 & 431200.260000 & \\
\hline NATOT & $-0: 016700$ & 3687308.670000 & 431200.260000 & \\
\hline NITOT & -0.006670 & 3687308.670000 & 431200.260000 & \\
\hline PBTOT & -0.005000 & 3687308.670000 & 431200.260000 & \\
\hline SETOT & -0.003330 & 3687308.670000 & 431200.260000 & \\
\hline SO4 & -1.670000 & 3687308.670000 & 431200.260000 & \\
\hline TOTRAD & & 3687308.670000 & 431200.260000 & \\
\hline TPO4 & -0.083300 & 3687308.670000 & 431200.260000 & \\
\hline UTOT & -0.033300 & 3687308.670000 & 431200.260000 & \\
\hline
\end{tabular}


WSRC-TR-00347

November, 1997

Appendix II-15

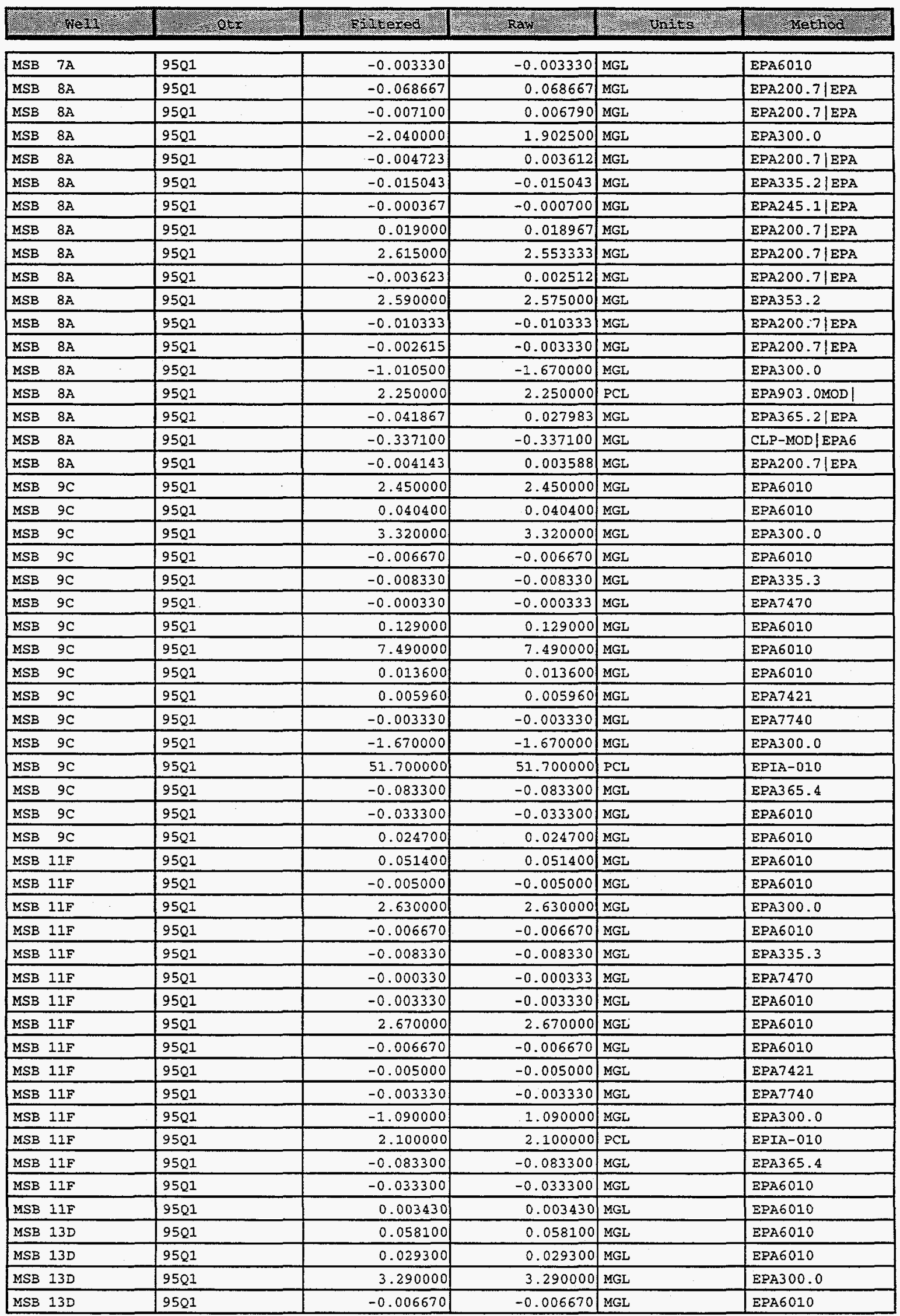


WSRC-TR-00347

November, 1997

Appendix II-16

\begin{tabular}{|c|c|c|c|c|}
\hline ZNTOT & -0.003330 & 3687308.670000 & 431200.260000 & \\
\hline ALTOT & 0.035683 & 3687466.880000 & 431298.830000 & 3.000000 \\
\hline BATOT & -0.006600 & 3687466.880000 & 431298.830000 & 3.000000 \\
\hline CL & -1.943500 & 3687466.880000 & 431298.830000 & 4.000000 \\
\hline CRTOT & -0.008890 & 3687466.880000 & 431298.830000 & 3.000000 \\
\hline CYN & -0.015043 & 3687466.880000 & 431298.830000 & 3.000000 \\
\hline HGTOT & 0.003609 & 3687466.880000 & 431298.830000 & 3.000000 \\
\hline MNTOT & -0.004910 & 3687466.880000 & 431298.830000 & 3.000000 \\
\hline NATOT & 0.022117 & 3687466.880000 & 431298.830000 & 3.000000 \\
\hline NITOT & -0.005823 & 3687466.880000 & 431298.830000 & 3.000000 \\
\hline NO3 & -0.060000 & 3687466.880000 & 431298.830000 & 2.000000 \\
\hline PBTOT & -0.066500 & 3687466.880000 & 431298.830000 & 3.000000 \\
\hline SETOT & -0.011165 & 3687466.880000 & 431298.830000 & 2.000000 \\
\hline 504 & -1.324500 & 3687466.880000 & 431298.830000 & 4.000000 \\
\hline TOTRAD & -0.100000 & 3687466.880000 & 431298.830000 & 3.000000 \\
\hline TPO4 & -0.047767 & 3687466.880000 & 431298.830000 & 3.000000 \\
\hline ЈTOT & -0.337100 & 3687466.880000 & 431298.830000 & 3.000000 \\
\hline ZNTOT & 0.002155 & 3687466.880000 & 431298.830000 & 3.000000 \\
\hline ALTOT & 0.020000 & 3687995.000000 & 431283.680000 & \\
\hline BATOT & -0.005000 & 3687995.000000 & 431283.680000 & \\
\hline CL & -0.417000 & 3687995.000000 & 431283.680000 & \\
\hline CRTOT & 0.004000 & 3687995.000000 & 431283.680000 & \\
\hline $\mathrm{CYN}$ & -0.008330 & 3687995.000000 & 431283.680000 & \\
\hline HGTOT & -0.000333 & 3687995.000000 & 431283.680000 & \\
\hline MNTOT & -0.003330 & 3687995.000000 & 431283.680000 & \\
\hline NATOT & 0.010000 & 3687995.000000 & 431283.680000 & \\
\hline NITOT & -0.006670 & 3687995.000000 & 431283.680000 & \\
\hline PBTOT & -0.005000 & 3687995.000000 & 431283.680000 & \\
\hline SETOT & -0.003330 & 3687995.000000 & 431283.680000 & \\
\hline SO4 & & 3687995.000000 & 431283.680000 & \\
\hline TOTRAD & -0.300000 & 3687995.000000 & 431283.680000 & \\
\hline TPO4 & -0.083300 & 3687995.000000 & 431283.680000 & \\
\hline UTOT & -0.033300 & 3687995.000000 & 431283.680000 & \\
\hline ZNTOT & 0.002000 & 3687995.000000 & 431283.680000 & \\
\hline ALTOT & -0.033300 & 3688144.040000 & 431289.770000 & \\
\hline BATOT & -0.005000 & 3688144.040000 & 431289.730000 & \\
\hline$C L$ & -0.417000 & 3688144.040000 & 431289.770000 & \\
\hline CRTOT & -0.006670 & 3688144.040000 & 431289.770000 & \\
\hline CYN & -0.008330 & 3688144.040000 & 431289.770000 & \\
\hline HGTOT & -0.000333 & 3688144.040000 & 431289.770000 & \\
\hline MNTOT & -0.003330 & 3688144.040000 & 431289.770000 & \\
\hline NATOT & -0.016700 & 3688144.040000 & 431289.770000 & \\
\hline NITOT & -0.006670 & 3688144.040000 & 431289.770000 & \\
\hline PBTOT & -0.005000 & 3688144.040000 & 431289.770000 & \\
\hline SETOT & -0.003330 & 3688144.040000 & 431289.770000 & \\
\hline SO4 & -1.670000 & 3688144.040000 & 431289.770000 & \\
\hline TOTRAD & 0.000000 & 3688144.040000 & 431289.770000 & \\
\hline TPO4 & -0.083300 & 3688144.040000 & 431289.770000 & \\
\hline UTOT & & 3688144.040000 & 431289.770000 & \\
\hline ZNTOT & -0.003330 & 3688144.040000 & 431289.770000 & \\
\hline ALTOT & -0.033300 & 3687744.350000 & 431181.400000 & \\
\hline BATOT & -0.005000 & 3687744.350000 & 431181.400000 & \\
\hline CL & -0.417000 & 3687744.350000 & 431181.400000 & \\
\hline CRTOT & -0.006670 & 3687744.350000 & 431181.400000 & \\
\hline
\end{tabular}


WSRC-TR-00347

November, 1997

Appendix II-17

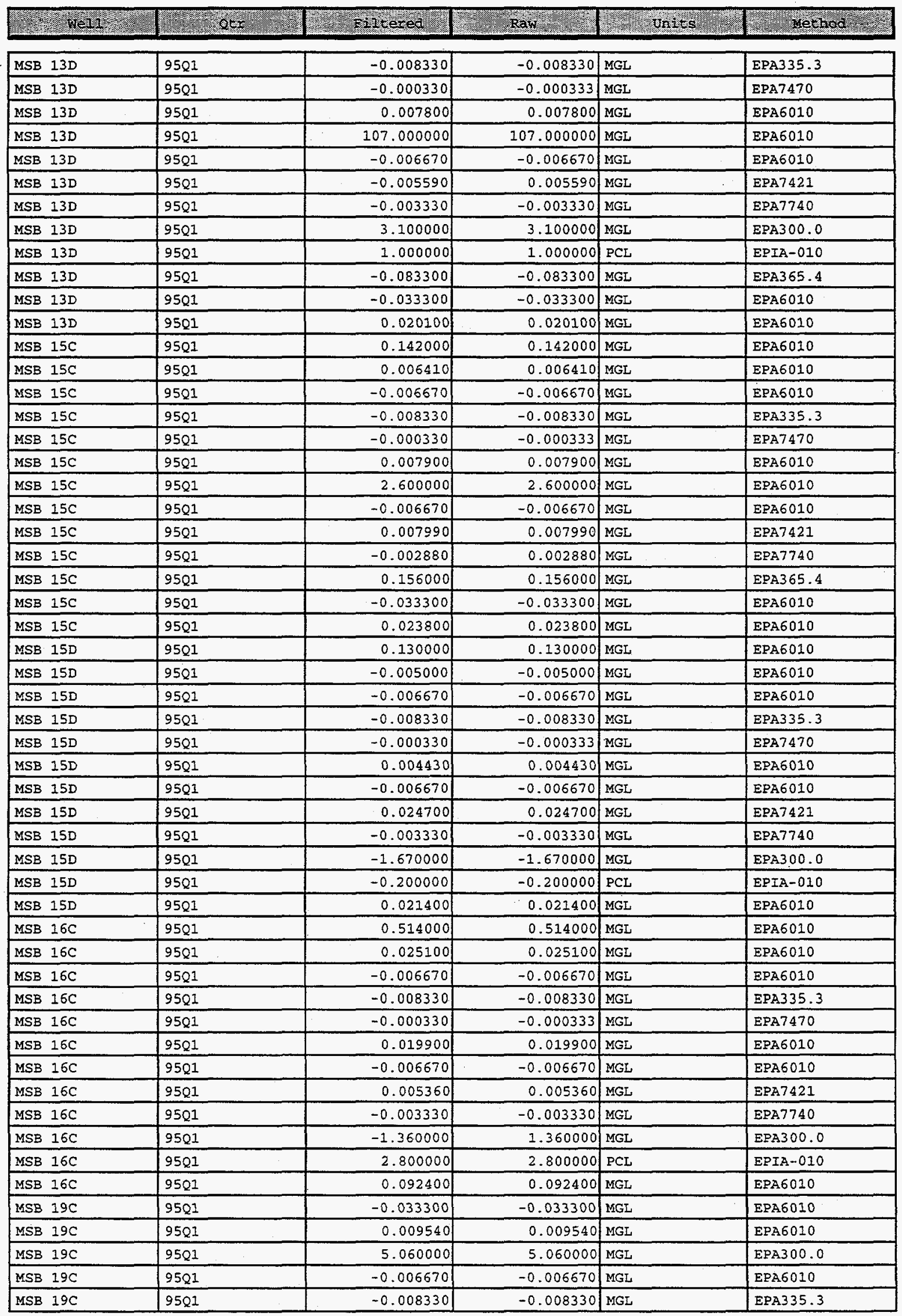


WSRC-TR-00347

November, 1997

Appendix II-18

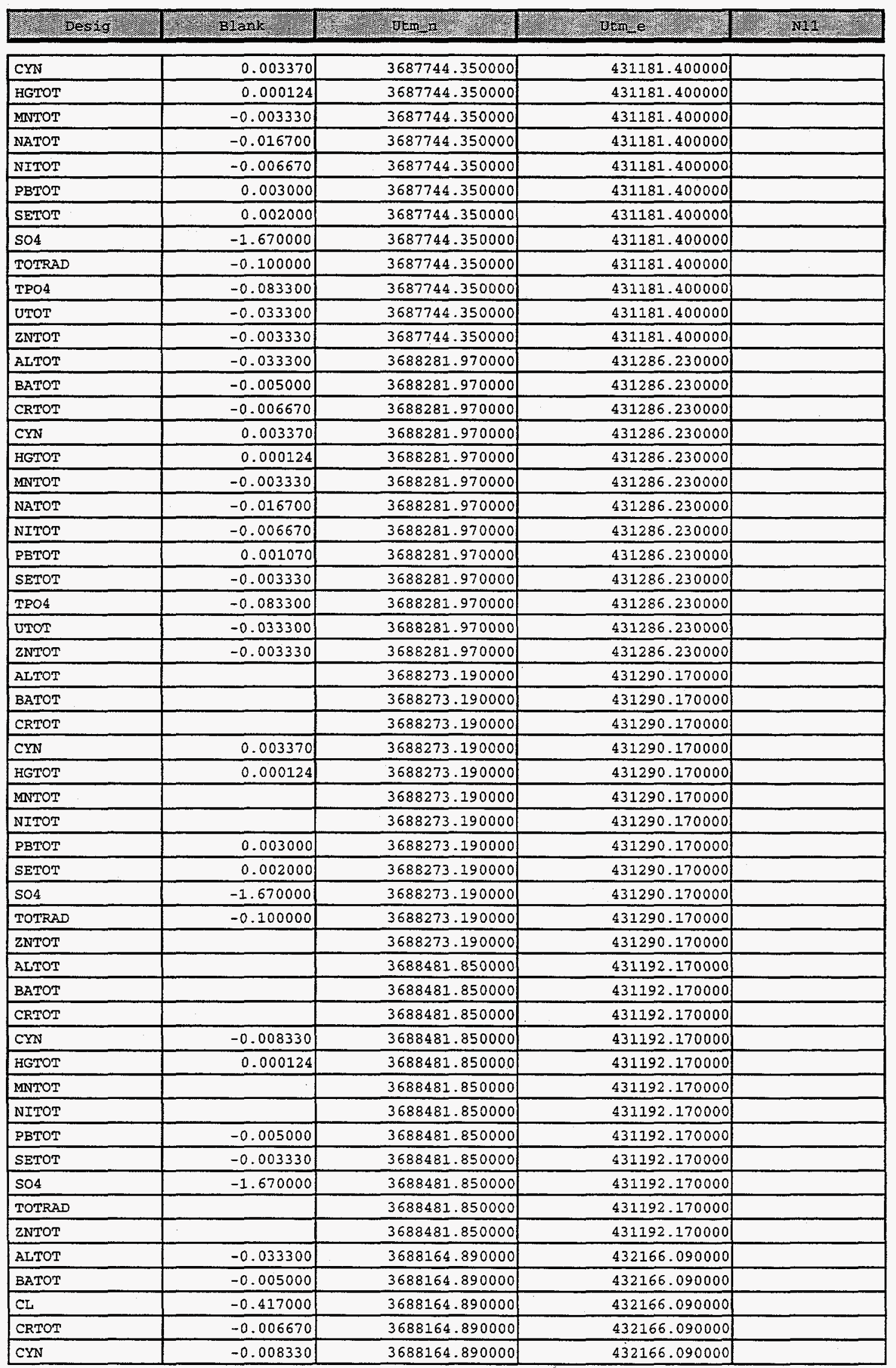




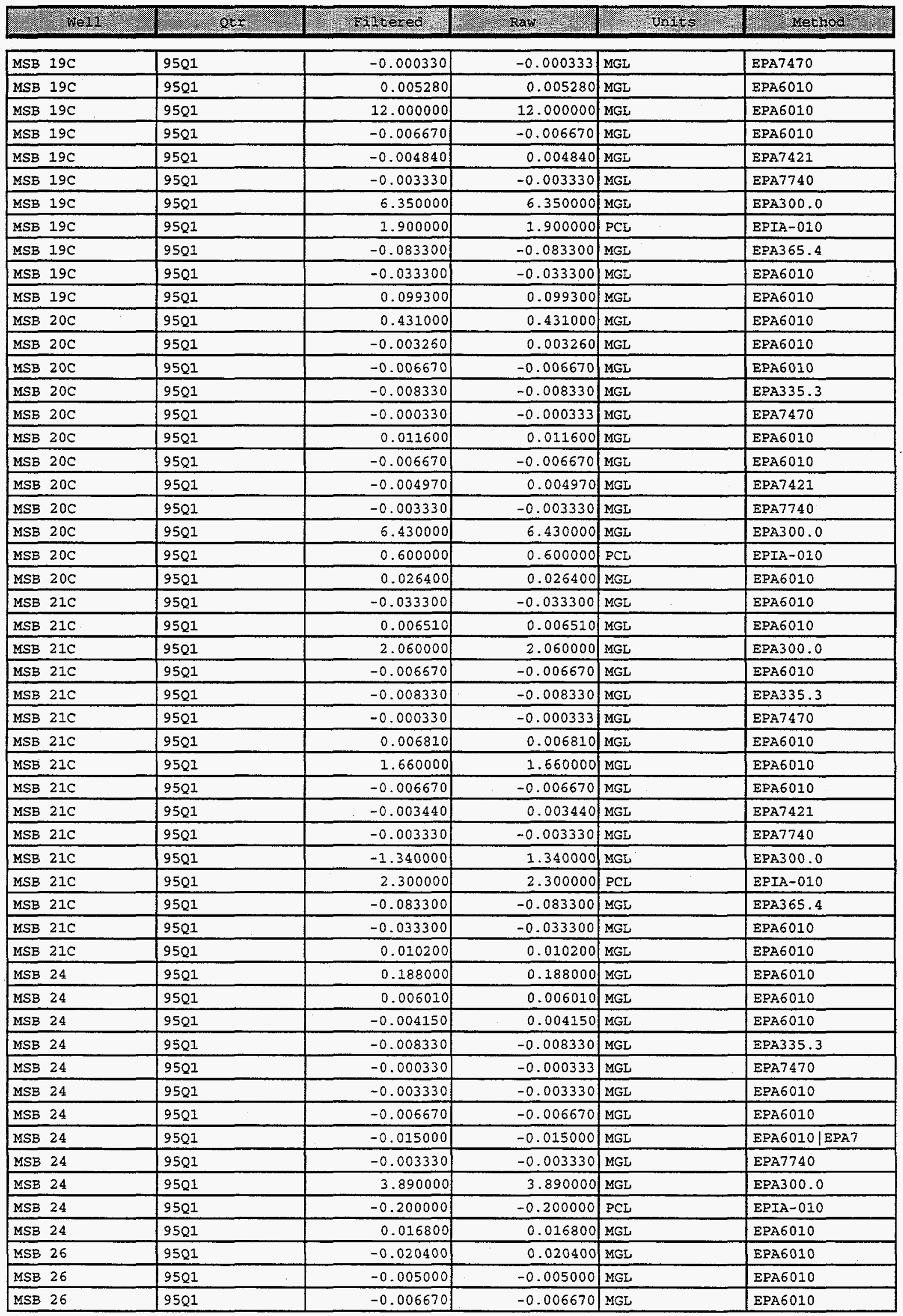




\begin{tabular}{|c|c|c|c|c|}
\hline 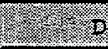 & $31 \mathrm{an} x$ & T. & 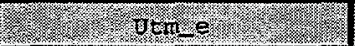 & 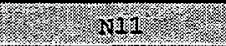 \\
\hline HGTOT & -0.000333 & 3688164.890000 & 432166.090000 & \\
\hline MNTOT & -0.003330 & 3688164.890000 & 432166.090000 & \\
\hline NATOT & -0.016700 & 3688164.890000 & 432166.090000 & \\
\hline NITOT & -0.006670 & 3688164.890000 & 432166.090000 & \\
\hline PBTOT & -0.005000 & 3688164.890000 & 432166.090000 & \\
\hline SETOT & -0.003330 & 3688164.890000 & 432166.090000 & \\
\hline 504 & -1.670000 & 3688164.890000 & 432166.090000 & \\
\hline TOTRAD & 0.000000 & 3688164.890000 & 432166.090000 & \\
\hline TPO4 & -0.083300 & 3688164.890000 & 432166.090000 & \\
\hline UTOT & -0.033300 & 3688164.890000 & 432166.090000 & \\
\hline ZNTOT & -0.003330 & 3688164.890000 & 432166.090000 & \\
\hline ALTOT & -0.033300 & 3687926.210000 & 430510.550000 & \\
\hline BATOT & -0.005000 & 3687926.210000 & 430510.550000 & \\
\hline CRTOT & -0.006670 & 3687926.210000 & 430510.550000 & \\
\hline CYN & -0.008330 & 3687926.210000 & 430510.550000 & \\
\hline HGTOT & -0.000333 & 3687926.210000 & 430510.550000 & \\
\hline MNTOT & -0.003330 & 3687926.210000 & 430510.550000 & \\
\hline NITOT & -0.006670 & 3687926.210000 & 430510.550000 & \\
\hline PBTOT & -0.005000 & 3687926.210000 & 430510.550000 & \\
\hline SETOT & -0.003330 & 3687926.210000 & 430510.550000 & \\
\hline SO4 & -1.670000 & 3687926.210000 & 430510.550000 & \\
\hline TOTRAD & -0.100000 & 3687926.210000 & 430510.550000 & \\
\hline ZNTOT & -0.003330 & 3687926.210000 & 430510.550000 & \\
\hline ALTOT & -0.033300 & 3688234.340000 & 430718.100000 & \\
\hline BATOT & -0.005000 & 3688234.340000 & 430718.100000 & \\
\hline CL & -0.417000 & 3688234.340000 & 430718.100000 & \\
\hline CRTOT & -0.006670 & 3688234.340000 & 430718.100000 & \\
\hline $\mathrm{CYN}$ & -0.008330 & 3688234.340000 & 430718.100000 & \\
\hline HGTOT & -0.000333 & 3688234.340000 & 430718.100000 & \\
\hline MNTOT & -0.003330 & 3688234.340000 & 430718.100000 & \\
\hline NATOT & -0.016700 & 3688234.340000 & 430718.100000 & \\
\hline NITOT & -0.006670 & 3688234.340000 & 430718.100000 & \\
\hline PBTOT & -0.005000 & 3688234.340000 & 430718.100000 & \\
\hline SETOT & -0.003330 & 3688234.340000 & 430718.100000 & \\
\hline SO4 & -1.670000 & 3688234.340000 & 430718.100000 & \\
\hline TOTRAD & 0.000000 & 3688234.340000 & 430718.100000 & \\
\hline TPO4 & -0.083300 & 3688234.340000 & 430718.100000 & \\
\hline UTOT & -0.033300 & 3688234.340000 & 430718.100000 & \\
\hline ZNTOT & -0.003330 & 3688234.340000 & 430718.100000 & \\
\hline ALTOT & -0.033300 & 3688860.090000 & 431245.650000 & \\
\hline BATOT & -0.005000 & 3688860.090000 & 431245.650000 & \\
\hline CRTOT & -0.006670 & 3688860.090000 & 431245.650000 & \\
\hline $\mathrm{CYN}$ & 0.003370 & 3688860.090000 & 431245.650000 & \\
\hline HGTOT & 0.000124 & 3688860.090000 & 431245.650000 & \\
\hline MNTOT & -0.003330 & 3688860.090000 & 431245.650000 & \\
\hline NITOT & -0.006670 & 3688860.090000 & 431245.650000 & \\
\hline PBTOT & -0.025000 & 3688860.090000 & 431245.650000 & 2.000000 \\
\hline SETOT & -0.003330 & 3688860.090000 & 431245.650000 & \\
\hline 504 & -1.670000 & 3688860.090000 & 431245.650000 & \\
\hline TOTRAD & -0.100000 & 3688860.090000 & 431245.650000 & \\
\hline ZNTOT & -0.003330 & 3688860.090000 & 431245.650000 & \\
\hline ALTOT & 0.020000 & 3688698.090000 & 431023.920000 & \\
\hline BATOT & -0.005000 & 3688698.090000 & 431023.920000 & \\
\hline CRTOT & 0.004000 & 3688698.090000 & 431023.920000 & \\
\hline
\end{tabular}


WSRC-TR-00347

November, 1997

Appendix II-21

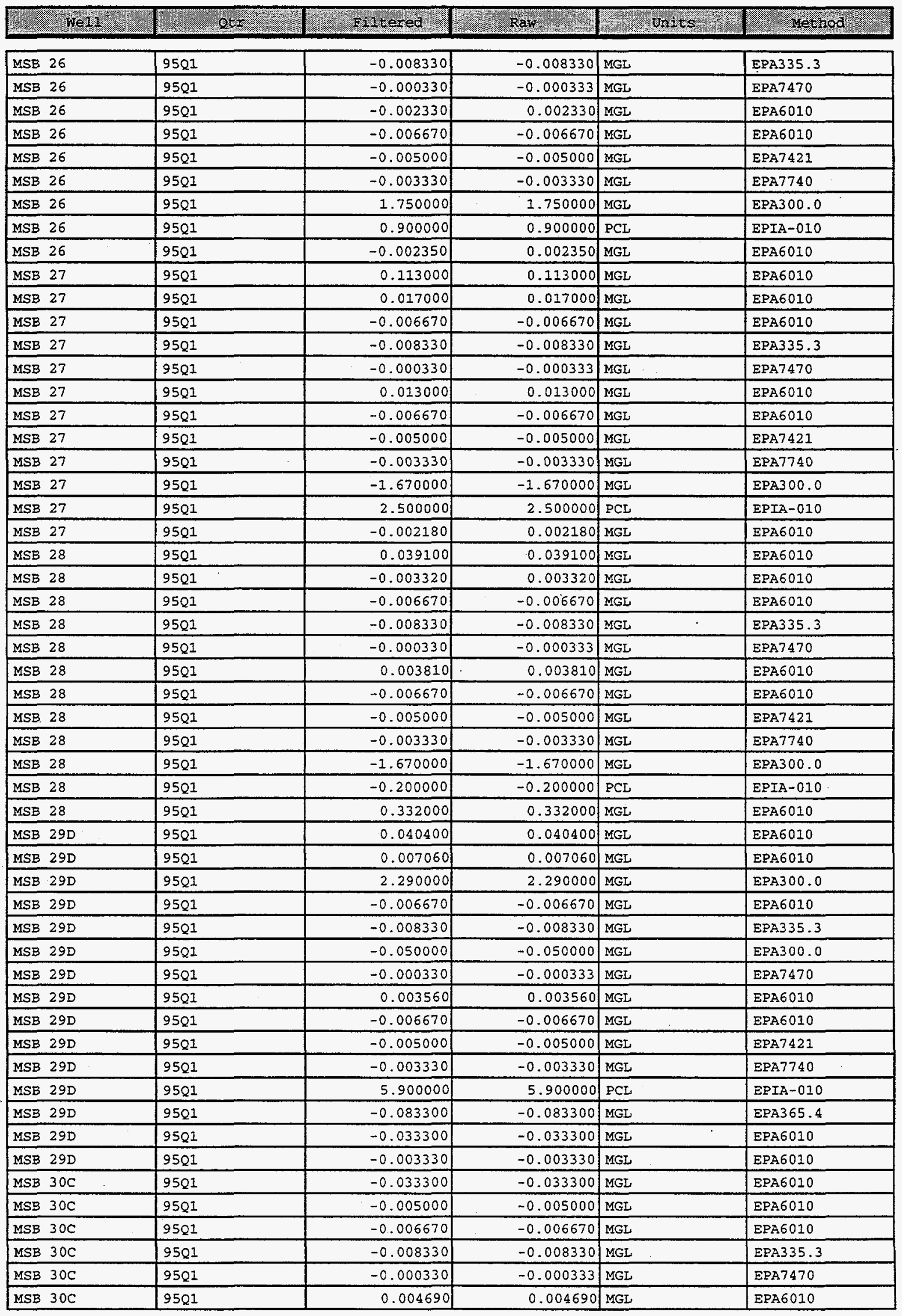


WSRC-TR-00347

November, 1997

Appendix II-22

\begin{tabular}{|c|c|c|c|c|}
\hline HGTOT & -0.000333 & 3688698.090000 & 431023.920000 & \\
\hline MNTOT & -0.003330 & 3688698.090000 & 431023.920000 & \\
\hline NITOT & -0.006670 & 3688698.090000 & 431023.920000 & \\
\hline PBTOT & -0.005000 & 3688698.090000 & 431023.920000 & \\
\hline SETOT & -0.003330 & 3688698.090000 & 431023.920000 & \\
\hline SO 4 & -1.670000 & 3688698.090000 & 431023.920000 & \\
\hline TOTRAD & 0.000000 & 3688698.090000 & 431023.920000 & \\
\hline ZNTOT & 0.002000 & 3688698.090000 & 431023.920000 & \\
\hline ALTOT & -0.033300 & 3688884.690000 & 431093.870000 & \\
\hline BATOT & -0.005000 & 3688884.690000 & 431093.870000 & \\
\hline CRTOT & -0.006670 & 3688884.690000 & 431093.870000 & \\
\hline CYN & -0.008330 & 3688884.690000 & 431093.870000 & \\
\hline HGTOT & -0.000333 & 3688884.690000 & 431093.870000 & \\
\hline MNTOT & -0.003330 & 3688884.690000 & 431093.870000 & \\
\hline NITOT & 0.009480 & 3688884.690000 & 431093.870000 & \\
\hline PBTOT & -0.005000 & 3688884.690000 & 431093.870000 & \\
\hline SETOT & -0.003330 & 3688884.690000 & 431093.870000 & \\
\hline SO4 & -1.670000 & 3688884.690000 & 431093.870000 & \\
\hline TOTRAD & -0.100000 & 3688884.690000 & 431093.870000 & 2.000000 \\
\hline ZNTOT & -0.003330 & 3688884.690000 & 431093.870000 & \\
\hline ALTOT & -0.033300 & 3688703.030000 & 430860.370000 & \\
\hline BATOT & -0.005000 & 3688703.030000 & 430860.370000 & \\
\hline CRTOT & -0.006670 & 3688703.030000 & 430860.370000 & \\
\hline CYN & -0.008330 & 3688703.030000 & 430860.370000 & \\
\hline HGTOT & -0.000333 & 3688703.030000 & 430860.370000 & \\
\hline MNTOT & -0.003330 & 3688703.030000 & 430860.370000 & \\
\hline NITOT & -0.006670 & 3688703.030000 & 430860.370000 & \\
\hline PBTOT & -0.005000 & 3688703.030000 & 430860.370000 & \\
\hline SETOT & -0.003330 & 3688703.030000 & 430860.370000 & \\
\hline SO4 & -1.670000 & 3688703.030000 & 430860.370000 & \\
\hline TOTRAD & & 3688703.030000 & 430860.370000 & \\
\hline ZNTOT & -0.003330 & 3688703.030000 & 430860.370000 & \\
\hline ALTOT & -0.033300 & 3689775.630000 & 431100.820000 & \\
\hline BATOT & -0.005000 & 3689775.630000 & 431100.820000 & \\
\hline $\mathrm{CL}$ & -0.417000 & 3689775.630000 & 431100.820000 & \\
\hline CRTOT & -0.006670 & 3689775.630000 & 431100.820000 & \\
\hline CYN & 0.003370 & 3689775.630000 & 431100.820000 & \\
\hline$F$ & -0.050000 & 3689775.630000 & 431100.820000 & \\
\hline HGTOT & 0.000124 & 3689775.630000 & 431100.820000 & \\
\hline MNTOT & -0.003330 & 3689775.630000 & 431100.820000 & \\
\hline NITOT & -0.005670 & 3689775.630000 & 431100.820000 & \\
\hline PBTOT & 0.003000 & 3689775.630000 & 431100.820000 & \\
\hline SETOT & -0.003330 & 3689775.630000 & 431100.820000 & \\
\hline TOTRAD & & 3689775.630000 & 431100.820000 & \\
\hline TPO4 & -0.083300 & 3689775.630000 & 431100.820000 & \\
\hline UTOT & -0.033300 & 3689775.630000 & 431100.820000 & \\
\hline ZNTOT & -0.003330 & 3689775.630000 & 431100.820000 & \\
\hline ALTOT & -0.033300 & 3688807.170000 & 430594.760000 & \\
\hline BATOT & -0.005000 & 3688807.170000 & 430594.760000 & \\
\hline CRTOT & -0.006670 & 3688807.170000 & 430594.760000 & \\
\hline CYN & & 3688807.170000 & 430594.760000 & \\
\hline HGTOT & -0.000333 & 3688807.170000 & 430594.760000 & \\
\hline MNTOT & -0.003330 & 3688807.170000 & 430594.760000 & \\
\hline
\end{tabular}


WSRC-TR-00347

November, 1997

Appendix II-23

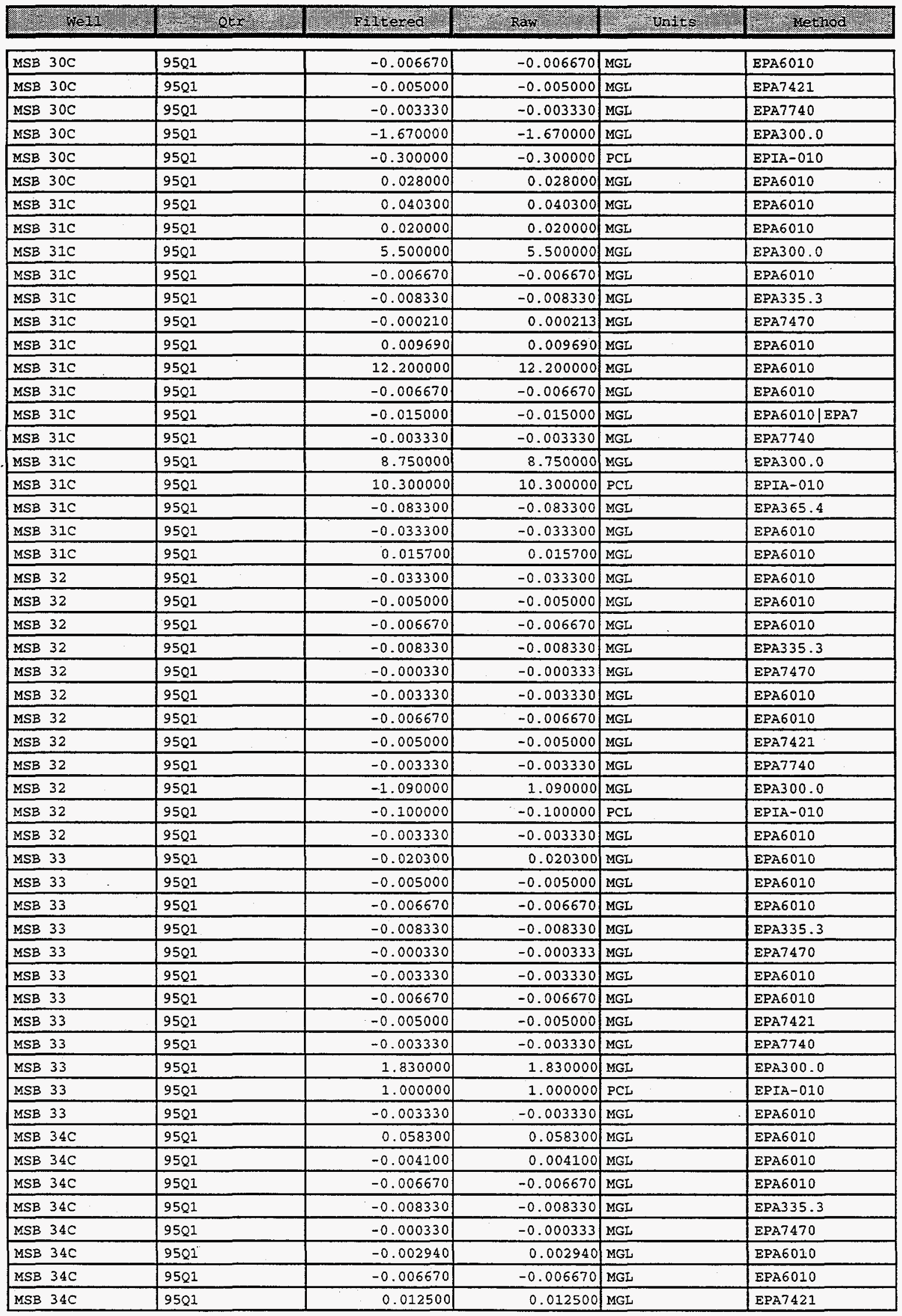


WSRC-TR-00347

November, 1997

Appendix II-24

\begin{tabular}{|c|c|c|c|c|}
\hline NITOT & -0.006670 & 3688807.170000 & 430594.760000 & \\
\hline PBTOT & -0.005000 & 3688807.170000 & 430594.760000 & \\
\hline SETOT & -0.003330 & 3688807.170000 & 430594.760000 & \\
\hline SO4 & -1.670000 & 3688807.170000 & 430594.760000 & \\
\hline TOTRAD & & 3688807.170000 & 430594.760000 & \\
\hline ZNTOT & -0.003330 & 3688807.170000 & 430594.760000 & \\
\hline ALTOT & -0.033300 & 3688255.290000 & 431778.990000 & \\
\hline BATOT & -0.005000 & 3688255.290000 & 431778.990000 & \\
\hline $\mathrm{CL}$ & -0.417000 & 3688255.290000 & 431778.990000 & \\
\hline CRTOT & -0.006670 & 3688255.290000 & 431778.990000 & \\
\hline $\mathrm{CYN}$ & -0.008330 & 3688255.290000 & 431778.990000 & \\
\hline HGTOT & -0.000333 & 3688255.290000 & 431778.990000 & \\
\hline MNTOT & -0.003330 & 3688255.290000 & 431778.990000 & \\
\hline NATOT & -0.016700 & 3688255.290000 & 431778.990000 & \\
\hline NITOT & -0.006670 & 3688255.290000 & 431778.990000 & \\
\hline PBTOT & -0.005000 & 3688255.290000 & 431778.990000 & 2.000000 \\
\hline SETOT & -0.003330 & 3688255.290000 & 431778.990000 & \\
\hline 504 & -1.670000 & 3688255.290000 & 431778.990000 & \\
\hline TOTRAD & -0.200000 & 3688255.290000 & 431778.990000 & \\
\hline TPO4 & -0.083300 & 3688255.290000 & 431778.990000 & \\
\hline UTOT & -0.033300 & 3688255.290000 & 431778.990000 & \\
\hline ZNTOT & -0.003330 & 3688255.290000 & 431778.990000 & \\
\hline ALTOT & -0.033300 & 3688156.900000 & 432847.110000 & \\
\hline BATOT & -0.005000 & 3688156.900000 & 432847.110000 & \\
\hline CRTOT & -0.006670 & 3688156.900000 & 432847.110000 & \\
\hline CYN & & 3688156.900000 & 432847.110000 & \\
\hline HGTOT & -0.000333 & 3688156.900000 & 432847.110000 & \\
\hline MNTOT & -0.003330 & 3688156.900000 & 432847.110000 & \\
\hline NITOT & -0.006670 & 3688156.900000 & 432847.110000 & \\
\hline PBTOT & -0.005000 & 3688156.900000 & 432847.110000 & \\
\hline SETOT & -0.003330 & 3688156.900000 & 432847.110000 & \\
\hline SO4 & -1.670000 & 3688156.900000 & 432847.110000 & \\
\hline TOTRAD & & 3688156.900000 & 432847.110000 & \\
\hline ZNTOT & -0.003330 & 3688156.900000 & 432847.110000 & \\
\hline ALTOT & 0.020000 & 3687577.780000 & 432892.680000 & \\
\hline ВАTOT & -0.005000 & 3687577.780000 & 432892.680000 & \\
\hline CRTOT & 0.004000 & 3687577.780000 & 432892.680000 & \\
\hline CYN & -0.008330 & 3687577.780000 & 432892.680000 & \\
\hline HGTOT & -0.000333 & 3687577.780000 & 432892.680000 & \\
\hline MNTOT & -0.003330 & 3687577.780000 & 432892.680000 & \\
\hline NITOT & -0.006670 & 3687577.780000 & 432892.680000 & \\
\hline PBTOT & 0.001070 & 3687577.780000 & 432892.680000 & \\
\hline SETOT & -0.003330 & 3687577.780000 & 432892.680000 & \\
\hline 504 & -1.670000 & 3687577.780000 & 432892.680000 & \\
\hline TOTRAD & 0.000000 & 3687577.780000 & 432892.680000 & \\
\hline ZNTOT & 0.002000 & 3687577.780000 & 432892.680000 & \\
\hline ALTOT & -0.033300 & 3689063.050000 & 431358.940000 & \\
\hline BATOT & -0.005000 & 3689063.050000 & 431358.940000 & \\
\hline CRTOT & -0.006670 & 3689063.050000 & 431358.940000 & \\
\hline CYN & -0.008330 & 3689063.050000 & 431358.940000 & \\
\hline HGTOT & -0.000333 & 3689063.050000 & 431358.940000 & \\
\hline MNTOT & -0.003330 & 3689063.050000 & 431358.940000 & \\
\hline NITOT & -0.006670 & 3689063.050000 & 431358.940000 & \\
\hline PBTOT & -0.005000 & 3689063.050000 & 431358.940000 & \\
\hline
\end{tabular}


WSRC-TR-00347

November, 1997

Appendix II-25

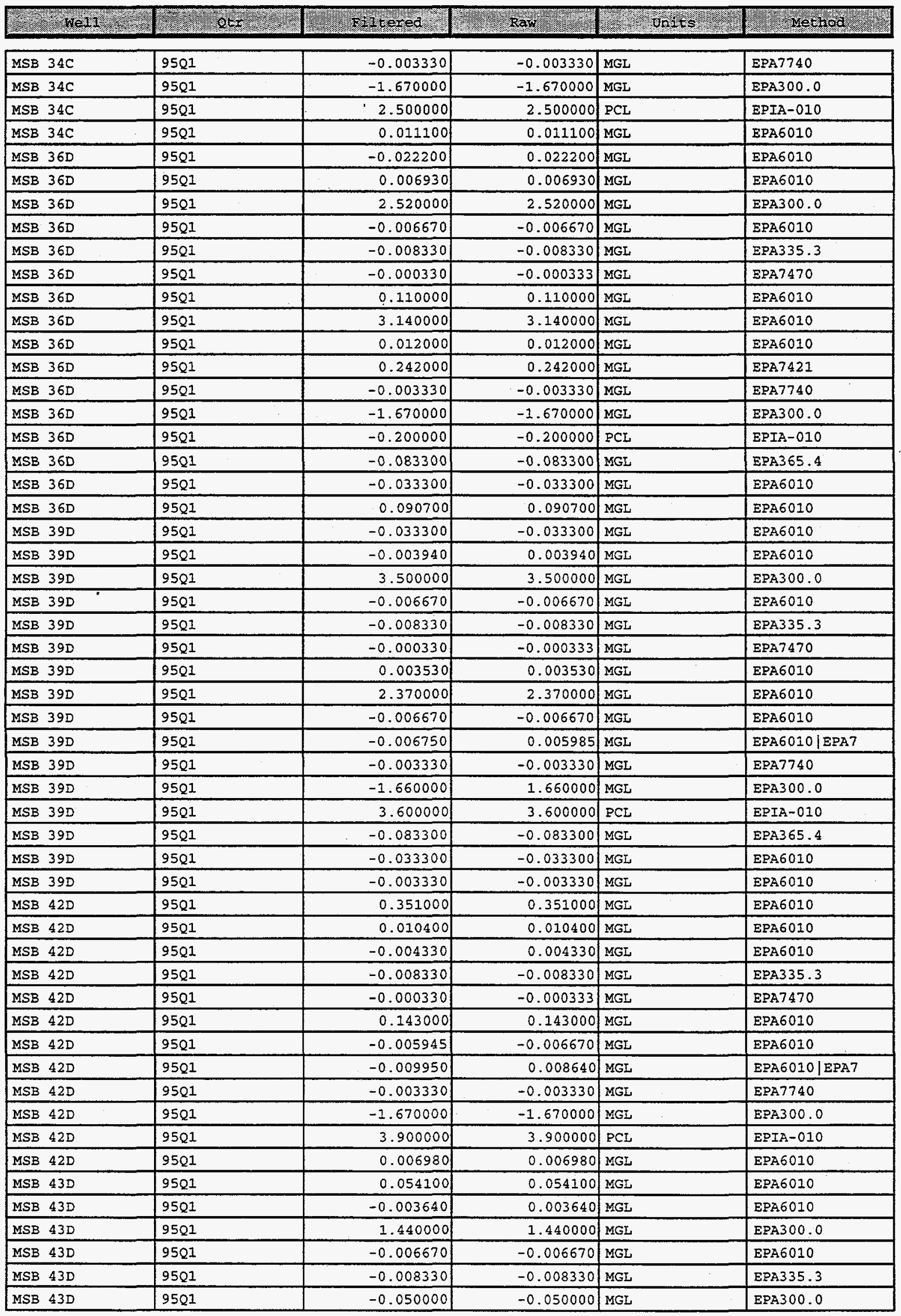


WSRC-TR-00347

November, 1997

Appendix II-26

\begin{tabular}{|c|c|c|c|c|}
\hline 504 & -1.670000 & 3689063.050000 & 431358.940000 & \\
\hline TOTRAD & -0.200000 & 3689063.050000 & 431358.940000 & \\
\hline ZNTOT & -0.003330 & 3689063.050000 & 431358.940000 & \\
\hline ALTOT & -0.033300 & 3687799.000000 & 431907.000000 & \\
\hline ВАTOT & -0.005000 & 3687799.000000 & 431907.000000 & \\
\hline CL & -0.417000 & 3687799.000000 & 431907.000000 & \\
\hline CRTOT & -0.006670 & 3687799.000000 & 431907.000000 & \\
\hline CYN & -0.008330 & 3687799.000000 & 431907.000000 & \\
\hline HGTOT & -0.000333 & 3687799.000000 & 431907.000000 & \\
\hline MNTOT & -0.003330 & 3687799.000000 & 431907.000000 & \\
\hline NATOT & -0.016700 & 3687799.000000 & 431907.000000 & \\
\hline NITOT & -0.006670 & 3687799.000000 & 431907.000000 & \\
\hline PBTOT & -0.005000 & 3687799.000000 & 431907.000000 & \\
\hline SETOT & -0.003330 & 3687799.000000 & 431907.000000 & \\
\hline 504 & -1.670000 & 3687799.000000 & 431907.000000 & \\
\hline TOTRAD & -0.100000 & 3687799.000000 & 431907.000000 & \\
\hline TPO4 & -0.083300 & 3687799.000000 & 431907.000000 & \\
\hline UTOT & -0.033300 & 3687799.000000 & 431907.000000 & \\
\hline ZNTOT & -0.003330 & 3687799.000000 & 431903.000000 & \\
\hline ALTOT & -0.033300 & 3687675.390000 & 431562.700000 & \\
\hline BATOT & -0.005000 & 3687675.390000 & 431562.700000 & \\
\hline CL & -0.417000 & 3687675.390000 & 431562.700000 & \\
\hline CRTOT & -0.006670 & 3687675.390000 & 431562.700000 & \\
\hline $\mathrm{CYN}$ & -0.008330 & 3687675.390000 & 431562.700000 & \\
\hline HGTOT & -0.000333 & 3687675.390000 & 431562.700000 & \\
\hline MNTOT & -0.003330 & 3687675.390000 & 431562.700000 & \\
\hline NATOT & -0.016700 & 3687675.390000 & 431562.700000 & \\
\hline NITOT & -0.006670 & 3687675.390000 & 431562.700000 & \\
\hline РВTOT & -0.005000 & 3687675.390000 & 431562.700000 & 2.000000 \\
\hline SETOT & -0.003330 & 3687675.390000 & 431562.700000 & \\
\hline SO4 & -1.670000 & 3687675.390000 & 431562.700000 & \\
\hline TOTRAD & & 3687675.390000 & 431562.700000 & \\
\hline TP04 & -0.083300 & 3687675.390000 & 431562.700000 & \\
\hline UTOT & -0.033300 & 3687675.390000 & 431562.700000 & \\
\hline ZNTOT & -0.003330 & 3687675.390000 & 431562.700000 & \\
\hline ALTOT & -0.033300 & 3689167.320000 & 431677.650000 & \\
\hline BATOT & -0.005000 & 3689167.320000 & 431677.650000 & \\
\hline CRTOT & -0.006670 & 3689167.320000 & 431577.650000 & \\
\hline $\mathrm{CYN}$ & -0.008330 & 3689167.320000 & 431677.650000 & \\
\hline HGTOT & -0.000333 & 3689167.320000 & 431677.650000 & \\
\hline MNTOT & -0.003330 & 3689167.320000 & 431677.650000 & \\
\hline NITOT & -0.006670 & 3689167.320000 & 431677.650000 & 2.000000 \\
\hline PBTOT & -0.005000 & 3689167.320000 & 431677.650000 & 2.000000 \\
\hline SETOT & -0.003330 & 3689167.320000 & 431677.650000 & \\
\hline 504 & -1.670000 & 3689167.320000 & 431677.650000 & \\
\hline TOTRAD & -0.400000 & 3689167.320000 & 431677.650000 & \\
\hline ZNTOT & -0.003330 & 3689167.320000 & 431677.650000 & \\
\hline ALTOT & -0.033300 & 3689421.940000 & 430540.350000 & \\
\hline BATOT & -0.005000 & 3689421.940000 & 430640.350000 & \\
\hline $\mathrm{CL}$ & -0.417000 & 3689421.940000 & 430640.350000 & \\
\hline CRTOT & -0.006670 & 3689421.940000 & 430640.350000 & \\
\hline$C Y N$ & 0.003370 & 3689421.940000 & 430640.350000 & \\
\hline $\mathbf{F}$ & -0.050000 & 3589421.940000 & 430640.350000 & \\
\hline
\end{tabular}


WSRC-TR-00347

November, 1997

Appendix II-27

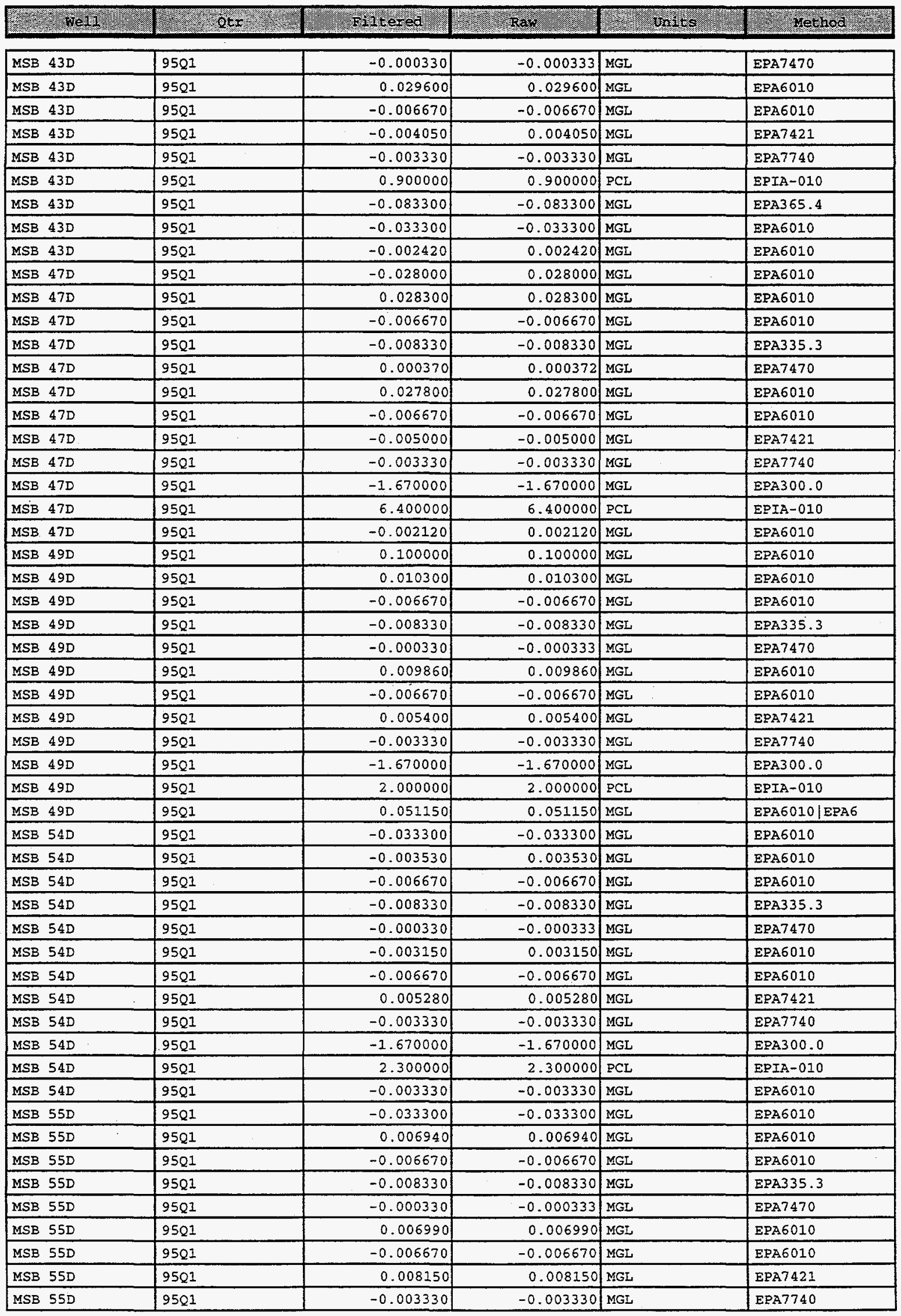


WSRC-TR-00347

November, 1997

Appendix II-28

\begin{tabular}{|c|c|c|c|c|}
\hline 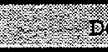 & Blark. & (3) vEm & Tut & 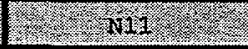 \\
\hline HGTOT & 0.000124 & 3689421.940000 & 430640.350000 & \\
\hline MNTOT & -0.003330 & 3689421.940000 & 430640.350000 & \\
\hline NITOT & -0.006670 & 3689421.940000 & 430640.350000 & \\
\hline PBTOT & 0.003000 & 3689421.940000 & 430640.350000 & \\
\hline SETOT & 0.002000 & 3689421.940000 & 430640.350000 & \\
\hline TOTRAD & & 3689421.940000 & 430640.350000 & \\
\hline TPO4 & -0.083300 & 3689421.940000 & 430640.350000 & \\
\hline UTOT & -0.033300 & 3689421.940000 & 430640.350000 & \\
\hline ZNTOT & -0.003330 & 3689421.940000 & 430640.350000 & \\
\hline ALTOT & -0.033300 & 3689857.790000 & 431401.740000 & \\
\hline BATOT & -0.005000 & 3689857.790000 & 431401.740000 & \\
\hline CRTOT & -0.006670 & 3689857.790000 & 431401.740000 & \\
\hline CYN & -0.008330 & 3689857.790000 & 431401.740000 & \\
\hline HGTOT & & 3689857.790000 & 431401.740000 & \\
\hline MNTOT & -0.003330 & 3689857.790000 & 431401.740000 & \\
\hline NITOT & -0.006670 & 3689857.790000 & 431401.740000 & \\
\hline PBTOT & -0.005000 & 3689857.790000 & 431401.740000 & \\
\hline SETOT & -0.003330 & 3689857.790000 & 431401.740000 & \\
\hline SO4 & -1.670000 & 3689857.790000 & 431401.740000 & \\
\hline TOTRAD & & 3689857.790000 & 431401.740000 & \\
\hline ZNTOT & -0.003330 & 3689857.790000 & 431401.740000 & \\
\hline ALTOT & -0.033300 & 3686940.650000 & 431140.370000 & \\
\hline BATOT & -0.005000 & 3686940.650000 & 431140.370000 & \\
\hline CRTOT & -0.006670 & 3686940.650000 & 431140.370000 & \\
\hline $\mathrm{CYN}$ & -0.008330 & 3686940.650000 & 431140.370000 & \\
\hline HGTOT & -0.000333 & 3686940.650000 & 431140.370000 & \\
\hline MNTOT & -0.003330 & 3686940.650000 & 431140.370000 & \\
\hline NITOT & -0.006670 & 3686940.650000 & 431140.370000 & \\
\hline PBTOT & -0.005000 & 3686940.650000 & 431140.370000 & \\
\hline SETOT & -0.003330 & 3686940.650000 & 431140.370000 & \\
\hline SO4 & -1.670000 & 3686940.650000 & 431140.370000 & \\
\hline TOTRAD & -0.200000 & 3686940.650000 & 431140.370000 & \\
\hline ZNTOT & -0.004165 & 3686940.650000 & 431140.370000 & 2.000000 \\
\hline ALTOT & -0.033300 & 3690371.220000 & 431329.710000 & \\
\hline BATOT & -0.005000 & 3690371.220000 & 431329.710000 & \\
\hline CRTOT & -0.006670 & 3690371.220000 & 431329.710000 & \\
\hline CYN & -0.008330 & 3690371.220000 & 431329.710000 & \\
\hline HGTOT & -0.000333 & 3690371.220000 & 431329.710000 & \\
\hline MNTOT & -0.003330 & 3690371.220000 & 431329.710000 & \\
\hline NITOT & -0.006670 & 3690371.220000 & 431329.710000 & \\
\hline PBTOT & -0.005000 & 3690371.220000 & 431329.710000 & \\
\hline SETOT & -0.003330 & 3690371.220000 & 431329.710000 & \\
\hline $\mathrm{SO} 4$ & -1.670000 & 3690371.220000 & 431329.710000 & \\
\hline TOTRAD & -0.100000 & 3690371.220000 & 431329.710000 & \\
\hline ZNTOT & -0.003330 & 3690371.220000 & 431329.710000 & \\
\hline ALTOT & -0.033300 & 3690183.230000 & 431107.750000 & \\
\hline BATOT & -0.005000 & 3690183.230000 & 431107.750000 & \\
\hline CRTOT & -0.006670 & 3690283.230000 & 431107.750000 & \\
\hline CYN & -0.008330 & 3690183.230000 & 431107.750000 & \\
\hline HGTOT & -0.000333 & 3690183.230000 & 431107.750000 & \\
\hline MNTOT & -0.003330 & 3690183.230000 & 431107.750000 & \\
\hline NITOT & -0.006670 & 3690183.230000 & 431107.750000 & \\
\hline PBTOT & -0.005000 & 3690183.230000 & 431107.750000 & \\
\hline SETOT & -0.003330 & 3690183.230000 & 431107.750000 & \\
\hline
\end{tabular}


WSRC-TR-00347

November, 1997

Appendix II-29

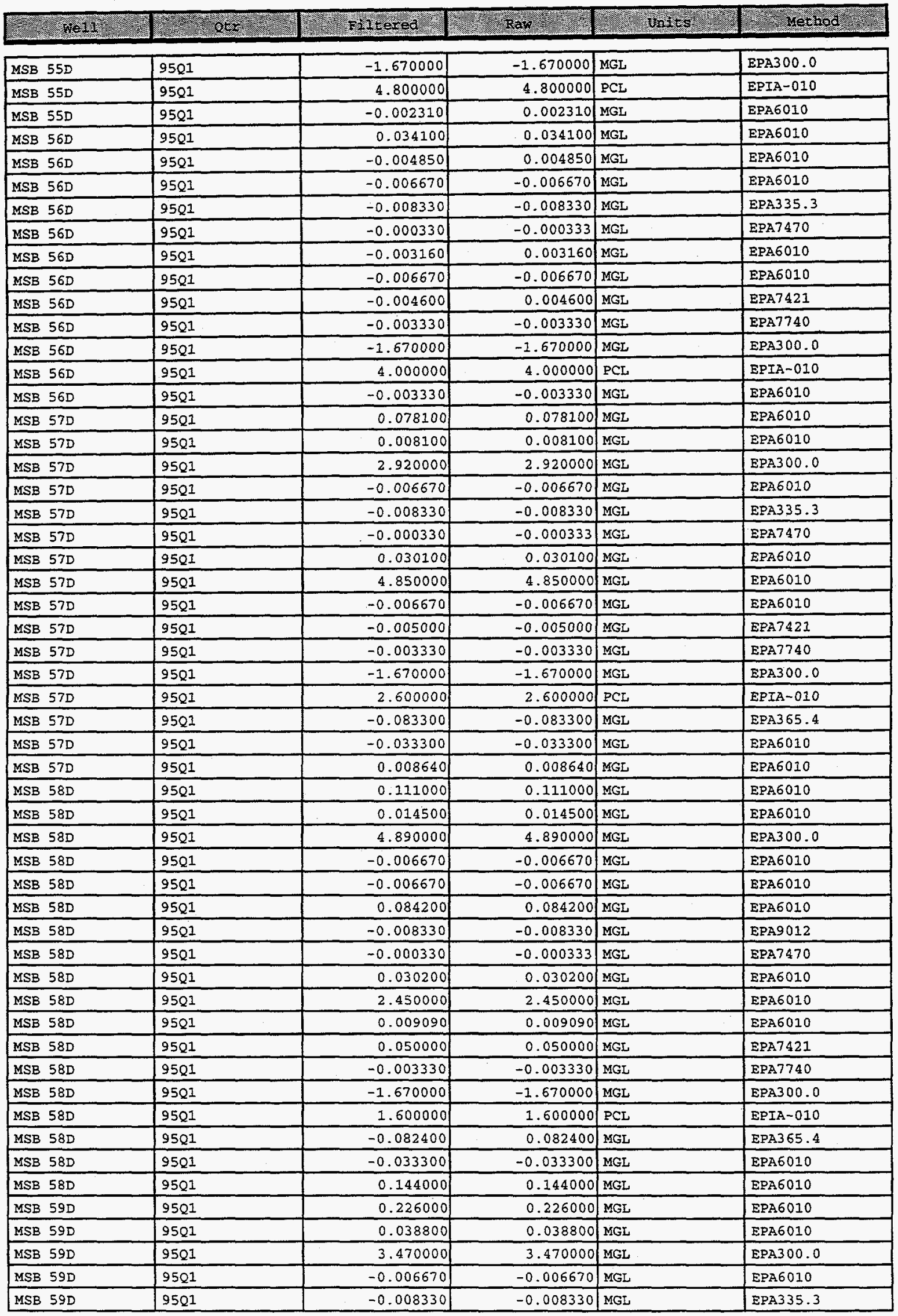


WSRC-TR-00347

November, 1997

Appendix II-30

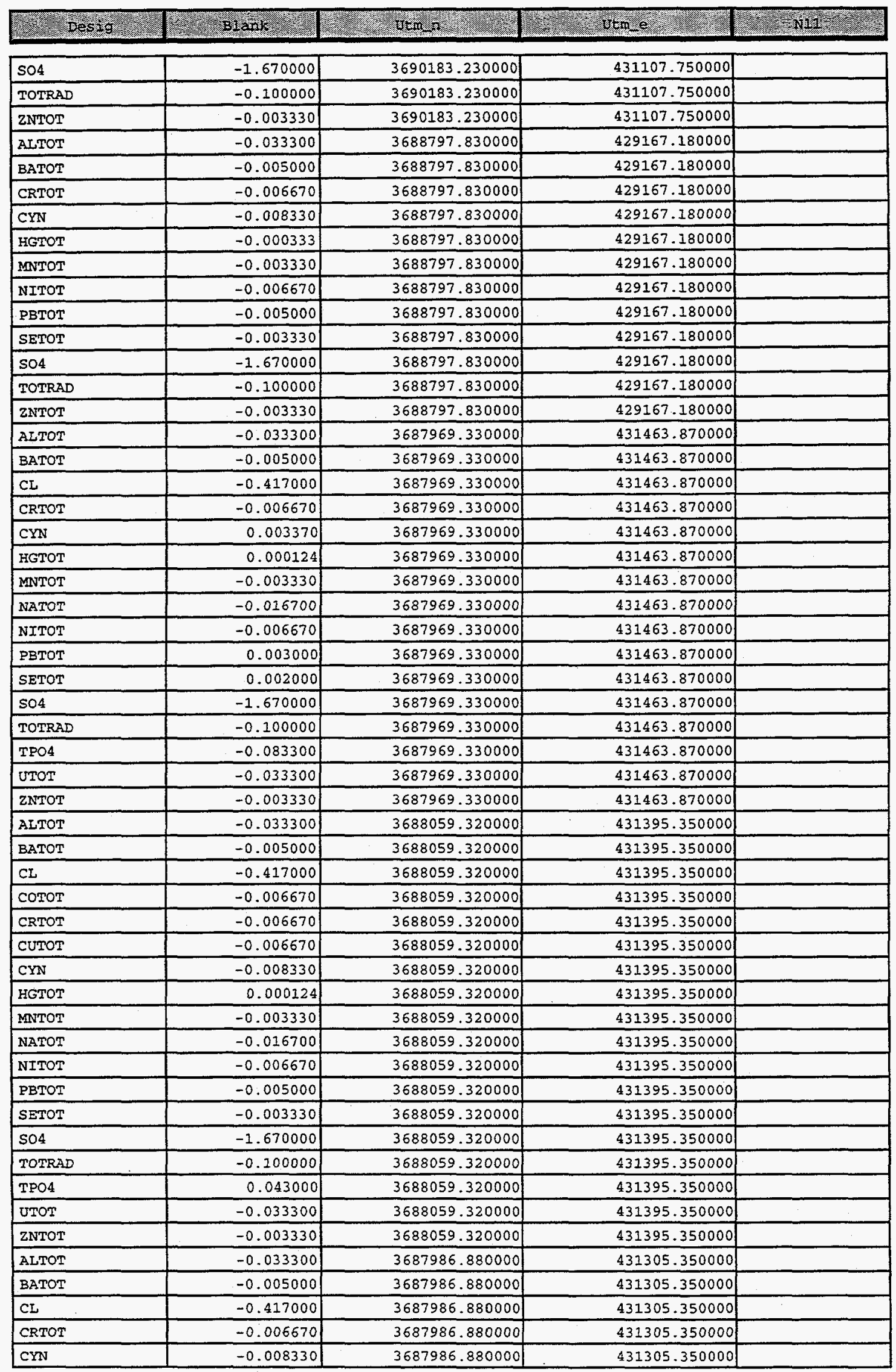




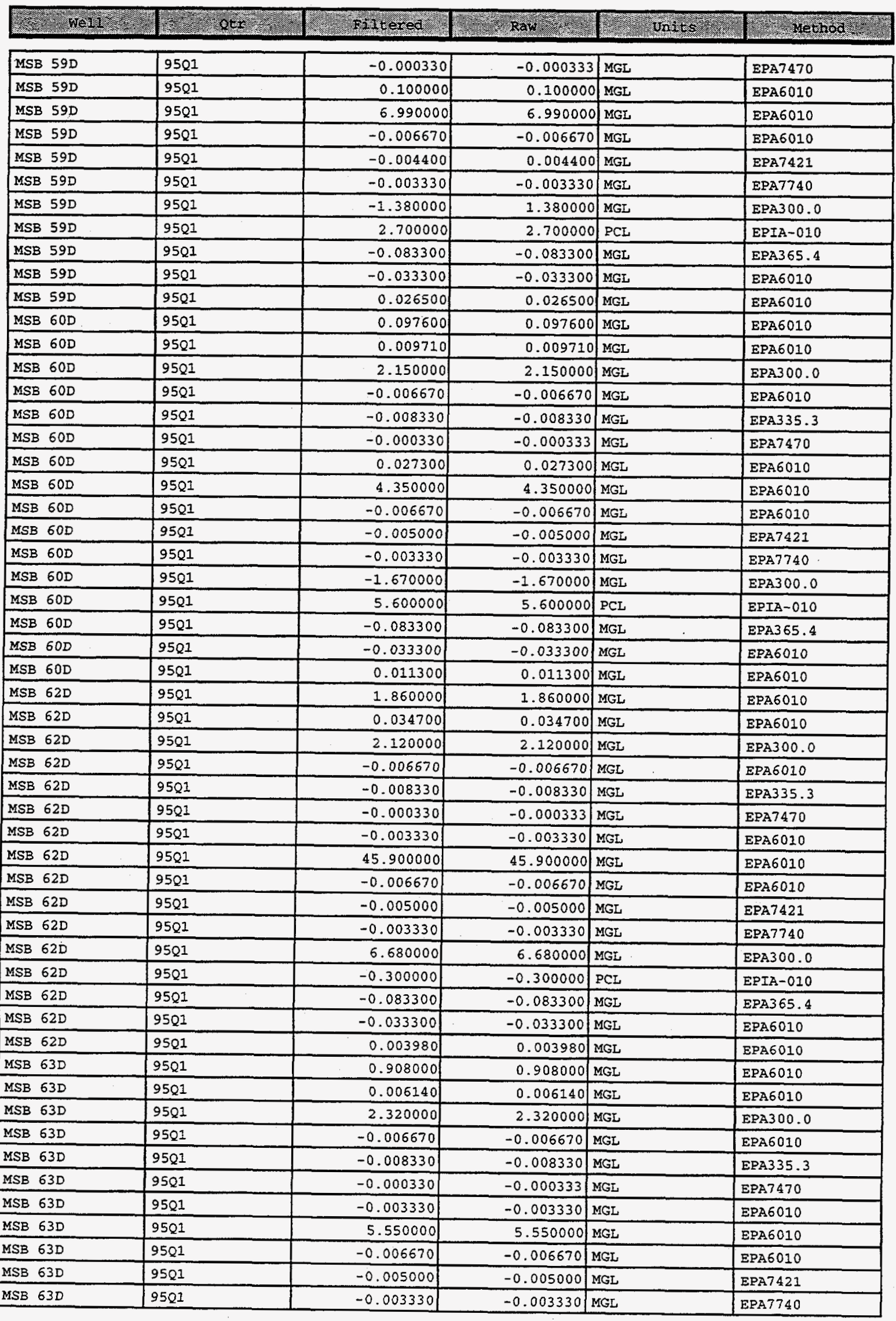


WSRC-TR-00347

November, 1997

Appendix II-32

\begin{tabular}{|c|c|c|c|c|}
\hline 87 pesig & IIs n n & 2040 & 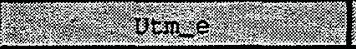 & nis \\
\hline HGTOT & -0.000333 & 3687986.880000 & 431305.350000 & \\
\hline MNTOT & -0.003330 & 3687986.880000 & 431305.350000 & \\
\hline NATOT & -0.016700 & 3687986.880000 & 431305.350000 & \\
\hline NITOT & -0.006670 & 3687986.880000 & 431305.350000 & \\
\hline PBTOT & -0.005000 & 3687986.880000 & 431305.350000 & \\
\hline SETOT & -0.003330 & 3687986.880000 & 431305.350000 & \\
\hline SO4 & -1.670000 & 3687986.880000 & 431305.350000 & \\
\hline TOTRAD & 0.000000 & 3687986.880000 & 431305.350000 & \\
\hline TPO4 & -0.083300 & 3687986.880000 & 431305.350000 & \\
\hline UTOT & -0.033300 & 3687986.880000 & 431305.350000 & \\
\hline ZNTOT & -0.003330 & 3687986.880000 & 431305.350000 & \\
\hline ALTOT & -0.033300 & 3687903.620000 & 431370.480000 & \\
\hline BATOT & -0.005000 & 3687903.620000 & 431370.480000 & \\
\hline $\mathrm{CL}$ & -0.417000 & 3687903.620000 & 431370.480000 & \\
\hline CRTOT & -0.006670 & 3687903.620000 & 431330.480000 & \\
\hline $\mathrm{CYN}$ & 0.003370 & 3687903.620000 & 431370.480000 & \\
\hline HGTOT & 0.000124 & 3687903.620000 & 431370.480000 & \\
\hline MNTOT & -0.003330 & 3687903.620000 & 431370.480000 & \\
\hline NATOT & -0.016700 & 3687903.620000 & 431370.480000 & \\
\hline NITOT & -0.006670 & 3687903.620000 & 431370.480000 & \\
\hline PBTOT & 0.003000 & 3687903.620000 & 431370.480000 & \\
\hline SETOT & 0.002000 & 3687903.620000 & 431370.480000 & \\
\hline 504 & -1.670000 & 3687903.620000 & 431370.480000 & \\
\hline TOTRAD & -0.100000 & 3687903.620000 & 431370.480000 & \\
\hline TPO4 & -0.083300 & 3687903.620000 & 431370.480000 & \\
\hline UTOT & -0.033300 & 3687903.620000 & 431370.480000 & \\
\hline ZNTOT & -0.003330 & 3687903.620000 & 431370.480000 & \\
\hline ALTOT & -0.033300 & 3687827.340000 & 431258.700000 & \\
\hline BATOT & -0.005000 & 3687827.340000 & 431258.700000 & \\
\hline $\mathrm{CL}$ & -0.417000 & 3687827.340000 & 431258.700000 & \\
\hline CRTOT & -0.006570 & 3687827.340000 & 431258.700000 & \\
\hline $\mathrm{CYN}$ & 0.003370 & 3687827.340000 & 431258.700000 & \\
\hline HGTOT & -0.000333 & 3687827.340000 & 431258.700000 & \\
\hline MNTOT & -0.003330 & 3687827.340000 & 431258.700000 & \\
\hline NATOT & -0.016700 & 3687827.340000 & 431258.700000 & \\
\hline NITOT & -0.006670 & 3687827.340000 & 431258.700000 & \\
\hline PBTOT & -0.005000 & 3687827.340000 & 431258.700000 & \\
\hline SETOT & -0.003330 & 3687827.340000 & 431258.700000 & \\
\hline SO4 & -1.670000 & 3687827.340000 & 431258.700000 & \\
\hline TOTRAD & & 3687827.340000 & 431258.700000 & \\
\hline TPO4 & -0.083300 & 3687827.340000 & 431258.700000 & \\
\hline UTOT & -0.033300 & 3687827.340000 & 431258.700000 & \\
\hline ZNTOT & -0.003330 & 3687827.340000 & 431258.700000 & \\
\hline ALTOT & -0.033300 & 3687650.720000 & 431370.120000 & \\
\hline BATOT & -0.005000 & 3687650.720000 & 431370.120000 & \\
\hline$C L_{1}$ & -0.417000 & 3687650.720000 & 431370.120000 & \\
\hline CRTOT & -0.006670 & 3687650.720000 & 431370.120000 & \\
\hline CYN & -0.008330 & 3687650.720000 & 431370.120000 & \\
\hline HGTOT & -0.000333 & 3687650.720000 & 431370.120000 & \\
\hline MNTOT & -0.003330 & 3687650.720000 & 431370.120000 & \\
\hline NATOT & -0.016700 & 3687650.720000 & 431370.120000 & \\
\hline NITOT & -0.006670 & 3687650.720000 & 431370.120000 & \\
\hline PBTOT & -0.005000 & 3687650.720000 & 431370.120000 & \\
\hline SETOT & -0.003330 & 3687650.720000 & 431370.120000 & \\
\hline
\end{tabular}


WSRC-TR-00347

November, 1997

Appendix II-33

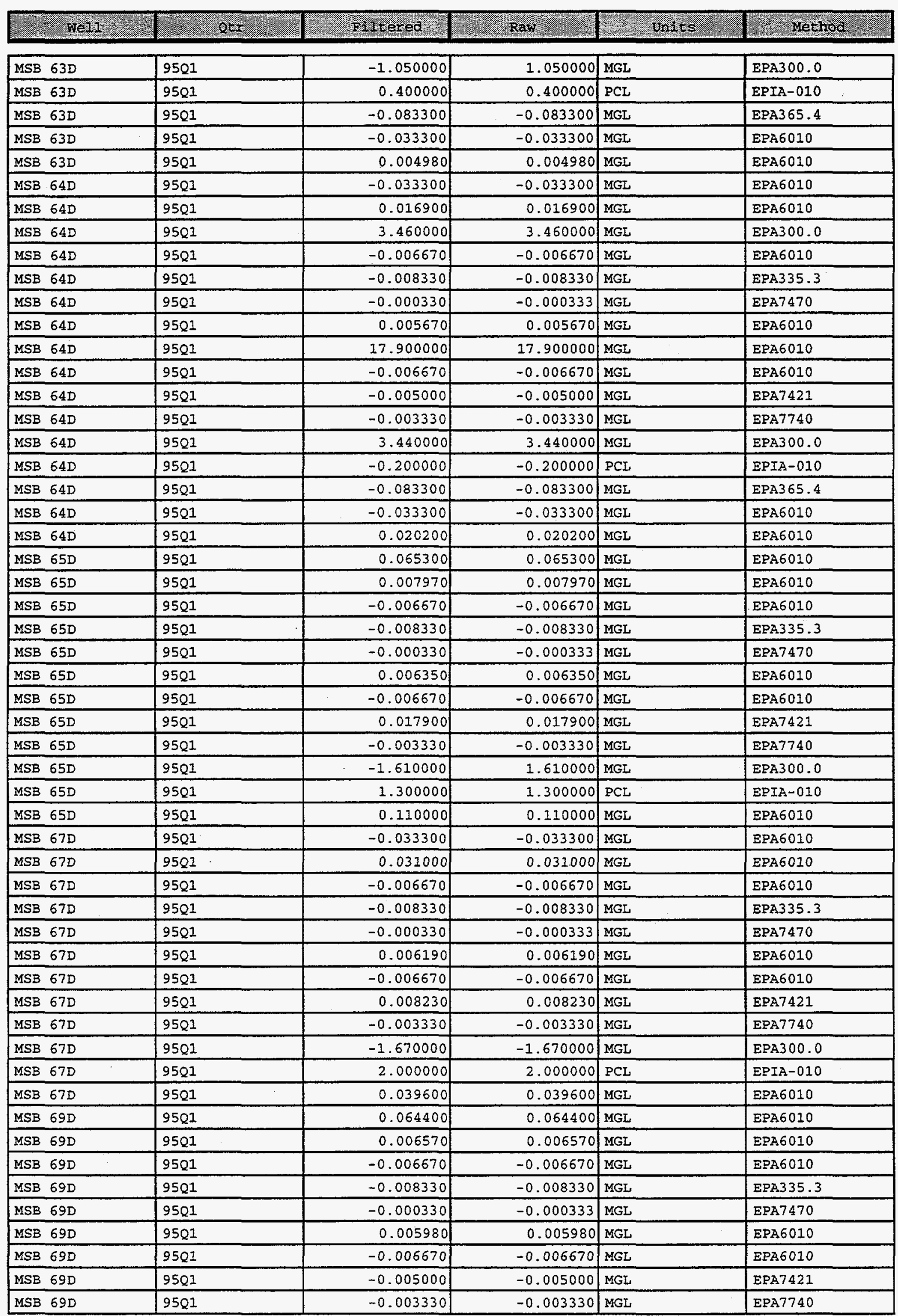


WSRC-TR-00347

November, 1997

Appendix II-34

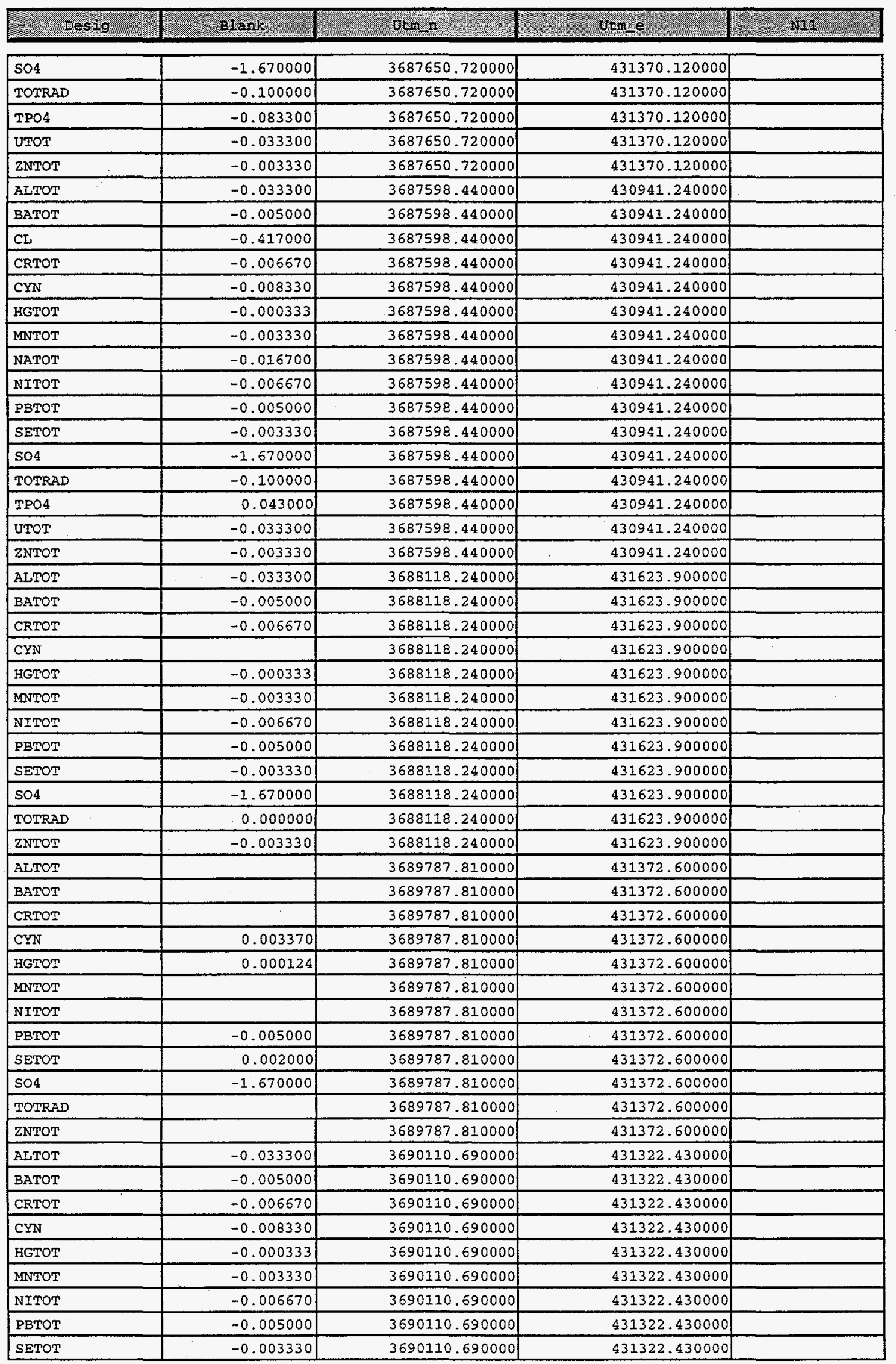


WSRC-TR-00347

November, 1997

Appendix II-35

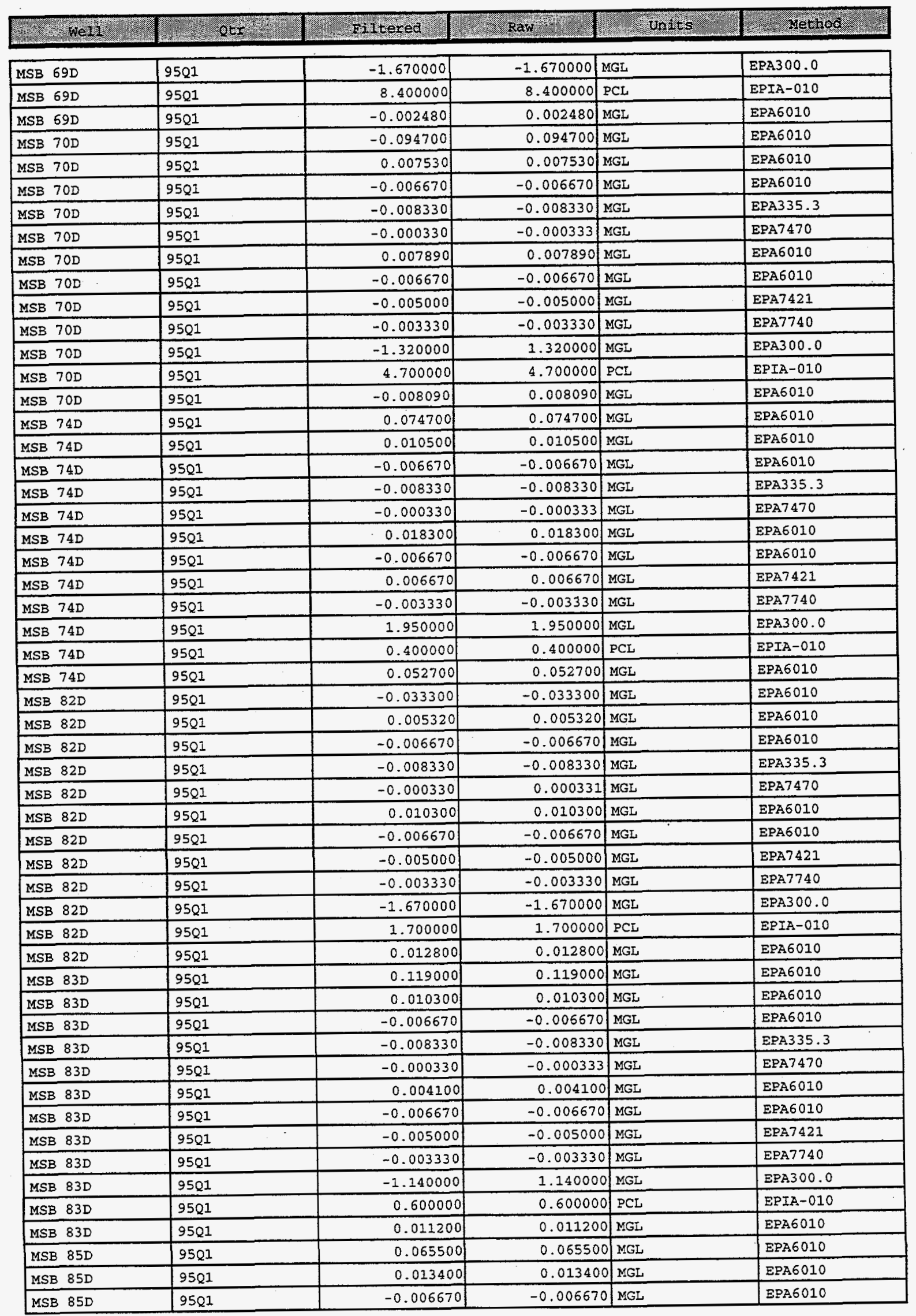


WSRC-TR-00347

November, 1997

Appendix II-36

\begin{tabular}{|c|c|c|c|c|}
\hline 10.2 .0 & Brank & Prespol & 7.7. & 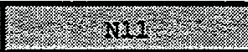 \\
\hline 504 & -1.670000 & 3690110.690000 & 431322.430000 & \\
\hline TOTRAD & -0.100000 & 3690110.690000 & 431322.430000 & \\
\hline ZNTOT & -0.003330 & 3690110.690000 & 431322.430000 & \\
\hline ALTOT & 0.020000 & 3687293.320000 & 430559.870000 & \\
\hline BATOT & -0.005000 & 3687293.320000 & 430559.870000 & \\
\hline CRTOT & 0.004000 & 3687293.320000 & 430559.870000 & \\
\hline CYN & & 3687293.320000 & 430559.870000 & \\
\hline HGTOT & -0.000333 & 3687293.320000 & 430559.870000 & \\
\hline MNTOT & -0.003330 & 3687293.320000 & 430559.870000 & \\
\hline NITOT & -0.006670 & 3687293.320000 & 430559.870000 & \\
\hline PBTOT & -0.005000 & 3687293.320000 & 430559.870000 & \\
\hline SETOT & -0.003330 & 3687293.320000 & 430559.870000 & \\
\hline 504 & -1.670000 & 3687293.320000 & 430559.870000 & \\
\hline TOTRAD & 0.000000 & 3687293.320000 & 430559.870000 & \\
\hline ZNTOT & 0.002000 & 3687293.320000 & 430559.870000 & \\
\hline ALTOT & -0.033300 & 3687635.010000 & 432373.650000 & \\
\hline BATOT & -0.005000 & 3687635.010000 & 432373.650000 & \\
\hline CRTOT & -0.006670 & 3687635.010000 & 432373.650000 & \\
\hline CYN & & 3687635.010000 & 432373.650000 & \\
\hline HGTOT & -0.000333 & 3687635.010000 & 432373.650000 & \\
\hline MNTOT & -0.003330 & 3687635.010000 & 432373.650000 & \\
\hline NITOT & -0.006670 & 3687635.010000 & 432373.650000 & \\
\hline РВTOT & -0.005000 & 3687635.010000 & 432373.650000 & \\
\hline SETOT & -0.003330 & 3687635.010000 & 432373.650000 & \\
\hline $\mathrm{SO4}$ & -1.670000 & 3687635.010000 & 432373.650000 & \\
\hline TOTRAD & 0.000000 & 3687635.010000 & 432373.650000 & \\
\hline ZNTOT & -0.003330 & 3687635.010000 & 432373.650000 & \\
\hline ALTOT & -0.033300 & 3689950.530000 & 431240.240000 & \\
\hline BATOT & -0.005000 & 3689950.530000 & 431240.240000 & \\
\hline CRTOT & -0.006670 & 3689950.530000 & 431240.240000 & \\
\hline CYN & -0.008330 & 3689950.530000 & 431240.240000 & \\
\hline HGTOT & -0.000333 & 3689950.530000 & 431240.240000 & \\
\hline MNTOT & -0.003330 & 3689950.530000 & 431240.240000 & \\
\hline NITOT & 0.009480 & 3689950.530000 & 431240.240000 & \\
\hline PBTOT & -0.005000 & 3689950.530000 & 431240.240000 & \\
\hline SETOT & -0.003330 & 3689950.530000 & 431240.240000 & \\
\hline $\mathrm{SO4}$ & -1.670000 & 3689950.530000 & 431240.240000 & \\
\hline TOTRAD & & 3689950.530000 & 431240.240000 & \\
\hline ZNTOT & -0.003330 & 3689950.530000 & 431240.240000 & \\
\hline ALTOT & -0.033300 & 3690254.210000 & 431191.280000 & \\
\hline BATOT & -0.005000 & 3690254.210000 & 431191.280000 & \\
\hline CRTOT & -0.006670 & 3690254.210000 & 431191.280000 & \\
\hline CYN & -0.008330 & 3690254.210000 & 431191.280000 & \\
\hline HGTOT & -0.000333 & 3690254.210000 & 431191.280000 & \\
\hline MNTOT & -0.003330 & 3690254.210000 & 431191.280000 & \\
\hline NITOT & 0.009480 & 3690254.210000 & 431191.280000 & \\
\hline PBTOT & -0.005000 & 3690254.210000 & 431191.280000 & \\
\hline SETOT & -0.003330 & 3690254.210000 & 431191.280000 & \\
\hline $\mathrm{SO} 4$ & -1.670000 & 3690254.210000 & 431191.280000 & \\
\hline TOTRAD & & 3690254.210000 & 431191.280000 & \\
\hline ZNTOT & -0.003330 & 3690254.210000 & 431191.280000 & \\
\hline ALTOT & -0.033300 & 3690236.160000 & 431474.870000 & \\
\hline BATOT & -0.005000 & 3690236.160000 & 431474.870000 & \\
\hline CRTOT & -0.006670 & 3690236.160000 & 431474.870000 & \\
\hline
\end{tabular}


WSRC-TR-00347

November, 1997

Appendix II-37

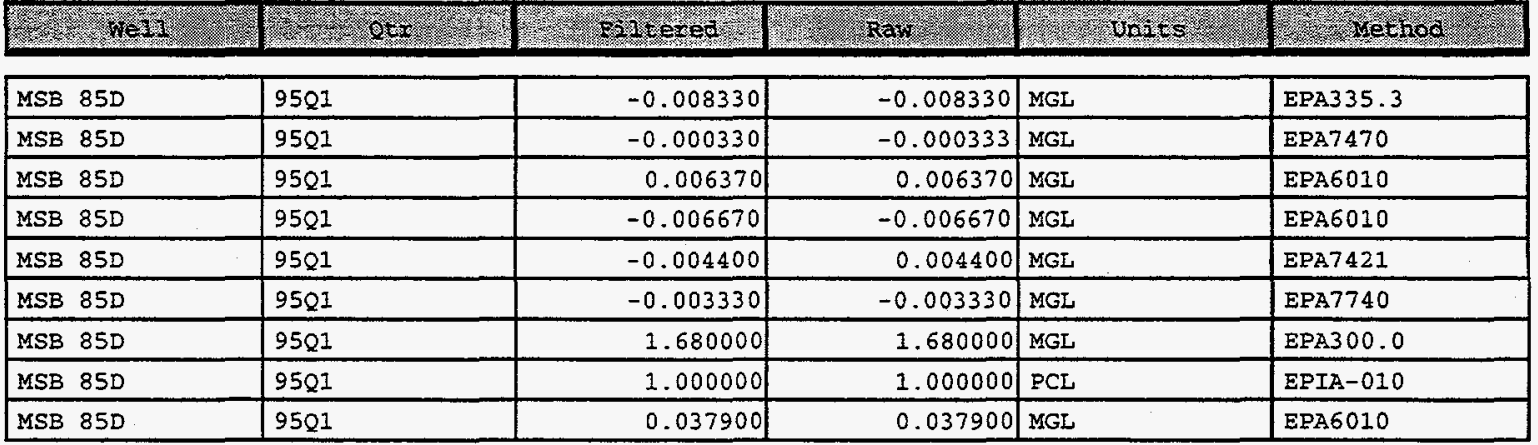


WSRC-TR-00347

November, 1997

Appendix II-38

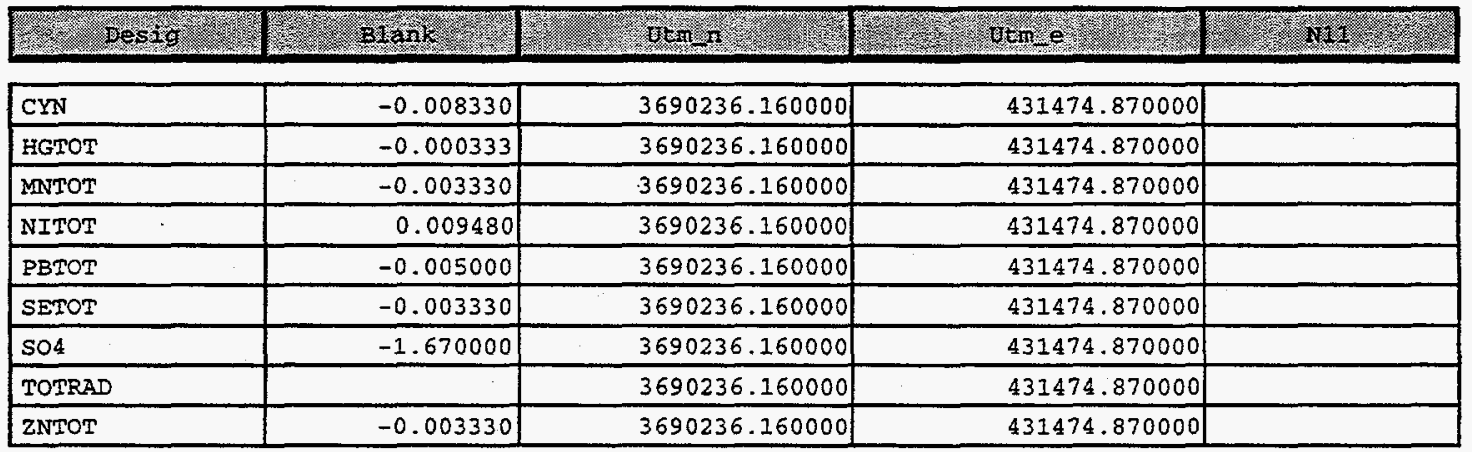


IVSRC-TR-00347

November, 1997

Appendix II-39

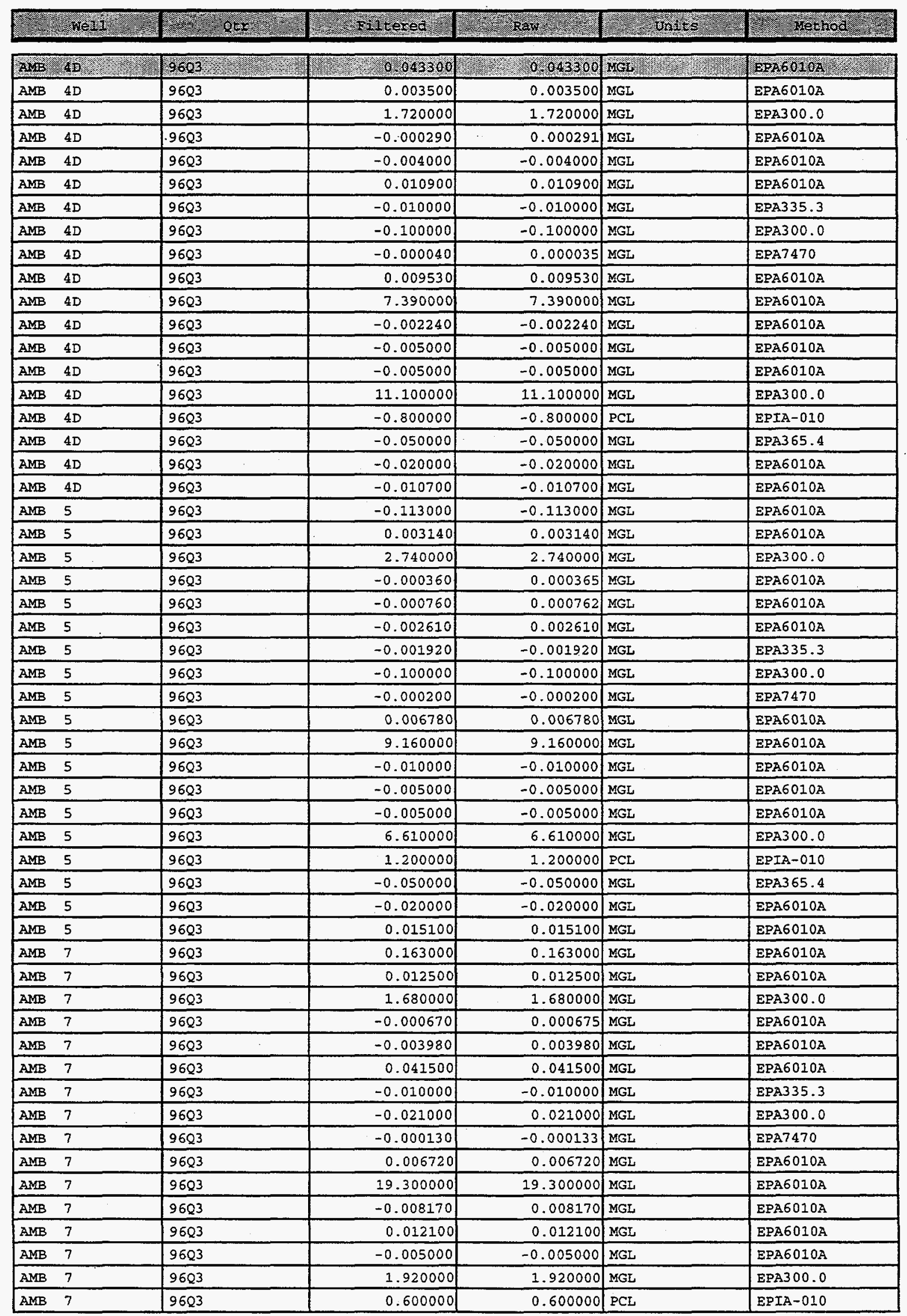


WSRC-TR-00347

November, 1997

Appendix II-40

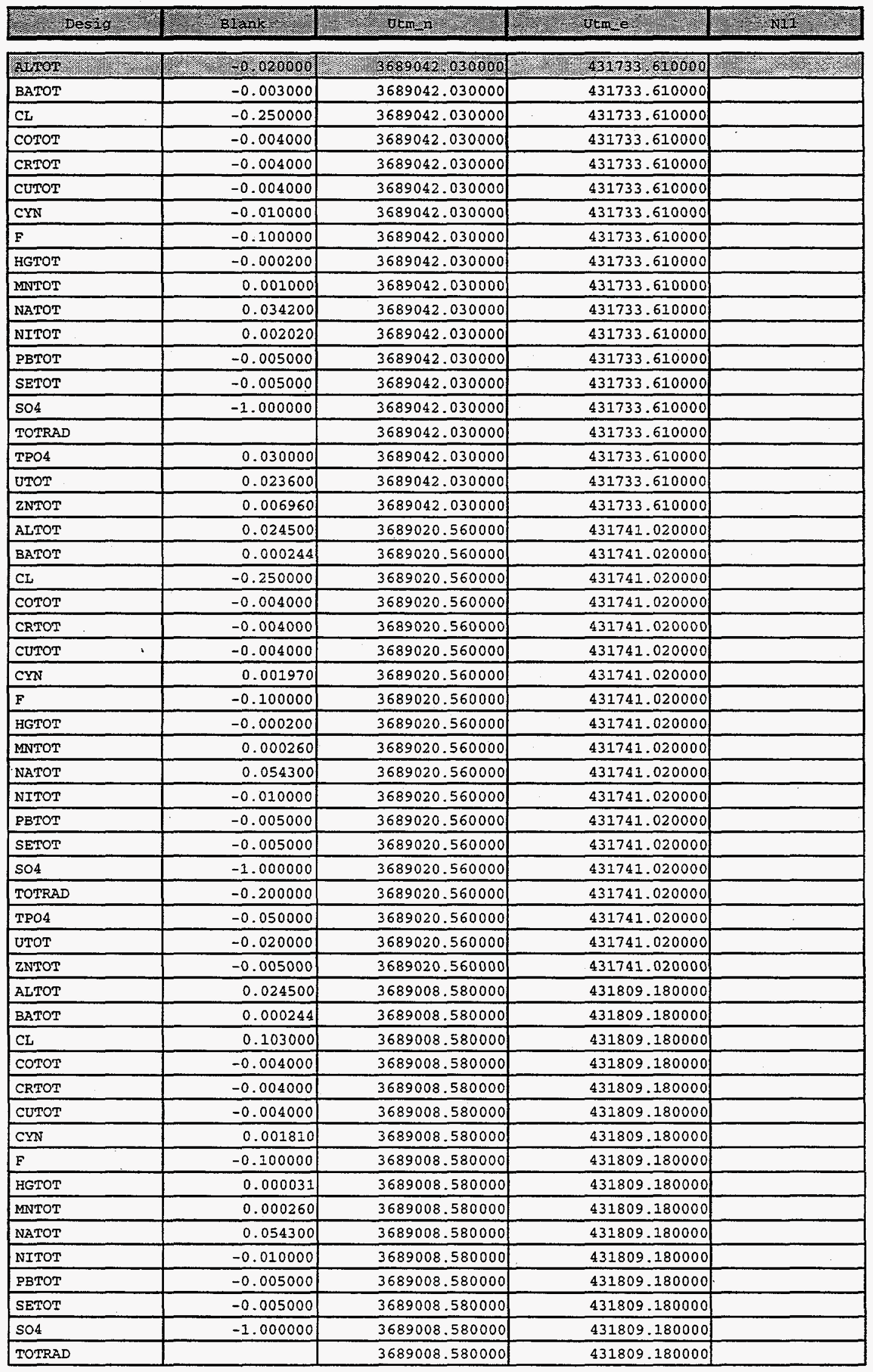




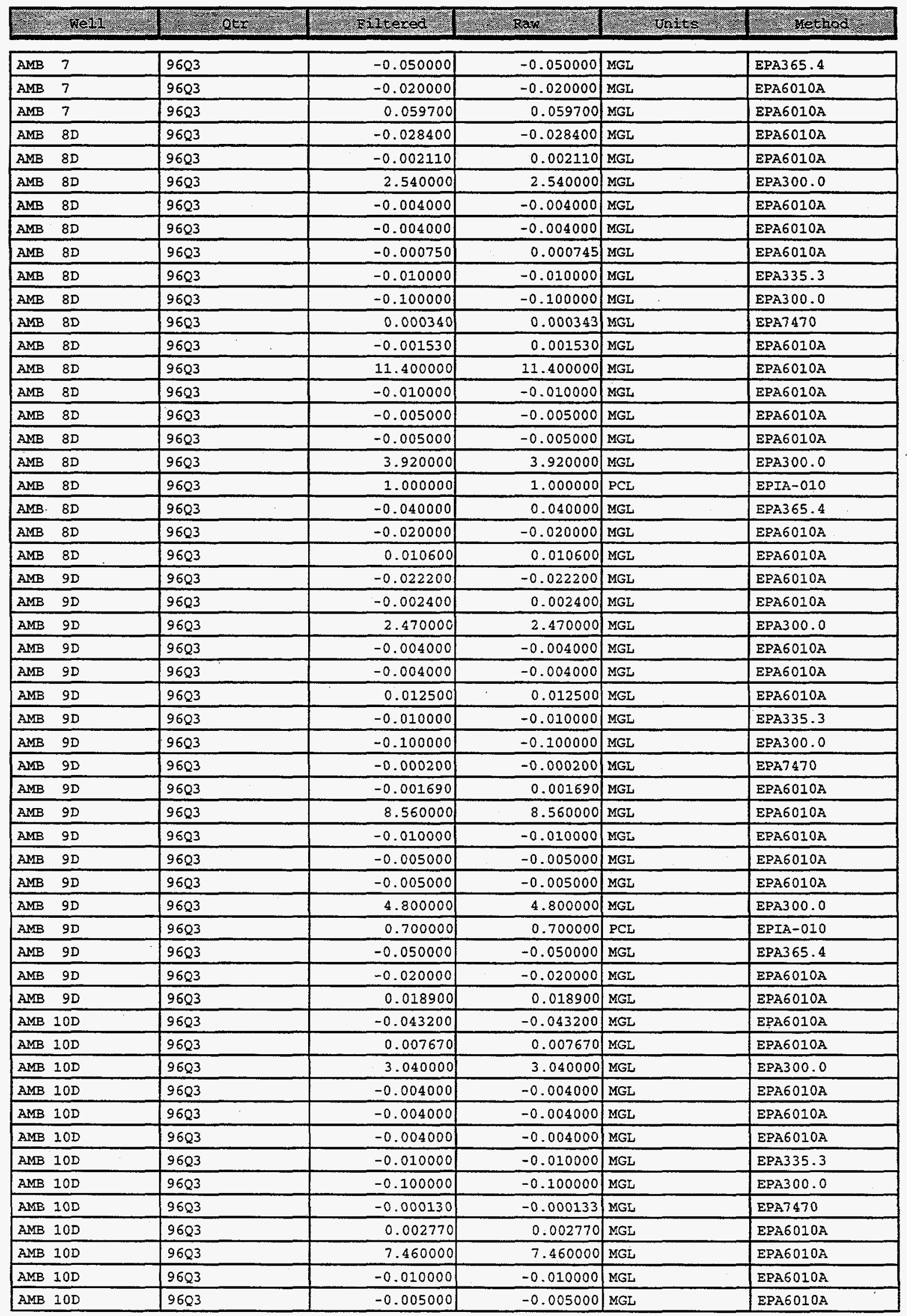


WSRC-TR-00347

November, 1997

Appendix 11-42

\begin{tabular}{|c|c|c|c|c|}
\hline 7ref & $2.3 \ln 2 \pi$ & (2) gtm & $6 \tan 2$ & 10.1111 \\
\hline TPO4 & -0.050000 & 3689008.580000 & 431809.180000 & \\
\hline UTOT & -0.020000 & 3689008.580000 & 431809.180000 & \\
\hline ZNTOT & -0.005000 & 3689008.580000 & 431809.180000 & \\
\hline ALTOT & 0.024500 & 3688957.180000 & 431762.020000 & \\
\hline BATOT & 0.000244 & 3688957.180000 & 431762.020000 & \\
\hline $\mathrm{CL}$ & 0.103000 & 3688957.180000 & 431762.020000 & \\
\hline COTOT & -0.004000 & 3688957.180000 & 431762.020000 & \\
\hline CRTOT & -0.004000 & 3688957.180000 & 431762.020000 & \\
\hline CUTOT & -0.004000 & 3688957.180000 & 431762.020000 & \\
\hline CYN & 0.001810 & 3688957.180000 & 431762.020000 & \\
\hline$E$ & -0.100000 & 3688957.180000 & 431762.020000 & \\
\hline HGTOT & 0.000031 & 3688957.180000 & 431762.020000 & \\
\hline MNTOT & 0.000260 & 3688957.180000 & 431762.020000 & \\
\hline NATOT & 0.054300 & 3688957.180000 & 431762.020000 & \\
\hline NITOT & -0.010000 & 3688957.180000 & $\$ 31762.020000$ & \\
\hline PBTOT & -0.005000 & 3688957.180000 & 431762.020000 & \\
\hline SETOT & -0.005000 & 3688957.180000 & 431762.020000 & \\
\hline $\mathrm{SO} 4$ & -1.000000 & 3688957.180000 & 431762.020000 & \\
\hline TOTRAD & & 3588957.180000 & 431762.020000 & \\
\hline TPOA & -0.050000 & 3688957.180000 & 431762.020000 & \\
\hline UTOT & -0.020000 & 3688957.180000 & 431762.020000 & \\
\hline ZNTOT & -0.005000 & 3688957.180000 & 431762.020000 & \\
\hline ALTOT & 0.024500 & 3688861.210000 & 431780.060000 & \\
\hline BATOT & 0.000244 & 3688861.210000 & 431780.060000 & \\
\hline$C L$ & 0.103000 & 3688861.210000 & 431780.050000 & \\
\hline COTOT & -0.004000 & 3688861.210000 & 431780.060000 & \\
\hline CRTOT & -0.004000 & 3688861.210000 & 431780.060000 & \\
\hline CUTOT & -0.004000 & 3688861.210000 & 431780.060000 & \\
\hline$C Y N$ & 0.001810 & 3688861.210000 & 431780.060000 & \\
\hline $\mathbf{F}$ & -0.100000 & 3688861.210000 & 431780.060000 & \\
\hline HGTOT & -0.000200 & 3688863.210000 & 431780.060000 & \\
\hline MNTOT & 0.000260 & 3688861.210000 & 431780.050000 & \\
\hline NATOT & 0.054300 & 3688861.210000 & 431780.060000 & \\
\hline NITOT & -0.010000 & 3688861.210000 & 431780.060000 & \\
\hline PBTOT & -0.005000 & 3688861.210000 & 431780.060000 & \\
\hline SETOT & -0.005000 & 3688861.210000 & 431780.060000 & \\
\hline $\mathrm{SO} 4$ & -1.000000 & 3688861.210000 & 431780.060000 & \\
\hline TOTRAD & & 3688861.210000 & 431780.060000 & \\
\hline TPO4 & -0.050000 & 3688861.210000 & 431780.060000 & \\
\hline UTOT & -0.020000 & 3688861.210000 & 431780.060000 & \\
\hline ZNTOT & -0.005000 & 3588861.210000 & 431780.060000 & \\
\hline ALTOT & 0.024500 & 3688823.930000 & 431879.930000 & \\
\hline BATOT & 0.000244 & 3688823.930000 & 431879.930000 & \\
\hline$C L$ & 0.103000 & 3588823.930000 & 431879.930000 & \\
\hline СОTOT & -0.004000 & 3688823.930000 & 431879.930000 & \\
\hline CRTOT & -0.004000 & 3688823.930000 & 431879.930000 & \\
\hline CUTOT & -0.004000 & 3688823.930000 & 431879.930000 & \\
\hline$C Y N$ & 0.001810 & 3688823.930000 & 431879.930000 & \\
\hline$F$ & -0.100000 & 3688823.930000 & 431879.930000 & \\
\hline HGTOT & 0.000031 & 3688823.930000 & 431879.930000 & \\
\hline MNTOT & 0.000260 & 3688823.930000 & 431879.930000 & \\
\hline NATOT & 0.054300 & 3688823.930000 & 431879.930000 & \\
\hline NITOT & -0.010000 & 3688823.930000 & 431879.930000 & \\
\hline PBTOT & -0.005000 & 3688823.930000 & 431879.930000 & \\
\hline
\end{tabular}


WSRC-TR-00347

November, 1997

Appendix II-43

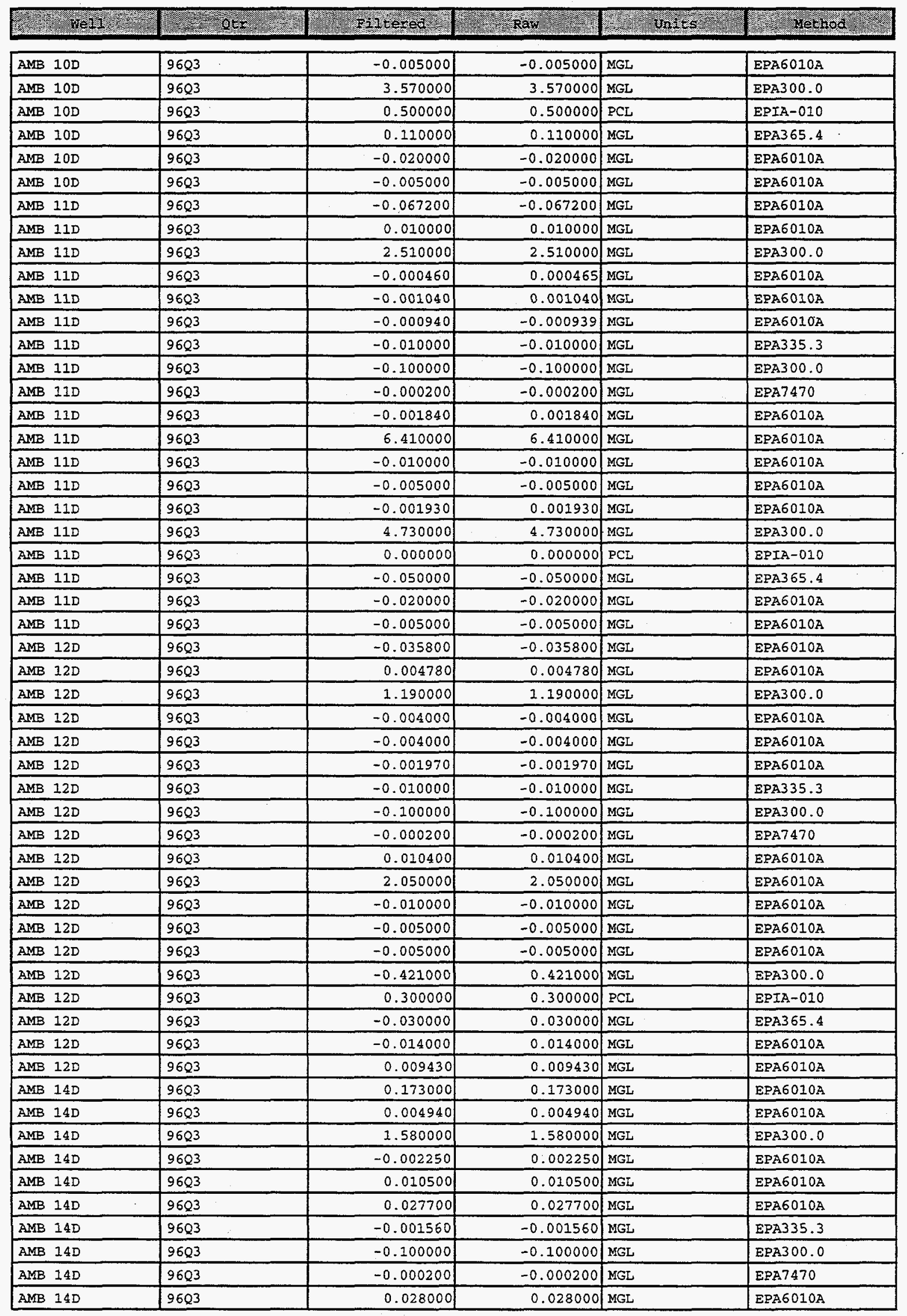




\begin{tabular}{|c|c|c|c|c|}
\hline 102 & Blant & ben & 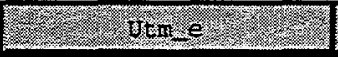 & (2) \\
\hline SETOT & -0.005000 & 3688823.930000 & 431879.930000 & \\
\hline SO4 & -1.000000 & 3688823.930000 & 431879.930000 & \\
\hline TOTRAD & & 3688823.930000 & 431879.930000 & \\
\hline TPO4 & -0.050000 & 3688823.930000 & 431879.930000 & \\
\hline UTOT & -0.020000 & 3688823.930000 & 431879.930000 & \\
\hline ZNTOT & -0.005000 & 3688823.930000 & 431879.930000 & \\
\hline ALTOT & 0.021700 & 3688869.710000 & 432026.240000 & \\
\hline BATOT & 0.000150 & 3688869.710000 & 432026.240000 & \\
\hline $\mathrm{CL}$ & 0.103000 & 3688869.710000 & 432026.240000 & \\
\hline COTOT & -0.004000 & 3688869.710000 & 432026.240000 & \\
\hline CRTOT & -0.004000 & 3688869.710000 & 432026.240000 & \\
\hline CUTOT & 0.001110 & 3688869.710000 & 432026.240000 & \\
\hline CYN & 0.001810 & 3688869.710000 & 432026.240000 & \\
\hline$F$ & -0.100000 & 3688869.710000 & 432026.240000 & \\
\hline HGTOT & -0.000200 & 3688869.710000 & 432026.240000 & \\
\hline MNTOT & 0.000139 & 3688869.710000 & 432026.240000 & \\
\hline NATOT & 0.074400 & 3688869.710000 & 432026.240000 & \\
\hline NITOT & -0.010000 & 3688869.710000 & 432026.240000 & \\
\hline PBTOT & -0.005000 & 3688869.710000 & 432026.240000 & \\
\hline SETOT & -0.005000 & 3688869.710000 & 432026.240000 & \\
\hline SO4 & -1.000000 & 3688869.710000 & 432026.240000 & \\
\hline TOTRAD & & 3688869.710000 & 432026.240000 & \\
\hline TPO4 & -0.050000 & 3688869.710000 & 432026.240000 & \\
\hline UTOT & -0.020000 & 3688869.710000 & 432026.240000 & \\
\hline ZNTOT & -0.005000 & 3688869.710000 & 432026.240000 & \\
\hline ALTOT & 0.021700 & 3688979.960000 & 431934.300000 & \\
\hline BATOT & 0.000150 & 3688979.960000 & 431934.300000 & \\
\hline $\mathrm{CL}$ & 0.103000 & 3688979.960000 & 431934.300000 & \\
\hline COTOT & -0.004000 & 3688979.960000 & 431934.300000 & \\
\hline CRTOT & -0.004000 & 3688979.960000 & 431934.300000 & \\
\hline CUTOT & 0.001110 & 3688979.960000 & 431934.300000 & \\
\hline CYN & 0.001810 & 3688979.960000 & 431934.300000 & \\
\hline$F$ & -0.100000 & 3688979.960000 & 431934.300000 & \\
\hline HGTOT & -0.000200 & 3688979.960000 & 431934.300000 & \\
\hline MNTOT & 0.000139 & 3688979.960000 & 431934.300000 & \\
\hline NATOT & 0.074400 & 3688979.960000 & 431934.300000 & \\
\hline NITOT & -0.010000 & 3688979.960000 & 431934.300000 & \\
\hline PBTOT & -0.005000 & 3688979.960000 & 431934.300000 & \\
\hline SETOT & -0.005000 & 3688979.960000 & 431934.300000 & \\
\hline $\mathrm{SO} 4$ & -1.000000 & 3688979.960000 & 431934.300000 & \\
\hline TOTRAD & & 3688979.960000 & 431934.300000 & \\
\hline TPO4 & -0.050000 & 3688979.960000 & 431934.300000 & \\
\hline UTOT & -0.020000 & 3688979.960000 & 431934.300000 & \\
\hline ZNTOT & -0.005000 & 3688979.960000 & 431934.300000 & \\
\hline ALTOT & 0.024500 & 3689049.590000 & 431679.790000 & \\
\hline BATOT & 0.000244 & 3689049.590000 & 431679.790000 & \\
\hline $\mathrm{CL}$ & -0.250000 & 3689049.590000 & 431679.790000 & \\
\hline COTOT & -0.004000 & 3689049.590000 & 431679.790000 & \\
\hline CRTOT & -0.004000 & 3689049.590000 & 431679.790000 & \\
\hline CUTOT & -0.004000 & 3689049.590000 & 431679.790000 & \\
\hline $\mathrm{CYN}$ & 0.001970 & 3689049.590000 & 431679.790000 & \\
\hline$F$ & -0.100000 & 3689049.590000 & 431679.790000 & \\
\hline HGTOT & -0.000200 & 3689049.590000 & 431679.790000 & \\
\hline MNTOT & 0.000260 & 3689049.590000 & 431679.790000 & \\
\hline
\end{tabular}


WSRC-TR-00347

November, 1997

Appendix II-45

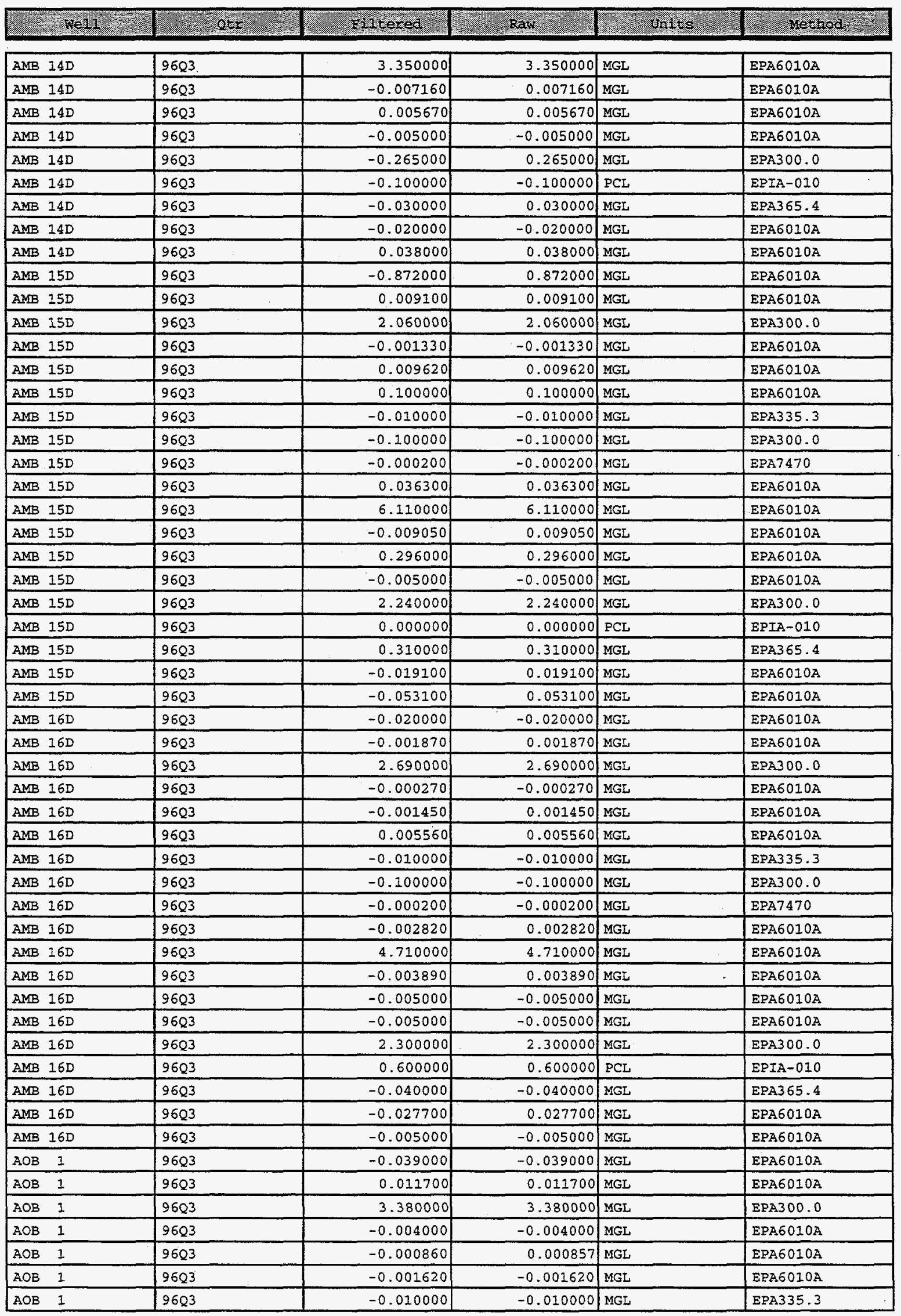


WSRC-TR-00347

November, 1997

Appendix II-46

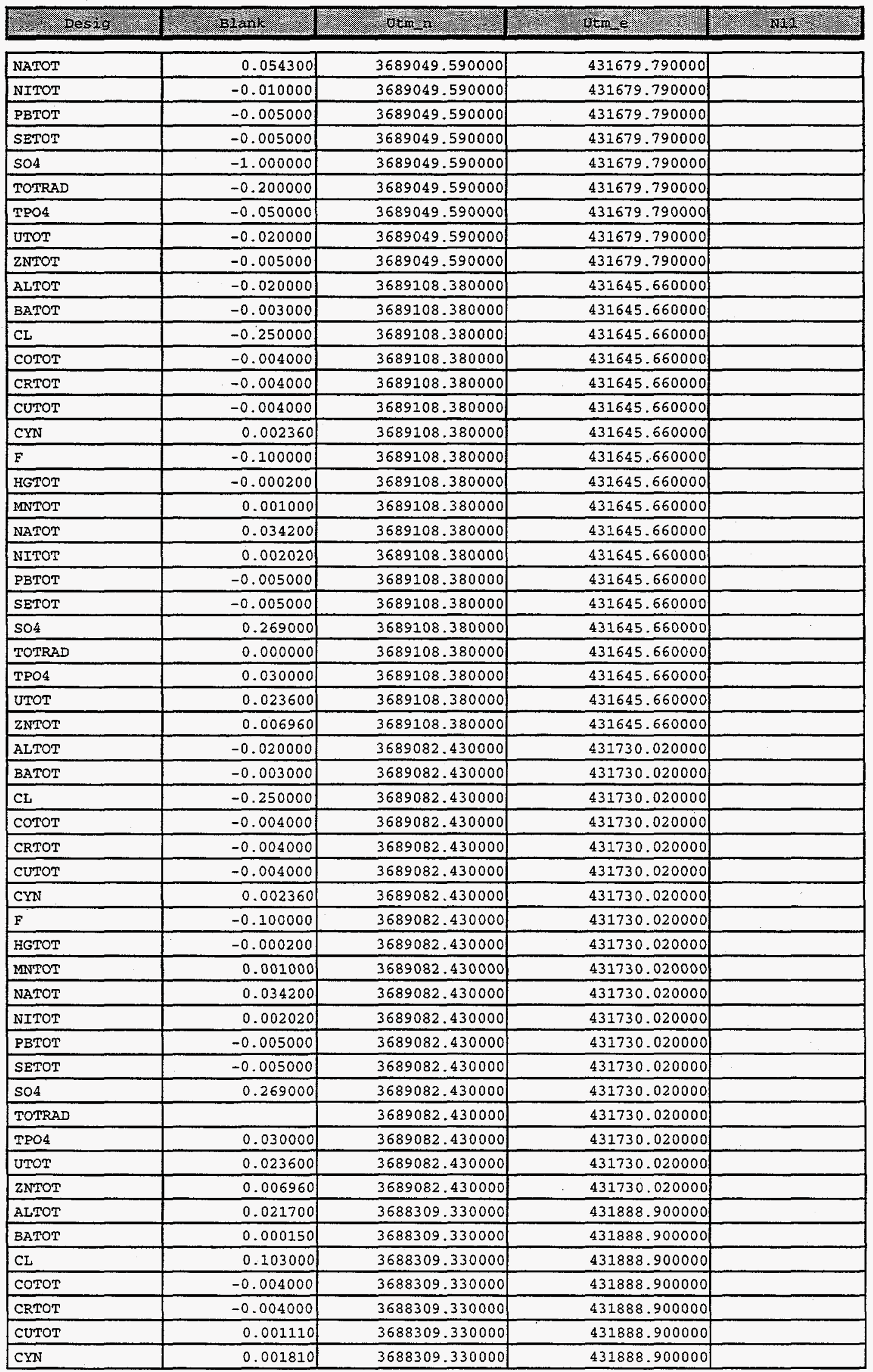


WSRC-TR-00347

November, 1997

Appendix II-47

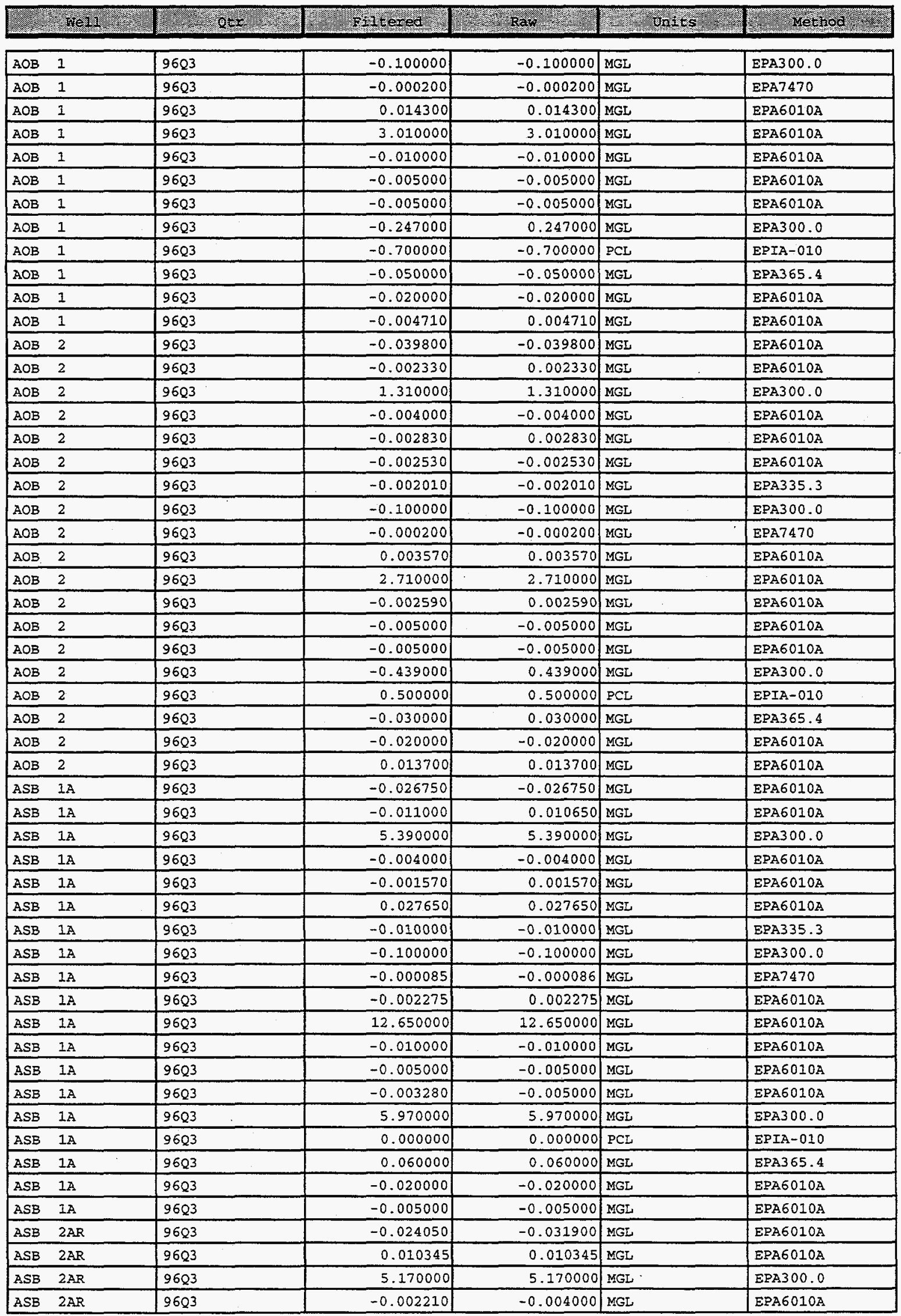




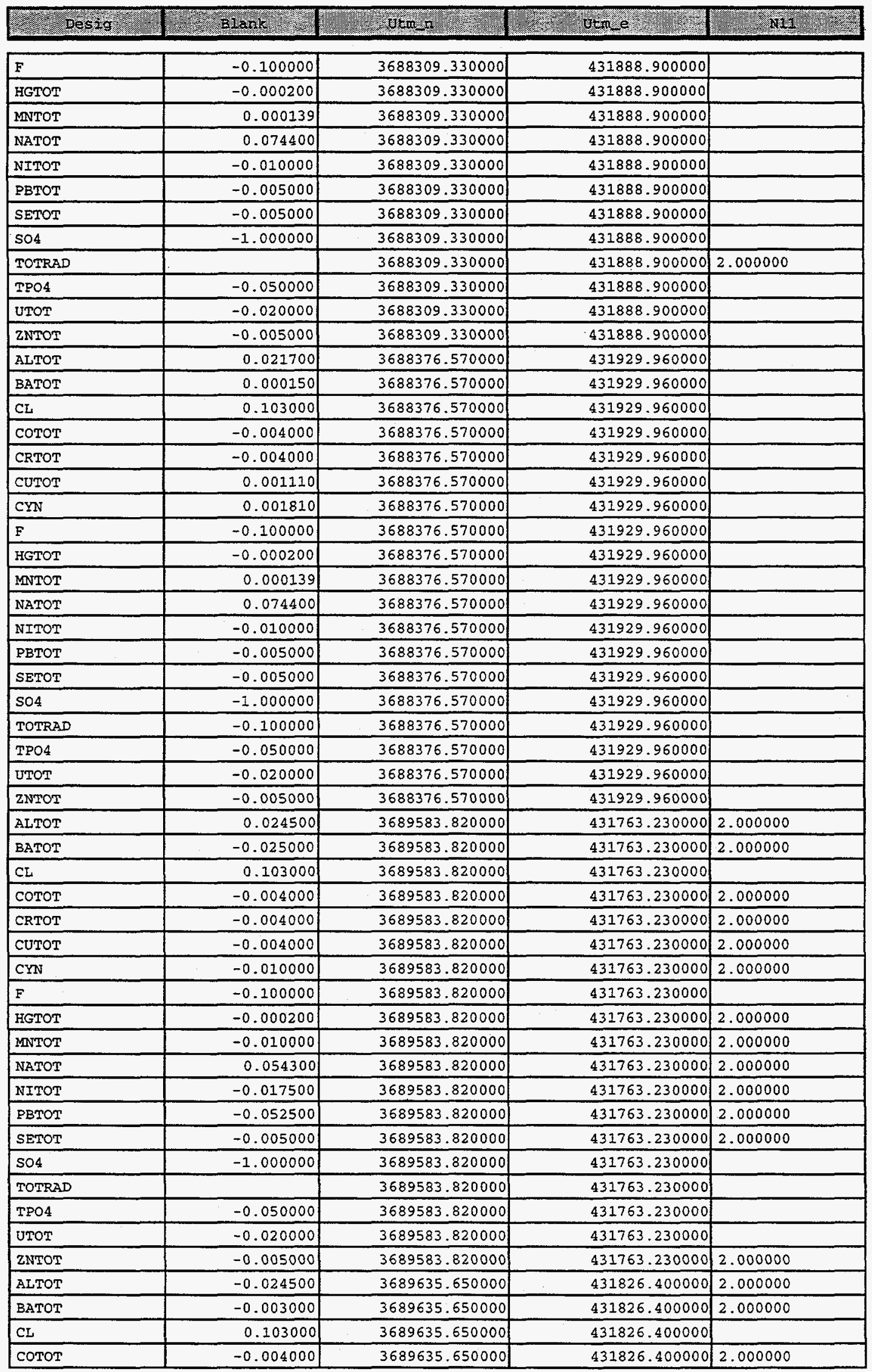




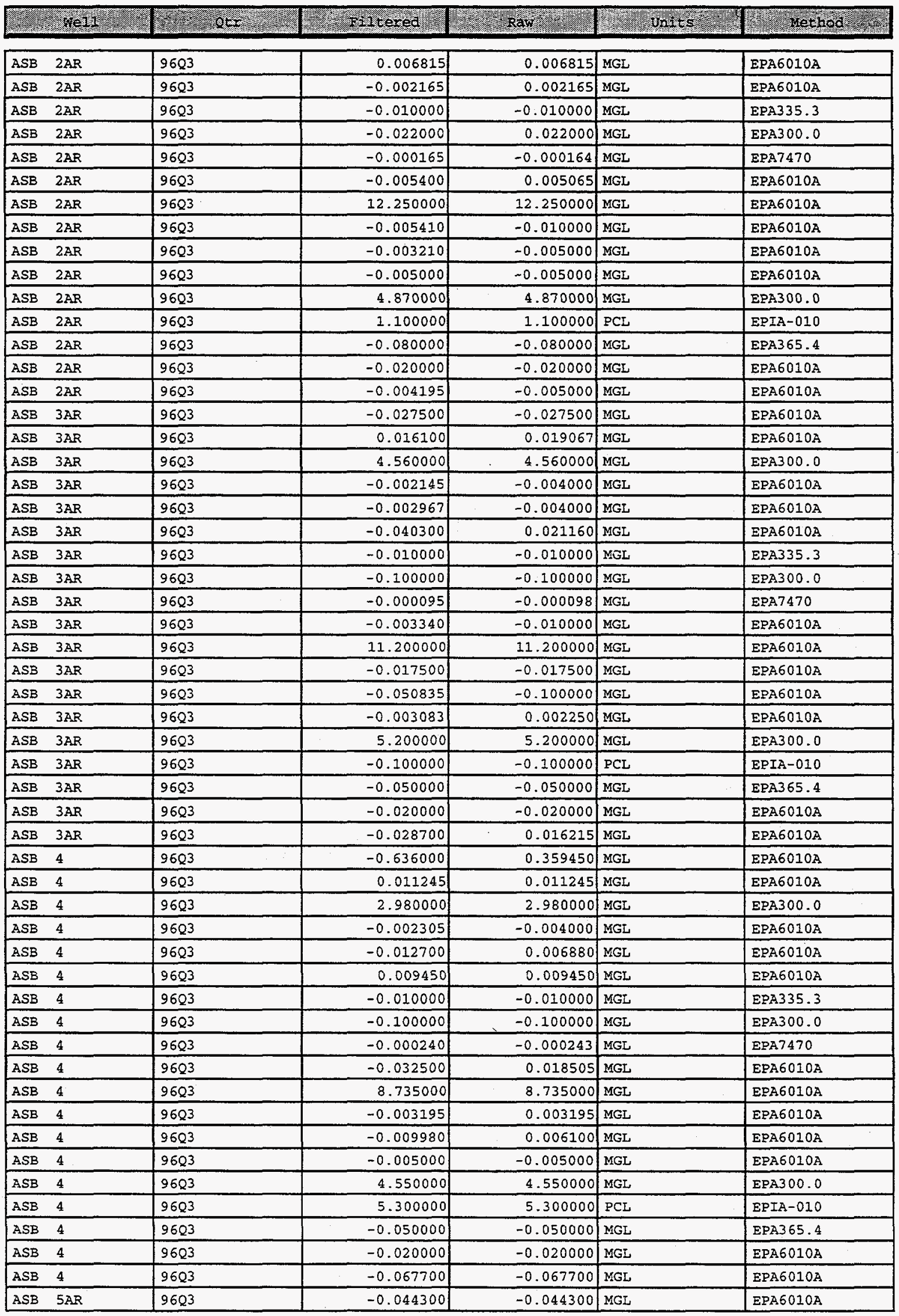


IVSRC-TR-00347

November, 1997

Appendix II-50

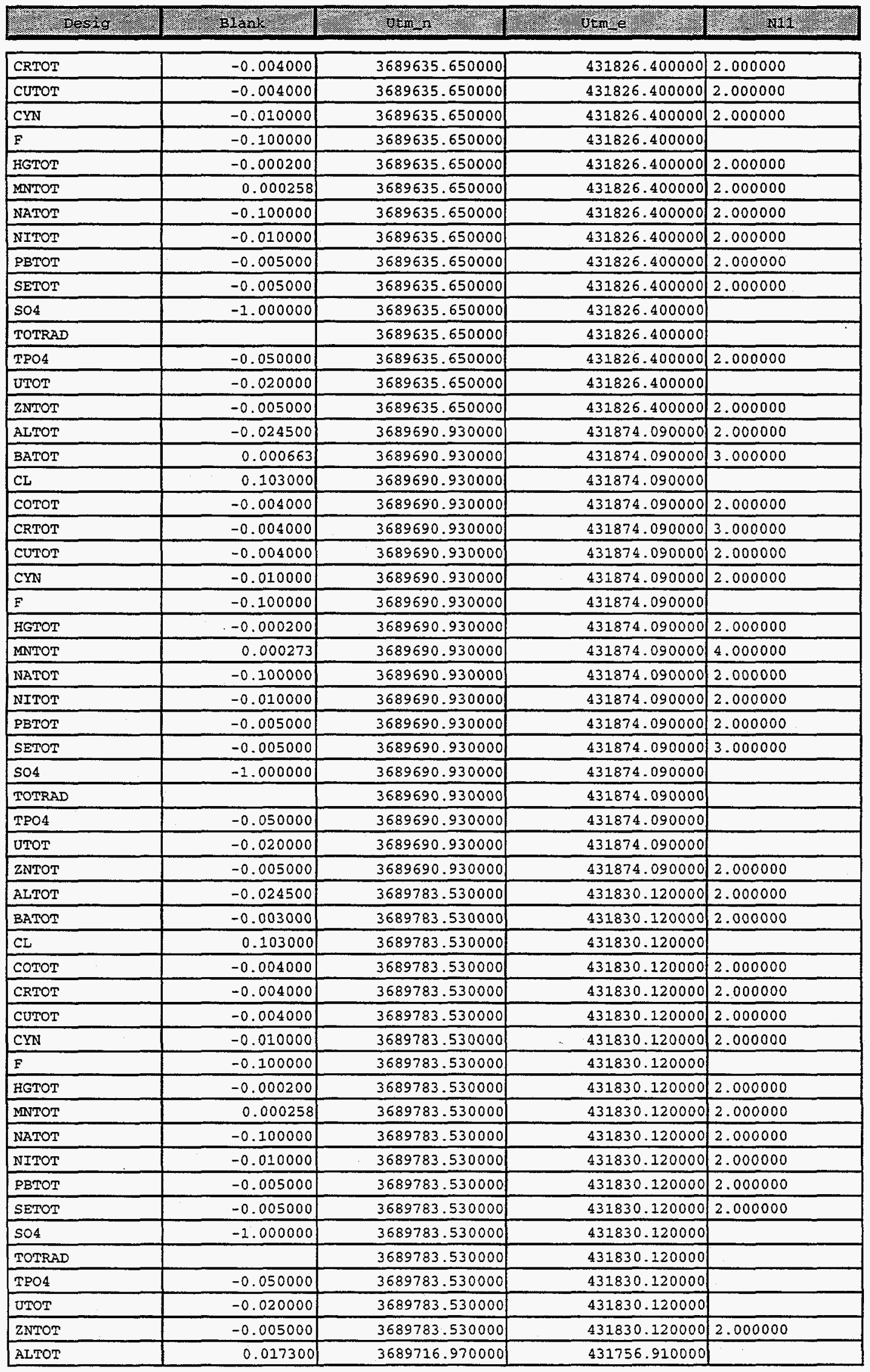




\begin{tabular}{|c|c|c|c|c|c|c|}
\hline ASB & $5 \mathrm{AR}$ & $96 Q 3$ & 0.004040 & 0.004040 & MGL & EPA $6010 \mathrm{~A}$ \\
\hline ASB & $5 A R$ & 9603 & 4.490000 & 4.490000 & MGL & EPA300.0 \\
\hline ASB & 5AR & 9603 & -0.004000 & -0.004000 & MGL & EPA6010A \\
\hline ASB & SAR & $96 Q 3$ & -0.001130 & 0.001130 & MGL & EPA6010A \\
\hline ASB & $5 A R$ & 9603 & 0.013800 & 0.013800 & MGL & EPA6010A \\
\hline ASB & $5 A R$ & $96 Q 3$ & -0.010000 & -0.010000 & MGL & EPA.335.3 \\
\hline ASB & $5 A R$ & 9603 & -0.100000 & -0.100000 & MGL & EPA300.0 \\
\hline ASB & SAR & 9603 & 0.000290 & 0.000292 & MGL & ERA7470 \\
\hline ASB & $5 A R$ & $96 Q 3$ & 0.005810 & 0.005810 & MGL & EPA6010A \\
\hline ASB & $5 A R$ & 9603 & 10.700000 & 10.700000 & MGL & EPA6010A \\
\hline ASB & $5 A R$ & $96 Q 3$ & -0.004590 & 0.004590 & MGL & EPA6010A \\
\hline ASB & SAR & 9603 & -0.003750 & 0.003750 & MGL & EPA6010A \\
\hline ASB & $5 A R$ & $96 Q 3$ & -0.005000 & -0.005000 & MGL & EPA6010A \\
\hline ASB & $5 A R$ & 9603 & 6.060000 & 6.060000 & MGL & EPA300.0 \\
\hline ASB & $5 A R$ & 9603 & -0.100000 & -0.100000 & PCL & EPIA-010 \\
\hline ASB & 5AR & $96 Q 3$ & -0.050000 & -0.050000 & MGL & EPA 365.4 \\
\hline ASB & $5 A R$ & 9603 & 0.020100 & 0.020100 & MGL & EPA6010A \\
\hline ASB & 5AR & 9603 & 0.013200 & 0.013200 & MGL & EPA6010A \\
\hline A.SB & $6 \mathrm{~A}$ & 9603 & -0.074200 & -0.120000 & MGL & ERA6010A \\
\hline ASB & $6 \mathrm{~A}$ & 9603 & -0.002780 & 0.002780 & MGL & EPA6010A \\
\hline ASB & $6 \mathrm{~A}$ & 9603 & 5.500000 & 5.500000 & MGL & EPA300.0 \\
\hline ASB & $6 \mathrm{~A}$ & $96 Q 3$ & -0.002325 & -0.004000 & MGL & EPA6010A \\
\hline ASB & $6 \mathrm{~A}$ & 9603 & -0.025800 & 0.014515 & MGL & EPA6010A \\
\hline ASB & $6 \mathrm{~A}$ & 9603 & -0.002505 & 0.002505 & MGL & EPA6010A \\
\hline ASB & $6 \mathrm{~A}$ & 9603 & -0.010000 & -0.010000 & MGI & EPA 335.3 \\
\hline ASB & $6 \mathrm{~A}$ & 9603 & -0.100000 & -0.100000 & MGL & EPA.300.0 \\
\hline ASB & $6 \mathrm{~A}$ & 9603 & -0.000120 & -0.000200 & MGL & EPA7470 \\
\hline ASB & $6 \mathrm{~A}$ & 9603 & -0.013800 & 0.010955 & MGL & EPA6010A \\
\hline ASB & $6 \mathrm{~A}$ & 9603 & 14.450000 & 14.450000 & MGL & EPA.6010A \\
\hline ASB & $6 \mathrm{~A}$ & 9603 & -0.015700 & 0.008535 & MGL & ERA6010A \\
\hline ASB & $6 \mathrm{~A}$ & 9603 & -0.005000 & -0.005000 & MGL & EPA6010A \\
\hline ASB & $6 \mathrm{~A}$ & 9603 & -0.005000 & -0.005000 & MGL & EPA6010A \\
\hline ASB & $6 \mathrm{~A}$ & 9603 & 7.520000 & 7.520000 & MGL & EPA300.0 \\
\hline ASB & $6 \mathrm{~A}$ & 9603 & -0.400000 & 0.400000 & PCL & EPIA-010 \\
\hline$A S B$ & $6 \mathrm{~A}$ & 9603 & -0.050000 & -0.050000 & MGL & EPA3 65.4 \\
\hline$A S B$ & $6 \mathrm{~A}$ & 9603 & -0.020000 & -0.020000 & MGL & EPA6010A \\
\hline ASB & $6 \mathrm{~A}$ & $96 Q 3$ & -0.004990 & -0.005000 & MGL & EPA6010A \\
\hline MSB & 1D & 9603 & 0.048000 & 0.048000 & MGL & EPA6010A \\
\hline MSB & 10 & 9603 & 0.012700 & 0.012700 & MGL & EPA6010A \\
\hline MSB & 1D & $96 Q 3$ & 3.270000 & 3.270000 & MGL & EPA300.0 \\
\hline MSB & 1D & $96 Q 3$ & -0.000640 & -0.000639 & MGL & EPA6010A \\
\hline MSB & $1 D$ & $96 Q 3$ & -0.004000 & -0.004000 & MGL & EPA6010A \\
\hline MSB & $1 D$ & 9603 & 0.022800 & 0.022800 & MGL & EPA6010A \\
\hline MSB & $1 D$ & 9603 & -0.010000 & -0.010000 & MGL & EPA.335.3 \\
\hline MSB & 10 & 9603 & -0.100000 & -0.100000 & MGL & $\operatorname{ErA} 300.0$ \\
\hline MSB & $1 D$ & 9603 & -0.000200 & -0.000200 & MGL & EPA7470 \\
\hline MSB & 1D & 9603 & 0.022300 & 0.022300 & MGL & EPA6010A \\
\hline MSB & 1D & $96 Q^{3}$ & 4.920000 & 4.920000 & MGL & EPA6010A \\
\hline MSB & 1D & $96 Q 3$ & -0.010000 & -0.010000 & MGL & EPA6010A \\
\hline MSB & 1D & $96 Q 3$ & -0.004350 & 0.004350 & MGL & ERA6010A \\
\hline MSB & 1D & $96 Q 3$ & -0.005000 & -0.005000 & MGL & EPA6010A \\
\hline MSB & 1D & $96 Q 3$ & -0.760000 & 0.760000 & MGL & ERA 300.0 \\
\hline MSB & 1D & 9603 & -1.100000 & -1.100000 & PCL & EPIA-010 \\
\hline MSB & ID & $96 Q 3$ & -0.030000 & 0.030000 & MGL & EPA365.4 \\
\hline
\end{tabular}




\begin{tabular}{|c|c|c|c|c|}
\hline 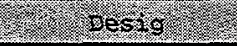 & ting & 1) $6 \operatorname{con} 5$ & 20.7. & V. \\
\hline BATOT & -0.003000 & 3689716.970000 & 431756.910000 & \\
\hline $\mathrm{CL}$ & -0.250000 & 3689726.970000 & 431756.910000 & \\
\hline COTOT & -0.004000 & 3689716.970000 & 431756.910000 & \\
\hline CRTOT & -0.004000 & 3689716.970000 & 431756.910000 & \\
\hline CUTOT & -0.004000 & 3689716.970000 & 431756.910000 & \\
\hline CYN & -0.010000 & 3689716.970000 & 431756.910000 & \\
\hline$F$ & -0.100000 & 3689716.970000 & 431756.910000 & \\
\hline HGTOT & -0.000200 & 3689716.970000 & 431756.910000 & \\
\hline MNTOT & 0.000219 & 3689716.970000 & 431756.910000 & \\
\hline NATOT & -0.100000 & 3689716.970000 & 431756.910000 & \\
\hline NITOT & -0.010000 & 3689716.970000 & 431756.910000 & \\
\hline PBTOT & -0.005000 & 3689716.970000 & 431756.910000 & \\
\hline SETOT & -0.005000 & 3689716.970000 & 431756.910000 & \\
\hline $\mathrm{SO4}$ & -1.000000 & 3689716.970000 & 431756.910000 & \\
\hline TOTRAD & -0.100000 & 3689716.970000 & 431756.910000 & \\
\hline TPO4 & -0.050000 & 3689716.970000 & 431756.910000 & \\
\hline UTOT & -0.020000 & 3689716.970000 & 431756.910000 & \\
\hline ZNTOT & -0.005000 & 3689716.970000 & 431756.910000 & \\
\hline ALTOT & 0.021700 & 3689639.510000 & 431746.030000 & 2.000000 \\
\hline BATOT & -0.025000 & 3689639.510000 & 431746.030000 & 2.000000 \\
\hline $\mathrm{CL}$ & -0.250000 & 3689639.510000 & 431746.030000 & \\
\hline COTOT & 0.000895 & 3689639.510000 & 431746.030000 & 2.000000 \\
\hline CRTOT & 0.000764 & 3689639.510000 & 431746.030000 & 2.000000 \\
\hline CUTOT & -0.004000 & 3689639.510000 & 431746.030000 & 2.000000 \\
\hline CYN & -0.010000 & 3689639.510000 & 431746.030000 & 2.000000 \\
\hline$F$ & -0.100000 & 3689639.510000 & 431746.030000 & \\
\hline HGTOT & -0.000200 & 3689639.510000 & 431746.030000 & 2.000000 \\
\hline MNTOT & -0.006000 & 3689639.510000 & 431746.030000 & 2.000000 \\
\hline NATOT & 0.026100 & 3689639.510000 & 431746.030000 & 2.000000 \\
\hline NITOT & -0.025000 & 3689639.510000 & 431746.030000 & 2.000000 \\
\hline PBTOT & -0.052500 & 3689639.510000 & 431746.030000 & 2.000000 \\
\hline SETOT & -0.005000 & 3689639.510000 & 431746.030000 & 2.000000 \\
\hline SO4 & -1.000000 & 3689639.510000 & 431746.030000 & \\
\hline TOTRAD & -0.200000 & 3689639.510000 & 431746.030000 & \\
\hline TPO4 & -0.050000 & 3689639.510000 & 431746.030000 & \\
\hline UTOT & -0.020000 & 3689639.510000 & 431746.030000 & \\
\hline ZNTOT & -0.005000 & 3689639.510000 & 431746.030000 & 2.000000 \\
\hline ALTOT & -0.020000 & 3687925.590000 & 431401.750000 & \\
\hline BATOT & 0.000133 & 3687925.590000 & 431401.750000 & \\
\hline $\mathrm{CL}$ & -0.250000 & 3687925.590000 & 431401.750000 & \\
\hline COTOT & 0.000425 & 3687925.590000 & 431401.750000 & \\
\hline CRTOT & -0.004000 & 3687925.590000 & 431401.750000 & \\
\hline CUTOT & 0.000925 & 3687925.590000 & 431401.750000 & \\
\hline $\mathrm{CYN}$ & 0.004770 & 3687925.590000 & 431401.750000 & \\
\hline$F$ & -0.100000 & 3687925.590000 & 431401.750000 & \\
\hline HGTOT & -0.000200 & 3687925.590000 & 431401.750000 & \\
\hline MNTOT & 0.000160 & 3687925.590000 & 431401.750000 & \\
\hline NATOT & -0.100000 & 3687925.590000 & 431401.750000 & \\
\hline NITOT & -0.010000 & 3687925.590000 & 431401.750000 & \\
\hline PBTOT & -0.005000 & 3687925.590000 & 431401.750000 & \\
\hline SETOT & -0.005000 & 3687925.590000 & 431401.750000 & \\
\hline SO4 & -1.000000 & 3687925.590000 & 431401.750000 & \\
\hline TOTRAD & & 3687925.590000 & 431401.750000 & \\
\hline TPO4 & -0.050000 & 3687925.590000 & 431401.750000 & \\
\hline
\end{tabular}




\begin{tabular}{|c|c|c|c|c|c|c|}
\hline \multicolumn{2}{|c|}{ 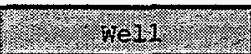 } & \multirow{2}{*}{$\begin{array}{l}962.06 \\
9603\end{array}$} & \multirow{2}{*}{$\frac{\mid \text { Piltered }}{\cdot-0.014200}$} & \multirow{2}{*}{$\frac{-0.014200}{-127}$} & \multirow{2}{*}{$\begin{array}{l}\text { MGL } \\
\text { MG }\end{array}$} & \multirow{2}{*}{ EPA6010A } \\
\hline MSB & ID & & & & & \\
\hline MSB & 10 & 9603 & 0.027800 & 0.027800 & MGL & EPA6010A \\
\hline MSB & $2 D$ & 9603 & 0.285000 & 0.285000 & MGL & EPA6010A \\
\hline MSB & $2 \mathrm{D}$ & 9603 & 0.018800 & 0.018800 & MGL & EPA6010A \\
\hline MSB & $2 D$ & 9603 & 2.620000 & 2.620000 & MGL & EPA300.0 \\
\hline MSB & $2 D$ & 9603 & -0.000450 & 0.000454 & MGL & EPA6010A \\
\hline MSB & $2 D$ & 9603 & -0.004000 & -0.004000 & MGL & EPA6010A \\
\hline MSB & 20 & 9603 & 0.004030 & 0.004030 & MGL & EPA6010A \\
\hline MSB & 20 & 9603 & -0.010000 & -0.010000 & MGL & EPA335.3 \\
\hline MSB & $2 D$ & 9603 & -0.051000 & 0.051000 & MGL & EPA300.0 \\
\hline MSB & 20 & 9683 & 0.000280 & 0.000279 & MGL & EPA7470 \\
\hline MSB & $2 \mathrm{D}$ & 9603 & 0.014200 & 0.014200 & MGL & EPA6010A \\
\hline MSB & $2 D$ & $96 Q 3$ & 2.500000 & 2.500000 & MGL & EPA6010A \\
\hline MSB & $2 \mathrm{D}$ & 9603 & -0.010000 & -0.010000 & MGL & EPA6010A \\
\hline MSB & $2 D$ & 9603 & -0.003420 & 0.003420 & MGL & EPA6010A \\
\hline MSB & 20 & 9603 & -0.005000 & -0.005000 & MGL & EPA6010A \\
\hline MSB & $2 D$ & 9603 & -0.118000 & 0.118000 & MGL & EPA 300.0 \\
\hline MSB & $2 D$ & 9603 & 8.900000 & 8.900000 & PCI & EPIA-010 \\
\hline MSB & $2 D$ & 9623 & -0.050000 & -0.050000 & MGL & EPA3 65.4 \\
\hline MSB & $2 \mathrm{D}$ & 9603 & -0.020000 & -0.020000 & MGL & EPA6010A \\
\hline MSB & $2 \mathrm{D}$ & 9603 & 0.008570 & 0.008570 & MGI & EPA6010A \\
\hline MSB & $4 \mathrm{D}$ & 9603 & 0.159000 & 0.159000 & MGL & EPA6010A \\
\hline MSB & $4 \mathrm{D}$ & 9603 & 0.013400 & 0.013400 & MGL & EPA6010A \\
\hline MSB & $4 \mathrm{D}$ & 9603 & 2.150000 & 2.150000 & MGL & EPA300.0 \\
\hline MSB & $4 \mathrm{D}$ & 9603 & -0.001210 & -0.001210 & MGL & EPA6010A \\
\hline MSB & $4 \mathrm{D}$ & 9603 & -0.002560 & -0.004000 & MGL & EPA6010A \\
\hline MSB & $4 \mathrm{D}$ & 9603 & -0.001410 & -0.001410 & MGL & EPA6010A \\
\hline MSB & $4 \mathrm{D}$ & 9603 & -0.010000 & -0.010000 & MGL & EPA335.3 \\
\hline MSB & 40 & 9603 & -0.026000 & 0.026000 & MGL & EPA300.0 \\
\hline MSB & 4D & 9603 & -0.000020 & 0.000020 & MGL & EPA7470 \\
\hline MSB & $4 \mathrm{D}$ & 9603 & 0.012900 & 0.012900 & MGL & EPA6010A \\
\hline MSB & $4 D$ & 9603 & 4.480000 & 4.480000 & MGI & EPA6010A \\
\hline MSB & $4 D$ & 9603 & -0.005790 & -0.010000 & MGE & EPA6010A \\
\hline MSB & $4 \mathrm{D}$ & 9603 & -0.005000 & -0.005000 & MGL & ERA6010A \\
\hline MSB & $4 D$ & 9603 & -0.003590 & -0.005000 & MGL & EPA6010A \\
\hline MSB & $4 D$ & 9603 & 1.440000 & 1.440000 & MGL & EPA300.0 \\
\hline MSB & $4 D$ & 9603 & 5.500000 & 5.500000 & PCL & EPIA-010 \\
\hline MSB & $4 D$ & 9603 & -0.050000 & -0.050000 & MGL & ERA365.4 \\
\hline MSB & $4 D$ & 9603 & -0.023800 & -0.023800 & MGL & EPA6010A \\
\hline MSB & $4 D$ & 9603 & 0.006020 & 0.006020 & MGL & EPA6010A \\
\hline MSB & $5 \mathrm{~A}$ & 9603 & -0.020000 & -0.020000 & MGL & EPA6010A \\
\hline MSB & $5 \mathrm{~A}$ & 9603 & 0.003390 & 0.003390 & MGL & EPA6010A \\
\hline MSB & $5 \mathrm{~A}$ & 9603 & 1.400000 & 1.400000 & MGL & EPA3 00.0 \\
\hline MSB & $5 \mathrm{~A}$ & 9603 & -0.000300 & -0.000297 & MGL & EPA6010A \\
\hline MSB & $5 \mathrm{~A}$ & 9603 & -0.000710 & 0.000706 & MGL & EPA6010A \\
\hline MSB & $5 \mathrm{~A}$ & 9603 & 0.018700 & 0.018700 & MGL & EPA6010A \\
\hline MSB & $5 \mathrm{~A}$ & 9603 & -0.010000 & -0.010000 & MGL & EPA335.3 \\
\hline MSB & $5 \mathrm{~A}$ & 9603 & -0.100000 & -0.100000 & MGL & EPA 300.0 \\
\hline MSB & $5 \mathrm{~A}$ & 9603 & -0.000200 & -0.000200 & MGL & EPA 7470 \\
\hline MSB & $5 \mathrm{~A}$ & 9603 & 0.005320 & 0.005320 & MGL & EPA6010A \\
\hline MSB & $5 \mathrm{~A}$ & 9603 & 4.420000 & 4.420000 & MGL & EPA6010A \\
\hline MSB & $5 \mathrm{~A}$ & 9603 & -0.010000 & -0.010000 & MGL & EPA6010A \\
\hline MSB & $5 \mathrm{~A}$ & 9603 & -0.002710 & 0.002710 & MGL & EEA6010A \\
\hline MSB & $5 \mathrm{~A}$ & 9603 & -0.005000 & -0.005000 & MGL & EPA6010A \\
\hline
\end{tabular}


WSRC-TR-00347

November, 1997

Appendix II-54

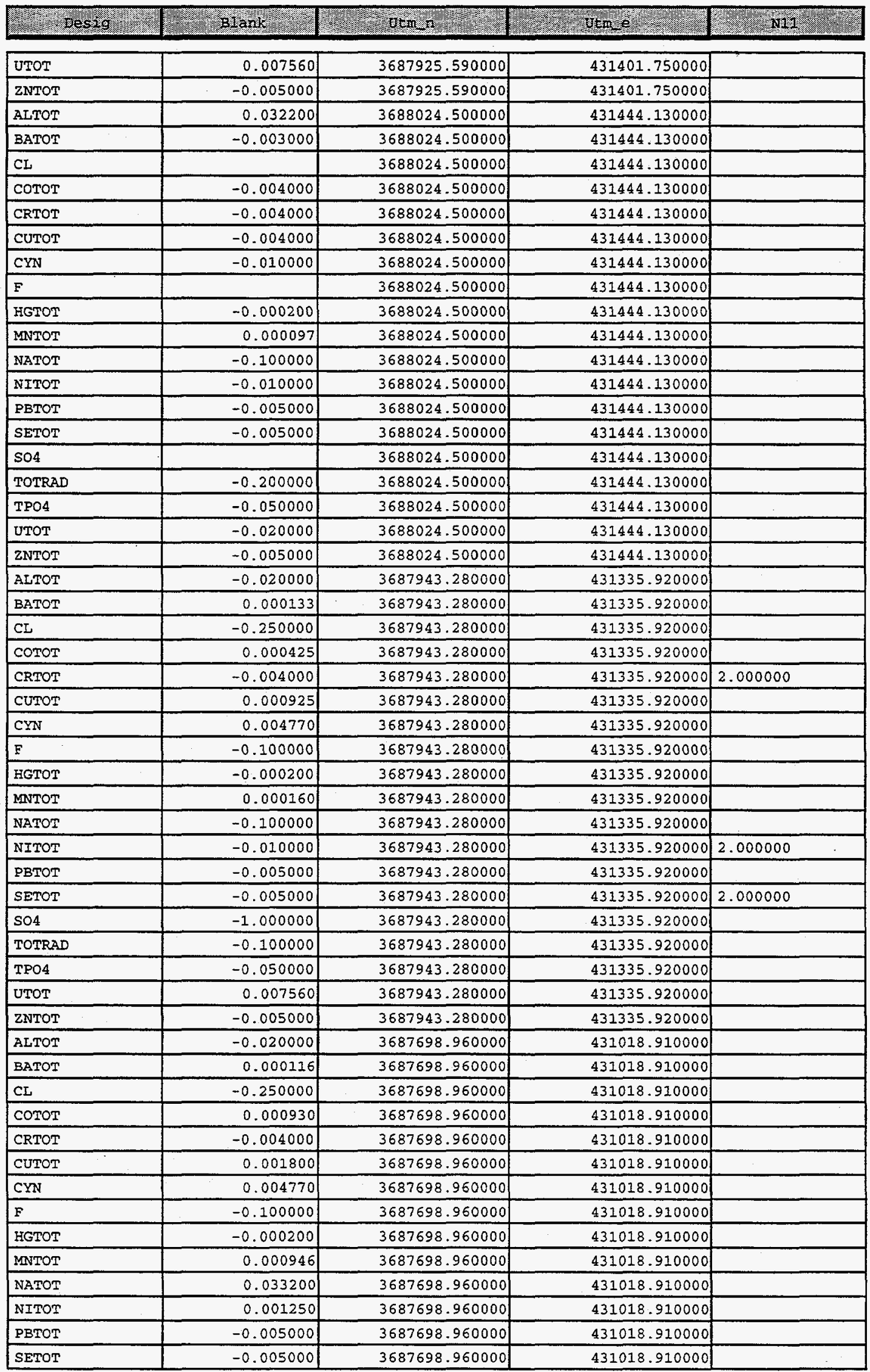


WSRC-TR-00347

November, 1997

Appendix II-55

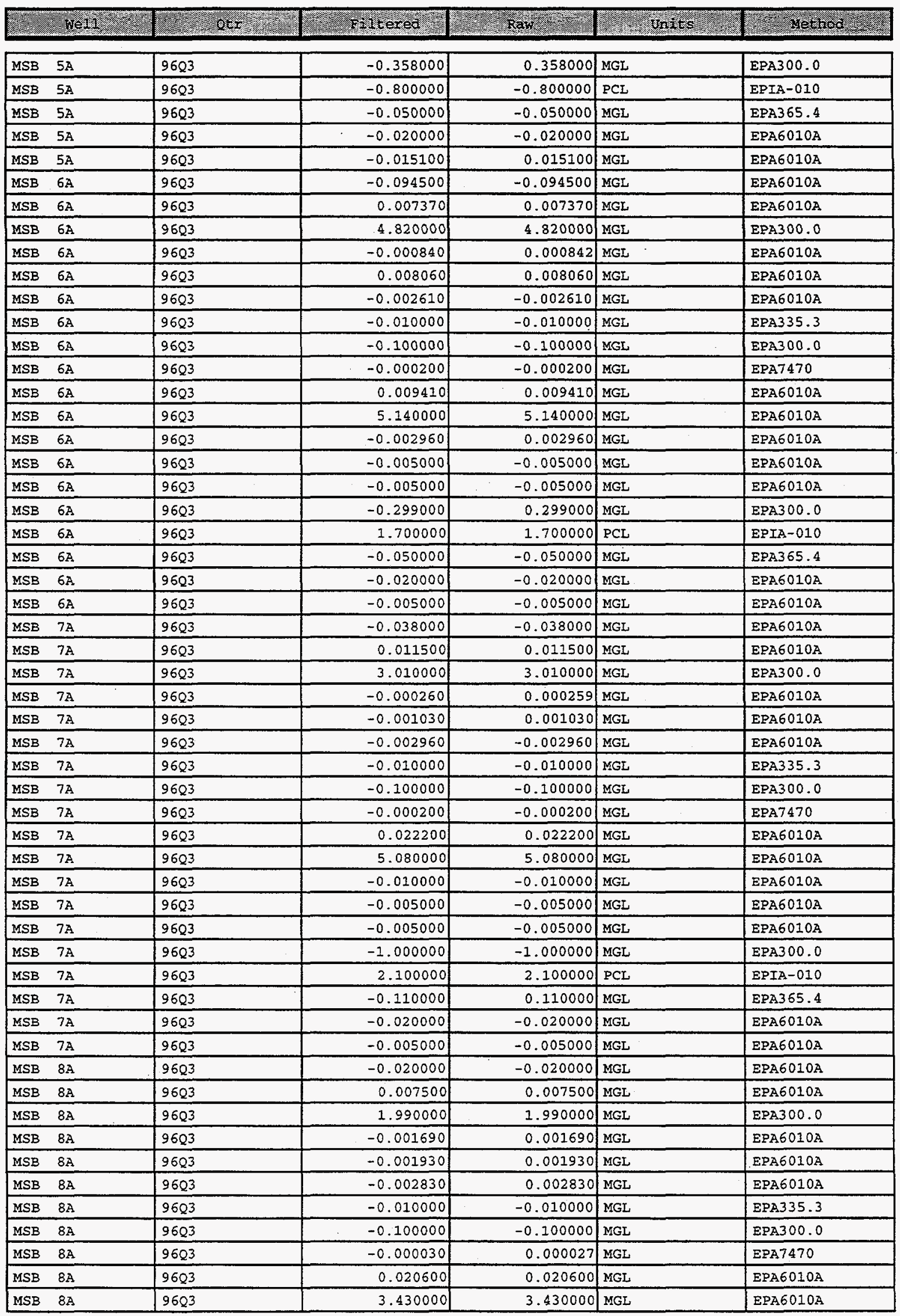


WSRC-TR-00347

November, 1997

Appendix II-56

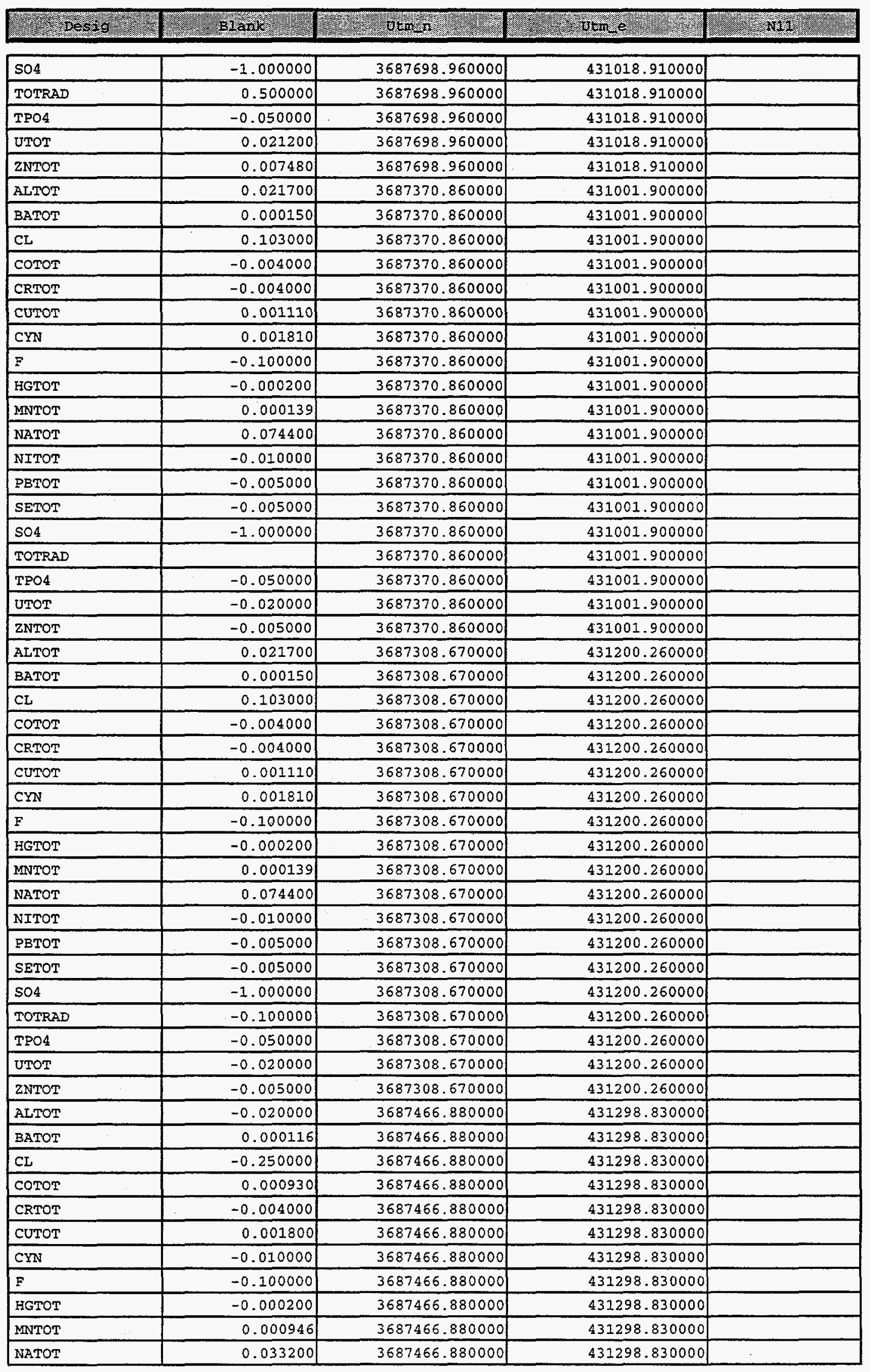


WSRC-TR-00347

November, 1997

Appendix II-57

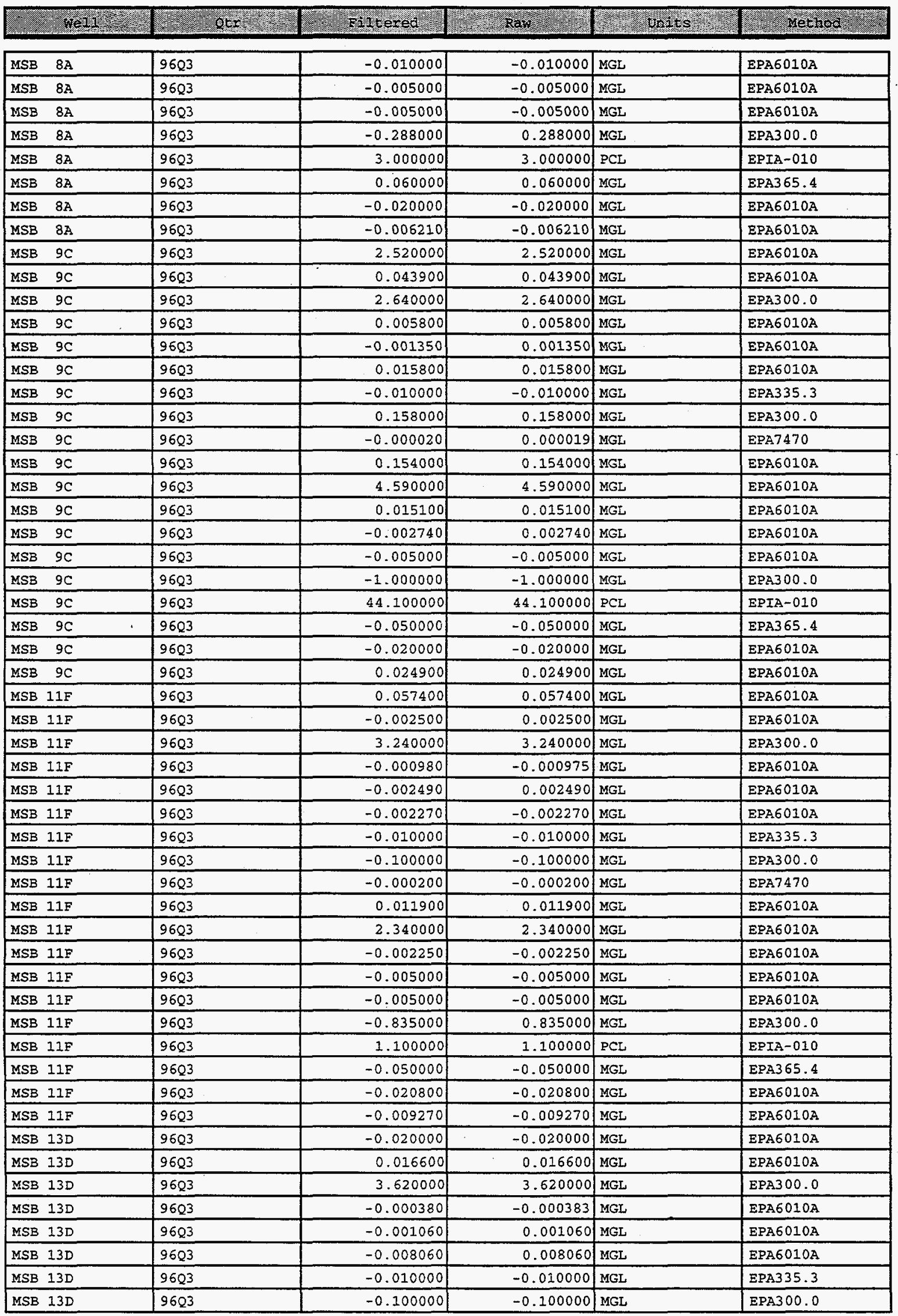


IVSRC-TR-00347

November, 1997

Appendix II-58

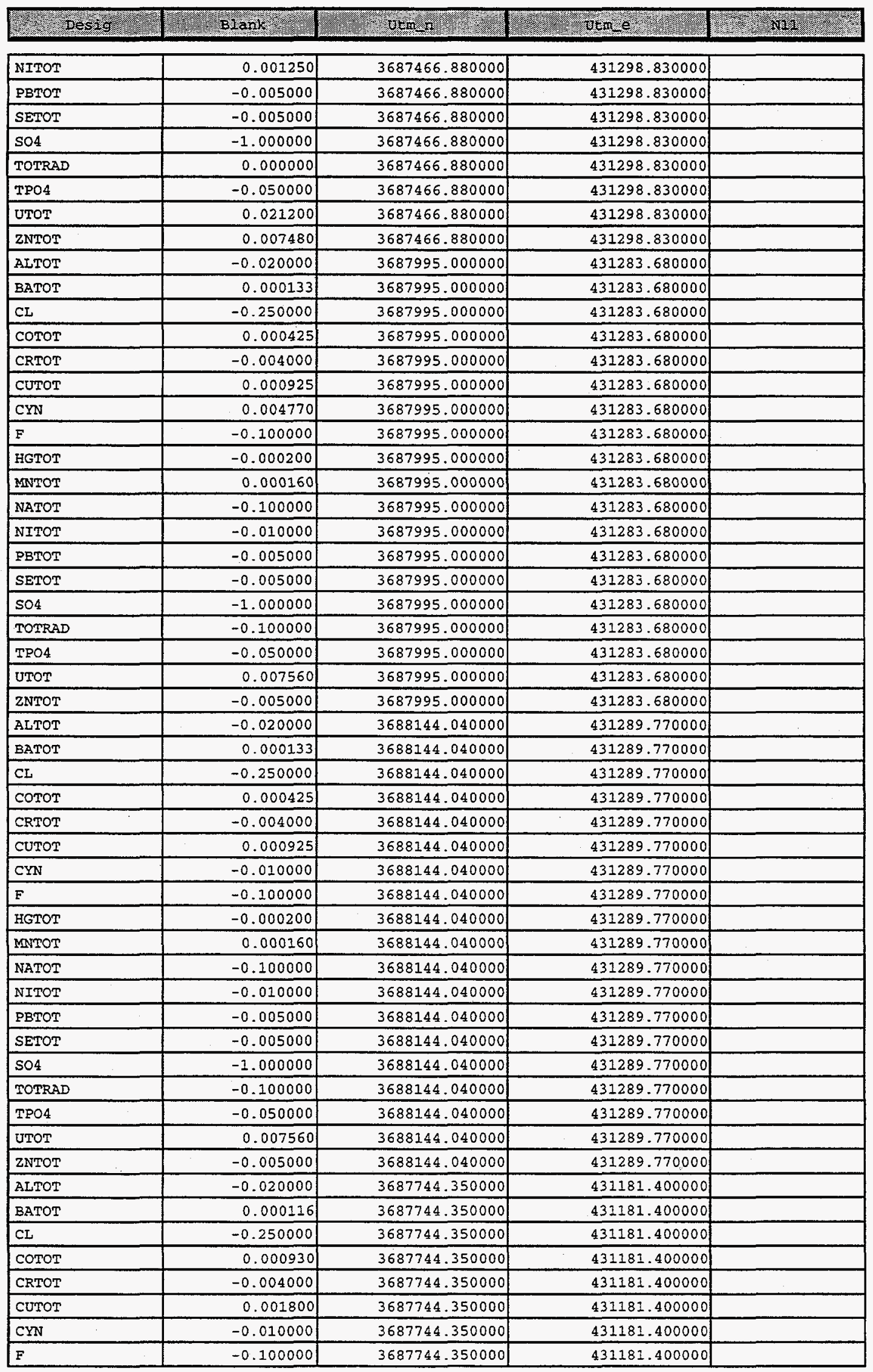




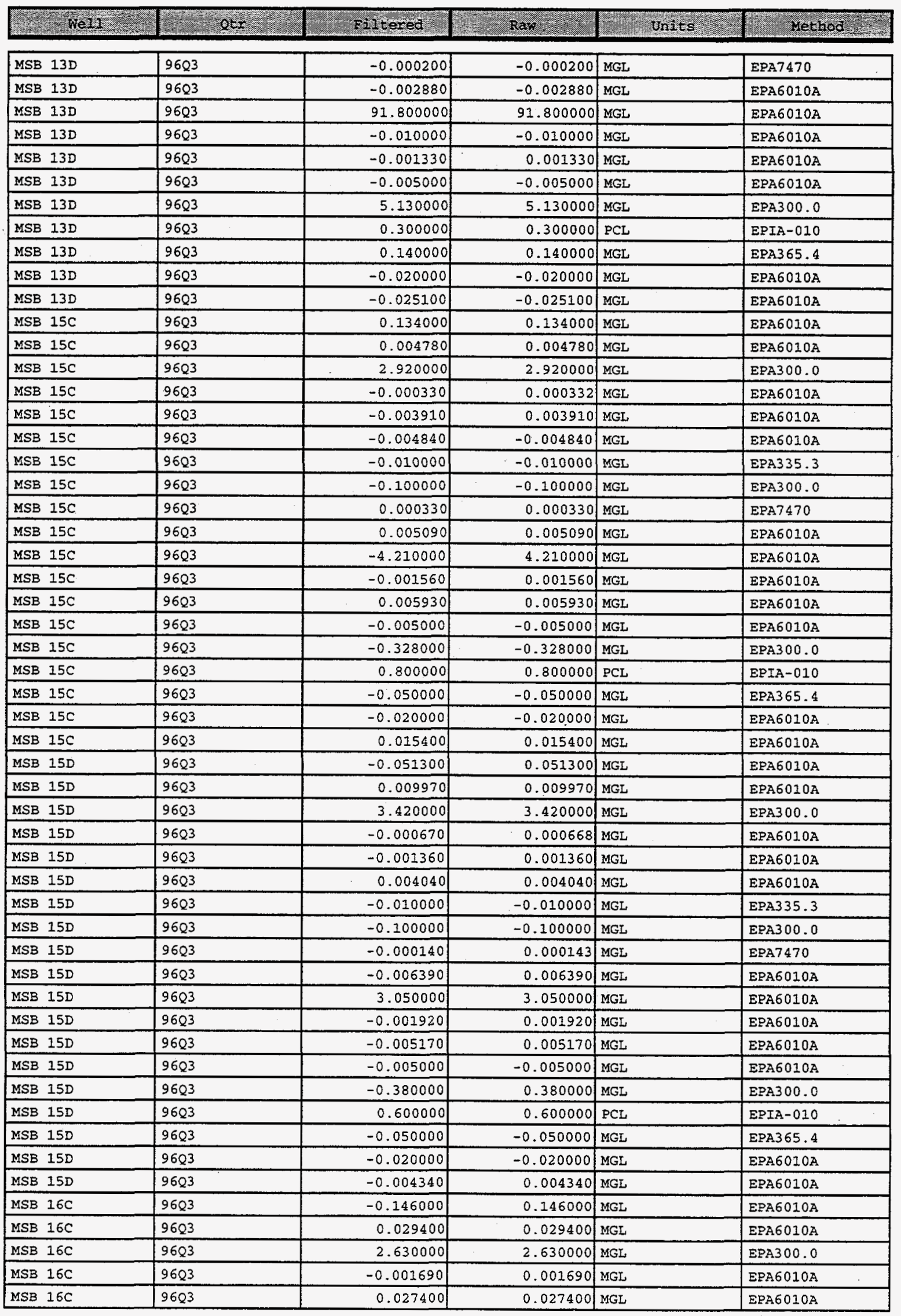




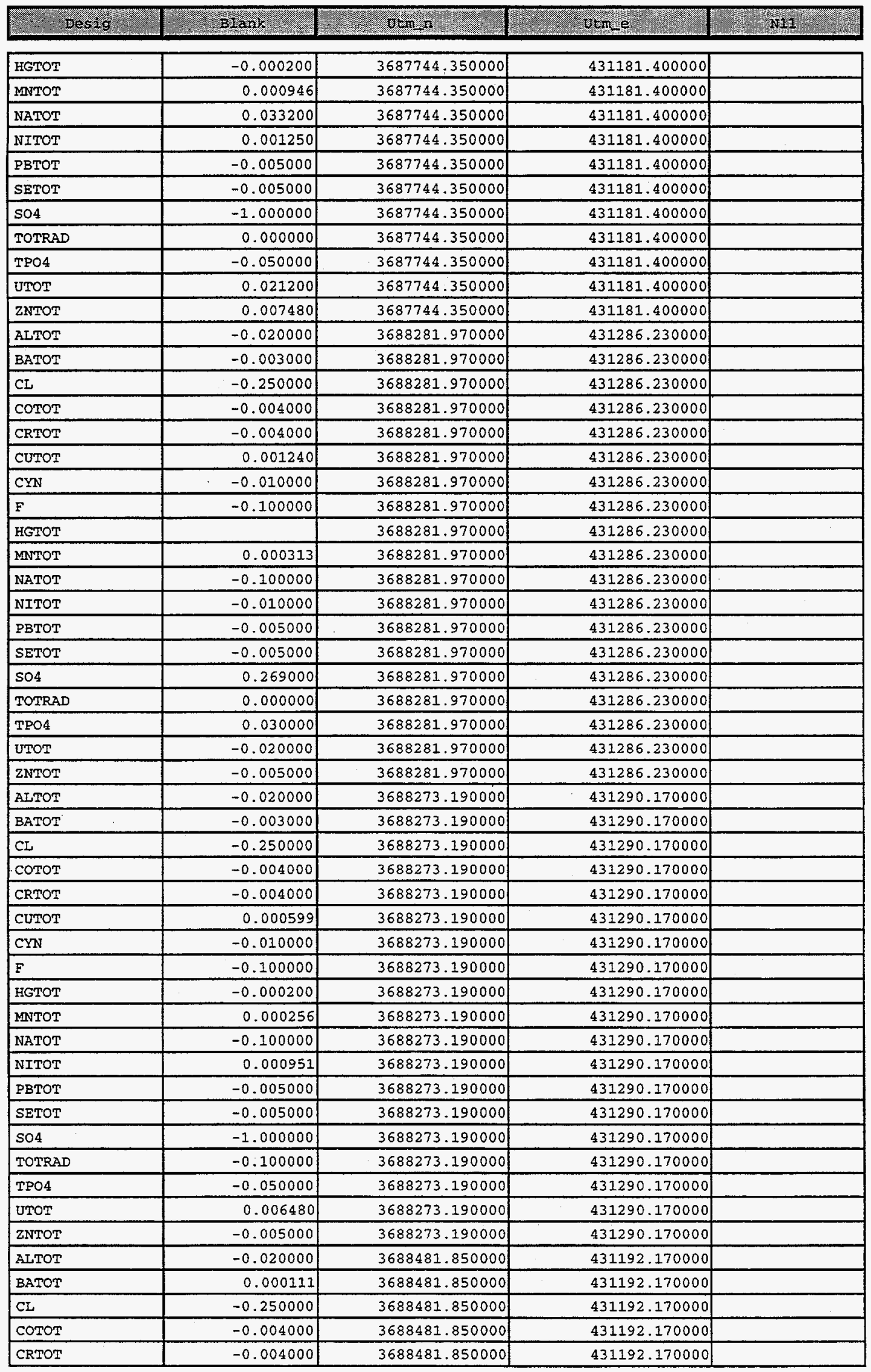




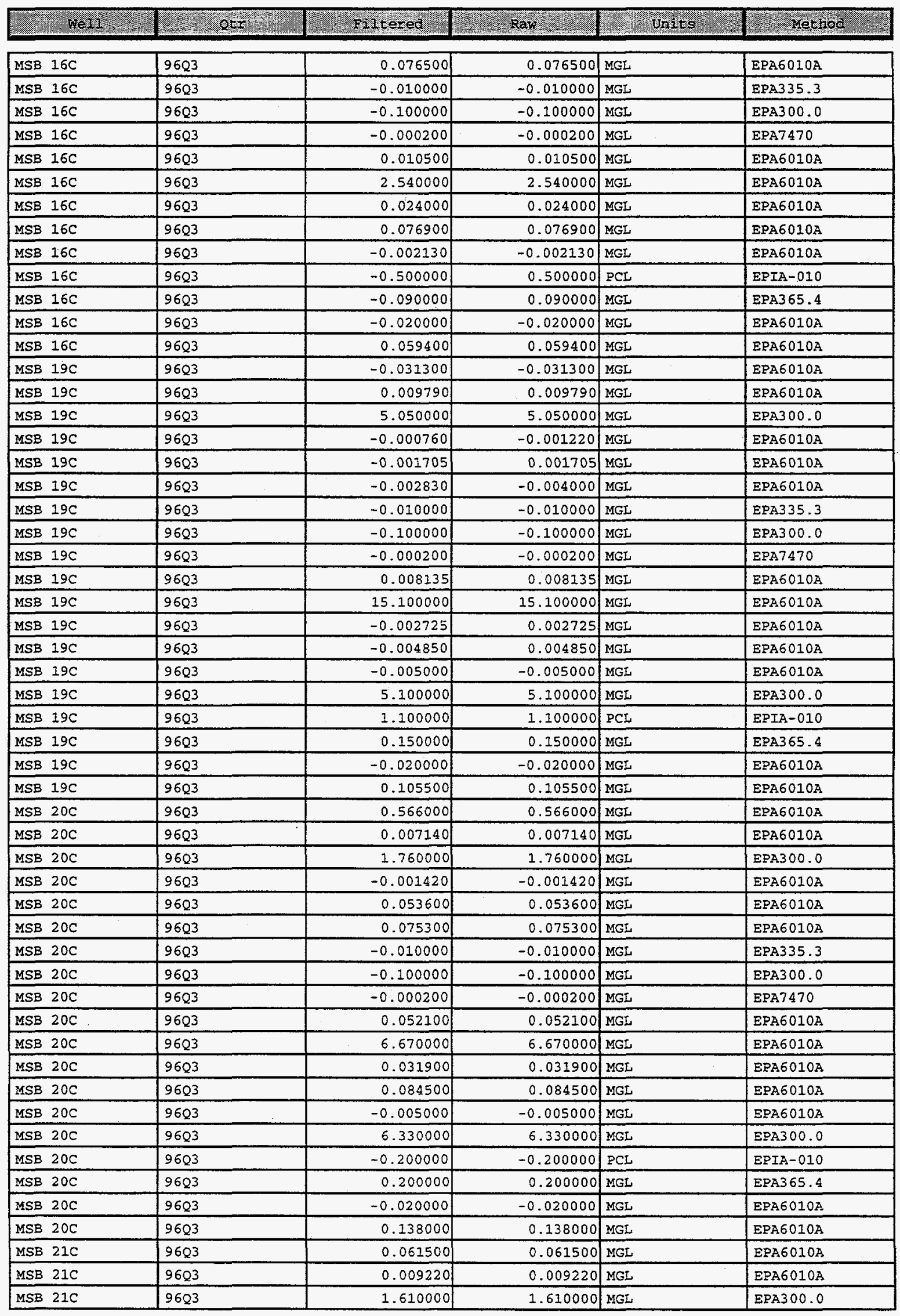




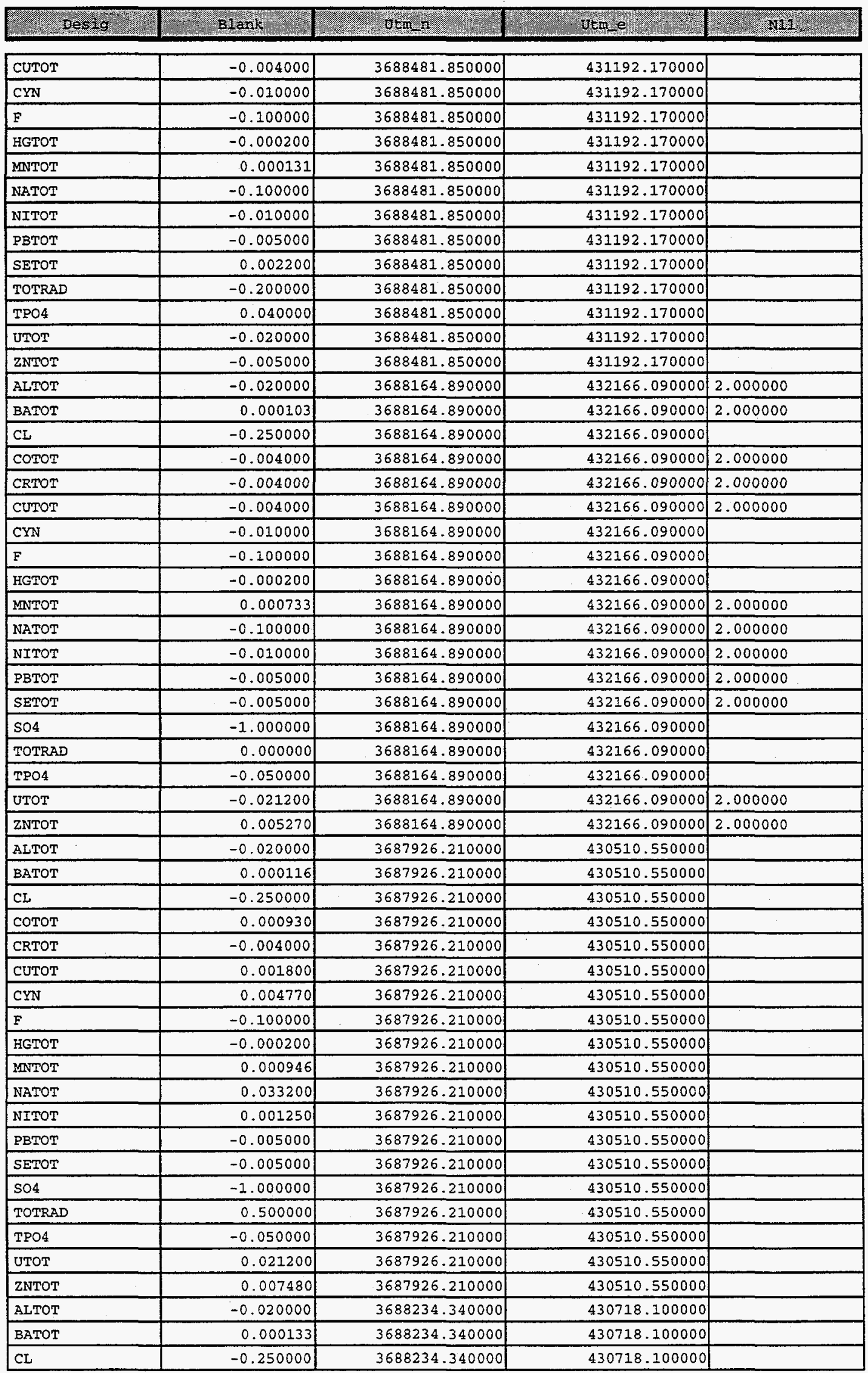


WSRC-TR-00347

November, 1997

Appendix II-63

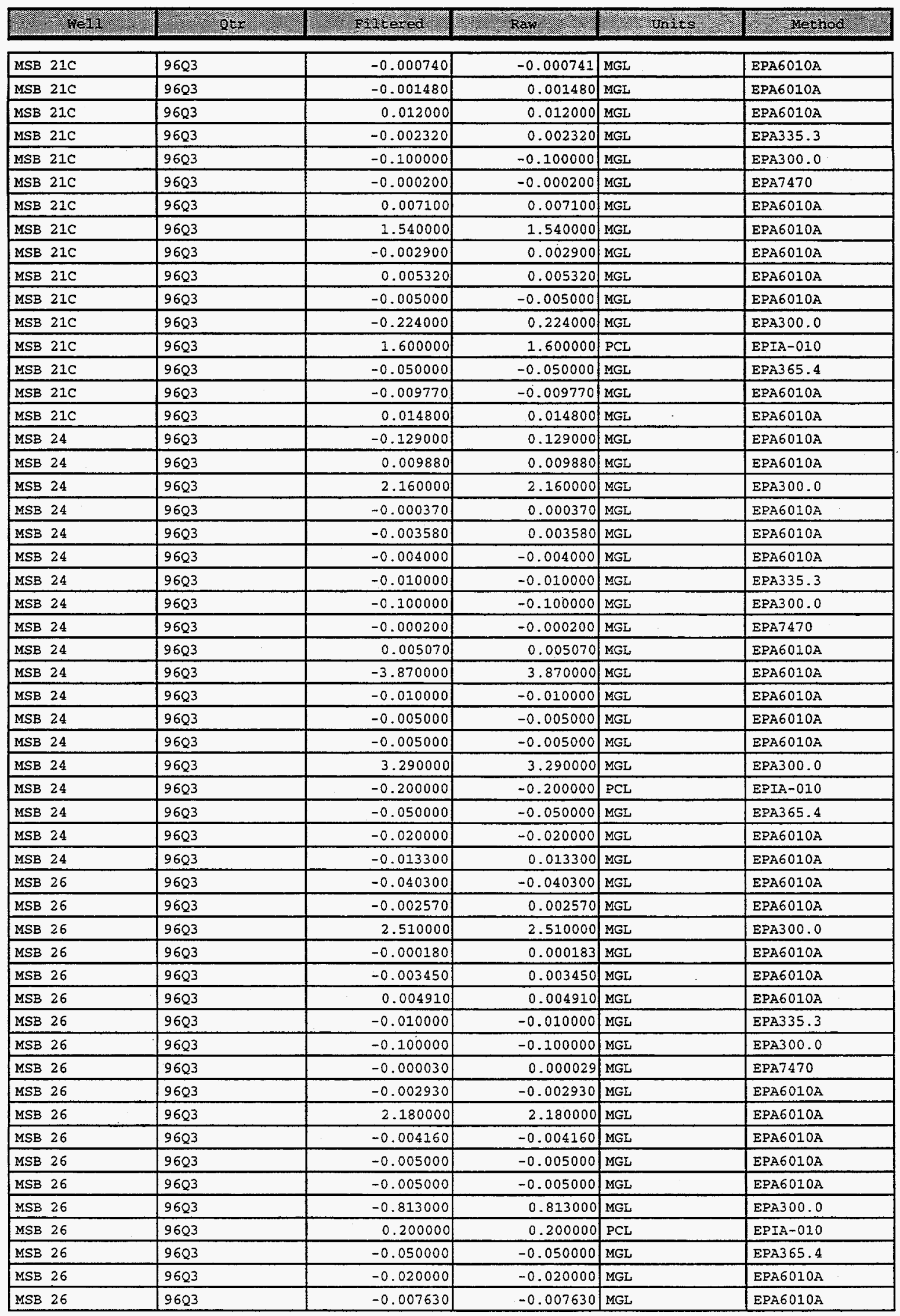


WSRC-TR-00347

November, 1997

Appendix II-64

\begin{tabular}{|c|c|c|c|c|}
\hline & 0.000425 & 3688234.340000 & 430718.100000 & \\
\hline CRTOT & -0.004000 & 3688234.340000 & 430718.100000 & \\
\hline CUTOT & 0.000925 & 3688234.340000 & 430718.100000 & \\
\hline $\mathrm{CYN}$ & 0.004770 & 3688234.340000 & 430718.100000 & \\
\hline $\mathrm{F}$ & -0.100000 & 3688234.340000 & 430718.100000 & \\
\hline HGTOT & -0.000200 & 3688234.340000 & 430718.100000 & \\
\hline MNTOT & 0.000160 & 3688234.340000 & 430728.100000 & \\
\hline NATOT & -0.100000 & 3688234.340000 & 430718.100000 & \\
\hline NITOT & -0.010000 & 3688234.340000 & 430718.100000 & \\
\hline PBTOT & -0.005000 & 3688234.340000 & 430718.100000 & \\
\hline SETOT & -0.005000 & 3688234.340000 & 430718.100000 & \\
\hline SO4 & -1.000000 & 3688234.340000 & 430718.100000 & \\
\hline TOTRAD & -0.100000 & 3688234.340000 & 430718.100000 & \\
\hline TPO4 & -0.050000 & 3688234.340000 & 430718.100000 & \\
\hline UTOT & 0.007560 & 3688234.340000 & 430718.100000 & \\
\hline ZNTOT & -0.005000 & 3688234.340000 & 430718.100000 & \\
\hline ALTOT & -0.020000 & 3688860.090000 & 431245.650000 & \\
\hline BATOT & 0.000216 & 3688860.090000 & 431245.650000 & \\
\hline $\mathrm{CL}$ & -0.250000 & 3688860.090000 & 431245.650000 & \\
\hline COTOT & 0.000930 & 3688860.090000 & 431245.650000 & \\
\hline CRTOT & -0.004000 & 3688860.090000 & 431245.650000 & \\
\hline CUTOT & 0.001800 & 3688860.090000 & 431245.650000 & \\
\hline $\mathrm{crn}$ & -0.010000 & 3688860.090000 & 431245.650000 & \\
\hline $\mathbf{F}$ & -0.100000 & 3688860.090000 & 431245.650000 & \\
\hline HGTOT & -0.000200 & 3688860.090000 & 431245.650000 & \\
\hline MNTOT & 0.000946 & 3688860.090000 & 431245.650000 & \\
\hline NATOT & 0.033200 & 3688860.090000 & 431245.650000 & \\
\hline NITOT & 0.001250 & 3688860.090000 & 431245.650000 & \\
\hline PBTOT & -0.005000 & 3688860.090000 & 431245.650000 & \\
\hline SETOT & -0.005000 & 3688860.090000 & 431245.650000 & \\
\hline 504 & -1.000000 & 3688860.090000 & 431245.650000 & \\
\hline TOTRAD & -0.100000 & 3688860.090000 & 431245.650000 & \\
\hline TPO4 & -0.050000 & 3688860.090000 & 431245.650000 & \\
\hline UTOT & 0.021200 & 3688860.090000 & 431245.650000 & \\
\hline ZNTOT & 0.007480 & 3688860.090000 & 431245.650000 & \\
\hline ALTOT & 0.012500 & 3688698.090000 & 431023.920000 & \\
\hline BATOT & 0.000107 & 3688698.090000 & 431023.920000 & \\
\hline$C L$ & -0.250000 & 3688698.090000 & 431023.920000 & \\
\hline COTOT & -0.004000 & 3688698.090000 & 431023.920000 & \\
\hline CRTOT & -0.004000 & 3688698.090000 & 431023.920000 & \\
\hline CUTOT & -0.004000 & 3688698.090000 & 431023.920000 & \\
\hline CYN & -0.010000 & 3688698.090000 & 431023.920000 & \\
\hline $\mathbf{F}$ & -0.100000 & 3688698.090000 & 431023.920000 & \\
\hline BGTOT & -0.000200 & 3688698.090000 & 431023.920000 & \\
\hline MNTOT & 0.005410 & 3688698.090000 & 431023.920000 & \\
\hline NATOT & 0.039900 & 3688698.090000 & 431023.920000 & \\
\hline NITOT & 0.000888 & 3688698.090000 & 431023.920000 & \\
\hline PBTOT & -0.005000 & 3688698.090000 & 431023.920000 & \\
\hline SETOT & -0.005000 & 3688698.090000 & 431023.920000 & \\
\hline SO4 & -1.000000 & 3688698.090000 & 431023.920000 & \\
\hline TOTRAD & & 3688698.090000 & 431023.920000 & \\
\hline TPO4 & -0.050000 & 3688698.090000 & 431023.920000 & \\
\hline UTOT & 0.009580 & 3688698.090000 & 431023.920000 & \\
\hline ZNTOT & 0.004200 & 3688698.090000 & 431023.920000 & \\
\hline
\end{tabular}




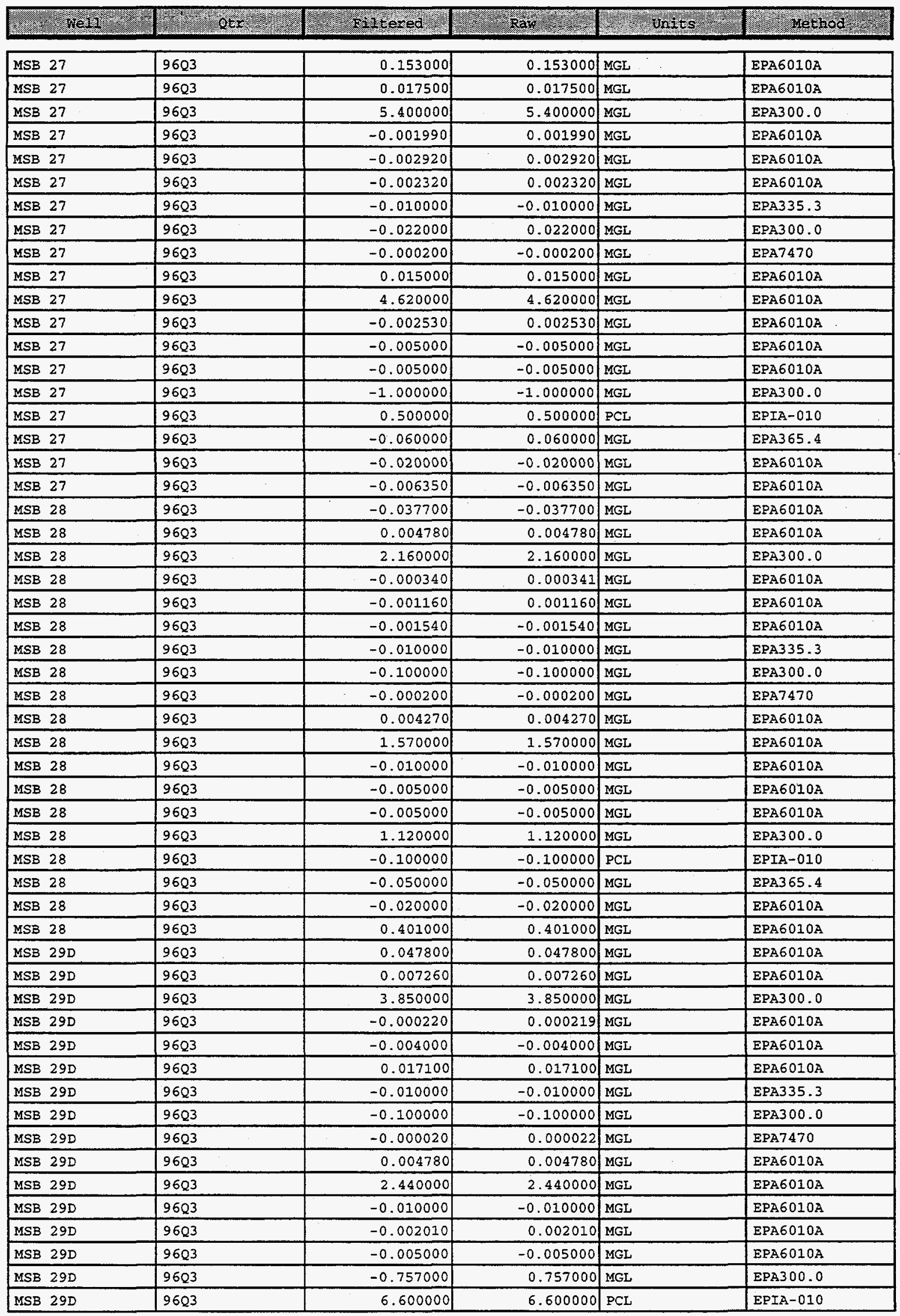


WSRC-TR-00347

November, 1997

Appendix II-66

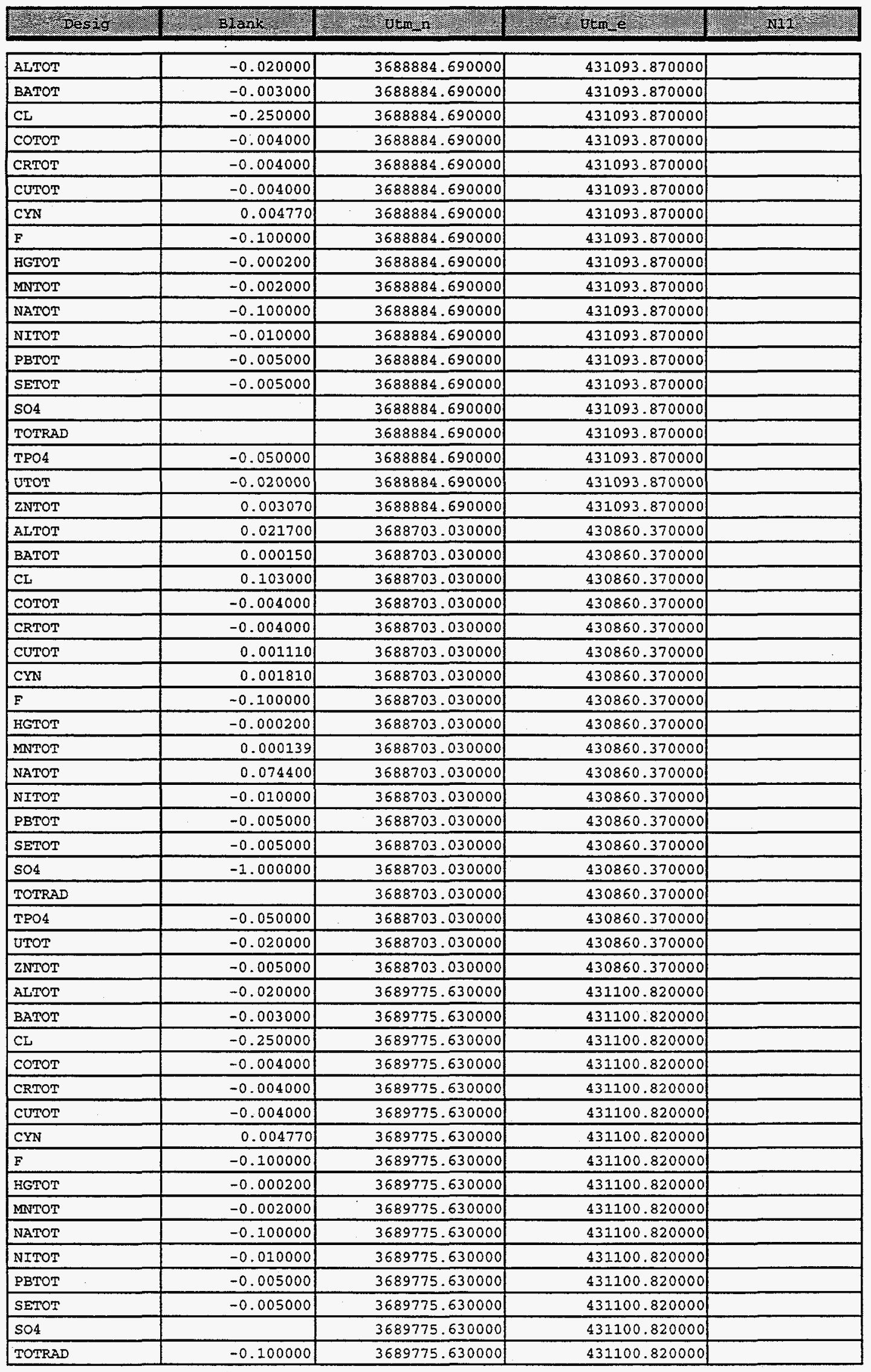




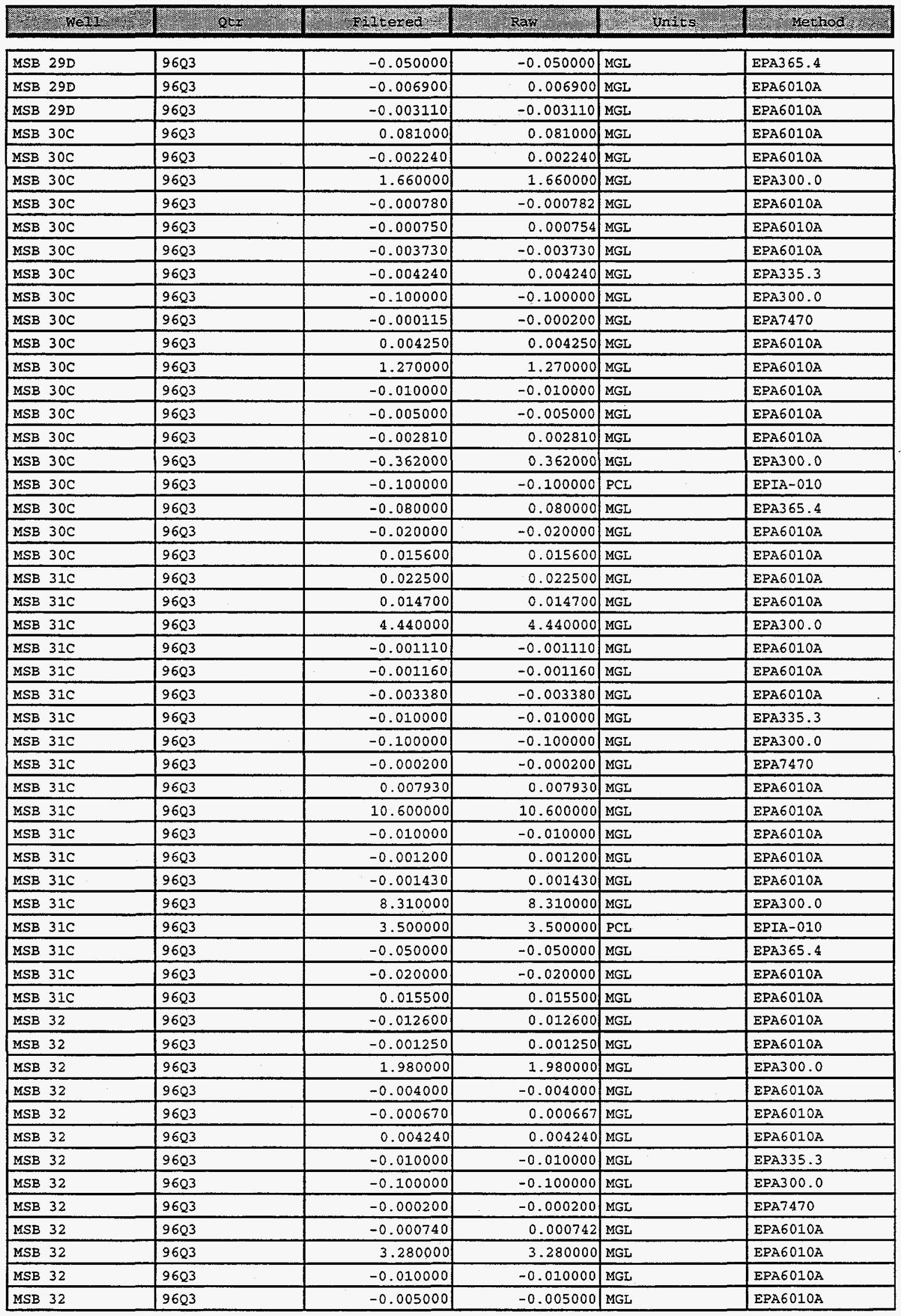




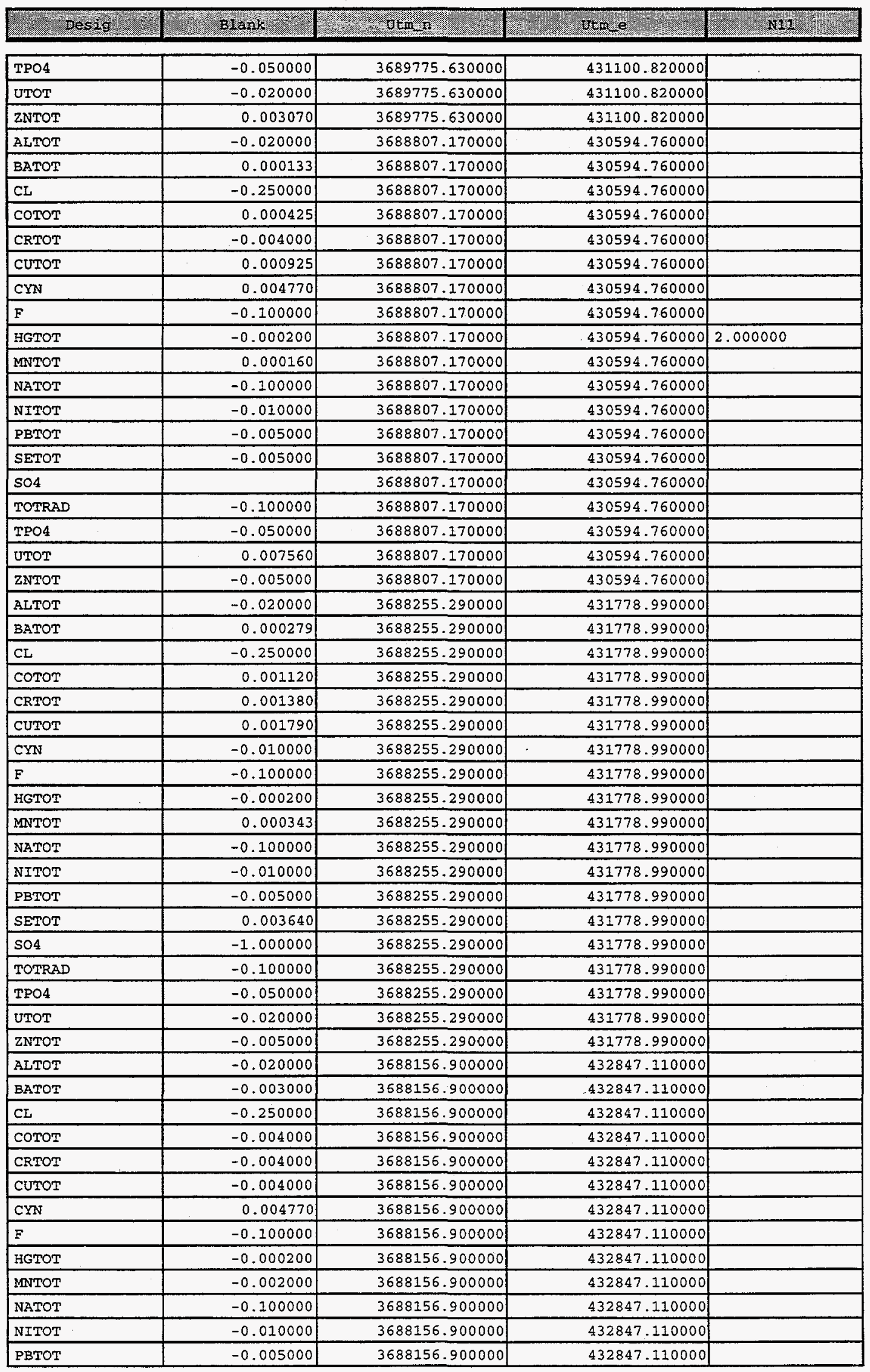


WSRC-TR-00347

November, 1997

Appendix II-69

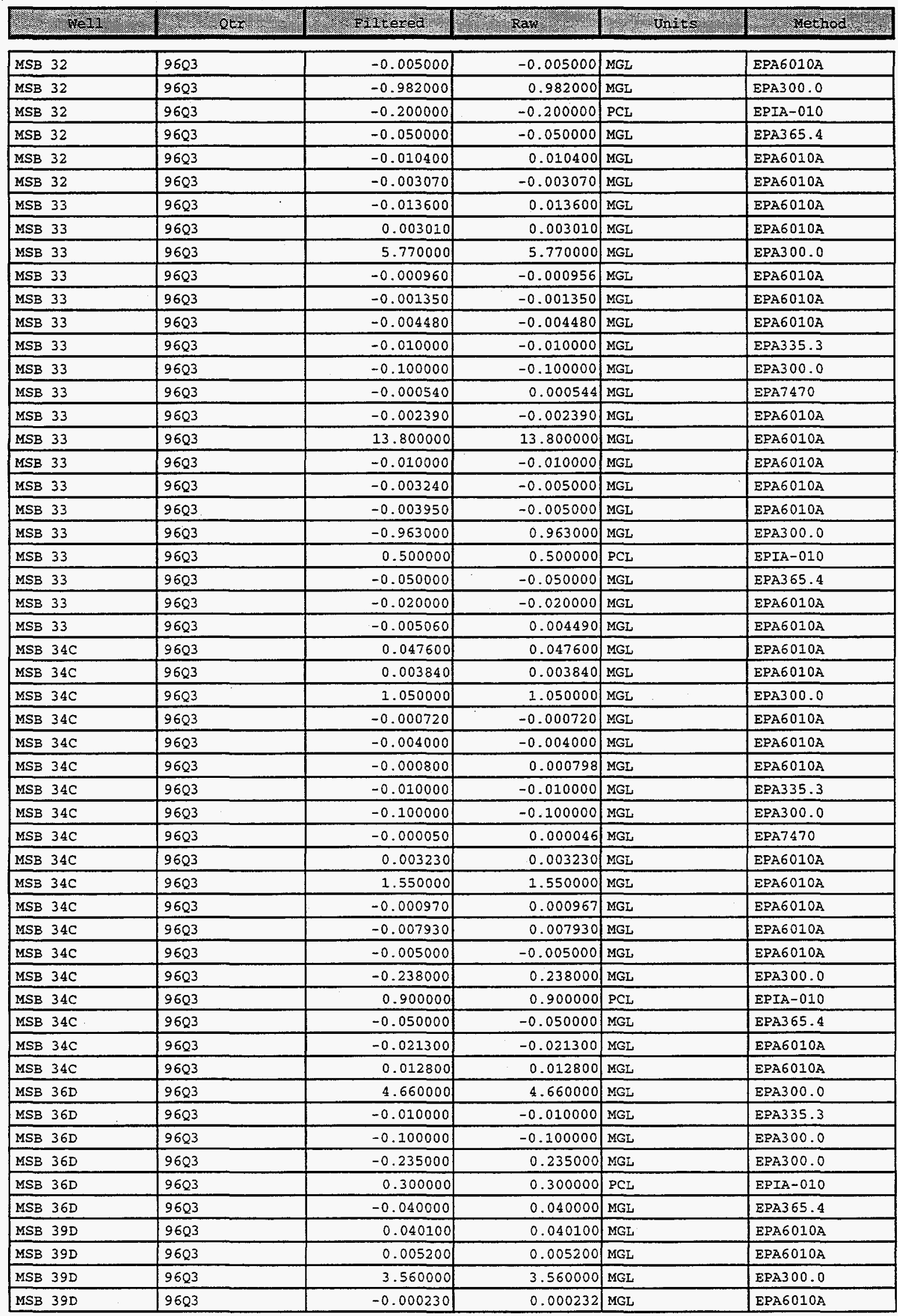


WSRC-TR-00347

November, 1997

Appendix II-70

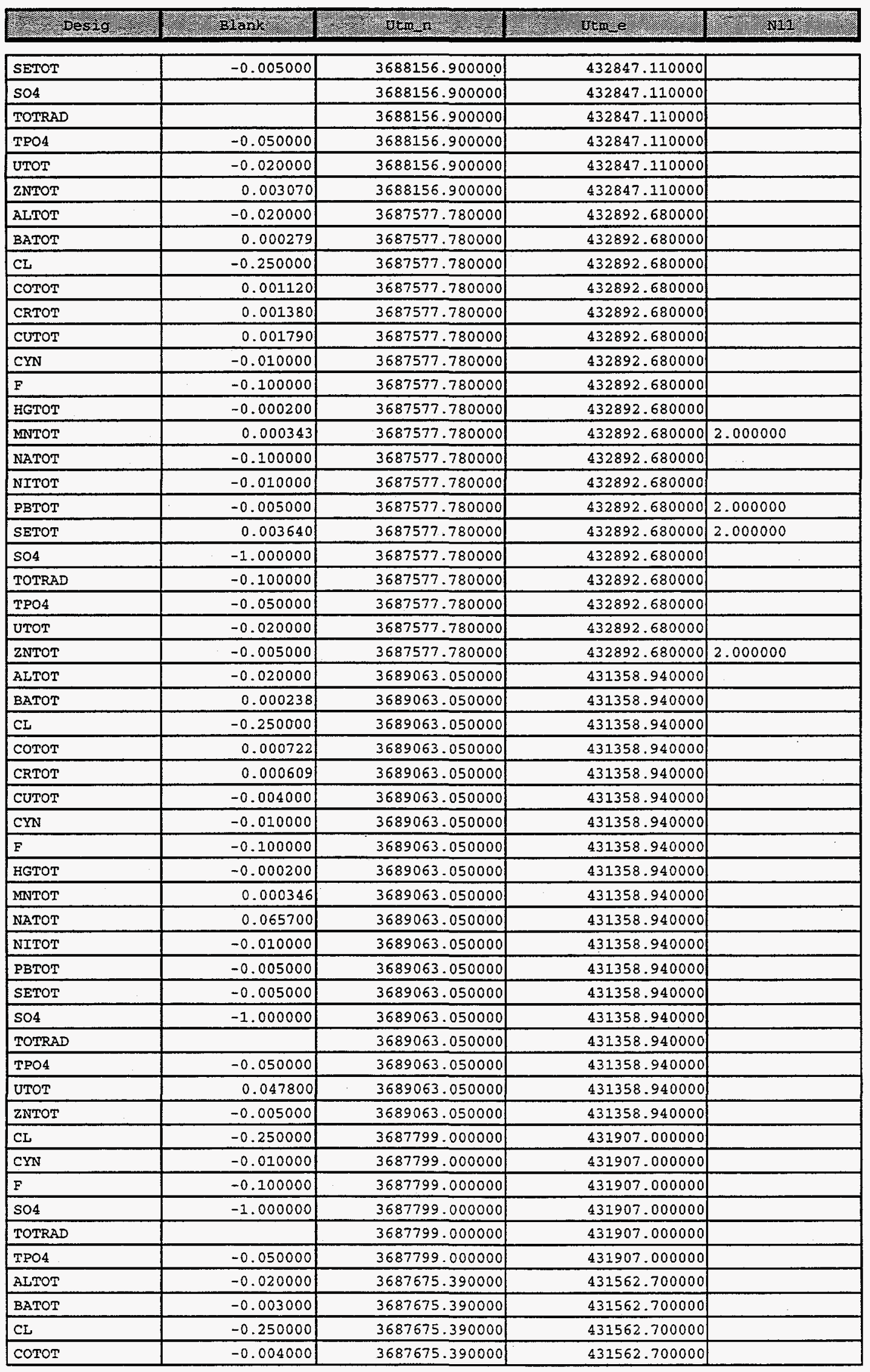




\begin{tabular}{|c|c|c|c|c|c|}
\hline $9-27$ nel1 & 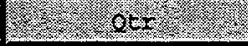 & 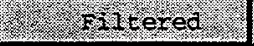 & zay & $\ln x=5-3$ & Yethod \\
\hline MSB $39 D$ & 9603 & 0.006070 & 0.006070 & MGL & EPA6010A \\
\hline MSB 39D & 9603 & 0.029500 & 0.029500 & MGL & EPA6010A \\
\hline MSB 39D & $96 Q 3$ & -0.010000 & -0.010000 & MGL & EPA335.3 \\
\hline MSB 39D & $96 Q 3$ & -0.100000 & -0.100000 & MGL & EPA300.0 \\
\hline MSB $39 D$ & $96 Q 3$ & -0.000200 & -0.000200 & MGL & EPA7470 \\
\hline MSB 39D & 9603 & 0.006410 & 0.006410 & MGL & EPA6010A \\
\hline MSB 39D & $96 Q 3$ & 2.370000 & 2.370000 & MGL & EPA6010A \\
\hline MSB 39D & 9603 & -0.002900 & 0.002900 & MGL & EPA6010A \\
\hline MSB 39D & 9603 & 0.008440 & 0.008440 & MGL & EPA6010A \\
\hline MSB 39D & 9603 & -0.005000 & -0.005000 & MGL & ERA6010A \\
\hline MSB $39 \mathrm{D}$ & 9603 & -0.204000 & 0.204000 & MGL & EPA300.0 \\
\hline MSB 39D & $96 Q 3$ & 2.100000 & 2.100000 & PCL & EPIA-010 \\
\hline MSB $39 D$ & 9603 & -0.050000 & -0.050000 & MGL & EPA.365.4 \\
\hline MSB 39D & 9603 & -0.020000 & -0.020000 & MGL & EPA6010A \\
\hline MSB 39D & 9603 & -0.005660 & -0.005660 & MGL & EPA.6010A \\
\hline$M S B \quad 42 D$ & 9603 & -0.182000 & 0.182000 & MGL & EPA6010A \\
\hline MSB $42 D$ & 9603 & 0.008700 & 0.008700 & MGL & EPA6010A \\
\hline MSB $42 D$ & 9603 & 1.670000 & 1.670000 & MGL & EPA300.0 \\
\hline MSB 42D & 9603 & -0.001430 & -0.001430 & MGL & EPA6010A \\
\hline MSB $42 D$ & $96 Q 3$ & -0.001860 & 0.001860 & MGL & EPA6010A \\
\hline MSB $42 D$ & $96 Q 3$ & 0.012300 & 0.012300 & MGL & EPA6010A \\
\hline MSB $42 \mathrm{D}$ & 9603 & -0.010000 & -0.010000 & MGL & EPA335.3 \\
\hline$M S B \quad 42 D$ & 9603 & -0.100000 & -0.100000 & MGL & EPA300.0 \\
\hline MSB 42D & 9603 & -0.000200 & -0.000200 & MGL & EPA7470 \\
\hline MSB $42 D$ & 9603 & 0.018100 & 0.018100 & MGL & EPA6010A \\
\hline$M S B \quad 42 D$ & 9603 & 2.030000 & 2.030000 & MGL & EPA $6010 \mathrm{~A}$ \\
\hline MSB $42 \mathrm{D}$ & 9603 & -0.003740 & 0.003740 & MGL & EPA6010A \\
\hline MSB $42 \mathrm{D}$ & 9503 & -0.002480 & 0.002480 & MGL & EPA6010A \\
\hline MSB 42D & $96 Q 3$ & -0.005000 & -0.005000 & MGL & EPA6010A \\
\hline MSB $42 D$ & $96 \mathrm{Q} 3$ & -0.272000 & 0.272000 & MGL & EPA300.0 \\
\hline MSB $42 D$ & 9603 & 1.300000 & 1.300000 & PCI & EPIA-010 \\
\hline MSB $42 D$ & 9603 & -0.030000 & -0.030000 & MGL & EPA365.4 \\
\hline MSB 42D & 9603 & -0.052500 & 0.052500 & MGL & EPA6010A \\
\hline MSB $42 D$ & $96 Q 3$ & -0.006780 & 0.006780 & MGL & EPA6010A \\
\hline MSB 43D & $96 Q 3$ & 0.062900 & 0.062900 & MGL & EPA6010A \\
\hline MSB $43 \mathrm{D}$ & 9603 & 0.003820 & 0.003820 & MGL & EPA6010A \\
\hline MSB 43D & 9603 & 1.660000 & 1.660000 & MGL & EPA300.0 \\
\hline MSB 43D & 9603 & -0.000920 & -0.000917 & MGL & EPA6010A \\
\hline MSB 43D & 9603 & -0.004000 & -0.004000 & MGL & EPA6010A \\
\hline MSB $43 D$ & $96 Q 3$ & 0.016400 & 0.016400 & MGL & EPA6010A \\
\hline MSB $43 D$ & 9603 & -0.006110 & 0.006110 & MGL & EPA335.3 \\
\hline MSB 43D & 9603 & -0.100000 & -0.100000 & MGL & EPA300.0 \\
\hline MSB $43 \mathrm{D}$ & $96 Q 3$ & -0.000200 & -0.000200 & MGL & EPA7470 \\
\hline MSB $43 D$ & $96 Q 3$ & 0.006660 & 0.006660 & MGL & EPA6010A \\
\hline MSB 43D & 9603 & 1.410000 & 1.410000 & MGI & EPA6010A \\
\hline MSB 43D & 9603 & -0.001800 & 0.001800 & MGL & EPA6010A \\
\hline MSB 43D & $96 Q 3$ & -0.003430 & 0.003430 & MGL & EPA6010A \\
\hline MSB 43D & $96 Q 3$ & -0.005000 & -0.005000 & MGL & EPA6010A \\
\hline$M S B \quad 43 D$ & 9603 & -0.398000 & 0.398000 & MGL & EPA300.0 \\
\hline MSB 43D & $96 Q 3$ & 1.000000 & 1.000000 & PCL & EPIA-010 \\
\hline MSB $43 D$ & $96 Q 3$ & -0.050000 & -0.050000 & MGL & EPA3 65.4 \\
\hline MSB $43 D$ & $96 Q 3$ & -0.013500 & -0.013500 & MGL & EPA6010A \\
\hline MSB 43D & 9603 & 0.006150 & 0.006150 & MGL & EPA6010A \\
\hline MSB $47 D$ & 9603 & -0.020000 & -0.020000 & MGL & EPA6010A \\
\hline
\end{tabular}


WSRC-TR-00347

November, 1997

Appendix I1-72

\begin{tabular}{|c|c|c|c|c|}
\hline Vy. & 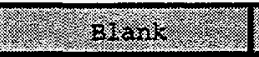 & 2.0. J & 1. & 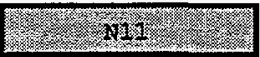 \\
\hline CRTOT & -0.004000 & 3687675.390000 & 431562.700000 & \\
\hline CUTOT & -0.004000 & 3687675.390000 & 431562.700000 & \\
\hline CYN & 0.004770 & 3687675.390000 & 431562.700000 & \\
\hline$F$ & -0.100000 & 3687675.390000 & 431562.700000 & \\
\hline HGTOT & -0.000200 & 3687675.390000 & 431562.700000 & \\
\hline MNTOT & -0.002000 & 3687675.390000 & 431562.700000 & \\
\hline NATOT & -0.100000 & 3687675.390000 & 431562.700000 & \\
\hline NITOT & -0.010000 & 3687675.390000 & 431562.700000 & \\
\hline PBTOT & -0.005000 & 3687675.390000 & 431562.700000 & \\
\hline SETOT & -0.005000 & 3687675.390000 & 431562.700000 & \\
\hline so 4 & & 3687675.390000 & 431562.700000 & \\
\hline TOTRAD & & 3687675.390000 & 431562.700000 & \\
\hline TPO4 & -0.050000 & 3687675.390000 & 431562.700000 & \\
\hline UTOT & -0.020000 & 3687675.390000 & 431562.700000 & \\
\hline ZNTOT & 0.003070 & 3687675.390000 & 431562.700000 & \\
\hline ALTOT & -0.020000 & 3689167.320000 & 433677.650000 & \\
\hline BATOT & -0.003000 & 3689167.320000 & 431677.650000 & \\
\hline$C L$ & -0.250000 & 3689167.320000 & 431677.650000 & \\
\hline COTOT & -0.004000 & 3689167.320000 & 431677.650000 & \\
\hline CRTOT & -0.004000 & 3689167.320000 & 431677.650000 & \\
\hline cutor & -0.004000 & 3689167.320000 & 431677.650000 & \\
\hline CYN & 0.002360 & 3689167.320000 & 431677.650000 & \\
\hline$F$ & -0.100000 & 3689167.320000 & 431677.650000 & \\
\hline HGTOT & -0.000200 & 3689167.320000 & 431677.650000 & \\
\hline MNTOT & 0.001000 & 3689167.320000 & 431677.650000 & \\
\hline NATOT & 0.034200 & 3689167.320000 & 431677.650000 & \\
\hline NITOT & 0.002020 & 3689167.320000 & 431677.650000 & \\
\hline PBTOT & -0.005000 & 3689167.320000 & 431677.650000 & \\
\hline SETOT & -0.005000 & 3689167.320000 & 431677.650000 & \\
\hline 504 & 0.269000 & 3689167.320000 & 431677.650000 & \\
\hline TOTRAD & 0.000000 & 3689167.320000 & 431677.650000 & \\
\hline TPO4 & 0.030000 & 3689167.320000 & 431677.650000 & \\
\hline UTOT & 0.023600 & 3689167.320000 & 431677.650000 & \\
\hline ZNTOT & 0.006960 & 3689167.320000 & 431677.650000 & \\
\hline ALTOT & -0.020000 & 3689421.940000 & 430640.350000 & \\
\hline BATOT & 0.000133 & 3689421.940000 & 430640.350000 & \\
\hline $\mathrm{CL}$ & -0.250000 & 3689421.940000 & 430640.350000 & \\
\hline COTOT & 0.000425 & 3689421.940000 & 430640.350000 & \\
\hline CRTOT & -0.004000 & 3689421.940000 & 430640.350000 & \\
\hline CUTOT & 0.000925 & 3689421.940000 & 430640.350000 & \\
\hline CYN & 0.004770 & 3689421.940000 & 430640.350000 & \\
\hline$F$ & -0.100000 & 3689421.940000 & 430640.350000 & \\
\hline HGTOT & -0.000200 & 3689421.940000 & 430640.350000 & \\
\hline MNTOT & 0.000160 & 3689421.940000 & 430640.350000 & \\
\hline NATOT & -0.100000 & 3689421.940000 & 430640.350000 & \\
\hline NITOT & -0.010000 & 3689421.940000 & 430640.350000 & \\
\hline PBTOT & -0.005000 & 3689421.940000 & 430640.350000 & \\
\hline SETOT & -0.005000 & 3689421.940000 & 430640.350000 & \\
\hline 504 & -1.000000 & 3689421.940000 & 430640.350000 & \\
\hline TOTRAD & -0.100000 & 3689421.940000 & 430640.350000 & \\
\hline TPO4 & -0.050000 & 3689421.940000 & 430640.350000 & \\
\hline UTOT & 0.007560 & 3689421.940000 & 430640.350000 & \\
\hline ZNTOT & -0.005000 & 3689421.940000 & 430640.350000 & \\
\hline ALTOT & -0.020000 & 3689857.790000 & 431401.740000 & \\
\hline
\end{tabular}




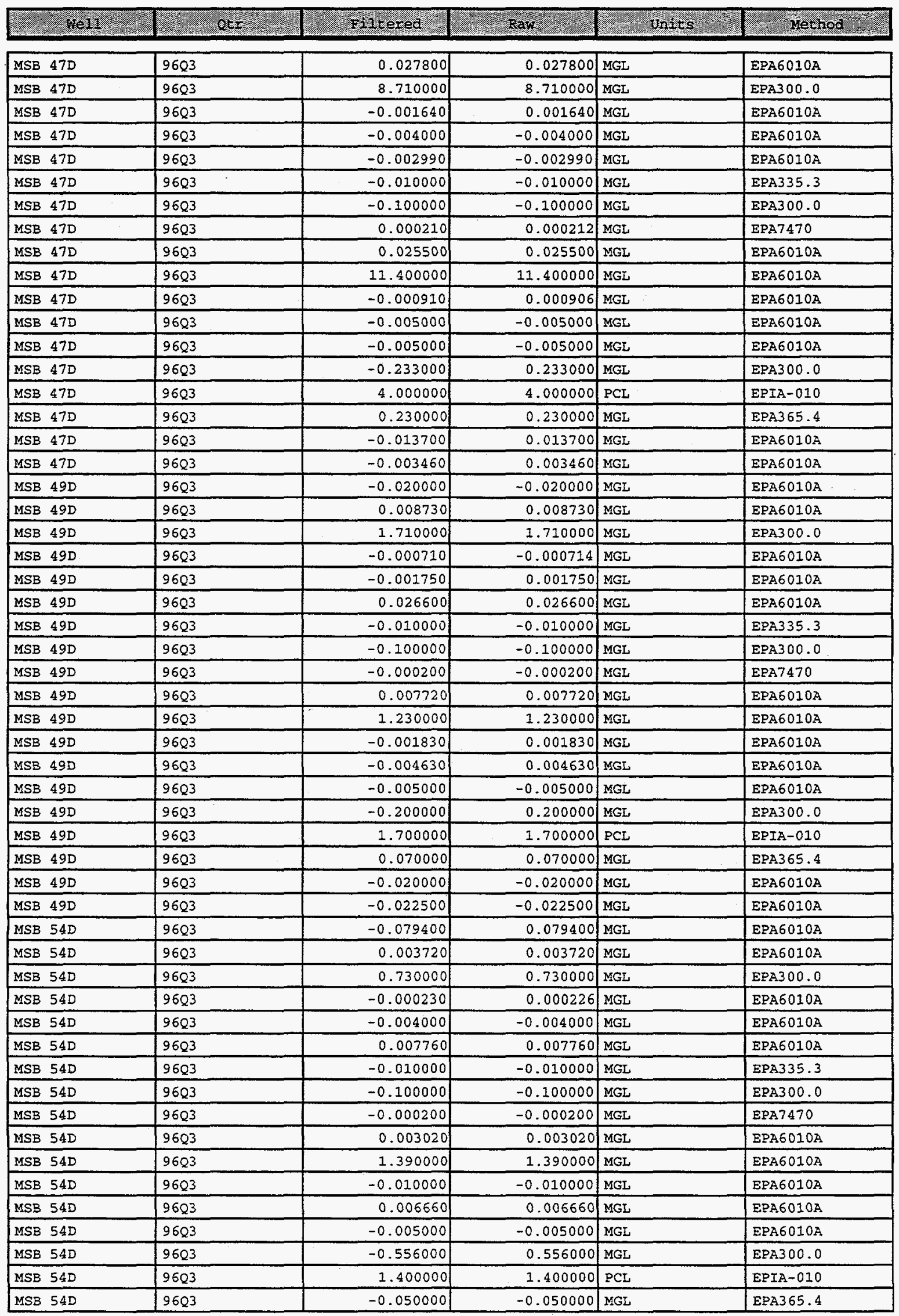




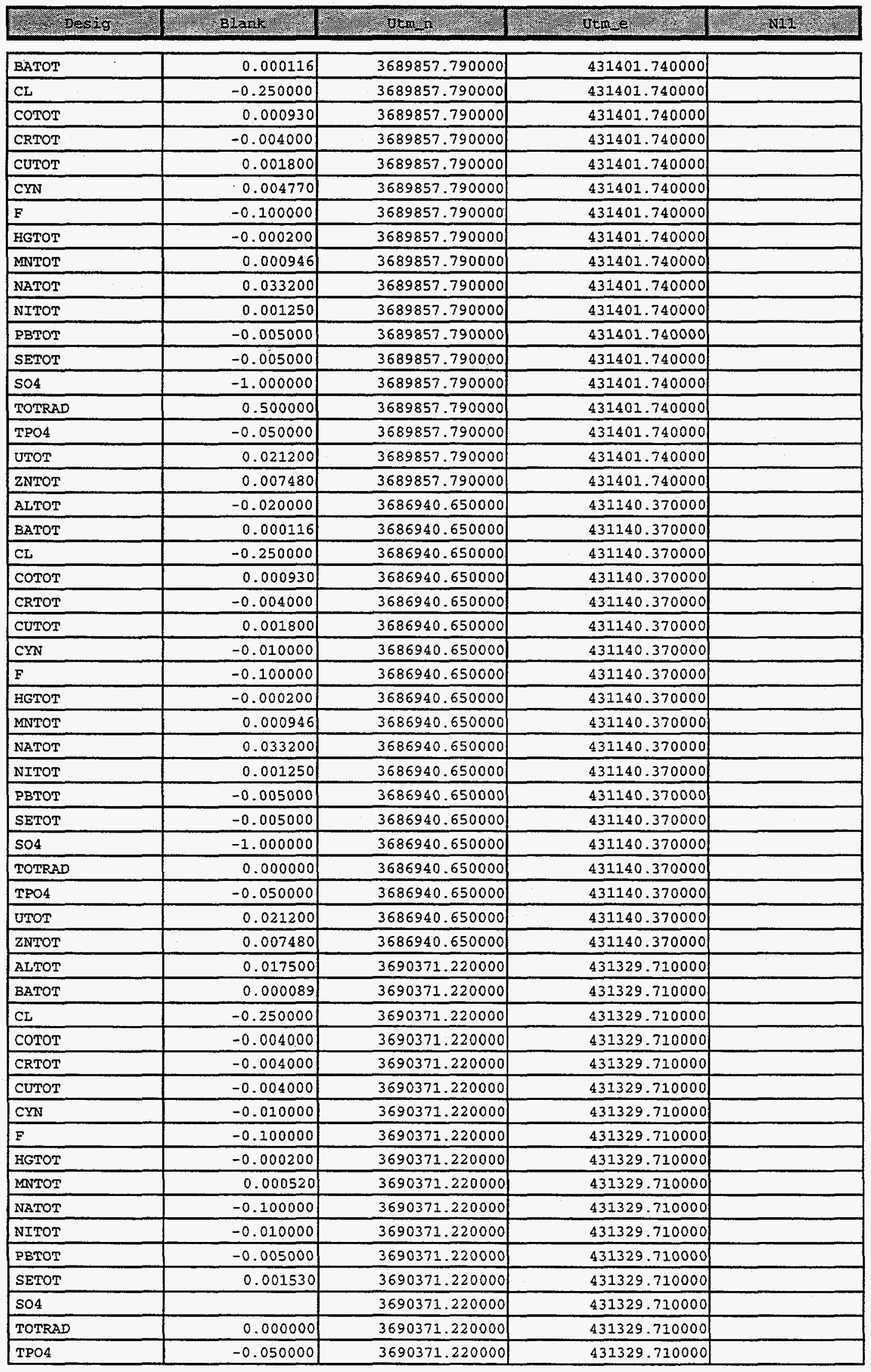


WSRC-TR-00347

November, 1997

Appendix I1-75

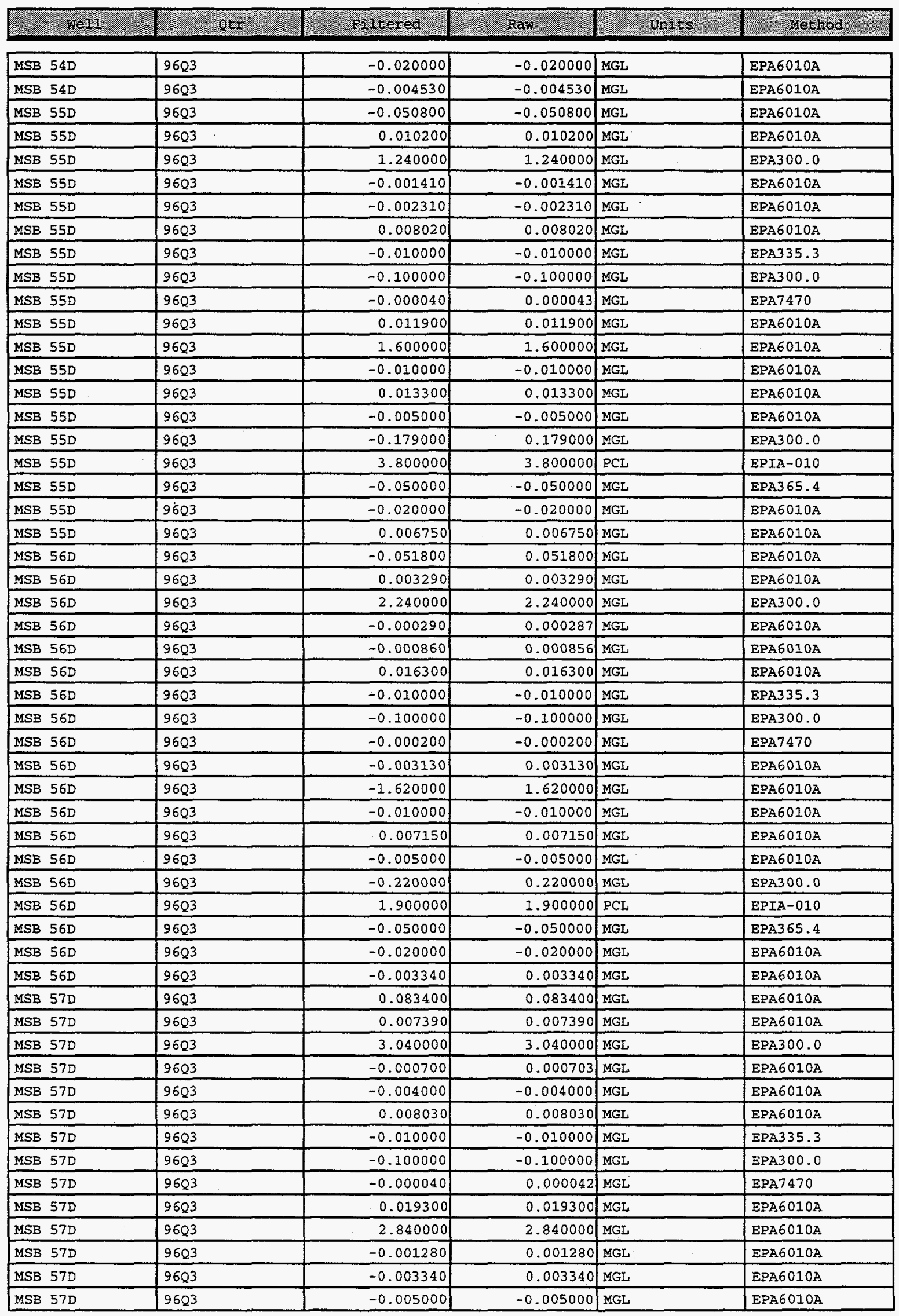




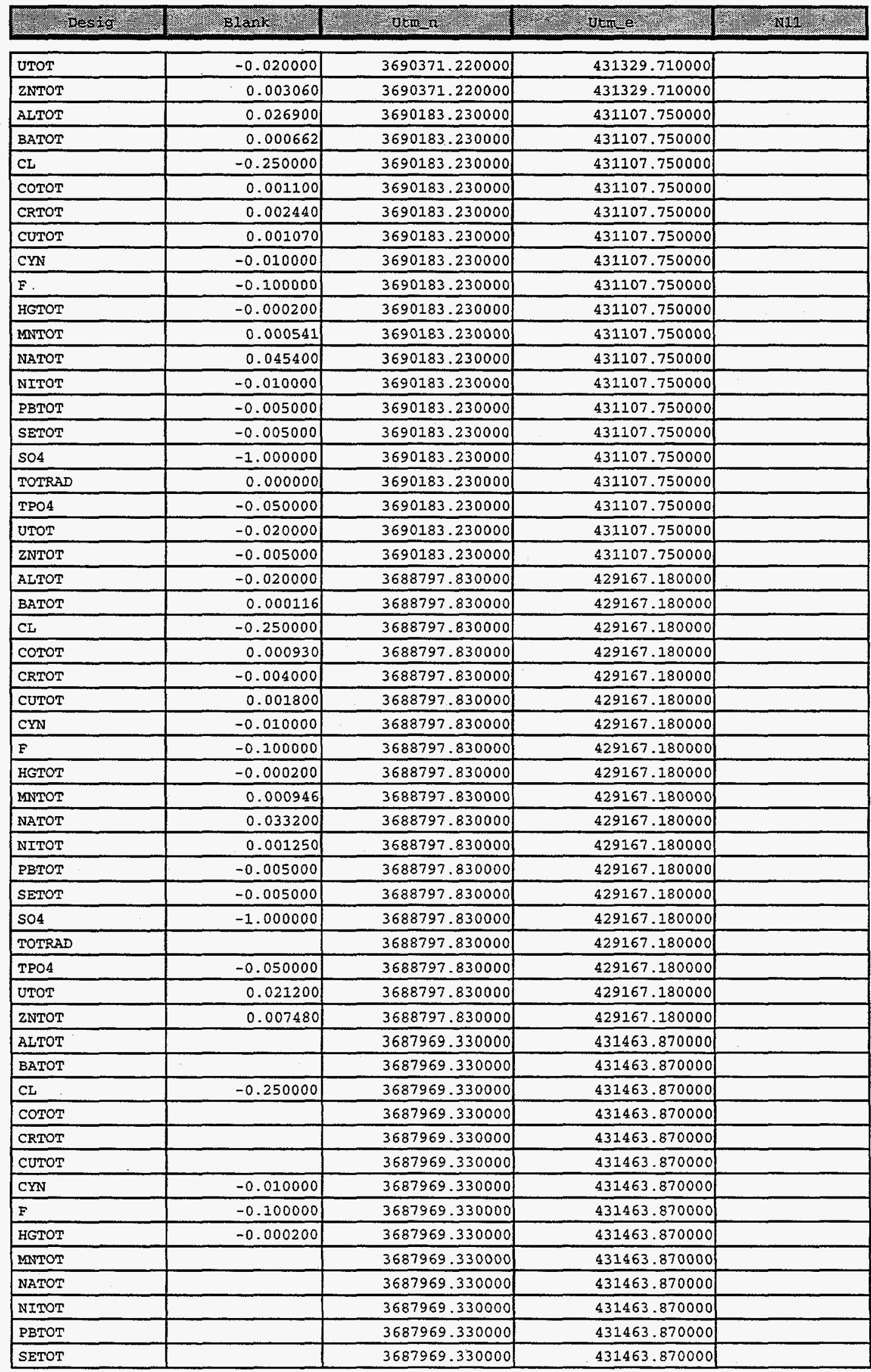




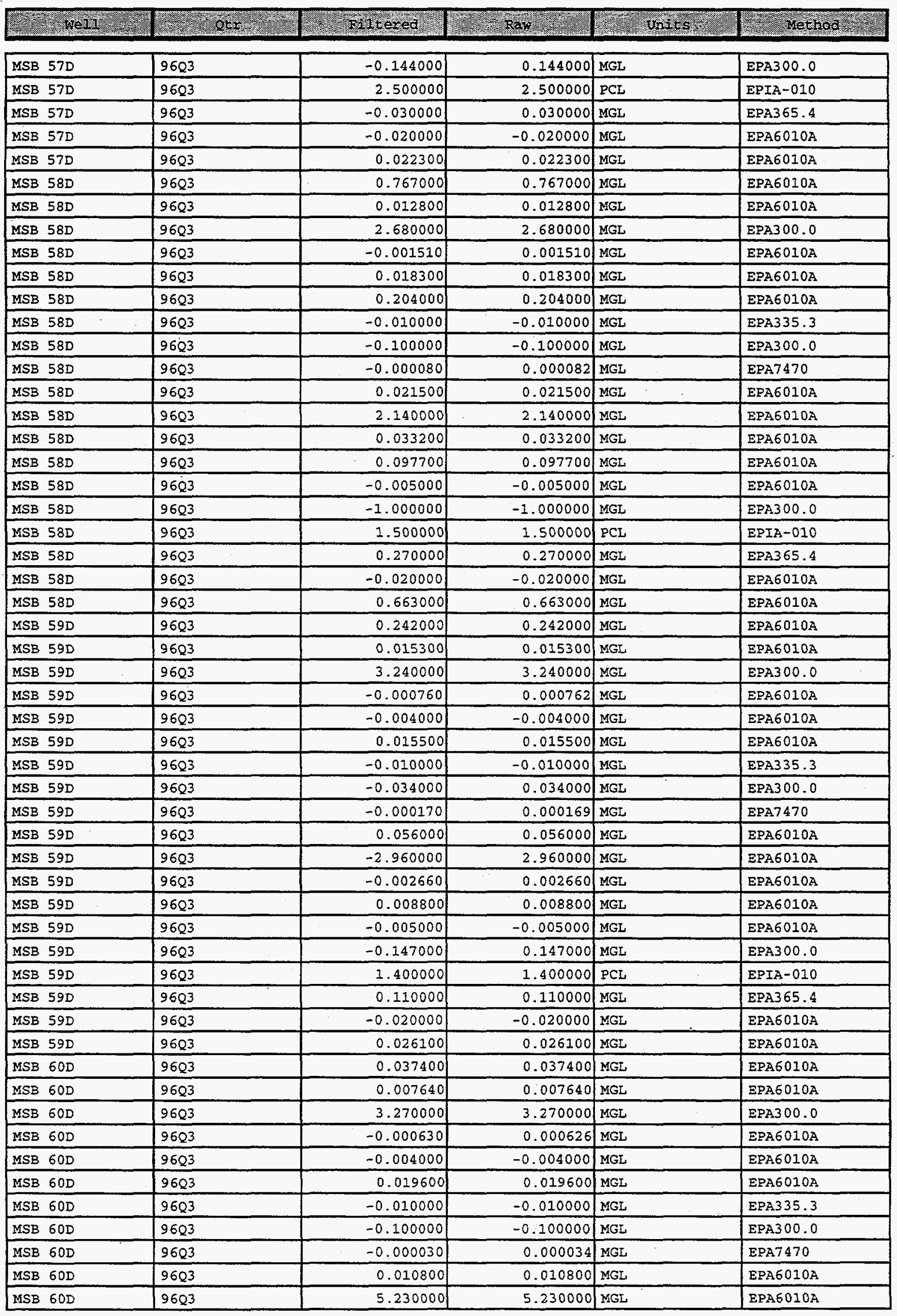


WSRC-TR-00347

November, 1997

Appendix II-78

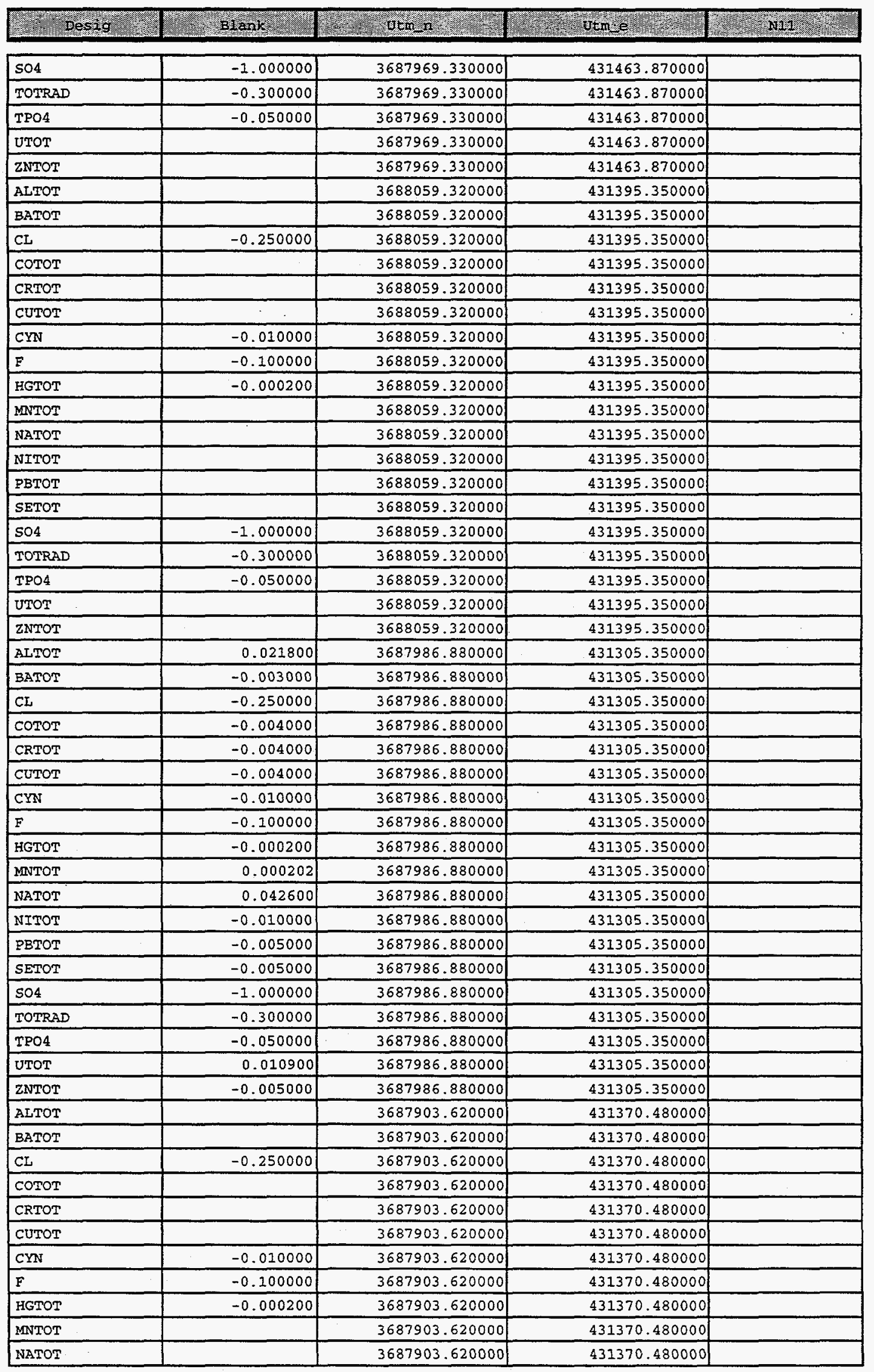


WSRC-TR-00347

November, 1997

Appendix II-79

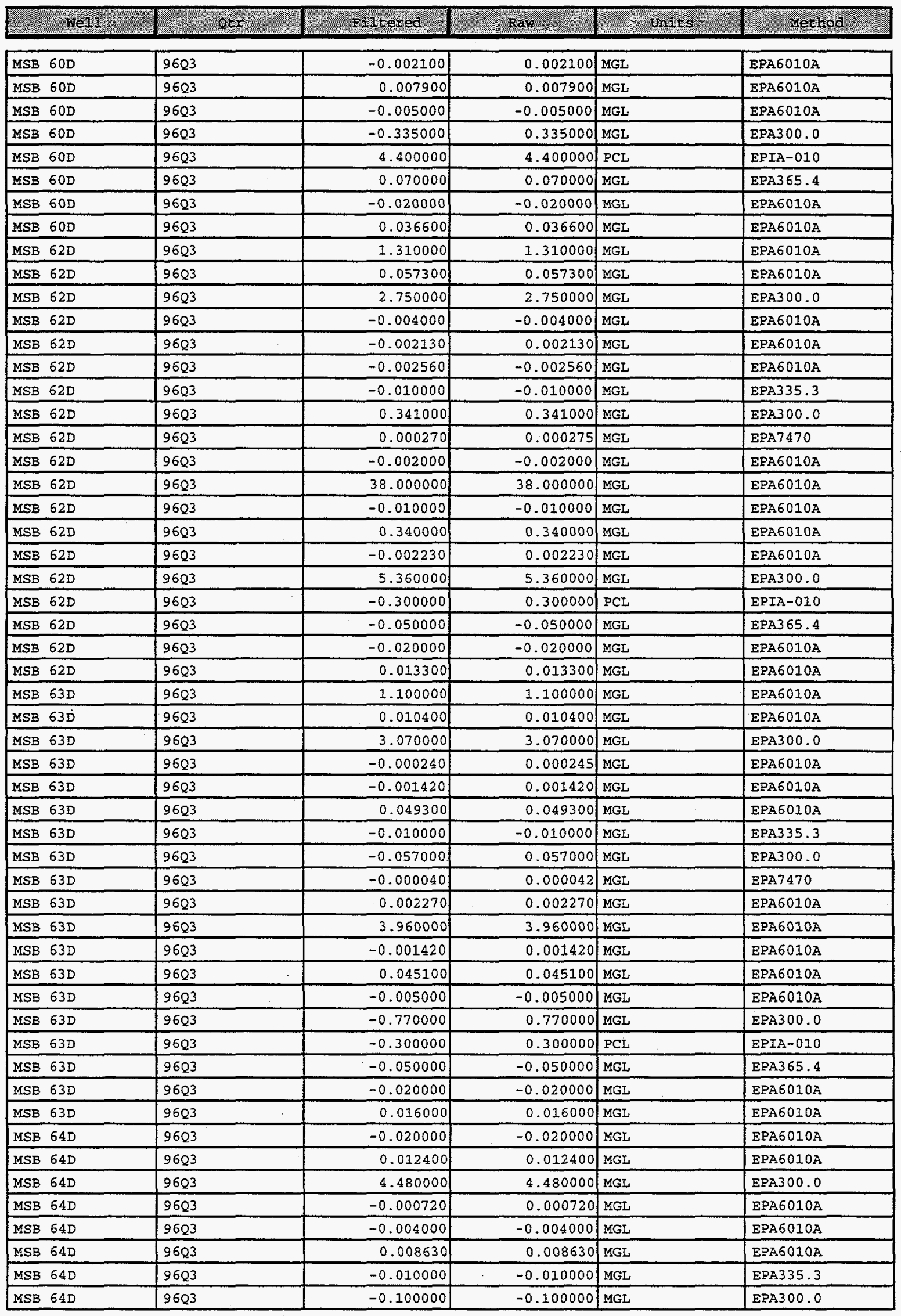


WSRC-TR-00347

November, 1997

Appendix II-80

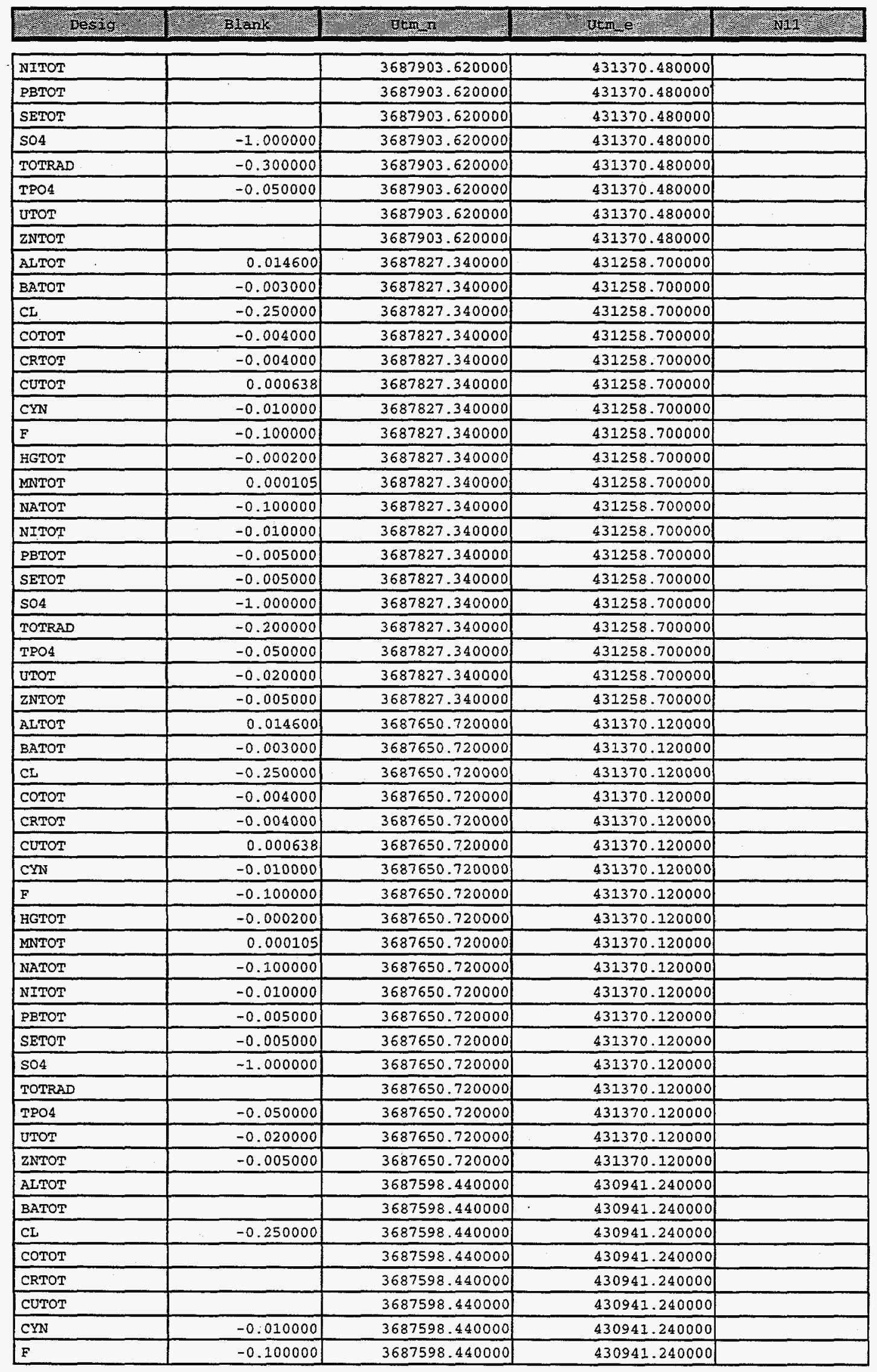


WSRC-TR-00347

November, 1997

Appendix II-81

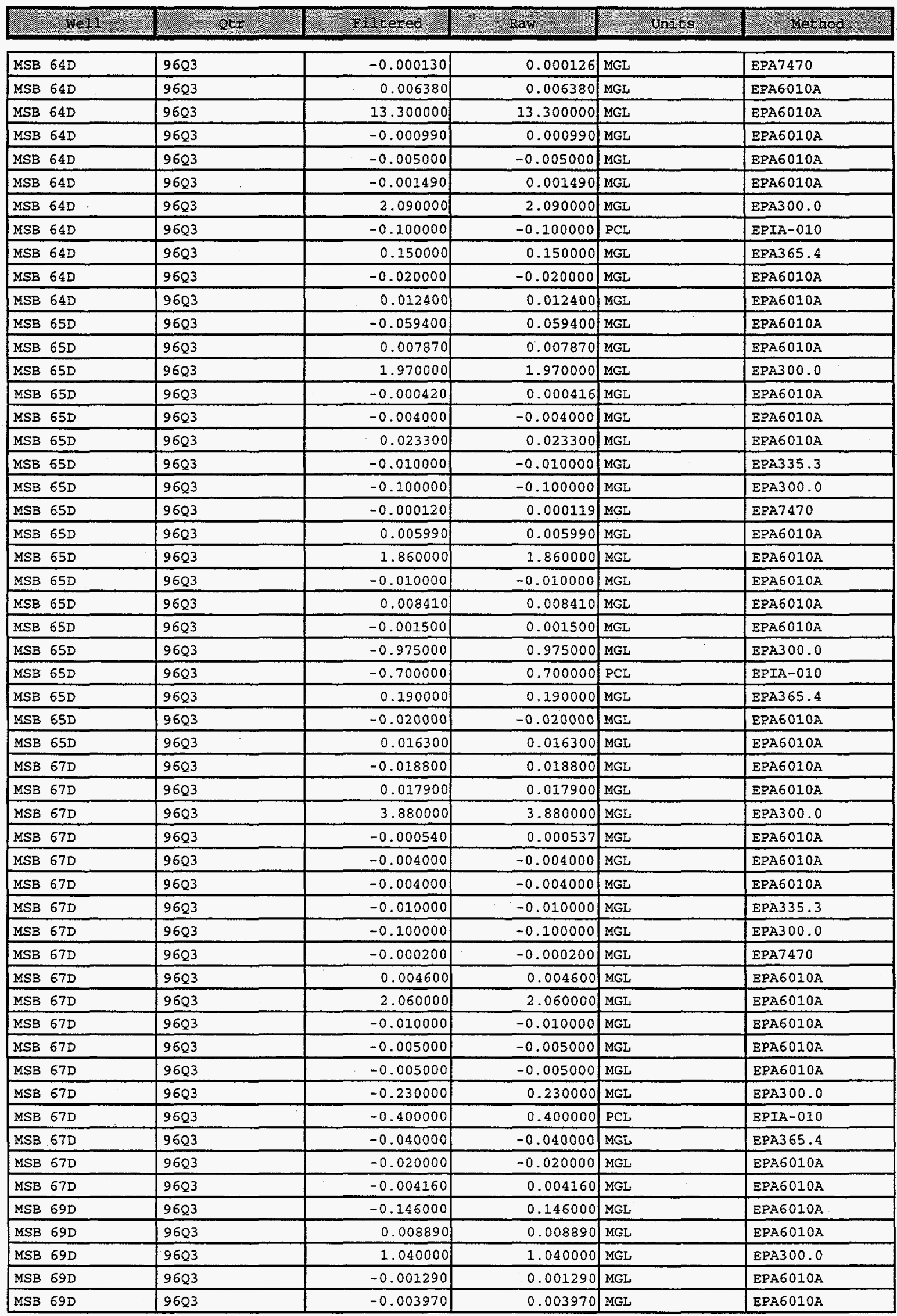




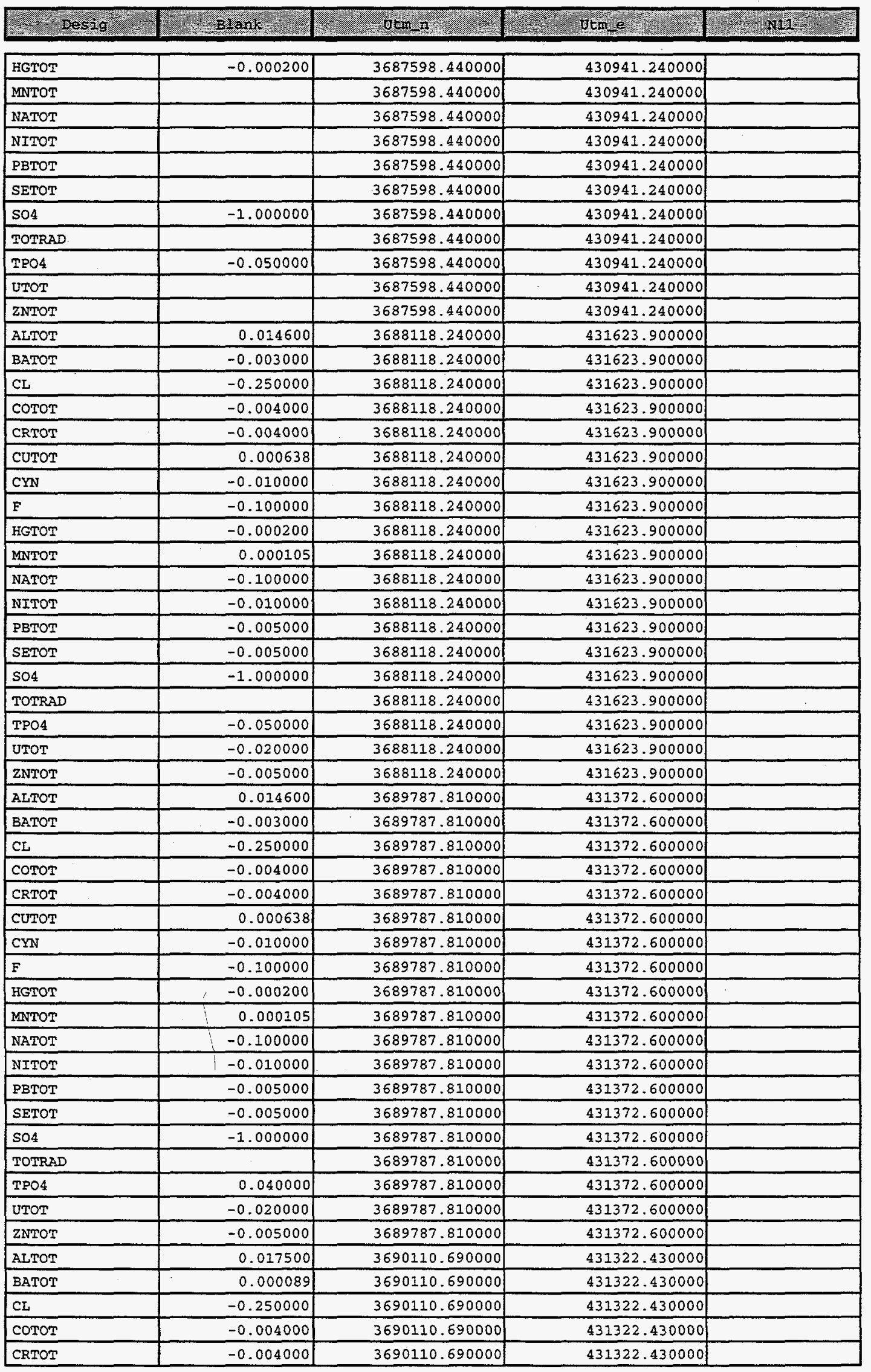




\begin{tabular}{|c|c|c|c|c|c|}
\hline 20 & (17. & 3ulcered & 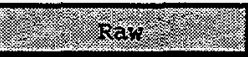 & 17nits & 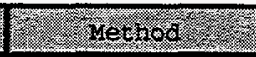 \\
\hline MSB 69D & 9603 & 0.008890 & 0.008890 & MGL & EPA6010A \\
\hline MSB 69D & 9603 & -0.010000 & -0.010000 & MGL & EPA335.3 \\
\hline MSB 69D & 9603 & -0.100000 & -0.100000 & MGL & EPA300.0 \\
\hline MSB $69 D$ & 9603 & -0.000200 & -0.000200 & MGL & EPA7470 \\
\hline MSB $69 D$ & 9603 & 0.008610 & 0.008610 & MGL & EPA6010A \\
\hline MSB 69D & $96 Q 3$ & 1.090000 & 1.090000 & MGL & EPA6010A \\
\hline MSB $69 \mathrm{D}$ & 9603 & -0.003110 & 0.003110 & MGL & EPA6010A \\
\hline MSB 690 & 9603 & -0.001690 & 0.001690 & MGL & EPA6010A \\
\hline MSB $69 D$ & $96 Q 3$ & -0.005000 & -0.005000 & MGL & EPA6010A \\
\hline MSB $69 \mathrm{D}$ & 9603 & -1.000000 & -1.000000 & MGL & EPA 300.0 \\
\hline MSB 69D & 9603 & 6.300000 & 6.300000 & PCL & EPIA-010 \\
\hline MSB 69D & 9603 & -0.050000 & -0.050000 & MGL & EPA365.4 \\
\hline MSB $69 \mathrm{D}$ & 9603 & -0.020000 & -0.020000 & MGL & EPA6010A \\
\hline MSB 69D & 9603 & -0.015000 & -0.015000 & MGL & EPA $6010 \mathrm{~A}$ \\
\hline MSB 70D & 9603 & 0.187000 & 0.187000 & MGL & EPA6010A \\
\hline MSB $70 D$ & 9603 & 0.007960 & 0.007960 & MGL & ERA $6010 \mathrm{~A}$ \\
\hline MSB 70D & 9603 & 2.120000 & 2.120000 & MGL & EPA300.0 \\
\hline MSB 70D & 9603 & -0.000330 & 0.000332 & MGL & EPA6010A \\
\hline MSB 70D & $96 Q^{3}$ & -0.002590 & 0.002590 & MGL & EPA6010A \\
\hline MSB 70D & $96 Q 3$ & 0.021600 & 0.021600 & MGL & EPA $6010 \mathrm{~A}$ \\
\hline MSB 7OD & 9603 & -0.010000 & -0.010000 & MGL & EPA335.3 \\
\hline MSB $70 \mathrm{D}$ & $96 Q 3$ & -0.100000 & -0.100000 & MGL & EPA300.0 \\
\hline MSB 70D & 9603 & -0.000200 & -0.000200 & MGL & EPA7470 \\
\hline MSB 7OD & 9603 & 0.006950 & 0.006950 & MGL & EPA6010A \\
\hline MSB $30 \mathrm{D}$ & 9603 & 2.960000 & 2.960000 & MGL & EPA6010A \\
\hline MSB 7OD & 9603 & -0.001220 & 0.001220 & MGL & EPA6010A \\
\hline MSB $7 O D$ & 9603 & -0.001490 & 0.001490 & MGL & EPA6010A \\
\hline MSB $70 D$ & 9603 & -0.002020 & 0.002020 & MGL & EPA6010A \\
\hline MSB 7OD & 9603 & -0.204000 & 0.204000 & MGL & EPA300.0 \\
\hline MSB 70D & 9603 & -1.600000 & 1.600000 & PCL & EPIA-010 \\
\hline MSB 70D & 9603 & -0.050000 & -0.050000 & MGL & EPA365.4 \\
\hline MSB 70D & 9603 & -0.020000 & -0.020000 & MGL & ERA6010A \\
\hline MSB 700 & 9603 & 0.015600 & 0.015600 & MGL & EPA6010A \\
\hline MSB 74D & 9603 & 0.559000 & 0.559000 & MGL & EPA6010A \\
\hline MSB 74D & 9603 & 0.018400 & 0.038400 & MGL & EPA6010A \\
\hline MSB $74 \mathrm{D}$ & 9603 & 2.040000 & 2.040000 & MGL. & EPA300.0 \\
\hline MSB $74 D$ & 9603 & -0.001120 & -0.001120 & MGL & EPA6010A \\
\hline MSB 74D & 9603 & -0.008950 & -0.008950 & MGL & EPA6010A \\
\hline MSB 74D & 9603 & 0.195000 & 0.195000 & MGL & EPA6010A \\
\hline$M S B \quad 74 D$ & 9603 & -0.010000 & -0.010000 & MGL & EPA335.3 \\
\hline MSB 74D & $96 Q^{3}$ & -0.100000 & -0.100000 & MGL & EPA300.0 \\
\hline MSB $74 \mathrm{D}$ & 9603 & -0.000200 & -0.000200 & MGL & EPA7470 \\
\hline MSB 74D & 9603 & 0.028100 & 0.028100 & MGL & EPA6010A \\
\hline MSB 74D & 9603 & 4.520000 & 4.520000 & MGL & EPA6010A \\
\hline MSB $74 D$ & 9603 & -0.003910 & 0.003910 & MGL & EPA6010A \\
\hline MSB 74D & 9603 & 0.031000 & 0.031000 & MGI & EPA6010A \\
\hline MSB 74D & $96 Q^{3}$ & -0.005000 & -0.005000 & MGL & EPA $6010 \mathrm{~A}$ \\
\hline MSB $74 \mathrm{D}$ & $96 Q 3$ & -0.455000 & 0.455000 & MGL & EPA300.0 \\
\hline MSB $74 \mathrm{D}$ & $96 Q 3$ & 0.800000 & 0.800000 & PCL & EPIA-010 \\
\hline MSB $74 D$ & 9603 & 0.080000 & 0.080000 & MGL & EPA3 35.4 \\
\hline MSB 74D & 9603 & -0.020000 & -0.020000 & MGL & EPA $6010 \mathrm{~A}$ \\
\hline MSB $74 D$ & $96 Q 3$ & 0.061200 & 0.061200 & MGL & EPA6010A \\
\hline MSB $82 D$ & $96 Q 3$ & -0.037000 & -0.037000 & MGL. & EPA6010A \\
\hline MSB $82 D$ & 9603 & 0.004220 & 0.004220 & MGL & EPA6010A \\
\hline
\end{tabular}




\begin{tabular}{|c|c|c|c|c|}
\hline CUTOT & -0.004000 & 3690110.690000 & 431322.430000 & \\
\hline CYN & -0.010000 & 3690110.690000 & 431322.430000 & \\
\hline F & -0.100000 & 3690110.690000 & 431322.430000 & \\
\hline HGTOT & -0.000200 & 3690110.690000 & 431322.430000 & \\
\hline MNTOT & 0.000520 & 3690110.690000 & 431322.430000 & \\
\hline NATOT & -0.100000 & 3690110.690000 & 431322.430000 & \\
\hline NITOT & -0.010000 & 3690110.690000 & 431322.430000 & \\
\hline PBTOT & -0.005000 & 3690110.690000 & 431322.430000 & \\
\hline SETOT & 0.001530 & 3690110.690000 & 431322.430000 & \\
\hline 504 & & 3590110.690000 & 431322.430000 & \\
\hline TOTRAD & & 3690110.690000 & 431322.430000 & \\
\hline TPO4 & -0.050000 & 3690110.690000 & 431322.430000 & \\
\hline UTOT & -0.020000 & 3690110.690000 & 431322.430000 & \\
\hline ZNTOT & 0.003060 & 3690110.690000 & 431322.430000 & \\
\hline ALTOT & 0.014600 & 3687293.320000 & 430559.870000 & \\
\hline BATOT & -0.003000 & 3687293.320000 & 430559.870000 & \\
\hline $\mathrm{CL}$ & -0.250000 & 3687293.320000 & 430559.870000 & \\
\hline COTOT & -0.004000 & 3687293.320000 & 430559.870000 & \\
\hline CRTOT & -0.004000 & 3687293.320000 & 430559.870000 & \\
\hline CUTOT & 0.000638 & 3687293.320000 & 430559.870000 & \\
\hline $\operatorname{CYN}$ & -0.010000 & 3687293.320000 & 430559.870000 & \\
\hline$F$ & -0.100000 & 3687293.320000 & 430559.870000 & \\
\hline HGTOT & -0.000200 & 3687293.320000 & 430559.870000 & \\
\hline MNTOT & 0.000105 & 3687293.320000 & 430559.870000 & \\
\hline NATOT & -0.100000 & 3687293.320000 & 430559.870000 & \\
\hline NITOT & -0.010000 & 3687293.320000 & 430559.870000 & \\
\hline PBTOT & -0.005000 & 3687293.320000 & 430559.870000 & \\
\hline SETOT & -0.005000 & 3687293.320000 & 430559.870000 & \\
\hline SO4 & -1.000000 & 3687293.320000 & 430559.870000 & \\
\hline TOTRAD & -0.200000 & 3687293.320000 & 430559.870000 & \\
\hline TPO4 & -0.050000 & 3687293.320000 & 430559.870000 & \\
\hline UTOT & -0.020000 & 3687293.320000 & 430559.870000 & \\
\hline ZNTOT & -0.005000 & 3687293.320000 & 430559.870000 & \\
\hline ALTOT & 0.026900 & 3687635.010000 & 432373.650000 & \\
\hline BATOT & 0.000662 & 3687635.010000 & 432373.650000 & \\
\hline $\mathrm{CL}$ & -0.250000 & 3687635.010000 & 432373.650000 & \\
\hline СОTOT & 0.001100 & 3687635.010000 & 432373.650000 & \\
\hline CRTOT & 0.002440 & 3687635.010000 & 432373.650000 & \\
\hline CUTOT & 0.001070 & 3687635.010000 & 432373.650000 & \\
\hline CYN & -0.010000 & 3687635.010000 & 432373.650000 & \\
\hline $\mathbf{F}$ & -0.100000 & 3687635.010000 & 432373.650000 & \\
\hline HGTOT & -0.000200 & 3687635.010000 & 432373.650000 & \\
\hline MNTOT & 0.000541 & 3687635.010000 & 432373.650000 & \\
\hline NATOT & 0.045400 & 3687635.010000 & 432373.650000 & \\
\hline NITOT & -0.010000 & 3687635.010000 & 432373.650000 & \\
\hline PBTOT & -0.005000 & 3687635.010000 & 432373.650000 & \\
\hline SETOT & -0.005000 & 3687635.010000 & 432373.650000 & \\
\hline $\mathrm{SO4}$ & -1.000000 & 3687635.010000 & 432373.650000 & \\
\hline TOTRAD & 0.000000 & 3687635.010000 & 432373.650000 & \\
\hline TPO4 & -0.050000 & 3687635.010000 & 432373.650000 & \\
\hline UTOT & -0.020000 & 3687635.010000 & 432373.650000 & \\
\hline ZNTOT & -0.005000 & 3687635.010000 & 432373.650000 & \\
\hline ALTOT & 0.026900 & 3689950.530000 & 431240.240000 & \\
\hline BATOT & 0.000662 & 3689950.530000 & 431240.240000 & \\
\hline
\end{tabular}


WSRC-TR-00347

November, 1997

Appendix II-85

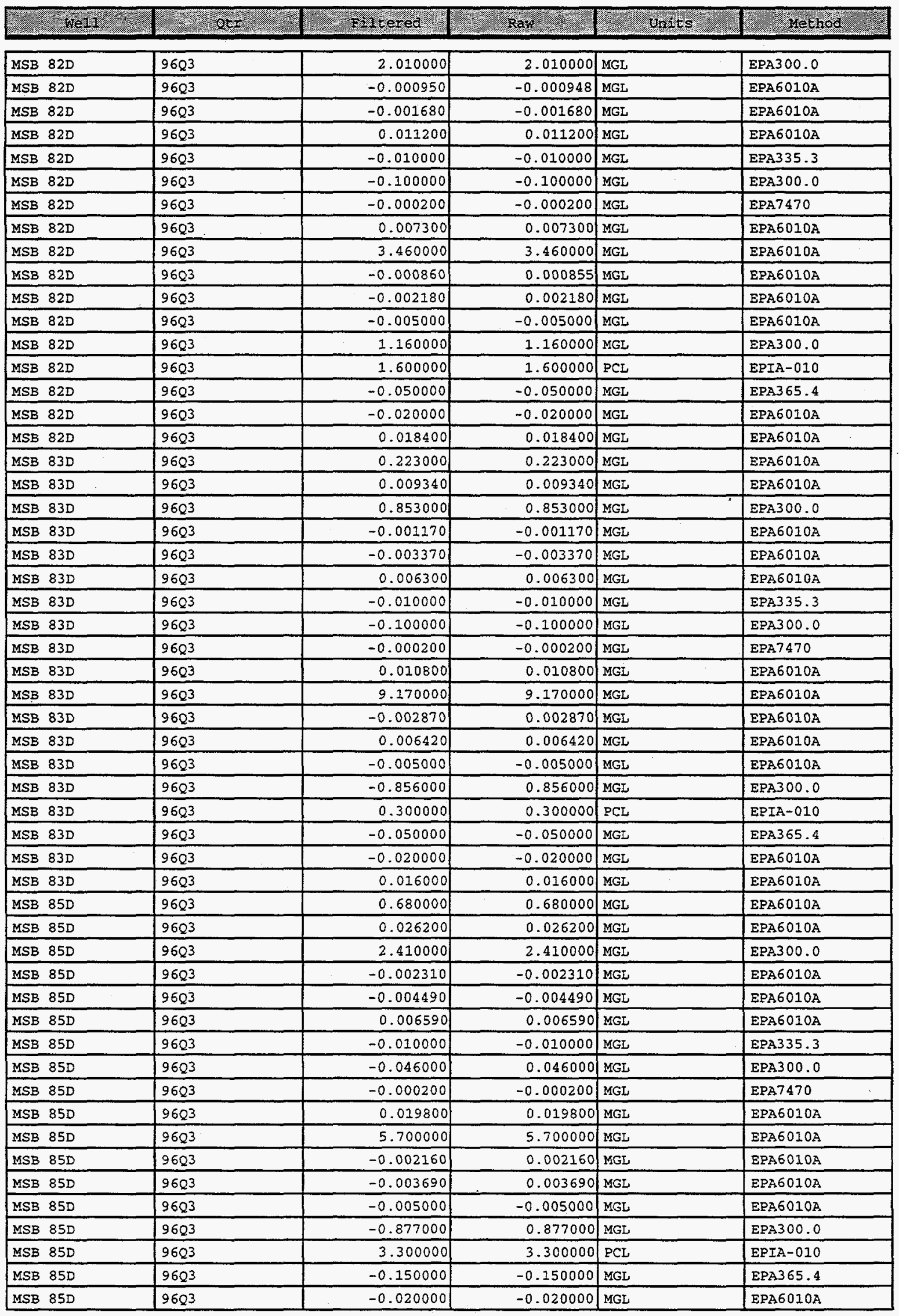


WSRC-TR-00347

November, 1997

Appendix II-86

\begin{tabular}{|c|c|c|c|c|}
\hline 10.0 & Biant & 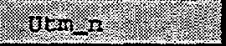 & 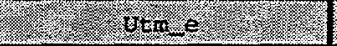 & (2) 111.10 \\
\hline $\mathrm{CL}$ & & 3689950.530000 & 431240.240000 & \\
\hline СОTOT & 0.001100 & 3689950.530000 & 431240.240000 & \\
\hline CRTOT & 0.002440 & 3689950.530000 & 431240.240000 & \\
\hline CUTOT & 0.001070 & 3689950.530000 & 431240.240000 & \\
\hline CYN & -0.010000 & 3689950.530000 & 431240.240000 & \\
\hline$F$ & -0.100000 & 3689950.530000 & 431240.240000 & \\
\hline HGTOT & -0.000200 & 3689950.530000 & 431240.240000 & \\
\hline MNTOT & 0.000541 & 3689950.530000 & 431240.240000 & \\
\hline NATOT & 0.045400 & 3689950.530000 & 431240.240000 & \\
\hline NITOT & -0.010000 & 3689950.530000 & 431240.240000 & \\
\hline PBTOT & -0.005000 & 3689950.530000 & 431240.240000 & \\
\hline SETOT & -0.005000 & 3689950.530000 & 431240.240000 & \\
\hline SO4 & & 3689950.530000 & 431240.240000 & \\
\hline TOTRAD & & 3689950.530000 & 431240.240000 & \\
\hline TPO4 & -0.050000 & 3689950.530000 & 431240.240000 & \\
\hline UTOT & -0.020000 & 3689950.530000 & 431240.240000 & \\
\hline ZNTOT & -0.005000 & 3689950.530000 & 431240.240000 & \\
\hline ALTOT & 0.026900 & 3690254.210000 & 431191.280000 & \\
\hline BATOT & 0.000662 & 3690254.210000 & 431191.280000 & \\
\hline $\mathrm{CL}$ & -0.250000 & 3690254.210000 & 431191.280000 & \\
\hline COTOT & 0.001100 & 3690254.210000 & 431191.280000 & \\
\hline CRTOT & 0.002440 & 3690254.210000 & 431191.280000 & \\
\hline CUTOT & 0.001070 & 3690254.210000 & 431191.280000 & \\
\hline $\mathrm{CYN}$ & -0.010000 & 3690254.210000 & 431191.280000 & \\
\hline F & -0.100000 & 3690254.210000 & 431191.280000 & \\
\hline HGTOT & -0.000200 & 3690254.210000 & 431191.280000 & \\
\hline MNTOT & 0.000541 & 3690254.210000 & 431191.280000 & \\
\hline NATOT & 0.045400 & 3690254.210000 & 431191.280000 & \\
\hline NITOT & -0.010000 & 3690254.210000 & 431191.280000 & \\
\hline PBTOT & -0.005000 & 3690254.210000 & 431191.280000 & \\
\hline SETOT & -0.005000 & 3690254.210000 & 431191.280000 & \\
\hline SO4 & -1.000000 & 3690254.210000 & 431191.280000 & \\
\hline TOTRAD & 0.000000 & 3690254.210000 & 431191.280000 & \\
\hline TPO4 & -0.050000 & 3690254.210000 & 431191.280000 & \\
\hline UTOT & -0.020000 & 3690254.210000 & 431191.280000 & \\
\hline ZNTOT & -0.005000 & 3690254.210000 & 431191.280000 & \\
\hline ALTOT & 0.026900 & 3690236.160000 & 431474.870000 & \\
\hline BATOT & 0.000662 & 3690236.160000 & 431474.870000 & \\
\hline CL & & 3690236.160000 & 431474.870000 & \\
\hline COTOT & 0.001100 & 3690236.160000 & 431474.870000 & \\
\hline CRTOT & 0.002440 & 3690236.160000 & 431474.870000 & \\
\hline CUTOT & 0.001070 & 3690236.160000 & 431474.870000 & \\
\hline CYN & -0.010000 & 3690236.160000 & 431474.870000 & \\
\hline $\mathrm{F}$ & -0.100000 & 3690236.160000 & 431474.870000 & \\
\hline HGTOT & -0.000200 & 3690236.160000 & 431474.870000 & \\
\hline MNTOT & 0.000541 & 3690236.160000 & 431474.870000 & \\
\hline NATOT & 0.045400 & 3690236.160000 & 431474.870000 & \\
\hline NITOT & -0.010000 & 3690236.160000 & 431474.870000 & \\
\hline PBTOT & -0.005000 & 3690236.160000 & 431474.870000 & \\
\hline SETOT & -0.005000 & 3690236.160000 & 431474.870000 & \\
\hline SO 4 & & 3690236.160000 & 431434.870000 & \\
\hline TOTRAD & 0.000000 & 3690236.160000 & 431474.870000 & \\
\hline TPO4 & 0.040000 & 3690236.160000 & 431474.870000 & \\
\hline UTOT & -0.020000 & 3690236.160000 & 431474.870000 & \\
\hline
\end{tabular}


Appendix 1I-87

20.2 neal

\begin{tabular}{|l|l|l|l|l|l|}
\hline MSB 85D & 9603 & 0.033300 & 0.033300 & MGL & EPA6010A \\
\hline
\end{tabular}


2. Besig

\begin{tabular}{|l|r|r|r|}
\hline ZNTOT & -0.005000 & 3690236.160000 & 431474.870000 \\
\hline
\end{tabular} 


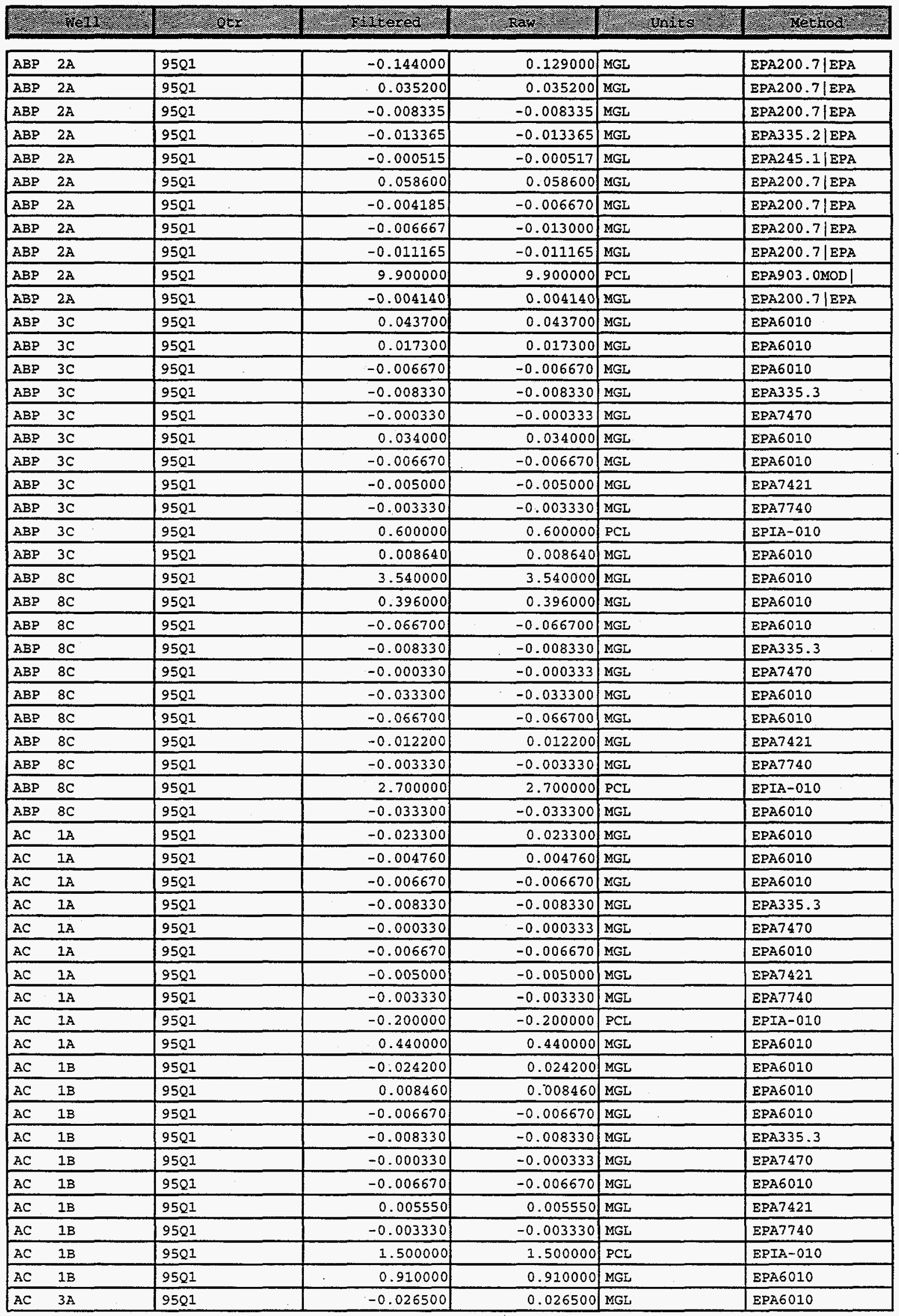




\begin{tabular}{|c|c|c|c|c|}
\hline N- bosig & 81ank & 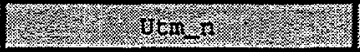 & ment & 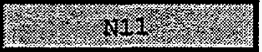 \\
\hline ALTOT & -0.0 .67900 & 3686146.040000 & 431063.900000 & 2.000000 \\
\hline BATOT & -0.006200 & 3686146.040000 & 431063.900000 & 2.000000 \\
\hline CRTOT & -0.008335 & 3686146.040000 & 431063.900000 & 2.000000 \\
\hline CYN & -0.013365 & 3686146.040000 & 431063.900000 & 2.000000 \\
\hline HGTOT & -0.005130 & 3686146.040000 & 431063.900000 & 2.000000 \\
\hline MNTOT & -0.004515 & 3686146.040000 & 431063.900000 & 2.000000 \\
\hline NITOT & -0.006035 & 3686146.040000 & 431063.900000 & 2.000000 \\
\hline PBTOT & 0.002033 & 3686146.040000 & 431063.900000 & 3.000000 \\
\hline SETOT & -0.011165 & 3686146.040000 & 431063.900000 & 2.000000 \\
\hline TOTRAD & -0.100000 & 3686146.040000 & 431063.900000 & 3.000000 \\
\hline ZNTOT & 0.325855 & 3686146.040000 & 431063.900000 & 3.000000 \\
\hline ALTOT & -0.033300 & 3686218.960000 & 431156.870000 & \\
\hline BATOT & -0.005000 & 3686218.960000 & 431156.870000 & \\
\hline CRTOT & -0.006670 & 3686218.960000 & 431156.870000 & \\
\hline CYN & -0.008330 & 3686218.960000 & 431156.870000 & \\
\hline HGTOT & -0.000333 & 3686218.960000 & 431156.870000 & \\
\hline MNTOT & -0.003330 & 3686218.960000 & 431156.870000 & \\
\hline NITOT & -0.006670 & 3686218.960000 & 431156.870000 & \\
\hline PBTOT & -0.005000 & 3686218.960000 & 431156.870000 & \\
\hline SETOT & -0.003330 & 3686218.960000 & 431156.870000 & \\
\hline TOTRAD & 0.000000 & 3686218.960000 & 431156.870000 & \\
\hline ZNTOT & -0.003330 & 3686218.960000 & 431156.870000 & \\
\hline ALTOT & -0.033300 & 3686141.620000 & 431010.550000 & \\
\hline BATOT & -0.005000 & 3686141.620000 & 431010.550000 & \\
\hline CRTOT & -0.006670 & 3686141.620000 & 431010.550000 & \\
\hline $\mathrm{CYN}$ & 0.003370 & 3686141.620000 & 431010.550000 & \\
\hline HGTOT & 0.000124 & 3686141.620000 & 431010.550000 & \\
\hline MNTOT & -0.003330 & 3686141.620000 & 431010.550000 & \\
\hline NITOT & -0.006670 & 3686141.620000 & 431010.550000 & \\
\hline PBTOT & 0.003000 & 3686141.620000 & 431010.550000 & \\
\hline SETOT & 0.002000 & 3686141.620000 & 431010.550000 & \\
\hline TOTRAD & -0.100000 & 3686141.620000 & 431010.550000 & \\
\hline ZNTOT & -0.005000 & 3686141.620000 & 431010.550000 & \\
\hline ALTOT & 0.020000 & 3687804.560000 & 429148.060000 & \\
\hline BATOT & -0.005000 & 3687804.560000 & 429148.060000 & \\
\hline CRTOT & 0.004000 & 3687804.560000 & 429148.060000 & \\
\hline $\mathrm{CYN}$ & -0.008330 & 3687804.560000 & 429148.060000 & \\
\hline HGTOT & -0.000333 & 3687804.560000 & 429148.060000 & \\
\hline NITOT & -0.006670 & 3687804.560000 & 429148.060000 & \\
\hline PBTOT & -0.025000 & 3687804.560000 & 429148.060000 & \\
\hline SETOT & -0.003330 & 3687804.560000 & 429148.060000 & \\
\hline TOTRAD & & 3687804.560000 & 429148.060000 & \\
\hline ZNTOT & -0.003330 & 3687804.560000 & 429148.060000 & \\
\hline ALTOT & 0.020000 & 3687806.120000 & 429151.340000 & \\
\hline BATOT & -0.005000 & 3687806.120000 & 429151.340000 & \\
\hline CRTOT & 0.004000 & 3687806.120000 & 429151.340000 & \\
\hline $\mathrm{CYN}$ & -0.008330 & 3687806.120000 & 429151.340000 & \\
\hline HGTOT & -0.000333 & 3687806.120000 & 429151.340000 & \\
\hline NITOT & -0.006670 & 3687806.120000 & 429151.340000 & \\
\hline PBTOT & -0.005000 & 3687806.120000 & 429151.340000 & \\
\hline SETOT & -0.003330 & 3687806.120000 & 429151.340000 & \\
\hline TOTRAD & & 3687806.120000 & 429151.340000 & \\
\hline ZNTOT & 0.002000 & 3687806.120000 & 429151.340000 & \\
\hline ALTOT & 0.020000 & 3686582.010000 & 429993.130000 & \\
\hline
\end{tabular}


WSRC-TR-00347

November, 1997

Appendix II-91

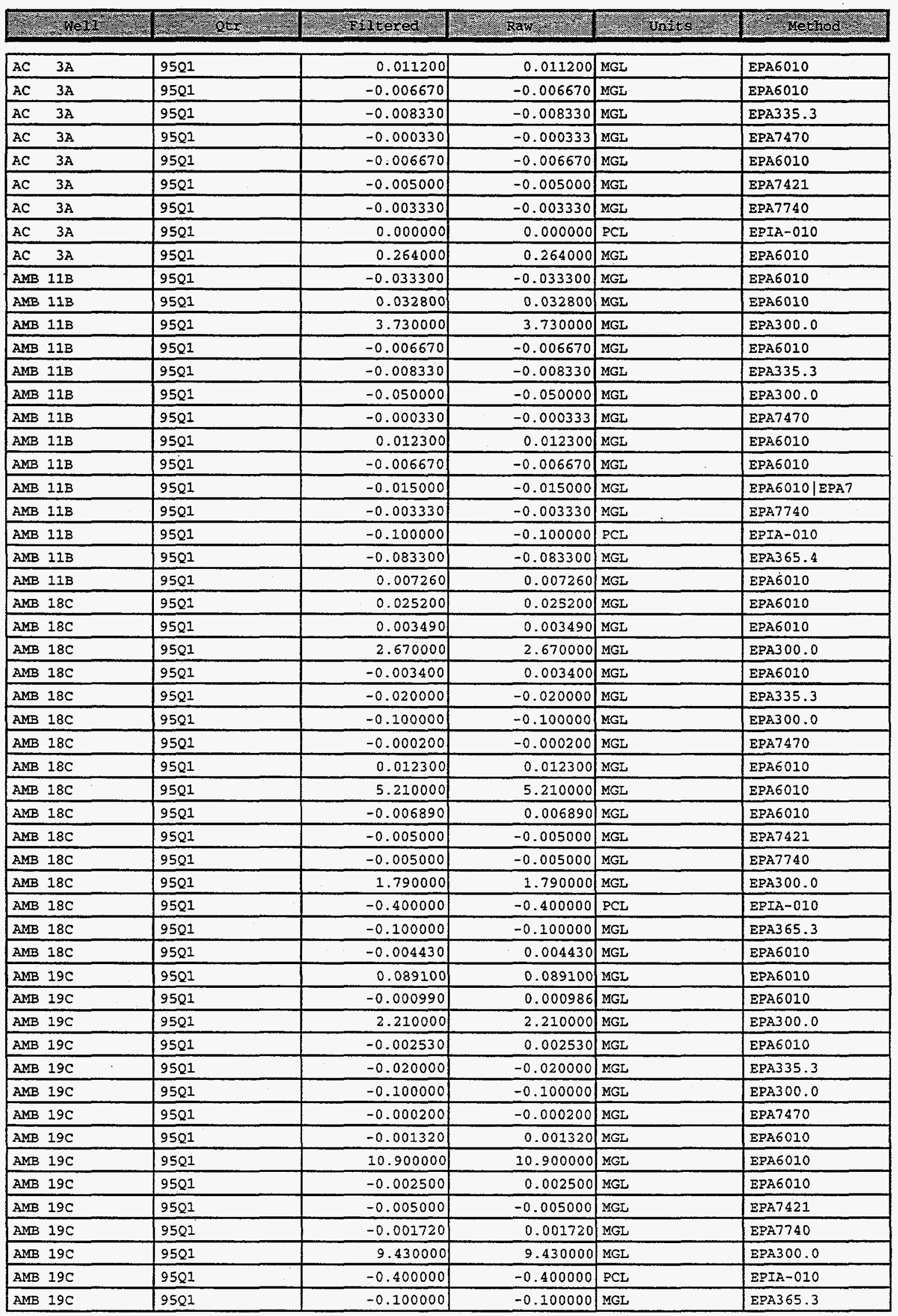




\begin{tabular}{|c|c|c|c|c|}
\hline BATOT & -0.005000 & 3686582.010000 & 429993.130000 & \\
\hline CRTOT & 0.004000 & 3686582.010000 & 429993.130000 & \\
\hline CYN & -0.008330 & 3686582.010000 & 429993.130000 & \\
\hline HGTOT & -0.000333 & 3686582.010000 & 429993.130000 & \\
\hline NITOT & -0.006670 & 3686582.010000 & 429993.130000 & \\
\hline PBTOT & -0.005000 & 3686582.010000 & 429993.130000 & \\
\hline SETOT & -0.003330 & 3686582.010000 & 429993.130000 & \\
\hline TOTRAD & & 3686582.010000 & 429993.130000 & \\
\hline ZNTOT & 0.002000 & 3686582.010000 & 429993.130000 & \\
\hline ALTOT & -0.033300 & 3688872.760000 & 432019.080000 & \\
\hline BATOT & -0.005000 & 3688872.760000 & 432019.080000 & \\
\hline CL & -0.417000 & 3688872.760000 & 432019.080000 & \\
\hline CRTOT & -0.006670 & 3688872.760000 & 432019.080000 & \\
\hline $\mathrm{CYN}$ & -0.008330 & 3688872.760000 & 432019.080000 & \\
\hline F & -0.050000 & 3688872.760000 & 432019.080000 & \\
\hline HGTOT & -0.000333 & 3688872.760000 & 432019.080000 & \\
\hline MNTOT & -0.003330 & 3688872.760000 & 432019.080000 & \\
\hline NITOT & -0.006670 & 3688872.760000 & 432019.080000 & \\
\hline PBTOT & -0.005000 & 3688872.760000 & 432019.080000 & 2.000000 \\
\hline SETOT & -0.003330 & 3688872.760000 & 432019.080000 & \\
\hline TOTRAD & -0.100000 & 3688872.760000 & 432019.080000 & \\
\hline TPO4 & 0.043000 & 3688872.760000 & 432019.080000 & \\
\hline ZNTOT & -0.003330 & 3688872.760000 & 432019.080000 & \\
\hline ALTOT & 0.004650 & 3688989.750000 & 431750.480000 & \\
\hline BATOT & 0.000258 & 3688989.750000 & 431750.480000 & \\
\hline CL & -0.250000 & 3688989.750000 & 431750.480000 & \\
\hline CRTOT & -0.004000 & 3688989.750000 & 431750.480000 & \\
\hline $\mathrm{CYN}$ & -0.020000 & 3688989.750000 & 431750.480000 & \\
\hline$F$ & -0.100000 & 3688989.750000 & 431750.480000 & \\
\hline HGTOT & -0.000200 & 3688989.750000 & 431750.480000 & \\
\hline MNTOT & 0.000240 & 3688989.750000 & 431750.480000 & \\
\hline NATOT & -0.100000 & 3688989.750000 & 431750.480000 & \\
\hline NITOT & -0.010000 & 3688989.750000 & 431750.480000 & \\
\hline PBTOT & -0.005000 & 3688989.750000 & 431750.480000 & \\
\hline SETOT & 0.001340 & 3688989.750000 & 431750.480000 & \\
\hline so4 & -1.000000 & 3688989.750000 & 431750.480000 & \\
\hline TOTRAD & & 3688989.750000 & 431750.480000 & \\
\hline TPO4 & -0.100000 & 3688989.750000 & 431750.480000 & \\
\hline ZNTOT & -0.005000 & 3688989.750000 & 433750.480000 & \\
\hline ALTOT & 0.004650 & 3688745.690000 & 431954.860000 & \\
\hline BATOT & 0.000258 & 3688745.690000 & 431954.860000 & \\
\hline CL & -0.250000 & 3688745.690000 & 431954.860000 & \\
\hline CRTOT & -0.004000 & 3688745.690000 & 431954.860000 & \\
\hline CYN & -0.020000 & 3688745.690000 & 431954.860000 & \\
\hline F & -0.100000 & 3688745.690000 & 431954.860000 & \\
\hline HGTOT & -0.000200 & 3688745.690000 & 431954.860000 & \\
\hline MNTOT & -0.002000 & 3688745.690000 & 431954.860000 & \\
\hline NATOT & -0.100000 & 3688745.690000 & 431954.860000 & \\
\hline NITOT & -0.010000 & 3688745.690000 & 431954.860000 & \\
\hline PBTOT & -0.005000 & 3688745.690000 & 431954.860000 & \\
\hline SETOT & 0.001340 & 3688745.690000 & 431954.860000 & \\
\hline 504 & -1.000000 & 3688745.690000 & 431954.860000 & \\
\hline TOTRAD & & 3688745.690000 & 431954.860000 & \\
\hline TPO4 & -0.100000 & 3688745.690000 & 431954.860000 & \\
\hline
\end{tabular}




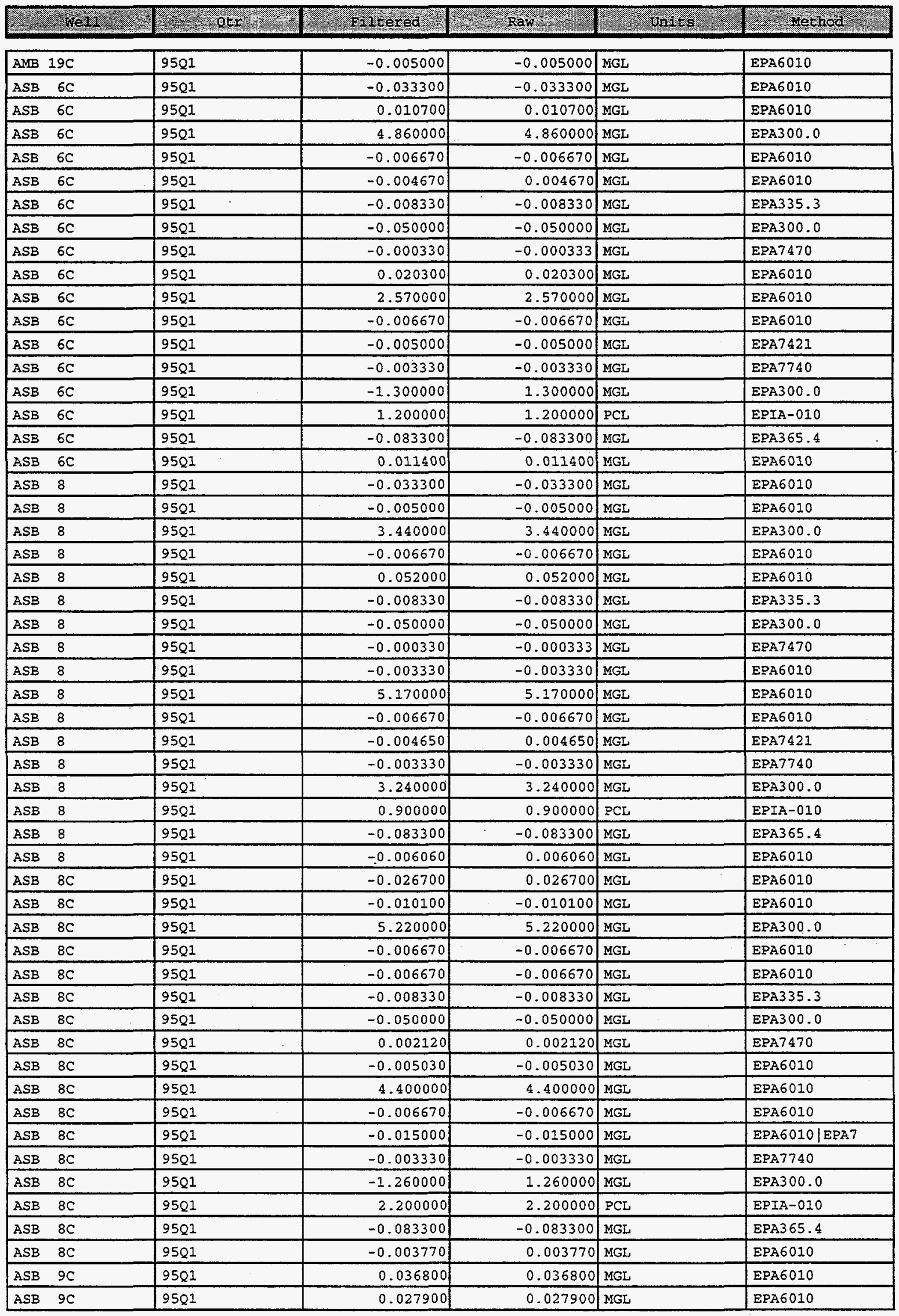




\begin{tabular}{|c|c|c|c|c|}
\hline ZNTOT & -0.005000 & 3688745.690000 & 431954.860000 & \\
\hline ALTOT & 0.020000 & 3689641.050000 & 431737.370000 & \\
\hline BATOT & -0.005000 & 3689641.050000 & 431737.370000 & \\
\hline CL & -0.417000 & 3689641.050000 & 431737.370000 & \\
\hline CRTOT & 0.004000 & 3689641.050000 & 431737.370000 & \\
\hline CUTOT & 0.004000 & 3689641.050000 & 431737.370000 & \\
\hline $\mathrm{CYN}$ & -0.008330 & 3689641.050000 & 431737.370000 & \\
\hline$F$ & -0.050000 & 3689641.050000 & 431737.370000 & \\
\hline HGTOT & -0.000333 & 3689641.050000 & 431737.370000 & \\
\hline MNTOT & -0.003330 & 3689641.050000 & 431737.370000 & \\
\hline NATOT & 0.010000 & 3689641.050000 & 431737.370000 & \\
\hline NITOT & -0.006670 & 3689641.050000 & 431737.370000 & \\
\hline PBTOT & -0.005000 & 3689641.050000 & 431737.370000 & \\
\hline SETOT & -0.003330 & 3689641.050000 & 431737.370000 & \\
\hline SO4 & -1.670000 & 3689641.050000 & 431737.370000 & \\
\hline TOTRAD & 0.000000 & 3689641.050000 & 431737.370000 & \\
\hline TPO4 & -0.083300 & 3689641.050000 & 431737.370000 & \\
\hline ZNTOT & 0.002000 & 3689641.050000 & 431737.370000 & \\
\hline ALTOT & 0.020000 & 3689886.100000 & 431740.160000 & \\
\hline BATOT & -0.005000 & 3689886.100000 & 431740.160000 & \\
\hline $\mathrm{CL}$ & -0.417000 & 3689886.100000 & 431740.160000 & \\
\hline CRTOT & 0.004000 & 3689886.100000 & 431740.160000 & \\
\hline CUTOT & -0.006670 & 3689886.100000 & 431740.160000 & \\
\hline CYN & -0.008330 & 3689886.100000 & 431740.160000 & \\
\hline$E$ & -0.050000 & 3689886.100000 & 431740.160000 & \\
\hline HGTOT & -0.000333 & 3689886.100000 & 431740.160000 & \\
\hline MNTOT & -0.003330 & 3689886.100000 & 431740.160000 & \\
\hline NATOT & 0.010000 & 3689886.100000 & 431740.160000 & \\
\hline NITOT & -0.006630 & 3689886.100000 & 431740.160000 & \\
\hline PBTOT & 0.003000 & 3689886.100000 & 431740.160000 & \\
\hline SETOT & 0.002000 & 3689886.100000 & 431740.160000 & \\
\hline SO4 & -1.670000 & 3689886.100000 & 431740.160000 & \\
\hline TOTRAD & 0.000000 & 3689886.100000 & 431740.160000 & \\
\hline TPO4 & -0.083300 & 3689886.100000 & 431740.160000 & \\
\hline ZNTOT & 0.002000 & 3689886.100000 & 431740.160000 & \\
\hline ALTOT & 0.020000 & 3689873.020000 & 431736.270000 & \\
\hline BATOT & -0.005000 & 3689873.020000 & 431736.270000 & 2.000000 \\
\hline$C L$ & -0.417000 & 3689873.020000 & 431736.270000 & \\
\hline CRTOT & 0.004000 & 3689873.020000 & 431736.270000 & \\
\hline CUTOT & 0.004000 & 3689873.020000 & 431736.270000 & \\
\hline CYN & -0.008330 & 3689873.020000 & 431736.270000 & \\
\hline F & -0.050000 & 3689873.020000 & 431736.270000 & \\
\hline HGTOT & -0.000333 & 3689873.020000 & 431736.270000 & \\
\hline MNTOT & -0.003330 & 3689873.020000 & 431736.270000 & 2.000000 \\
\hline NATOT & 0.010000 & 3689873.020000 & 431736.270000 & \\
\hline NITOT & -0.006670 & 3689873.020000 & 431736.270000 & 2.000000 \\
\hline PBTOT & -0.011300 & 3689873.020000 & 431736.270000 & 2.000000 \\
\hline SETOT & -0.003330 & 3689873.020000 & 431736.270000 & \\
\hline SO4 & -1.670000 & 3689873.020000 & 431736.270000 & \\
\hline TOTRAD & -0.200000 & 3689873.020000 & 431736.270000 & \\
\hline TPO4 & -0.083300 & 3689873.020000 & 431736.270000 & \\
\hline ZNTOT & 0.002000 & 3689873.020000 & 431736.270000 & \\
\hline ALTOT & -0.033300 & 3689630.230000 & 432327.610000 & \\
\hline BATOT & -0.005000 & 3689630.230000 & 432327.610000 & \\
\hline
\end{tabular}




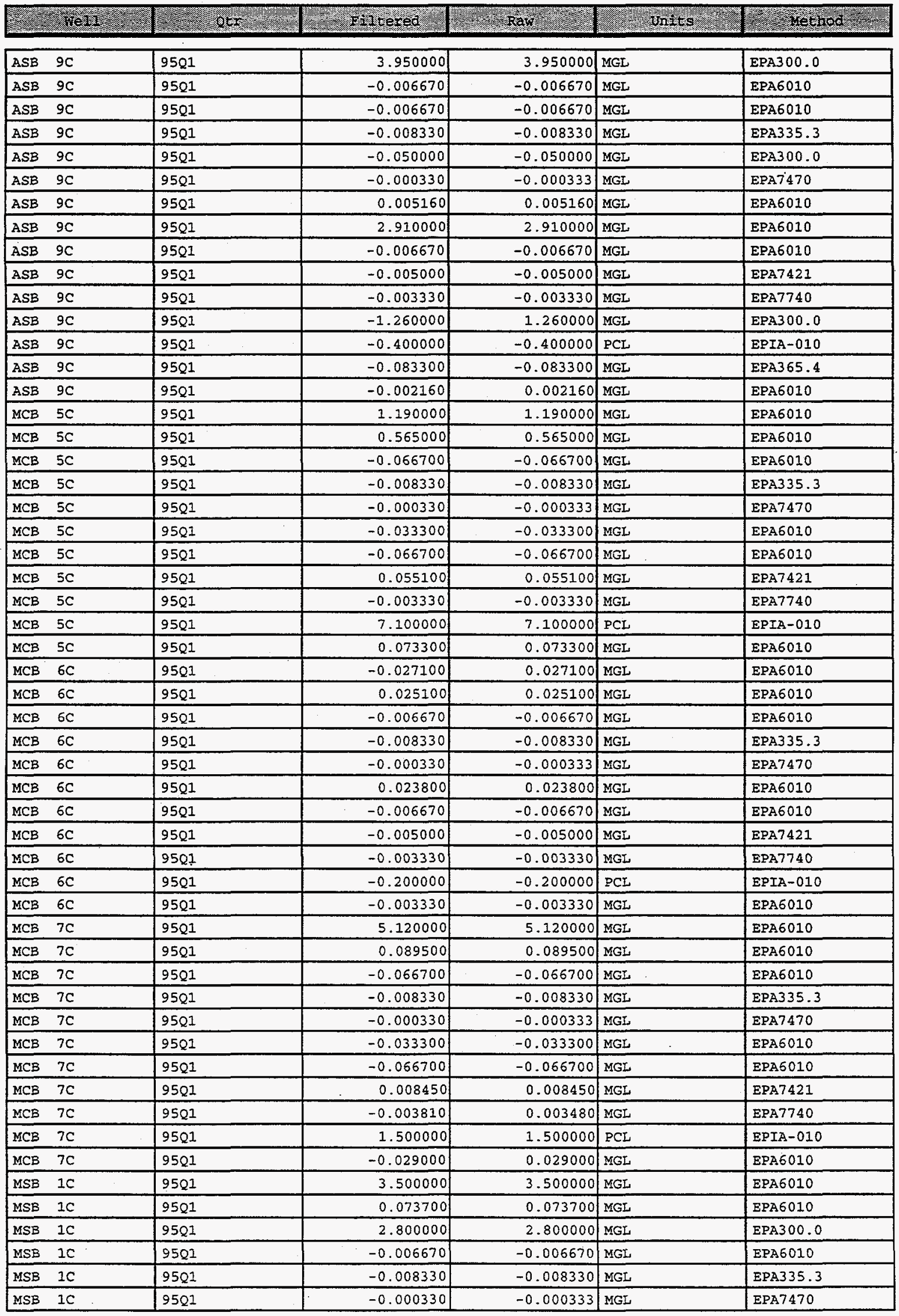




\begin{tabular}{|c|c|c|c|c|}
\hline 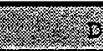 & 10 & 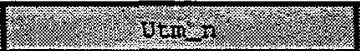 & (2) & 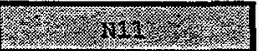 \\
\hline CL & -0.417000 & 3689630.230000 & 432327.610000 & \\
\hline CRTOT & -0.006670 & 3689630.230000 & 432327.610000 & \\
\hline CUTOT & -0.006670 & 3689630.230000 & 432327.610000 & \\
\hline $\mathrm{CYN}$ & -0.008330 & 3689630.230000 & 432327.610000 & 2.000000 \\
\hline F & -0.050000 & 3689630.230000 & 432327.610000 & \\
\hline HGTOT & -0.000333 & 3689630.230000 & 432327.610000 & \\
\hline MNTOT & -0.003330 & 3689630.230000 & 432327.610000 & \\
\hline NATOT & -0.016700 & 3689630.230000 & 432327.610000 & \\
\hline NITOT & -0.006670 & 3689630.230000 & 432327.610000 & \\
\hline PBTOT & -0.005000 & 3689630.230000 & 432327.610000 & \\
\hline SETOT & -0.003330 & 3689630.230000 & 432327.610000 & \\
\hline SO4 & -1.670000 & 3689630.230000 & 432327.610000 & \\
\hline TOTRAD & -0.200000 & 3689630.230000 & 432327.610000 & \\
\hline TPO4 & -0.083300 & 3689630.230000 & 432327.610000 & \\
\hline ZNTOT & -0.003330 & 3689630.230000 & 432327.610000 & \\
\hline ALTOT & -0.033300 & 3686168.800000 & 431327.740000 & \\
\hline BATOT & -0.005000 & 3686168.800000 & 431327.740000 & \\
\hline CRTOT & -0.006670 & 3686168.800000 & 431327.740000 & \\
\hline CYN & 0.003370 & 3686168.800000 & 431327.740000 & \\
\hline HGTOT & 0.000124 & 3686168.800000 & 431327.740000 & \\
\hline MNTOT & -0.003330 & 3686168.800000 & 431327.740000 & \\
\hline NITOT & -0.006670 & 3686168.800000 & 431327.740000 & \\
\hline PBTOT & 0.003000 & 3686168.800000 & 431327.740000 & \\
\hline SETOT & 0.002000 & 3686168.800000 & 431327.740000 & \\
\hline TOTRAD & -0.100000 & 3686168.800000 & 431327.740000 & \\
\hline ZNTOT & -0.003330 & 3686168.800000 & 431327.740000 & \\
\hline ALTOT & -0.033300 & 3686254.790000 & 431395.130000 & \\
\hline BATOT & -0.005000 & 3686254.790000 & 431395.130000 & \\
\hline CRTOT & -0.006670 & 3686254.790000 & 431395.130000 & \\
\hline CYN & -0.008330 & 3686254.790000 & 431395.130000 & \\
\hline HGTOT & -0.000333 & 3686254.790000 & 431395.130000 & \\
\hline MNTOT & -0.003330 & 3686254.790000 & 431395.130000 & \\
\hline NITOT & -0.006670 & 3686254.790000 & 431395.130000 & \\
\hline PBTOT & -0.005000 & 3686254.790000 & 431395.130000 & \\
\hline SETOT & -0.003330 & 3686254.790000 & 431395.130000 & \\
\hline TOTRAD & -0.100000 & 3686254.790000 & 431395.130000 & \\
\hline ZNTOT & -0.003330 & 3686254.790000 & 431395.130000 & \\
\hline ALTOT & -0.033300 & 3686127.040000 & 431361.080000 & \\
\hline BATOT & -0.005000 & 3686127.040000 & 431361.080000 & \\
\hline CRTOT & -0.006670 & 3686127.040000 & 431361.080000 & \\
\hline CYN & 0.003370 & 3686127.040000 & 431361.080000 & \\
\hline HGTOT & -0.000333 & 3686127.040000 & 431361.080000 & \\
\hline MNTOT & -0.003330 & 3686127.040000 & 431361.080000 & \\
\hline NITOT & -0.006670 & 3686127.040000 & 431361.080000 & \\
\hline PBTOT & 0.001070 & 3686127.040000 & 431361.080000 & \\
\hline SETOT & -0.003330 & 3686127.040000 & 431361.080000 & 2.000000 \\
\hline TOTRAD & -0.100000 & 3686127.040000 & 431361.080000 & \\
\hline ZNTOT & -0.005000 & 3686127.040000 & 431361.080000 & \\
\hline ALTOT & 0.020000 & 3687936.210000 & 431416.820000 & \\
\hline BATOT & -0.005000 & 3687936.210000 & 431416.820000 & \\
\hline CL & -0.417000 & 3687936.210000 & 431416.820000 & \\
\hline CRTOT & 0.004000 & 3687936.210000 & 431416.820000 & \\
\hline $\mathrm{CYN}$ & -0.008330 & 3687936.210000 & 431416.820000 & \\
\hline HGTOT & -0.000333 & 3687936.210000 & 431416.820000 & \\
\hline
\end{tabular}




\begin{tabular}{|c|c|c|c|c|c|c|}
\hline \multicolumn{3}{|c|}{ 20. nem.1. } & \multirow{2}{*}{\begin{tabular}{|c|}
-0.003330 \\
-0.002
\end{tabular}} & \multirow{2}{*}{\begin{tabular}{|r|}
-0.003330 \\
\end{tabular}} & \multirow{2}{*}{ 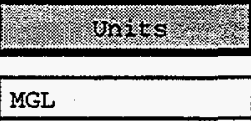 } & \multirow{2}{*}{ 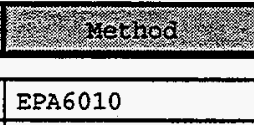 } \\
\hline MSB & $1 C$ & $95 Q 1$ & & & & \\
\hline MSB & $1 C$ & 9501 & 37.000000 & 37.000000 & MGL & EPA6010 \\
\hline MSB & 1C & $95 Q 1$ & -0.006670 & -0.006630 & MGL & EPA 6010 \\
\hline MSB & $1 C$ & 9501 & -0.005000 & -0.005000 & MGL & ERA7421 \\
\hline MSB & $1 \mathrm{c}$ & $95 Q 1$ & -0.002020 & 0.002020 & MGL & EPA7740 \\
\hline MSB & $1 C$ & 9501 & 5.170000 & 5.170000 & MGL & EPA300.0 \\
\hline MSB & $1 \mathrm{C}$ & 9501 & 2.100000 & 2.100000 & PCL & EPIA-010 \\
\hline MSB & IC & 9501 & -0.083300 & -0.083300 & MGL & EPA3 65.4 \\
\hline MSB & IC & $95 Q 1$ & -0.033300 & -0.033300 & MGL & EPA6010 \\
\hline MSB & $1 \mathrm{C}$ & $95 Q 1$ & -0.003330 & -0.003330 & MGL & EPA6010 \\
\hline MSB & $1 \mathrm{CC}$ & $95 Q 1$ & -0.042800 & 0.042800 & MGL & EPA6010 \\
\hline MSB & $1 \mathrm{CC}$ & 9501 & 0.019500 & 0.019500 & MGL & EPA 6010 \\
\hline MSB & $1 \mathrm{CC}$ & 9501 & 2.050000 & 2.050000 & MGL & EPA 300.0 \\
\hline MSB & $1 \mathrm{CC}$ & $95 Q 1$ & -0.006670 & -0.006670 & MGL & EPA6010 \\
\hline MSB & $1 \mathrm{CC}$ & $95 Q 1$ & -0.008330 & -0.008330 & MGL & EPA335.3 \\
\hline MSB & $1 \mathrm{CC}$ & $95 Q 1$ & -0.000330 & -0.000333 & MGL & EPA7470 \\
\hline MSB & $1 \mathrm{CC}$ & 95Q1 & 0.009520 & 0.009520 & MGL & EPA6010 \\
\hline MSB & $1 C C$ & $95 Q 1$ & 16.100000 & 16.100000 & MGL & EPA6010 \\
\hline MSB & $2 \mathrm{CC}$ & 9501 & -0.006670 & -0.006670 & MGL & EPA6010 \\
\hline MSB & $1 \mathrm{CC}$ & 9501 & 0.007350 & 0.007350 & MGL & EPA7421 \\
\hline MSB & $1 \mathrm{CC}$ & 9501 & -0.003330 & -0.003330 & MGL & EPA7740 \\
\hline MSB & $1 C C$ & 9501 & -1.670000 & -1.670000 & MGL & EPA300.0 \\
\hline MSB & $1 C C$ & 9501 & 2.500000 & 2.500000 & PCL & EPIA-010 \\
\hline MSB & $1 \mathrm{CC}$ & 9501 & -0.083300 & -0.083300 & MGL & EPA365.4 \\
\hline MSB & $1 C C$ & 9501 & -0.033300 & -0.033300 & MGL & EPA6010 \\
\hline MSB & $1 \mathrm{CC}$ & $95 Q 1$ & 0.030400 & 0.030400 & MGL & EPA6010 \\
\hline MSB & $2 \mathrm{C}$ & 9501 & 0.535000 & 0.535000 & MGL & EPA6010 \\
\hline MSB & $2 \mathrm{C}$ & 9501 & 0.046500 & 0.046500 & MGL & EPA6010 \\
\hline MSB & $2 C$ & 9501 & 2.000000 & 2.000000 & MGL & EPA300.0 \\
\hline MSB & $2 C$ & 9501 & 0.012700 & 0.012700 & MGL & EPA6010 \\
\hline MSB & $2 C$ & $95 Q 1$ & -0.008330 & -0.008330 & MGL & EPA335.3 \\
\hline MSB & $2 \mathrm{C}$ & 9501 & -0.000330 & -0.000333 & MGL & EPA7470 \\
\hline MSB & $2 \mathrm{C}$ & 9501 & 0.011300 & 0.011300 & MGL & EPA6010 \\
\hline MSB & $2 \mathrm{C}$ & 9501 & 63.900000 & 63.900000 & MGL & EPA6010 \\
\hline MSB & $2 \mathrm{C}$ & $95 Q 1$ & -0.006670 & -0.006670 & MGL & EPA6010 \\
\hline MSB & $2 C$ & 9501 & -0.005000 & -0.005000 & MGL & EPA7421 \\
\hline MSB & $2 \mathrm{C}$ & 9501 & -0.003330 & 0.003330 & MGL & EPA7740 \\
\hline MSB & $2 C$ & $95 Q 1$ & 2.850000 & 2.850000 & MGL & EPA300.0 \\
\hline MSB & $2 C$ & 9501 & 1.600000 & 1.600000 & PCL & EPIA-010 \\
\hline MSB & $2 c$ & 9501 & -0.083300 & -0.083300 & MGL & EPA365.4 \\
\hline MSB & $2 C$ & 9501 & -0.033300 & -0.033300 & MGL & EPA 6010 \\
\hline MSB & $2 C$ & $95 Q 1$ & -0.004140 & 0.004140 & MGL & EPA6010 \\
\hline MSB & $4 C$ & 9501 & -0.032600 & 0.032600 & MGL & EPA6010 \\
\hline MSB & $4 \mathrm{C}$ & 9501 & 0.183000 & 0.183000 & MGL & EPA6010 \\
\hline MSB & $4 \mathrm{C}$ & 9501 & 3.630000 & 3.630000 & MGL & EPA300.0 \\
\hline MSB & $4 \mathrm{C}$ & 9501 & -0.006670 & -0.006670 & MGL & EPA6010 \\
\hline MSB & $4 \mathrm{C}$ & 9501 & -0.008330 & -0.008330 & MGL & EPA335.3 \\
\hline MSB & $4 \mathrm{C}$ & 9501 & -0.000330 & -0.000333 & MGL & EPA7470 \\
\hline MSB & $4 \mathrm{C}$ & 9501 & -0.002140 & 0.002140 & MGL & EPA6010 \\
\hline MSB & $4 \mathrm{C}$ & 9501 & 23.000000 & 23.000000 & MGL & EPA6010 \\
\hline MSB & $4 \mathrm{C}$ & 9501 & -0.006670 & -0.006670 & MGL & EPA6010 \\
\hline MSB & $4 C$ & 9501 & -0.005000 & -0.005000 & MGL & EPA7421 \\
\hline MSB & $4 \mathrm{C}$ & 9501 & -0.002510 & 0.002510 & MGL & EPA7740 \\
\hline MSB & $4 \mathrm{C}$ & 9501 & 2.230000 & 2.230000 & MGL & EPA3 300.0 \\
\hline
\end{tabular}




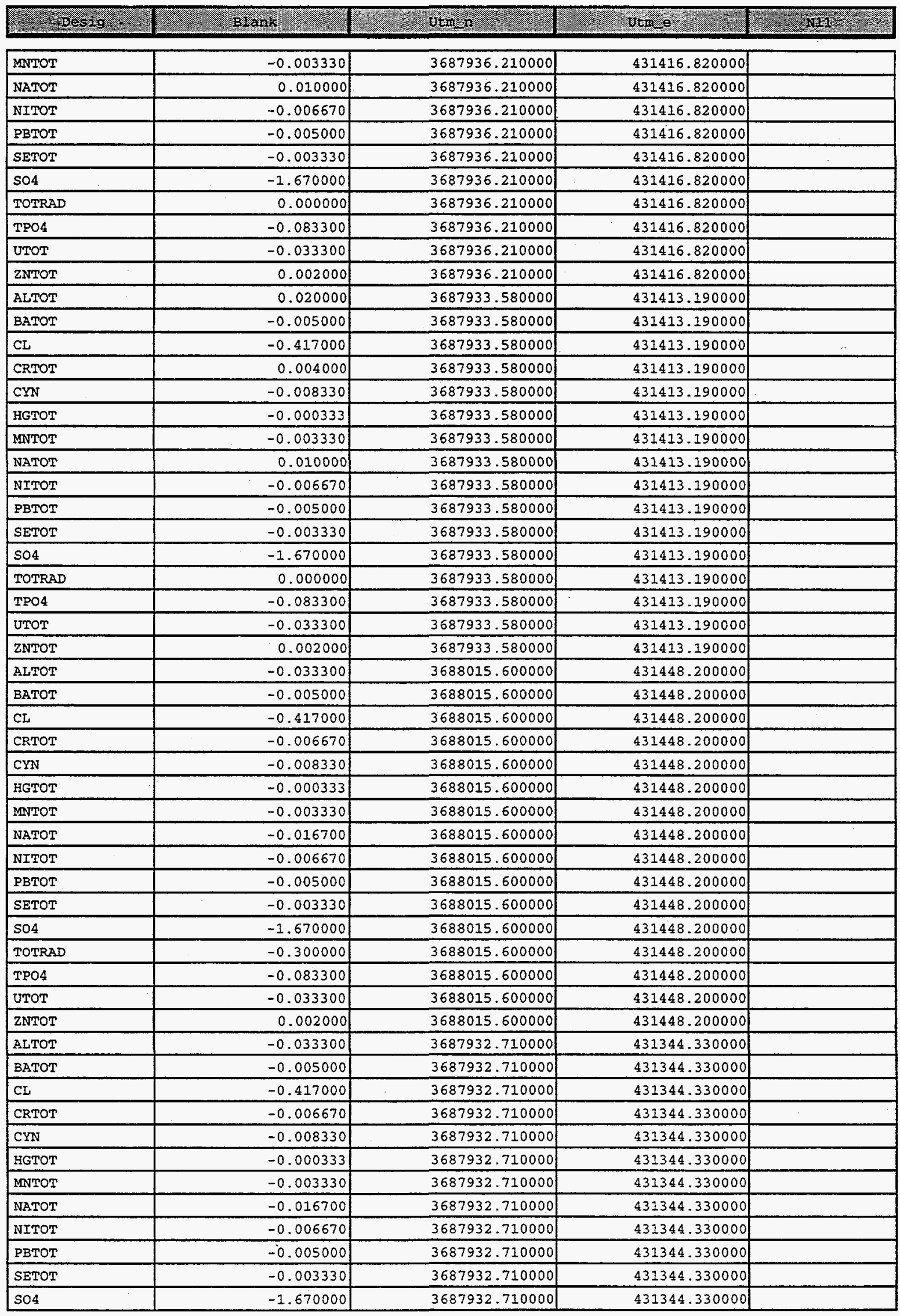




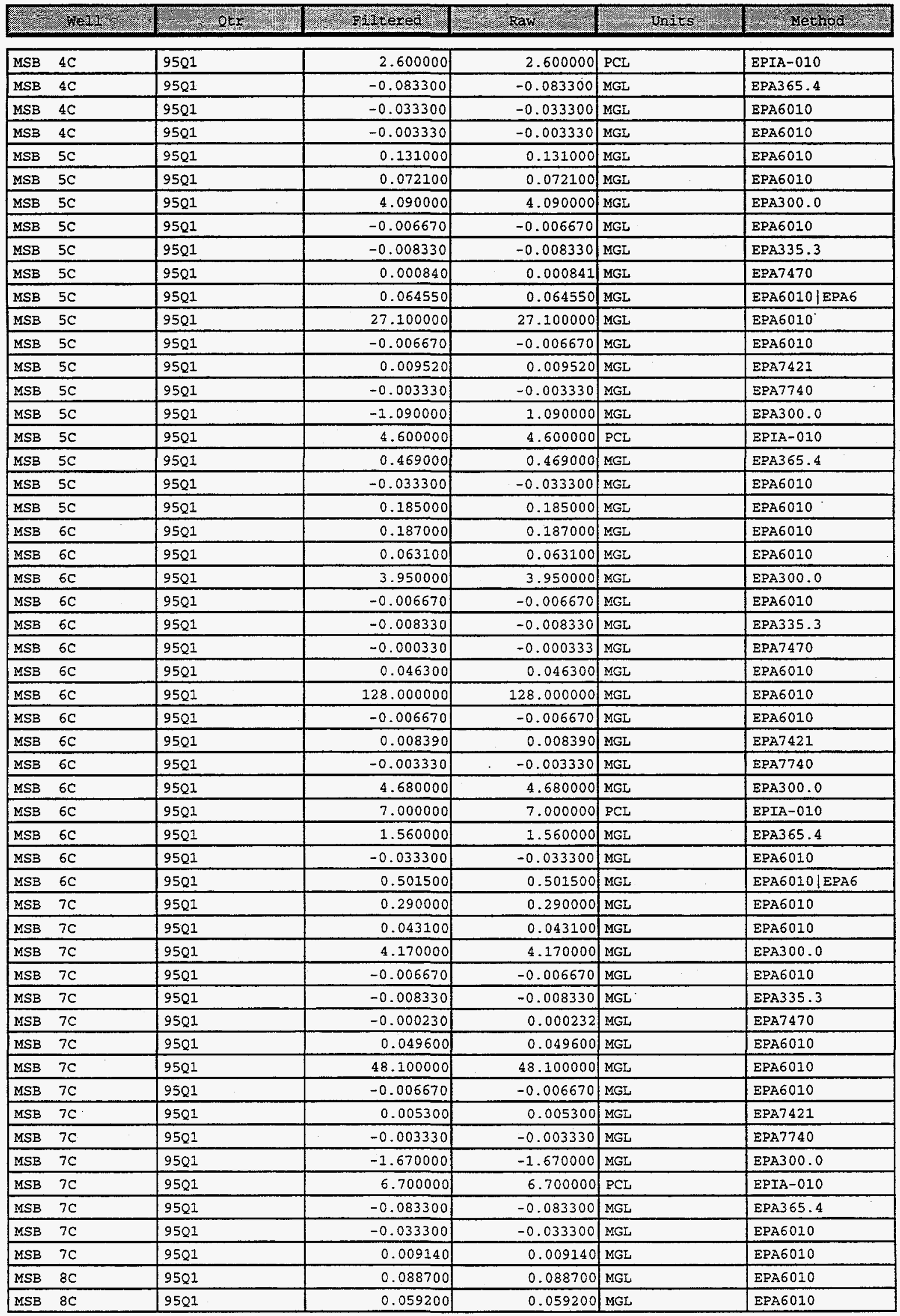




\begin{tabular}{|c|c|c|c|c|}
\hline TPO4 & -0.083300 & 3687932.710000 & 431344.330000 & \\
\hline UTOT & -0.033300 & 3687932.710000 & 431344.330000 & \\
\hline ZNTOT & -0.003330 & 3687932.710000 & 431344.330000 & \\
\hline ALTOT & -0.033300 & 3687693.290000 & 431011.690000 & \\
\hline BATOT & -0.005000 & 3687693.290000 & 431011.690000 & \\
\hline CE & -0.417000 & 3687693.290000 & 431011.690000 & \\
\hline CRTOT & -0.006670 & 3687693.290000 & 431011.690000 & \\
\hline CYN & 0.003370 & 3687693.290000 & 431011.690000 & \\
\hline HGTOT & -0.000333 & 3687693.290000 & 431011.690000 & \\
\hline MNTOT & -0.002665 & 3687693.290000 & 431011.690000 & 2.000000 \\
\hline NATOT & -0.016700 & 3687693.290000 & 431011.690000 & \\
\hline NITOT & -0.006670 & 3687693.290000 & 431011.690000 & \\
\hline PBTOT & -0.005000 & 3687693.290000 & 431011.690000 & \\
\hline SETOT & -0.003330 & 3687693.290000 & 431011.690000 & \\
\hline SO4 & -1.670000 & 3687693.290000 & 431011.690000 & \\
\hline TOTRAD & 0.000000 & 3687693.290000 & 431011.690000 & \\
\hline TPO4 & -0.083300 & 3687693.290000 & 431011.690000 & \\
\hline UTOT & -0.033300 & 3687693.290000 & 431011.690000 & \\
\hline ZNTOT & -0.003330 & 3687693.290000 & 431011.690000 & \\
\hline ALTOT & -0.033300 & 3687380.310000 & 430996.610000 & \\
\hline BATOT & -0.005000 & 3687380.310000 & 430996.610000 & \\
\hline $\mathrm{CL}$ & -0.417000 & 3687380.310000 & 430996.610000 & \\
\hline CRTOT & -0.006670 & 3687380.310000 & 430996.610000 & \\
\hline CYN & 0.003370 & 3687380.310000 & 430996.610000 & \\
\hline HGTOT & -0.000333 & 3687380.310000 & 430996.610000 & \\
\hline MNTOT & -0.003330 & 3687380.310000 & 430996.610000 & \\
\hline NATOT & -0.016700 & 3687380.310000 & 430996.610000 & \\
\hline NITOT & -0.006670 & 3687380.310000 & 430996.610000 & \\
\hline PBTOT & -0.005000 & 3687380.310000 & 430996.610000 & \\
\hline SETOT & -0.003330 & 3687380.310000 & 430996.610000 & \\
\hline $\mathrm{SO4}$ & -1.670000 & 3687380.310000 & 430996.610000 & \\
\hline TOTRAD & 0.000000 & 3687380.310000 & 430996.610000 & \\
\hline TPO4 & -0.083300 & 3687380.310000 & 430996.610000 & \\
\hline UTOT & -0.033300 & 3687380.310000 & 430996.610000 & \\
\hline ZNTOT & -0.003330 & 3687380.310000 & 430996.610000 & 2.000000 \\
\hline ALTOT & -0.033300 & 3687311.410000 & 431191.860000 & \\
\hline ВАТОT & -0.005000 & 3687311.410000 & 431191.860000 & \\
\hline CL & -0.417000 & 3687311.410000 & 431191.860000 & \\
\hline CRTOT & -0.006670 & 3687311.410000 & 431191.860000 & \\
\hline CYN & 0.003370 & 3687311.410000 & 431191.860000 & \\
\hline HGTOT & -0.000333 & 3687311.410000 & 431191.860000 & \\
\hline MNTOT & -0.003330 & 3687311.410000 & 431191.860000 & \\
\hline NATOT & -0.016700 & 3687311.410000 & 431191.860000 & \\
\hline NITOT & -0.006670 & 3687311.410000 & 431191.860000 & \\
\hline PBTOT & -0.005000 & 3687311.410000 & 431191.860000 & \\
\hline SETOT & -0.003330 & 3687311.410000 & 431191.860000 & \\
\hline SO4 & -1.670000 & 3687311.410000 & 431191.860000 & \\
\hline TOTRAD & 0.000000 & 3687311.410000 & 431191.860000 & \\
\hline TPO4 & -0.083300 & 3687311.410000 & 431191.860000 & \\
\hline UTOT & -0.033300 & 3687311.410000 & 431191.860000 & \\
\hline ZNTOT & -0.003330 & 3687311.410000 & 431191.860000 & \\
\hline ALTOT & -0.033300 & 3687456.360000 & 431295.710000 & \\
\hline BATOT & -0.005000 & 3687456.360000 & 431295.710000 & \\
\hline
\end{tabular}


WSRC-TR-00347

November, 1997

Appendix II-101

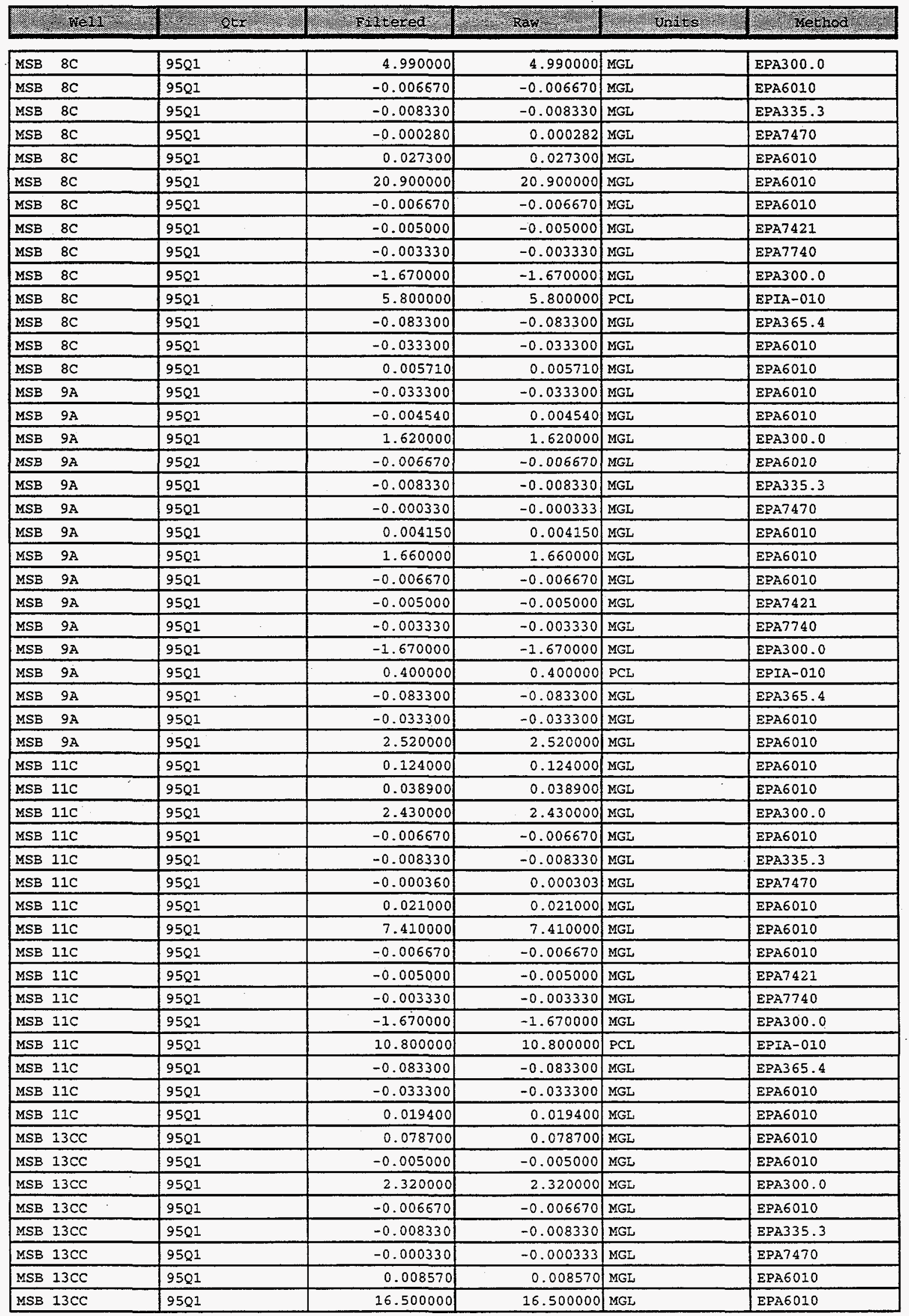




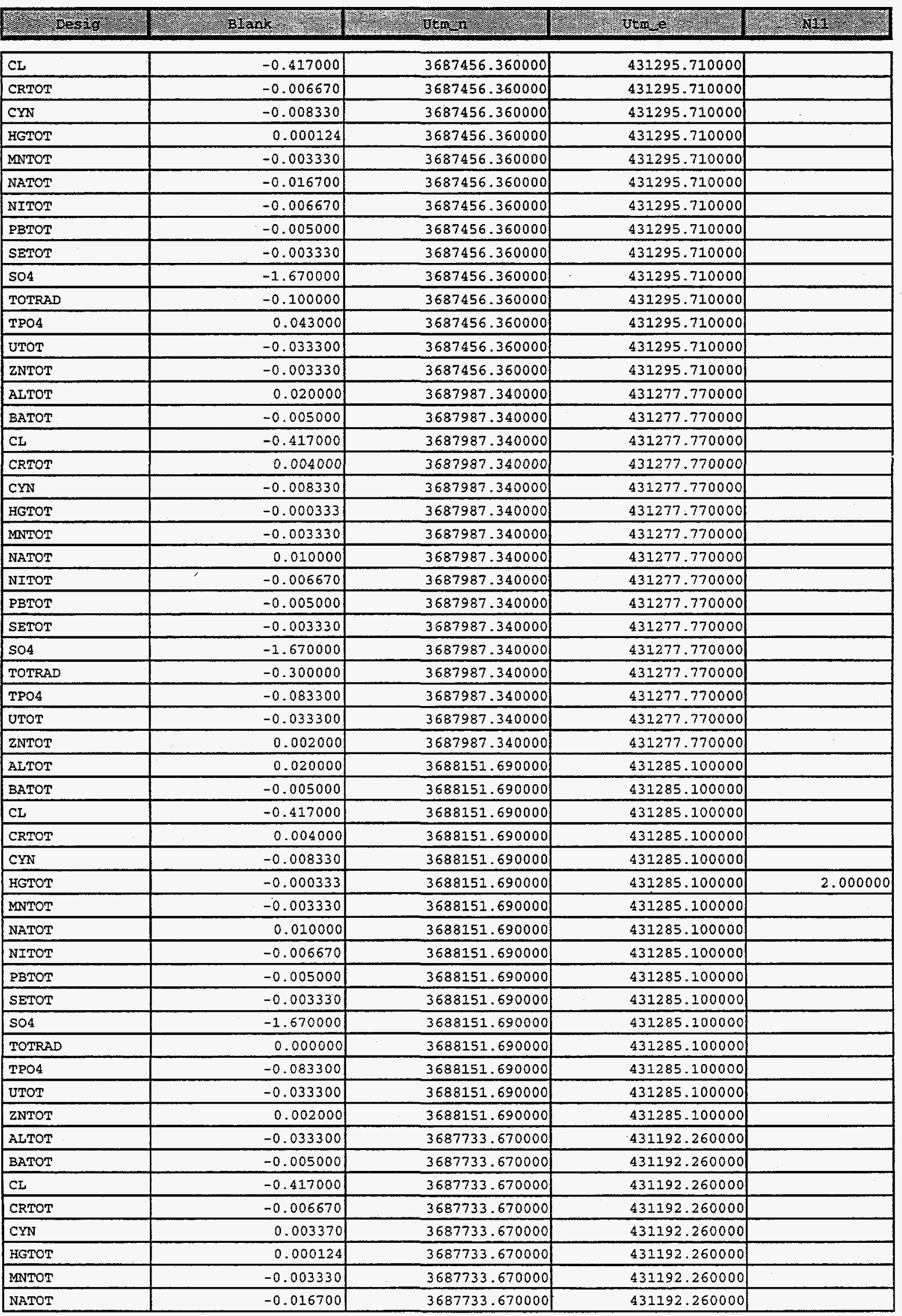


WSRC-TR-00347

November, 1997

Appendix II-103

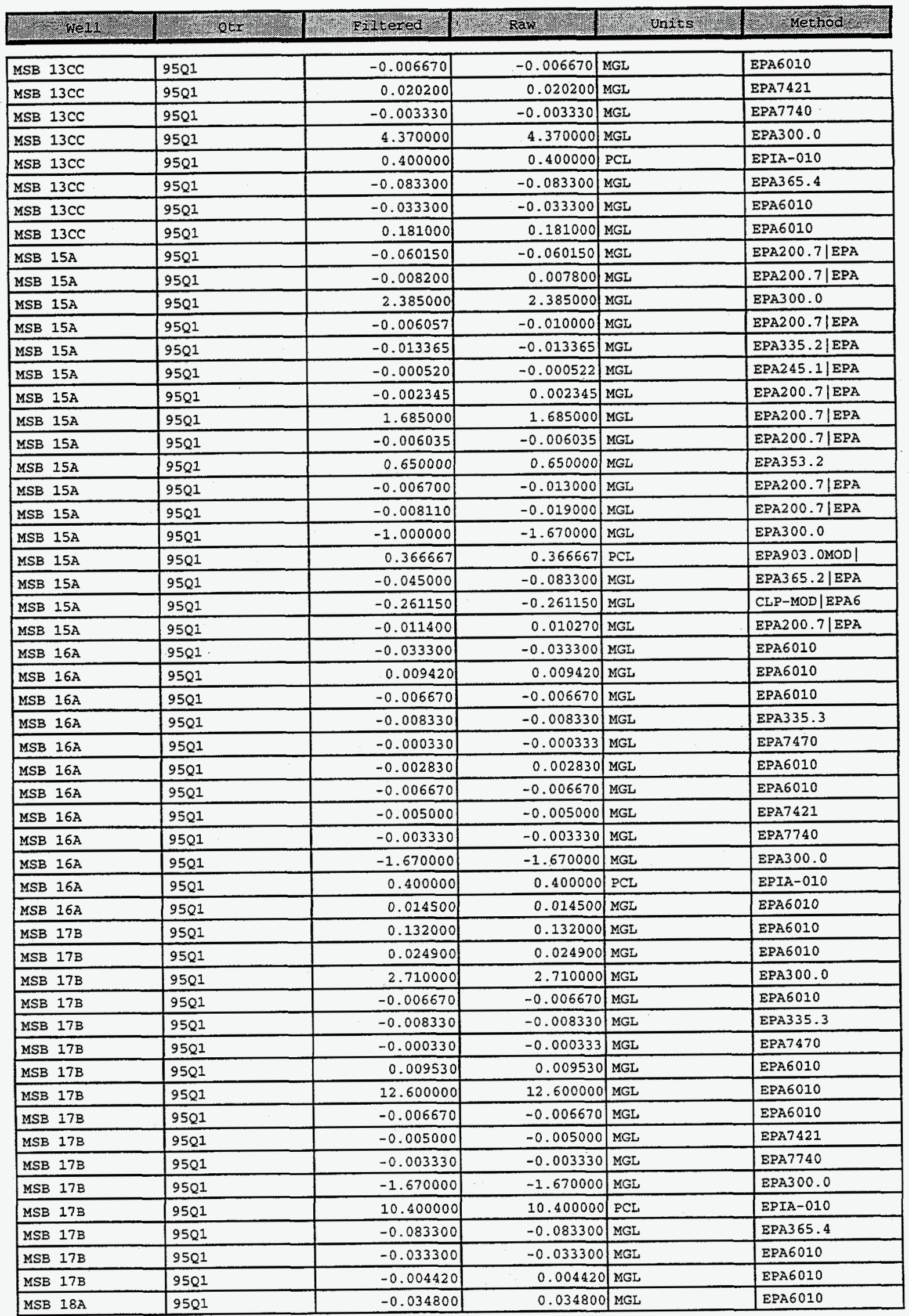


WSRC-TR-00347

November, 1997

Appendix II-104

\begin{tabular}{|c|c|c|c|c|}
\hline 2.28 & 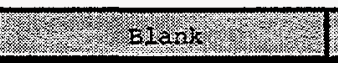 & 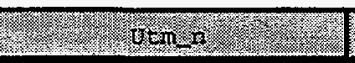 & 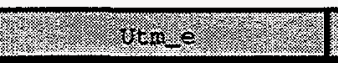 & 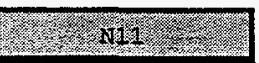 \\
\hline NITOT & -0.006670 & 3687733.670000 & 431192.260000 & \\
\hline PBTOT & 0.003000 & 3687733.670000 & 431192.260000 & \\
\hline SETOT & 0.002000 & 3687733.670000 & 431192.260000 & \\
\hline 504 & -1.670000 & 3687733.670000 & 431192.260000 & \\
\hline TOTRAD & -0.100000 & 3687733.670000 & 431192.260000 & \\
\hline TPO4 & -0.083300 & 3687733.670000 & 431192.260000 & \\
\hline UTOT & -0.033300 & 3687733.670000 & 431192.260000 & \\
\hline ZNTOT & -0.003330 & 3687733.670000 & 431192.260000 & \\
\hline ALTOT & -0.034100 & 3688276.130000 & 431287.840000 & 2.000000 \\
\hline BATOT & -0.006200 & 3688276.130000 & 431287.840000 & 2.000000 \\
\hline $\mathrm{CL}$ & -0.308500 & 3688276.130000 & 431287.840000 & 2.000000 \\
\hline CRTOT & -0.008890 & 3688276.130000 & 431287.840000 & 3.000000 \\
\hline $\mathrm{CYN}$ & -0.018400 & 3688276.130000 & 431287.840000 & 2.000000 \\
\hline HGTOT & 0.002727 & 3688276.130000 & 431287.840000 & 2.000000 \\
\hline MNTOT & -0.004515 & 3688276.130000 & 431287.840000 & 2.000000 \\
\hline NATOT & -0.067900 & 3688276.130000 & 431287.840000 & 2.000000 \\
\hline NITOT & -0.006035 & 3688276.130000 & 431287.840000 & 2.000000 \\
\hline $\mathrm{NO} 3$ & -0.060000 & 3688276.130000 & 431287.840000 & \\
\hline PBTOT & -0.013000 & 3688276.130000 & 431287.840000 & 3.000000 \\
\hline SETOT & 0.002133 & 3688276.130000 & 431287.840000 & 3.000000 \\
\hline $\mathrm{SO4}$ & -1.185000 & 3688276.130000 & 431287.840000 & 2.000000 \\
\hline TOTRAD & -0.100000 & 3688276.130000 & 431287.840000 & 3.000000 \\
\hline TPO4 & -0.056650 & 3688276.130000 & 431287.840000 & 2.000000 \\
\hline UTOT & -0.261150 & 3688276.130000 & 431287.840000 & 2.000000 \\
\hline ZNTOT & -0.006000 & 3688276.130000 & 431287.840000 & 2.000000 \\
\hline ALTOT & & 3688475.910000 & 431194.470000 & \\
\hline BATOT & & 3688475.910000 & 431194.470000 & \\
\hline CRTOT & & 3688475.910000 & 431194.470000 & \\
\hline $\mathrm{CYMN}$ & 0.003370 & 3688475.910000 & 431194.470000 & \\
\hline HGTOT & 0.000124 & 3688475.910000 & 431194.470000 & \\
\hline MNTOT & & 3688475.910000 & 431194.470000 & \\
\hline NITOT & & 3688475.910000 & 431194.470000 & \\
\hline PBTOT & 0.003000 & 3688475.910000 & 431194.470000 & \\
\hline SETOT & -0.003330 & 3688475.910000 & 431194.470000 & \\
\hline 504 & -1.670000 & 3688475.910000 & 431194.470000 & \\
\hline TOTRAD & & 3688475.910000 & 431194.470000 & \\
\hline ZNTOT & & 3688475.910000 & 431194.470000 & \\
\hline ALTOT & 0.020000 & 3687568.180000 & 430827.290000 & \\
\hline BATOT & -0.005000 & 3687568.180000 & 430827.290000 & \\
\hline $\mathrm{CL}$ & -0.417000 & 3687568.180000 & 430827.290000 & \\
\hline CRTOT & 0.004000 & 3687568.180000 & 430827.290000 & \\
\hline $\mathrm{CYN}$ & -0.008330 & 3687568.180000 & 430827.290000 & \\
\hline FGTOT & -0.000333 & 3687568.180000 & 430827.290000 & \\
\hline MNTOT & -0.003330 & 3687568.180000 & 430827.290000 & \\
\hline NATOT & 0.010000 & 3687568.180000 & 430827.290000 & \\
\hline NITOT & -0.006670 & 3687568.180000 & 430827.290000 & \\
\hline PBTOT & -0.005000 & 3687568.180000 & 430827.290000 & \\
\hline SETOT & -0.003330 & 3687568.180000 & 430827.290000 & \\
\hline SO4 & -1.670000 & 3687568.180000 & 430827.290000 & \\
\hline TOTRAD & -0.300000 & 3687568.180000 & 430827.290000 & \\
\hline TPO4 & -0.083300 & 3687568.180000 & 430827.290000 & \\
\hline UTOT & -0.033300 & 3687568.180000 & 430827.290000 & \\
\hline ZNTOT & 0.002000 & 3687568.180000 & 430827.290000 & \\
\hline ALTOT & 0.020000 & 3687156.480000 & 431079.000000 & \\
\hline
\end{tabular}




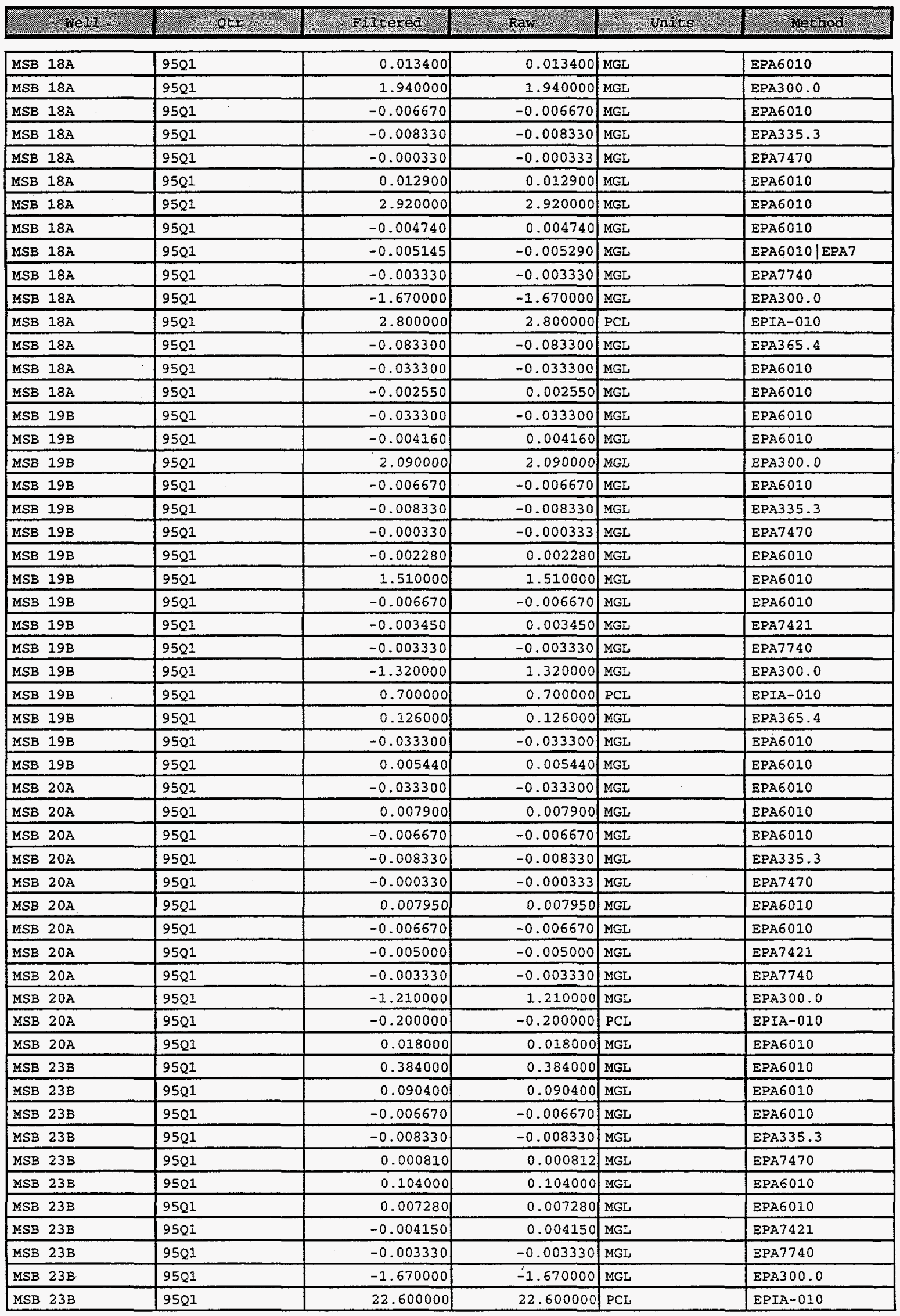


WSRC-TR-00347

November, 1997

Appendix II-106

\begin{tabular}{|c|c|c|c|c|}
\hline $\mathrm{CL}$ & -0.417000 & 3687156.480000 & 431079.000000 & \\
\hline CRTOT & 0.004000 & 3687156.480000 & 431079.000000 & \\
\hline CYN & -0.008330 & 3687156.480000 & 431079.000000 & \\
\hline HGTOT & -0.000333 & 3687156.480000 & 431079.000000 & \\
\hline MNTOT & -0.003330 & 3687156.480000 & 431079.000000 & \\
\hline NATOT & 0.010000 & 3687156.480000 & 431079.000000 & \\
\hline NITOT & -0.006670 & 3687156.480000 & 431079.000000 & \\
\hline PBTOT & -0.005000 & 3687156.480000 & 431079.000000 & 2.000000 \\
\hline SETOT & -0.003330 & 3687156.480000 & 431079.000000 & \\
\hline $\mathrm{SO} 4$ & -1.670000 & 3687156.480000 & 431079.000000 & \\
\hline TOTRAD & 0.000000 & 3687156.480000 & 431079.000000 & \\
\hline TPO4 & -0.083300 & 3687156.480000 & 431079.000000 & \\
\hline UTOT & -0.033300 & 3687156.480000 & 431079.000000 & \\
\hline ZNTOT & 0.002000 & 3687156.480000 & 431079.000000 & \\
\hline ALTOT & -0.033300 & 3688165.300000 & 432162.930000 & \\
\hline BATOT & -0.005000 & 3688165.300000 & 432162.930000 & \\
\hline$C L$ & -0.417000 & 3688165.300000 & 432162.930000 & \\
\hline CRTOT & -0.006670 & 3688165.300000 & 432162.930000 & \\
\hline CYN & -0.008330 & 3688165.300000 & 432162.930000 & \\
\hline HGTOT & -0.000333 & 3688165.300000 & 432162.930000 & \\
\hline MNTOT & -0.003330 & 3688165.300000 & 432162.930000 & \\
\hline NATOT & -0.016700 & 3688165.300000 & 432162.930000 & \\
\hline NITOT & -0.006670 & 3688165.300000 & 432162.930000 & \\
\hline PBTOT & -0.005000 & 3688165.300000 & 432162.930000 & \\
\hline SETOT & -0.003330 & 3688165.300000 & 432162.930000 & \\
\hline 504 & -1.670000 & 3688165.300000 & 432162.930000 & \\
\hline TOTRAD & 0.000000 & 3688165.300000 & 432162.930000 & \\
\hline TPO4 & -0.083300 & 3688165.300000 & 432162.930000 & \\
\hline UTOT & -0.033300 & 3688165.300000 & 432162.930000 & \\
\hline ZNTOT & -0.003330 & 3688165.300000 & 432162.930000 & \\
\hline ALTOT & -0.033300 & 3687918.380000 & 430505.590000 & \\
\hline BATOT & -0.005000 & 3687918.380000 & 430505.590000 & \\
\hline CRTOT & -0.006670 & 3687918.380000 & 430505.590000 & \\
\hline $\mathrm{CYN}$ & -0.008330 & 3687918.380000 & 430505.590000 & \\
\hline HGTOT & -0.000333 & 3687918.380000 & 430505.590000 & \\
\hline MNTOT & -0.003330 & 3687918.380000 & 430505.590000 & \\
\hline NITOT & -0.006670 & 3687918.380000 & 430505.590000 & \\
\hline PBTOT & -0.005000 & 3687918.380000 & 430505.590000 & \\
\hline SETOT & -0.003330 & 3687918.380000 & 430505.590000 & \\
\hline $\mathrm{SO4}$ & -1.670000 & 3687918.380000 & 430505.590000 & \\
\hline TOTRAD & 0.000000 & 3687918.380000 & 430505.590000 & \\
\hline ZNTOT & -0.003330 & 3687918.380000 & 430505.590000 & \\
\hline ALTOT & 0.020000 & 3688691.860000 & 431158.370000 & \\
\hline BATOT & -0.005000 & 3688691.860000 & 431158.370000 & \\
\hline CRTOT & 0.004000 & 3688691.860000 & 431158.370000 & \\
\hline $\mathrm{CYN}$ & -0.008330 & 3688691.860000 & 431158.370000 & \\
\hline HGTOT & -0.000333 & 3688691.860000 & 431158.370000 & \\
\hline MNTOT & -0.003330 & 3688691.860000 & 431158.370000 & \\
\hline NITOT & -0.006670 & 3688691.860000 & 431158.370000 & \\
\hline PBTOT & -0.005000 & 3688691.860000 & 431158.370000 & \\
\hline SETOT & -0.003330 & 3688691.860000 & 431158.370000 & \\
\hline SO4 & -1.670000 & 3688691.860000 & 431158.370000 & \\
\hline TOTRAD & -0.300000 & 3688691.860000 & 431158.370000 & \\
\hline
\end{tabular}


WSRC-TR-00347

November, 1997

Appendix II-107

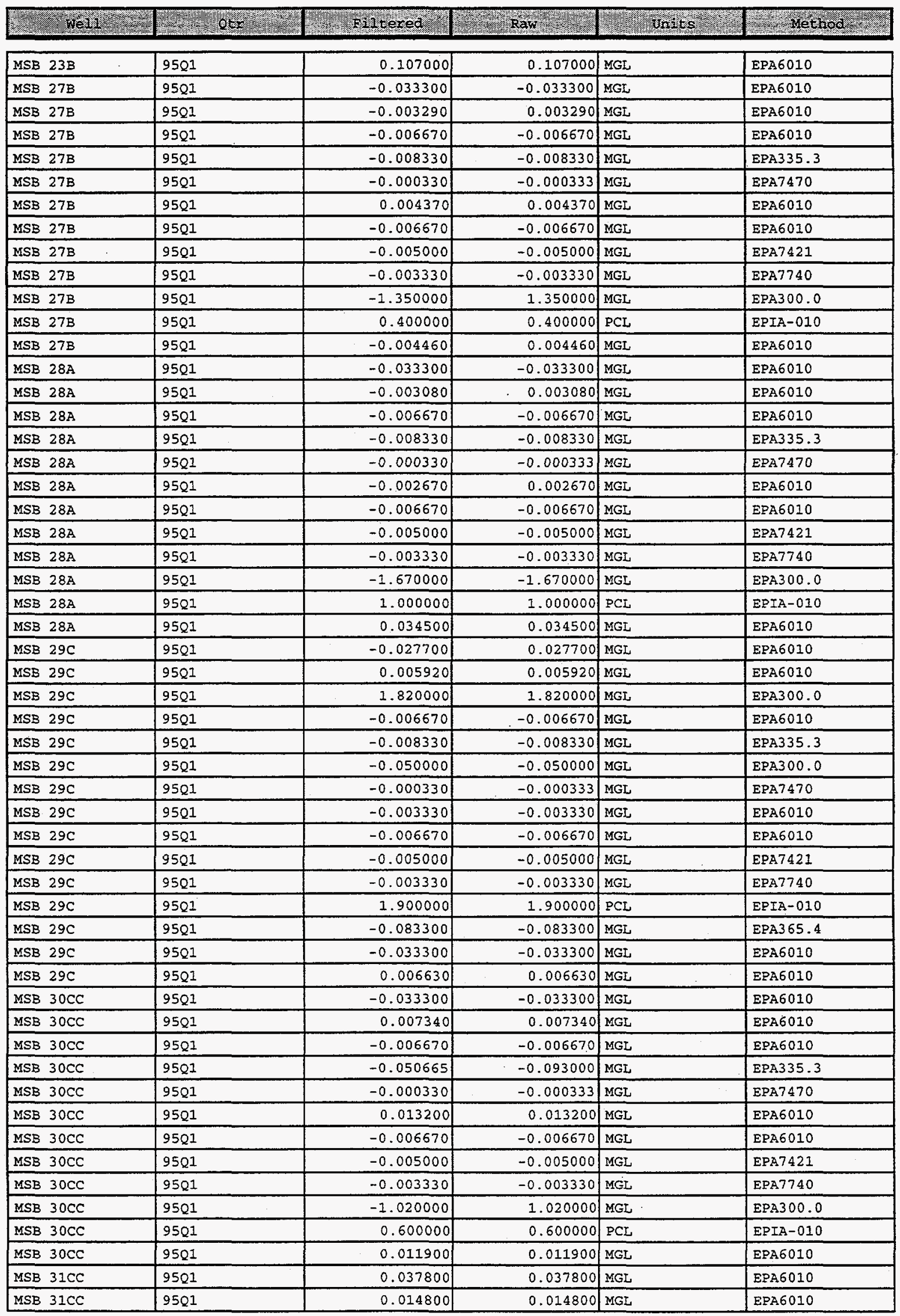




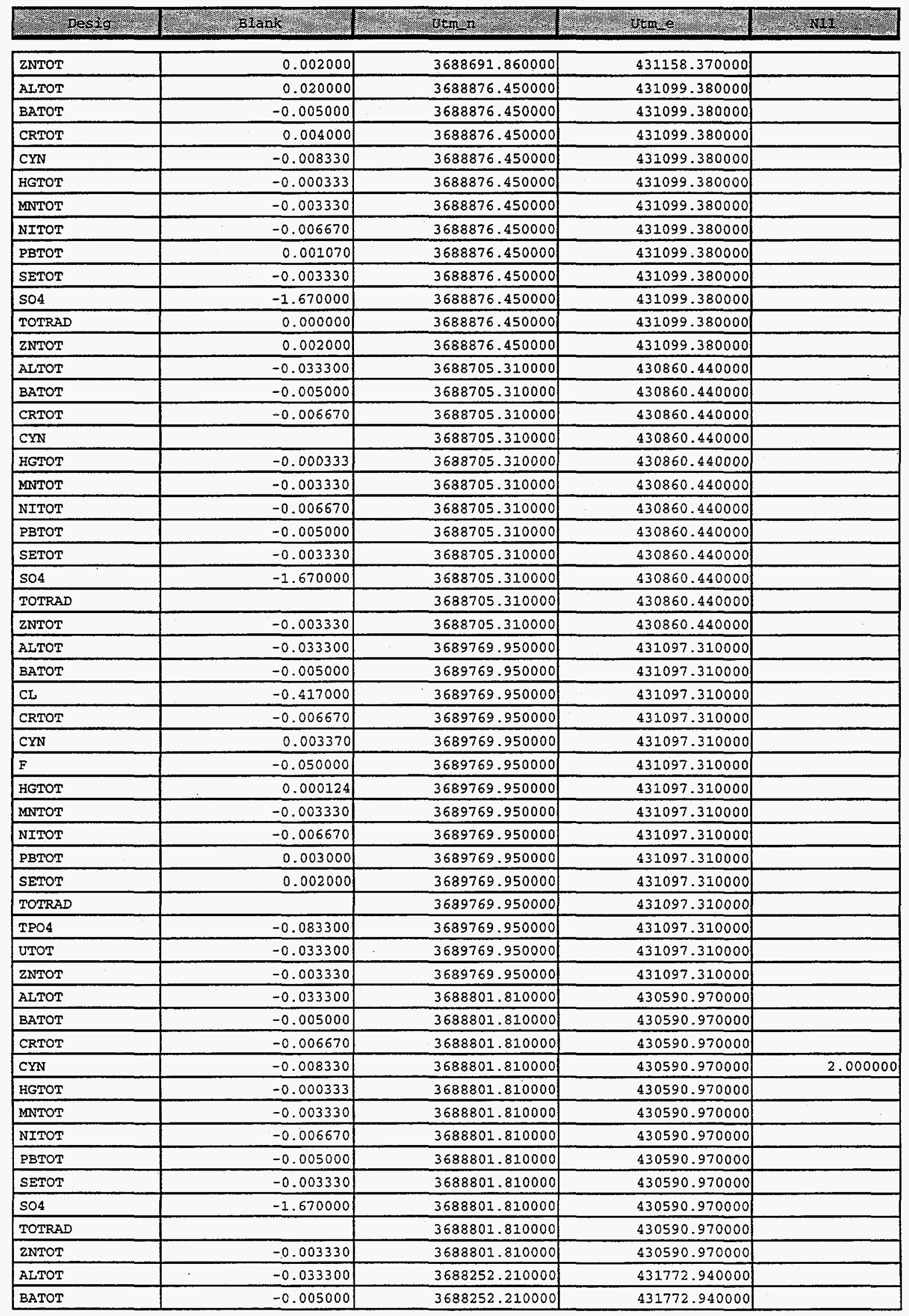




\begin{tabular}{|c|c|c|c|c|c|}
\hline (t) & 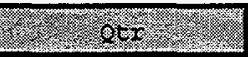 & Thtered & (7r) & d8. & (t. \\
\hline MSB $31 C C$ & 9501 & -0.006670 & -0.006670 & MGL & EPA6010 \\
\hline MSB $31 \mathrm{CC}$ & $95 Q 1$ & -0.008330 & -0.008330 & MGL & ERA335.3 \\
\hline MSB $31 C C$ & $95 Q 1$ & -0.000330 & -0.000333 & MGL & EPA7470 \\
\hline MSB $31 C C$ & $95 Q 1$ & 0.008820 & 0.008820 & MGL & EPA6010 \\
\hline$M S B$ & $95 Q 1$ & -0.006670 & -0.006670 & MGL & EPA6010 \\
\hline MSB $31 \mathrm{CC}$ & 9501 & -0.015000 & -0.015000 & MGL & EPA6010|EPA7 \\
\hline MSB $31 \mathrm{CC}$ & 9501 & -0.003330 & -0.003330 & MGL & EPA7740 \\
\hline MSB $31 \mathrm{CC}$ & 9501 & 2.300000 & 2.300000 & MGL & EPA 300.0 \\
\hline MSB $31 \mathrm{CC}$ & 9501 & 0.400000 & 0.400000 & PCI & EPIA-010 \\
\hline MSB $31 C C$ & 9501 & 0.009850 & 0.009850 & MGL & EPA6010 \\
\hline MSB $32 \mathrm{C}$ & $950 ?$ & -0.033300 & -0.033300 & MGL & EPA6010 \\
\hline MSB $32 \mathrm{C}$ & 9501 & 0.007200 & 0.007200 & MGL. & EPA6010 \\
\hline MSB $32 \mathrm{C}$ & 9501 & -0.006670 & -0.006670 & MGL & EPA6010 \\
\hline MSB $32 \mathrm{C}$ & 9501 & -0.008330 & -0.008330 & MGL & EPA335.3 \\
\hline MSB $32 \mathrm{C}$ & 9501 & -0.000330 & -0.000333 & MGL & EPA7470 \\
\hline MSB $32 \mathrm{C}$ & 9501 & 0.003370 & 0.003370 & MGL & EPA 6010 \\
\hline MSB $32 \mathrm{C}$ & 9501 & -0.006670 & -0.006670 & MGL & EPA6010 \\
\hline MSB $32 \mathrm{C}$ & 9501 & 0.012500 & 0.012500 & MGL & EPA7421 \\
\hline MSB $32 \mathrm{C}$ & 9501 & -0.003330 & -0.003330 & MGL & EPA7740 \\
\hline MSB $32 \mathrm{C}$ & 9501 & -1.670000 & -1.670000 & MGL & EPA 300.0 \\
\hline MSB $32 \mathrm{C}$ & 9501 & 0.700000 & 0.700000 & PCL & EPIA-010 \\
\hline MSB $32 \mathrm{C}$ & 9501 & 0.005510 & 0.005510 & MGL & EPA6010 \\
\hline MSB $33 C$ & 9501 & -0.033300 & -0.033300 & MGL & EPA6010 \\
\hline MSB $33 \mathrm{C}$ & $95 \mathrm{QI}$ & -0.003700 & 0.003700 & MGL & EPA 6010 \\
\hline MSB $33 \mathrm{C}$ & $95 Q 1$ & -0.006670 & -0.006670 & MGL & EPA6010 \\
\hline MSB $33 \mathrm{C}$ & 9501 & -0.008330 & -0.008330 & MGL & EPA335.3 \\
\hline MSB $33 \mathrm{C}$ & 9501 & -0.000330 & -0.000333 & MGL & EPA7470 \\
\hline MSB $33 \mathrm{C}$ & 9501 & -0.002070 & 0.002070 & MGL & EPA6010 \\
\hline MSB $33 \mathrm{C}$ & 9501 & -0.006670 & -0.006670 & MGL & EPA6010 \\
\hline MSB $33 C$ & 9501 & -0.005000 & -0.005000 & MGL & EPA7421 \\
\hline MSB $33 \mathrm{C}$ & 9501 & -0.003330 & -0.003330 & MGL & EPA7740 \\
\hline MSB $33 \mathrm{C}$ & 9501 & -1.530000 & 1.530000 & MGL & EPA300.0 \\
\hline MSB $33 \mathrm{C}$ & 9501 & -0.300000 & -0.300000 & PCI & EPIA-010 \\
\hline MSB $33 \mathrm{C}$ & 9501 & -0.003330 & -0.003330 & MGL & EPA6010 \\
\hline MSB $34 B$ & 9501 & -0.029800 & 0.029800 & MGL & EPA6010 \\
\hline MSB $34 \mathrm{~B}$ & 9501 & 0.005250 & 0.005250 & MGI & EPA6010 \\
\hline MSB $34 \mathrm{~B}$ & 9501 & -0.006670 & -0.006670 & MGL & EPA6010 \\
\hline MSB $34 B$ & 9501 & -0.008330 & -0.008330 & MGL & EPA335.3 \\
\hline MSB $34 \mathrm{~B}$ & $95 \mathrm{Q} 1$ & -0.000230 & 0.000230 & MGL & EPA7470 \\
\hline MSB $34 \mathrm{~B}$ & 9501 & -0.002920 & 0.002920 & MGL & EPA6010 \\
\hline MSB $34 \mathrm{~B}$ & 9501 & -0.006670 & -0.006670 & MGL & EPA6010 \\
\hline MSB $34 B$ & 9501 & -0.005000 & -0.005000 & MGL & EPA7421 \\
\hline MSB $34 B$ & 9501 & -0.003330 & -0.003330 & MGL & EPA7740 \\
\hline MSB $34 B$ & 9501 & -1.670000 & -1.670000 & MGL & EPA300.0 \\
\hline MSB $34 \mathrm{~B}$ & 9501 & 2.800000 & 2.800000 & PCL & EPIA-010 \\
\hline MSB $34 \mathrm{~B}$ & 9501 & 0.014200 & 0.014200 & MGL & EPA6010 \\
\hline MSB $35 B$ & 9501 & -0.033300 & -0.033300 & MGL & EPA6010 \\
\hline MSB 35B & 9501 & -0.003760 & 0.003760 & MGL & EPA6010 \\
\hline MSB $35 B$ & $95 \mathrm{Q} 1$ & -0.006670 & -0.006670 & MGL & EPA 6010 \\
\hline MSB $35 B$ & 9501 & -0.008330 & -0.008330 & MGL & EPA335.3 \\
\hline MSB $35 B$ & 9501 & -0.000330 & -0.000333 & MGL & EPA7470 \\
\hline MSB $35 B$ & 9501 & -0.003330 & -0.003330 & MGL & EPA.6010 \\
\hline MSB $35 B$ & 9501 & -0.006670 & -0.006670 & MGL & EPA6010 \\
\hline MSB $35 \mathrm{~B}$ & 9501 & -0.005000 & -0.005000 & MGL & EPA7 721 \\
\hline
\end{tabular}


WSRC-TR-00347

November, 1997

Appendix II-110

\begin{tabular}{|c|c|c|c|c|}
\hline 20 & P. & 10.7) & 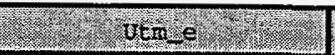 & W \\
\hline CRTOT & -0.006670 & 3688252.210000 & 431772.940000 & \\
\hline CYN & -0.008330 & 3688252.210000 & 431772.940000 & \\
\hline HGTOT & 0.000124 & 3688252.210000 & 431772.940000 & \\
\hline MNTOT & -0.003330 & 3688252.210000 & 431772.940000 & \\
\hline NITOT & -0.006670 & 3688252.210000 & 431772.940000 & \\
\hline PBTOT & -0.005000 & 3688252.210000 & 431772.940000 & 2.000000 \\
\hline SETOT & -0.003330 & 3688252.210000 & 431772.940000 & \\
\hline $\mathrm{SO4}$ & -1.670000 & 3688252.210000 & 431772.940000 & \\
\hline TOTRAD & & 3688252.210000 & 431772.940000 & \\
\hline ZNTOT & -0.003330 & 3688252.210000 & 431772.940000 & \\
\hline ALTOT & -0.033300 & 3688166.450000 & 432845.060000 & \\
\hline BATOT & -0.005000 & 3688166.450000 & 432845.060000 & \\
\hline CRTOT & -0.006670 & 3688166.450000 & 432845.060000 & \\
\hline CYN & & 3688166.450000 & 432845.060000 & \\
\hline HGTOT & -0.000333 & 3688166.450000 & 432845.060000 & \\
\hline MNTOT & -0.003330 & 3688166.450000 & 432845.060000 & \\
\hline NITOT & -0.006670 & 3688166.450000 & 432845.060000 & \\
\hline PBTOT & -0.005000 & 3688166.450000 & 432845.060000 & \\
\hline SETOT & -0.003330 & 3688166.450000 & 432845.060000 & \\
\hline 504 & -1.670000 & 3688166.450000 & 432845.060000 & \\
\hline TOTRAD & & 3688166.450000 & 432845.060000 & \\
\hline ZNTOT & -0.003330 & 3688166.450000 & 432845.060000 & \\
\hline ALTOT & & 3687568.260000 & 432903.530000 & \\
\hline BATOT & & 3687568.260000 & 432903.530000 & \\
\hline CRTOT & & 3687568.260000 & 432903.530000 & \\
\hline CYN & -0.008330 & 3687568.260000 & 432903.530000 & \\
\hline HGTOT & -0.000333 & 3687568.260000 & 432903.530000 & \\
\hline MNTOT & & 3687568.250000 & 432903.530000 & \\
\hline NITOT & & 3687568.260000 & 432903.530000 & \\
\hline PBTOT & -0.005000 & 3687568.260000 & 432903.530000 & \\
\hline SETOT & -0.003330 & 3687568.260000 & 432903.530000 & \\
\hline SO4 & -1.670000 & 3687568.260000 & 432903.530000 & \\
\hline TOTRAD & 0.000000 & 3687568.260000 & 432903.530000 & \\
\hline ZNTOT & & 3687568.260000 & 432903.530000 & \\
\hline ALTOT & -0.033300 & 3689065.560000 & 431356.890000 & \\
\hline BATOT & -0.005000 & 3689065.560000 & 431356.890000 & \\
\hline CRTOT & -0.006670 & 3689065.560000 & 431356.890000 & \\
\hline CYN & -0.008330 & 3689065.560000 & 431356.890000 & \\
\hline HGTOT & -0.000333 & 3689065.560000 & 431356.890000 & \\
\hline MNTOT & -0.003330 & 3689065.560000 & 431356.890000 & \\
\hline NITOT & -0.006670 & 3689065.560000 & 431356.890000 & \\
\hline PBTOT & -0.005000 & 3689065.560000 & 431356.890000 & \\
\hline SETOT & -0.003330 & 3689065.560000 & 431356.890000 & \\
\hline SO4 & -1.670000 & 3689065.560000 & 431356.890000 & \\
\hline TOTRAD & -0.200000 & 3689065.560000 & 431356.890000 & \\
\hline ZNTOT & -0.003330 & 3689065.560000 & 431356.890000 & \\
\hline ALTOT & -0.033300 & 3688441.470000 & 431966.830000 & \\
\hline BATOT & -0.005000 & 3688441.470000 & 431966.830000 & \\
\hline CRTOT & -0.006670 & 3688441.470000 & 431966.830000 & \\
\hline CYN & -0.008330 & 3688441.470000 & 431966.830000 & \\
\hline HGTOT & -0.000333 & 3688441.470000 & 431966.830000 & \\
\hline MNTOT & -0.003330 & 3688441.470000 & 431966.830000 & \\
\hline NITOT & 0.009480 & 3688441.470000 & 431966.830000 & \\
\hline PBTOT & -0.005000 & 3688441.470000 & 431966.830000 & \\
\hline
\end{tabular}


WSRC-TR-00347

November, 1997

Appendix II-111

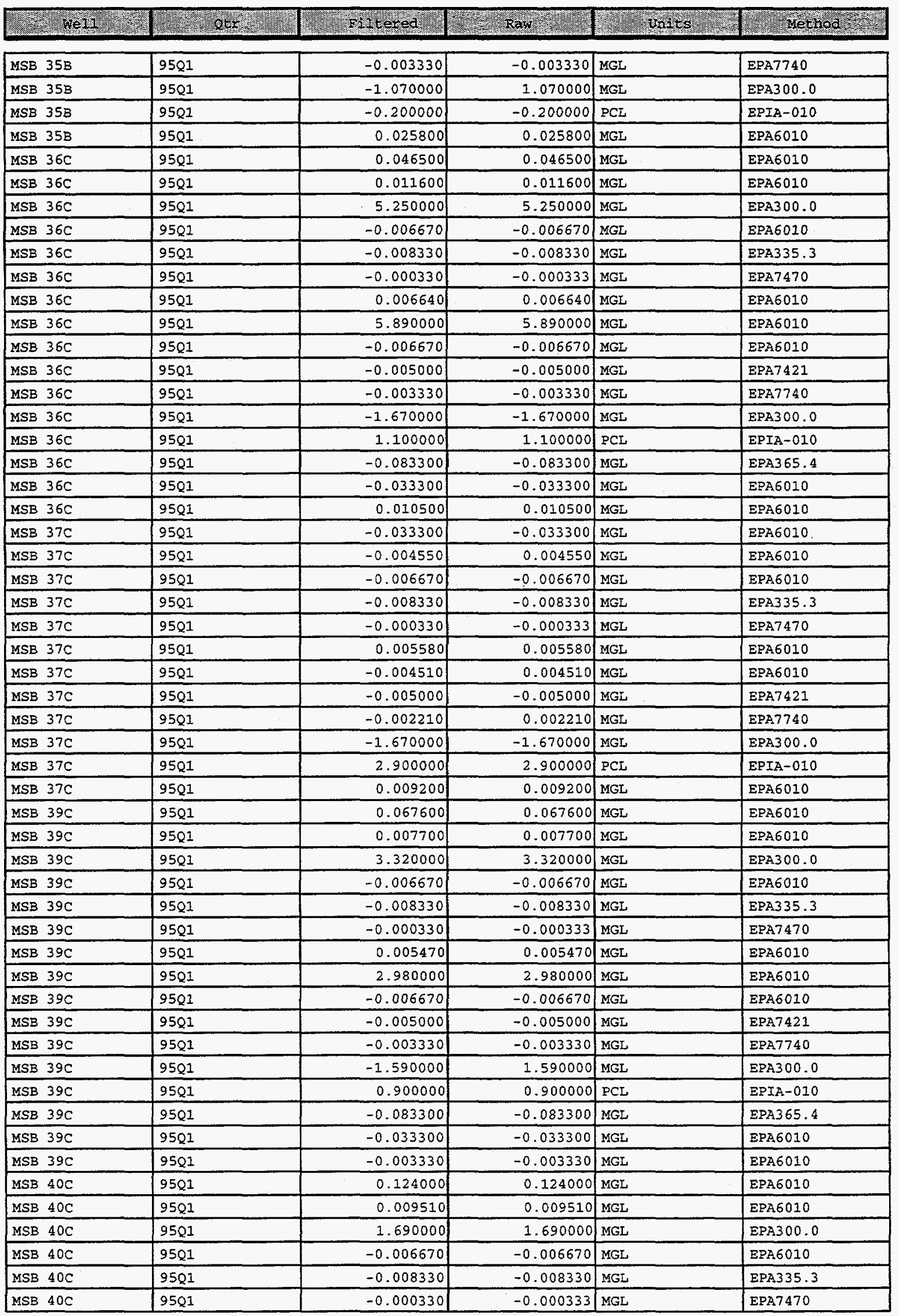




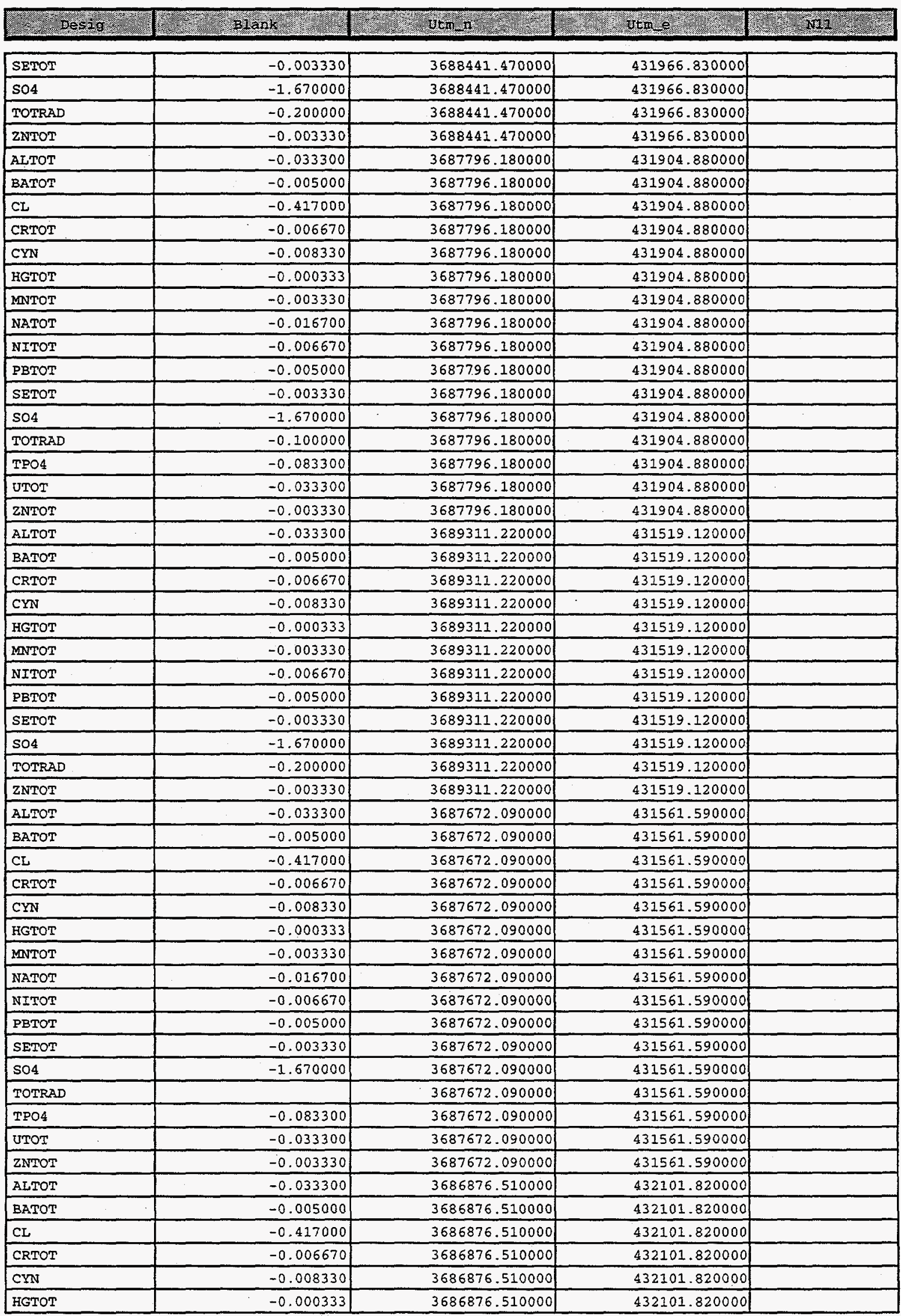




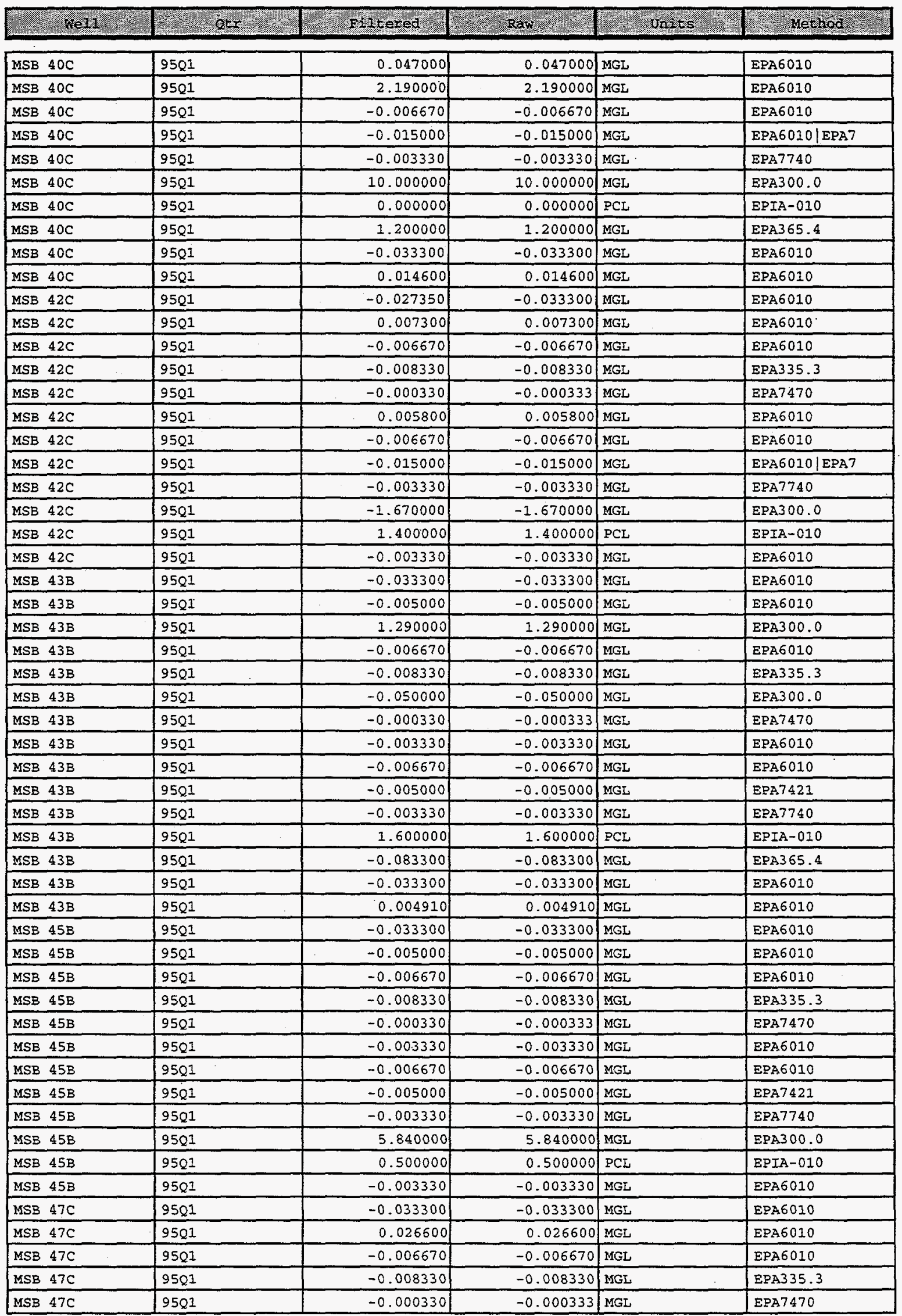




\begin{tabular}{|c|c|c|c|c|}
\hline MNTOT & -0.003330 & 3686876.510000 & 432101.820000 & \\
\hline NATOT & -0.016700 & 3686876.510000 & 432101.820000 & \\
\hline NITOT & -0.006670 & 3686876.510000 & 432101.820000 & \\
\hline PBTOT & -0.005000 & 3686876.510000 & 432101.820000 & 2.000000 \\
\hline SETOT & -0.003330 & 3686876.510000 & 432101.820000 & \\
\hline 504 & -1.670000 & 3686876.510000 & 432101.820000 & \\
\hline TOTRAD & -0.100000 & 3686876.510000 & 432101.820000 & \\
\hline TPO4 & -0.083300 & 3686876.510000 & 432101.820000 & \\
\hline UTOT & -0.033300 & 3686876.510000 & 432101.820000 & \\
\hline ZNTOT & -0.003330 & 3686876.510000 & 432101.820000 & \\
\hline ALTOT & -0.033300 & 3689164.100000 & 431680.110000 & 2.000000 \\
\hline BATOT & -0.005000 & 3689164.100000 & 431680.110000 & \\
\hline CRTOT & -0.006670 & 3689164.100000 & 431680.110000 & \\
\hline CYN & -0.008330 & 3689164.100000 & 431680.110000 & \\
\hline HGTOT & -0.000333 & 3689164.100000 & 431680.110000 & \\
\hline MNTOT & -0.003330 & 3689164.100000 & 431680.110000 & \\
\hline NITOT & -0.006670 & 3689164.100000 & 431680.110000 & \\
\hline PBTOT & -0.005000 & 3689164.100000 & 431680.110000 & 2.000000 \\
\hline SETOT & -0.003330 & 3689164.100000 & 431680.110000 & \\
\hline 504 & -1.670000 & 3689164.100000 & 431680.110000 & \\
\hline TOTRAD & -0.400000 & 3689164.100000 & 431680.110000 & \\
\hline ZNTOT & -0.003330 & 3689164.100000 & 431680.110000 & \\
\hline ALTOT & -0.033300 & 3689420.210000 & 430637.760000 & \\
\hline BATOT & -0.005000 & 3689420.210000 & 430637.760000 & \\
\hline $\mathrm{CL}$ & -0.417000 & 3689420.210000 & 430637.760000 & \\
\hline CRTOT & -0.006670 & 3689420.210000 & 430637.760000 & \\
\hline $\mathrm{CYN}$ & 0.003370 & 3689420.210000 & 430637.760000 & \\
\hline$F$ & -0.050000 & 3689420.210000 & 430637.760000 & \\
\hline HGTOT & 0.000124 & 3689420.210000 & 430637.760000 & \\
\hline MNTOT & -0.003330 & 3689420.210000 & 430637.760000 & \\
\hline NITOT & -0.006670 & 3689420.210000 & 430637.360000 & \\
\hline PBTOT & 0.003000 & 3689420.210000 & 430637.760000 & \\
\hline SETOT & 0.002000 & 3689420.210000 & 430637.760000 & \\
\hline TOTRAD & & 3689420.210000 & 430637.760000 & \\
\hline TPO4 & -0.083300 & 3689420.210000 & 430637.760000 & \\
\hline UTOT & -0.033300 & 3689420.210000 & 430637.760000 & \\
\hline ZNTOT & -0.003330 & 3689420.210000 & 430637.760000 & \\
\hline ALTOT & -0.033300 & 3688833.500000 & 431533.500000 & \\
\hline BATOT & -0.005000 & 3688833.500000 & 431533.500000 & \\
\hline CRTOT & -0.006670 & 3688833.500000 & 431533.500000 & \\
\hline $\mathrm{CYN}$ & -0.008330 & 3688833.500000 & 431533.500000 & \\
\hline HGTOT & -0.000333 & 3688833.500000 & 431533.500000 & \\
\hline MNTOT & -0.003330 & 3688833.500000 & 431533.500000 & \\
\hline NITOT & -0.006670 & 3688833.500000 & 431533.500000 & \\
\hline PBTOT & -0.005000 & 3688833.500000 & 431533.500000 & \\
\hline SETOT & -0.003330 & 3688833.500000 & 431533.500000 & \\
\hline $\mathrm{SO} 4$ & -1.670000 & 3688833.500000 & 431533.500000 & \\
\hline TOTRAD & -0.100000 & 3688833.500000 & 431533.500000 & \\
\hline ZNTOT & -0.003330 & 3688833.500000 & 431533.500000 & \\
\hline ALTOT & 0.020000 & 3689862.100000 & 431402.940000 & \\
\hline BATOT & -0.005000 & 3689862.100000 & 431402.940000 & \\
\hline CRTOT & 0.004000 & 3689862.100000 & 431402.940000 & \\
\hline CYN & -0.008330 & 3689862.100000 & 431402.940000 & \\
\hline HGTOT & -0.000333 & 3689862.100000 & 431402.940000 & \\
\hline
\end{tabular}


WSRC-TR-00347

November, 1997

Appendix II-115

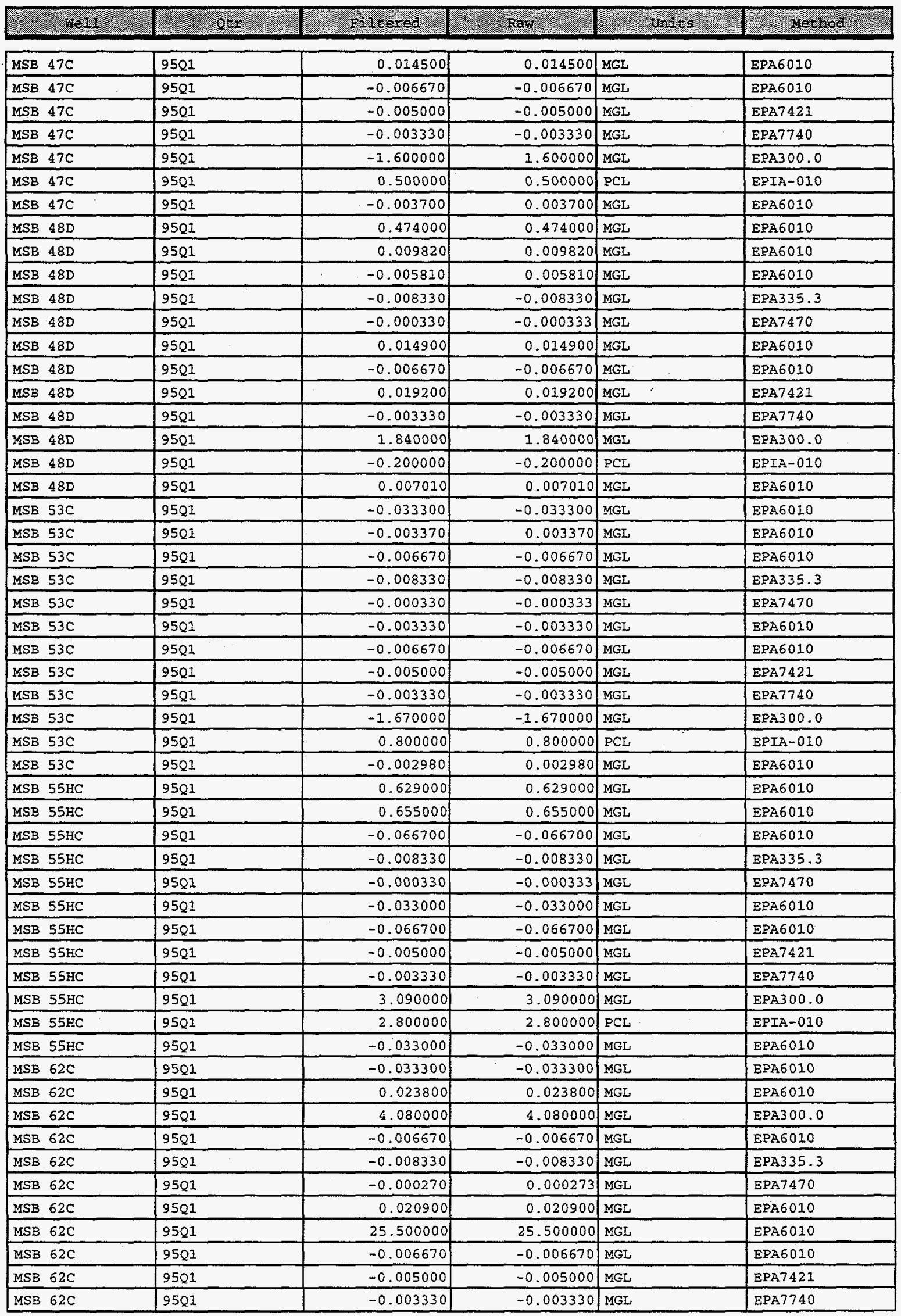




\begin{tabular}{|c|c|c|c|c|}
\hline MNTOT & -0.003330 & 3689862.100000 & 431402.940000 & \\
\hline NITOT & -0.006670 & 3689862.100000 & 431402.940000 & \\
\hline PBTOT & 0.003190 & 3689862.100000 & 431402.940000 & \\
\hline SETOT & -0.003330 & 3689862.100000 & 431402.940000 & \\
\hline so4 & -1.670000 & 3689862.100000 & 431402.940000 & \\
\hline TOTRAD & & 3689862.100000 & 431402.940000 & \\
\hline ZNTOT & 0.002000 & 3689862.100000 & 431402.940000 & \\
\hline ALTOT & -0.033300 & 3690428.640000 & 431691.860000 & \\
\hline BATOT & -0.005000 & 3690428.640000 & 431691.860000 & \\
\hline CRTOT & -0.006670 & 3690428.640000 & 431691.860000 & \\
\hline $\mathrm{CYN}$ & & 3690428.640000 & 431691.860000 & \\
\hline HGTOT & -0.000333 & 3690428.640000 & 431691.860000 & \\
\hline MNTOT & -0.003330 & 3690428.640000 & 431691.860000 & \\
\hline NITOT & -0.006670 & 3690428.640000 & 431691.860000 & \\
\hline PBTOT & -0.005000 & 3690428.640000 & 431691.860000 & \\
\hline SETOT & -0.003330 & 3690428.640000 & 431691.860000 & \\
\hline 504 & -1.670000 & 3690428.640000 & 431691.860000 & \\
\hline TOTRAD & & 3690428.640000 & 431691.860000 & \\
\hline ZNTOT & -0.003330 & 3690428.640000 & 431691.860000 & \\
\hline ALTOT & -0.033300 & 3690156.240000 & 432072.640000 & \\
\hline BATOT & -0.005000 & 3690156.240000 & 432072.640000 & \\
\hline CRTOT & -0.006670 & 3690156.240000 & 432072.640000 & \\
\hline $\mathrm{CYN}$ & -0.008330 & 3690156.240000 & 432072.640000 & \\
\hline HGTOT & -0.000333 & 3690156.240000 & 432072.640000 & \\
\hline MNTOT & -0.003330 & 3690156.240000 & 432072.640000 & \\
\hline NITOT & -0.006670 & 3690156.240000 & 432072.640000 & \\
\hline PBTOT & -0.005000 & 3690156.240000 & 432072.640000 & \\
\hline SETOT & -0.003330 & 3690156.240000 & 432072.640000 & \\
\hline 504 & -1.670000 & 3690156.240000 & 432072.640000 & \\
\hline TOTRAD & -0.200000 & 3690156.240000 & 432072.640000 & \\
\hline ZNTOT & -0.003330 & 3690156.240000 & 432072.640000 & \\
\hline ALTOT & -0.033300 & 3690168.030000 & 431114.150000 & \\
\hline BATOT & -0.005000 & 3690168.030000 & 431114.150000 & \\
\hline CRTOT & -0.006670 & 3690168.030000 & 431114.150000 & \\
\hline CYN & 0.003370 & 3690168.030000 & 431114.150000 & \\
\hline HGTOT & -0.000333 & 3690168.030000 & 431114.150000 & \\
\hline MNTOT & -0.003330 & 3690168.030000 & 431114.150000 & \\
\hline NITOT & -0.006670 & 3690168.030000 & 431114.150000 & \\
\hline PBTOT & -0.005000 & 3690168.030000 & 431114.150000 & \\
\hline SETOT & -0.003330 & 3690168.030000 & 431114.150000 & \\
\hline 504 & -1.670000 & 3690168.030000 & 431114.150000 & \\
\hline TOTRAD & & 3690168.030000 & 431114.150000 & \\
\hline ZNTOT & -0.005000 & 3690168.030000 & 431114.150000 & \\
\hline ALTOT & -0.033300 & 3687831.530000 & 431260.210000 & \\
\hline BATOT & -0.005000 & 3687831.530000 & 431260.210000 & \\
\hline $\mathrm{CL}$ & -0.417000 & 3687831.530000 & 431260.210000 & \\
\hline CRTOT & -0.006670 & 3687831.530000 & 431260.210000 & \\
\hline CYN & -0.008330 & 3687831.530000 & 431260.210000 & \\
\hline HGTOT & 0.000124 & 3687831.530000 & 431260.210000 & \\
\hline MNTOT & -0.003330 & 3687831.530000 & 431260.210000 & \\
\hline NATOT & -0.016700 & 3687831.530000 & 431260.210000 & \\
\hline NITOT & -0.006670 & 3687831.530000 & 431260.210000 & \\
\hline PBTOT & -0.005000 & 3687831.530000 & 431260.210000 & \\
\hline SETOT & -0.003330 & 3687831.530000 & 431260.210000 & \\
\hline
\end{tabular}


WSRC-TR-00347

November, 1997

Appendix II-117

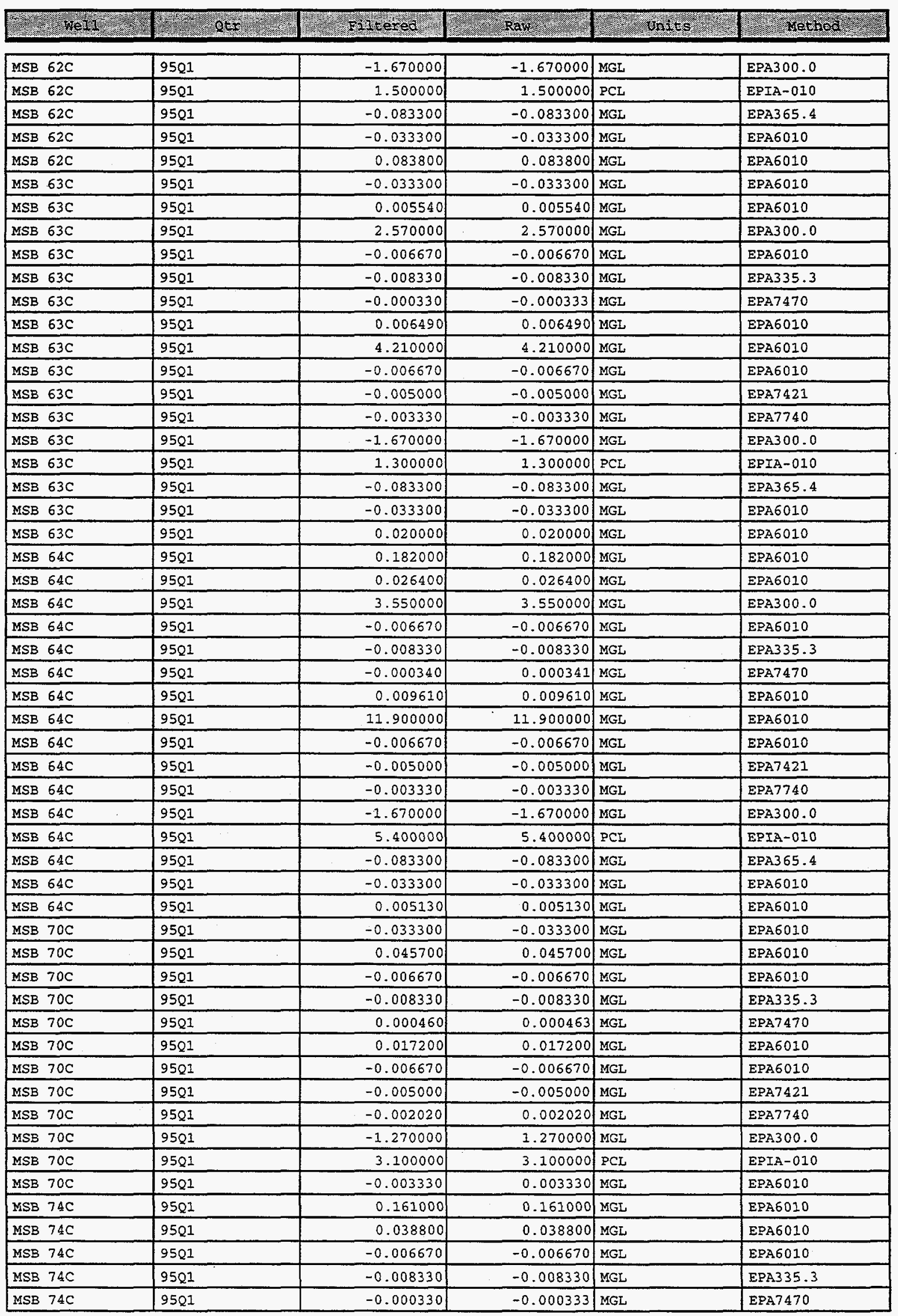


WSRC-TR-00347

November, 1997

Appendix I1-118

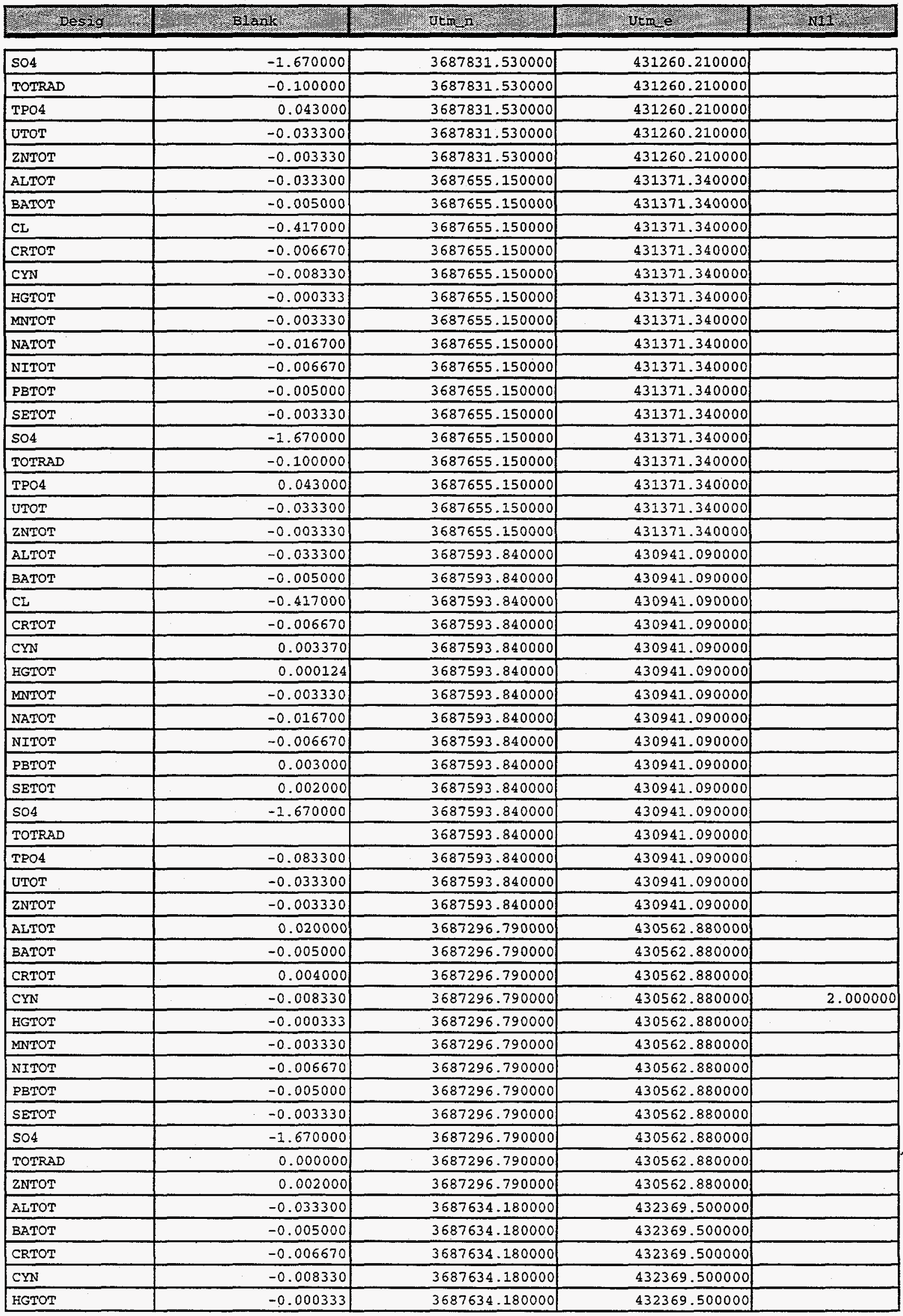


WSRC-TR-00347

November, 1997

Appendix II-119

\begin{tabular}{|c|c|c|c|c|c|}
\hline MSB $74 \mathrm{C}$ & $95 Q 1$ & -0.003200 & 0.003200 & MGL & EPA6010 \\
\hline MSB $74 \mathrm{C}$ & 9501 & -0.006670 & -0.006670 & MGL & EPA6010 \\
\hline MSB $74 \mathrm{C}$ & 9521 & -0.005000 & -0.005000 & MGL & EPA7421 \\
\hline MSB $74 \mathrm{C}$ & 9501 & -0.003330 & -0.003330 & MGL & EPA7740 \\
\hline MSB $74 \mathrm{C}$ & 9501 & 10.800000 & 10.800000 & MGL & EPA300.0 \\
\hline MSB $74 \mathrm{C}$ & 9501 & -0.200000 & -0.200000 & PCL & EPIA-010 \\
\hline MSB $74 \mathrm{C}$ & 9501 & 0.006890 & 0.006890 & MGL & EPA 6010 \\
\hline MSB $75 \mathrm{C}$ & 9521 & -0.027800 & 0.027800 & MGL & EPA6010 \\
\hline MSB $75 \mathrm{C}$ & 9501 & 0.017900 & 0.017900 & MGL & EPA 6010 \\
\hline MSB $75 \mathrm{C}$ & 9501 & -0.006670 & -0.006670 & MGL & EPA 6010 \\
\hline MSB $75 \mathrm{C}$ & 9501 & -0.008330 & -0.008330 & MGL & EPA335.3 \\
\hline MSB $75 \mathrm{C}$ & 9501 & -0.000330 & -0.000333 & MGL, & EPA7470 \\
\hline MSB $75 C$ & 9501 & 0.035500 & 0.035500 & MGL & EPA 6010 \\
\hline MSB $75 \mathrm{C}$ & 9501 & -0.006670 & -0.006670 & MGL & EPA6010 \\
\hline MSB $75 \mathrm{C}$ & 9501 & 0.007950 & 0.007950 & MGL & EPA7421 \\
\hline MSB $75 \mathrm{C}$ & 9501 & -0.003330 & -0.003330 & MGL & EPA7740 \\
\hline MSB $75 \mathrm{C}$ & 9501 & 14.000000 & 14.000000 & MGL & ERA 300.0 \\
\hline MSB $75 \mathrm{C}$ & 9501 & -0.200000 & -0.200000 & PCL & EPIA-010 \\
\hline MSB $75 \mathrm{C}$ & 9501 & 0.012400 & 0.012400 & MGL & EPA 6010 \\
\hline MSB $76 \mathrm{C}$ & 9501 & 0.131000 & 0.131000 & MGL & EPA6010 \\
\hline MSB $76 \mathrm{C}$ & 9521 & 0.013900 & 0.013900 & MGL & EPA6010 \\
\hline MSB $76 \mathrm{C}$ & 9501 & -0.006670 & -0.006670 & MGL & EPA6010 \\
\hline MSB $76 \mathrm{C}$ & 9501 & -0.008330 & -0.008330 & MGL & EPA335.3 \\
\hline MSB $76 \mathrm{C}$ & $95 Q 1$ & -0.000330 & -0.000333 & MGL & EPA7470 \\
\hline MSB $76 \mathrm{C}$ & $95 Q 1$ & -0.003290 & 0.003290 & MGL & EPA 6010 \\
\hline MSB $76 \mathrm{C}$ & 9501 & -0.006670 & -0.006670 & MGL & EPA 6010 \\
\hline MSB $76 \mathrm{C}$ & 9501 & -0.005000 & -0.005000 & MGL & EPA.7421 \\
\hline MSB $76 \mathrm{C}$ & 9501 & -0.003330 & -0.003330 & MGL & EPA7740 \\
\hline MSB $76 \mathrm{C}$ & 9581 & 1.760000 & 1.760000 & MGL & EPA 300.0 \\
\hline MSB $76 \mathrm{C}$ & 9501 & -0.100000 & -0.100000 & PCL & EPIA-010 \\
\hline MSB $76 \mathrm{C}$ & 9501 & 0.010300 & 0.010300 & MGL & EPA 6010 \\
\hline MSB $79 \mathrm{C}$ & 9521 & 0.167000 & 0.167000 & MGL & EPA $6010 \mid$ EPA 6 \\
\hline MSB $79 \mathrm{C}$ & 9501 & 0.010250 & 0.010250 & MGL & EPA6010|EPA6 \\
\hline MSB $79 \mathrm{C}$ & 9501 & -0.006670 & -0.006670 & MGL & EPA6010 \\
\hline MSB $79 C$ & 9501 & -0.008330 & -0.008330 & MGL, & EPA335.3 \\
\hline MSB $79 \mathrm{C}$ & 9501 & -0.000330 & -0.000333 & MGL & EPA7470 \\
\hline MSB $79 \mathrm{C}$ & 9501 & 0.003700 & 0.003700 & MGL & EPA6010|EPA6 \\
\hline MSB $79 \mathrm{C}$ & 9501 & -0.006670 & -0.006670 & MGL & EPA6010/EPA6 \\
\hline MSB $79 \mathrm{C}$ & 9521 & -0.005000 & -0.005000 & MGL & EPA7421 \\
\hline MSB $79 \mathrm{C}$ & 9501 & -0.002160 & 0.002160 & MGL & EPA7740 \\
\hline MSB $79 \mathrm{C}$ & 9501 & 1.960000 & 1.960000 & MGL & EPA 300.0 \\
\hline MSB $79 \mathrm{C}$ & 9501 & 1.400000 & 1.400000 & PCL & EPIA-010 \\
\hline MSB $79 \mathrm{C}$ & 9501 & -0.002300 & 0.002300 & MGL & EPA6010 \\
\hline MSB $87 \mathrm{C}$ & 9501 & -0.033300 & -0.033300 & MGL & EPA6010 \\
\hline MSB $87 \mathrm{C}$ & 9501 & -0.004610 & 0.004610 & MGL & EPA6010 \\
\hline MSB $87 C$ & 9501 & -0.006670 & -0.006670 & MGL & EPA.6010. \\
\hline MSB $87 \mathrm{C}$ & 9501 & -0.008330 & -0.008330 & MGL & ERA335.3 \\
\hline MSB $87 \mathrm{C}$ & 9501 & -0.000330 & -0.000333 & MGL & EPA7470 \\
\hline MSB $87 C$ & 9501 & 0.005490 & 0.005490 & MGL & EPA6010 \\
\hline MSB $87 \mathrm{C}$ & 9501 & -0.006670 & -0.006670 & MGL & EPA6010 \\
\hline MSB $87 C$ & 9501 & -0.005000 & -0.005000 & MGL & EPA7421 \\
\hline MSB $87 \mathrm{C}$ & 9501 & -0.003330 & -0.003330 & MGL & EPA7740 \\
\hline MSB $87 \mathrm{C}$ & 9501 & -1.670000 & -1.670000 & MGL & EPA3 00.0 \\
\hline MSB $87 \mathrm{C}$ & 9501 & -0.300000 & -0.300000 & PCL & EPIA-010 \\
\hline
\end{tabular}


WSRC-TR-00347

November, 1997

Appendix II-120

\begin{tabular}{|c|c|c|c|c|}
\hline 1007 & (2) & f & 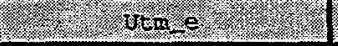 & 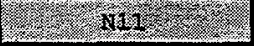 \\
\hline MNTOT & -0.003330 & 3687634.180000 & 432369.500000 & \\
\hline NITOT & -0.006670 & 3687634.180000 & 432369.500000 & \\
\hline PBTOT & -0.005000 & 3687634.180000 & 432369.500000 & \\
\hline SETOT & -0.003330 & 3687634.180000 & 432369.500000 & \\
\hline $\mathrm{SO} 4$ & -1.670000 & 3687634.180000 & 432369.500000 & \\
\hline TOTRAD & & 3687634.180000 & 432369.500000 & \\
\hline ZNTOT & -0.003330 & 3687634.180000 & 432369.500000 & \\
\hline AITOT & -0.033300 & 3687286.430000 & 432020.590000 & \\
\hline BATOT & -0.005000 & 3687286.430000 & 432020.590000 & \\
\hline CRTOT & -0.006670 & 3687286.430000 & 432020.590000 & \\
\hline CYN & -0.008330 & 3687286.430000 & 432020.590000 & \\
\hline HGTOT & -0.000333 & 3687286.430000 & 432020.590000 & \\
\hline MNTOT & -0.003330 & 3687286.430000 & 432020.590000 & \\
\hline NITOT & -0.006670 & 3687286.430000 & 432020.590000 & \\
\hline PBTOT & -0.005000 & 3687286.430000 & 432020.590000 & \\
\hline SETOT & -0.003330 & 3687286.430000 & 432020.590000 & \\
\hline $\mathrm{SO} 4$ & -1.670000 & 3687286.430000 & 432020.590000 & \\
\hline TOTRAD & 0.000000 & 3687286.430000 & 432020.590000 & \\
\hline ZNTOT & -0.003330 & 3687286.430000 & 432020.590000 & \\
\hline ALTOT & -0.033300 & 3687670.770000 & 430415.780000 & \\
\hline BATOT & -0.005000 & 3687670.770000 & 430415.780000 & \\
\hline CRTOT & -0.006670 & 3687670.770000 & 430415.780000 & \\
\hline CYN & -0.008330 & 3687670.770000 & 430415.780000 & \\
\hline HGTOT & -0.000333 & 3687670.770000 & 430415.780000 & \\
\hline MNTOT & -0.003330 & 3687670.770000 & 430415.780000 & \\
\hline NITOT & -0.006670 & 3687670.770000 & 430415.780000 & \\
\hline PBTOT & -0.005000 & 3687670.770000 & 430415.780000 & \\
\hline SETOT & -0.003330 & 3687670.770000 & 430415.780000 & \\
\hline so4 & -1.670000 & 3687670.770000 & 430415.780000 & \\
\hline TOTRAD & 0.000000 & 3687670.770000 & 430415.780000 & \\
\hline ZNTOT & -0.003330 & 3687670.770000 & 430415.780000 & \\
\hline ALTOT & -0.033300 & 3687090.070000 & 431570.710000 & 2.000000 \\
\hline BATOT & -0.005000 & 3687090.070000 & 431570.710000 & 2.000000 \\
\hline CRTOT & 0.004000 & 3687090.070000 & 431570.710000 & \\
\hline CYN & & 3687090.070000 & 431570.710000 & \\
\hline HGTOT & -0.000333 & 3687090.070000 & 431570.710000 & \\
\hline MNTOT & -0.002665 & 3687090.070000 & 431570.710000 & 2.000000 \\
\hline NITOT & -0.006670 & 3687090.070000 & 431570.710000 & 2.000000 \\
\hline PBTOT & -0.005000 & 3687090.070000 & 431570.710000 & \\
\hline SETOT & -0.003330 & 3687090.070000 & 431570.710000 & \\
\hline SO4 & -1.570000 & 3687090.070000 & 431570.710000 & \\
\hline TOTRAD & 0.000000 & 3687090.070000 & 431570.710000 & \\
\hline ZNTOT & 0.002000 & 3687090.070000 & 431570.710000 & \\
\hline ALTOT & -0.033300 & 3688352.340000 & 432276.090000 & \\
\hline BATOT & -0.005000 & 3688352.340000 & 432276.090000 & \\
\hline CRTOT & -0.006670 & 3688352.340000 & 432276.090000 & \\
\hline CYN & & 3688352.340000 & 432276.090000 & \\
\hline HGTOT & -0.000333 & 3688352.340000 & 432276.090000 & \\
\hline MNTOT & -0.003330 & 3688352.340000 & 432276.090000 & \\
\hline NITOT & 0.009480 & 3688352.340000 & 432276.090000 & \\
\hline PBTOT & -0.005000 & 3688352.340000 & 432276.090000 & \\
\hline SETOT & -0.003330 & 3688352.340000 & 432276.090000 & \\
\hline 504 & -1.670000 & 3688352.340000 & 432276.090000 & \\
\hline TOTRAD & & 3688352.340000 & 432276.090000 & \\
\hline
\end{tabular}


WSRC-TR-00347

November, 1997

Appendix II-121

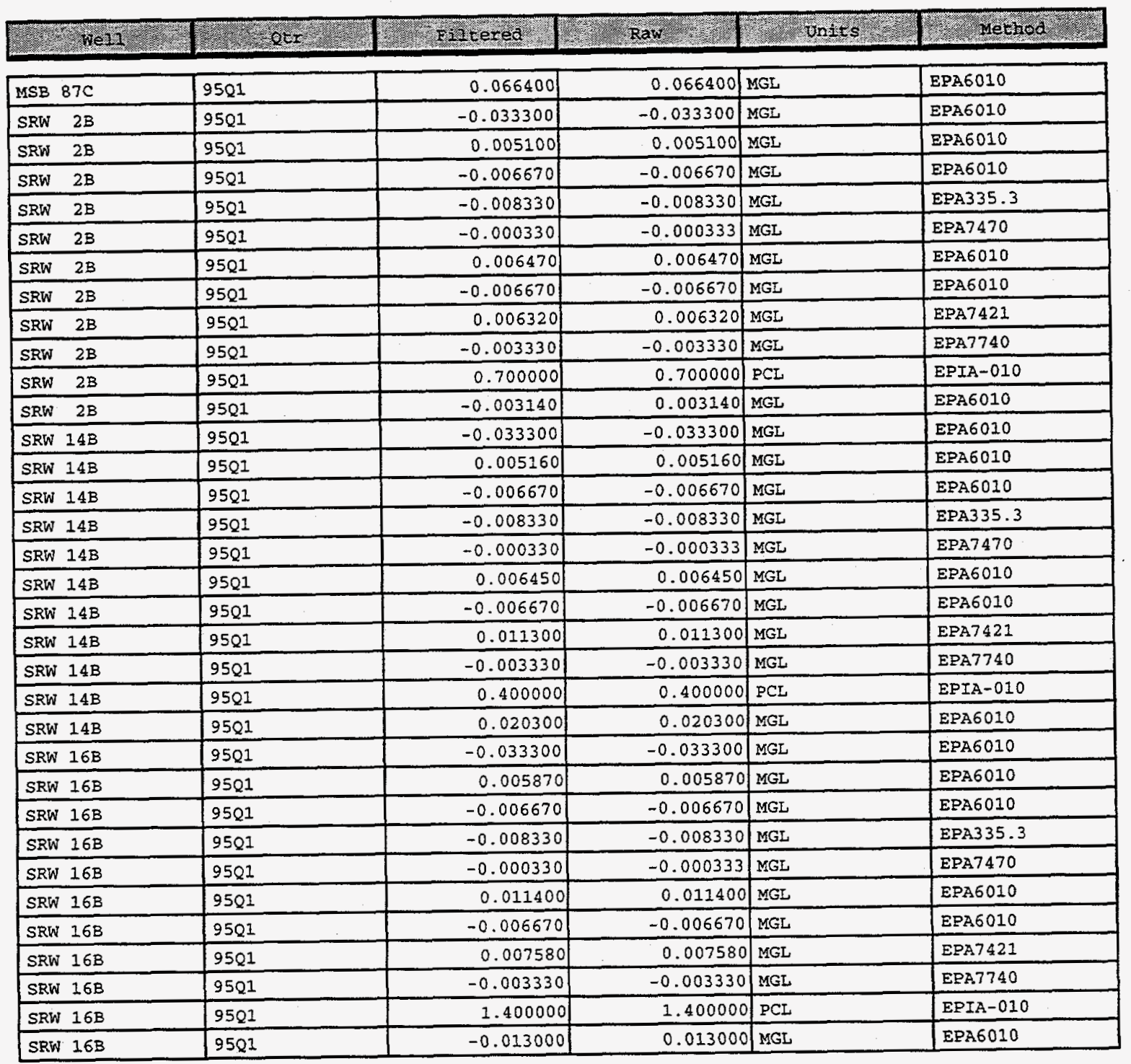


WSRC-TR-00347

November, 1997

Appendix II-122

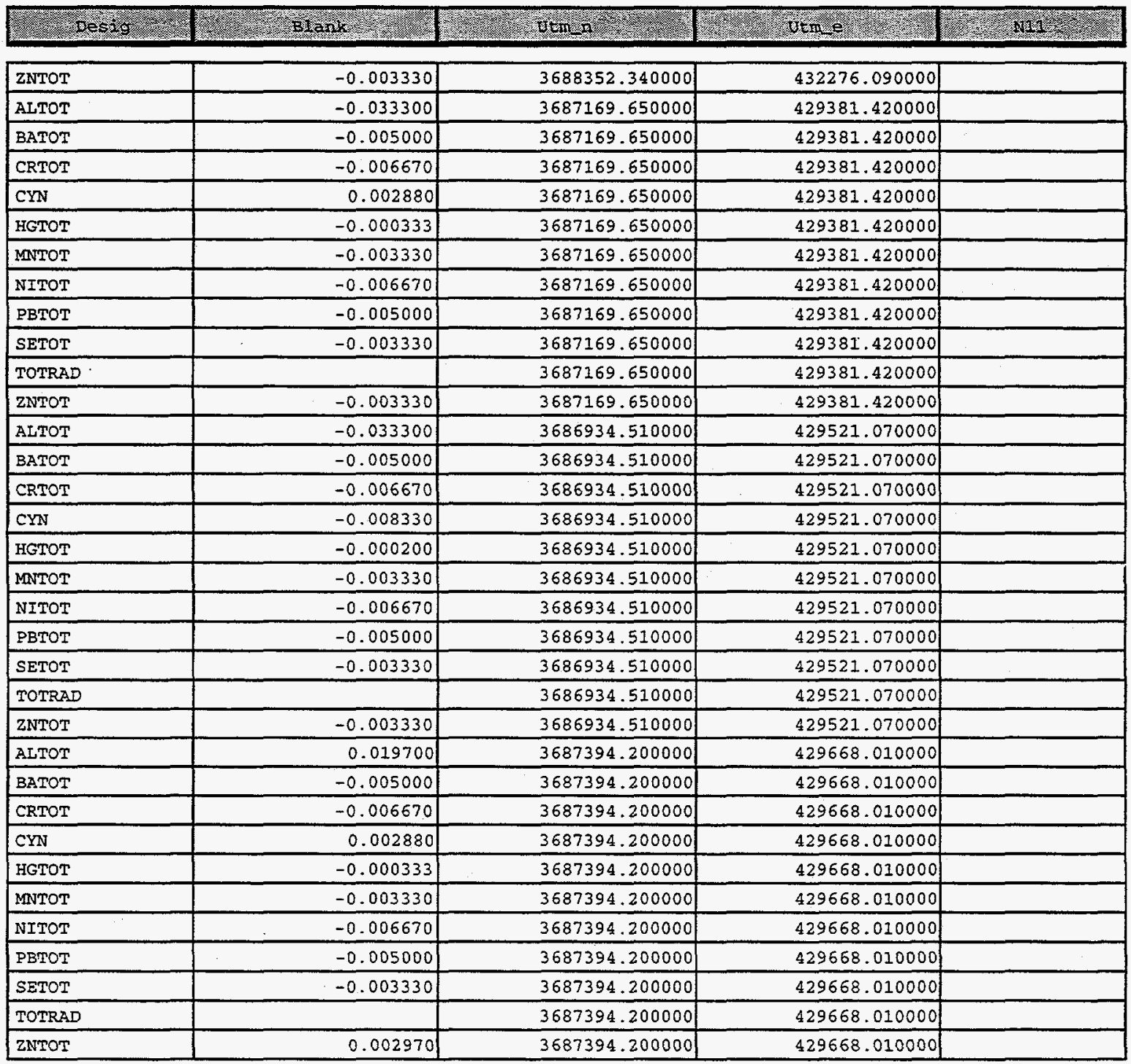


WSRC-TR-00347

November, 1997

Appendix II-123

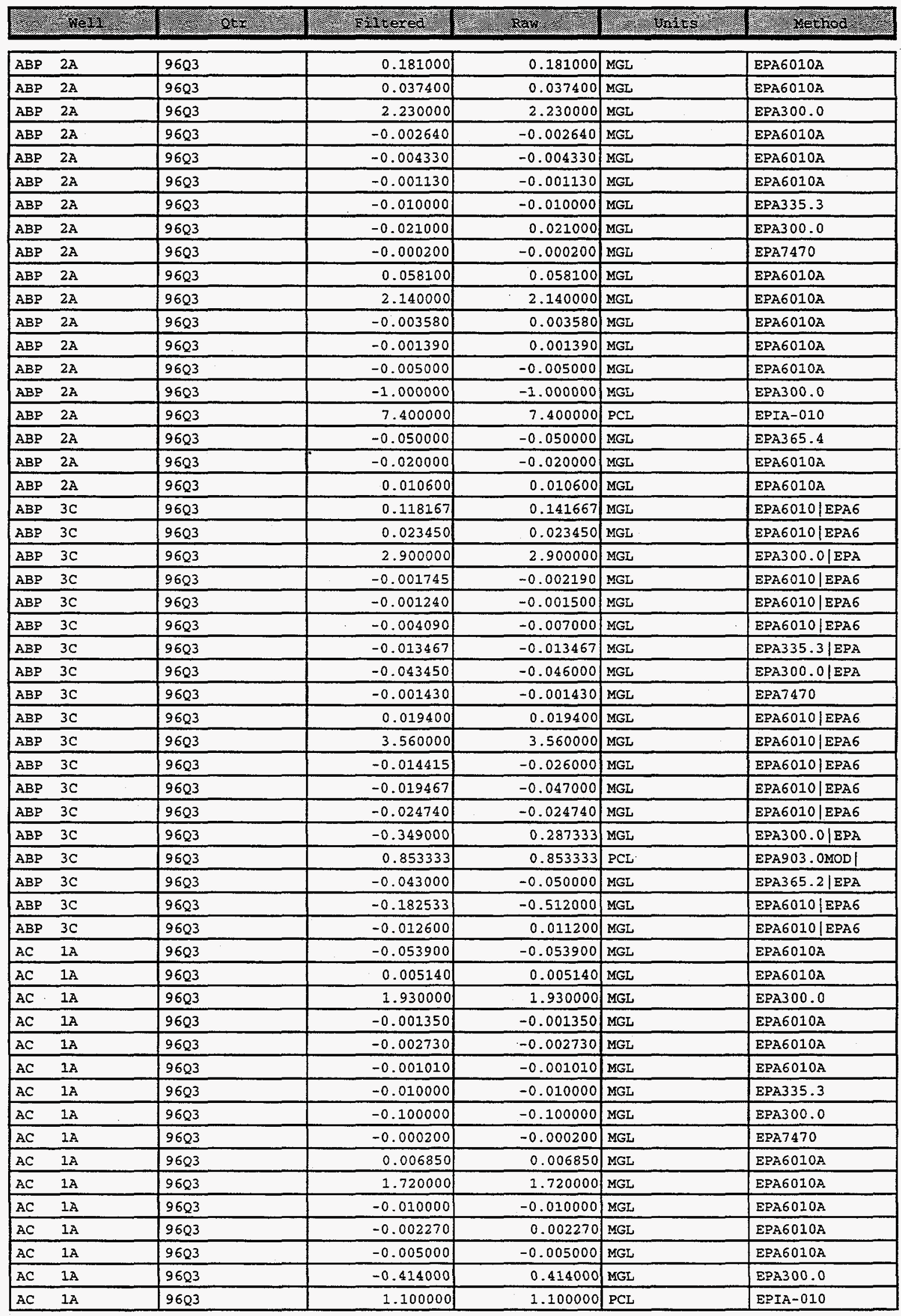




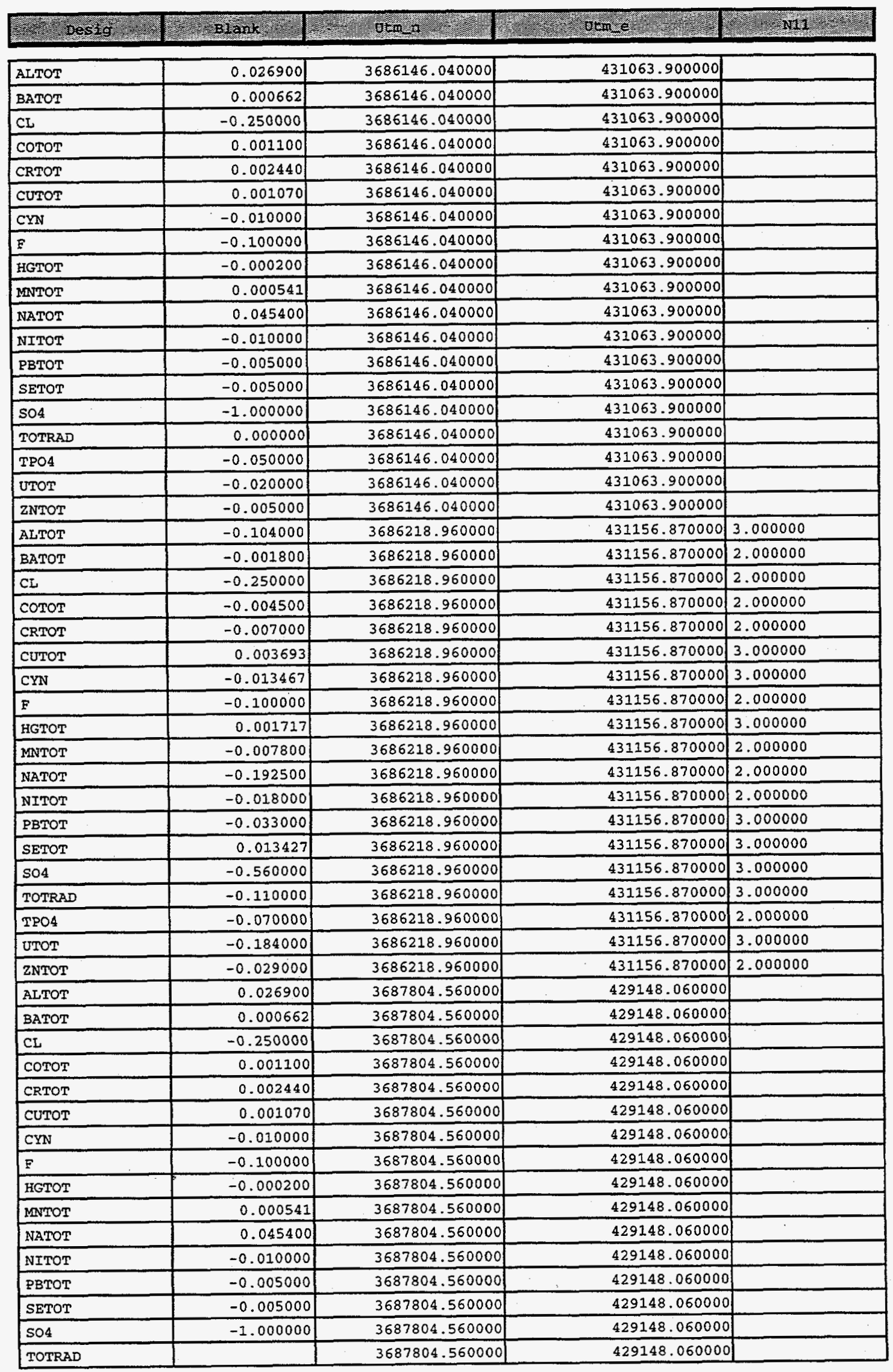




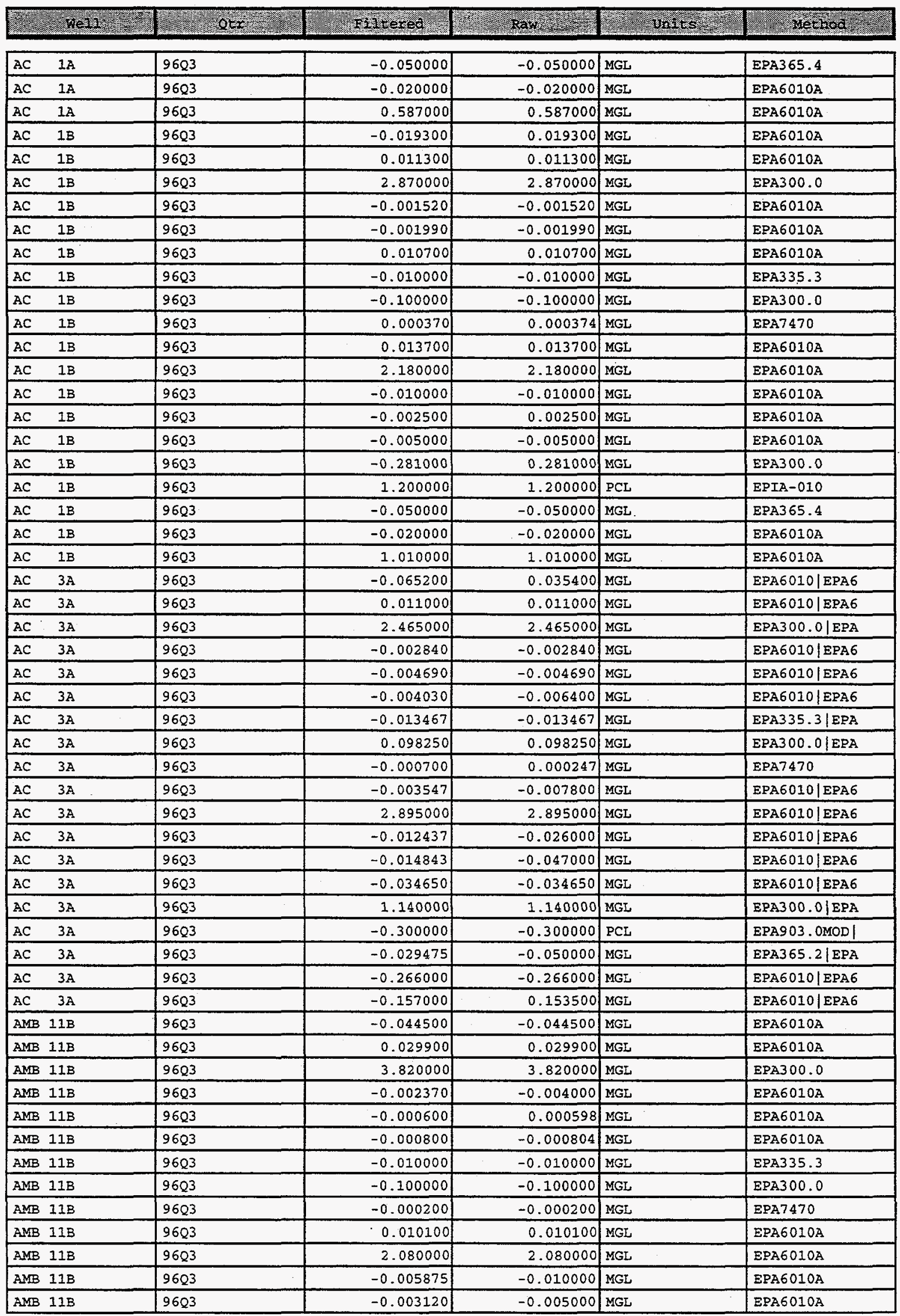




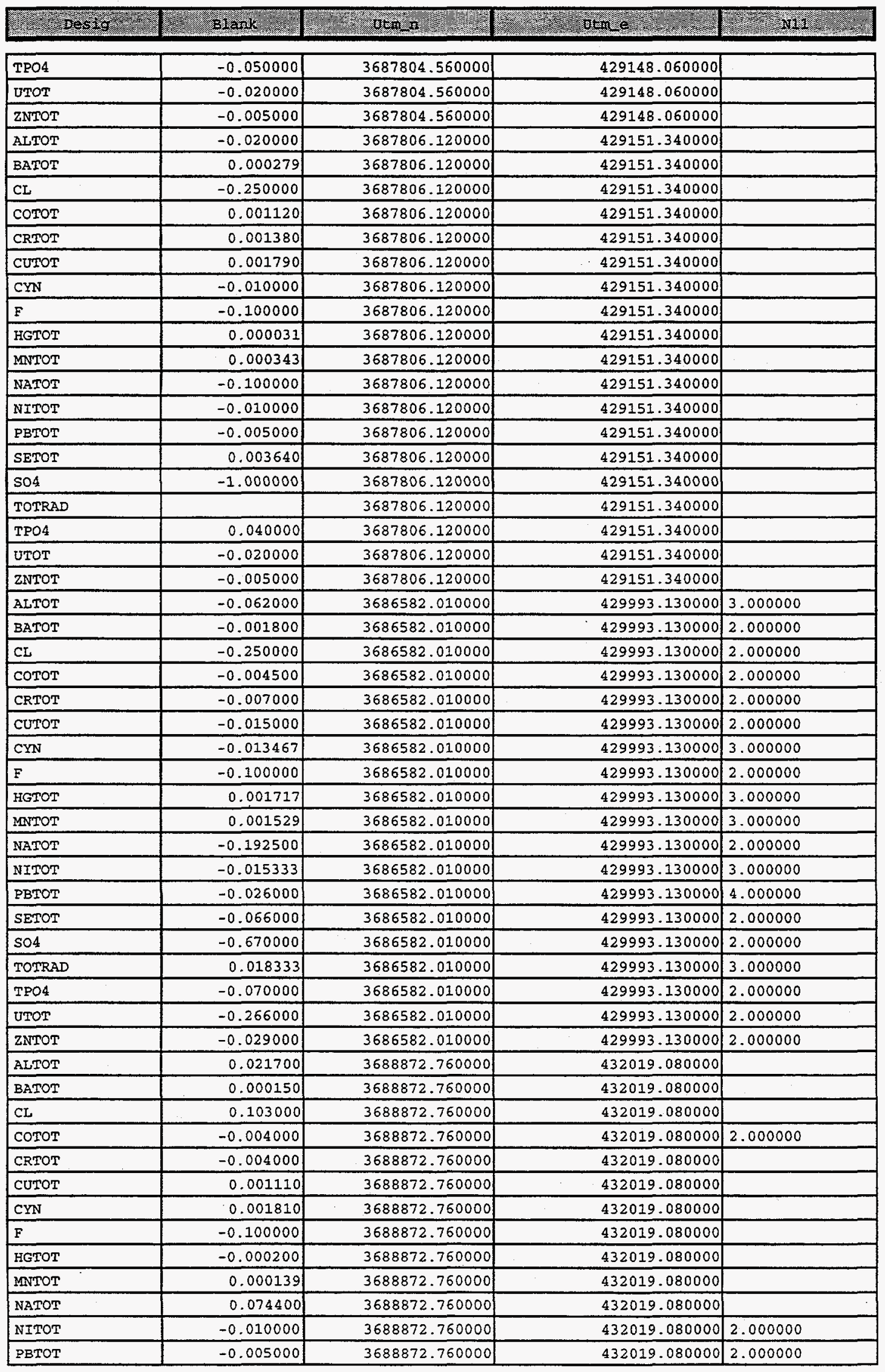




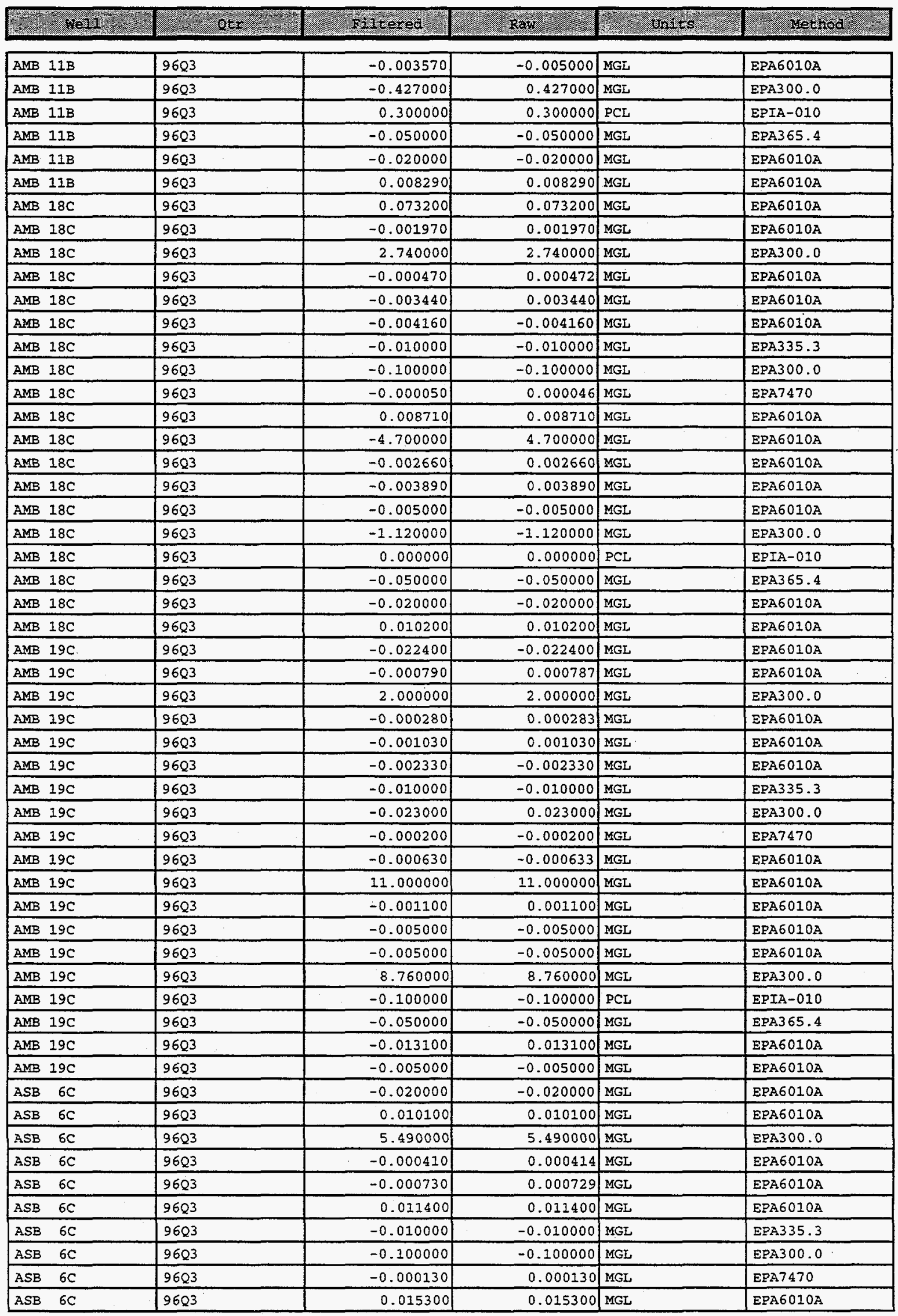


WSRC-TR-00347

November, 1997

Appendix II-128

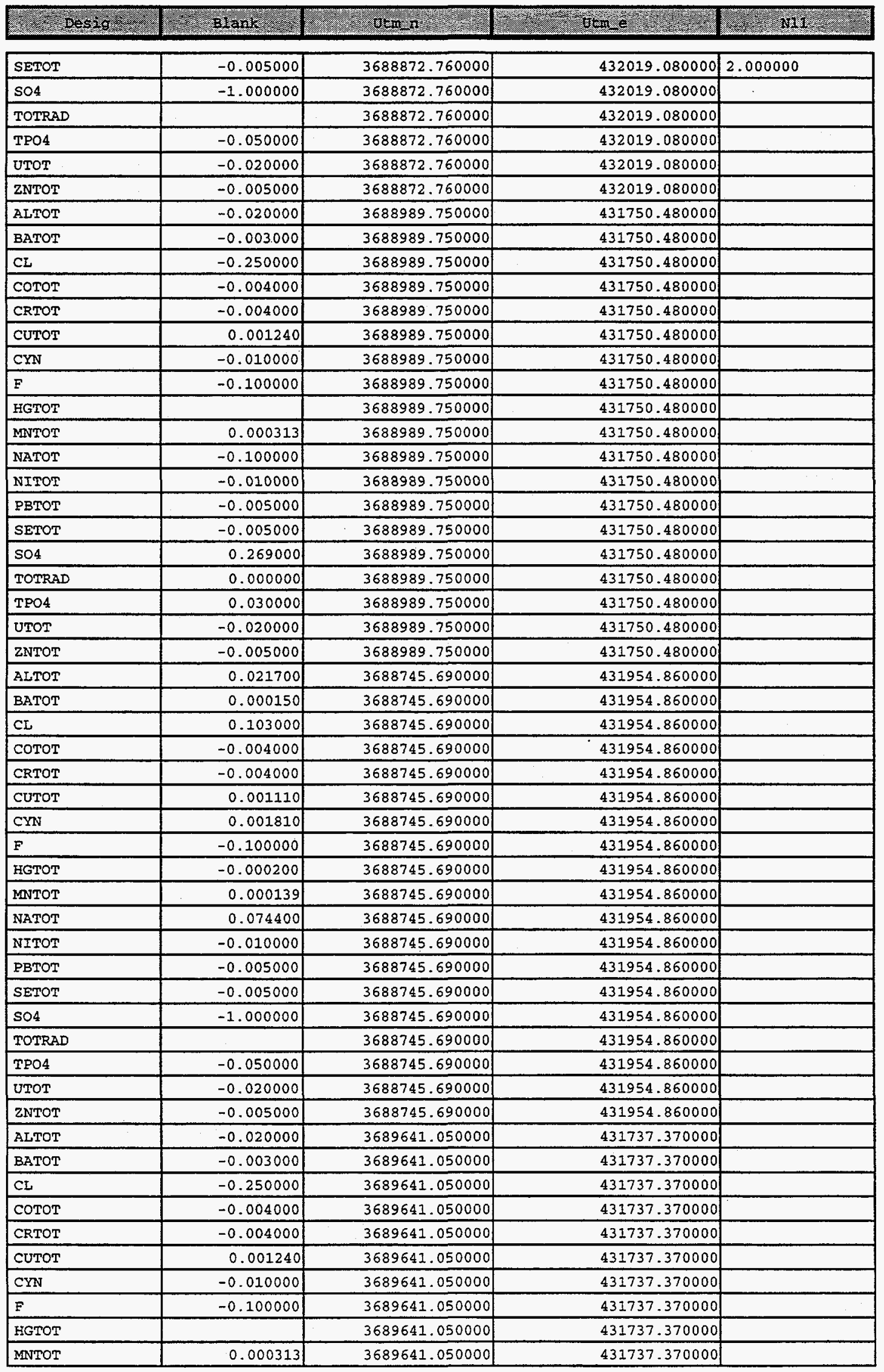


WSRC-TR-00347

November, 1997

Appendix 11-129

\begin{tabular}{|c|c|c|c|c|c|c|}
\hline \multicolumn{2}{|c|}{2.0 .861} & 10 & $3 t$ tered & Rar & (2) & Sustrod \\
\hline ASB & $6 c$ & $96 Q 3$ & -2.500000 & 2.500000 & MGL & EPA6010A \\
\hline A.SB & $6 \mathrm{C}$ & 9603 & -0.010000 & -0.010000 & MGL & EPA6010A \\
\hline A.SB & $6 \mathrm{C}$ & $96 Q 3$ & -0.001730 & 0.001730 & MGL & EPA6010A \\
\hline ASB & $6 \mathrm{C}$ & $96 Q 3$ & -0.005000 & -0.005000 & MGL & EPA $6010 \mathrm{~A}$ \\
\hline ASB & $6 \mathrm{c}$ & $96 Q 3$ & -0.211000 & -0.211000 & MGL & EPA 300.0 \\
\hline ASB & $6 \mathrm{C}$ & $96 Q 3$ & 0.600000 & 0.600000 & PCL & EPIA-010 \\
\hline ASB & $6 \mathrm{C}$ & 9603 & -0.050000 & -0.050000 & MGL & EPA3 65.4 \\
\hline ASB & $6 \mathrm{C}$ & $96 Q 3$ & -0.020000 & -0.020000 & MGL & EPA6010A \\
\hline ASB & $6 \mathrm{C}$ & 9603 & 0.017900 & 0.017900 & MGL & EPA $6010 \mathrm{~A}$ \\
\hline ASB & 8 & 9603 & -0.042600 & -0.042600 & MGL & EPA6010A \\
\hline A.SB & 8 & 9603 & -0.001140 & 0.001140 & MGL & EPA $6010 \mathrm{~A}$ \\
\hline ASB & 8 & $96 Q 3$ & 2.770000 & 2.770000 & MGL & EPA300.0 \\
\hline A.SB & 8 & $96 Q 3$ & -0.002565 & -0.002565 & MGL & EPA6010A \\
\hline ASB & 8 & 9603 & -0.002525 & -0.002525 & MGL & EPA6010A \\
\hline ASB & 8 & $96 Q 3$ & 0.077700 & 0.077700 & MGL & EPA6010A \\
\hline ASB & 8 & 9603 & -0.005775 & -0.005775 & MGL & EPA335.3 \\
\hline ASB & 8 & 9603 & -0.100000 & -0.100000 & MGL & EPA300.0 \\
\hline ASB & 8 & 9603 & -0.000250 & 0.000171 & MGL & EPA7470 \\
\hline ASB & 8 & 9603 & -0.000515 & -0.000941 & MGL & EPA6010A \\
\hline A.SB & 8 & 9603 & 6.035000 & 6.035000 & MGL & EPA6010A \\
\hline ASB & 8 & 9623 & -0.010000 & -0.010000 & MGL & EPA6010A \\
\hline ASB & 8 & $96 Q 3$ & 0.010030 & 0.010030 & MGL & EPA6010A \\
\hline ASB & 8 & $96 Q 3$ & -0.005000 & -0.005000 & MGL & EPA6010A \\
\hline ASB & 8 & 9603 & 4.550000 & 4.550000 & MGL & ERA 300.0 \\
\hline ASB & 8 & 9603 & 0.600000 & 0.600000 & PCL & EPIA-010 \\
\hline A.SB & 8 & 9603 & -0.050000 & -0.050000 & MGL & EPA 365.4 \\
\hline A.SB & 8 & $96 Q 3$ & -0.020000 & -0.020000 & MGL & EPA6010A \\
\hline ASB & 8 & $96 Q 3$ & 0.015530 & 0.015530 & MGL & EPA6010A \\
\hline ASB & $8 \mathrm{C}$ & $96 Q 3$ & -0.043500 & 0.043500 & MGL & EPA6010A \\
\hline ASB & $8 \mathrm{C}$ & 9603 & 0.011800 & 0.011800 & MGL & EPA6010A \\
\hline ASB & $8 \mathrm{C}$ & 9603 & 6.840000 & 6.840000 & MGL & EPA300.0 \\
\hline A.SB & $8 c$ & $96 Q 3$ & -0.000200 & 0.000200 & MGL & EPA6010A \\
\hline A.SB & $8 \mathrm{C}$ & $96 Q 3$ & 0.005020 & 0.005020 & MGL & EPA6010A \\
\hline ASB & $8 \mathrm{C}$ & $96 Q 3$ & 0.007550 & 0.007550 & MGL & EPA6010A \\
\hline ASB & $8 \mathrm{C}$ & 9603 & -0.010000 & -0.010000 & MGL & EPA335.3 \\
\hline ASB & $8 c$ & 9603 & -0.100000 & -0.100000 & MGL & EPA 300.0 \\
\hline ASB & $8 \mathrm{C}$ & 9603 & 0.002680 & 0.002680 & MGL & EPA7470 \\
\hline A.SB & $8 \mathrm{C}$ & 9603 & -0.005720 & 0.005720 & MGL & EPA6010A \\
\hline ASB & $8 \mathrm{C}$ & $96 Q 3$ & 4.470000 & 4.470000 & MGL & EPA6010A \\
\hline ASB & $8 \mathrm{C}$ & 9603 & -0.002950 & 0.002950 & MGL & EPA6010A \\
\hline ASB & $8 C$ & 9603 & -0.002300 & 0.002300 & MGL & EPA6010A \\
\hline ASB & $8 C$ & 9603 & -0.005000 & -0.005000 & MGL & EPA6010A \\
\hline ASB & $8 \mathrm{C}$ & $96 Q 3$ & -0.150000 & 0.150000 & MGL & EPA 300.0 \\
\hline ASB & $8 \mathrm{C}$ & 9603 & 1.200000 & 1.200000 & PCL & EPIA-010 \\
\hline ASB & $8 C$ & 9603 & 0.230000 & 0.230000 & MGL & EPA 365.4 \\
\hline ASB & $8 \mathrm{C}$ & 9603 & -0.014500 & -0.014500 & MGL & EPA6010A \\
\hline ASB & $8 \mathrm{C}$ & 9603 & -0.005000 & -0.005000 & MGL & EPA6010A \\
\hline ASB & $9 c$ & 9603 & -0.020000 & -0.020000 & MGL & EPA6010A \\
\hline ASB & $9 \mathrm{C}$ & $96 Q 3$ & 0.024000 & 0.024000 & MGL & EPA6010A \\
\hline ASB & $9 \mathrm{C}$ & $96 Q 3$ & 4.340000 & 4.340000 & MGL & EPA 300.0 \\
\hline ASB & $9 \mathrm{C}$ & $96 Q 3$ & -0.004000 & -0.004000 & MGL & EPA6010R \\
\hline ASB & $9 \mathrm{C}$ & $96 Q 3$ & -0.004000 & -0.004000 & MGL & EPA6010A \\
\hline ASB & $9 \mathrm{C}$ & 9603 & -0.002370 & -0.002370 & MGL & EPA $6010 \mathrm{~A}$ \\
\hline ASB & $9 \mathrm{C}$ & $96 Q 3$ & -0.010000 & -0.010000 & MGL & EPA335.3 \\
\hline
\end{tabular}


WSRC-TR-00347

November, 1997

Appendix II-130

\begin{tabular}{|c|c|c|c|c|}
\hline $2: 79 \operatorname{ses} 19$ & 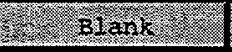 & 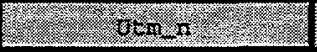 & ns. & (2) \\
\hline NATOT & -0.100000 & 3689641.050000 & 431737.370000 & \\
\hline NITOT & -0.010000 & 3689641.050000 & 431737.370000 & \\
\hline PBTOT & -0.005000 & 3689641.050000 & 431737.370000 & \\
\hline SETOT & -0.005000 & 3689641.050000 & 431737.370000 & \\
\hline 504 & 0.269000 & 3689641.050000 & 431737.370000 & \\
\hline TOTRAD & -0.100000 & 3689641.050000 & 431737.370000 & \\
\hline TPO4 & -0.050000 & 3689641.050000 & 431737.370000 & \\
\hline UTOT & -0.020000 & 3689641.050000 & 431737.370000 & \\
\hline ZNTOT & -0.005000 & 3689641.050000 & 431737.370000 & \\
\hline ALTOT & 0.023100 & 3689886.100000 & 431740.160000 & 2.000000 \\
\hline BATOT & 0.000180 & 3689886.100000 & 431740.160000 & 2.000000 \\
\hline CL & 0.103000 & 3689886.100000 & 431740.160000 & \\
\hline COTOT & -0.004000 & 3689886.100000 & 431740.160000 & 2.000000 \\
\hline CRTOT & -0.004000 & 3689886.100000 & 431740.160000 & 2.000000 \\
\hline CUTOT & -0.004000 & 3689886.100000 & 431740.160000 & 2.000000 \\
\hline CYN & -0.010000 & 3689886.100000 & 431740.160000 & 2.000000 \\
\hline E & -0.100000 & 3689886.100000 & 431740.160000 & \\
\hline HGTOT & -0.000200 & 3689886.100000 & 431740.160000 & 2.000000 \\
\hline MNTOT & -0.002000 & 3689886.100000 & 431740.160000 & 2.000000 \\
\hline NATOT & 0.040200 & 3689886.100000 & 431740.160000 & 2.000000 \\
\hline NITOT & -0.010000 & 3689886.100000 & 431740.160000 & 2.000000 \\
\hline PBTOT & -0.005000 & 3689886.100000 & 431740.160000 & 2.000000 \\
\hline SETOT & -0.005000 & 3689886.100000 & 431740.160000 & 2.000000 \\
\hline SO4 & -1.000000 & 3689886.100000 & 431740.160000 & \\
\hline TOTRAD & & 3689886.100000 & 431740.160000 & \\
\hline TPO4 & -0.050000 & 3689886.100000 & 431740.160000 & \\
\hline UTOT & -0.020000 & 3689886.100000 & 431740.160000 & \\
\hline ZNTOT & -0.005000 & 3689886.100000 & 431740.160000 & 2.000000 \\
\hline ALTOT & -0.020000 & 3689873.020000 & 431736.270000 & \\
\hline BATOT & -0.003000 & 3689873.020000 & 431736.270000 & \\
\hline $\mathrm{CL}$ & -0.250000 & 3689873.020000 & 431736.270000 & \\
\hline COTOT & -0.004000 & 3689873.020000 & 431736.270000 & \\
\hline CRTOT & -0.004000 & 3689873.020000 & 431736.270000 & \\
\hline CUTOT & 0.000599 & 3689873.020000 & 431736.270000 & \\
\hline CYN & -0.010000 & 3689873.020000 & 431736.270000 & \\
\hline$F$ & -0.100000 & 3689873.020000 & 431736.270000 & \\
\hline HGTOT & -0.000200 & 3689873.020000 & 431736.270000 & \\
\hline MATOT & 0.000256 & 3689873.020000 & 431736.270000 & \\
\hline NATOT & -0.100000 & 3689873.020000 & 431736.270000 & \\
\hline NITOT & 0.000951 & 3689873.020000 & 431736.270000 & \\
\hline PBTOT & -0.005000 & 3689873.020000 & 431736.270000 & \\
\hline SETOT & -0.005000 & 3689873.020000 & 431736.270000 & \\
\hline $\mathrm{SO} 4$ & -1.000000 & 3689873.020000 & 431736.270000 & \\
\hline TOTRAD & -0.100000 & 3689873.020000 & 431736.270000 & \\
\hline TPO4 & -0.050000 & 3689873.020000 & 431736.270000 & \\
\hline UTOT & 0.006480 & 3689873.020000 & 431736.270000 & \\
\hline ZNTOT & -0.005000 & 3689873.020000 & 431736.270000 & \\
\hline ALTOT & -0.020000 & 3689630.230000 & 432327.610000 & \\
\hline BATOT & -0.003000 & 3689630.230000 & 432327.610000 & \\
\hline $\mathrm{CL}$ & -0.250000 & 3689630.230000 & 432327.610000 & \\
\hline COTOT & -0.004000 & 3689630.230000 & 432327.610000 & \\
\hline CRTOT & -0.004000 & 3689630.230000 & 432327.610000 & \\
\hline CUTOT & 0.001240 & 3689630.230000 & 432327.610000 & \\
\hline CYN & -0.010000 & 3689630.230000 & 432327.610000 & \\
\hline
\end{tabular}


WSRC-TR-00347

November, 1997

Appendix II-131

\begin{tabular}{|c|c|c|c|c|c|c|}
\hline tor & 8 & 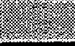 & S1 rerealu & 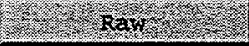 & (2) & $10-10 \mathrm{~d}$ \\
\hline ASE & $9 c$ & $96 Q 3$ & -0.100000 & -0.100000 & MGL & EPA300.0 \\
\hline ASE & $9 \mathrm{C}$ & $96 Q 3$ & -0.000200 & -0.000200 & MGL & EPA 7470 \\
\hline ASE & 9c & $96 Q 3$ & 0.006550 & 0.006550 & MGL & EPA6010A \\
\hline ASE & $9 \mathrm{C}$ & $96 Q 3$ & -2.830000 & 2.830000 & MGL & EPA6010A \\
\hline ASE & $9 \mathrm{C}$ & $96 Q 3$ & -0.010000 & -0.010000 & MGL & EPA6010A \\
\hline ASE & $9 \mathrm{C}$ & 9603 & -0.005000 & -0.005000 & MGL & EPA6010A \\
\hline ASE & $9 \mathrm{C}$ & 9603 & -0.005000 & -0.005000 & MGL & EPA6010A \\
\hline ASE & $9 c$ & $96 Q^{3}$ & -0.151000 & 0.151000 & MGL & EPA 300.0 \\
\hline ASE & $9 \mathrm{C}$ & $96 Q 3$ & 0.700000 & 0.700000 & PCL & EPIA-010 \\
\hline ASE & $9 \mathrm{C}$ & $96 Q 3$ & -0.050000 & -0.050000 & MGL & EPA 365.4 \\
\hline ASE & $9 c$ & 9603 & -0.020000 & -0.020000 & MGL & EPA6010A \\
\hline ASE & $9 C$ & 9603 & 0.008460 & 0.008460 & MGL & EPA6010A \\
\hline & $5 C$ & $96 Q 3$ & -0.128000 & 0.128000 & MGL & EPA6010A \\
\hline $\mathrm{MCE}$ & $5 c$ & 9603 & 0.031300 & 0.031300 & MGL & EPA $6010 A$ \\
\hline $\mathrm{MCE}$ & $5 c$ & 9603 & 2.910000 & 2.910000 & MGL & EPA300.0 \\
\hline$M C E$ & $5 c$ & 9603 & -0.000560 & -0.000563 & MGL & EPA6010A \\
\hline $\mathrm{MCE}$ & $5 \mathrm{C}$ & 9603 & -0.000620 & 0.000623 & MGL & EPA6010A \\
\hline & $5 C$ & 9603 & 0.004080 & 0.004080 & MGL & EPA6010A \\
\hline $\mathrm{MCE}$ & $5 c$ & $96 Q 3$ & -0.010000 & -0.010000 & MGL & EPA335.3 \\
\hline $\mathrm{MCE}$ & $5 c$ & 9603 & -0.100000 & -0.100000 & MGL & EPA300.0 \\
\hline & $5 C$ & 9603 & -0.000100 & 0.000103 & MGL & EPA7470 \\
\hline $\mathrm{MCE}$ & $5 c$ & 9603 & 0.008470 & 0.008470 & MGL & EPA6010A \\
\hline $\mathrm{MCE}$ & $5 c$ & $96 Q 3$ & 6.210000 & 6.210000 & MGL & EPA6010A \\
\hline $\mathrm{MCE}$ & $5 C$ & $96 Q 3$ & -0.003210 & 0.003210 & MGL & EPA6010A \\
\hline $\mathrm{MCE}$ & $5 c$ & 9603 & -0.005000 & -0.005000 & MGL & EPA6010A \\
\hline & $5 c$ & $96 Q 3$ & -0.005000 & -0.005000 & MGL & EPA6010A \\
\hline & $5 c$ & $96 Q 3$ & -0.881000 & 0.881000 & MGL & EPA300.0 \\
\hline & $5 C$ & $96 Q 3$ & -0.200000 & -0.200000 & PCL & EPIA-010 \\
\hline & $5 \mathrm{C}$ & 9603 & 0.160000 & 0.160000 & MGL & EPA 365.4 \\
\hline MCE & $5 \mathrm{C}$ & $96 Q 3$ & -0.020000 & -0.020000 & MGL & EPA $6010 \mathrm{~A}$ \\
\hline MCE & $5 c$ & 9603 & -0.006700 & 0.006700 & MGL & EPA $6010 \mathrm{~A}$ \\
\hline MCE & $6 \mathrm{C}$ & $96 Q 3$ & -0.110000 & 0.047088 & MGL & EPA6010|EPA6 \\
\hline MCE & $6 \mathrm{C}$ & 9603 & 0.034933 & 0.034933 & MGL & EPA6010|EPA6 \\
\hline MCE & $6 \mathrm{C}$ & 9603 & 2.480000 & 2.480000 & MGL & EPA3 $00.0 \mid$ EPA \\
\hline$M C E$ & $6 \mathrm{C}$ & 9603 & -0.001787 & -0.004000 & MGL & EPA6010|EPA6 \\
\hline & $6 \mathrm{C}$ & 9603 & -0.003333 & -0.004000 & MGI & EPA6010|EPA6 \\
\hline & $6 \mathrm{C}$ & 9603 & -0.015000 & -0.015000 & MGL & EPA6010|EPA6 \\
\hline & $6 \mathrm{C}$ & $96 Q 3$ & -0.012600 & -0.012600 & MGL & EPA335.3/EPA \\
\hline MCE & $6 C$ & 9603 & -0.057350 & -0.057350 & MGL & EPA300.0|EPA \\
\hline MCE & $6 \mathrm{C}$ & $96 Q 3$ & -0.000223 & -0.000700 & MGL & EPA7470 \\
\hline MCE & $6 C$ & 9603 & 0.016400 & 0.018633 & MGL & EPA6010/EPA6 \\
\hline MCE & $6 \mathrm{C}$ & $96 Q 3$ & 2.683333 & 3.710000 & MGL & EPA6010|EPA6 \\
\hline & $6 \mathrm{C}$ & 9603 & -0.005573 & -0.010000 & MGL & EPA6010|EPA6 \\
\hline & $6 \mathrm{C}$ & 9603 & -0.017870 & -0.047000 & MGL & EPA6010|EPA6 \\
\hline & $6 C$ & 9603 & -0.025333 & -0.025333 & MGL & EPA6010|EPA6 \\
\hline MCE & $6 C$ & 9603 & 2.426667 & 2.426667 & MGI & EPA $300.0 \mid$ EPA \\
\hline $\mathrm{MCE}$ & $6 \mathrm{C}$ & $96 Q 3$ & -0.316667 & -0.450000 & PCL & EPA903.0MOD \\
\hline MCE & $6 \mathrm{C}$ & 9603 & -0.032950 & -0.050000 & MGL & EPA3 65.2 |EPA \\
\hline MCE & $6 \mathrm{C}$ & $96 Q 3$ & -0.181733 & -0.181733 & MGL & EPA6010/EPA6 \\
\hline & $6 \mathrm{C}$ & 9603 & -0.053000 & -0.053000 & MGL & EPA6010|EPA6 \\
\hline $\mathrm{MCF}$ & $7 C$ & 9603 & 4.070000 & 4.070000 & MGL & EPA6010A \\
\hline $\mathrm{MCF}$ & $7 c$ & $96 Q 3$ & 0.076200 & 0.076200 & MGL & EPA6010A \\
\hline & $7 C$ & $96 Q 3$ & & 2.100000 & MGL & EPA300.0 \\
\hline MCE & $7 c$ & $96 Q 3$ & -0.000600 & 0.000602 & MGL & EPA6010A \\
\hline
\end{tabular}




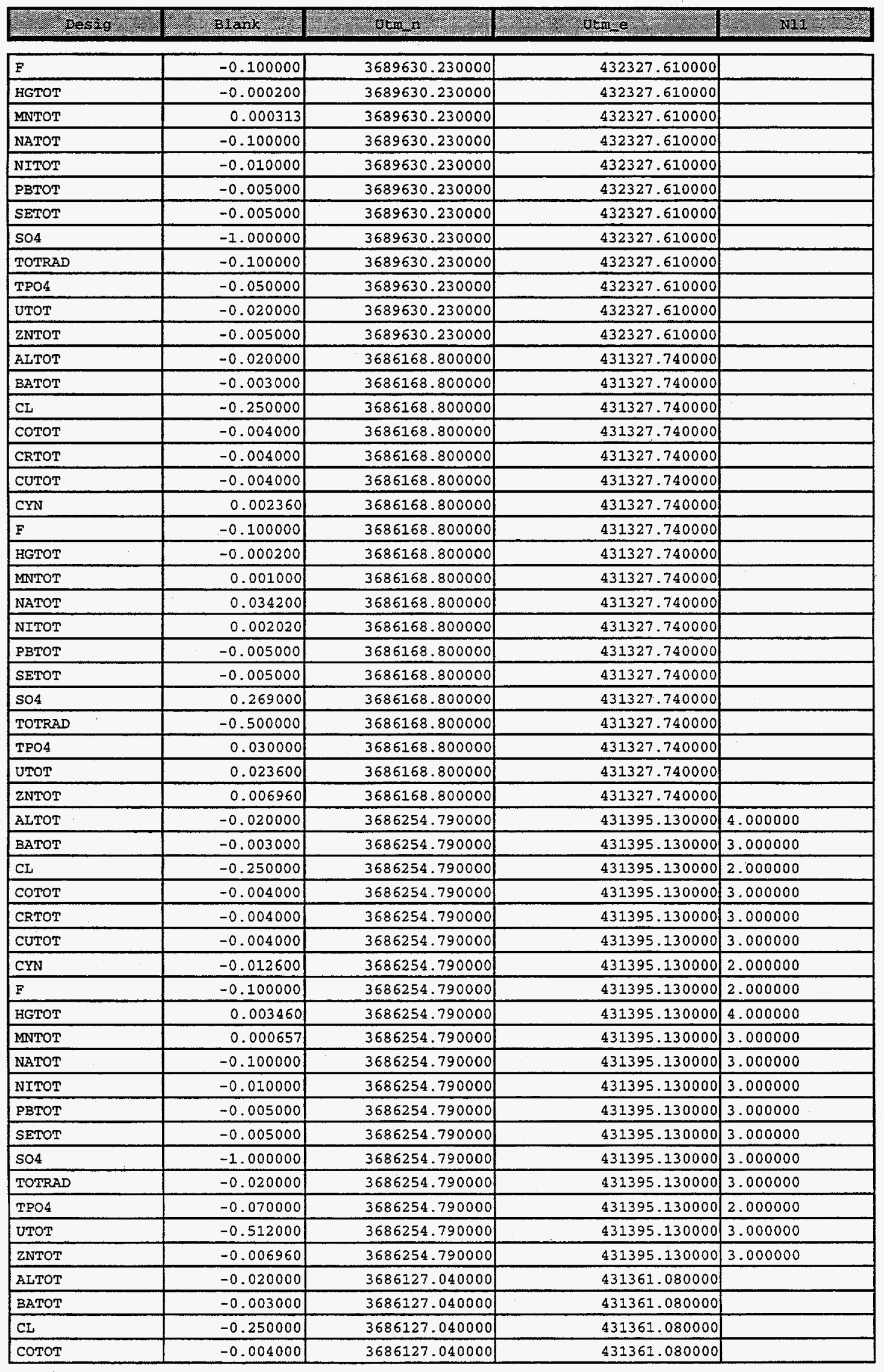




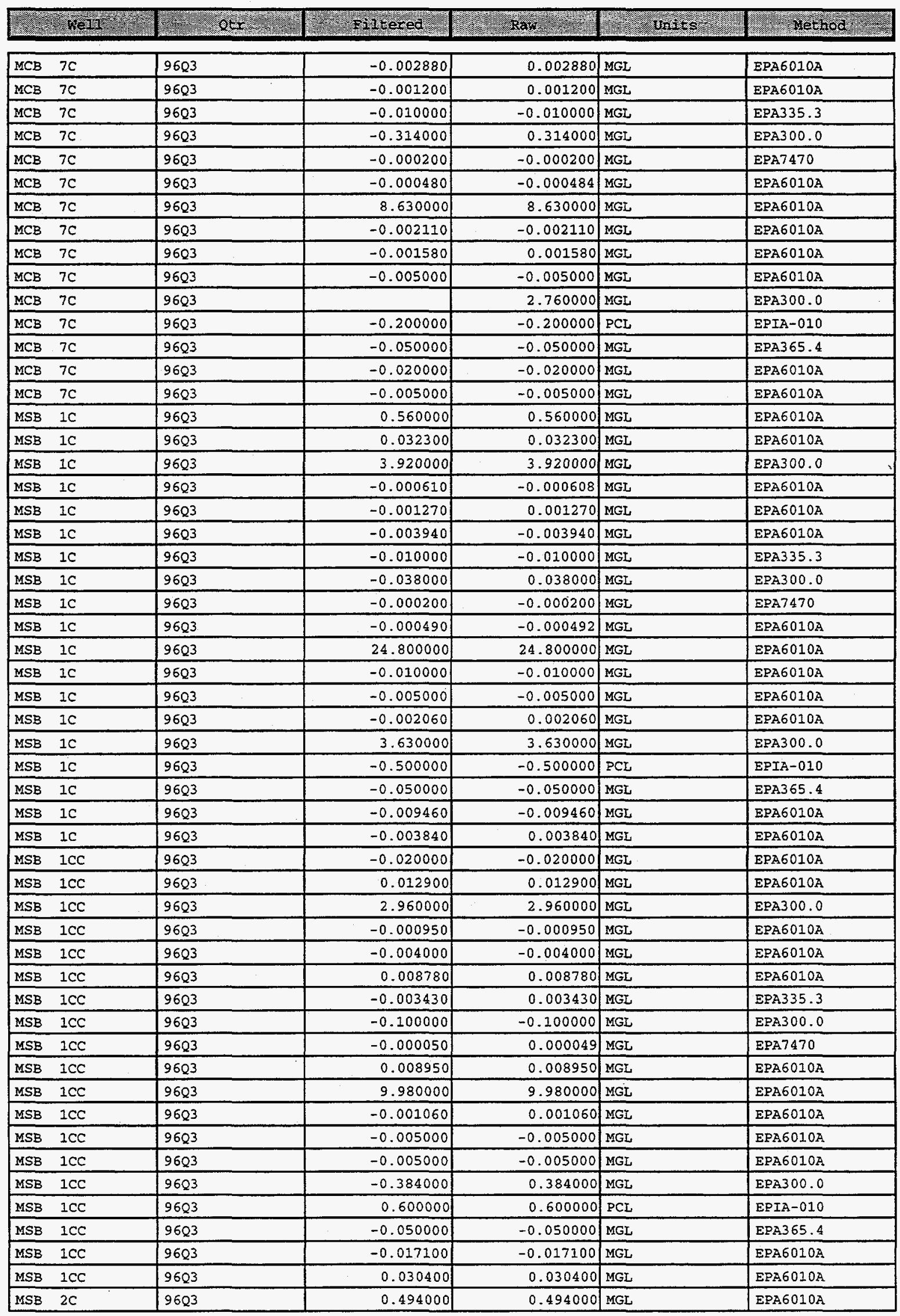




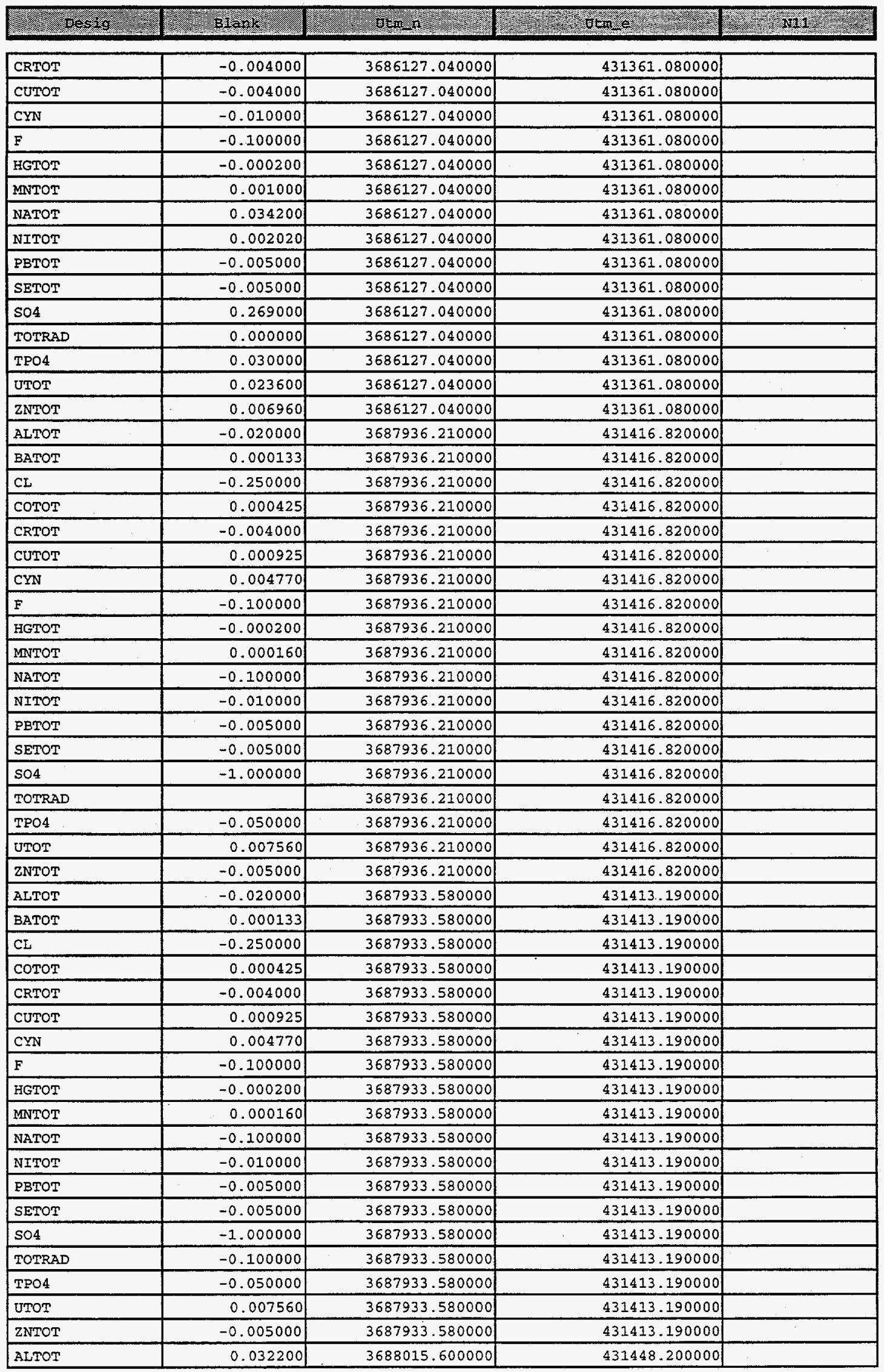




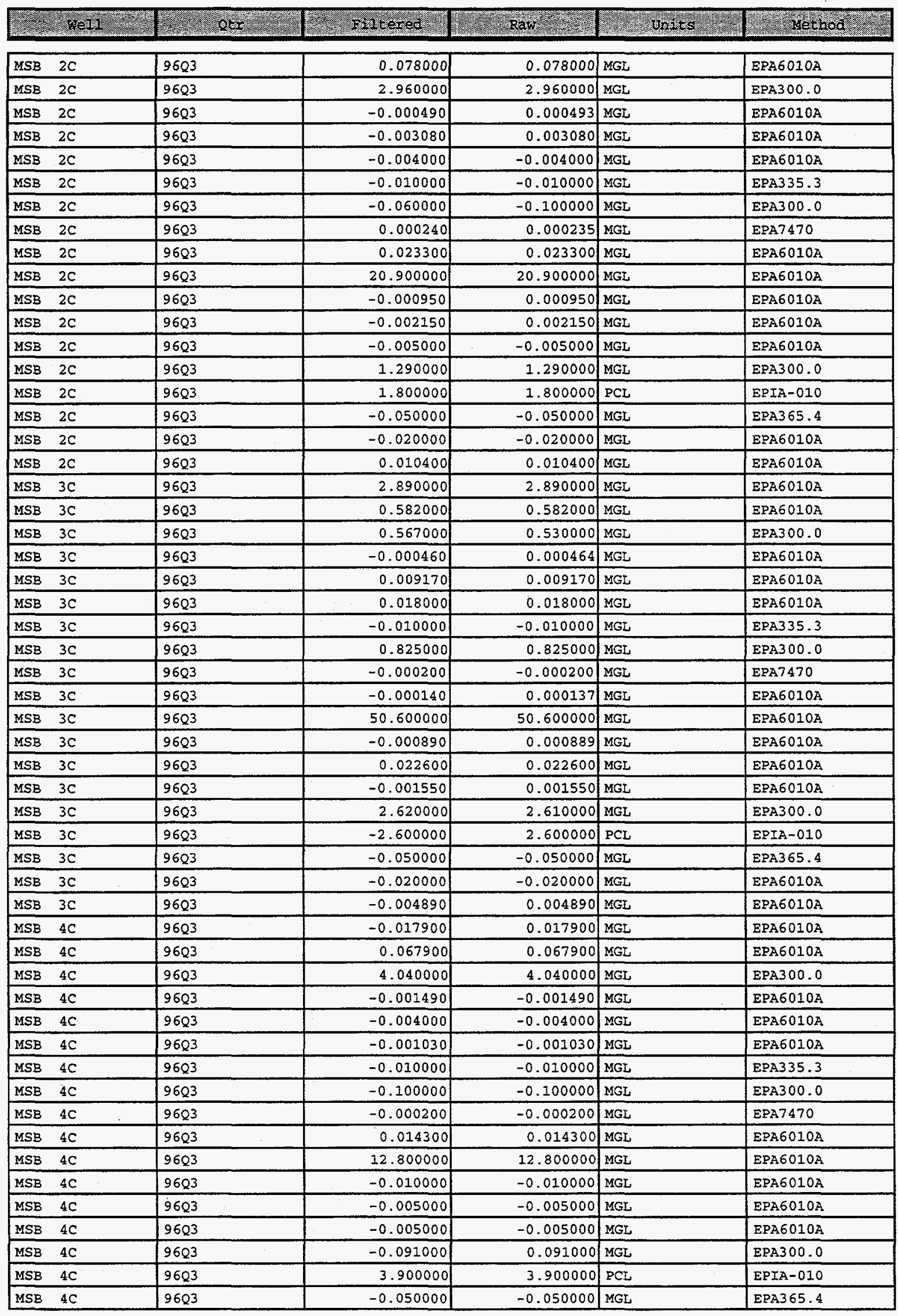




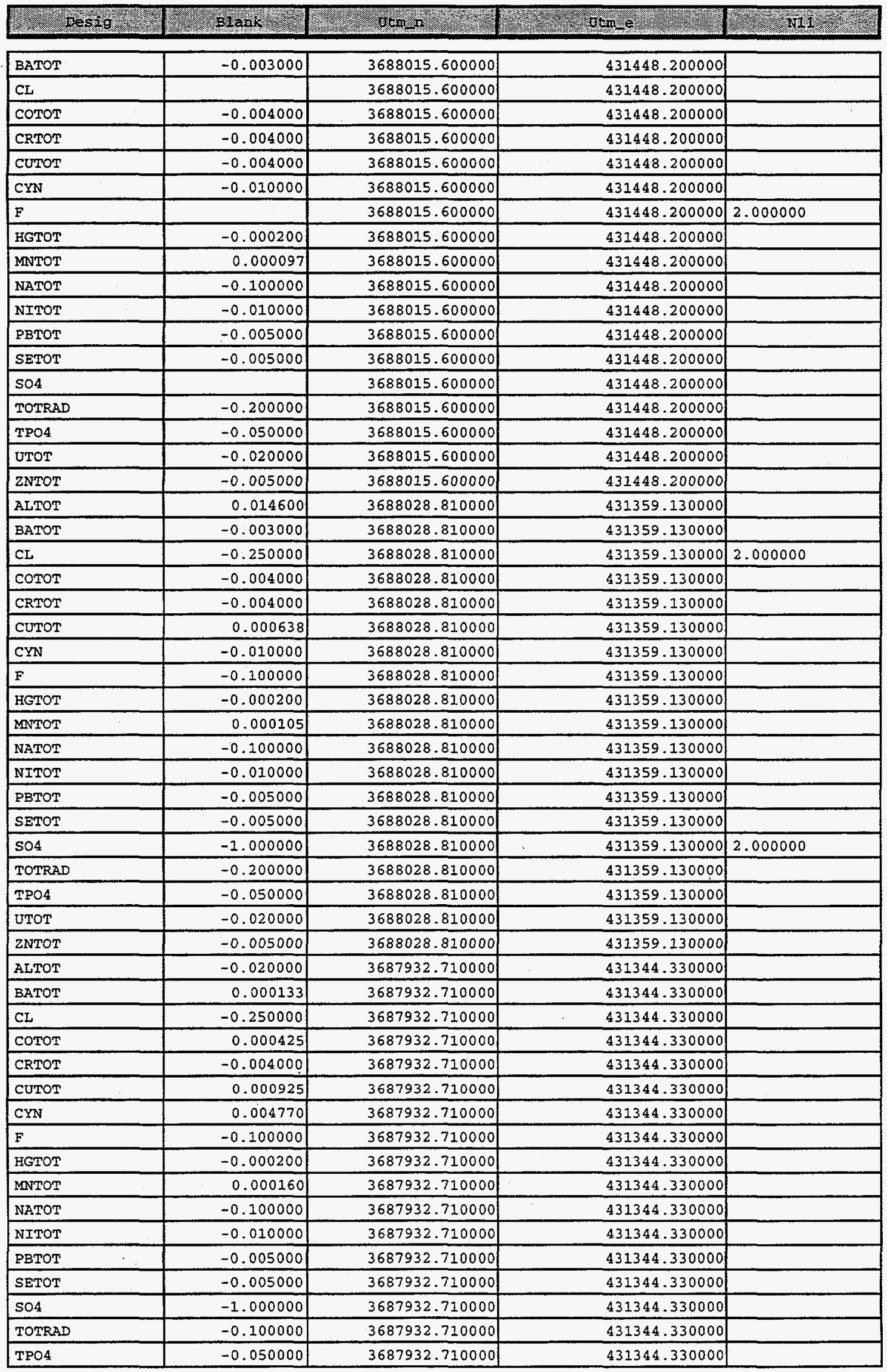


WSRC-TR-00347

November, 1997

Appendix 11-137

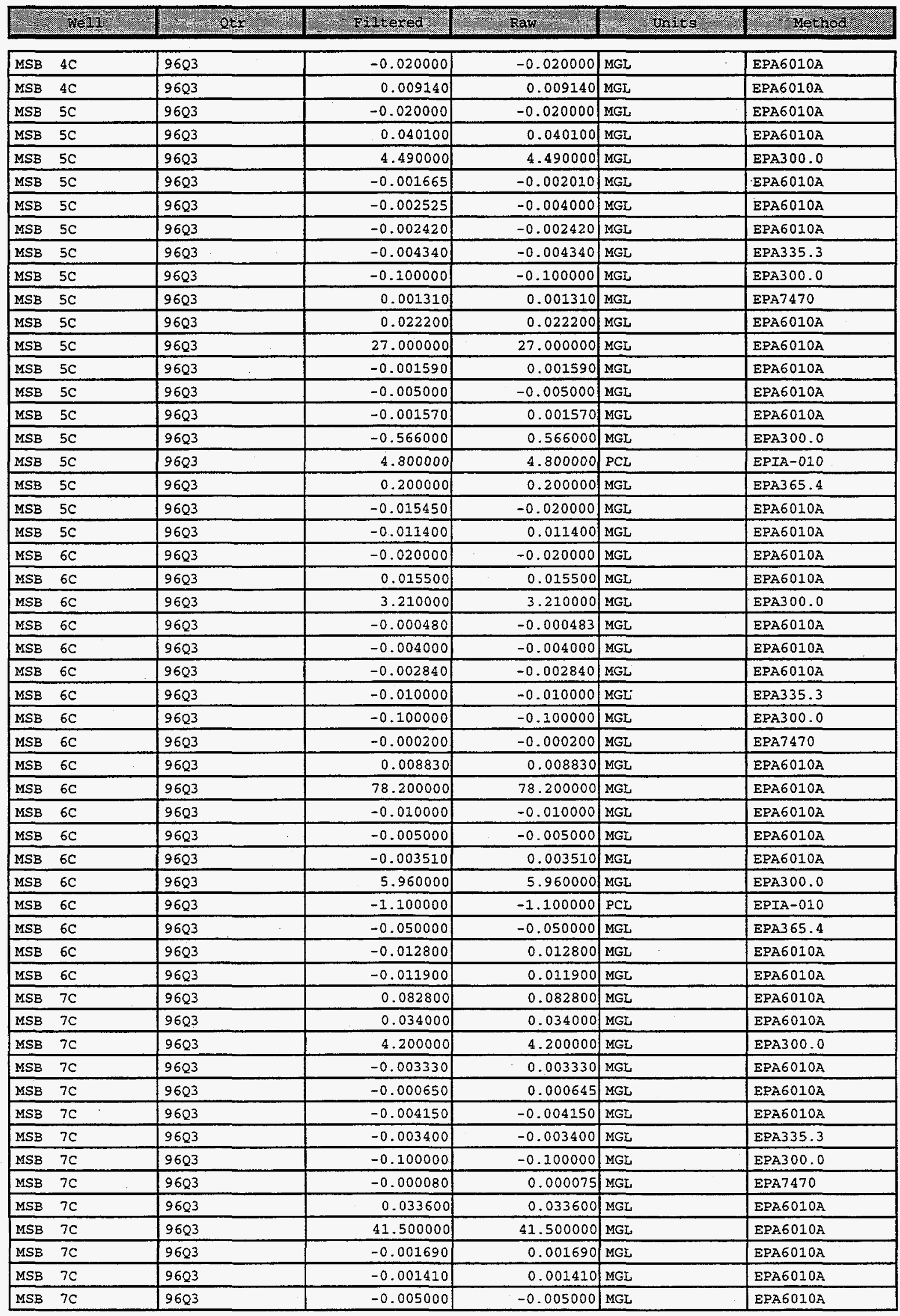




\begin{tabular}{|c|c|c|c|c|}
\hline 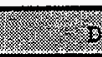 & Blans & 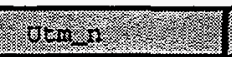 & N7 & 3.7. \\
\hline UTOT & 0.007560 & 3687932.710000 & 431344.330000 & \\
\hline ZNTOT & -0.005000 & 3687932.710000 & 431344.330000 & \\
\hline ALTOT & -0.020000 & 3687693.290000 & 431011.690000 & \\
\hline BATOT & 0.000116 & 3687693.290000 & 431011.690000 & \\
\hline$\overline{C L}$ & -0.250000 & 3687693.290000 & 431011.690000 & \\
\hline COTOT & 0.000930 & 3687693.290000 & 431011.690000 & 2.000000 \\
\hline CRTOT & -0.004000 & 3687693.290000 & 431011.690000 & 2.000000 \\
\hline CUTOT & 0.001800 & 3687693.290000 & 431011.690000 & \\
\hline $\mathrm{CYN}$ & 0.004770 & 3687693.290000 & 431011.690000 & \\
\hline $\boldsymbol{F}$ & -0.100000 & 3687693.290000 & 431011.690000 & \\
\hline HGTOT & -0.000200 & 3687693.290000 & 431011.690000 & \\
\hline MNTOT & 0.000946 & 3687693.290000 & 431011.690000 & \\
\hline NATOT & 0.033200 & 3687693.290000 & 431011.690000 & \\
\hline NITOT & 0.001250 & 3687693.290000 & 431011.690000 & \\
\hline PBTOT & -0.005000 & 3687693.290000 & 431011.690000 & \\
\hline SETOT & -0.005000 & 3687693.290000 & 431011.690000 & \\
\hline SO4 & -1.000000 & 3687693.290000 & 431011.690000 & \\
\hline TOTRAD & 0.500000 & 3687693.290000 & 431011.690000 & \\
\hline TPO4 & -0.050000 & 3687693.290000 & 431011.690000 & \\
\hline UTOT & 0.021200 & 3687693.290000 & 431011.690000 & 2.000000 \\
\hline ZNTOT & 0.007480 & 3687693.290000 & 431011.690000 & \\
\hline ALTOT & -0.020000 & 3687380.310000 & 430996.610000 & \\
\hline BATOT & 0.000116 & 3687380.310000 & 430996.610000 & \\
\hline $\mathrm{CL}$ & -0.250000 & 3687380.310000 & 430996.610000 & \\
\hline COTOT & 0.000930 & 3687380.310000 & 430996.610000 & \\
\hline CRTOT & -0.004000 & 3687380.310000 & 430996.610000 & \\
\hline CUTOT & 0.001800 & 3687380.310000 & 430996.610000 & \\
\hline $\mathrm{CYN}$ & 0.004770 & 3687380.310000 & 430996.610000 & \\
\hline $\mathrm{F}$ & -0.100000 & 3687380.310000 & 430996.610000 & \\
\hline HGTOT & -0.000200 & 3687380.310000 & 430996.610000 & \\
\hline MNTOT & 0.000946 & 3687380.310000 & 430996.610000 & \\
\hline NATOT & 0.033200 & 3687380.310000 & 430996.610000 & \\
\hline NITOT & 0.001250 & 3687380.310000 & 430996.610000 & \\
\hline PBTOT & -0.005000 & 3687380.310000 & 430996.610000 & \\
\hline SETOT & -0.005000 & 3687380.310000 & 430996.610000 & \\
\hline 504 & -1.000000 & 3687380.310000 & 430996.610000 & \\
\hline TOTRAD & 0.500000 & 3687380.310000 & 430996.610000 & \\
\hline TPO4 & -0.050000 & 3687380.310000 & 430996.610000 & \\
\hline UTOT & 0.021200 & 3687380.310000 & 430996.610000 & \\
\hline ZNTOT & 0.007480 & 3687380.310000 & 430996.610000 & \\
\hline ALTOT & -0.020000 & 3687311.410000 & 431191.860000 & - \\
\hline BATOT & 0.000116 & 3687311.410000 & 431191.860000 & \\
\hline $\mathrm{CL}$ & -0.250000 & 3687311.410000 & 431191.860000 & \\
\hline COTOT & 0.000930 & 3687311.410000 & 431191.860000 & \\
\hline CRTOT & -0.004000 & 3687311.410000 & 431191.860000 & \\
\hline CUTOT & 0.001800 & 3687311.410000 & 431191.860000 & \\
\hline $\mathrm{CYN}$ & 0.004770 & 3687311.410000 & 431191.860000 & \\
\hline$E$ & -0.100000 & 3687311.410000 & 431191.860000 & \\
\hline HGTOT & -0.000200 & 3687311.410000 & 431191.860000 & \\
\hline MNTOT & 0.000946 & 3687311.410000 & 431191.860000 & \\
\hline NATOT & 0.033200 & 3687311.410000 & 431191.860000 & \\
\hline NITOT & 0.001250 & 3687311.410000 & 431191.860000 & \\
\hline PBTOT & -0.005000 & 3687311.410000 & 431191.860000 & \\
\hline SETOT & -0.005000 & 3687311.410000 & 431191.860000 & \\
\hline
\end{tabular}




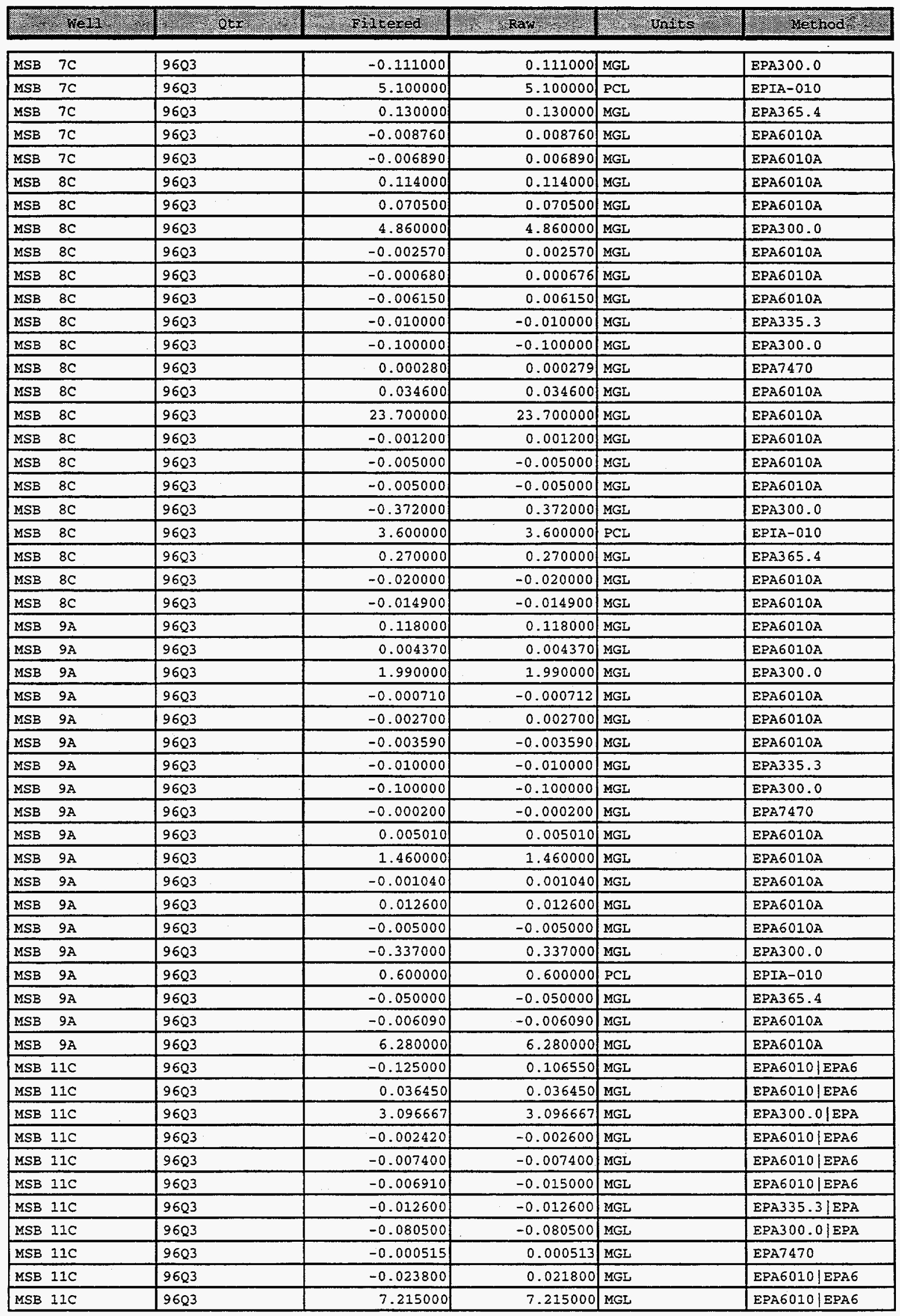




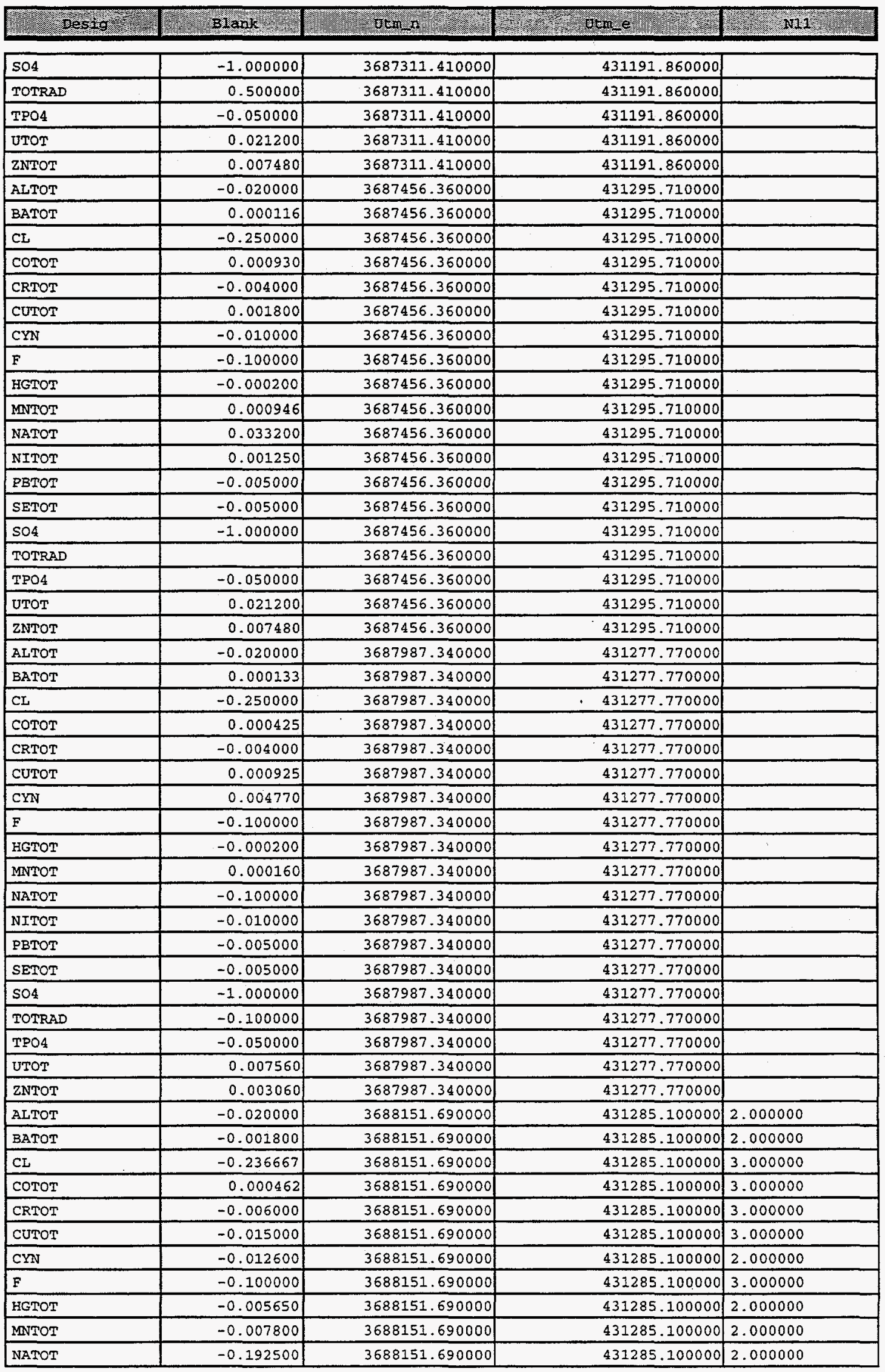


WSRC-TR-00347

November, 1997

Appendix II-141

\begin{tabular}{|c|c|c|c|c|c|}
\hline 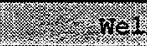 & 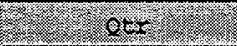 & 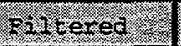 & P. Tra & 1 brits & 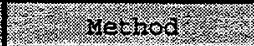 \\
\hline MSB 11C & $96 Q 3$ & -0.010090 & -0.026000 & MGL & EPA6010|EPA6 \\
\hline MSB $11 C$ & 9603 & -0.026000 & -0.026000 & MGL & EPA6010|EPA6 \\
\hline MSB 11C & $96 Q 3$ & -0.035500 & -0.035500 & MGL & EPA6010|EPA6 \\
\hline MSB $11 \mathrm{C}$ & $96 Q 3$ & -0.751000 & -1.000000 & MGL & EPA300.0|EPA \\
\hline MSB $11 \mathrm{C}$ & $96 Q 3$ & 3.290000 & 3.290000 & PCL & EPA903.0MOD| \\
\hline MSB $11 \mathrm{C}$ & $96 Q 3$ & -0.060000 & -0.060000 & MGL & EPA365.2|EPA \\
\hline MSB $11 \mathrm{C}$ & 9603. & -0.181233 & -0.181233 & MGL & EPA6010|EPA6 \\
\hline MSB 11C & $96 Q 3$ & -0.015730 & -0.022200 & MGL & EPA6010|EPA6 \\
\hline MSB 12B & 9623 & -0.095400 & 0.095400 & MGL & EPA6010A \\
\hline MSB $12 B$ & 9603 & 0.020100 & 0.020100 & MGL & EPA6010A \\
\hline MSB $12 B$ & 9603 & 3.480000 & 3.480000 & MGL & EPA300.0 \\
\hline MSB $12 B$ & $96 Q 3$ & -0.001440 & 0.001440 & MGL & EPA6010A \\
\hline MSB $12 \mathrm{~B}$ & 9603 & -0.000790 & 0.000794 & MGL & EPA6010A \\
\hline MSB $12 B$ & 9623 & -0.008560 & 0.008560 & MGL & EPA $6010 \mathrm{~A}$ \\
\hline MSB $12 \mathrm{~B}$ & 9603 & -0.010000 & -0.010000 & MGL & EPA335.3 \\
\hline MSB $12 \mathrm{~B}$ & 9603 & -0.100000 & -0.100000 & MGL & EPA 300.0 \\
\hline MSB $12 B$ & 9603 & 0.000410 & 0.000413 & MGL & EPA7470 \\
\hline MSB 12B. & 9603 & 0.007480 & 0.007480 & MGL & EPA6010A \\
\hline MSB $12 B$ & $96 Q 3$ & -13.900000 & 13.900000 & MGL & EPA6010A \\
\hline MSB $12 B$ & $96 Q 3$ & -0.010000 & -0.010000 & MGL & EPA6010A \\
\hline MSB $12 B$ & $96 Q 3$ & -0.002780 & 0.002780 & MGL & EPA6010A \\
\hline MSB $12 B$ & 9683 & -0.005000 & -0.005000 & MGL & EPA6010A \\
\hline MSB $12 B$ & $96 Q 3$ & -0.249000 & 0.249000 & MGL & EPA300.0 \\
\hline MSB $12 B$ & $96 Q 3$ & 3.000000 & 3.000000 & PCL & EPIA-010 \\
\hline MSB $12 \mathrm{~B}$ & $96 Q 3$ & -0.050000 & -0.050000 & MGL & EPA3 65.4 \\
\hline MSB 12B & 9603 & -0.020000 & -0.020000 & MGL & EPA6010A \\
\hline MSB $12 \mathrm{~B}$ & $96 Q 3$ & -0.022100 & 0.022100 & MGL & EPA6010A \\
\hline MSB $13 \mathrm{CC}$ & 9603 & -0.020000 & -0.020000 & MGL & EPA6010A \\
\hline MSB $13 \mathrm{CC}$ & 9603 & -0.001210 & 0.001210 & MGL & EPA6010A \\
\hline MSB $13 \mathrm{CC}$ & $96 Q 3$ & 2.300000 & 2.300000 & MGL & EPA300.0 \\
\hline MSB $13 \mathrm{CC}$ & 9603 & -0.000420 & -0.000416 & MGL & EPA6010A \\
\hline MSB $13 \mathrm{CC}$ & $96 Q 3$ & -0.004000 & -0.004000 & MGL & EPA6010A \\
\hline MSB $13 \mathrm{CC}$ & 9603 & 0.012500 & 0.012500 & MGL & EPA6010A \\
\hline MSB $13 \mathrm{CC}$ & 9603 & -0.010000 & -0.010000 & MGL & EPA335.3 \\
\hline MSB $13 C C$ & $96 Q 3$ & -0.100000 & -0.100000 & MGL & ERA300.0 \\
\hline MSB $13 \mathrm{CC}$ & 9603 & -0.000200 & -0.000200 & MGL & EPA7470 \\
\hline MSB $13 \mathrm{CC}$ & $96 Q 3$ & -0.002570 & 0.002570 & MGL & EPA6010A \\
\hline MSB $13 \mathrm{CC}$ & 9603 & 19.600000 & 19.600000 & MGL & EPA6010A \\
\hline MSB $13 \mathrm{CC}$ & $96 Q 3$ & -0.000970 & 0.000965 & MGL & EPA6010A \\
\hline MSB $13 C C$ & 9603 & -0.003960 & 0.003960 & MGL & EPA6010A \\
\hline MSB $13 \mathrm{CC}$ & 9603 & -0.002030 & 0.002030 & MGL & EPA6010A \\
\hline MSB $13 C C$ & $96 Q 3$ & 6.010000 & 6.010000 & MGL & EPA300.0 \\
\hline MSB $13 \mathrm{CC}$ & $96 Q 3$ & 0.000000 & 0.000000 & PCL & EPIA-010 \\
\hline MSB $13 \mathrm{CC}$ & 9603 & 0.080000 & 0.080000 & MGL & EPA3 65.4 \\
\hline MSB $13 \mathrm{CC}$ & $96 Q 3$ & -0.012000 & 0.012000 & MGL & EPA6010A \\
\hline MSB $13 \mathrm{CC}$ & $96 Q 3$ & -0.012000 & 0.012000 & MGL & EPA6010A \\
\hline MSB $15 \mathrm{~A}$ & $96 Q 3$ & -0.029600 & -0.029600 & MGL & EPA6010A \\
\hline MSB $15 \mathrm{~A}$ & $96 Q 3$ & 0.018100 & 0.018100 & MGL & EPA6010A \\
\hline MSB 15A & $96 Q 3$ & 2.740000 & 2.740000 & MGL & EPA 300.0 \\
\hline MSB $15 \mathrm{~A}$ & 9603 & -0.004000 & -0.004000 & MGL & EPA6010A \\
\hline MSB $15 \mathrm{~A}$ & $96 Q 3$ & 0.004610 & 0.004610 & MGL & EPA6010A \\
\hline MSB 15A & 9623 & -0.004370 & 0.004370 & MGL & EPA6010A \\
\hline MSB $15 \mathrm{~A}$ & 9603 & -0.010000 & -0.010000 & MGL & EPA335.3 \\
\hline MSB $15 \mathrm{~A}$ & $96 Q 3$ & -0.041000 & 0.041000 & MGL & EPA300.0 \\
\hline
\end{tabular}




\begin{tabular}{|c|c|c|c|c|}
\hline 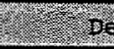 & matis & (2) & 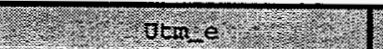 & $x+1$ \\
\hline NITOT & -0.020667 & 3688151.690000 & 431285.100000 & 3.000000 \\
\hline PBTOT & -0.026000 & 3688151.690000 & 431285.100000 & 2.000000 \\
\hline SETOT & -0.035500 & 3688151.690000 & 431285.100000 & 2.000000 \\
\hline $\mathrm{SO4}$ & -0.380000 & 3688151.690000 & 431285.100000 & 3.000000 \\
\hline TOTRAD & -0.230000 & 3688151.690000 & 431285.100000 & 2.000000 \\
\hline TPO4 & -0.060000 & 3688151.690000 & 431285.100000 & 2.000000 \\
\hline UTOT & 0.090373 & 3688151.690000 & 431285.100000 & 3.000000 \\
\hline ZNTOT & -0.029000 & 3688151.690000 & 431285.100000 & 2.000000 \\
\hline ALTOT & -0.020000 & 3687793.280000 & 431003.350000 & \\
\hline BATOT & 0.000116 & 3687793.280000 & 431003.350000 & \\
\hline $\mathrm{CL}$ & -0.250000 & 3687793.280000 & 431003.350000 & \\
\hline COTOT & 0.000930 & 3687793.280000 & 431003.350000 & \\
\hline CRTOT & -0.004000 & 3687793.280000 & 431003.350000 & \\
\hline CUTOT & 0.001800 & 3687793.280000 & 431003.350000 & \\
\hline $\mathrm{CYN}$ & -0.010000 & 3687793.280000 & 431003.350000 & \\
\hline $\boldsymbol{F}$ & -0.100000 & 3687793.280000 & 431003.350000 & \\
\hline HGTOT & -0.000200 & 3687793.280000 & 431003.350000 & \\
\hline MNTOT & 0.000946 & 3687793.280000 & 431003.350000 & \\
\hline NATOT & 0.033200 & 3687793.280000 & 431003.350000 & \\
\hline NITOT & 0.001250 & 3687793.280000 & 431003.350000 & \\
\hline PBIOT & -0.005000 & 3687793.280000 & 431003.350000 & \\
\hline SETOT & -0.005000 & 3687793.280000 & 431003.350000 & \\
\hline $\mathrm{SO} 4$ & -1.000000 & 3687793.280000 & 431003.350000 & \\
\hline TOTRAD & -0.100000 & 3687793.280000 & 431003.350000 & \\
\hline TPO4 & -0.050000 & 3687793.280000 & 431003.350000 & \\
\hline UTOT & 0.021200 & 3687793.280000 & 431003.350000 & \\
\hline ZNTOT & 0.007480 & 3687793.280000 & 431003.350000 & \\
\hline ALTOT & -0.020000 & 3687733.670000 & 431192.260000 & \\
\hline BATOT & 0.000116 & 3687733.670000 & 431192.250000 & \\
\hline $\mathrm{CL}$ & -0.250000 & 3687733.670000 & 431192.260000 & \\
\hline COTOT & 0.000930 & 3687733.670000 & 431192.250000 & \\
\hline CRTOT & -0.004000 & 3687733.670000 & 431192.260000 & \\
\hline CUTOT & 0.001800 & 3687733.670000 & 431192.260000 & \\
\hline CYN & 0.004770 & 3687733.670000 & 431192.260000 & \\
\hline$F$ & -0.100000 & 3687733.670000 & 431192.260000 & \\
\hline EGTOT & -0.000200 & 3687733.670000 & 431192.260000 & \\
\hline MNTOT & 0.000946 & 3687733.670000 & 431192.260000 & \\
\hline NATOT & 0.033200 & 3687733.670000 & 431192.260000 & \\
\hline NITOT & 0.001250 & 3687733.670000 & 431192.260000 & \\
\hline PETOT & -0.005000 & 3687733.670000 & 431192.260000 & \\
\hline SETOT & -0.005000 & 3687733.670000 & 431192.260000 & \\
\hline $\mathrm{SO} 4$ & -1.000000 & 3687733.670000 & 431192.260000 & \\
\hline TOTRAD & 0.500000 & 3687733.670000 & 431192.260000 & \\
\hline TPO4 & -0.050000 & 3687733.670000 & 431192.260000 & \\
\hline UTOT & 0.021200 & 3687733.670000 & 431192.260000 & \\
\hline ZNTOT & 0.007480 & 3687733.670000 & 431192.260000 & \\
\hline ALTOT & -0.020000 & 3688276.130000 & 431287.840000 & \\
\hline BATOT & -0.003000 & 3688276.130000 & 431287.840000 & \\
\hline $\mathrm{CL}$ & -0.250000 & 3688276.130000 & 432287.840000 & \\
\hline COTOT & -0.004000 & 3688276.130000 & 431287.840000 & \\
\hline CRTOT & -0.004000 & 3688276.130000 & 431287.840000 & \\
\hline CUTOT & 0.001240 & 3688276.130000 & 431287.840000 & \\
\hline $\mathrm{CYN}$ & -0.010000 & 3688276.130000 & 431287.840000 & \\
\hline$F$ & -0.100000 & 3688276.130000 & 431287.840000 & \\
\hline
\end{tabular}




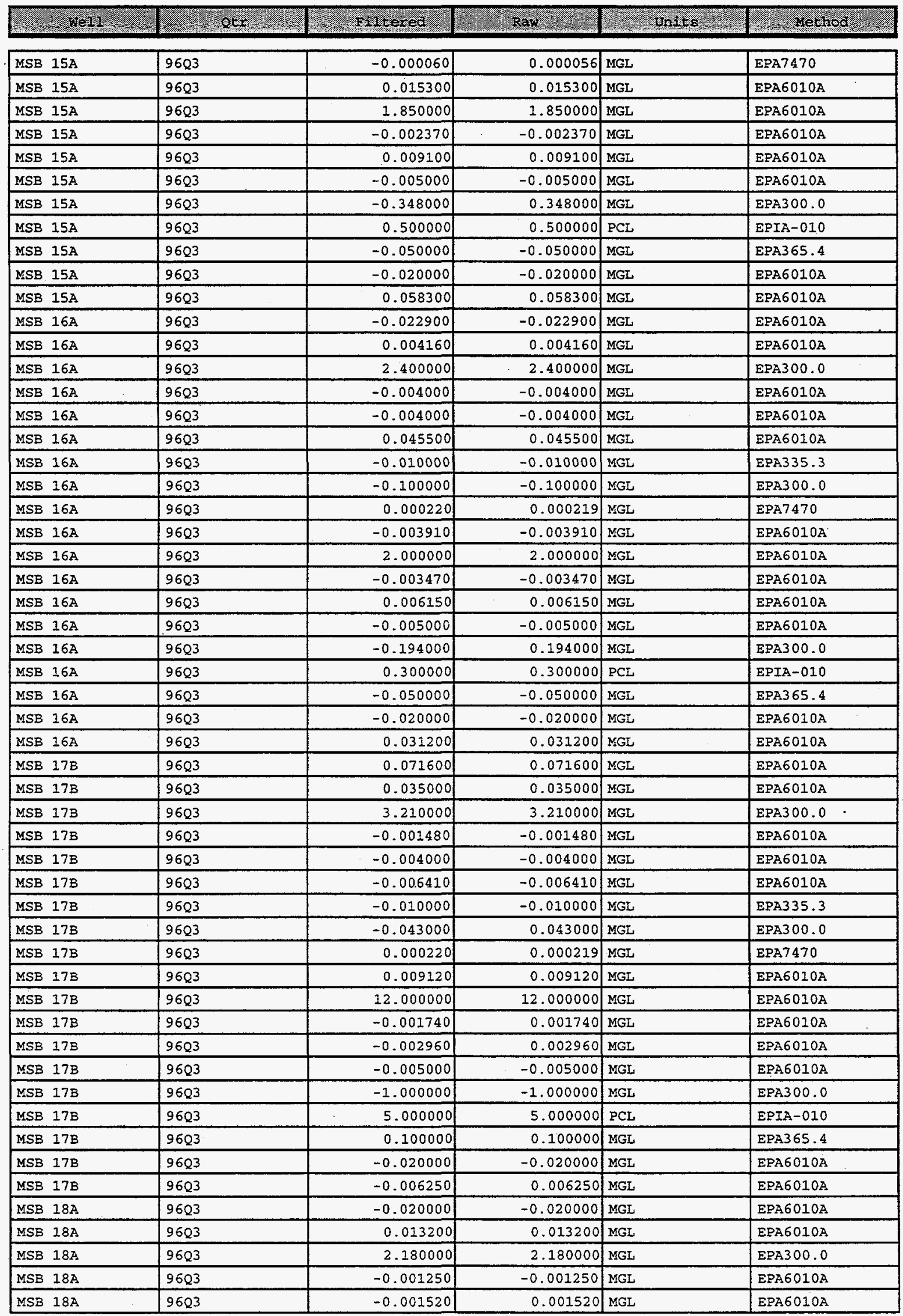




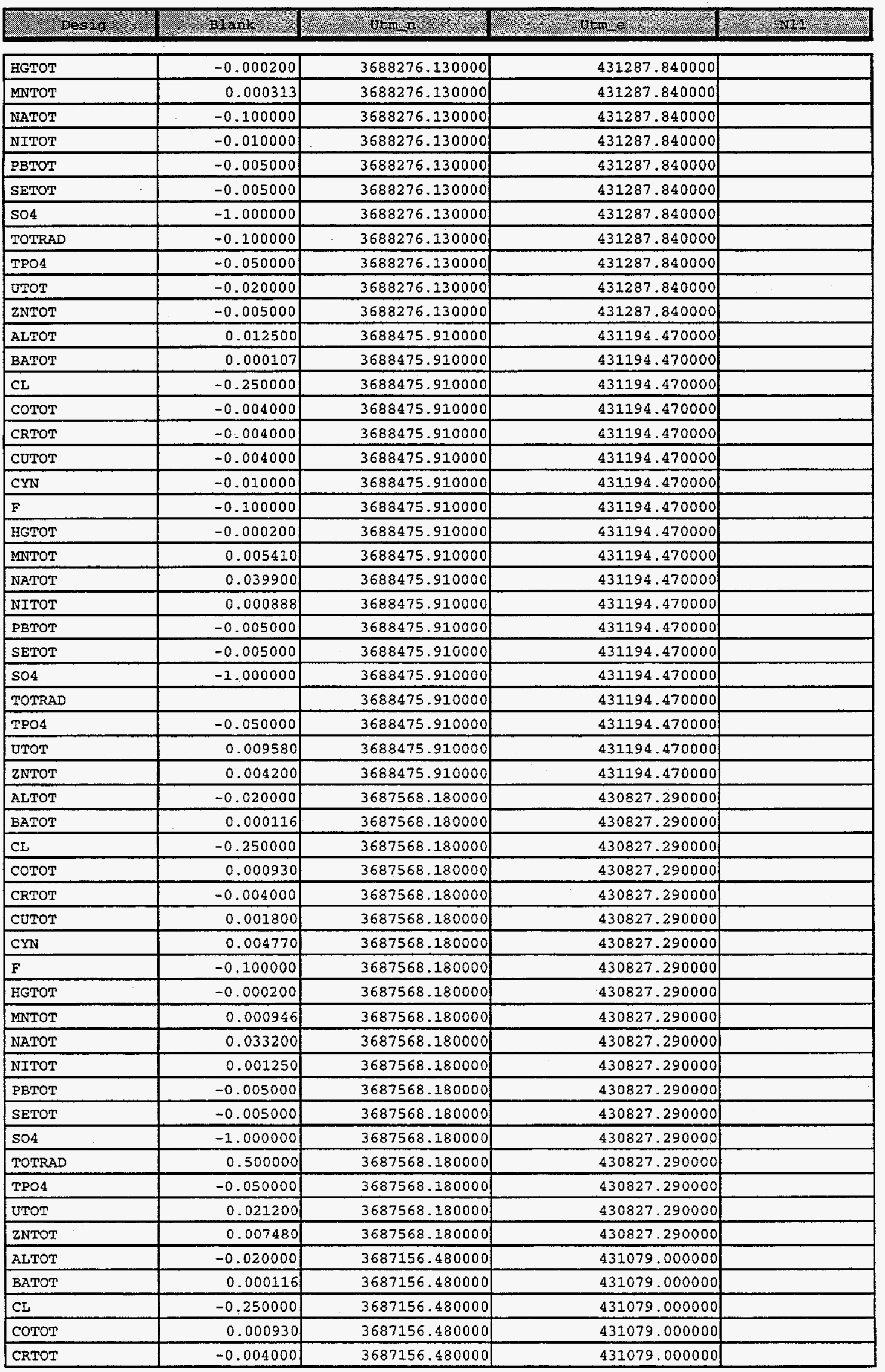


WSRC-TR-00347

November, 1997

Appendix II-145

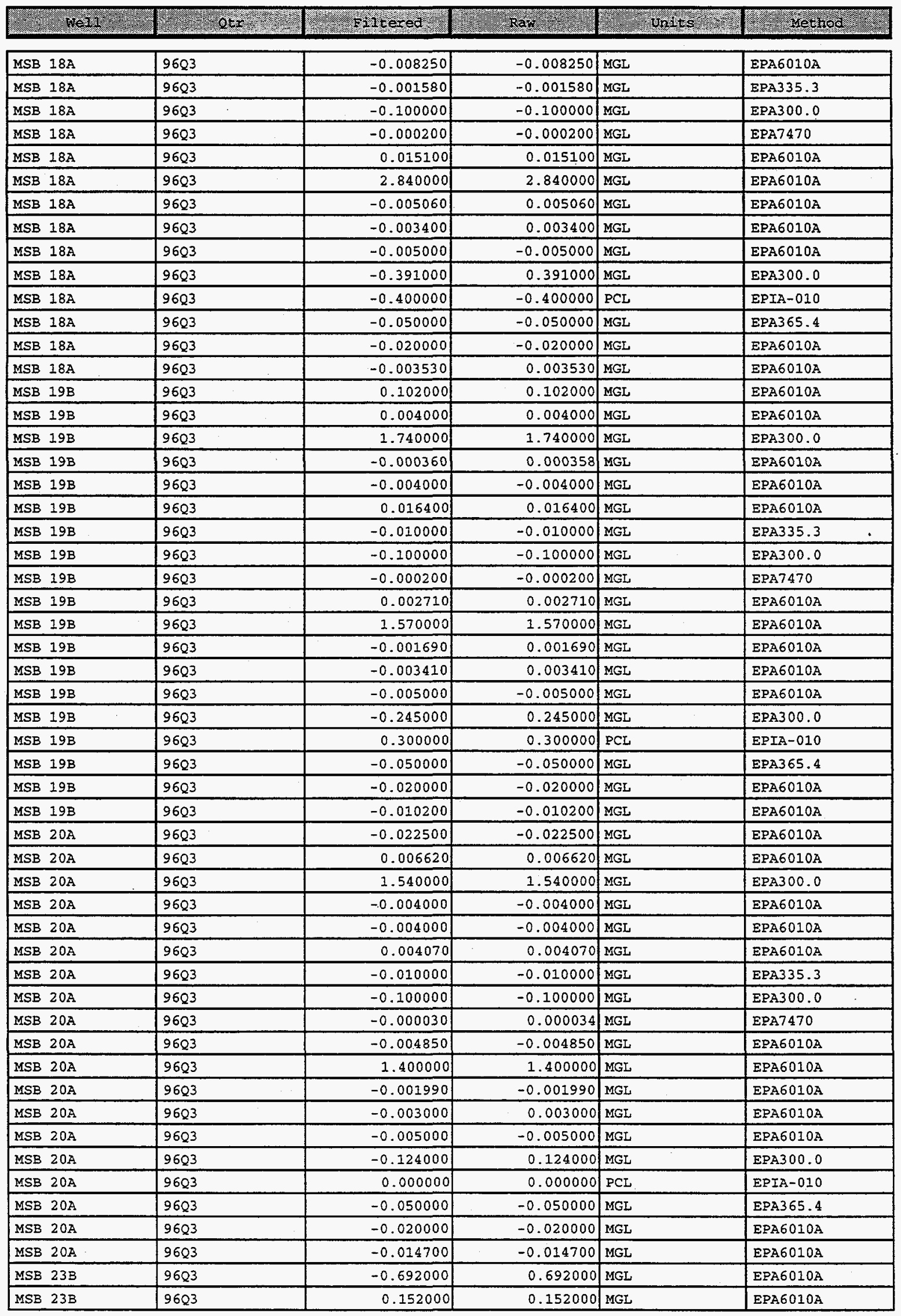




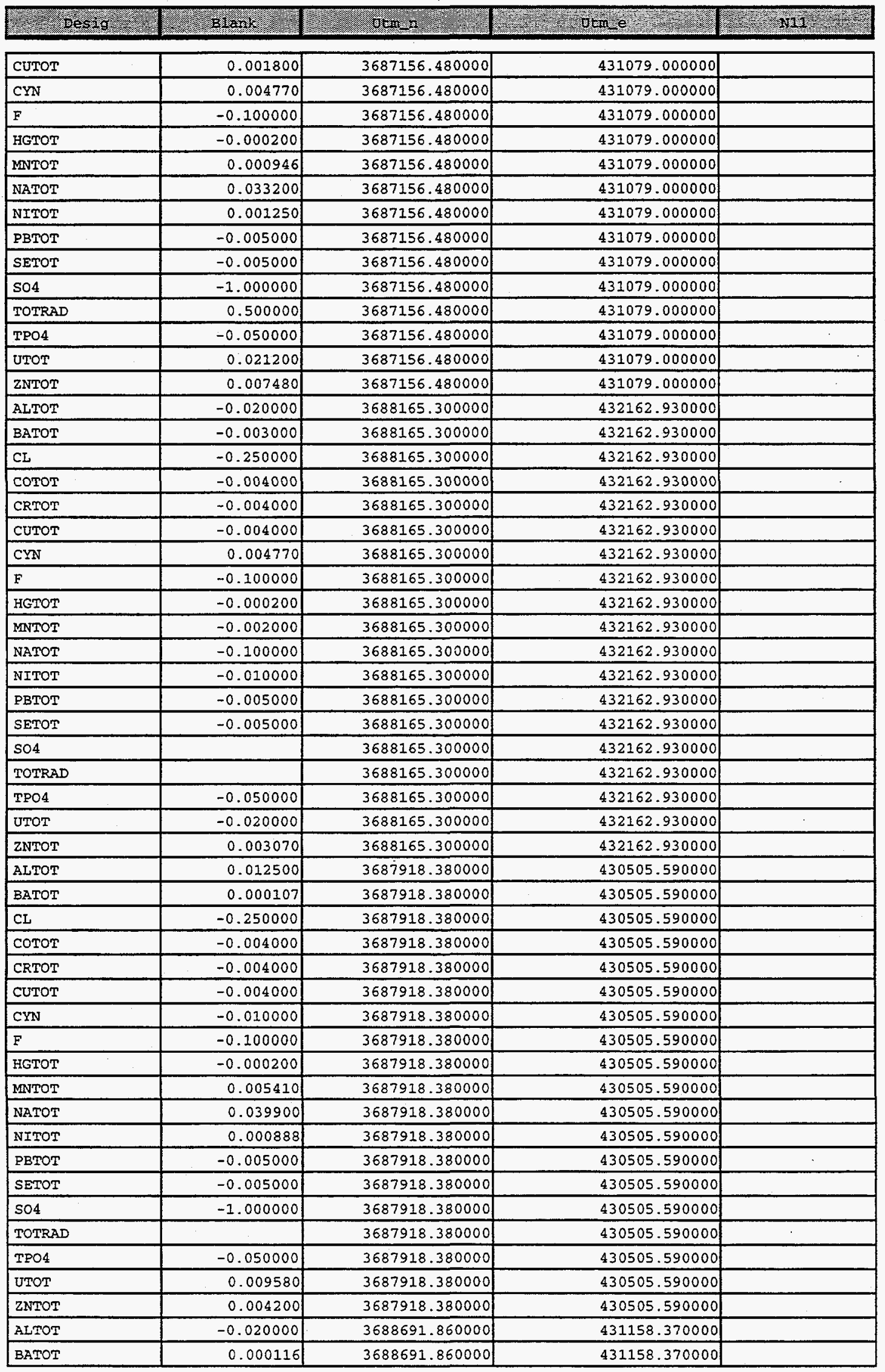




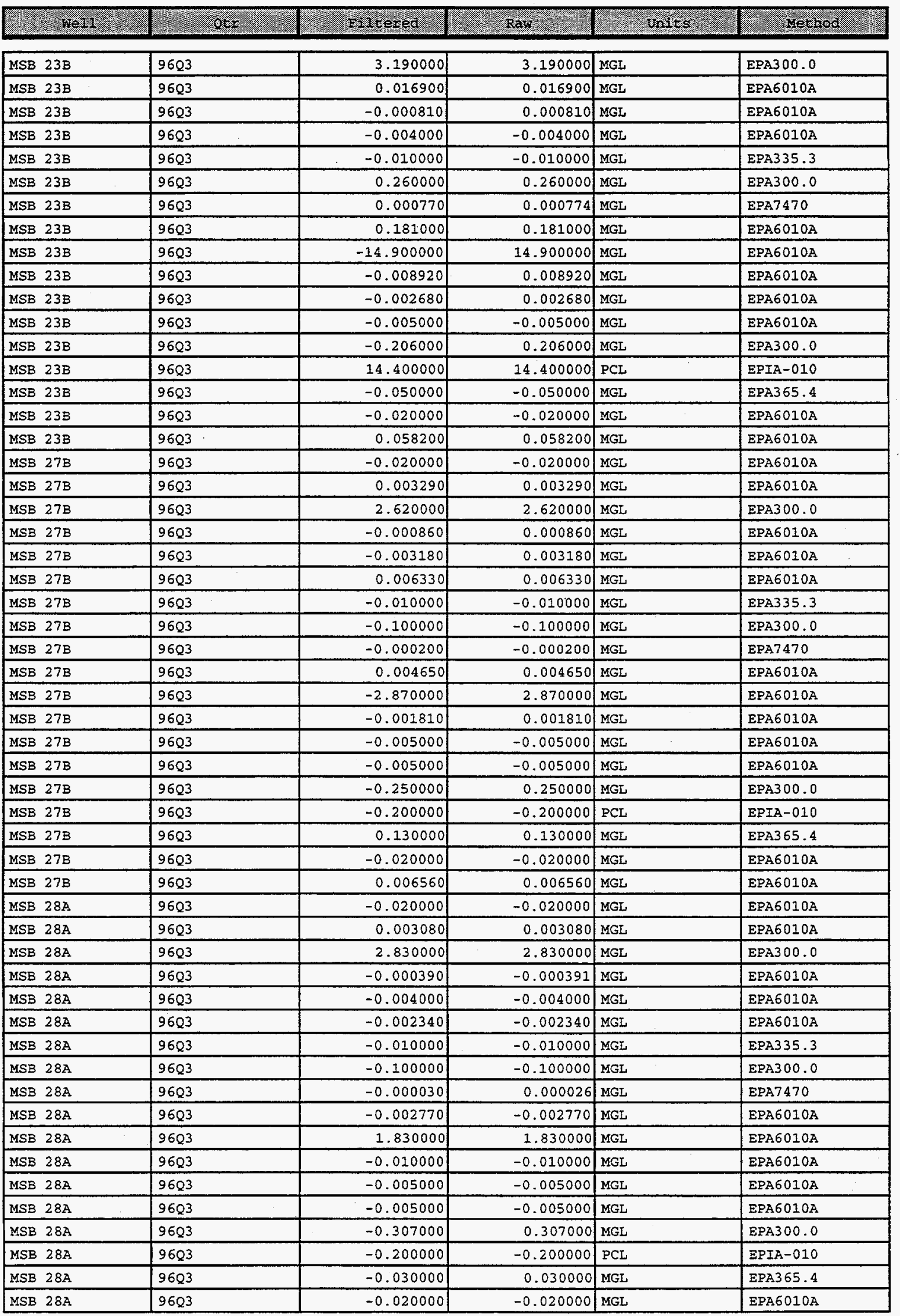




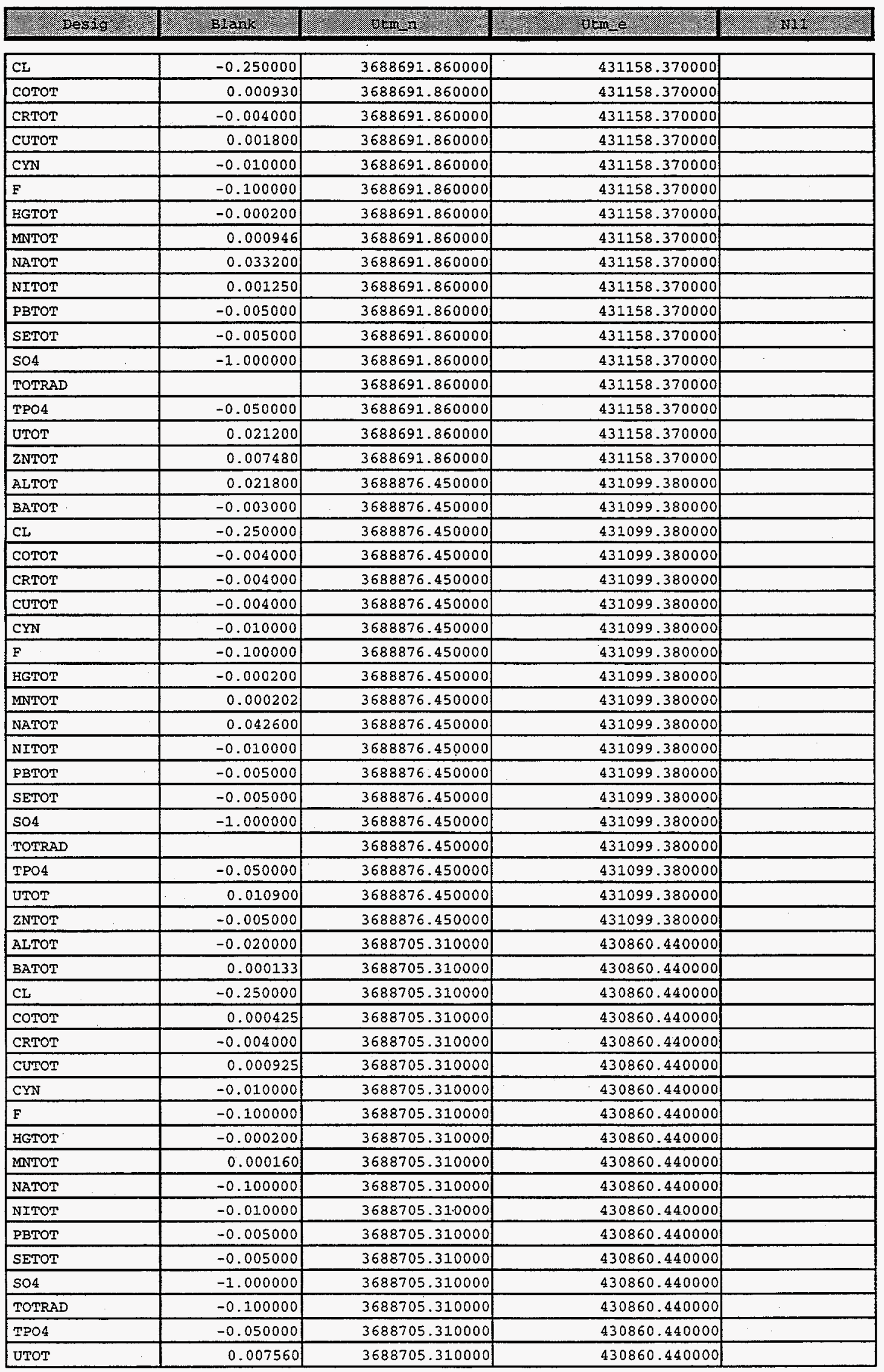


WSRC-TR-00347

November, 1997

Appendix II-149

\begin{tabular}{|c|c|c|c|c|c|}
\hline 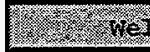 & & Eltrexed & $\mathrm{Rat}$ & & 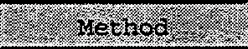 \\
\hline MSB 28A & $96 Q 3$ & -0.019600 & -0.019600 & MGL & EPA6010A \\
\hline MSB $29 \mathrm{C}$ & $96 Q 3$ & 0.041000 & 0.041000 & MGL & EPA6010A \\
\hline MSB $29 \mathrm{C}$ & 9603 & 0.005860 & 0.005860 & MGL & EPA6010A \\
\hline MSB $29 \mathrm{C}$ & $96 Q 3$ & 2.260000 & 2.260000 & MGL & EPA300.0 \\
\hline MSB $29 \mathrm{C}$ & $96 Q 3$ & -0.000730 & 0.000730 & MGL & EPA6010A \\
\hline MSB $29 \mathrm{C}$ & 9603 & -0.001830 & 0.001830 & MGL & EPA6010A \\
\hline MSB $29 \mathrm{C}$ & 9603 & -0.003740 & 0.003740 & MGL & EPA6010A \\
\hline MSB $29 \mathrm{C}$ & $96 Q 3$ & -0.010000 & -0.010000 & MGL & EPA335.3 \\
\hline MSB $29 C$ & 9603 & -0.100000 & -0.100000 & MGL & EPA300.0 \\
\hline MSB 29C & 9603 & -0.000200 & -0.000200 & MGL & EPA7470 \\
\hline MSB $29 \mathrm{C}$ & 9603 & 0.002300 & 0.002300 & MGL & EPA6010A \\
\hline MSB 29C & 9603 & 2.320000 & 2.320000 & MGL & EPA6010A \\
\hline MSB $29 \mathrm{C}$ & $96 Q 3$ & -0.001220 & 0.001220 & MGL & EPA6010A \\
\hline MSB $29 \mathrm{C}$ & 9603 & -0.003200 & 0.003200 & MGL & EPA6010A \\
\hline MSB $29 \mathrm{C}$ & $96 Q 3$ & -0.005000 & -0.005000 & MGL & EPA6010A \\
\hline MSB $29 \mathrm{C}$ & $96 Q 3$ & -0.128000 & 0.128000 & MGL & EPA300.0 \\
\hline MSB 29C & $96 Q 3$ & 1.400000 & 1.400000 & $\mathrm{PCL}$ & EPIA -010 \\
\hline MSB 29C & $96 Q 3$ & -0.050000 & -0.050000 & MGI & EPA 365.4 \\
\hline MSB $29 \mathrm{C}$ & $96 Q 3$ & -0.020000 & -0.020000 & MGL & EPA6010A \\
\hline MSB 29C & 9603 & -0.008080 & -0.008080 & MGL & EPA6010A \\
\hline MSB $30 \mathrm{CC}$ & $96 Q 3$ & -0.020000 & -0.020000 & MGL & EPA6010A \\
\hline MSB $30 \mathrm{CC}$ & $96 Q 3$ & 0.006350 & 0.006350 & MGL & EPA6010A \\
\hline MSB $30 \mathrm{CC}$ & $96 Q 3$ & 1.330000 & 1.330000 & MGL & ERA 300.0 \\
\hline MSB $30 \mathrm{CC}$ & $96 Q 3$ & -0.000270 & 0.000269 & MGL & EPA6010A \\
\hline MSB $30 \mathrm{CC}$ & $96 Q 3$ & -0.004000 & -0.004000 & MGL & EPA6010A \\
\hline MSB $30 \mathrm{CC}$ & $96 Q 3$ & 0.004030 & 0.004030 & MGL & EPA6010A \\
\hline MSB $30 C C$ & $96 Q 3$ & -0.001890 & 0.001890 & MGL & EPA335.3 \\
\hline MSB $30 \mathrm{CC}$ & 9603 & -0.100000 & -0.100000 & MGL & EPA300.0 \\
\hline MSB $30 \mathrm{CC}$ & $96 Q 3$ & -0.000200 & -0.000200 & MGL & EPA7470 \\
\hline MSB $30 \mathrm{CC}$ & $96 Q 3$ & 0.013000 & 0.013000 & MGL & EPA6010A \\
\hline MSB $30 C C$ & $96 Q 3$ & 1.670000 & 1.670000 & MGL & EPA6010A \\
\hline MSB $30 \mathrm{CC}$ & $96 Q 3$ & -0.000960 & 0.000958 & MGL & EPA6010A \\
\hline MSB $30 C C$ & $96 Q 3$ & -0.005000 & -0.005000 & MGL & EPA6010A \\
\hline MSB $30 \mathrm{CC}$ & $96 Q 3$ & -0.005000 & -0.005000 & MGL & EPA6010A \\
\hline MSB $30 \mathrm{CC}$ & 9603 & -0.941000 & 0.941000 & MGL & EPA300.0 \\
\hline MSB $30 \mathrm{CC}$ & 9603 & 0.000000 & 0.000000 & PCL & EPIA-010 \\
\hline MSB $30 \mathrm{CC}$ & $96 Q 3$ & -0.050000 & -0.050000 & MGL & EPA365.4 \\
\hline MSB $30 \mathrm{CC}$ & 9603 & -0.020000 & -0.020000 & MGL & EPA6010A \\
\hline MSB $30 \mathrm{CC}$ & 9603 & -0.011800 & -0.011800 & MGL & EPA6010A \\
\hline MSB $31 C C$ & 9603 & 0.033300 & 0.033300 & MGL & EPA6010A \\
\hline MSB 31CC & 9603 & 0.015000 & 0.015000 & MGL & EPA6010A \\
\hline MSB $31 C C$ & $96 Q 3$ & & 1.990000 & MGI & EPA300.0 \\
\hline MSB $31 \mathrm{CC}$ & 9603 & -0.000740 & -0.000738 & MGL & EPA6010A \\
\hline MSB $31 \mathrm{CC}$ & 9603 & -0.001000 & -0.001000 & MGL & EPA6010A \\
\hline MSB $31 C C$ & 9603 & -0.002020 & -0.002020 & MGL & EPA6010A \\
\hline MSB $31 \mathrm{CC}$ & 9603 & -0.010000 & -0.010000 & MGL & EPA 335.3 \\
\hline MSB $31 \mathrm{CC}$ & 9603 & -0.100000 & -0.100000 & MGL & EPA 300.0 \\
\hline MSB $31 \mathrm{CC}$ & 9603 & -0.000200 & -0.000200 & MGE & EPA7470 \\
\hline MSB $31 C C$ & 9603 & 0.006220 & 0.006220 & MGL & EPA6010A \\
\hline MSB $31 \mathrm{CC}$ & 9603 & 2.180000 & 2.180000 & MGL & EPA.6010A \\
\hline MSB $31 \mathrm{CC}$ & 9603 & -0.001220 & 0.001220 & MGL & EPA6010A \\
\hline MSB $31 C C$ & 9603 & -0.005000 & -0.005000 & MGL & EPA6010A \\
\hline MSB $31 \mathrm{CC}$ & 9603 & -0.002740 & 0.002740 & MGL & EPA6010A \\
\hline MSB $31 \mathrm{CC}$ & 9603 & & 1.150000 & MGL & EPA300.0 \\
\hline
\end{tabular}


IVSRC-TR-00347

November, 1997

Appendix II-150

\begin{tabular}{|c|c|c|c|c|}
\hline 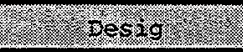 & (miark & $2.15 \mathrm{~ms}$ r & 20. & 1.0.7. \\
\hline 2NTOT & -0.005000 & 3688705.310000 & 430860.440000 & \\
\hline ALTOT & -0.020000 & 3689769.950000 & 431097.310000 & \\
\hline BATOT & -0.003000 & 3689769.950000 & 431097.310000 & \\
\hline CL & -0.250000 & 3689769.950000 & 431097.310000 & \\
\hline COTOT & -0.004000 & 3689769.950000 & 431097.310000 & \\
\hline CRTOT & -0.004000 & 3689769.950000 & 431097.310000 & \\
\hline CUTOT & -0.004000 & 3689769.950000 & 431097.310000 & \\
\hline $\mathrm{CYN}$ & 0.004770 & 3689769.950000 & 431097.310000 & \\
\hline$F$ & -0.100000 & 3689769.950000 & 431097.310000 & \\
\hline HGTOT & -0.000200 & 3689769.950000 & 431097.310000 & \\
\hline MNTOT & -0.002000 & 3689769.950000 & 431097.310000 & \\
\hline NATOT & -0.100000 & 3689769.950000 & 431097.310000 & \\
\hline NITOT & -0.010000 & 3689769.950000 & 431097.310000 & \\
\hline PBTOT & -0.005000 & 3689769.950000 & 431097.310000 & \\
\hline SETOT & -0.005000 & 3689769.950000 & 431097.310000 & \\
\hline so4 & & 3689769.950000 & 431097.310000 & \\
\hline TOTRAD & -0.100000 & 3689769.950000 & 431097.310000 & \\
\hline TPO4 & -0.050000 & 3689769.950000 & 431097.310000 & \\
\hline UTOT & -0.020000 & 3689769.950000 & 431097.310000 & \\
\hline ZNTOT & 0.003070 & 3689769.950000 & 431097.310000 & \\
\hline ALTOT & -0.020000 & 3688801.810000 & 430590.970000 & \\
\hline BATOT & -0.003000 & 3688801.810000 & 430590.970000 & \\
\hline CL & -0.250000 & 3688801.810000 & 430590.970000 & \\
\hline СОTOT & -0.004000 & 3688801.810000 & 430590.970000 & \\
\hline CRTOT & -0.004000 & 3688801.810000 & 430590.970000 & \\
\hline CUTOT & -0.004000 & 3688801.810000 & 430590.970000 & \\
\hline CYN & 0.004770 & 3688801.810000 & 430590.970000 & \\
\hline$F$ & -0.100000 & 3688801.810000 & 430590.970000 & \\
\hline HGTOT & -0.000200 & 3688801.810000 & 430590.970000 & \\
\hline MNTOT & -0.002000 & 3688801.810000 & 430590.970000 & \\
\hline NATOT & -0.100000 & 3688801.810000 & 430590.970000 & \\
\hline NITOT & -0.010000 & 3688801.810000 & 430590.970000 & \\
\hline PBTOT & -0.005000 & 3688801.810000 & 430590.970000 & \\
\hline SETOT & -0.005000 & 3688801.810000 & 430590.970000 & \\
\hline SO4 & & 3688801.810000 & 430590.970000 & \\
\hline TOTRAD & & 3688801.810000 & 430590.970000 & \\
\hline TPO4 & -0.050000 & 3688802.810000 & 430590.970000 & \\
\hline UTOT & -0.020000 & 3688801.810000 & 430590.970000 & \\
\hline ZNTOT & 0.003070 & 3688801.810000 & 430590.970000 & \\
\hline ALTOT & -0.020000 & 3688252.210000 & 431772.940000 & \\
\hline BATOT & 0.000279 & 3688252.210000 & 431772.940000 & \\
\hline $\mathrm{CL}$ & -0.250000 & 3688252.210000 & 431772.940000 & \\
\hline СОTOT & 0.001120 & 3688252.210000 & 431772.940000 & \\
\hline CRTOT & 0.001380 & 3688252.210000 & 431772.940000 & \\
\hline CUTOT & 0.001790 & 3688252.210000 & 431772.940000 & \\
\hline $\mathrm{CYN}$ & -0.010000 & 3688252.210000 & 431772.940000 & \\
\hline F & -0.100000 & 3688252.210000 & 431772.940000 & \\
\hline HGTOT & -0.000200 & 3688252.210000 & 431772.940000 & \\
\hline MNTOT & 0.000343 & 3688252.210000 & 431772.940000 & \\
\hline NATOT & -0.100000 & 3688252.210000 & 431772.940000 & \\
\hline NITOT & -0.010000 & 3688252.210000 & 431772.940000 & \\
\hline PBTOT & -0.005000 & 3688252.210000 & 431772.940000 & \\
\hline SETOT & 0.003640 & 3688252.210000 & 431772.940000 & \\
\hline SO4 & -1.000000 & 3688252.210000 & 431772.940000 & \\
\hline
\end{tabular}




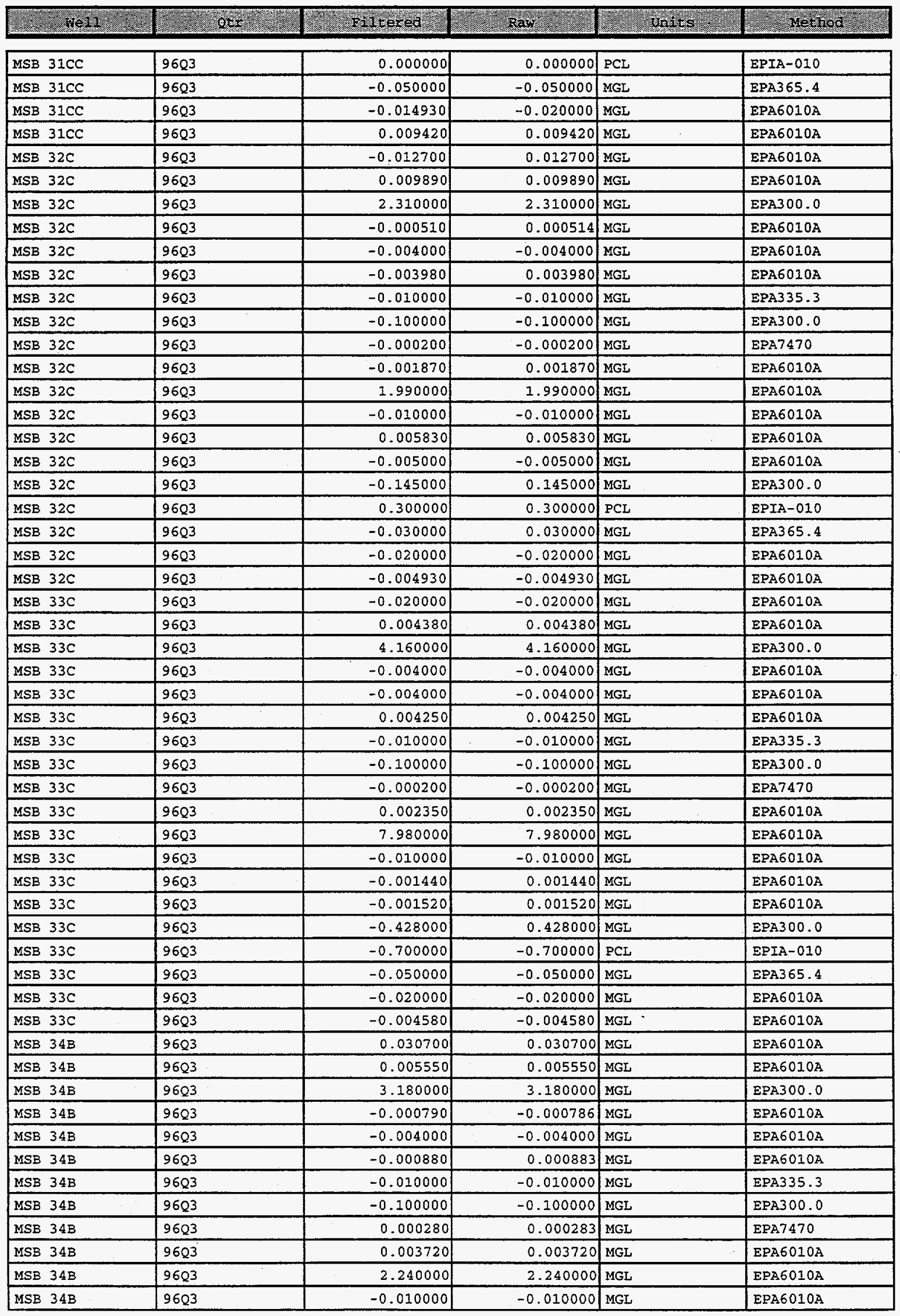




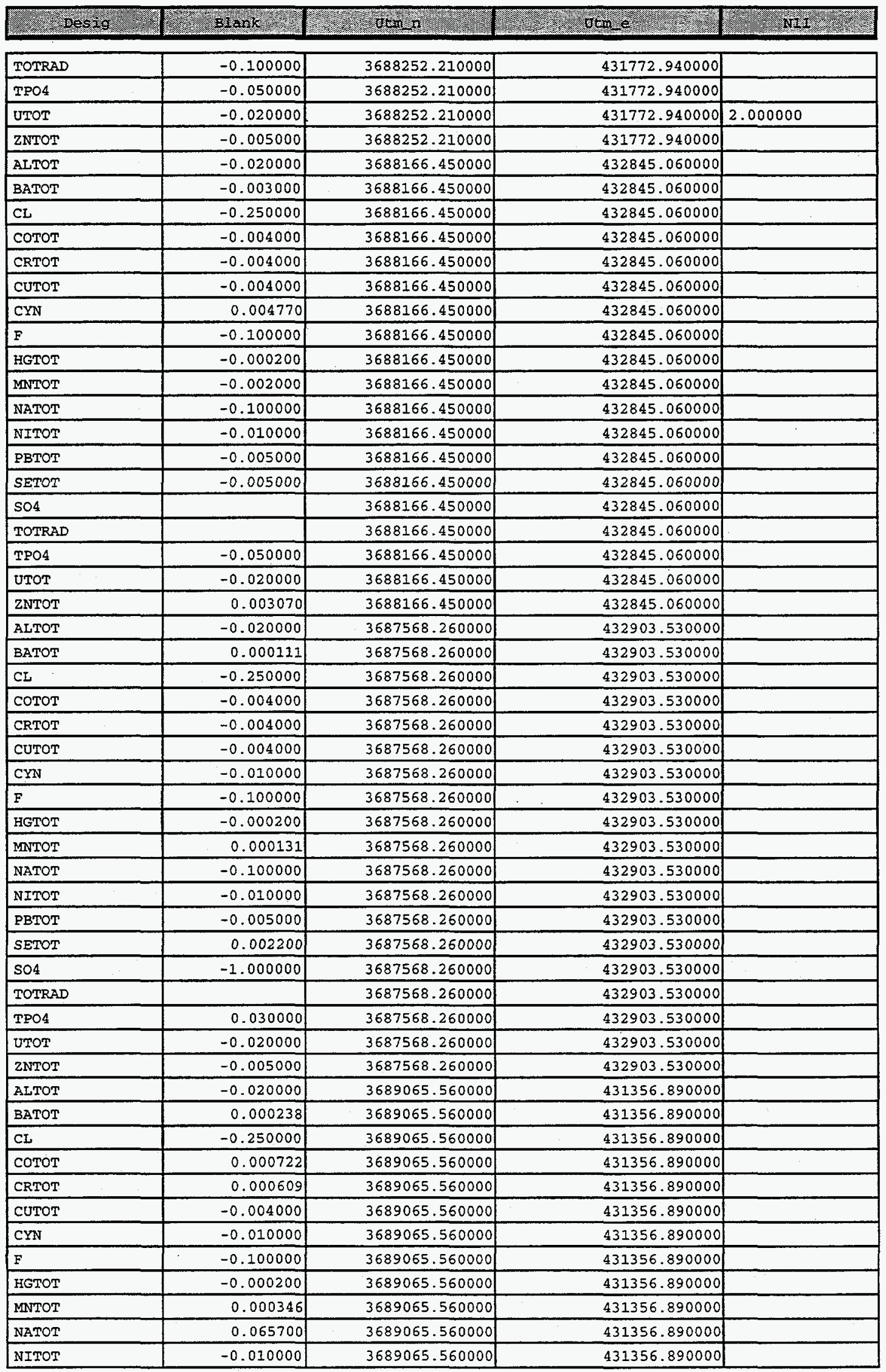


WSRC-TR-00347

November, 1997

Appendix 11-153

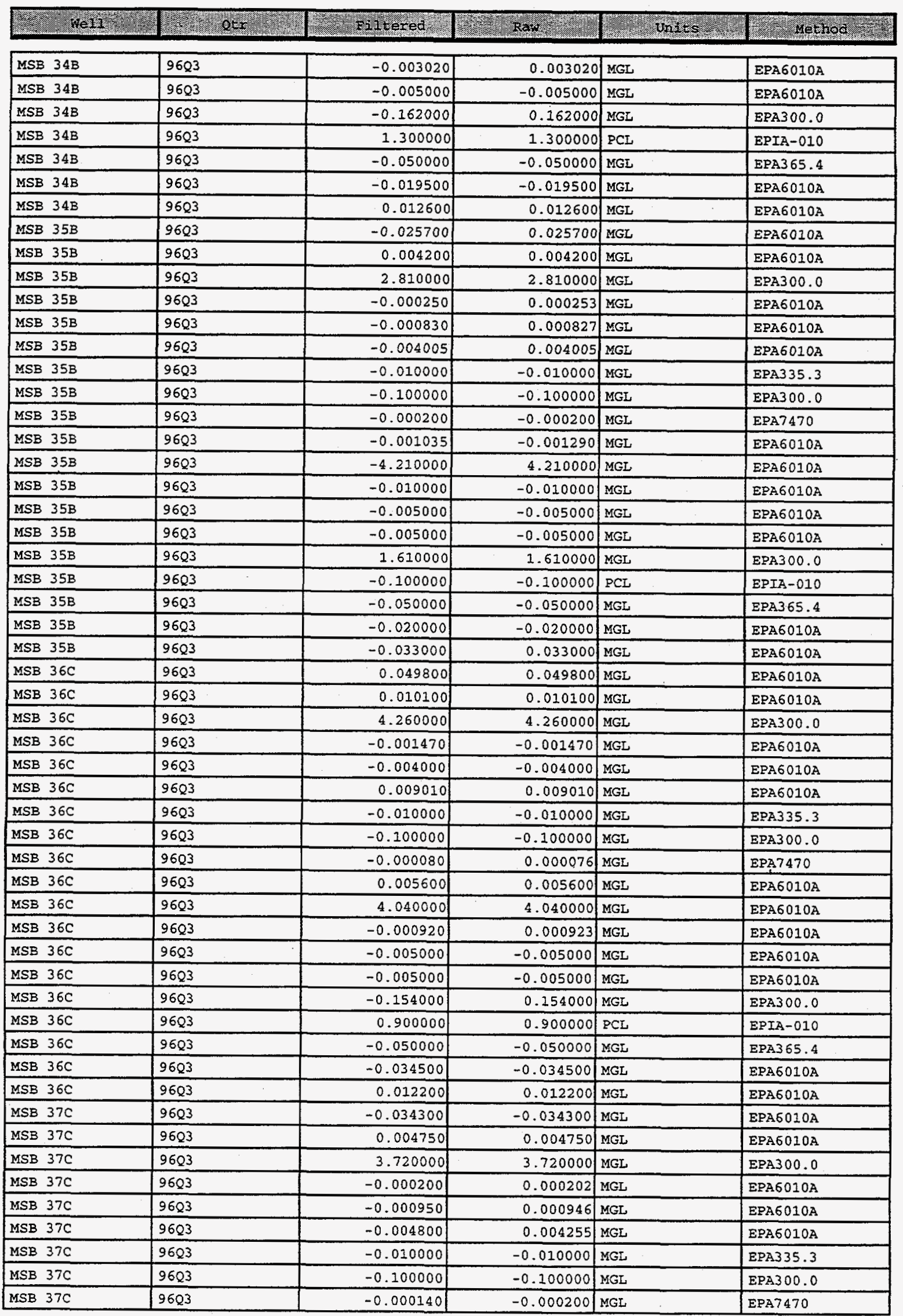




\begin{tabular}{|c|c|c|c|c|}
\hline PBTOT & -0.005000 & 3689065.560000 & 431356.890000 & \\
\hline SETOT & -0.005000 & 3689065.560000 & 431356.890000 & \\
\hline 504 & -1.000000 & 3689065.560000 & 431356.890000 & \\
\hline TOTRAD & -0.300000 & 3689065.560000 & 431356.890000 & \\
\hline TPO4 & -0.050000 & 3689065.560000 & 431356.890000 & \\
\hline UTOT & 0.047800 & 3689065.560000 & 431356.890000 & \\
\hline ZNTOT & -0.005000 & 3689065.560000 & 431356.890000 & \\
\hline ALTOT & -0.020000 & 3688441.470000 & 431966.830000 & 2.000000 \\
\hline BATOT & 0.000116 & 3688441.470000 & 431966.830000 & \\
\hline$C L$ & -0.250000 & 3688441.470000 & 431966.830000 & \\
\hline Cotor & 0.000930 & 3688441.470000 & 431966.830000 & \\
\hline CRTOT & -0.004000 & 3688441.470000 & 431966.830000 & \\
\hline CUTOT & 0.001800 & 3688441.470000 & 431966.830000 & 2.000000 \\
\hline $\mathrm{CYN}$ & -0.010000 & 3688441.470000 & 431966.830000 & \\
\hline$F$ & -0.100000 & 3688441.470000 & 431966.830000 & \\
\hline HGTOT & -0.000200 & 3688441.470000 & 431966.830000 & \\
\hline MNTOT & 0.000946 & 3688441.470000 & 431966.830000 & 2.000000 \\
\hline NATOT & 0.033200 & 3688441.470000 & 431966.830000 & \\
\hline NITOT & 0.001250 & 3688441.470000 & 431966.830000 & \\
\hline PBTOT & -0.005000 & 3688441.470000 & 431966.830000 & \\
\hline SETOT & -0.005000 & 3688441.470000 & 431966.830000 & \\
\hline 504 & -1.000000 & 3688441.470000 & 431966.830000 & \\
\hline TOTRAD & & 3688441.470000 & 431966.830000 & \\
\hline TPO4 & -0.050000 & 3688441.470000 & 431966.830000 & \\
\hline UTOT & 0.021200 & 3688441.470000 & 431966.830000 & \\
\hline ZNTOT & 0.007480 & 3688441.470000 & 431966.830000 & \\
\hline ALTOT & -0.020000 & 3687796.180000 & 431904.880000 & \\
\hline BATOT & 0.000238 & 3687796.180000 & 431904.880000 & \\
\hline$C L$ & -0.250000 & 3687796.180000 & 431904.880000 & \\
\hline COTOT & 0.000722 & 3687796.180000 & 431904.880000 & \\
\hline CRTOT & 0.000609 & 3687796.180000 & 431904.880000 & \\
\hline CUTOT & -0.004000 & 3687796.180000 & 431904.880000 & \\
\hline CYN & -0.010000 & 3687796.180000 & 431904.880000 & \\
\hline$F$ & -0.100000 & 3687796.180000 & 431904.880000 & \\
\hline HGTOT & -0.000200 & 3687796.180000 & 431904.880000 & \\
\hline MNTOT & 0.000346 & 3687796.180000 & 431904.880000 & \\
\hline NATOT & 0.065700 & 3687796.180000 & 431904.880000 & \\
\hline NITOT & -0.010000 & 3687796.180000 & 431904.880000 & \\
\hline PBTOT & -0.005000 & 3687796.180000 & 431904.880000 & \\
\hline SETOT & -0.005000 & 3687796.180000 & 431904.880000 & \\
\hline 504 & -1.000000 & 3687796.180000 & 431904.880000 & \\
\hline TOTRAD & & 3687796.180000 & 431904.880000 & \\
\hline TPO4 & -0.050000 & 3687796.180000 & 431904.880000 & \\
\hline UTOT & 0.047800 & 3687796.180000 & 431904.880000 & \\
\hline ZNTOT & -0.005000 & 3687796.180000 & 431904.880000 & \\
\hline ALTOT & 0.012500 & 3689311.220000 & 431519.120000 & \\
\hline BATOT & 0.000107 & 3689311.220000 & 431519.120000 & \\
\hline $\mathrm{CL}$ & -0.250000 & 3689311.220000 & 431519.120000 & \\
\hline COTOT & -0.004000 & 3689311.220000 & 431519.120000 & \\
\hline CRTOT & -0.004000 & 3689311.220000 & 431519.120000 & \\
\hline CUTOT & -0.004000 & 3689311.220000 & 431519.120000 & $2 . .000000$ \\
\hline CYN & -0.010000 & 3689311.220000 & 431519.120000 & \\
\hline$F$ & -0.100000 & 3689311.220000 & 431519.120000 & \\
\hline HGTOT & -0.000200 & 3689311.220000 & 431519.120000 & 2.000000 \\
\hline
\end{tabular}


WSRC-TR-00347

November, 1997

Appendix II-155

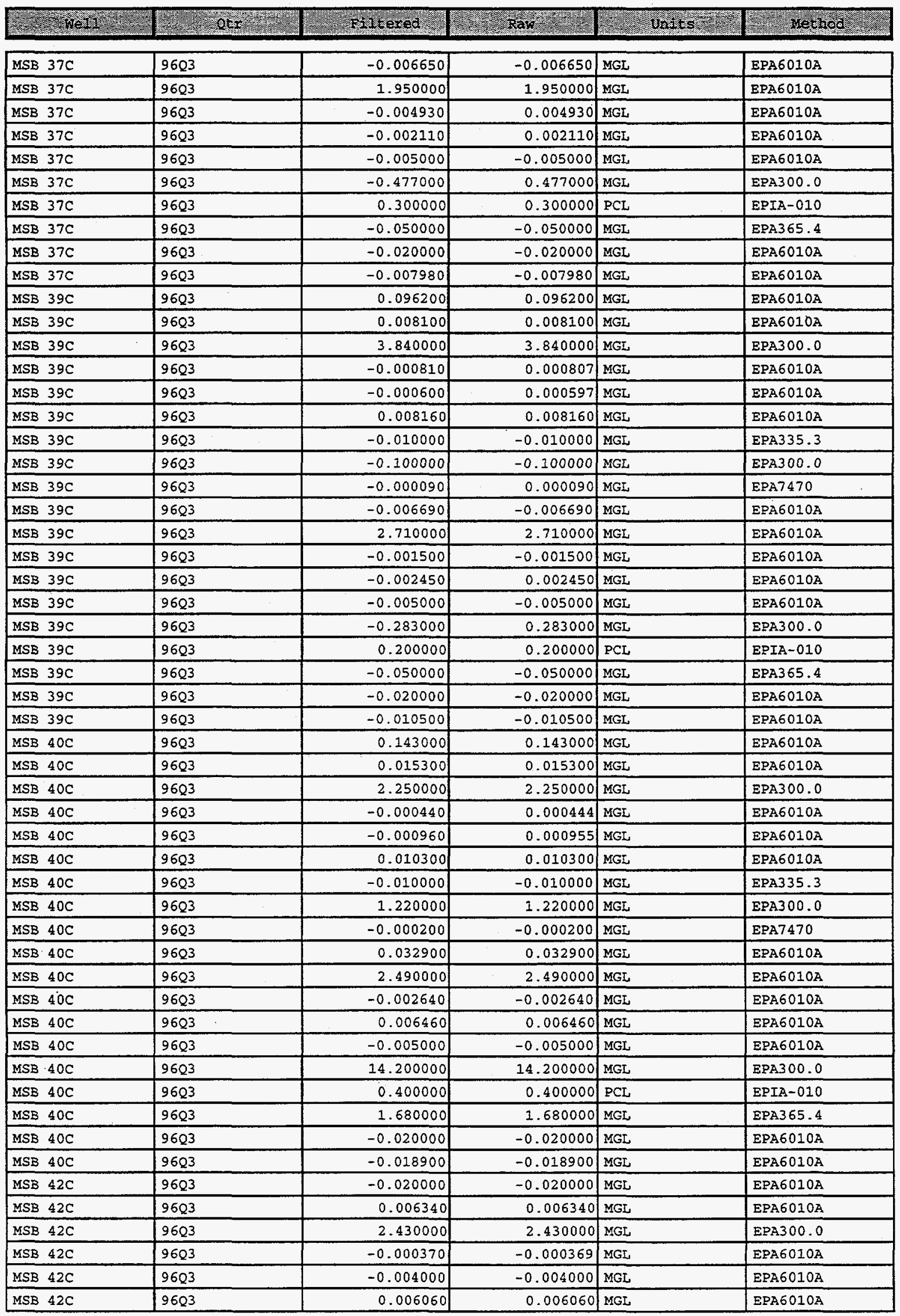


WSRC-TR-00347

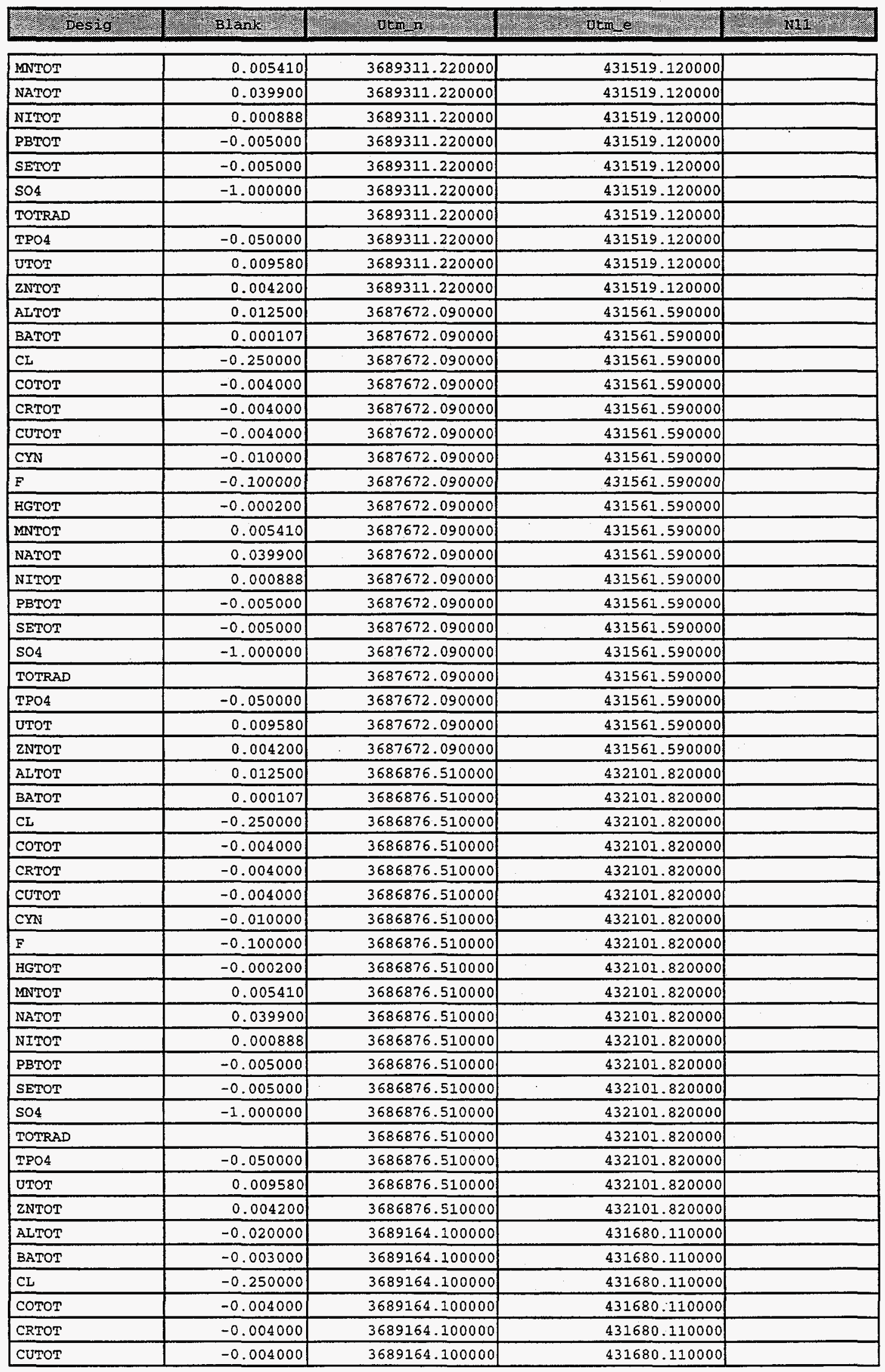


WSRC-TR-00347

November, 1997

Appendix II-157

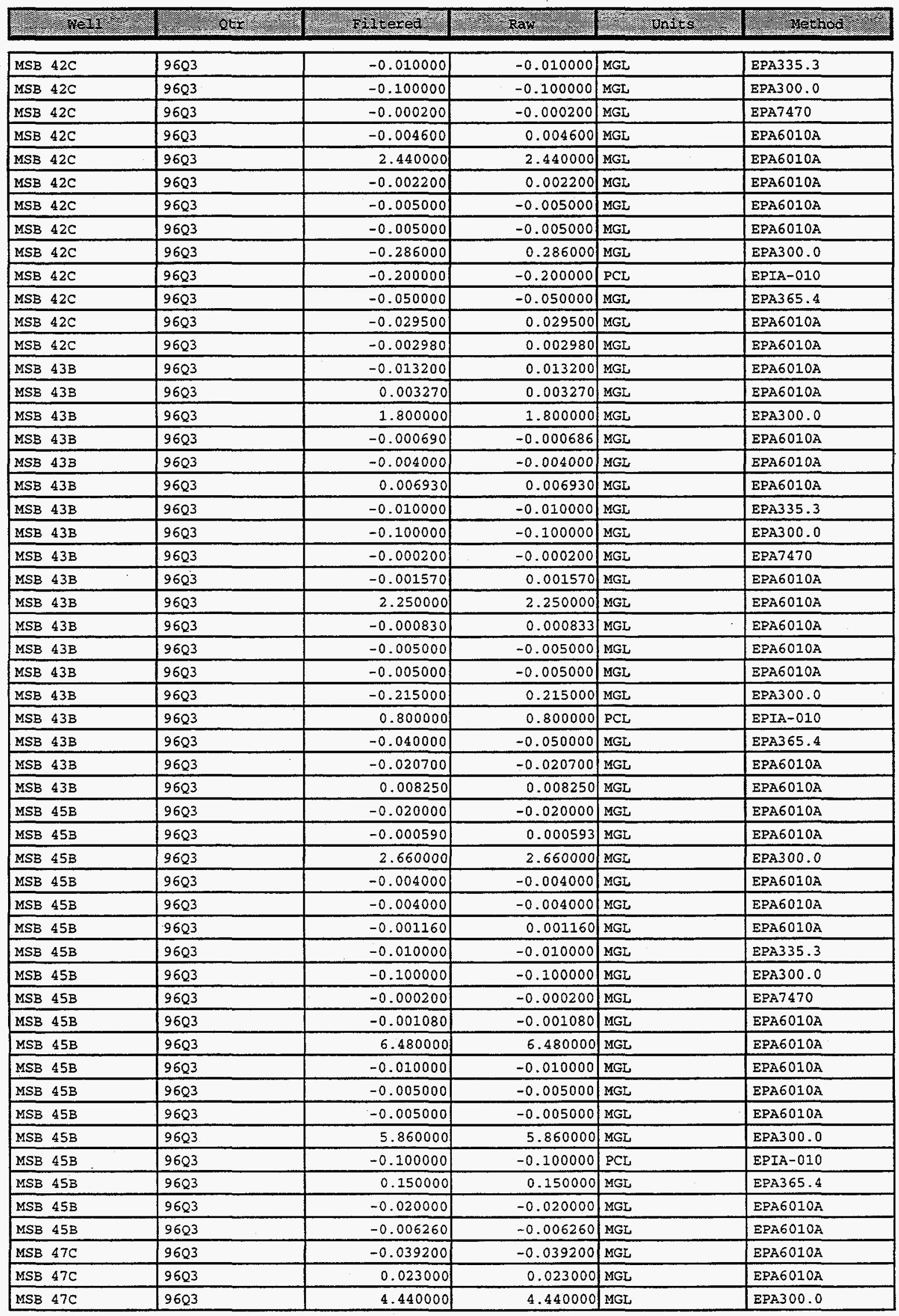




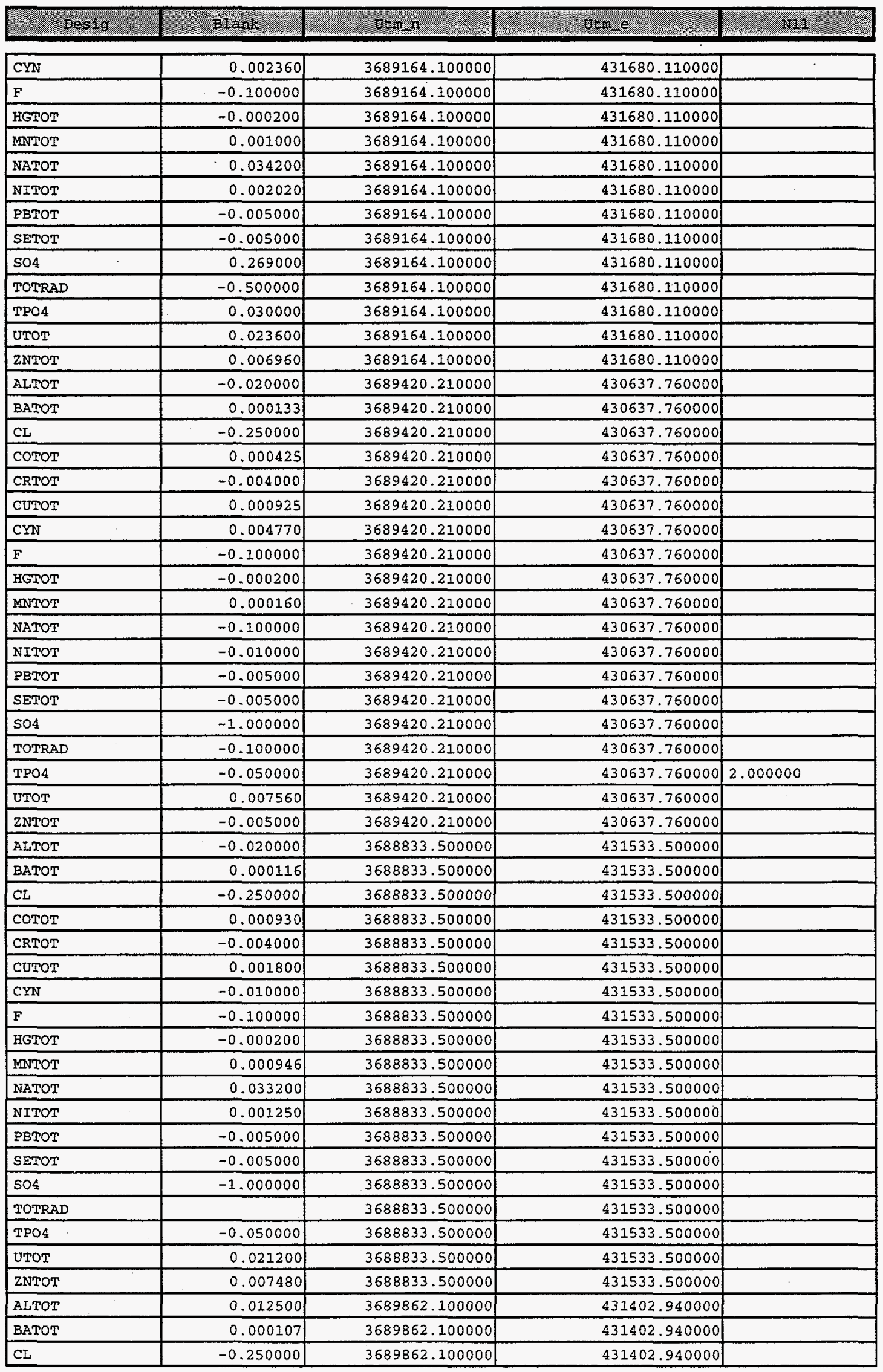




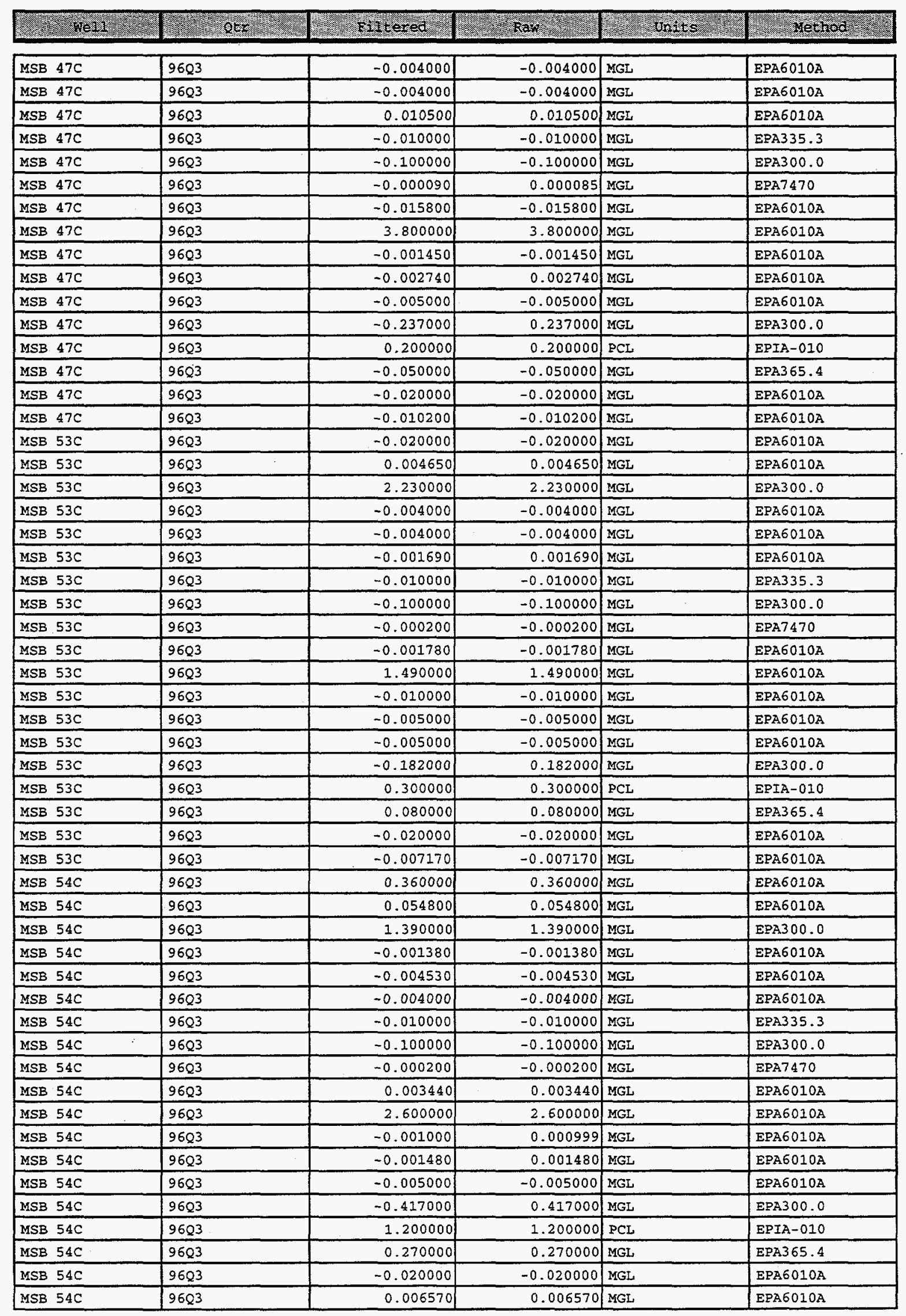


WSRC-TR-00347

November, 1997

Appendix II-160

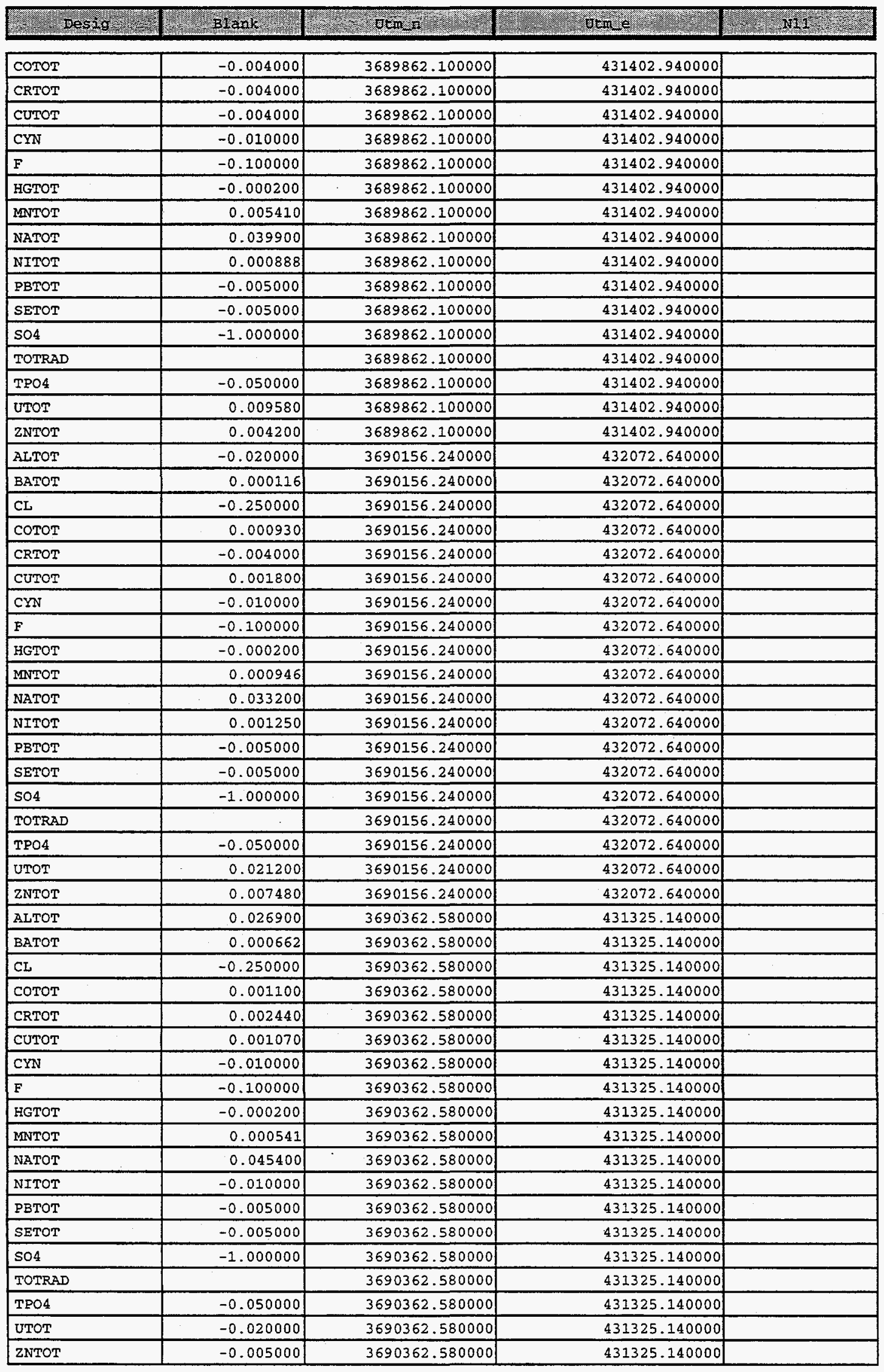


WSRC-TR-00347

November, 1997

Appendix II-161

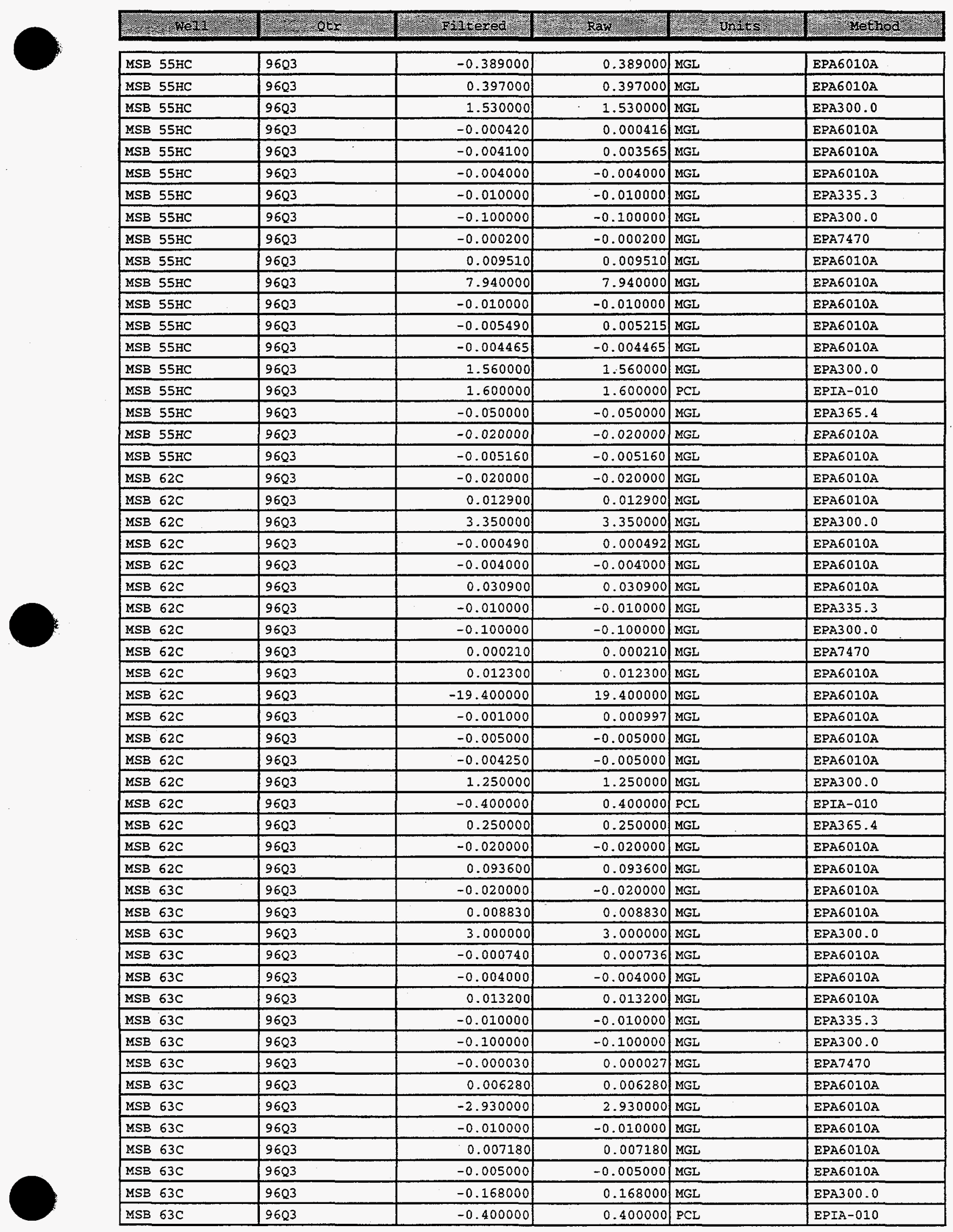


WSRC-TR-00347

November, 1997

Appendix II-162

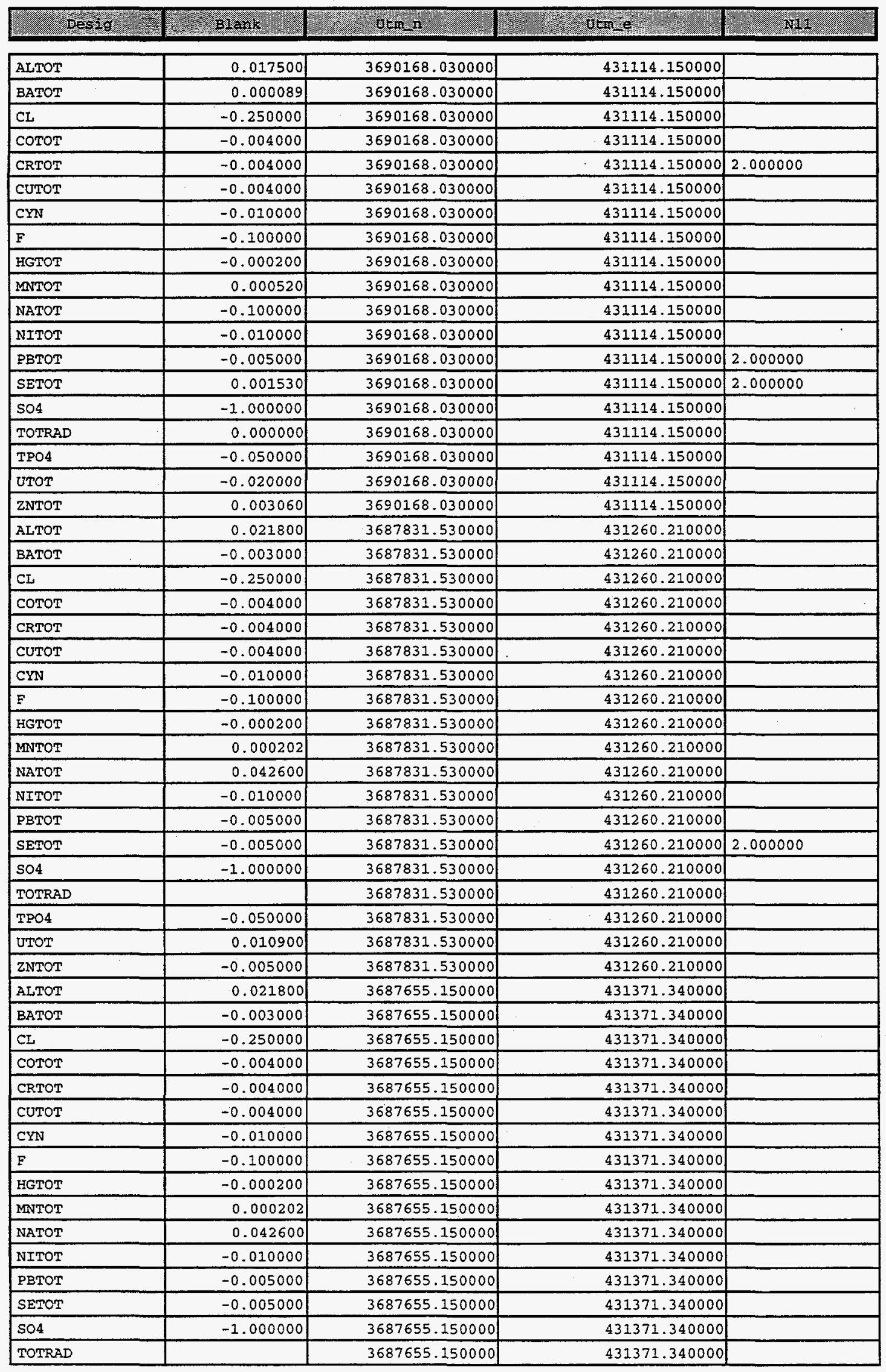




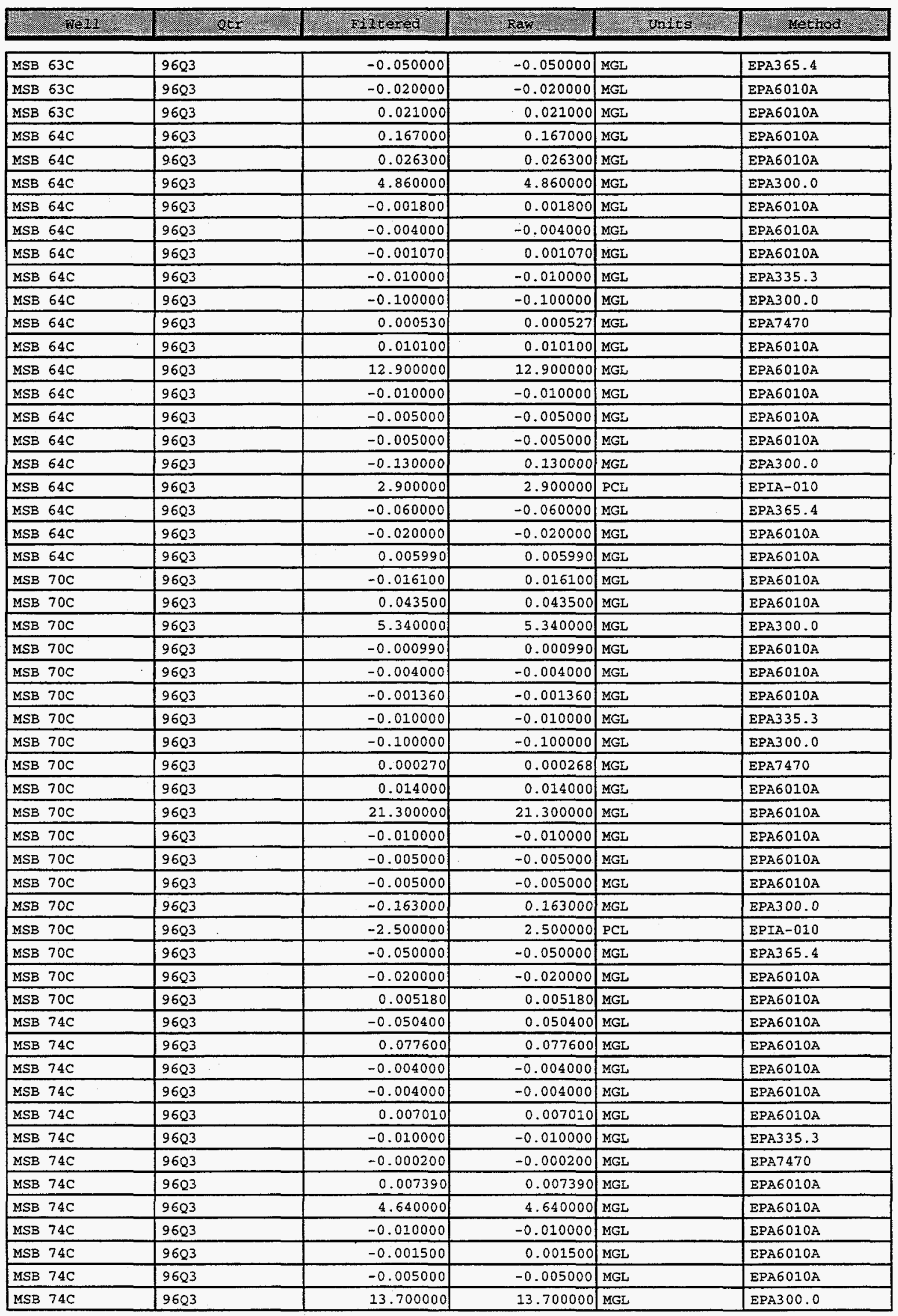




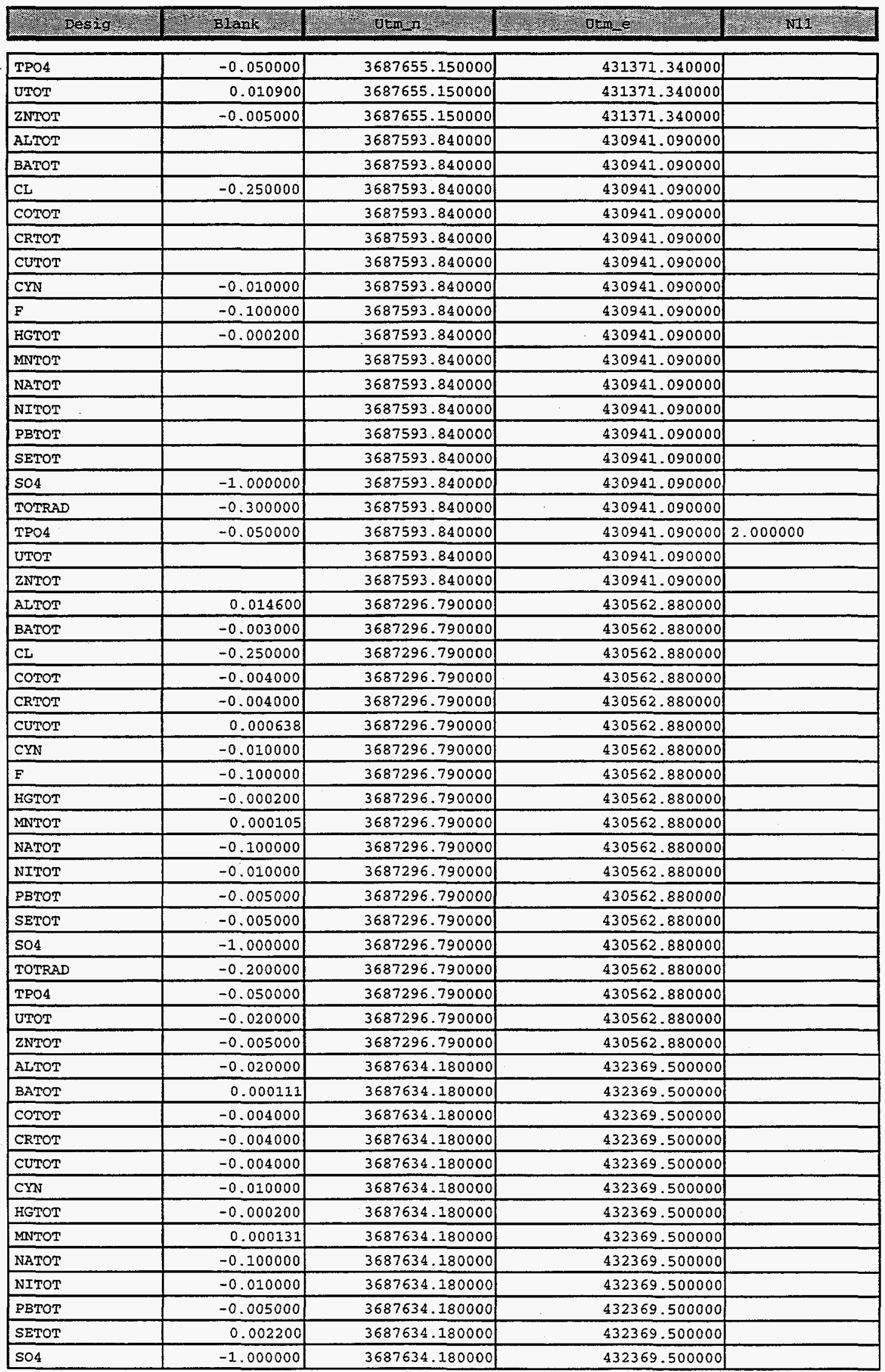




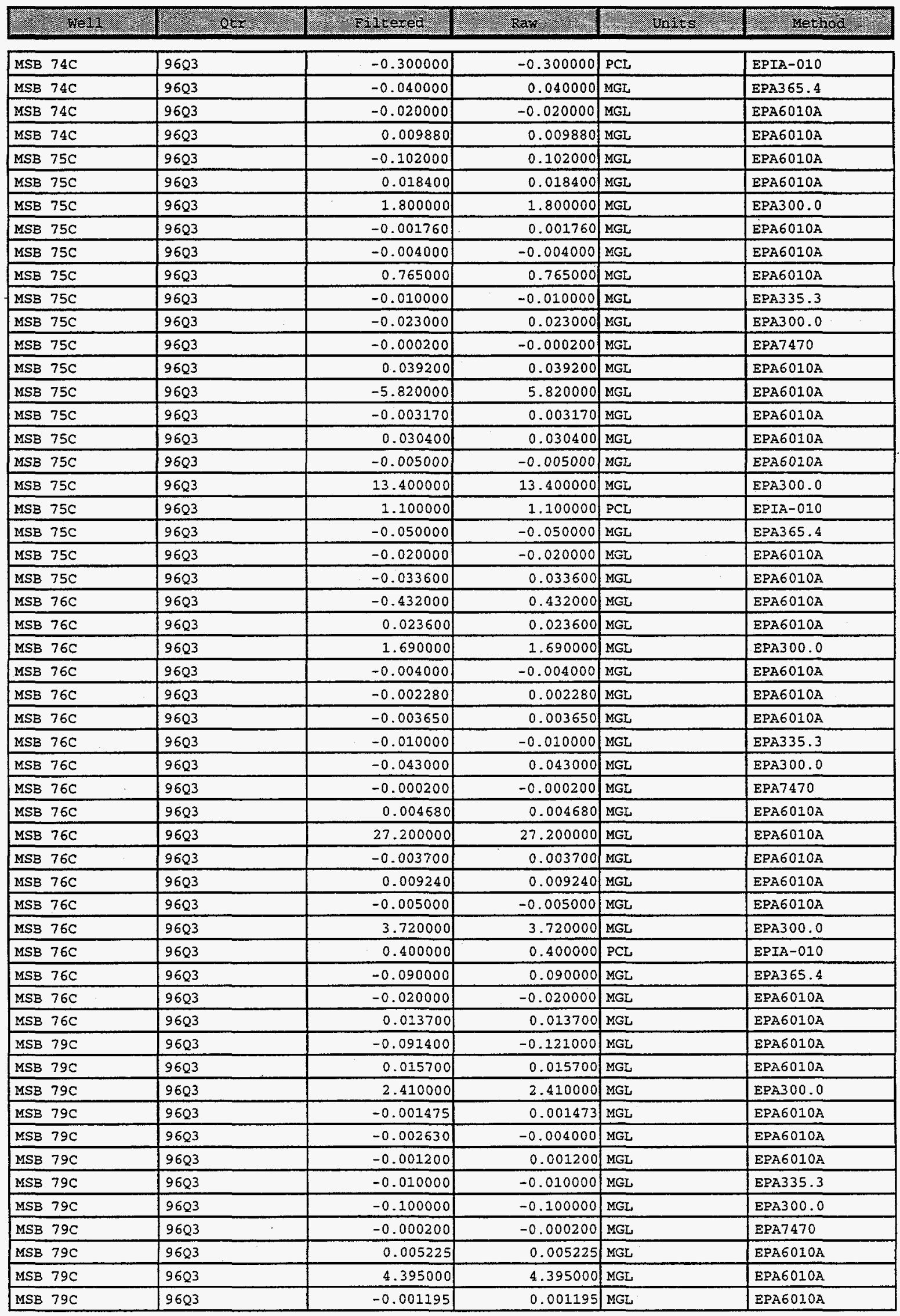




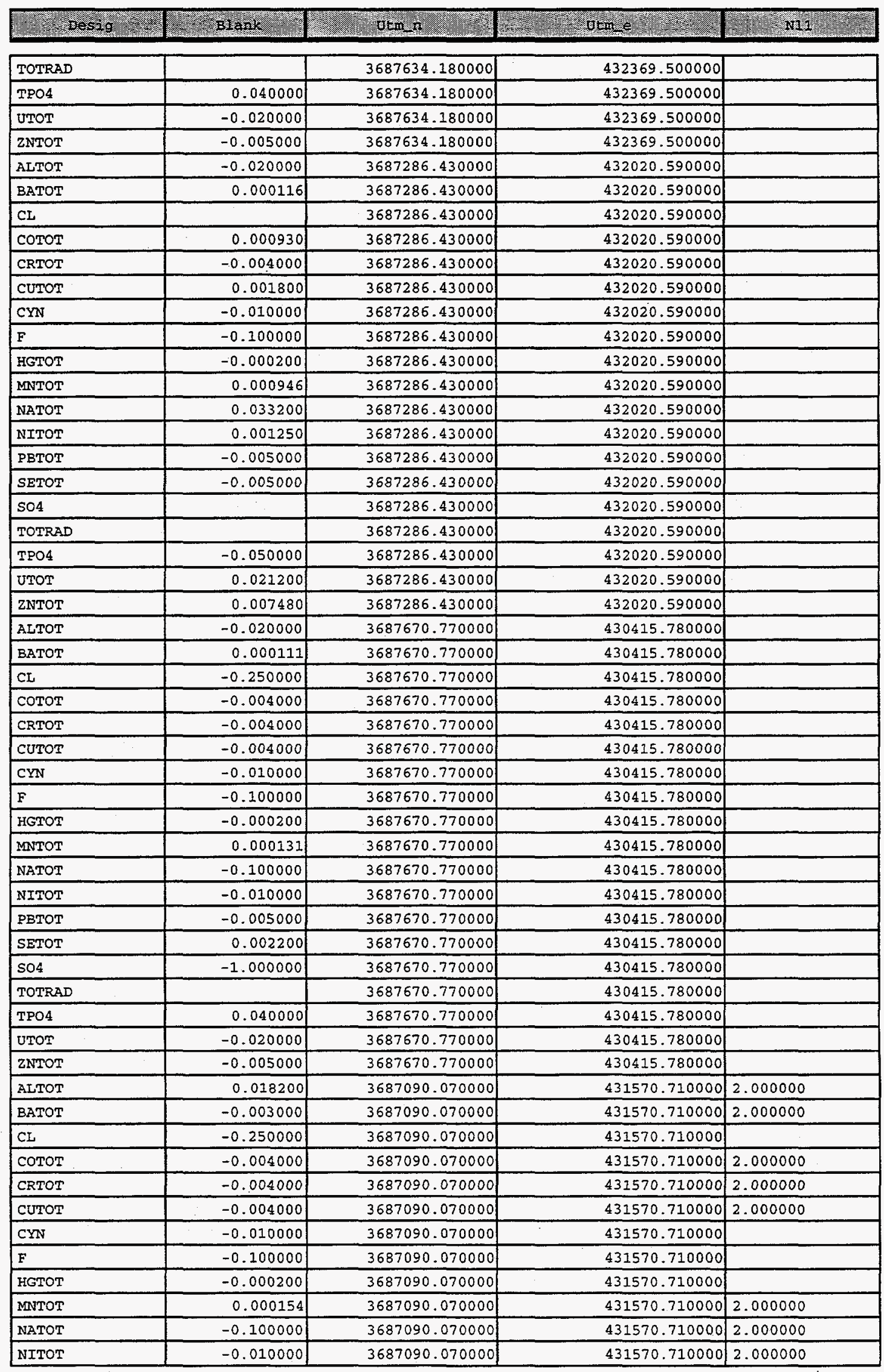


WSRC-TR-00347

November, 1997

Appendix II-167

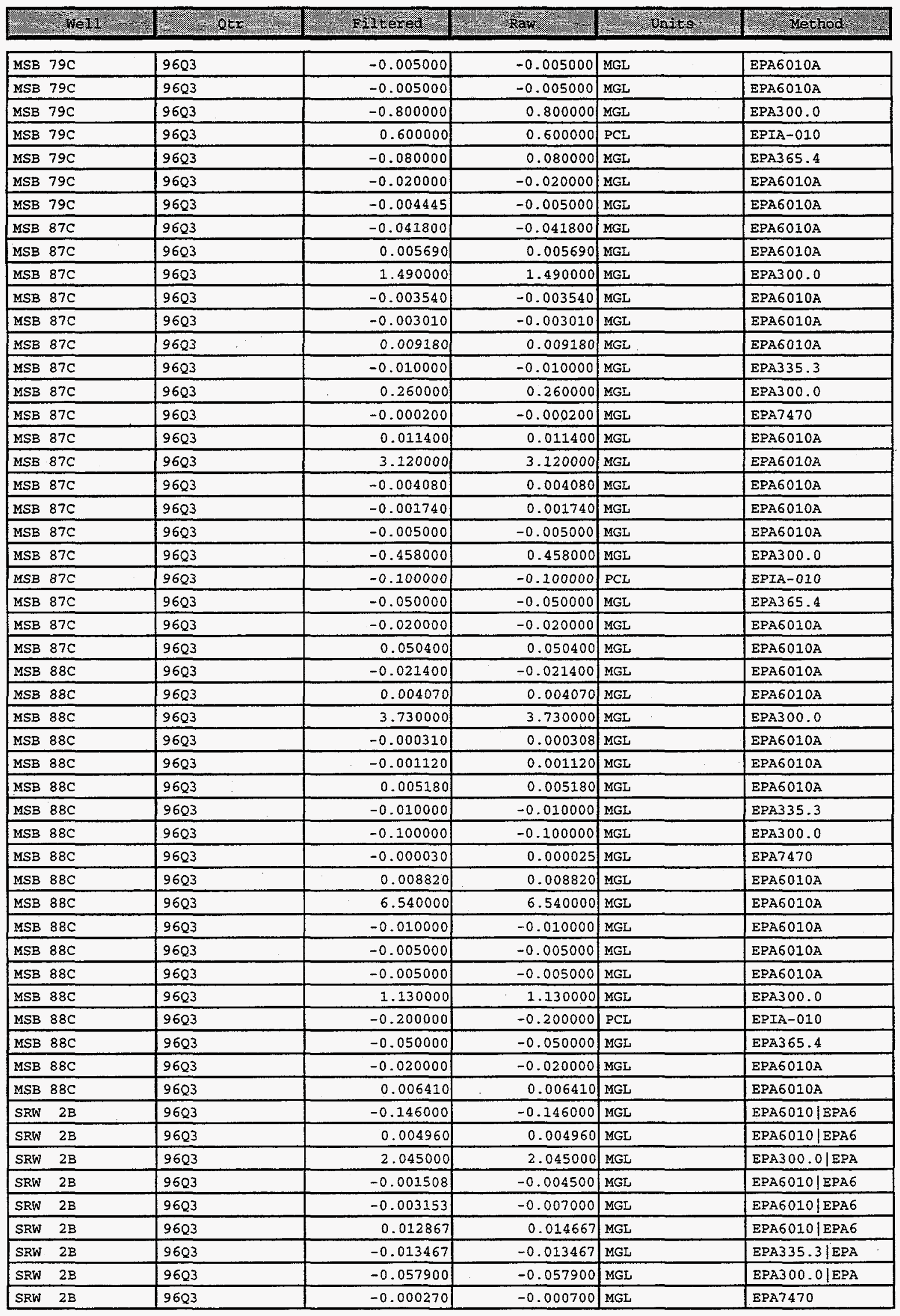


WSRC-TR-00347

November, 1997

Appendix II-168

\begin{tabular}{|c|c|c|c|c|}
\hline 2 & Blank & $\operatorname{con} 2$ & 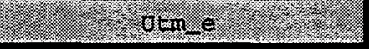 & Int \\
\hline PBTOT & -0.005000 & 3687090.070000 & 431570.710000 & 2.000000 \\
\hline SETOT & -0.005000 & 3687090.070000 & 431570.710000 & 2.000000 \\
\hline SO4 & -1.000000 & 3687090.070000 & 431570.710000 & \\
\hline TOTRAD & -0.100000 & 3687090.070000 & 431570.710000 & \\
\hline TPO4 & 0.030000 & 3687090.070000 & 431570.710000 & \\
\hline UTOT & -0.020000 & 3687090.070000 & 431570.710000 & 2.000000 \\
\hline ZNTOT & -0.005000 & 3687090.070000 & 431570.710000 & 2.000000 \\
\hline ALTOT & 0.026900 & 3688352.340000 & 432276.090000 & \\
\hline BATOT & 0.000662 & 3688352.340000 & 432276.090000 & \\
\hline $\mathrm{CL}$ & -0.250000 & 3688352.340000 & 432276.090000 & \\
\hline СОTOT & 0.001100 & 3688352.340000 & 432276.090000 & \\
\hline CRTOT & 0.002440 & $3688352: 340000$ & 432276.090000 & \\
\hline CUTOT & 0.001070 & 3688352.340000 & 432276.090000 & \\
\hline CYN & -0.010000 & 3688352.340000 & 432276.090000 & \\
\hline$F$ & -0.100000 & 3688352.340000 & 432276.090000 & \\
\hline HGTOT & -0.000200 & 3688352.340000 & 432276.090000 & \\
\hline MNTOT & 0.000541 & 3688352.340000 & 432276.090000 & \\
\hline NATOT & 0.045400 & 3688352.340000 & 432276.090000 & \\
\hline NITOT & -0.010000 & 3688352.340000 & 432276.090000 & \\
\hline PBTOT & -0.005000 & 3688352.340000 & 432276.090000 & \\
\hline SETOT & -0.005000 & 3688352.340000 & 432276.090000 & \\
\hline $\mathrm{SO4}$ & -1.000000 & 3688352.340000 & 432276.090000 & \\
\hline TOTRAD & & 3688352.340000 & 432276.090000 & \\
\hline TPO4 & -0.050000 & 3688352.340000 & 432276.090000 & \\
\hline UTOT & -0.020000 & 3688352.340000 & 432276.090000 & \\
\hline 2NTOT & -0.005000 & 3688352.340000 & 432276.090000 & \\
\hline ALTOT & 0.021800 & 3687156.140000 & 432840.690000 & \\
\hline BATOT & -0.003000 & 3687156.140000 & 432840.690000 & \\
\hline $\mathrm{CL}$ & -0.250000 & 3687156.140000 & 432840.690000 & \\
\hline COTOT & -0.004000 & 3687156.140000 & 432840.690000 & \\
\hline CRTOT & -0.004000 & 3687156.140000 & 432840.690000 & \\
\hline CUTOT & -0.004000 & 3687156.140000 & 432840.690000 & \\
\hline $\mathrm{CYN}$ & -0.010000 & 3687156.140000 & 432840.690000 & \\
\hline$F$ & -0.100000 & 3687156.140000 & 432840.690000 & \\
\hline HGTOT & -0.000200 & 3687156.140000 & 432840.690000 & \\
\hline MNTOT & 0.000202 & 3687156.140000 & 432840.690000 & \\
\hline NATOT & 0.042600 & 3687156.140000 & 432840.690000 & \\
\hline NITOT & -0.010000 & 3687156.140000 & 432840.690000 & \\
\hline PBTOT & -0.005000 & 3687156.140000 & 432840.690000 & \\
\hline SETOT & -0.005000 & 3687156.140000 & 432840.690000 & \\
\hline 504 & -1.000000 & 3687156.140000 & 432840.690000 & \\
\hline TOTRAD & & 3687156.140000 & 432840.690000 & \\
\hline TPO4 & 0.040000 & 3687156.140000 & 432840.690000 & \\
\hline UTOT & 0.010900 & 3687156.140000 & 432840.690000 & \\
\hline ZNTOT & -0.005000 & 3687156.140000 & 432840.690000 & \\
\hline ALTOT & -0.020000 & 3687169.650000 & 429381.420000 & 3.000000 \\
\hline BATOT & -0.003000 & 3687169.650000 & 429381.420000 & 3.000000 \\
\hline CL & -0.250000 & 3687169.650000 & 429381.420000 & 2.000000 \\
\hline СОTOT & -0.004000 & 3687169.650000 & 429381.420000 & 4.000000 \\
\hline CRTOT & -0.004000 & 3687169.650000 & 429381.420000 & 4.000000 \\
\hline CUTOT & -0.004000 & 3687169.650000 & 429381.420000 & 3.000000 \\
\hline CYN & -0.013467 & 3687169.650000 & 429381.420000 & 3.000000 \\
\hline$F$ & -0.100000 & 3687169.650000 & 429381.420000 & 2.000000 \\
\hline HGTOT & 0.003460 & 3687169.650000 & 429381.420000 & 4.000000 \\
\hline
\end{tabular}




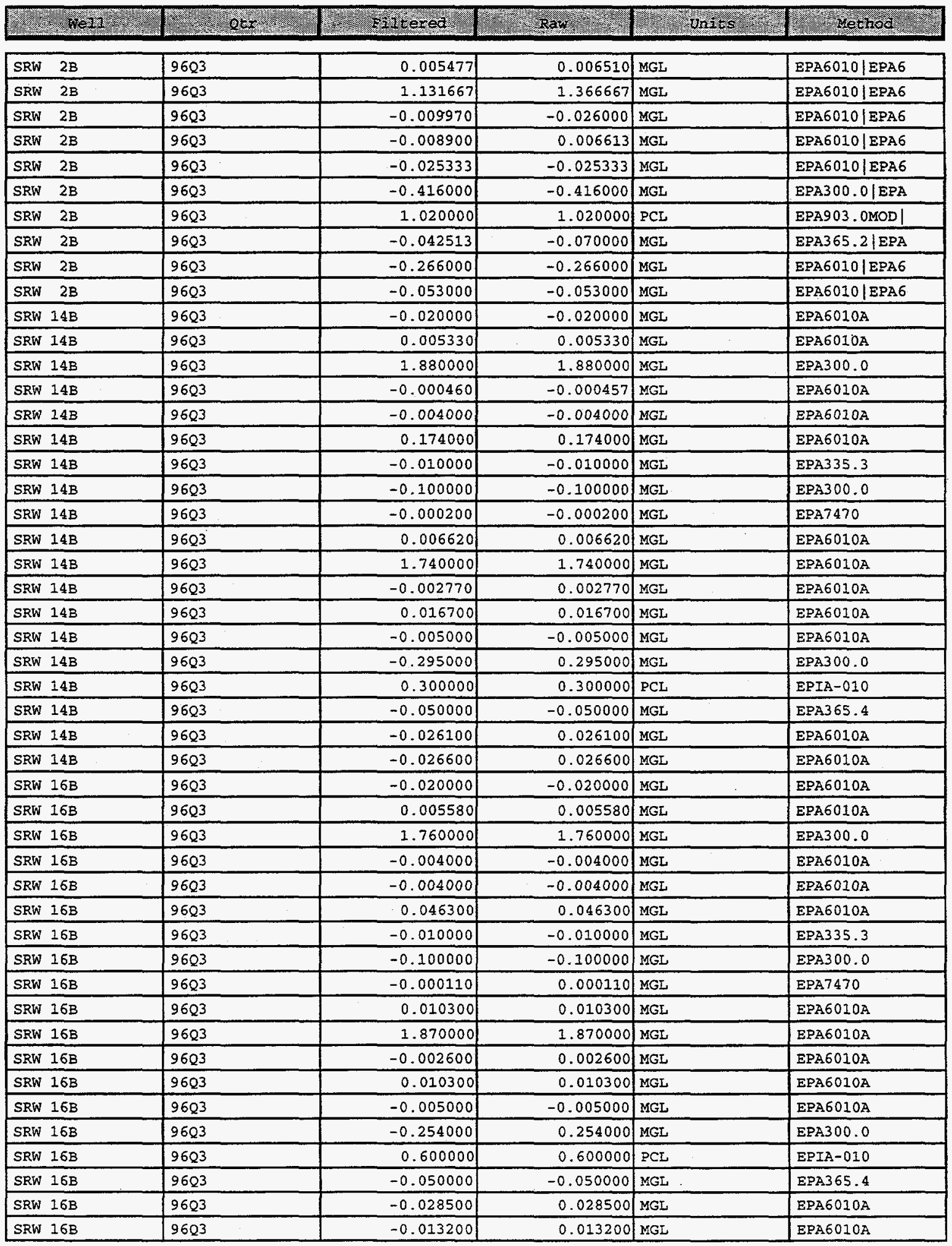




\begin{tabular}{|c|c|c|c|c|}
\hline MNTOT & 0.000657 & 3687169.650000 & 429381.420000 & 3.000000 \\
\hline NATOT & -0.100000 & 3687169.650000 & 429381.420000 & 3.000000 \\
\hline NITOT & -0.010000 & 3687169.650000 & 429381.420000 & 4.000000 \\
\hline PBTOT & -0.005000 & 3687169.650000 & 429381.420000 & 3.000000 \\
\hline SETOT & -0.005000 & 3687169.650000 & 429381.420000 & 3.000000 \\
\hline SO4 & -0.340000 & 3687169.650000 & 429381.420000 & 2.000000 \\
\hline TOTRAD & -0.020000 & 3687169.650000 & 429381.420000 & 2.000000 \\
\hline TPO4 & -0.070000 & 3687169.650000 & 429381.420000 & 3.000000 \\
\hline UTOT & -0.266000 & 3687169.650000 & 429381.420000 & 2.000000 \\
\hline ZNTOT & -0.006960 & 3687169.650000 & 429381.420000 & 4.000000 \\
\hline ALTOT & -0.020000 & 3686934.510000 & 429521.070000 & \\
\hline BATOT & -0.003000 & 3686934.510000 & 429521.070000 & \\
\hline $\mathrm{CL}$ & -0.250000 & 3686934.510000 & 429521.070000 & \\
\hline COTOT & -0.004000 & 3686934.510000 & 429521.070000 & \\
\hline CRTOT & -0.004000 & 3686934.510000 & 429521.070000 & \\
\hline CUTOT & -0.004000 & 3686934.510000 & 429521.070000 & \\
\hline $\mathrm{CYN}$ & 0.002360 & 3686934.510000 & .429521 .070000 & \\
\hline$F$ & -0.100000 & 3686934.510000 & 429521.070000 & \\
\hline HGTOT & -0.000200 & 3686934.510000 & 429521.070000 & \\
\hline MNTOT & 0.001000 & 3686934.510000 & 429521.070000 & \\
\hline NATOT & 0.034200 & 3686934.510000 & 429521.070000 & \\
\hline NITOT & 0.002020 & 3686934.510000 & 429521.070000 & \\
\hline PBTOT & -0.005000 & 3686934.510000 & 429521.070000 & \\
\hline SETOT & -0.005000 & 3686934.510000 & 429521.070000 & \\
\hline SO4 & -1.000000 & 3686934.510000 & 429521.070000 & \\
\hline TOTRAD & & 3686934.510000 & 429521.070000 & \\
\hline TPO4 & 0.030000 & 3686934.510000 & 429521.070000 & \\
\hline UTOT & 0.023600 & 3686934.510000 & 429521.070000 & \\
\hline ZNTOT & 0.006960 & 3686934.510000 & 429521.070000 & \\
\hline ALTOT & -0.020000 & 3687394.200000 & 429668.010000 & \\
\hline ВATOT & -0.003000 & 3687394.200000 & 429668.010000 & \\
\hline $\mathrm{CL}$ & -0.250000 & 3687394.200000 & 429668.010000 & \\
\hline СоTот & -0.004000 & 3687394.200000 & 429668.010000 & \\
\hline CRTOT & -0.004000 & 3687394.200000 & 429668.010000 & \\
\hline CUTOT & -0.004000 & 3687394.200000 & 429668.010000 & \\
\hline $\mathrm{CYN}$ & 0.002350 & 3687394.200000 & 429668.010000 & \\
\hline$E$ & -0.100000 & 3687394.200000 & 429668.010000 & \\
\hline HGTOT & -0.000200 & 3687394.200000 & 429668.010000 & \\
\hline MNTOT & 0.001000 & 3687394.200000 & 429668.010000 & \\
\hline NATOT & 0.034200 & 3687394.200000 & 429668.010000 & \\
\hline NITOT & 0.002020 & 3687394.200000 & 429668.010000 & \\
\hline PBTOT & -0.005000 & 3687394.200000 & 429668.010000 & \\
\hline SETOT & -0.005000 & 3687394.200000 & 429668.010000 & \\
\hline 504 & 0.269000 & 3687394.200000 & 429668.010000 & \\
\hline TOTRAD & -0.500000 & 3687394.200000 & 429668.010000 & \\
\hline TP04 & 0.030000 & 3687394.200000 & 429668.010000 & \\
\hline UTOT & 0.023600 & 3687394.200000 & 429668.010000 & \\
\hline 2NTOT & 0.006960 & 3687394.200000 & 429668.010000 & \\
\hline
\end{tabular}

